\title{
WestVirginiaUniversity
}

THE RESEARCH REPOSITORY @ WVU

Graduate Theses, Dissertations, and Problem Reports

2014

\section{Assisted history matching using pattern recognition technology}

Alireza Shahkarami

West Virginia University

Follow this and additional works at: https://researchrepository.wvu.edu/etd

\section{Recommended Citation}

Shahkarami, Alireza, "Assisted history matching using pattern recognition technology" (2014). Graduate Theses, Dissertations, and Problem Reports. 448.

https://researchrepository.wvu.edu/etd/448

This Dissertation is protected by copyright and/or related rights. It has been brought to you by the The Research Repository @ WVU with permission from the rights-holder(s). You are free to use this Dissertation in any way that is permitted by the copyright and related rights legislation that applies to your use. For other uses you must obtain permission from the rights-holder(s) directly, unless additional rights are indicated by a Creative Commons license in the record and/ or on the work itself. This Dissertation has been accepted for inclusion in WVU Graduate Theses, Dissertations, and Problem Reports collection by an authorized administrator of The Research Repository @ WVU.

For more information, please contact researchrepository@mail.wvu.edu. 


\title{
Assisted History Matching Using Pattern RECOGNITION TECHNOLOGY
}

\author{
Alireza Shahkarami \\ Dissertation submitted \\ to the Benjamin M. Statler College of Engineering and Mineral Resources \\ at West Virginia University \\ in partial fulfillment of the requirements for the degree of \\ Doctor of Philosophy in \\ Petroleum and Natural Gas Engineering \\ Shahab D. Mohaghegh, Ph.D., Chair \\ Samuel Ameri, M.S. \\ Khashayar Aminian, Ph.D. \\ Peter Dzurman, M.S. \\ Razi Gaskari, Ph.D. \\ Thomas H. Wilson, Ph.D. \\ Department of Petroleum and Natural Gas Engineering \\ Morgantown, West Virginia \\ 2014
}

Keywords: Assisted History Matching, Pattern Recognition Technology, Surrogate Reservoir Models, Artificial Intelligence and Data Mining

Copyright 2014 Alireza Shahkarami 


\title{
ABSTRACT \\ Assisted History Matching Using Pattern Recognition Technology
}

\begin{abstract}
Alireza Shahkarami
Reservoir simulation and modeling is utilized throughout field development in different capacities. Sensitivity analysis, history matching, operations optimization and uncertainty assessment are the conventional analyses in full field model studies. Realistic modeling of the complexities of a reservoir requires a large number of grid blocks. As the complexity of a reservoir increases and consequently the number of grid blocks, so does the time required to accomplish the abovementioned tasks.

This study aims to examine the application of pattern recognition technologies to improve the time and efforts required for completing successful history matching projects. The pattern recognition capabilities of Artificial Intelligence and Data Mining (AI\&DM) techniques are used to develop a Surrogate Reservoir Model (SRM) and use it as the engine to drive the history matching process. SRM is a prototype of the full field reservoir simulation model that runs in fractions of a second. SRM is built using a small number of geological realizations.
\end{abstract}

To accomplish the objectives of this work, a three step process was envisioned:

- Part one, a proof of concept study: The goal of first step was to prove that SRM is able to substitute the reservoir simulation model in a history matching project. In this part, the history match was accomplished by tuning only one property (permeability) throughout the reservoir.

- Part two, a feasibility study: This step aimed to study the feasibility of SRM as an effective tool to solve a more complicated history matching problem, particularly when the degrees of uncertainty in the reservoir increase. Therefore, the number of uncertain reservoir properties increased to three properties (permeability, porosity, and thickness). The SRM was trained, calibrated, and validated using a few geological realizations of the base reservoir model. In order to complete an automated history matching workflow, the SRM was coupled with a global optimization algorithm called Differential Evolution (DE). DE optimization method is considered as a novel and robust optimization algorithm from the class of evolutionary algorithm methods.

- Part three, a real-life challenge: The final step was to apply the lessons learned in order to achieve the history match of a real-life problem. The goal of this part was to challenge the strength of SRM in a more complicated case study. Thus, a standard test reservoir model, known as PUNQ-S3 reservoir model in the petroleum engineering literature, was selected. The PUNQ-S3 reservoir model represents a small size industrial reservoir engineering model. This model has been formulated to test the ability of various methods in the history matching and uncertainty quantification. The surrogate reservoir model was developed using ten geological realizations of the model. The uncertain properties in this model are distributions of porosity, horizontal, and vertical permeability. Similar to the second part of this study, the DE optimization method was connected to the SRM to form an automated workflow in order to perform the history matching. This automated workflow is able to produce multiple realizations of the reservoir which match the past performance. The successful matches were utilized to quantify the uncertainty in the prediction of cumulative oil production. 
The results of this study prove the ability of the surrogate reservoir models, as a fast and accurate tool, to address the practical issues of reservoir simulation models in the history matching workflow. Nevertheless, the achievements of this dissertation are not only aimed at the history matching procedure, but also benefit the other time-consuming operations in the reservoir management workflow (such as sensitivity analysis, production optimization, and uncertainty assessment). 
"Raise your words, not voice. It is rain that grows flowers, not thunder."

\section{Rumi}

I dedicate this dissertation to my parents and the memory of many in my beloved motherland. 


\section{Acknowledgment}

The final and probably the hardest part that I have to write for my dissertation, is this part. I am not sure if I can thoroughly fulfill it, but let's do it.

None of these things were existed and I might have been a different person than what I am now, if my advisor, Dr. Shahab D. Mohaghegh, were not giving me the learning opportunity at his research group. I would like to thank Shahab for believing in me. To me he is a dear friend at first, then an excellent example of mentor, researcher, instructor, and advisor. My endless gratitude goes to him.

I would like to thank my dissertation committee members for all their guidance during this process. Many thanks go to Dr. Razi Gaskari for being a good friend and providing brilliant feedback. My gratitude goes to Mr. Pete Dzurman for the great technical discussions and positive comments I have received. It was a great honor to have Dr. Aminian, Prof. Ameri, and Dr. Wilson in my dissertation committee and I would like to thank them for their significant contributions to this work.

I wish to thank my amazing friends and colleagues at PEARL research group for providing a friendly environment and helpful discussions about different issues during the past four years. Special thanks go to Vida Gholami, Mohammad Maysami, and Alireza Haghighat at first for being awesome friends, then having great discussions on this work. I also wish to thank all my friends; ones who have been beside me and ones who are distant, without their support studying all long days and nights far away from home would not be ever possible. I would like to state my special thanks to Aracely Rivero for making my life happier during my study in Morgantown.

In addition, I would like to thank Computer Modeling Group and Intelligent Solution Inc., for making the $\mathrm{CMG}$ reservoir simulator and IDEA software available to me to perform the reservoir modeling in this work.

Last but not least, my profound gratitude is expended to my dear parents and siblings. Although they have been miles away from me, I have always been supported by their understanding, trust and wholehearted help they have been giving to me. 


\section{Table of Contents}

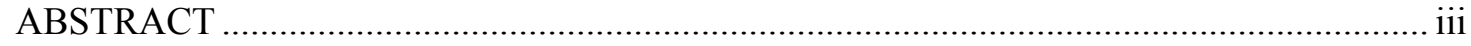

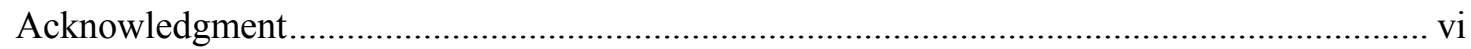

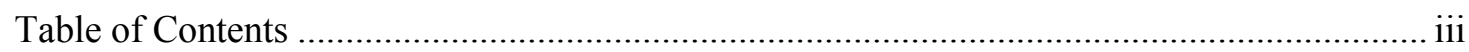

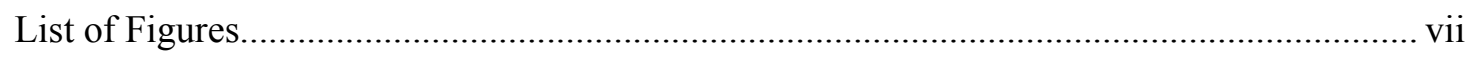

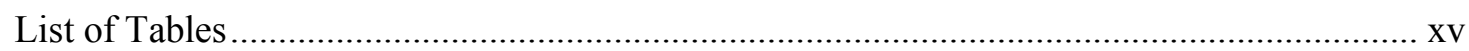

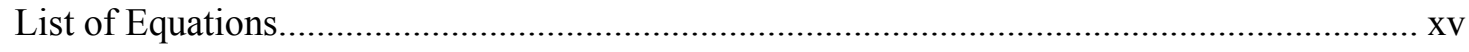

Chapter 1: Problem Statement .......................................................................................... 1

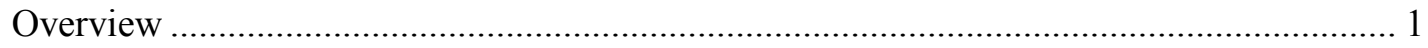

$1.1 \quad$ Problem statement ………………………………………………………. 1

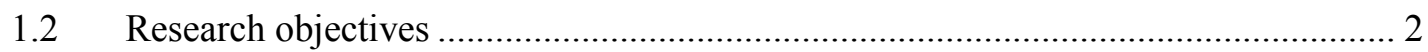

$1.3 \quad$ Audience of the study ................................................................................ 3

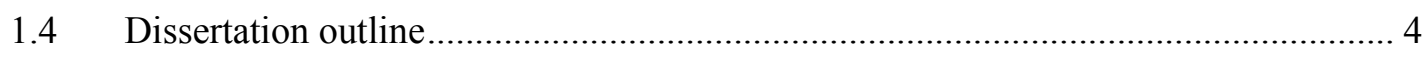

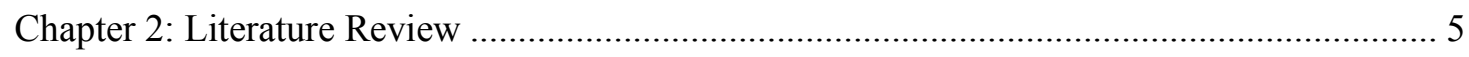

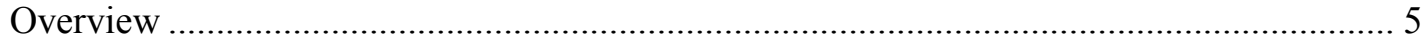

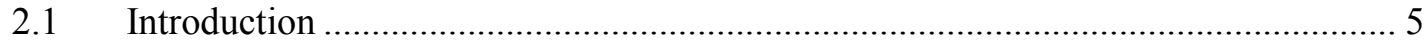

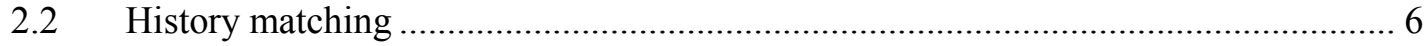

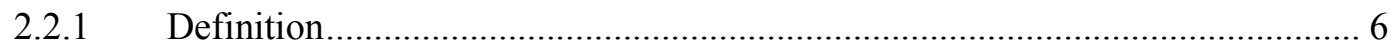

2.2.2 Objectives of history matching .............................................................. 8

2.2.3 Practical approaches ……………………….................................. 9

2.2.4 A literature review on history matching: ……………................................ 13

2.3 Alternative approaches to simulation models ........................................................ 19

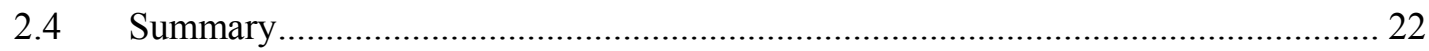

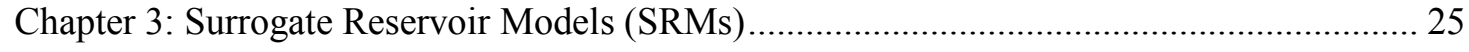

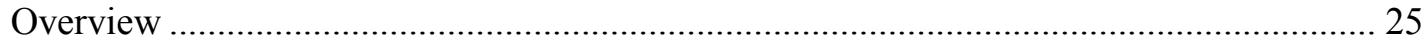

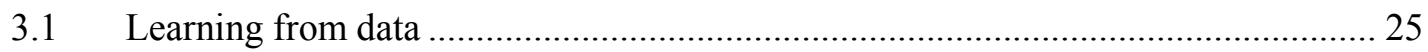

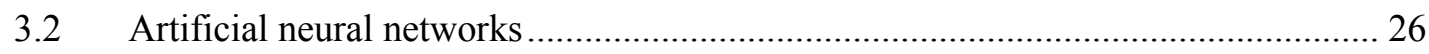

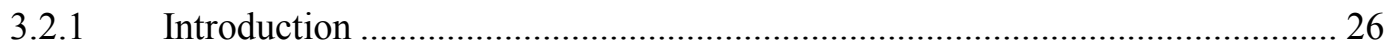

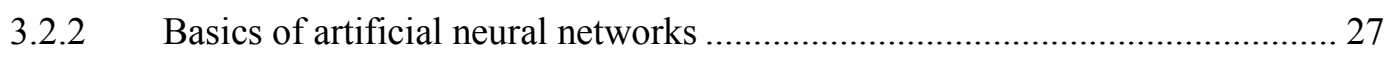

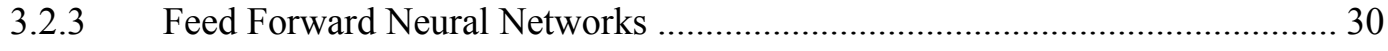

3.2.4 Applications of artificial neural networks …………………………………... 31

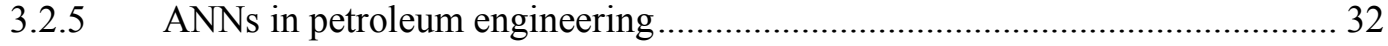




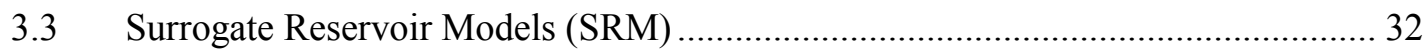

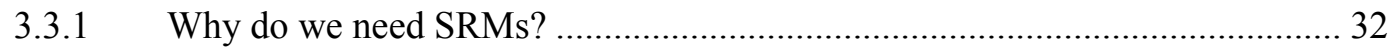

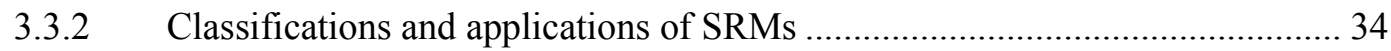

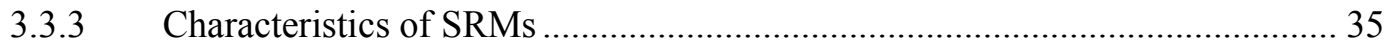

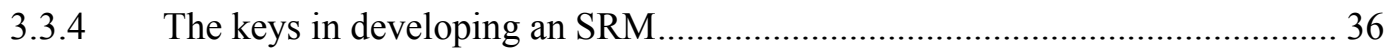

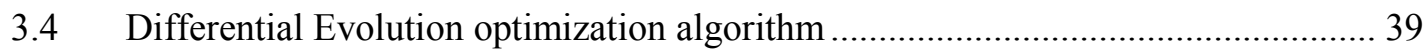

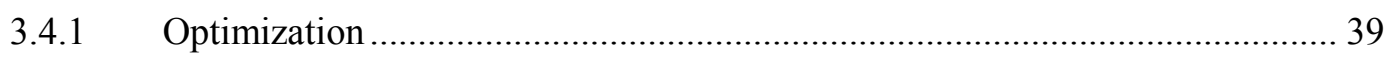

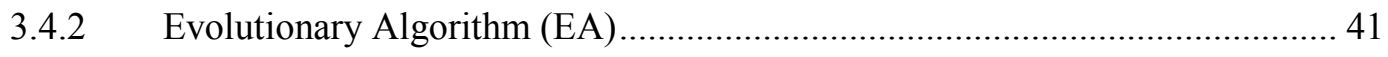

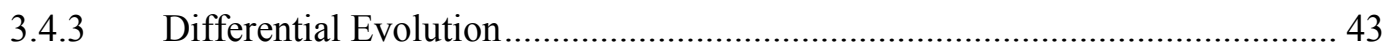

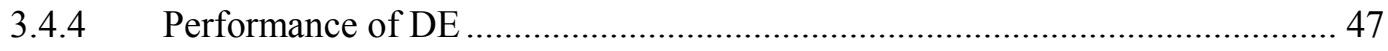

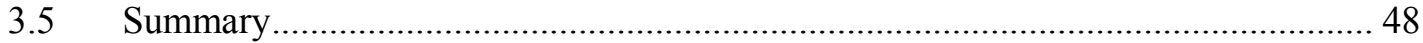

Chapter 4: Surrogate Reservoir Model based history matching: A proof of concept

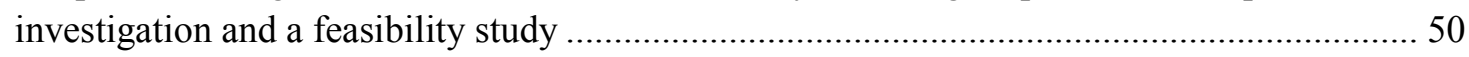

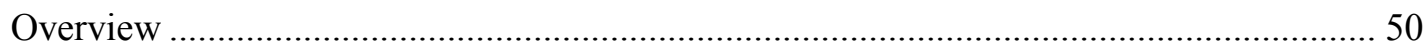

4.1 General steps in development and application of SRM for history matching .......... 51

4.2 Model generation and spatio-temporal database preparation .................................. 52

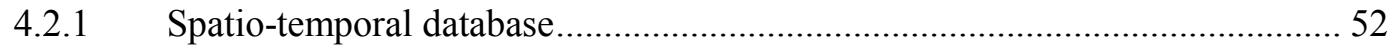

4.2.2 Informative simulation runs representation of reservoir uncertainties ............. 54

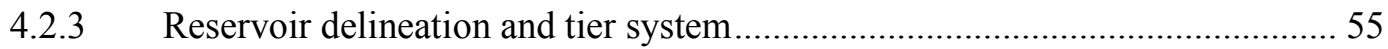

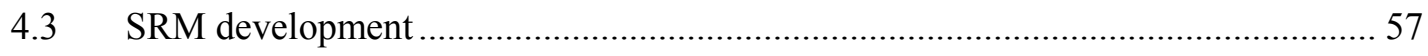

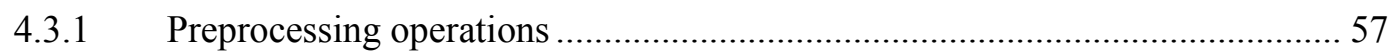

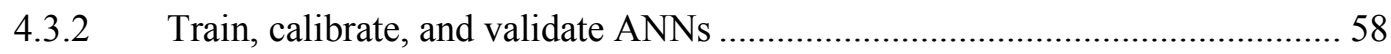

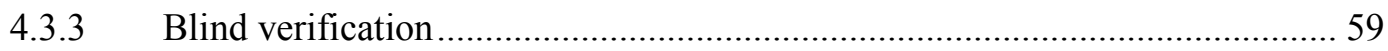

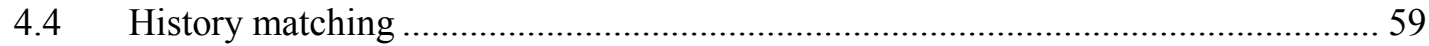

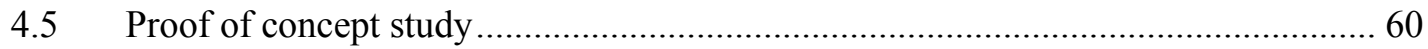

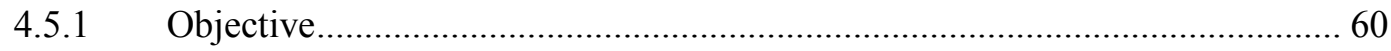

4.5.2 Model generation and spatio-temporal database preparation ........................... 61

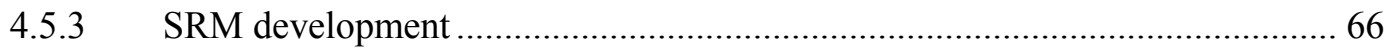

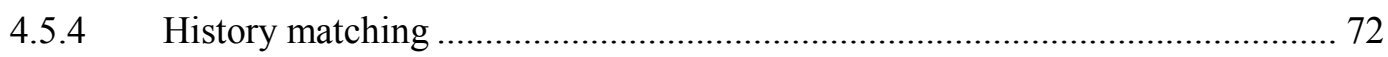

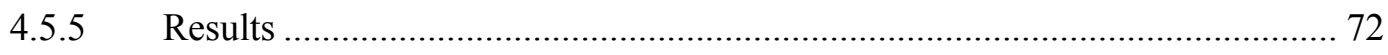

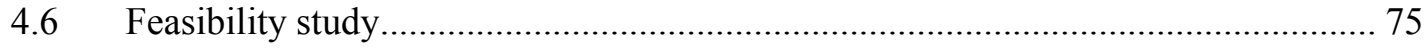

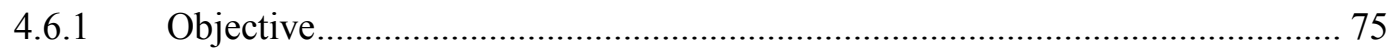

4.6.2 Model generation and spatio-temporal database preparation ........................... 76

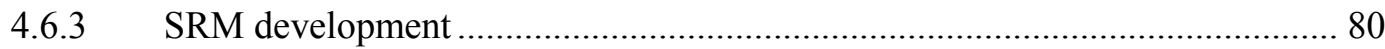




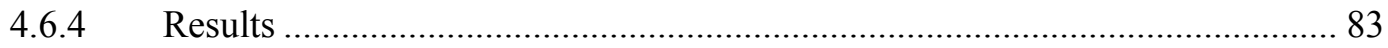

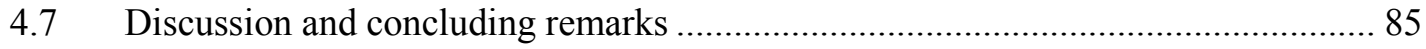

Chapter 5: A real-life case study, implementation of SRM on PUNQ-S3 problem................. 87

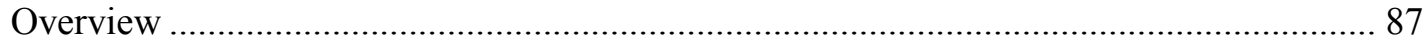

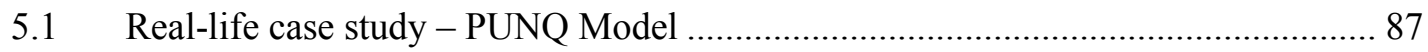

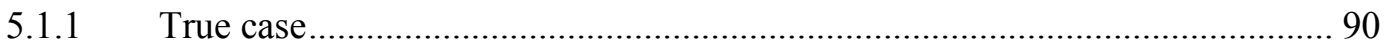

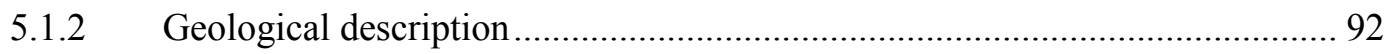

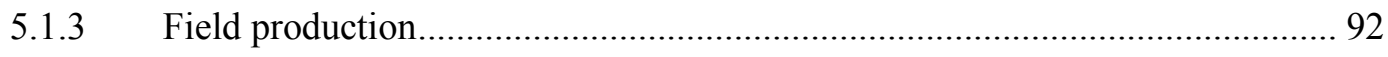

5.1.4 Literature review on PUNQ-S3 reservoir model ............................................ 94

5.2 Model generation and spatio-temporal database preparation .................................. 96

5.2.1 Informative simulation runs representation of reservoir uncertainties ............. 96

5.2.2 Reservoir delineation and tier system........................................................ 100

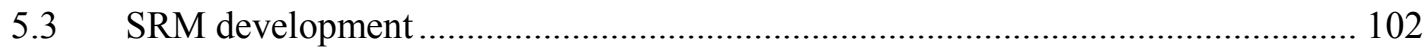

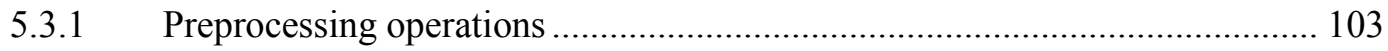

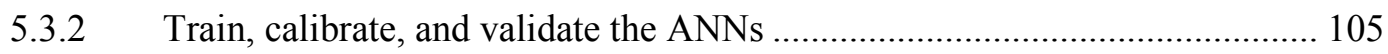

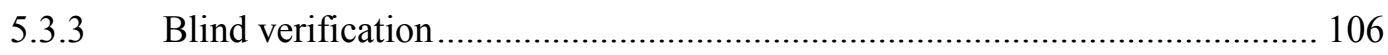

5.4 Automated SRM-based history matching............................................................. 106

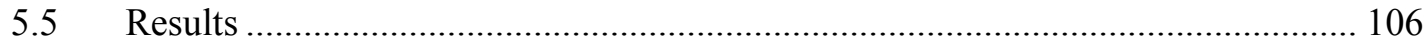

5.5.1 Importing the matched characteristics into the simulator ............................... 114

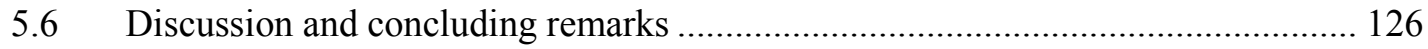

Chapter 6: Summary, conclusions and recommendations ..................................................... 129

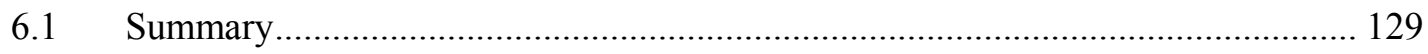

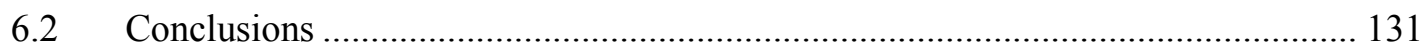

6.3 Recommendations for future studies …........................................................... 133

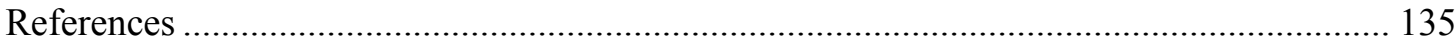

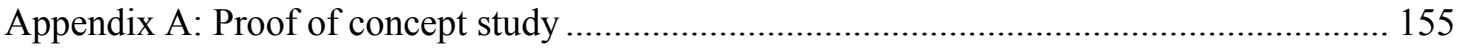

a. Production history performance for the reservoir model in proof of concept and

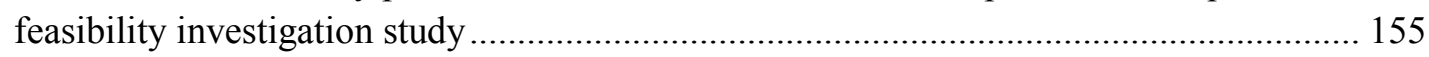

b. Ten created permeability distributions for training the SRM ................................. 158

c. The results of SRM for a training realizations- Run \# 1 ............................................. 159

d. The results of SRM - blind verification realization ................................................. 162

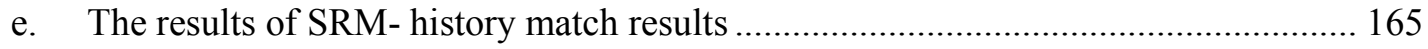

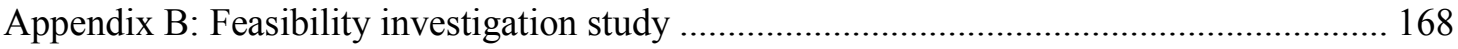

a. Variable reservoir characteristics for training realizations ........................................ 168 


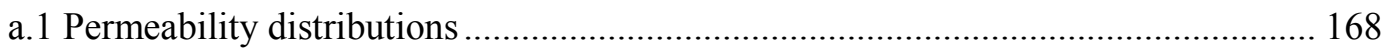

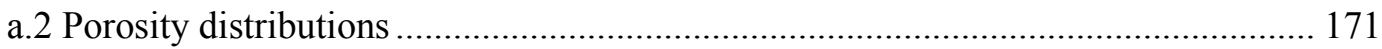

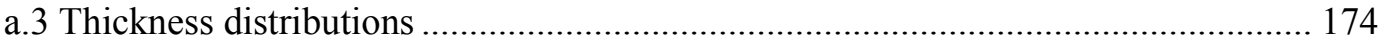

b. The results of SRM for a training realization- Run \#1 …....................................... 178

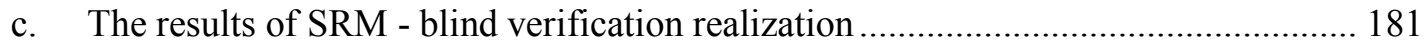

d. The results of SRM - automated history matching results....................................... 187

Appendix C: A real-life case study, implementation of SRM on PUNQ-S3 problem ............ 190

a. Variable reservoir characteristics for training realizations ........................................ 190

a.1 Horizontal permeability distributions .................................................................... 190

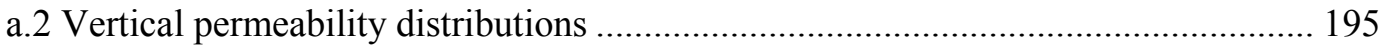

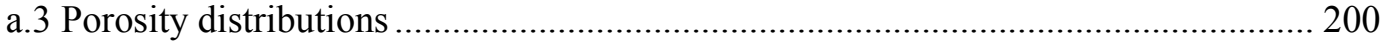

b. Variable reservoir characteristics for the blind realization ......................................... 205

c. The results of training and blind realizations compared with the observed data........ 207

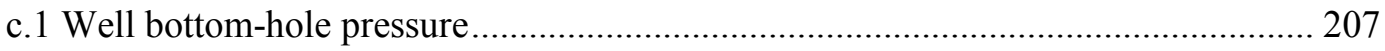

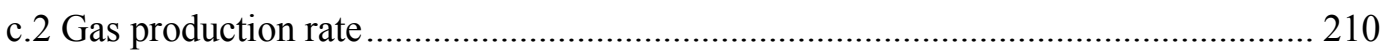

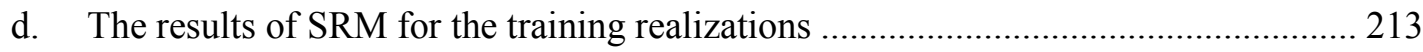

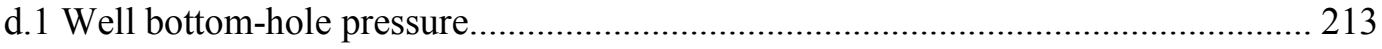

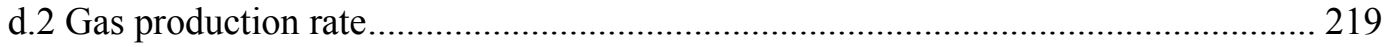

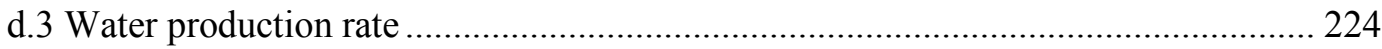




\section{List of Figures}

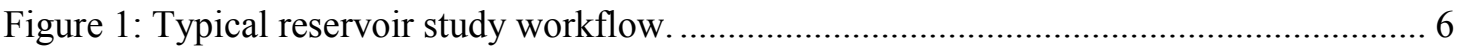

Figure 2: Forward problem verses inverse type of problem.

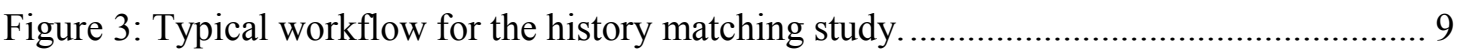

Figure 4: Matching pressure behavior (Toronyi and Saleri 1988, Cosentino 2001)............... 11

Figure 5: Matching saturation performance of reservoir (Toronyi and Saleri 1988, Cosentino

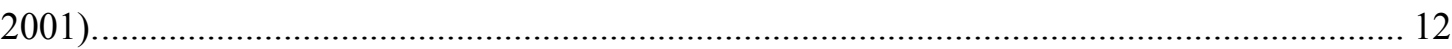

Figure 6: Number of papers on the history matching topic between 1990 to 2010

(Rwechungura, Dadashpour and Kleppe 2011).

Figure 7: The scheme of neuron (cell) and its components (AGH University of Science and

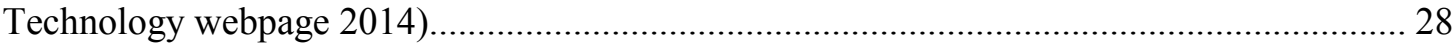

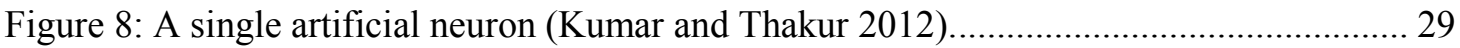

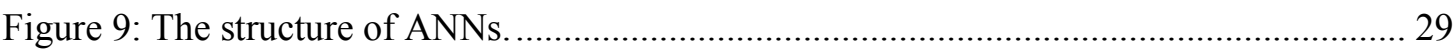

Figure 10: Feed forward neural network architecture with three hidden layers (Roberts 2000).

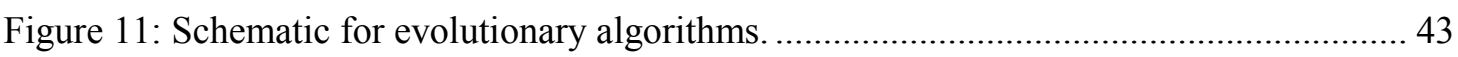

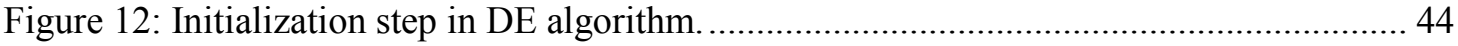

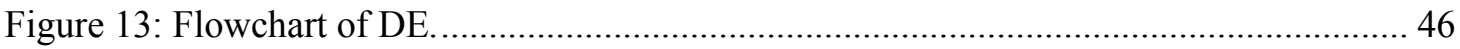

Figure 14: The steps of DE (Hajizadeh, Christie and Demyanov 2009) ............................... 46

Figure 15: Three steps were defined to prove the concept of SRM, show the feasibility of the

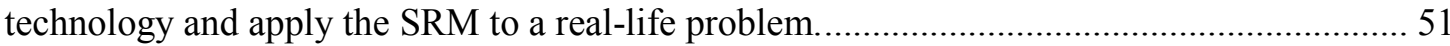

Figure 16: The steps in developing an SRM for history matching purpose. ............................ 52

Figure 17: Different types of data in the spatio-temporal database......................................... 53

Figure 18: Reservoir delineation based on Voronoi graph theory.......................................... 55

Figure 19: Dividing each Voronoi polygon (drainage area) to tiers. This tier system includes

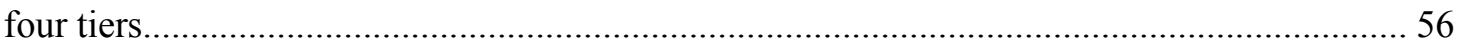

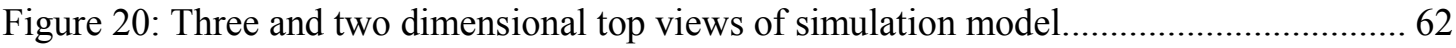

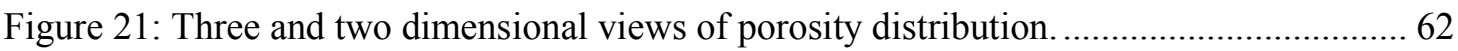

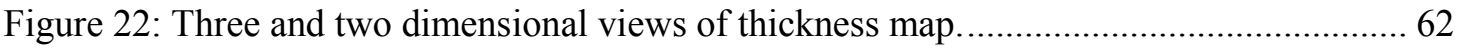

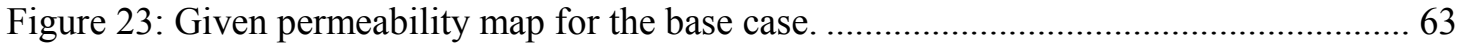

Figure 24: Bottom-hole pressure as constraints for the production wells. ............................ 63

Figure 25: Production history data for some wells, the rest of wells are available in Appendix

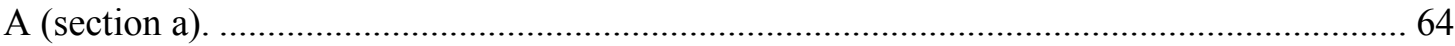

Figure 26: Different permeabilty distributions created to train the SRM (th rest of maps are

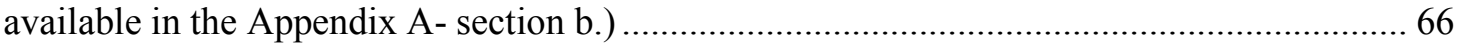

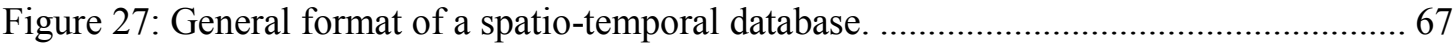

Figure 28: ANN structure for the Initialization (left) and Main (right) networks. .................. 70

Figure 29: A snapshot of the training procedure............................................................... 71

Figure 30: Scatter plots for the training, calibration and verification sets during the training

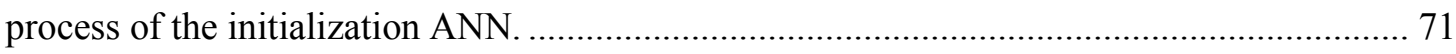

Figure 31: Scatter plots for the training, calibration, and verification sets during the training

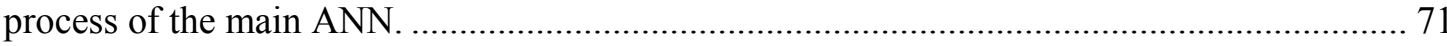

Figure 32: Three (left) and two (right) dimensional views of permeability distribution for the

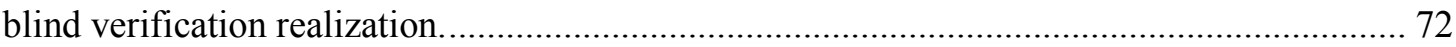


Figure 33: Comparison between the results of SRM and simulator for well \#20 in a training realization

Figure 34: Comparison between the results of SRM and simulator for well \#20 in a blind

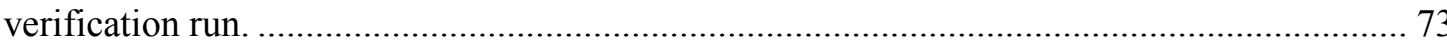

Figure 35: The outcome of history matching for well \#20 comparing the best match (SRM

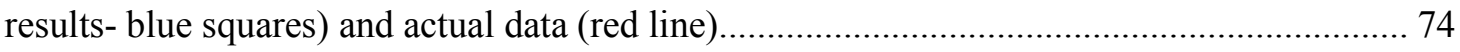

Figure 36: Comparison of matched and actual Permeability distributions.............................. 74

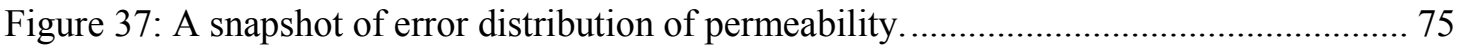

Figure 38: Flow chart to generate different realizations by altering property values at well

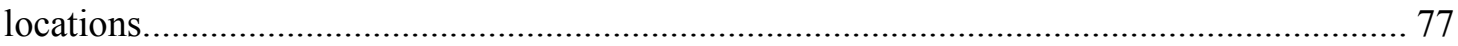

Figure 39: Comparison of permeability distributions between realizations \#1 and \#2. The property distributions for the rest of realizations are provided in Appendix B (section a). ..... 77

Figure 40: Comparison of porosity distributions between realizations \#1 and \#2. ................. 78

Figure 41: Comparison of thickness distributions between realizations \#1 and \#2................. 78

Figure 42: Permeability, porosity, and thickness distributions for two blind verification

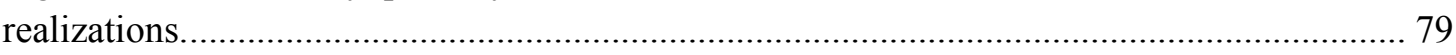

Figure 43: Permeability, porosity, and thickness distribution for actual simulation model, $23^{\text {rd }}$

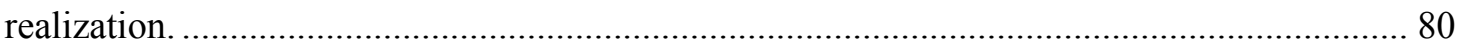

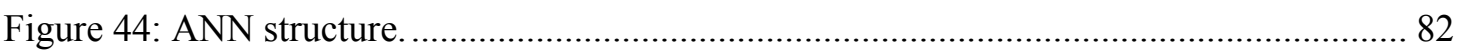

Figure 45: Scatter plots for the training, calibration and verification sets during the training

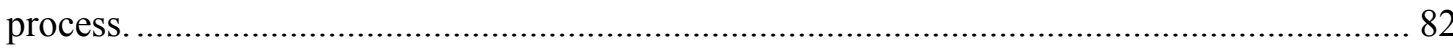

Figure 46: Comparison between the results of SRM and simulator for well \#2 in a training

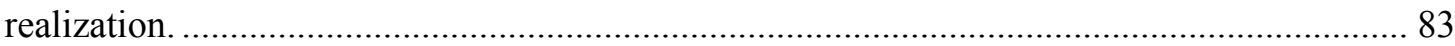

Figure 47: Comparison between the results of SRM and simulator for well \#2 in a blind

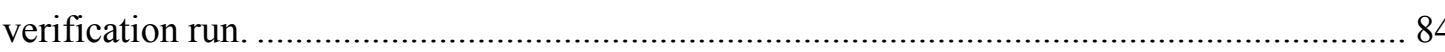

Figure 48: The outcome of history matching for well \#2 comparison between ten best matches

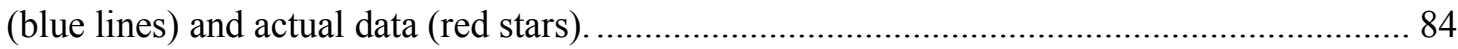

Figure 49: European companies, universities, and research centers involved in the PUNQ

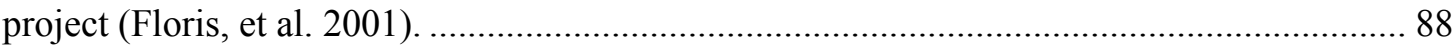

Figure 50: Search results for "PUNQ model" key word in the SPE database (access date:

March 2014). The figure summarizes the number of publications by different publishers, the type of publications, the affiliation of authors, and the authors involved in highest number of publications (OnePetro 2014).

Figure 51: The top structure of PUNQ-S3 model. The field is bounded to the east and south by a fault, and links to the north and west to a fairly strong aquifer. In addition there is a small gas cap in the center of the dome shaped structure (Floris, et al. 2001) ..................................... 90

Figure 52: Horizontal permeability distributions for PUNQ-S3 true case. ............................. 91

Figure 53: Vertical permeability distributions for PUNQ-S3 true case.................................... 91

Figure 54: Porosity distributions for PUNQ-S3 true case. ................................................. 92

Figure 55: Provided field performance for PUNQ-S3 reservoir model. Bottom-hole pressure (BHP), well oil production (WOPR), gas oil ratio (GOR), and water cut (WCT) for each well and field are available for eight years (Floris, et al. 2001)..................................................... 94 Figure 56: Comparing the total oil recovery of the true model with low-median-high ranges of cumulative production after 16.5 years for all the approaches in Floris et al. article. Floris et al. 
(2001) published the description and results of eleven approaches using PUNQ-S3 as the test

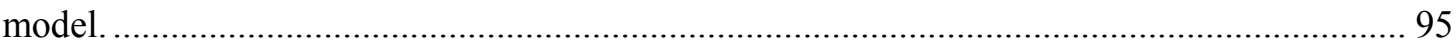

Figure 57: Horizontal permeability distributions for the realization $\# 1$................................ 97

Figure 58: Vertical permeability distributions for the realization \#1 ................................... 98

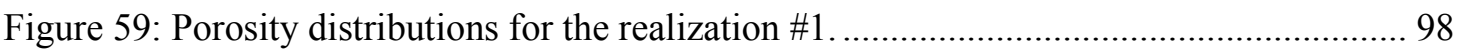

Figure 60: Comparison of well bottom-hole pressure for the ten training realizations and blind

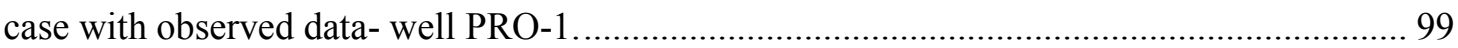

Figure 61: Comparison of gas rate production for the ten training realizations and blind case

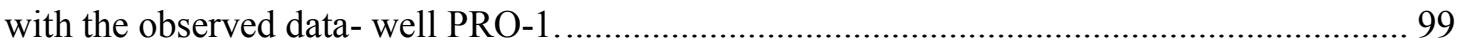

Figure 62: Comparison of water rate production for the ten training realizations and blind case

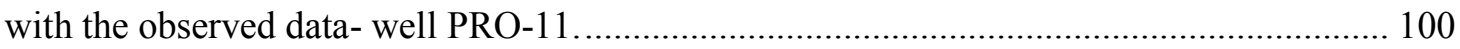

Figure 63: The drainage areas assigned to the wells in layers two and four based on Voronoi

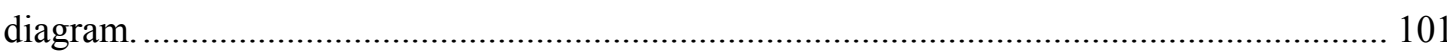

Figure 64: A modified version of Voronoi diagram used to define the drainage areas for the

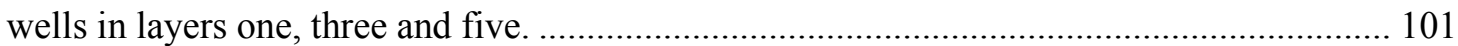

Figure 65: The designed tier system for the PUNQ-S3 reservoir model............................... 102

Figure 66: Inputs and outputs available in the spatio-temporal database for PUNQ-S3 SRM.

Figure 67: ANN structure used for training the SRM. ..................................................... 106

Figure 68: Comparison of well bottom-hole pressure profile generated by the SRM (blue markers) with the similar results from a numerical simulator (CMG) for a training realization of PUNQ-S3 reservoir model.

Figure 69: Comparison of gas production rates generated by the SRM (blue markers) with the similar results from a numerical simulator $(\mathrm{CMG})$ for a training realization of PUNQ-S3

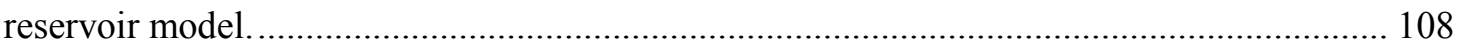

Figure 70: Comparison of water production rate generated by the SRM (blue markers) with the similar results from a numerical simulator $(\mathrm{CMG})$ for a training realization of PUNQ-S3 reservoir model 108

Figure 71: Validating the SRM using a blind run. The comparison of well bottom-hole pressure from the SRM with the results of numerical simulation model for PUNQ-S3 problem.

Figure 72: Validating the SRM using a blind run. The comparison of gas production rate profile from the SRM with the results of numerical simulation model for PUNQ-S3 problem.

Figure 73: Validating the SRM using a blind run. The comparison of water production rate profile (Well PRO-11) from the SRM with the results of numerical simulation model for PUNQ-S3 problem.

Figure 74: History matching results of well bottom-hole pressure. The comparision of 10 best matches (blue lines) coming from SRM with the actual data (red circles).

Figure 75: History matching results of gas production (rates are on the left side and cumulative production are on the right side of the figure). Comparision of 10 best matches (blue lines) coming from the SRM with the actual data (red circles). The data belong to the wells PRO-1, PRO-11, PRO-12 and PRO-15.

Figure 76: History matching results for gas production (rates are on the left side and cumulative production are on the right side of the figure). Comparision of 10 best matches 
(blue lines) coming from the SRM with the actual data (red circles). The data belong to wells PRO-4 and PRO-5.

Figure 77: History matching results of water production (rates are on the left side and cumulative production are on the right side of the figure). Comparision of 10 best matches (blue lines) coming from the SRM with the actual data (red circles). In this study we have just one well (PRO-11) with water breakthrough during eight years of production history.

Figure 78: Comparison between the SRM based history matching results (match \#1) and actual data for cumulative oil production. The red line represents the matched realization and the blue circles are the actual field data (eight years of production history). The green dot also displays the cumulative production for the true case after 16.5 years.

Figure 79: Comparison between the SRM based history matching results (match \#1) and actual data for cumulative gas production. The red line represents the matched realization and the blue circles are the actual field data (eight years of production history). The green dot also displays the cumulative production for the true case after 16.5 years................................. 115 Figure 80: Comparison between the SRM based history matching results (match \#1) and actual data for cumulative water production. The red line represents the matched realization and the blue circles are the actual field data (eight years of production history). The green dot also displays the cumulative production for the true case after 16.5 years. 116 Figure 81: Comparison between the SRM history matching results and actual data. The graph compares the gas rate and cumulative gas production for eight years of available field data for different wells in the PUNQ-S3 reservoir model.

Figure 82: Comparison between the SRM history matching results and actual data. The graph compares the well bottom-hole pressure for eight years of available field data for different wells in the PUNQ-S3 reservoir model.

Figure 83: Comparison between the SRM history matching results and actual data. The graph compares the water rate and cumulative water production for eight years of available field data for different wells in the PUNQ-S3 reservoir model.

Figure 84: Comparison of actual data and the result of a realization of PUNQ-S3 reservoir model. This realization was made using the average properties values of ten best matches. This graph represents the field cumulative oil production, comparing the actual data (blue circles) with the simulator results (red line). Also the green dot is the actual cumulative oil production after 16.5 years, provided in PUNQ project for the forecast comparison. 120

Figure 85: Comparison of actual data and the result of a realization of PUNQ-S3 reservoir model. This realization was made using the average properties values of ten best matches. This graph represents the field cumulative gas production, comparing the actual data (blue circles) with the simulator results (red line). Also the green dot is the actual cumulative gas production after 16.5 years, provided in PUNQ project for the forecast comparison. 121

Figure 86: Comparison of actual data and the result of a realization of PUNQ-S3 reservoir model. This realization was made using the average properties values of ten best matches. This graph represents the field cumulative water production, comparing the actual data (blue circles) with the simulator results (red line). Also the green dot is the actual cumulative water production after 16.5 years, provided in PUNQ project for the forecast comparison. 121 Figure 87: Comparison between the SRM history matching results and actual data. The graph compares the gas rate and cumulative gas production for eight years of available field data for 
different wells in the PUNQ-S3 reservoir model. The results belong to a realization of PUNQS3 reservoir which its characteristics were the average of properties of ten best matches. ... 122 Figure 88: Comparison between the SRM history matching results and actual data. The graph compares the well bottom-hole pressure for eight years of available field data for different wells in the PUNQ-S3 reservoir model. The results belong to a realization of PUNQ-S3 reservoir which its characteristics were the average of properties of ten best matches. 123

Figure 89: Comparison between the SRM history matching results and actual data. The graph compares the water rate and cumulative water production for eight years of available field data for the well PRO-11 in PUNQ-S3 reservoir model. The results belong to a realization of PUNQ-S3 reservoir which its characteristics were the average of properties of ten best matches.

Figure 90: Comparison of future production forecast of the best matched models with the true case.

Figure 91: Box plot of future production forecast for 11 selected matches (ten best matches and the average of these matches). The upper level of box represents P90, the middle is P50

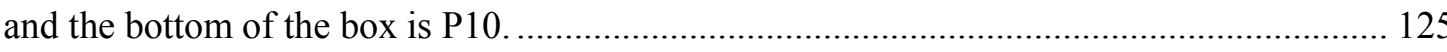

Figure 92: Actual oil rate and cumulative production for different wells. ............................ 155

Figure 93: Actual oil rate and cumulative production for different wells. ............................ 156

Figure 94: Actual oil rate and cumulative production for different wells. ............................ 157

Figure 95: Permeability Map for different Training Realizations. ..................................... 158

Figure 96: Comparison of the SRM results with simulator outputs after the training process-

Run \# 1.

Figure 97: Comparison of the SRM results with simulator outputs after the training process-

Run \# 1.

Figure 98: Comparison of the SRM results with simulator outputs after the training process-

Run \# 1.

Figure 99: Comparison of the SRM results with simulator outputs, the blind verification run.

Figure 100: Comparison of the SRM results with simulator outputs, the blind verification run.

Figure 101: Comparison of the SRM results with simulator outputs, the blind verification run.

Figure 102: Comparison of the SRM results with field data, history match results............... 165

Figure 103: Comparison of the SRM results with field data, history match results................ 166

Figure 104: Comparison of the SRM results with field data, history match results............... 167

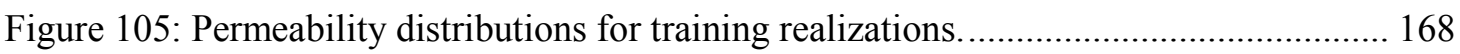

Figure 106: Permeability distributions for training realizations......................................... 169

Figure 107: Permeability distributions for training realizations.......................................... 170

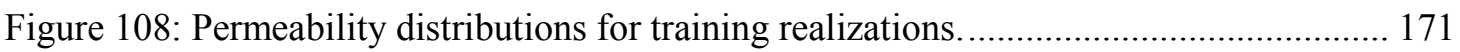

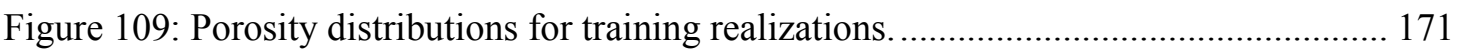

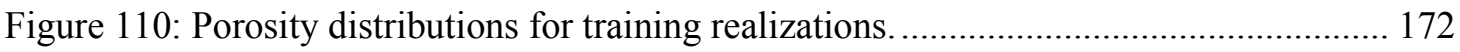

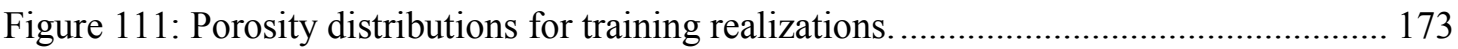

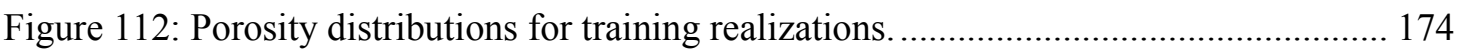

Figure 113: Thickness distributions for training realizations......................................... 174

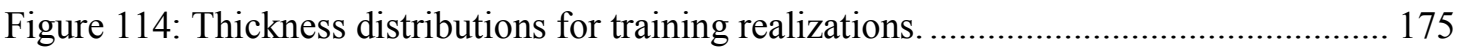




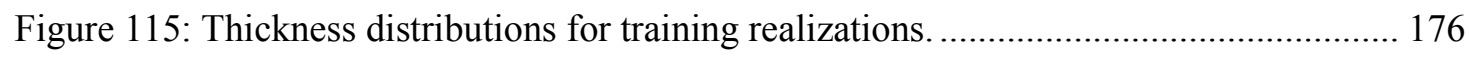

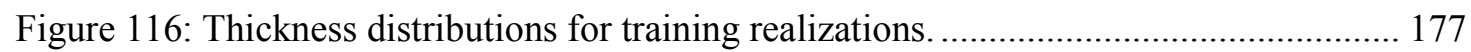

Figure 117: Comparison between the results of SRM and simulator for wells in a training

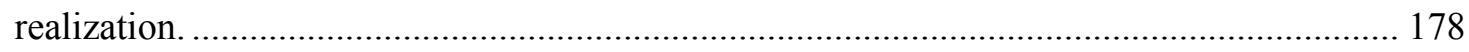

Figure 118: Comparison between the results of SRM and simulator for wells in a training realization.

Figure 119: Comparison between the results of SRM and simulator for wells in a training realization 180

Figure 120: Comparison between the results of SRM and simulator for wells in a blind realization. 181

Figure 121: Comparison between the results of SRM and simulator for wells in a blind realization.

Figure 122: Comparison between the results of SRM and simulator for wells in a blind realization.

Figure 123: Comparison between the results of SRM and simulator for wells in a blind realization.

Figure 124: Comparison between the results of SRM and simulator for wells in a blind realization.

Figure 125: Comparison between the results of SRM and simulator for wells in a blind realization.

Figure 126: Comparison between ten best history matching cases (blue lines) and actual data

(red stars) for different wells.

Figure 127: Comparison between ten best history matching cases (blue lines) and actual data (red stars) for different wells.

Figure 128: Comparison between ten best history matching cases (blue lines) and actual data (red stars) for different wells 189

Figure 129: Horizontal permeability distributions for the training realizations. 190

Figure 130: Horizontal permeability distributions for the training realizations. 191

Figure 131: Horizontal permeability distributions for the training realizations. 192

Figure 132: Horizontal permeability distributions for the training realizations. 193

Figure 133: Horizontal permeability distributions for the training realizations. .................... 194

Figure 134: Vertical permeability distributions for the training realizations. ........................ 195

Figure 135: Vertical permeability distributions for the training realizations. ............................ 196

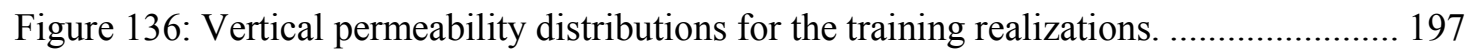

Figure 137: Vertical permeability distributions for the training realizations. ........................ 198

Figure 138: Vertical permeability distributions for the training realizations. ......................... 199

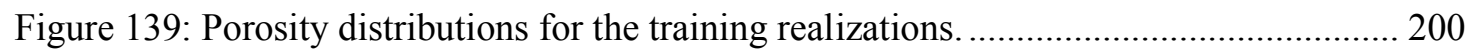

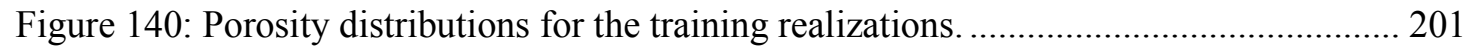

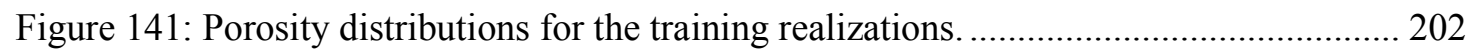

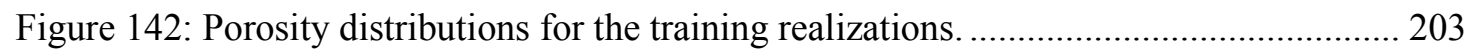

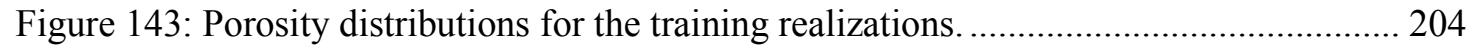

Figure 144: Horizontal permeability distributions for the blind realization.......................... 205

Figure 145: Vertical permeability distributions for the blind realization............................. 205

Figure 146: Porosity distributions for the blind realization.............................................. 206 
Figure 147: Comparison of well bottom-hole pressure for the ten training realizations and

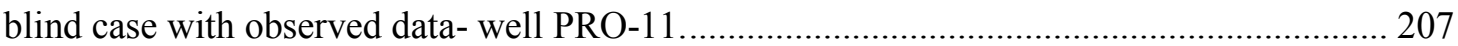

Figure 148: Comparison of well bottom-hole pressure for the ten training realizations and

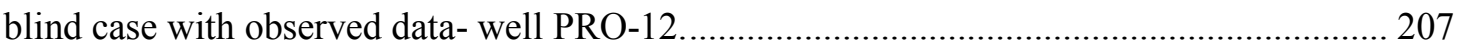

Figure 149: Comparison of well bottom-hole pressure for the ten training realizations and

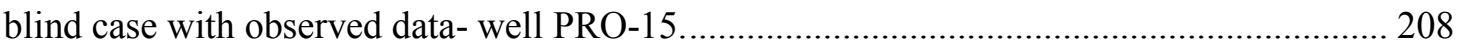

Figure 150: Comparison of well bottom-hole pressure for the ten training realizations and

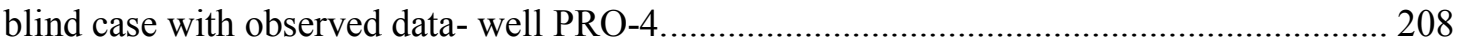
Figure 151: Comparison of well bottom-hole pressure for the ten training realizations and blind case with observed data- well PRO-5.

Figure 152: Comparison of gas rate production for the ten training realizations and blind case

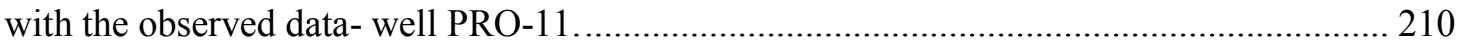
Figure 153: Comparison of gas rate production for the ten training realizations and blind case with the observed data- well PRO-12. 210 Figure 154: Comparison of gas rate production for the ten training realizations and blind case with the observed data- well PRO-15.

Figure 155: Comparison of gas rate production for the ten training realizations and blind case with the observed data- well PRO-4.

Figure 156: Comparison of gas rate production for the ten training realizations and blind case with the observed data- well PRO-5.

Figure 157: Comparison of the well bottom-hole pressure results for SRM and simulator for training run $\# 2$.

Figure 158: Comparison of the well bottom-hole pressure results for SRM and simulator for training run $\# 3$.

Figure 159: Comparison of the well bottom-hole pressure results for SRM and simulator for training run \#4.

Figure 160: Comparison of the well bottom-hole pressure results for SRM and simulator for training run $\# 5$.

Figure 161: Comparison of the well bottom-hole pressure results for SRM and simulator for training run \#6.

Figure 162: Comparison of the well bottom-hole pressure results for SRM and simulator for training run \#7.

Figure 163: Comparison of the well bottom-hole pressure results for SRM and simulator for training run $\# 8$.

Figure 164: Comparison of the well bottom-hole pressure results for SRM and simulator for training run $\# 9$.

Figure 165: Comparison of the well bottom-hole pressure results for SRM and simulator for training run \#10.

Figure 166: Comparison of the gas production rate results for SRM and simulator for training run $\# 2$.

Figure 167: Comparison of the gas production rate results for SRM and simulator for training run \#3. 219

Figure 168: Comparison of the gas production rate results for SRM and simulator for training run \#4. 
Figure 169: Comparison of the gas production rate results for SRM and simulator for training run \#5

Figure 170: Comparison of the gas production rate results for SRM and simulator for training run \#6. 221

Figure 171: Comparison of the gas production rate results for SRM and simulator for training run \#7. 221

Figure 172: Comparison of the gas production rate results for SRM and simulator for training run \#8. 222 Figure 173: Comparison of the gas production rate results for SRM and simulator for training run $\# 9$. 222 Figure 174: Comparison of the gas production rate results for SRM and simulator for training run \#10. 223

Figure 175: Comparison of the water production rate results for SRM and simulator for training runs \#2 to \#7 224 Figure 176: Comparison of the water production rate results for SRM and simulator for training runs \#8 to \#10. 


\section{List of Tables}

Table 1: Required data for a simulation study (Fanchi 2006)............................................... 7

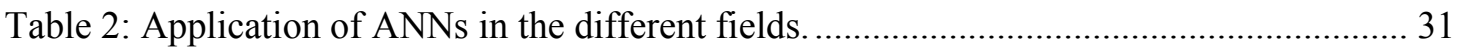

Table 3: Porosity, thickness and permeability values at the well sites.................................... 64

Table 4: Permeability (md) values designed at well locations for generating permeability maps. The permeability values for each well have been ranged by color, which red and blue

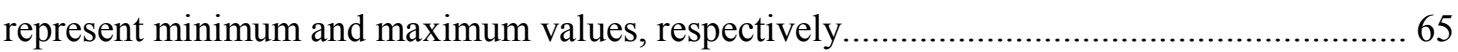

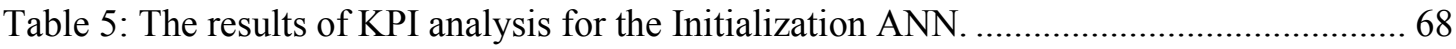

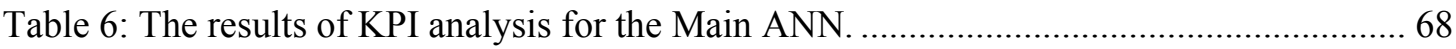

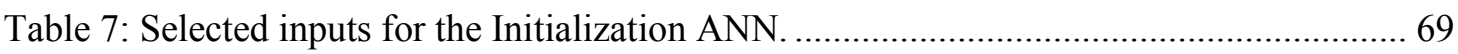

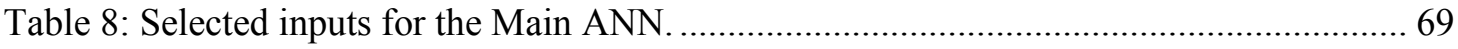

Table 9: The results of KPI analysis for the SRM in the "Feasibility Study"........................ 81

Table 10: Selected inputs for training the SRM in the "Feasibility Study".............................. 81

Table 11: The results of KPI analysis for the SRM in the PUNQ-S3 problem. ..................... 104

Table 12: Selected inputs for the well bottom-hole pressure network. ................................... 105

Table 13: Selected inputs for the gas production rate network. ........................................... 105

Table 14: Selected inputs for the water production rate network........................................ 105

\section{List of Equations}

Equation 1: Sigmoid function as the activation function used in the ANNs algorithm............ 29

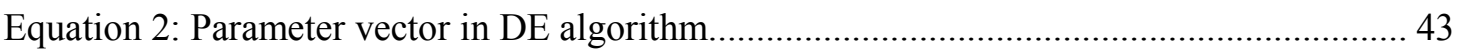

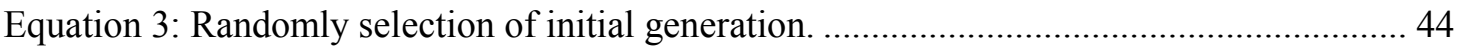

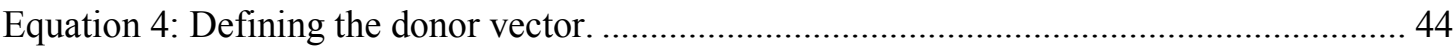

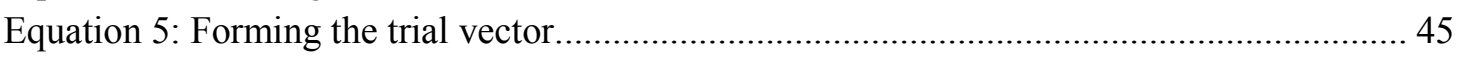

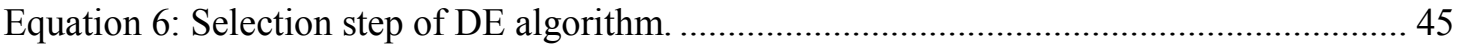

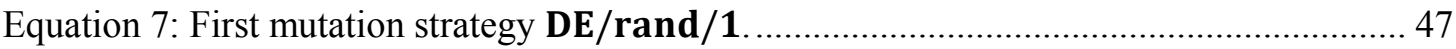

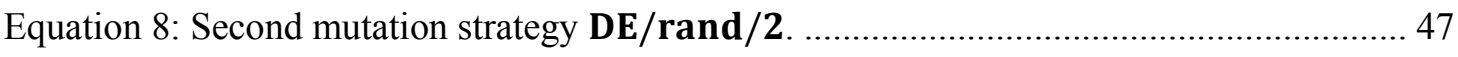

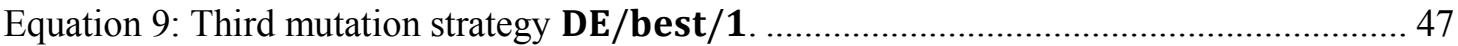

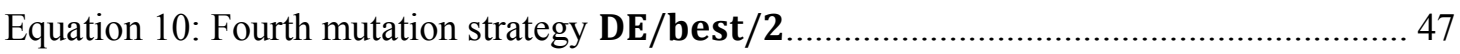

Equation 11: Fifth mutation strategy DE/target - to - best/1 .................................... 47

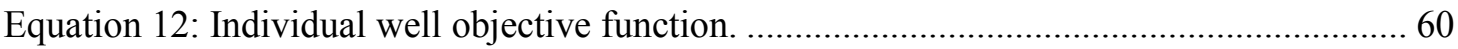

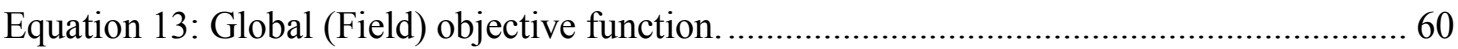




\section{Chapter 1: Problem Statement}

\section{Overview}

The first chapter of this dissertation starts with the problem statement of the study. Then it discusses main objectives behind this research and different steps to obtain them. In continuation it explains the main attractions of this research and introduces the specific people who might be interested in this study. Finally the chapter ends up with the outline of this dissertation.

\subsection{Problem statement}

The purpose of reservoir management is to develop strategies to maximize hydrocarbon recovery. Reservoir simulation is usually the standard decision making tool used by industry in this workflow. The common concern of reservoir simulation and modeling is accuracy. It is generally believed that models with higher resolution (in both time and space) are more accurate. Based on what our industry used to do, any increase in resolution (in time and space) translates to increase in computational time and cost. Therefore a well-known dichotomy arises. On one hand the model must satisfy the accuracy requirements (high resolution), and on the other hand it needs to be fast enough to become practical.

The new improvements in reservoir data acquisition have raised the complexity of the reservoir model and therefore the time required to run it. At the same time, typical reservoir modeling tasks such as sensitivity analysis, history matching, field development optimization, and uncertainty assessment require a large number of simulation runs. The challenge now is to keep the complexity of the reservoir model while shortening its run-time.

The main objective of history matching is to improve and validate the reservoir simulation model by incorporating the observed data into the characterization process, in order to obtain a reliable production forecast. A simulation model which has been tuned to match the past performance of a reservoir offers a higher degree of confidence to predict the future. Having a trustworthy prediction of field performance has a direct impact on the technical and financial performance of operators. 
History matching, by nature, is an ill-posed inverse problem. Correspondingly, classical history matching where reservoir parameters are adjusted manually in a trial-and-error fashion makes this scenario more tedious and time-consuming. Assisted (automated) history matching was proposed to decrease the amount of labor required during the manual history matching. During the last two decades there have been efforts to improve assisted history matching in a way that could be applicable in the real world. Despite all the attempts, due to increasing rate of complexity and resulotion in the reservoir models, there is still hesitation about the practicality and potential of these methods to handle highly complicated real reservoir models. This makes assisted history matching still a challenging and hot research topic.

The novelty of the idea in this study is to examine a new application of pattern recognition technologies to improve the time and efforts required for completing successful history matching projects. The pattern recognition capabilities of Artificial Intelligence and Data Mining (AI\&DM) techniques are used to develop a Surrogate Reservoir Model (SRM) and use it as the engine to drive the history matching process. SRM is a prototype of the full field reservoir simulation model that runs in fractions of a second. SRM is built using a small number of geological realizations. The results of this study are intended to prove the potential of artificial intelligence based reservoir models to ease the obstacles involved in conventional history matching tools.

\subsection{Research objectives}

The primary objective of this dissertation is to examine the application of an artificial intelligence based reservoir model known as surrogate reservoir model (SRM) as an efficient tool for history matching projects. In order to achieve the goal of this study, the larger objective is divided into three main parts:

- Part one, a proof of concept study: The goal of first step is to prove that SRM is able to substitute the reservoir simulation model in a history matching project. In this part, the history match was accomplished by tuning only one property (permeability) throughout the reservoir.

- Part two, a feasibility study: This step aims to study the feasibility of SRM as an efficient tool to solve a more complicated history matching problem. Therefore the number of uncertain reservoir properties increased to three properties (permeability, porosity, and thickness). The SRM was trained, calibrated, and validated using a few geological realizations of the base reservoir model. In order to complete an automated history matching workflow, the SRM was coupled with a global optimization 
algorithm called Differential Evolution (DE). DE optimization method is considered as a novel and robust optimization algorithm from the class of evolutionary algorithm methods.

- Part three, a real-life challenge: The final step is to apply the lessons learned in order to achieve the history match of a real-life problem. The goal of this part is to challenge the strength of SRM in a more complicated case study. Therefore, a standard test model that is known as PUNQ-S3 in literature was selected. The PUNQ-S3 reservoir model represents a small size industrial reservoir engineering model which was formulated to test the ability of various methods and research groups to quantify the uncertainty in the prediction of cumulative oil production. The PUNQ-S3 model has a "true" case which is designed to compare the results of different methods of history matching and uncertainty assessment. Eight years of production and 16.5 years cumulative production are available. Eight years of production was used to match the model and 16.5 years of cumulative production was utilized to compare the future production. The surrogate reservoir model was developed using ten geological realizations of the model. The uncertain properties in this model are distributions of porosity, horizontal, and vertical permeability. Similar to part two, DE optimization method was connected to the SRM to form an automated workflow in order to perform history matching. This automated workflow is able to produce multiple realizations of the reservoir which match the past performance.

\subsection{Audience of the study}

This dissertation concentrates on developing a new class of reservoir models which suits the era of "data intensive" science. These types of reservoir models are purely based on streams of data. They are able to capture the complex and non-linear behavior of fluid flow through the porous media by only using a few examples of the system. They are relatively easy to develop and simple to analyze. The computational cost for developing these models is not comparable with conventional reservoir models (numerical simulation models). Their most important characteristic is very low computational time; they run in fractions of second and can reproduce the outputs of reservoir simulation models with a high accuracy. The main audience of this dissertation is the reservoir modelers who are struggling with drawbacks associated with the high resolution and complex reservoir simulation models. A single realization of these reservoir simulation models takes hours to run even with advanced and parallel computational powers. These problems are magnified when the models are involved in reservoir management tasks which require hundreds of realizations of these models. History 
matching is an example of these kinds of tasks. Therefore this dissertation looks forward to exciting those who suffer the tedious and time-consuming features of conventional history matching tools. The achievements of this dissertation are not only aimed at the history matching procedure, but also beneficiate the other time-consuming operations in reservoir management workflow (such as sensitivity analysis, production optimization, and uncertainty assessment).

\subsection{Dissertation outline}

Chapter 2 of this dissertation defines the history matching problem and discusses the objectives and different approaches of history matching. It continues with a chronological literature review on history matching and finally the chapter ends with presenting the alternative methods available for simulation and modeling.

Chapter 3 is set to prepare the readers with the technology (SRMs) used in this dissertation. It starts with describing artificial neural networks used in the SRMs. The basics of artificial neural networks and their applications are reviewed. Then the surrogate reservoir model is introduced as the novel technology utilized to perform history matching. Finally an optimization method used in automated history matching workflow is presented.

Chapter 4 includes three main sections. The first section describes the general steps required to build a surrogate reservoir model. The second and third sections present two case studies used to prove and analyze the ability of SRM in history matching projects.

Chapter 5 is dedicated to the application of SRM on a real-life case study. A surrogate reservoir model is developed and validated for a standard reservoir model. Then the developed SRM is coupled with the optimization algorithm to build an automated history matching workflow. The performance of SRM for this case study is presented.

Finally chapter 6 provides the summary, discusses the findings and major contributions, and makes recommendation for future work. 


\section{Chapter 2: Literature Review}

\section{Overview}

In order to recognize the real values behind the objectives of this dissertation, first a comprehensive study regarding the history matching should be performed. The chapter initially starts with defining history matching and reviewing different objectives of it. Then it goes through the traditional and modern ways of performing a history matching process. In addition it gives a chronological review of the history matching problem. The chapter ends with a brief review about application of proxy models in the history matching problem.

\subsection{Introduction}

Reservoir simulation is an area of reservoir engineering which computer models are used to predict the flow of fluids (typically oil, water, and gas) through porous media. Reservoir simulation is a mixture of physics, mathematics, reservoir engineering, and computer programming and its objective is building a tool to predict interaction between hydrocarbon and the reservoir under different operational conditions.

Reservoir simulation began in 1954 with the radial gas-flow computations (Watts 1997). Since that time, computational advancements have caused significant improvements in reservoir simulation. These improvements mainly happened in the size and resolution of the reservoir model. Consequently these improvements lead to a huge expansion in the type of users of reservoir simulation and their role in industry and business. The introduction of high-speed computers and the electronic explosion in the last two decades have changed reservoir simulation from a fancy and costly approach to a practical toolbox, which could be available on the laptop of a student.

Today, although reservoir simulation is an established and standard technology, it has not stopped improving. Nowadays, feedback from reservoir simulation models are used in almost all reservoir development decisions. Simulating reservoirs easily and realistically makes them a primary and reasonable choice for oil and gas companies in the development of new (green) fields. Similarly, they are used in developed (brown or mature) fields where production forecasts are needed to help make future investment decisions.

A typical reservoir model includes any of the geological, fluid, or other characteristics of the reservoir and a large number of grid blocks. This model simulates a detailed process of fluid 
flow from reservoir to the surface. The following are the typical reservoir study tasks performed by a reservoir model:

- Sensitivity analysis of uncertain parameters

- Conditioning a simulation model to history data (history matching)

- Field-development planning and production optimization

- Probabilistic forecasting and risk analysis (uncertainty assessment)

A typical reservoir study workflow contains these tasks. This workflow is summarized in Figure 1.

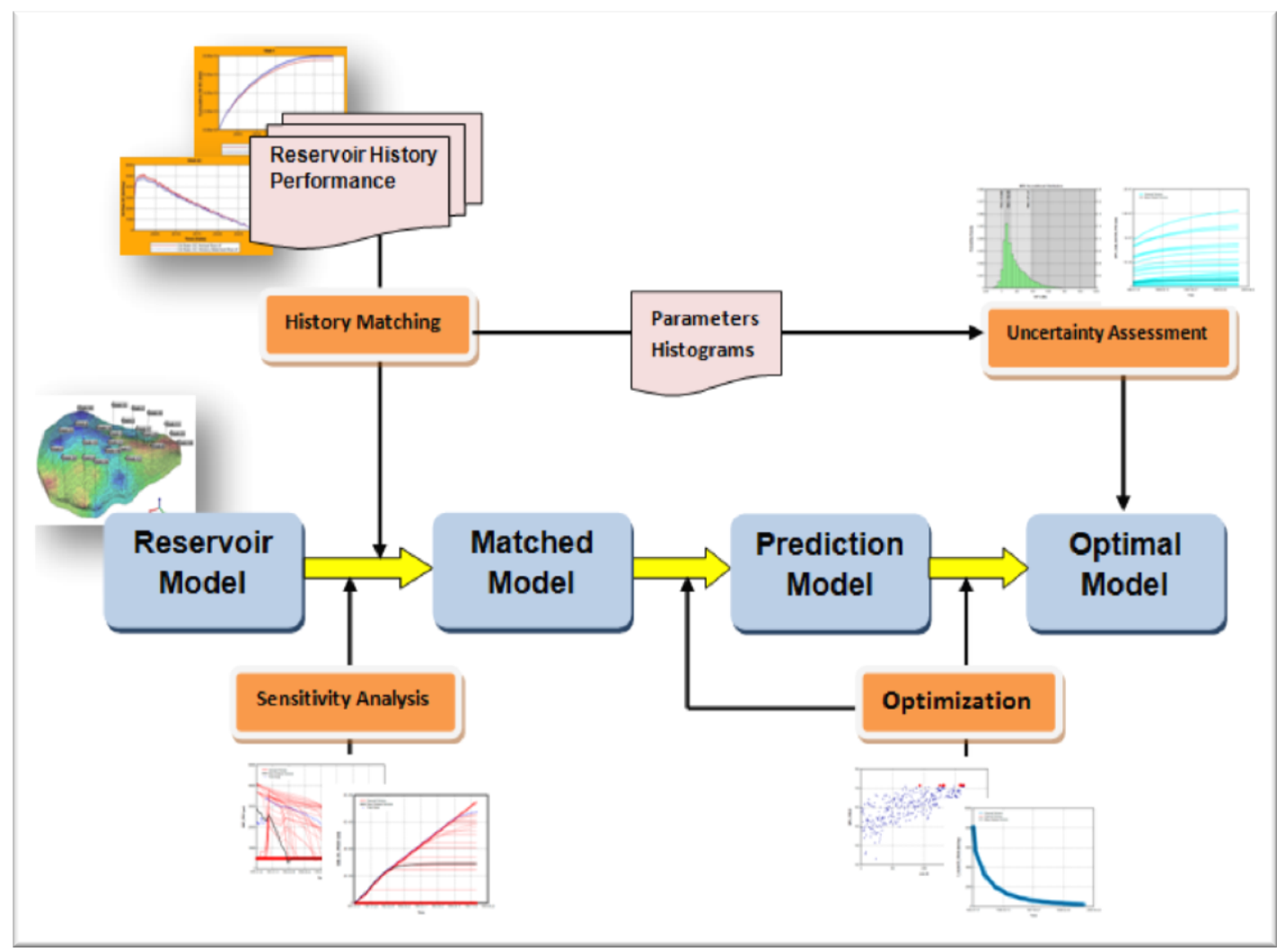

Figure 1: Typical reservoir study workflow.

\subsection{History matching}

\subsubsection{Definition}

Most of the required input data to build a simulation model comes from the samples taken from wellbore or near wellbore areas (Table 1). These data usually are acquired using techniques such as well testing, well logs, core sample analyses, laboratory studies of reservoir fluids, and wellbore performance. Compared to the actual size of a reservoir (acres in the areal dimension) the sources of reservoir simulation model input data represent a very limited portion of reservoir. However these data are the only information provided for the reservoir 
simulation model of the field under study. The point to take is that a huge part of the reservoir remains unknown to the engineers and geologists working on the simulation model. Therefore there is no doubt that the base (first version) simulation model is not very trustworthy due to the uncertainties involved in its input data. A reservoir simulation model goes through modification throughout the life a reservoir. Once new data are acquired, these data are used to modify the reservoir model. A common type of data is the performance of the reservoir. The performance of the reservoir is what we refer to it as the output of reservoir simulation model. Having the real performance of the reservoir, it is very common to calibrate the simulation model to match the real behavior of the reservoir. This calibration process includes the conditioning of geological or static model, which covers the uncertain parameters, to the field performance. This process is typically known as history matching.

History matching is known to be an ill-posed inverse problem. The inverse problem is the opposite of a forward or direct problem wherein the model parameters are used to predict the data. While inverse problem the observed data is used to conclude (adjust) the model parameters (Figure 2). On the other hand, a problem is ill-poised (not well-posed) when there are multiple non-unique solutions for a certain problem. History matching is an ill-posed problem because many possible combinations of reservoir parameters can result in almost same behavior of the reservoir (and match the history data).

Table 1: Required data for a simulation study (Fanchi 2006).

\begin{tabular}{|c|c|}
\hline Property & Sources \\
\hline Permeability & $\begin{array}{l}\text { Pressure transient testing, core analyses, correlations, } \\
\text { well performance }\end{array}$ \\
\hline Porosity, rock compressibility & Core analyses, well logs \\
\hline Relative permeability and capillary pressure & Laboratory core flow tests \\
\hline Saturations & $\begin{array}{l}\text { Well logs, core analyses, pressure cores, single well } \\
\text { tracer tests }\end{array}$ \\
\hline Fluid property (PVT) data & Laboratory analyses of reservoir fluid samples \\
\hline Faults, boundaries, fluid contacts & Seismic, pressure transient testing \\
\hline Aquifers & $\begin{array}{c}\text { Seismic, material balance calculations, regional } \\
\text { exploration studies }\end{array}$ \\
\hline Fracture spacing, orientation, connectivity & $\begin{array}{c}\text { Core analyses, well logs, seismic study, pressure } \\
\text { transient tests, interference testing, wellbore } \\
\text { performance }\end{array}$ \\
\hline $\begin{array}{l}\text { Rate and pressure data, completion and } \\
\text { work-over data }\end{array}$ & Field performance history \\
\hline
\end{tabular}




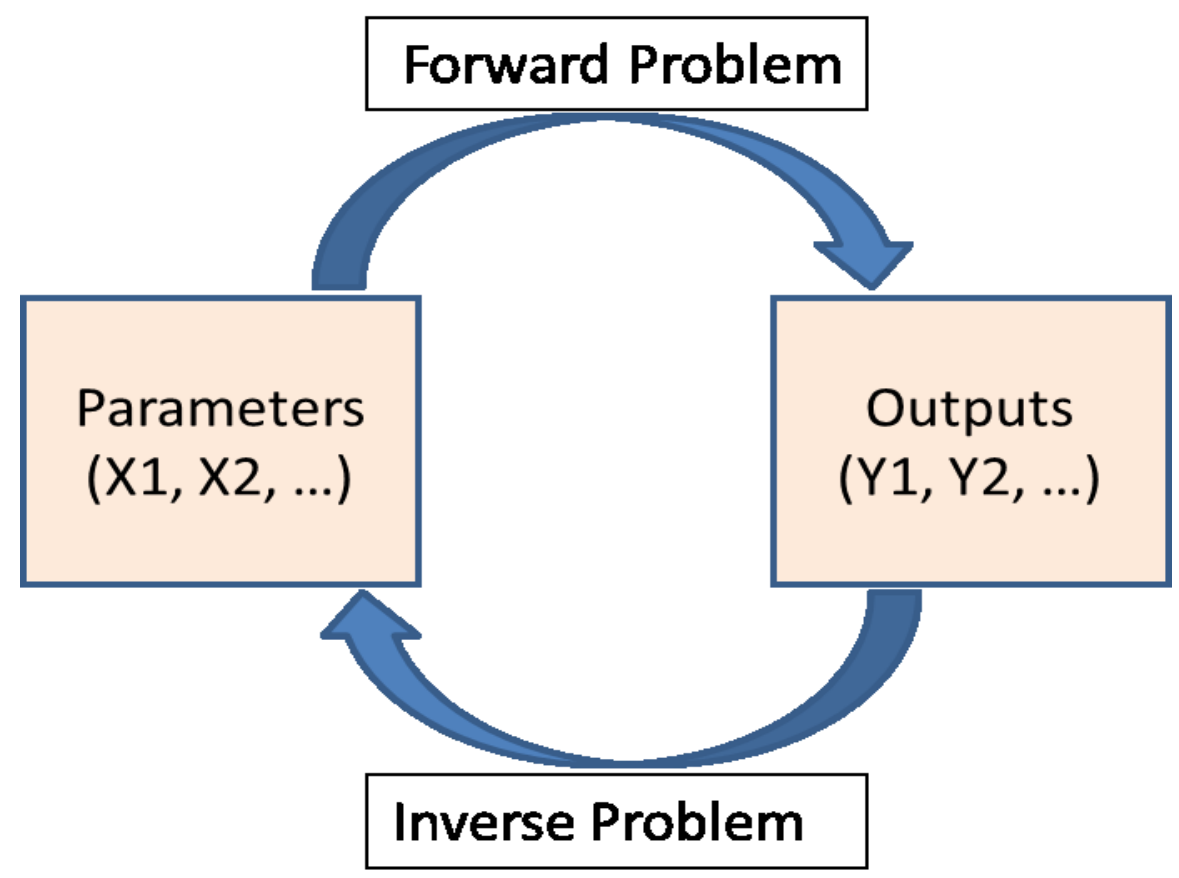

Figure 2: Forward problem verses inverse type of problem.

\subsubsection{Objectives of history matching}

The first objective of history matching is verifying the performance of the simulation model. If a simulation model is able to match the observed field data, then model is a close approximation to the actual subsurface reservoir. Such a verified model is more likely to predict reliable future behavior of the field. Additionally once the most uncertain reservoir parameters have been estimated by matching the observed reservoir behavior, the tuned model can be used to predict the performance of the reservoir under different operating scenarios. On the other hand, a successful history match process has the potential to give a better perception of the available mechanism in the reservoir. Therefore, there is a chance to improve the future development plans of the reservoir and modify the different reservoir characterization and data acquisition programs (Ertekin, Abou-Kassem and King 2001). The new data coming from these plans will help to improve the reservoir model and subsequently improve the history match, model forecasts, and the general understanding of the reservoir. Another valuable impact of a well-done history matching process is identifying abnormal behavior of reservoir. This kind of behavior could be due to unusual operating constraints or some hidden reservoir properties. For example, problems such as casing leaks, improper allocation of fluids to wells, etcetera could be identified during a history matching process (Mattax and Dalton 1990). 


\subsubsection{Practical approaches}

Probably the work performed by Kruger (1960) to adjust the reservoir parameters was the first history matching research which is documented. It has been more than five decades since that time; still the objective of history matching remains the same (a try to minimize the error between the reservoir model output and observed data). Figure 3 is the general workflow to perform a history matching process. Various methods of history matching may be different in many aspects, but they follow the same workflow. This workflow starts with a base geological realization, runs the fluid model and basically compares the results of reservoir model with the field data. This comparison usually is accomplished by calculating misfit values using set of objective functions. Next is to decide if the results are satisfactory; if the answer is positive then the reservoir model can be used for further analysis such as future forecast. In case the results need to be improved, then we go back to the geological model and try to adjust the reservoir properties. Adjusted model is then executed and by having new results whole process is repeated until a satisfied match is acquired. However there are different approaches to perform each step of this workflow.

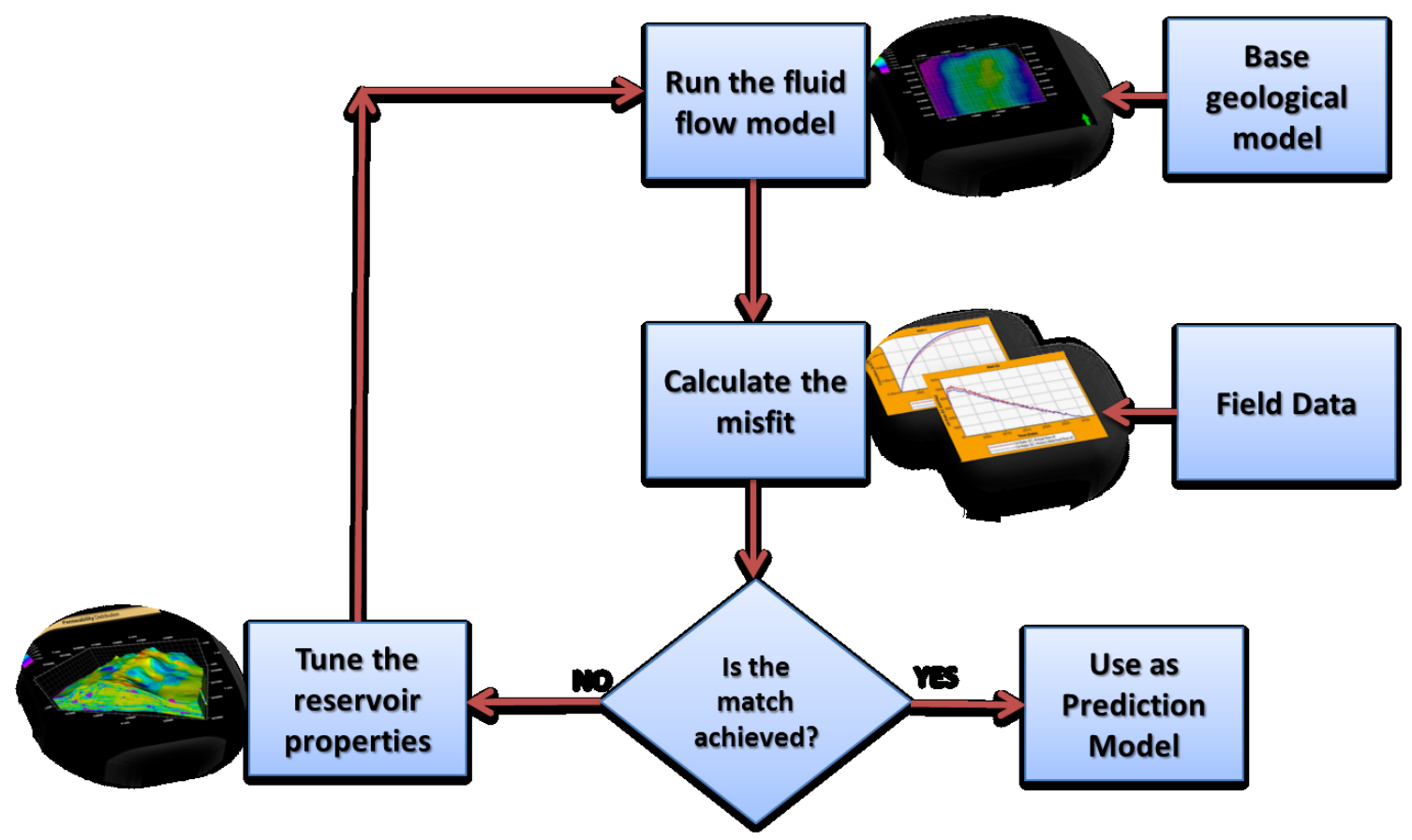

Figure 3: Typical workflow for the history matching study.

Everybody agrees that history matching is not a simple procedure. There are multiple criteria which determine degree of success in this process. The most important items are quality and quantity of available data, specific characteristic of the reservoir under study, time and 
resources allocated to the study, and finally experience and knowledge of the research group working on the problem. As a result each one of these criteria give the history matching problem its most important characteristic which is the non-uniqueness character of results. In addition there are many items which make history matching a problem without specific and distinct method to solve. General approaches to perform history matching might be implemented on different reservoirs. However the success of history matching is very subjective to the characteristics of problem. The main reason behind this claim is that each reservoir has its particular specifications and behavior. The geological characterization, performance history, production development and operation plans, reservoir drive mechanism, and well specifications are the properties that make each reservoir a unique and completely different unit (Cosentino 2001).

\subsubsection{Manual History Matching:}

Traditionally history matching has been performed in a trial and error process. In this process which is referred to as manual history matching, a reservoir engineer tries to evaluate the error between the observed data and modeled data and manually adjust the reservoir parameters seeking a better match. The quality of match in manual history matching is subjective and mainly depends on the experience and technical knowledge of the user. This is the oldest way to perform a history matching. There are some similar workflows they have been developed through the years (Mattax and Dalton 1990, Cosentino 2001, Ertekin, Abou-Kassem and King 2001). In a widely discussed approach, reservoir parameters are tuned manually and in a two steps process:

- The first step, called pressure match, tries to achieve a match for pressure behavior of the field. At this step, the objective is to adjust the global energy balance. Figure 4 (Toronyi and Saleri 1988, Cosentino 2001) describes the procedure at this level. Permeability is a vital parameter affecting flow in the reservoir. Therefore permeability could be modified globally and locally to achieve the pressure match. 


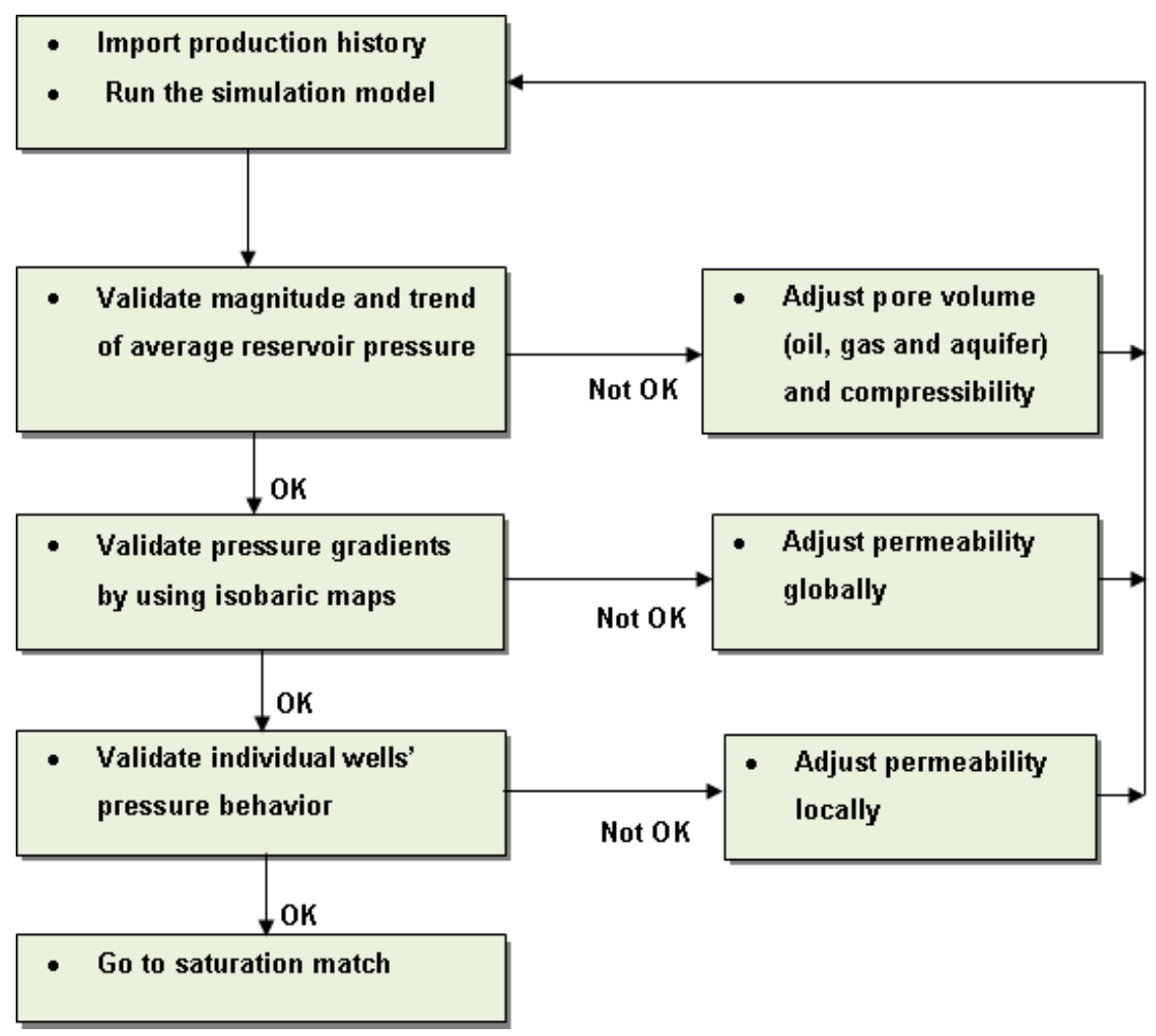

Figure 4: Matching pressure behavior (Toronyi and Saleri 1988, Cosentino 2001).

- The second phase, called saturation match, attempts to match the fluid saturation in the reservoir. In the real case, there is no saturation distribution to be showed as a reservoir performance, but breakthrough of fluids (water and gas) and evolution of the relevant production profiles after that could be considered as saturation history. In the same way, permeability as well as relative permeability curves and functions are the critical parameters in order to get a match. Figure 5 demonstrates the steps to obtain a saturation match. 


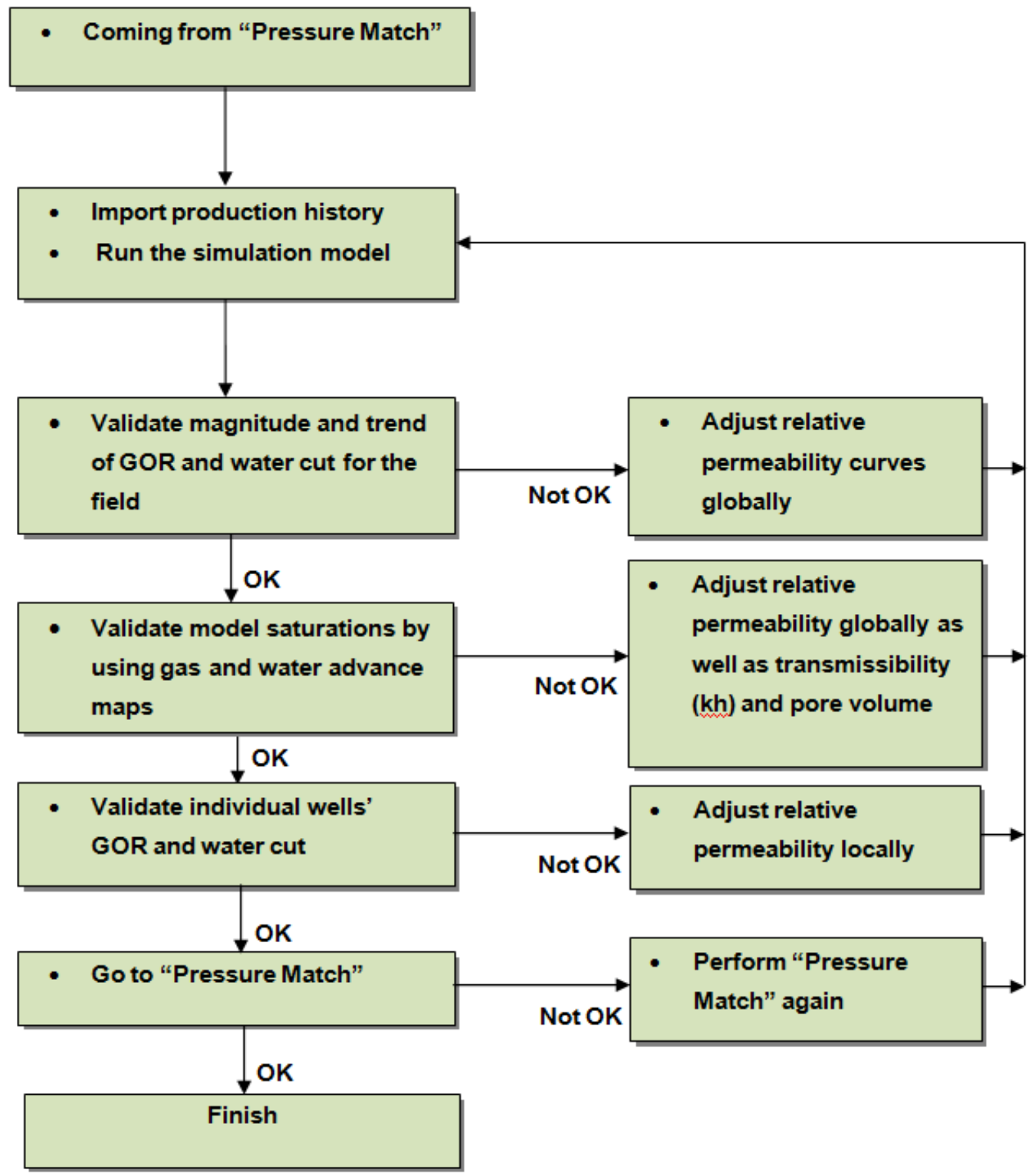

Figure 5: Matching saturation performance of reservoir (Toronyi and Saleri 1988, Cosentino 2001).

\subsubsection{Automated History Matching}

Today, the traditional manual history matching is not the most popular approach to perform a history matching project. This approach is usually time-consuming, tedious, inefficient, and computationally expensive. The results mainly lack geological realism and therefore they are weak in prediction. The necessity of quantifying uncertainties is also growing in industry. In order to capture the uncertainties involved in the outcomes, the industry seeks a range of predictions instead of a single prediction. These predations are in the form of probabilities, like $10^{\text {th }}, 50^{\text {th }}$, and $90^{\text {th }}$ percentiles. For these purposes, the traditional trial-and-error approach of history matching is considered outdated. 
The modern approaches of history matching try to address the shortfalls of old fashioned history matching methods (Romeu 2010). Although advancements in computational power and software have attempted to automate the process, still human-expert intervention seems inevitable. In general, a collection of these approaches which aim to decrease the time and efforts required for history matching are referred to as automated (assisted) history matching. The most important characteristics of these methods are:

- Getting rid of trial and error approach by coupling the objective functions with optimization algorithms.

- Having multiple scenarios for prediction study instead of single matched model.

- Variation in objective functions.

- Implementation of alternative models instead of reservoir simulation models. These models are known as proxy (surrogate) models and they are the approximation of full field reservoir simulation model. There are different logics behind using these models which this and upcoming chapter will discuss.

In the following, a chronological review of history matching approaches is presented. This literature review includes examples of manual history matching and different methods of automated (assisted) history matching.

\subsubsection{A literature review on history matching:}

The earliest studies in the field of history matching started in 1960's. Kruger (1961) presented a calculation procedure to determine the areal permeability distribution in a reservoir. His method allowed the verification of the basic reservoir data by matching the past performance of the reservoir. The proposed calculation method was based on a mathematical model of the reservoir. He used the matched model to predict the future. Wahl et al. (1962) used an electrical model to represent the reservoir model and used it in order to match the performance of Saudi Arabian oil fields. Jacquard (1964) showed that there is a mathematical relationship between an electric resistance-capacity network and a reservoir model. Using this relationship he developed a method for interpreting pressure measurements. Later the results of this work helped Jacquard and Jain (1965) to develop an automated system to interpret the field data. Their procedure was based on variation analysis in electric networking for a two dimensional case. Jahns (1966) developed a systematic search which was able to adjust the properties of the reservoir model automatically and find the match between the measured and calculated pressure data. The reservoir model was a single-phase, compressible, two-dimensional model. Jahns utilized the same search method used by Jacquard and Jain which was based on an 
adoption of steepest descent method. Coats et al. (1970) proposed a method which used least square and linear programming techniques to determine the reservoir characteristics by matching the given reservoir performance. They applied the method on examples of the reservoirs with single and two phase flow. Later Solorzano et al. (1973) modified the method of Coats et al. and used it as the base for their automated history matching method. The use of gradient optimization method started in the early 1970's. Slater and Durrer (1971) used a balanced error-weighted gradient method to match the reservoir performance data.

Chen et al. (1974) and Wasserman et al. (1975) were among the first researchers to formulate history matching as an optimal control problem. An optimal control is a set of differential equations describing the paths of control variables that minimize the objective function. At that time, the use of optimal control theory with first-derivative optimization methods provided an effective approach for automatic history matching. Dougherty and Khairkhah (1975) used optimal control theory for history matching a gas reservoir.

Gavalas et al. (1976) looked at history matching as an underdetermined statistical problem. They used a Bayesian framework to reduce the number of unknowns. They assumed that the unknown vector of reservoir parameter is a random variable with a mean value of $\alpha$ and prior covariance of C. $\alpha$ and $\mathrm{C}$ values are achieved from the available geological data. Also they utilized gradient and Gauss-Newton optimization methods in their work.

Watson et al. (1980) studied history matching in two-phase petroleum reservoirs. In addition to porosity and permeability, they changed the coefficients of relative permeability functions for hypothetical case studies of water flooding. Pruess et al. (1980) probably were the first researchers to use software similar to today's numerical simulator to perform history matching. They used the SHAFT 79 simulator developed in the Lawrence Berkley Laboratory for history matching. Watson and Lee (1986) introduced a new algorithm for automated history matching based on a modification of Gauss-Newton method for minimization of least square functions.

With the advent of geo-statistics, a new class of parameterization approaches which is known as pilot point appeared. In these approaches, the reservoir properties are estimated for a limited number of points (controlling points) and the rest of reservoir are interpolated using geostatistical and mathematical methods. These methods led to a significant decrease in the number of parameters. Marsily et al. (1987) were the pioneers in using geo-statistical methods in the history matching problem. They started by just using kriging. Later RamaRao et al. 
(1995) and Gomez-Hernandez et al. (1997) used Gaussian Random. Fasanino et al. (1986) also used the kriging method to create a distribution of reservoir properties obtained from history matching.

Zuber et al. (1987) utilized a dual-porosity and two-phase reservoir simulator to match the methane and water production for coal-bed methane reservoirs.

The gradient of objective function can hold information regarding the sensitivity of reservoir parameters. Therefore gradient based optimization methods were among the first approaches used in automatic history matching. Different methods to calculate the gradients (first or second order) have been proposed. Yang and Watson (1988) used a method based on a solution of the adjoint equations. Anterion et al. (1989) developed an analytical approach to calculate the gradients for an optimization method. They coupled this gradient based optimization method with a three-phase, three-dimensional simulator (SCORE). Although they recommended that the use of this method leads to a decrease in processing time and number of runs, still the experience of an engineer was required to make a decision in changing the parameters. The concerns regarding the role of engineering input to, and interaction with, algorithms in history matching have been the center of arguments for many years (Watkins, Parish and Modine 1992). The engineering knowledge and experience can be an additional help in providing the initial values for parameters or even in the optimization framework.

In the early 90's we observe the arrival of experimental design and surface methods in petroleum engineering. The theory behind experimental design goes back to 1920 and 1930 in the application for agricultural purposes. Simply, it can be said that experimental design looks for constructing a design setting which is able to give maximum possible information from a minimum number of experiments. Damsleth et al. (1992) claimed that instead of changing one parameter at a time, using a well-designed setup makes it possible to achieve the same information with considerably fewer realizations of the simulation model. They applied the technique for a North Sea gas field in order to decrease the number of simulation runs in sensitivity analysis study. They mentioned that the technique has reduced the number of runs by 30 to 40 percent compared to the framework in which one parameter at a time was varied. Based on their finding the technique would not give good results when it comes to extrapolation.

Response surface investigates the relationships between the inputs and output(s). This statistical method was introduced by Box and Wilson (1951). Eide et al. (1994) used 
experimental design to develop a response surface which gives the output of reservoir simulation. The response surface was used as an approximation of the reservoir simulation model to perform automated history matching for a synthetic reservoir model. Basically, they used experimental design to create a set of simulation runs with different combinations of reservoir parameters. These runs were used to develop the response surface, which in their case, was a regression and kriging method.

In 1992 the first type of global optimization methods was applied in history matching. Ouenes et al. (1992a) introduced the application of a new optimization algorithm which did not require calculating the gradients of objective function. The algorithm is known as Simulated Annealing. The method was independently proposed by Kirkpatrick, et al. (1983) and two years later by Černý (1985) for finding the global minima of a cost function which may have several local minima. Ouenes and his coworkers used this method for history matching of several case studies with a high number of variables (1992b, 1993a, 1993b). Another important characteristic of global optimization methods such as simulated annealing was their ability to be used in a parallel computation workflow. Ouenes et al. were among the first ones which used parallel computing in history matching. By using parallel computing techniques, large problems which require high computational power can be divided into smaller ones and carried out simultaneously or in parallel.

The years after early 90 's could be referred to as the modern era of history matching. As the computational advancements were happening quickly, the reservoir simulation models were getting complicated as well. Therefore simple optimization methods were not good enough to address complex history matching problems (Bush and Carter 1996). Significant works were done to move history matching from a labor intensive engineer-based framework to a fully or semi-fully automated approach. During this time we observe the experiment of different optimization algorithms trying to reduce the cost of finding global minima. Single matched realization of the simulation model switched to multiple representatives of a simulation model (Tyler, Svanes and Omdal 1993, Palatnic, et al. 1993). These multiple solutions were useful for understanding the uncertainty of future production and reduce the risk involved in cost and benefit.

Another type of technique which has been used in automated history matching is streamline simulation (Emanuel and Milliken 1998, Vasco, Yoon and Datta-Gupta 1998, Wang and Kovscek 2000). Originally developed in the 1960's, the streamline simulation is an alternative to block-based finite difference simulation. In conventional finite difference simulation flow 
transports from cell to cell, while in a streamline simulation model, fluids are transported along streamlines (Baker 2001). Streamline simulation is a fast and efficient forward model particularly for history matching purposes. Furthermore its ability to highlight the flow paths between the producer/injector wells can be used to identify the parts of a reservoir which are critical to match the past performance. Nevertheless this technique has its own assumptions and limitations (Thiele, Batycky and Fenwick 2010).

Baker (2001) believed that during a history matching deciding which cell in a reservoir model should be adjusted is very important; therefore regrouping a bunch of cells might be necessary. In this case, streamline simulation can be helpful in grouping cells that need to be adjusted.

Vasco et al. (1998) and Emanuel and Milliken (1998) were the first to use streamline simulation in history matching. Wang and Kovscek (2000) developed a streamline approach to modify the effective permeability along the streamlines in order to match the dynamic production data including producer water-cut curve, well pressures, and rates. The idea was to connect water-cut curve at a producer to the water breakthrough of individual streamlines; thus by changing the permeability associated with the streamlines, the breakthrough time of streamlines and producer fractional-flow curve can be found. On synthetic data sets, they achieved rapid history matches, often in only two or three simulations. Later Agarwal and Blunt (2003) extended the work of Wang and Kovscek to a real field case in the North Sea. Fenwick et al. (2005) used streamline simulation coupled with a combination of geo-statistical tools to history match a giant Middle Eastern oil field. They just changed the permeability to achieve the match. Batycky et al. (2007) used the approach of Fenwick et al. to apply it to a reservoir with 1.4 million grid blocks. They used streamlines as a guide to find the location and amount of changes in the reservoir.

As it was reviewed, gradient based optimization methods were the first algorithms used to minimize the objective function in history matching. Generally these approaches are computationally expensive and also very prone to fall into a local minima rather than a global minimum. On the other hand, most of the history matching problems are multi-dimensional, non-linear optimization problems which often include multiple local minima. In order to address these problems, global optimization methods such as simulated annealing and Evolutionary Algorithms such as Genetic Algorithms (GAs) and Evolution Strategy were proposed. Another advantage of global optimization methods over gradient based methods is the ability of parallel computing, which plays a critical role in dealing with complex models. 
Sen et al. (1995) compared the performance of genetic algorithms with simulated annealing. Genetic algorithms belong to the larger class of evolutionary algorithms. The idea behind these algorithms is to evolve a group of solutions using the operators inspired by natural evolution, such as inheritance, mutation, selection, and crossover. Genetic algorithms and evolutionary strategies (ES) are two of the most common types of evolutionary algorithms. Romero et al. (2000a, 2000b) coupled geo-statistical modeling with genetic algorithms to match the production data on a realistic synthetic case study. They concluded that genetic algorithms are capable of handling large full field reservoir simulation models with many parameters. Schulze-Riegert et al. (2001) applied evolutionary strategies to complex history matching problems. They claimed that evolutionary strategies are robust and less sensitive to non-linearities and discontinuities of the solution space. Nevertheless one challenging problem was convergence improvement. Genetic algorithms were the optimization tool for assisted history matching in Top-Down Reservoir Modeling workflow, a reservoir management workflow proposed and used by BP (Williams, et al. 2004). Al-Shammaand Teigland (2006) used evolutionary algorithms (GAs) for history matching a complex reservoir model. They had 46 different parameters including porosity, permeability, and transmissibility at different regions. The results of history matching then were used to perform uncertainty assessment. Takuda et al. (2004) used a genetic algorithm to match the results of core flooding experiments. The adjustable parameters were relative permeability and capillary pressure curves. The water saturation of the grid block was measured during the core water flooding and then was used as the measured data, which should be matched. Castellini et al. (2006) combined designs and response surface techniques with genetic algorithms to perform history matching and assess the uncertainty for future forecast. Ballester and Carter (2007) applied a modified genetic algorithm and parallel computing to perform history matching for a real reservoir case study. In a classic genetic algorithm, the data are converted to binary (zero and one) codes; while Ballester and Carter used a modified genetic algorithm in which data were an array of real numbers. GAs and evolutionary algorithms have proven to be a great optimization tools for history matching and other types of problem in petroleum engineering (Stephen and Arwini 2010).

During the last decade application of a number of global optimization methods have gained popularity in the automated history matching process. Among the successful methods we can include the ensemble Kalman filter (Van Leeuwen 1999, Evensen 2003, Haugen, et al. 2006, Aanonsen, et al. 2009, Hanea, et al. 2010, Szklarz, Hanea and Peters 2011), Neighborhood Algorithm (Christie, MacBeth and Subbey 2002, Stephen, et al. 2006, Rotondi, et al. 2006, 
Subbey and Christie 2003), Genetic Algorithms (Erbas and Christie 2007) (Castellini 2005), Scatter search (Sousa 2007), Tabu Search (Yang, Ngheim and Card 2007), Hamiltonian Monte Carlo (HMC) (Mohamed, Christie and Demyanov, Comparison of Stochastic Sampling Algorithms for Uncertainty Quantification 2009), Particle Swarm Optimization (PSO) (Eberhart and Yuhui Shi 2001, Mohamed, Christie and Demyanov, Comparison of Stochastic Sampling Algorithms for Uncertainty Quantification 2009, 2010, Rwechungura, Dadashpour and Kleppe 2011, Kathrada 2009) Ant Colony Optimization (ACO) algorithm (Razavi and Jalai-Farahani 2008, Hajizadeh, Christie and Demyanov 2009, 2010), Markov chain Monte Carlo (Maucec 2007), and Chaotic Optimization (Mantica 2002).

Rwechungura et al. (2011) have published fascinating information regarding the increasing interest in history matching since 1990. Figure 6 which is taken from their article summarizes the number of papers on the history matching topic between the years of 1990 to 2010.

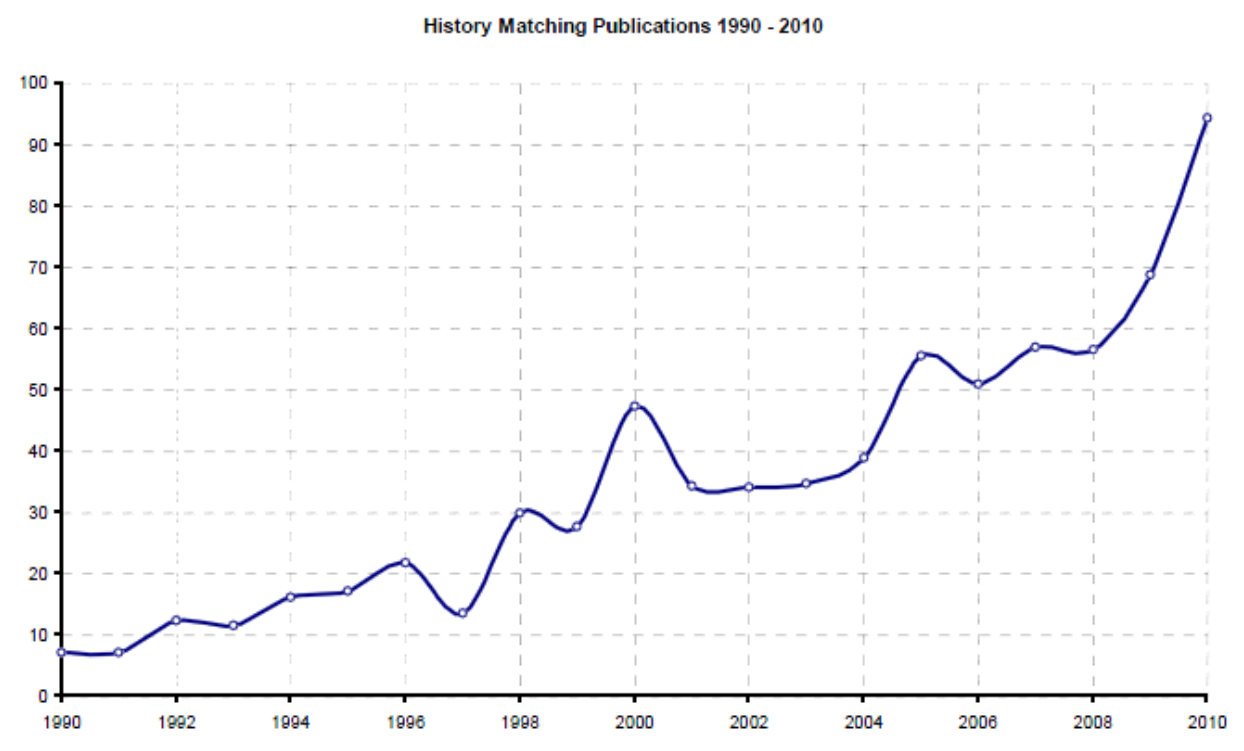

Figure 6: Number of papers on the history matching topic between 1990 to 2010 (Rwechungura, Dadashpour and Kleppe 2011).

\subsection{Alternative approaches to simulation models}

In the previous section we presented a brief review covering more than 50 years applications of history matching. As the brilliant era of history matching, the last 20 years have observed a lot of improvements in the way we deal with this problem. Probably the most notable improvement is the attempt to switch history matching from an individual knowledge-based approach to an automated (or semi-automated) workflow. Today, although the interpretation of engineer is still required, the main portion of history matching is performed through an 
automated machine-based workflow. However, almost all the methods covered in the previous section suffer from one common drawback. This drawback is the significant computational cost associated with the complex and high resolution simulation models (S. D. Mohaghegh 2011). During the last two decades the main approach to address this problem was to find an optimization method which can reach the solution with the minimum number of runs (Hajizadeh, Christie and Demyanov 2009). Huge advancements happened in this area. Optimization algorithms moved from gradient based methods to stochastic global optimization approaches. At the same time, improvements in simulation models led to models with higher resolutions. Furthermore, it seems like the problem still remains the same, because as the proposed solutions are improving the problem is getting bigger.

Different attempts have been made to speed up the simulation models. One is the application of different computational methods in order to decrease the size of the system. Techniques such as IMPES (Zhangxin, Guanren and Baoyan 2004), Sequential, and IMPSAT (Haukas, Aavatsmark and Espedal 2004) are used whenever there is no need for a fully solved model (Yang, et al. 2009). Up-scaling the static and dynamic models, as much as possible, are another way to reduce the size of the problem. Clearly, using coarser grid blocks results in the simpler and smaller mathematical model to solve; therefore this leads to a faster modeling process. Up-scaling the detailed fine-scale geo-models to a lower-order, coarser representation is a common practice in reservoir simulation and modeling (Jansen, Brouwer and Douma 2009, Vakili-Ghahani and Jansen 2010). Some (Yang, et al. 2009) even go further and consider the famous Material Balance model as the extreme version of up-scaling, where the reservoir is assumed as a huge volumetric tank. Beside the assumptions associated with Material Balance Equation, as a matter of fact this method is very effective to estimate reservoir pressure decline after production, or for back-computing initial amount of hydrocarbons through a simple history match of production pressure data (Hurst 1974, Ojo and Osisanya 2006, Yang, et al. 2009).

Another alternative method that has been frequently used in petroleum engineering is decline curve analysis. Many articles are available on this subject (Agarwal, et al. 1999, Li and Horne 2003, Cheng, Lee and McVay 2008, Ilk, et al. 2010). Decline curves are able to quickly predict the future performance of the reservoir based on the past production history. The use of this method goes back to even before the availability of computers in petroleum engineering. At that period, decline curve analysis was performed manually on semi-log plot papers. The early decline curve was based on empirical rate-time equation of Arps (1945). Later different 
people modified Arps's equation for various purposes (Fetkovich 1980, Fetkovich, Vienot, et al. 1987, Agarwal, et al. 1999, Li and Horne 2003, Cheng, Lee and McVay 2008, Ilk, et al. 2010). Although decline curve analysis is still a fast and cheap way for predicting the future performance and finding the well's problems, it has its own limitation and simplifications (Ilk, et al. 2010). For example, the common assumptions for this method are single phase, homogeneous, and slightly compressible plane radial flow systems (Yang, et al. 2009).

Advent of global optimization methods such as simulated annealing, genetic algorithms, and evolutionary strategies was associated with using parallel computation (Saleri 1993, Ouenes, Brefort, et al. 1993b, Sen, et al. 1995). Parallel processing enabled a faster history matching process, but as the size and complexity of reservoir model increased the problem basically remains the same (S. D. Mohaghegh, J. S. Liu, et al. 2012b).

An efficient and popular, but at the same time debatable approach, to address the high computational cost of a simulation model is to substitute the full field simulation model with inexpensive approximation or proxy models. By the rise of super complicated, heavy to perform simulation models, proxy or surrogate models showed up as an alternative tool over full scale engineering simulation models. Basically proxy models are fast approximations to the high fidelity simulation models. They have been widely used in different industries such as aerospace in order to substitute the time-consuming and high computational expense simulation models (Queipo, et al. 2005, Forrester and Keane 2009). Their efficiency to reproduce the results of simulation models with a high speed makes them popular. Using proxy models in petroleum engineering goes back to almost two decades ago (Eide, et al. 1994). However, they still remain arguable because of practical reasons (Zubarev 2009, S. D. Mohaghegh 2011, Goodwin and Powell 2012). Proxy models obtain higher computational efficiency at the expense of losing the resolution and accuracy associated with full-physics simulation models.

Mathematical and statistical based techniques such as response surface models combined with sampling methods (Experimental Design) are popular types of proxy models which have been used in the petroleum industry for a long time (Damsleth, Hage and Volden 1992, Eide, et al. 1994). Response surface methods can be a suitable alternative to simulation models; however, it is essential to correctly apply the statistical design methodology to capture the fluid flow behavior. Response surface models have been frequently used in petroleum engineering. There are number of articles available in uncertainty analysis of reservoir behavior (Damsleth, Hage and Volden 1992, Manceau, et al. 2001, Friedmann, Chawathe and Larue 2003, Cheong and 
Gupta 2005), well optimization (Zabalza, et al. 2000, Landa and Güyagüler 2003, Valladao, et al. 2013), and history matching (Eide, et al. 1994, White and Royer 2003, Alessio, Bourdon and Coca 2005, Gupta, et al. 2008, Cheng, Dehghani and Billiter 2008, Arwini and Stephen 2011).

Reduced order modeling is another attempt to transfer the high dimensional models into a meaningful representation of reduced dimensionality. They have been applied in many application areas including petroleum engineering. It is believed that the nonlinear dimensionality reduction techniques of reduced order modeling are able to transform the high resolution and complex geological models into low dimensional representatives. Although, in reality, many well-known reduced order modeling algorithms were developed for linear systems (Amsallem and Farhat 2008). On the other hand, while most of reduced order models can perform with high efficiency, they require a large number of model realizations to capture the relationships between the inputs (S. D. Mohaghegh 2011, S. Amini, et al. 2012). Another drawback is the lack of robustness when it comes to the parameter variations. Therefore, they need to be rebuilt in order to capture the changes in the inputs (Amsallem and Farhat 2008). In recent years there has been some attempts in using reduced order models for history matching, uncertainty quantification, and optimization (Cardoso 2009, Cardoso and Durlofsky 2010, He, Sarma and Durlofsky 2011, Bazargan and Christie 2012, Bazargan, Christie and Tchelepi 2013, Wu, et al. 2013) (Gildin, Ibrahim and Ghasemi 2014).

Another relatively new type of proxy models which are considered as an efficient alternative to reservoir simulation models are proxy models based on artificial intelligence and data mining techniques. These models are known as Surrogate Reservoir Models (SRMs) and have gained popularity in recent years to address many time-consuming operations (such as history matching) traditionally performed by reservoir simulation models. Different types of techniques to build these models give them different characteristics compared to regular proxy models (statistical and mathematical based models). Surrogate reservoir modeling technology is the modeling technique used in this dissertation. Therefore, the next chapter separately concentrates on this type of modeling.

\subsection{Summary}

Reservoir management is dedicated to design strategies to maximize the hydrocarbon recovery. This goal is obtained through the completion of various reservoir studies. In order to achieve this goal, reservoir simulation models are the common and standard tools used by industry in reservoir management workflow. Typical tasks performed by simulation models 
include sensitivity analysis, history matching, production optimization, and uncertainty assessment. Among these tasks history matching plays a vital role. History matching is a calibration and verification step in this workflow. The feedback from history matching enhances the simulation model reliability and decreases the uncertainty involved in future prediction. Moreover, history matching provides a better understanding of the reservoir.

History matching has more than half a century background in the oil and gas industry. The first history matching case studies were simply adjusting parameters manually and observing the impact of changes by comparing the outputs with field performance. Manually history matching workflow, although is not the most efficient way to perform history matching, it is still conducted in the industry. Many researchers tried different optimization methods to automate the process of history matching. Gradient based optimization algorithms were among the first optimization approaches utilized in the automated history matching workflows. The advent of geo-statistical approaches, such as pilot point method, resulted in huge changes in reservoir parameterization. Using experimental design methods started in the early 90's to decrease the number of runs required for history matching. These approaches were then used to develop proxy models, particularly response surface models, in order to approximate the results of the simulation model. Computational advancements led to the simulation models with higher resolution and accuracy. Global optimization methods took advantage of parallel processing to speed up the process of history matching. Also global optimization methods showed multiple advantages over gradient based optimization algorithms. At this time single matched realization of the simulation model switched to multiple representatives of the simulation model.

By end of the 90's and start of last decade streamline simulation showed as a fast and efficient forward model particularly for history matching. However, this approach might be limited by its own assumptions. Application of global optimization algorithms in history matching enjoyed significant improvements by this time. Evolutionary Algorithms such as Genetic Algorithms (GAs) and Evolution Strategy proposed a higher efficiency in automated history matching workflow. During the last decade, numerous history matching case studies were accomplished using these methods.

Proxy models as an inexpensive approximation of high computational cost full field simulation models are frequently used in different areas of engineering. By increasing the time and cost required to run the reservoir simulation models, proxy models appeared in petroleum engineering. They are fast and relatively easy to develop; however, due to practicality 
concerns there is a long way to completely surpass full field reservoir simulation models in reservoir management workflow. Response surface models and reduced order models are the most famous types of proxy models used in petroleum engineering.

Other relatively new types of models to approximate the reservoir simulation models are the proxy models based on artificial intelligence and data mining techniques. The most famous example of these models is surrogate reservoir models. The unique characteristics of these models are provided by pattern recognition capabilities of artificial intelligence and data mining techniques used to develop them. The next chapter will introduce these models in a deeper context. 


\section{Chapter 3: Surrogate Reservoir Models (SRMs)}

\section{Overview}

In the previous chapter a brief review about alternative methods to substitute the reservoir simulation models in the modeling process was presented. In the same context, one new approach is the proxy models constructed based on artificial intelligence and data mining techniques. A successful example of the techniques to build these proxy models is Artificial Neural Networks (ANNs). In this dissertation ANNs are used as the core of a novel reservoir modeling approach known as Surrogate Reservoir Modeling. This chapter concentrates on introducing Surrogate Reservoir Models (SRMs). For this purpose, a review on artificial neural networks is presented. Next the definition, classification, and characteristics of an SRM will be discussed. In order to use the SRM in an automated history matching workflow, an optimization method is utilized. The last section of this chapter goes through this optimization method.

\subsection{Learning from data}

In the pattern recognition concept, the data analysis process deals with the predictive modeling. By having a high dimensional database, the objective is to learn the underlying behavior in the data and forecast the performance of unforeseen examples. The learning process refers to some form of algorithm to reduce the error on the set of training data (R. O. Duda, P. E. Hart, and D. G. Stork 2001). The learning procedures could be distinguished into (i) supervised learning or (ii) unsupervised learning (recently another set of learning has been discussed in the literature called semi-supervised) (Jain 2009). Supervised learning generally represents a learning procedure which takes down an available set of inputs and also known corresponding outputs. The efforts will be made to build a predictive model by matching the available responses with the inputs. This predictive model is able to generate reasonable predictions for the response to novel data. The most important characteristic of this learning technique is that the responses (outputs) are recognized or labeled in the training database. On the other hand, unsupervised learning involves only unlabeled data which makes the process 
more challenging than the previous one. In other words, unsupervised learning forms clusters or natural patterns underlying in the structure of data. In our case we develop surrogate models using the available training examples of reservoir simulation models. In other words, we face examples with known outputs; therefore this case mainly involves the supervised learning process. This is valid for the modeling style available in the artificial neural networks, which will be covered in this chapter.

\subsection{Artificial neural networks}

\subsubsection{Introduction}

One of the most famous pattern recognition techniques, which has a long and prosperous history in a variety of scientific fields, is Artificial Neural Networks (ANNs), usually called Neural Networks (NNs). There are characteristics which distinguish ANNs from the traditional modeling methods. ANNs are non-linear data driven, fact, and example based and most importantly a self-adaptive approach (Kriesel 2011). Their pattern recognition ability is more highlighted when there are unknown and complex relationships between different parameters. Similar to the way that the human brain processes the non-linear and complex data, the ANNs can identify and learn the patterns between the sets of inputs and the corresponding output sets (S. D. Mohaghegh, Virtual intelligence applications in petroleum engineering: part 3 - fuzzy logic 2000). After the training step (learning from the provided examples known as training set), ANNs are able to predict the outcome of independent data which have not been used during the training process. One important feature of ANNs is that they can process the problems which even have incomplete and noisy data (S. D. Mohaghegh 2009). Therefore, they are ideally suited for the modeling the problems from the oil and gas industry which are known to have uncertain and imprecise data. Another important characteristic of ANNs is their self-adaptive nature. An ANN adjusts its structure based on the output and input information that flows through the network during the learning phase (S. D. Mohaghegh 1995). This feature plays a vital role in application areas where learning by example is dominant.

Historically ANNs were motivated by the goal of having machines that are able to mimic the brain's behavior (Haykin 2008). In fact, ANN includes an interconnected group of artificial neurons and its structure is an idea coming originally from the human neural system. However, the "network" in ANNs is more a mathematical/statistical concept than neuroscience. ANNs are physical cellular systems capable of obtaining, storing information, 
and using experimental knowledge (S. D. Mohaghegh 2009). There are several distinguished characteristics of ANNs which make them an efficient tool to solve the non-linear and complex problems (Haykin 2008, S. D. Mohaghegh 2011, Kriesel 2011, S. D. Mohaghegh 2014):

- Non-linear nature of neural networks is suitable for many real-life problems. Stock market prediction and weather forecast are examples in which non-linear characteristic of ANNs has been successfully implemented.

- Unlike the statistical and mathematical based methods, artificial neural networks are not limited by predetermined functions. In fact they are data driven models. Thus they are able to identify the non-linear relations among different parameters in the database without a prior assumption of the relation between the input and target values (Hagan, Demuth and Beale 1996).

- The ability of ANNs to learn by examples. ANNs can learn the hidden patterns and trends of the problem from the known examples.

- The generalization ability of ANNS; this helps to predict new outcomes from the past performance.

- The capability of ANNs to catch the full patterns from the incomplete and noisy data.

- Parallel processing capability of ANNs gives the option to process information faster.

- ANNs are constrained to the data used for training them. That means they may not show a good performance on the data outside of the range of inputs which they have seen during the training process. However, neural networks have the capability to adapt the network parameters to the adjustments in the system. A trained network can be easily retrained by introducing new information. This feature is important when the system under study is dynamic (for example stock market or receiving new pressure data from the field). Therefore, neural networks can be simply trained again by having new data.

\subsubsection{Basics of artificial neural networks}

The phrase of "neural network" consists of two words: the first word "neural" is the adjective form of neuron which in the field of medical science is the basic unit of the nervous system and describes a cell that transmits the messages from the brain to other parts of body (Merriam Webster Dictionery Online 2014). The second word, "network", is referred to as a group or system of interconnected people or objects. The terminology of artificial neural networks has originated from the analogy of biological neural networks (Kriesel 2011). A biological neuron 
is composed of different parts; each neuron receives the signals through synapses which are located on the dendrites or membrane of the neuron. As the received signals are strong enough to pass a threshold, the neuron is activated and sends out a signal to the other neurons through the axon (Kriesel 2011). The human brain is made up of $10^{11}$ cells which are connected with approximately $10^{15}$ connections (synapses) (Kriesel 2011). Figure 7 depicts the structure of a biological neuron. Each neuron works with the frequency of 1 to $100 \mathrm{~Hz}$; therefore, the human neural network is able to handle $10^{18}$ operations per second. This rate is much higher than the performance of the best available computers that the human being has created so far (AGH University of Science and Technology webpage 2014). This structure gives the learning and pattern recognition abilities to the human brain.

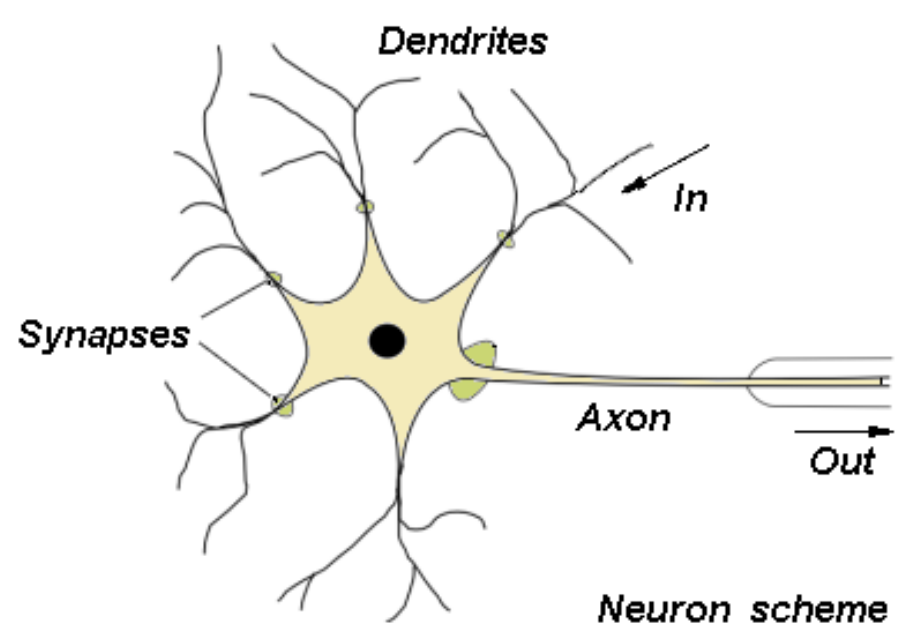

Figure 7: The scheme of neuron (cell) and its components (AGH University of Science and Technology webpage 2014).

The artificial neuron is much simpler than the biological one. It is basically consists of several inputs, $\left[y_{1}, \ldots, y_{k}\right]$ (similar to synapses); the input signals are multiplied by weights, $\left[w_{1 j}, \ldots, w_{k j}\right]$ (representing the strength of respective signals) and then makes a summation. Next, a mathematical function known as activation function, $f\left(x_{j}\right)$, determines the activations of the neuron. Finally, another function calculates the output. The artificial neural networks are a combination of neurons which process the information in parallel. Figure 8 demonstrates the structure of an artificial neuron. 


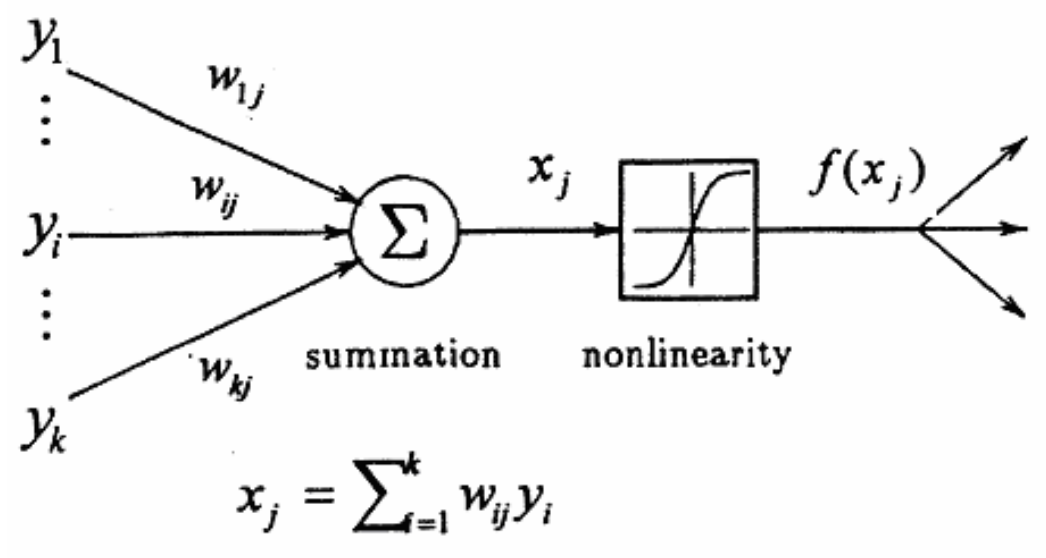

Figure 8: A single artificial neuron (Kumar and Thakur 2012).

The most common activation function is sigmoid function defined as the following equation (Kriesel 2011). The output of sigmoid function is between zero and one.

Equation 1: Sigmoid function as the activation function used in the ANNs algorithm.

$$
f(x)=\frac{1}{1+e^{-x}}
$$

The learning procedure in ANNs is supervised learning; ANNs have an input layer and an output layer (R. O. Duda, P. E. Hart, and D. G. Stork, 2001). In addition to the input and output layers, ANNs have at least one more layer known as the hidden layer as it is shown in Figure 9. Usually all the computations are performed in the neurons located in the hidden layer.

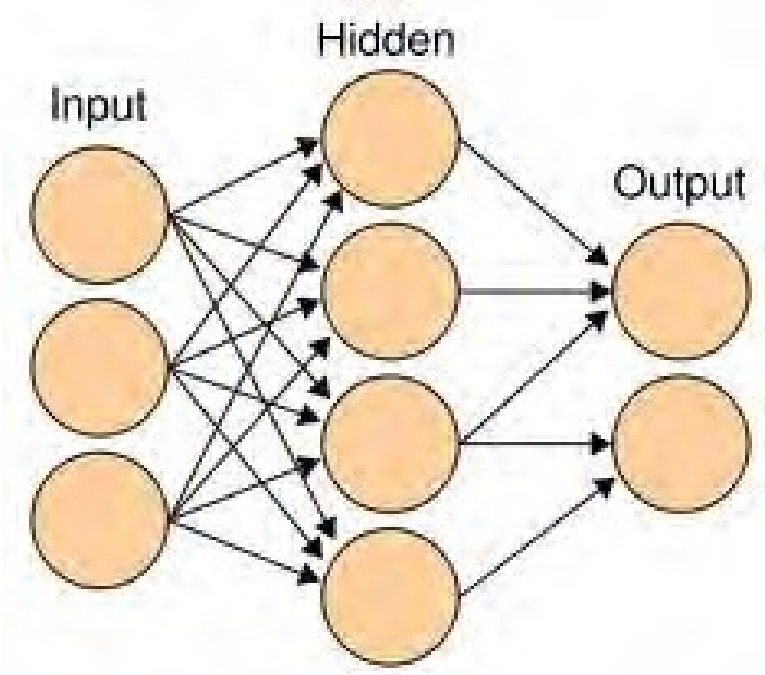

Figure 9: The structure of ANNs. 


\subsubsection{Feed Forward Neural Networks}

An ANN is a data processing system including a network of interconnected processing elements (artificial neurons). The way that these neurons are arranged and connected to each other is defined as the architecture of neural network. There are several types of architecture available in the applications and literature. The most commonly used architecture is feed forward neural network (Kriesel 2011). As the name of this architecture indicates, the flow of information is "forward" and in one direction. Figure 10 shows the structure of a feed forward neural network with three hidden layers. There is always one input layer and one output layer; also one hidden layer is required. The hidden layers are disconnected with the external world and therefore are called hidden layers. The neurons in each layer are connected to the neurons on the next layer. The flow of information is from the input layer to the hidden layers and finally to the output layer. In addition in feed forward architecture neurons in the same layer are not connected to each other or to the neurons in the previous layers. If we recall the definition of supervised learning, this type of learning is associated with labels (outputs), therefore, we have supervised learning in feed forward neural networks (R. O. Duda, P. E. Hart, and D. G. Stork, 2001).

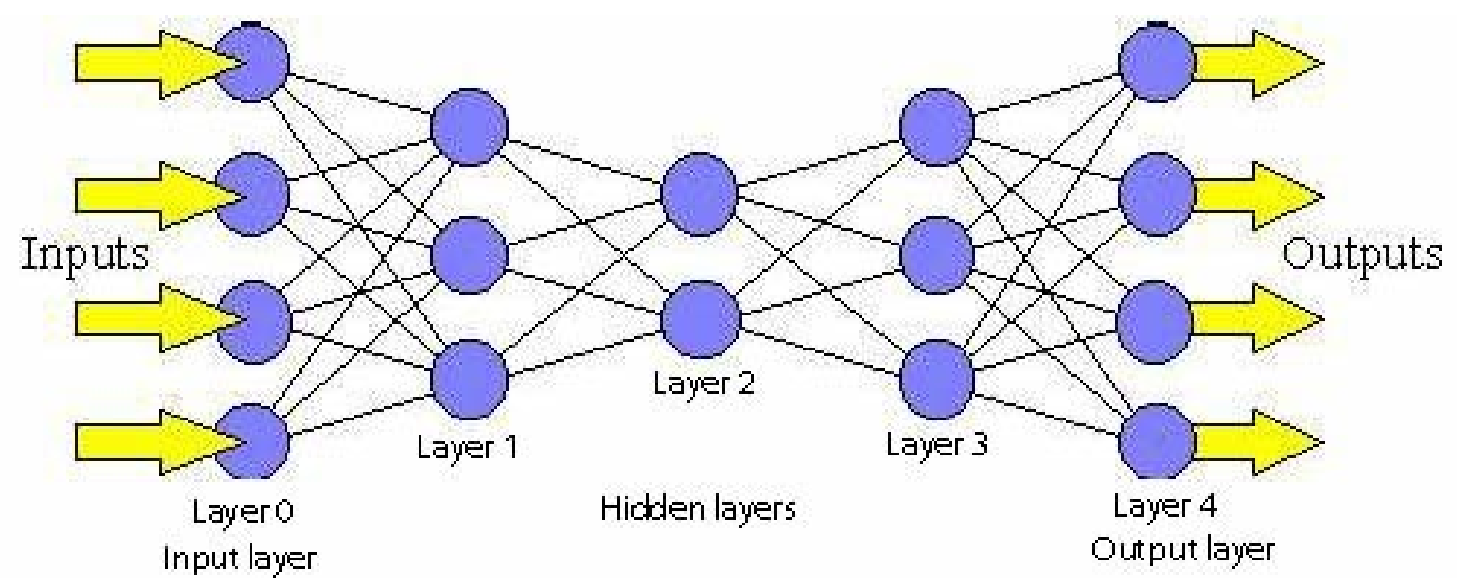

Figure 10: Feed forward neural network architecture with three hidden layers (Roberts 2000).

The learning process from the provided data is an iteration approach of adjusting the weights between the inputs and known output values. The most common learning algorithm for feed forward networks is back propagation (BP) (Fausett 1993, Rojas and Feldman. 1996).When the training data set is ready, the samples are fed to the network. Then the network produces some outputs based on the available weights (randomly selected at the initial step). The outputs are compared to the known-correct outputs and the mean squared error is calculated. Next the error value is propagated backwards (this is the reason for calling the process back 
propagation) and the weights are adjusted. By having the new set of weights, the whole process is repeated until the optimization process meets the stoppage criteria. The stoppage criteria could be an error threshold, time, or user intervention. It should be noted that the optimization process during the training step is unable to find the global optima and therefore the stoppage criteria is required.

Those who interested in further reading about neural networks are encouraged to refer to available reference materials (Fausett 1993, Rojas and Feldman. 1996, Haykin 2008, Kriesel 2011).

\subsubsection{Applications of artificial neural networks}

Since the advent of ANNs (W.S. McCulloch and W.H. Pitts 1943), they have seen different stages of rise and fall; however, in the recent years, ANNs have appeared as a practical technology with many successful applications in sale and marketing, finance, energy, geology, etc. Some applied applications of ANNs are listed in Table 2.

Table 2: Application of ANNs in the different fields.

\begin{tabular}{|ll|}
\hline Sales forecasting & (D.H.F. Yip, E.L. Hines, and W.W.H. Yu 1997) \\
\hline Industrial process & (G.Glan Devadhas, S. Pushpakumar, and D.M.Mary Synthia Regis Prabha \\
& $2012)$ \\
\hline Customer research & (M. Chattopadhyay, P. K. Dan, S. Majumdar, and P. S.Chakraborty 2012) \\
\hline Risk management & (S. Aless, R. Sarcià , G. Cantone, and V. R. Basili 2007) \\
\hline Credit evaluation & (B. Baesens, R. Setiono, C. Mues, and J Vanthienen 2003) \\
\hline Energy cost prediction & (M. Yalcintas, S. Akkurt 2005) \\
\hline Medical diagnosis & (F. Amato, A. López, E. María Peña-Méndez, P. Van̆hara, and A. Hampl \\
& 2013) (S. Lei and W. Xing-cheng 2010) \\
\hline Business applications & (E. Y. Li 1994) \\
\hline Financial applications & (Tan 2004) \\
\hline Stock & \\
\hline markediction & (A. A., et al. 2012) \\
\hline
\end{tabular}




\subsubsection{ANNs in petroleum engineering}

Although ANNs have been around for a long time, their popularity in petroleum engineering started two decades ago (J. K. Ali 1994). Since that time, the applications of ANNs in addressing conventional problems of the petroleum industry have been widely studied. Some examples found in literature are: well log interpretation (Baldwin, Otte and Whealtley 1989, Masoud 1998, Jong-Se and Jungwhan 2004), well test data analysis (Al-Kaabi and Lee 1990, Ershaghi, et al. 1993, Athichanagorn and Horne 1995, Sultanp and Al-Kaabi 2002), reservoir characterization (Mohaghegh, et al. 1995, Ahmed, et al. 1997, Singh, et al. 2008), calibration of seismic attributes (David 1993), seismic pattern recognition (Yang and Huang 1991), inversion of seismic waveforms (Roth and Tarantoia 1992), prediction of PVT data (Briones, et al. 1994, Gharbi and Elsharkawy 1997, Osman, Abdel-Wahhab and Al-Marhoun 2001, Oloso, et al. 2009), fractures and faults identification (L.Thomas and Pointe 1995, Key, et al. 1997, Sadiq and I.S. Nashawi 2000, Aminzadeh and deGroot 2005), hydrocarbons detection (Cheng-Dang, et al. 1994, Aminzadeh and deGroot 2005), formation damage forecast (Nikravesh, et al. 1996, Kalam, Al-Alawi and Al-Mukheini 1996), and more.

\subsection{Surrogate Reservoir Models (SRM)}

Surrogate reservoir modeling is the terminology used to describe the new way of reservoir modeling and simulation which is based on using artificial intelligence and data mining (AI\&DM) techniques. Nevertheless, it is not correct to generalize the concept of surrogate reservoir modeling to any type of experiment that is involving AI\&DM (S. D. Mohaghegh 2014). This approach is compared to the conventional methods which the oil and gas industry have used during the last half of century. The numerical simulation and modeling techniques are the leader of the conventional approach in our industry.

Surrogate Reservoir Models (SRMs) are relatively new tools for fast track and comprehensive reservoir analysis which originate from the existing reservoir simulation models. In other words, SRMs are approximations of the full field three dimensional numerical reservoir models and are capable of accurately mimicking the behavior of these full field models (S. D. Mohaghegh 2014).

\subsubsection{Why do we need SRMs?}

The question which may arise is why we should make a model from the numerical reservoir simulation model. In order to answer this question, we should investigate the characteristics of numerical simulation models and their applications in reservoir study. Reservoir simulation is 
known as the standard tool used by industry for reservoir management and study (Ertekin, Abou-Kassem and King 2001). The reservoir simulation model is developed by the integration of static and dynamic reservoir data. This simulation model is the major reference of information when a decision should be made in the reservoir management workflow. The full field reservoir simulation models are utilized in industry to perform sensitivity analysis, history matching, production optimization, and uncertainty assessment. These are the typical tasks in a reservoir study in order to secure the investment and also increase the rate of return for an asset. Each one of these tasks requires hundreds realizations of the reservoir. On the other hand, during the life of a reservoir when the new data are acquired, the reservoir simulation model usually goes through modifications. These modifications commonly lead to resolution enhancement or increases in the number of layers and grid blocks. As the number of grid blocks increases, so does the time required to make a run. As we talked about it earlier, using techniques such as optimization algorithms and applying parallel processing might reduce the number of runs to achieve the goal of simulation. However, the number of simulation runs is still too high to be practical. Furthermore, with the growth of smart field technology in the oil and gas industry, the necessity for a real time reservoir modeling tool is inevitable. Consequently the answer to all the issues associated with reservoir simulation models is to find an alternative tool. At the same time this tool should keep the accuracy and resolution of simulation models and also have a high speed to perform the tasks required in a reservoir management workflow.

Here the pattern recognition capabilities of SRM come into play. Despite the geo-statistical based approaches (such as response surfaces, reduced order models, etcetera) that require hundreds of simulation runs (Carreras 2006, B. Li 2005, Salhi 2005), SRM is created by a few simulation runs (S. D. Mohaghegh 2011). The accuracy of SRM is guaranteed by validating over the realizations of the reservoir model which are not used during the SRM development. After the training and validation of SRM, it is able to reproduce the outputs of full field model with the high accuracy and same resolution but with a very fast pace (usually takes fractions of a second for a single run). The reasons behind the accuracy and high speed of SRM to replicate the behavior of the simulation model go back to the characteristics of neuro-fuzzy system used in SRM development. The neuro-fuzzy system in SRM could be different AI\&DM techniques. In this study this technique is artificial neural networks, which we covered earlier. 


\subsubsection{Classifications and applications of SRMs}

Different types of SRMs are categorized based on the outputs or functionality of these models. Two of the most common ones are grid-based and well-based SRMs. The well-based SRM is developed to give the outputs of the reservoir simulation model at the well such as production and injection profiles. In addition, grid-based SRMs are particularly designed to generate the outputs at the grid level like pressure and phase saturation distributions. Obviously for a full field model, well-based and grid-based SRMs could be coupled in order to generate the full results of simulation models. For a study like history matching, since we deal with production and pressure profiles at the well, we need to develop the well-based SRM. Therefore, we have well-based surrogate reservoir models in this study.

SRM has been successfully tested with several commercial reservoir simulators such as ECLIPSE $^{\mathrm{TM}}$ (Schlumberger 2014), IMEX ${ }^{\mathrm{TM}}$ and GEM $^{\mathrm{TM}}$ (CMG 2013) with models up to 6.5 million cell blocks (Intelligent Solutions, Inc. 2014). In 2006 (S. D. Mohaghegh, C. A. Modavi, et al.), SRM was presented for the first time by Shahab Mohaghegh to solve the problem of time-consuming runs for an uncertainty analysis of a giant oil field with 165 horizontal wells in the Middle East. The reservoir simulation model included about one million grid blocks and took 10 hours to run using a cluster of twelve $3.2 \mathrm{GHz}$ processors. Therefore, using the simulation model was a major issue for the uncertainty analysis study which requires hundreds of realizations of the reservoir simulation model. In his study, SRM was used as an objective function for a Monte Carlo Simulation to build hundreds and thousands of simulation runs in a very short time compared to the numerical simulator (S. D. Mohaghegh, C. A. Modavi, et al. 2006). Later in another publication (2009), Mohaghegh et al. presented the results of the SRM prediction which were made in 2006. The results showed the accuracy of SRM predictions.

In another study performed by Jalali and Mohaghegh (2009) an SRM was trained, calibrated, and validated only using only eight simulation runs. The SRM was used to substitute the reservoir simulation model for the uncertainty quantification of a complex coal-bed methane reservoir model.

Amini et al. $(2012,2014)$ presented the results of application of a grid-based SRM to perform a real case $\mathrm{CO}_{2}$ sequestration project. In this project $\mathrm{CO}_{2}$ was injected into a depleted gas reservoir located in Otway, Australia. They used SRM to predict the distribution of pressure and $\mathrm{CO}_{2}$ throughout the reservoir in a matter of seconds. The SRM was applied to analyze the geological uncertainty and also study the performance of the reservoir under different 
operational constraints. The numerical reservoir simulation model was made using a commercial simulator and consisted of $100 * 100 * 10$ grid blocks.

In projects, like $\mathrm{CO}_{2}$ storage and sequestration, a comprehensive study is required to assure a safe and efficient process. This study usually includes short and long term periods of monitoring $\mathrm{CO}_{2}$ plume in the formation. The intricacy of simulating multiphase flow, having a large number of time steps required to study injection and post-injection periods of $\mathrm{CO}_{2}$ sequestration, highly heterogeneous reservoir, large number of wells, etcetera result in a highly complicated reservoir model. A single realization for such a reservoir takes hours to run. Additionally, a thorough understanding of the $\mathrm{CO}_{2}$ sequestration process requires multiple realizations of the reservoir model. Consequently, using a conventional numerical simulator makes the computational cost of the analysis too high to be practical. Application of SRM in this type of study could be very valuable. Shahkarami and his colleagues (2014) developed a surrogate reservoir model for a carbon storage project which requires injection and postinjection monitoring of $\mathrm{CO}_{2}$ for a thousand year period. Each realization of the reservoir simulation model took 4 to 24 hours (depending on the convergence) to run; while the developed SRM ran in a matter of seconds (a single run). The authors used less than 15 simulation runs to develop the SRM.

Mohaghegh et al. (2012a, 2012b) have discussed the results of several projects involving surrogate reservoir models for fast track analysis of numerical simulation models. Other publications regarding the SRM can be found in variety of reference materials (S. D. Mohaghegh, J. S. Liu, et al. 2012b) (S. D. Mohaghegh 2009, S. D. Mohaghegh 2011, S. D. Mohaghegh 2014).

\subsubsection{Characteristics of SRMs}

SRM as a relatively new technology has proved itself as an effective tool to address many time-consuming processes performed by numerical simulation models. The main characteristics of SRM are listed as (S. D. Mohaghegh 2014):

- Low development cost and labor. Unlike the traditional proxy models used in the oil and gas industry (response surfaces, reduced order models ...) which require hundreds of simulation runs, SRM is created using only a few realizations of the reservoir simulation model. There are two main reasons behind this unique ability of SRM. First is the pattern recognition ability of AI\&DM techniques used to develop SRM. The second reason is the way in which a single realization of the reservoir simulation 
model is presented to SRM.

- High implementation pace with low computational cost. When it comes to the implementation of SRM, it has remarkably low computational cost with a high pace. The computational cost of SRM can be divided into two phases: the development phase and the execution phase. Literally the main computational cost of SRMs belongs to the development stage. For the execution phase, SRM can easily be implemented on a normal PC workstation or a laptop and get involved in the reservoir management workflow (S. D. Mohaghegh 2014).

- Short development time. Compared to the traditional reservoir simulation model it takes a short time to develop a surrogate reservoir model. This time, depending on the size and complexity of the problem, varies from weeks to months (S. D. Mohaghegh 2014).

- Example based and case-subjective. The learning ability of SRM is conditioned to the training examples. Depending on the nature of the examples and the strength of the provided database you can generally expect an SRM to be trained reasonably well. The main limitation of SRM could be the dependency of SRM on the training examples (database). That means you cannot train an SRM for an example and expect it to give the same accuracy for a completely irrelevant example. Giving an instance related to petroleum engineering, an SRM trained for a specific reservoir does not necessarily have a good performance for another reservoir.

- Honor and preserve the physics behind the problem. The nature of geo-statistical based proxy models disconnects them from the physics of the problem. The reason behind this issue is because these types of proxy models are limited by the strict assumptions of normality, linearity, variable dependence, and etcetera. Therefore, geostatistical based proxy models are not able to capture the physics and the non-linear relations which exist among the parameters in real-life. This is not the case for the SRMs. The AI\&DM techniques (such as ANNs), used to develop an SRM, utilize a different type of learning and that makes it easy for SRM to understand the physics hidden in the training examples.

\subsubsection{The keys in developing an SRM}

SRMs are developed using the data extracted from the realizations of the simulation model. Depending on the objective of the study, these realizations can vary in geological properties or/and operational conditions. For example, for a history matching study of reservoir 
characteristics and for an uncertainty analysis study a combination of reservoir properties and operational constraints can be changed. The data then are extracted to form a spatio-temporal database. Building this database is the first step in developing surrogate reservoir models. The main objective of this database is to teach the model the process of fluid flow phenomena in the reservoir. Therefore, meticulous efforts should be considered in this part of process. The quality and quantity of this database determine the degree of success in developing a successful SRM. Not dedicating enough attention to this part of the process is the main reason behind unsuccessful attempts at applying artificial intelligence based models in the literature (Zubarev 2009). In this section we will go through different steps in building a surrogate reservoir model. Additionally, Mohaghegh has thoroughly discussed different steps of SRM development in his paper (2011).

In order to create the spatio-temporal database, the first step is to identify the number of runs that are required to develop the SRM. The purpose of having different realizations of a reservoir simulation model is to introduce the uncertainties involved in the model to the SRM. This is a common step in building SRM and developing response surface methods; however, there is a key difference between these two methods and that is the functional forms behind these models. Response surface and other reduced order models are developed using statistical approaches, which use predetermined functional forms. For example, response surface proxy models which use regression have a fixed structure with different components and coefficients. These coefficients then are adjusted during the training process; however, the structure of function (for example polynomial function) is fixed (Eide, et al. 1994, White and Royer 2003, Alessio, Bourdon and Coca 2005, Gupta, et al. 2008, Cheng, Dehghani and Billiter 2008, Arwini and Stephen 2011). In order to match these functional forms, hundreds of runs are needed. Therefore SRM, due its learning structure, is not constrained to these types of functions.

The pattern recognition characteristics of SRMs enable them to be developed by having a small number of simulation runs. Nevertheless, there is no algorithm to find the optimum number of simulation runs to build an SRM. The common practice when choosing the best number to train the SRM is to use rules of thumb based on the intricacy and heterogeneity of the reservoir model, which might vary. Yet, it is obvious that if the number of simulation runs is too small, the SRM may not be able to catch the uncertainty and the variation in the parameters. In this situation the surrogate reservoir model might even show good results for the training samples; here is where the validation sample plays an important role. Although 
SRM might have a good performance on the training sample, they will fail to create the same quality for the validation set. Therefore, the validation examples will expose the lack of required information in the training samples (Mohaghegh, Liu, et al. 2012a, 2012b). Alternatively, if the number of simulation runs is too large, there is no reason to develop an SRM since the solution is close to the original problem, which is a high number of simulation runs. This is a problem which occurs frequently for case studies involved geo-statistical based proxy models. In these cases the cost of developing the proxy model is too high and does not justify using them.

After running the realizations, the static and dynamic data are extracted in order to build the representative spatio-temporal database. The database includes different types of data such as static and dynamic reservoir characteristics, operational constraints, etc. Static data refers to properties of the reservoir that are not changing overtime, such as permeability, porosity, top depth, and thickness. Dynamic data refer to any data such as well constraints or pressure and phase saturations that are not necessarily constant overtime (S. D. Mohaghegh, J. S. Liu, et al. 2012b).

Once the database is prepared, it is ready to be used to train the SRM. Usually some preprocessing steps are performed before the training starts. The inputs to train the SRM then are selected. The training process includes training the neuro-fuzzy agents which form the SRM. In this study we have artificial neural networks. Training process of artificial neural networks has three main parts: training (learning), calibration, and validation. Therefore, the spatio-temporal database is divided into three corresponding sets. The training set is used to train the ANNs. The calibration set is used to control the learning process and finally the validation set is for testing the trained network. A further validation step in the SRM development is utilized to assure its robustness. This step is referred to as "Blind Verification". It is called "blind" because it is a set of realizations that has not been used during the training process. These blind testing sets are complete realizations of the reservoir, while the verification set used in the training process is a randomly selected portion of the spatio-temporal database.

We will cover each step required to build an SRM in a practical way in the next chapter. 


\subsection{Differential Evolution optimization algorithm}

Nowadays an important option in history matching workflow is the automation ability. In other words, the developed workflow should be able to perform following items without human involvement:

1. Select the value of adjustable parameters; range of parameters can be provided by the engineer.

2. Call the reservoir model to compute the output; the reservoir model could be the simulation model or an approximation of simulation model such as SRM.

3. Calculate the objective functions; the objective functions can be provided by the user.

4. Measure the misfit; quantify the error between the measured data and simulated data.

5. Finally, decide if the misfit value is satisfying; the stoppage criteria here can be design by the engineer.

For accomplishing the mentioned items two main tools are required. The first one is a reservoir model (simulation model or an approximation of it such as SRM) and the second tool is an optimization algorithm. The reservoir model produces the response of the reservoir by having the input data. However, other items in the above list are done by the optimization algorithm. In the previous chapter we reviewed numerous applications of optimization methods in history matching. In this study the reservoir model is an approximation of reservoir simulation model (a surrogate reservoir model) and the optimization tool is a method from the class of evolutionary algorithms (EAs) known as Differential Evolution (DE). DE recently has shown great performance for petroleum engineering case studies (Wang and Buckley 2006) (Decker and Mauldon 2006, Jahangiri 2007, Hajizadeh, Christie and Demyanov 2009, 2010, Wang and Gao 2010, Wang, Gao and Yang, et al. 2011, Mirzabozorg, et al. 2013) (Okano 2013). In this section we talk about the necessity of having an optimization method and then introduce DE as a novel optimization algorithm.

\subsubsection{Optimization}

Optimization is a daily life problem. Selecting the best option in our decisions are optimization problems; these problems could be selecting the best route of transportation, buying the best brand of milk, choosing the best hobby to do, etc. In each one of these decisions we look for the best solution to reach a certain goal or satisfy a necessity. Merriam Webster dictionary defines the word "optimization" as "an act, process, or methodology of making something (as a design, system, or decision) as fully perfect, functional, or effective as possible" (Merriam Webster Dictionery Online 2014). In mathematics and computer science, optimization is the 
process of selecting the best item from the group of different options (Wikipedia 2014). In the simplest case, we are seeking the largest (maximum) value or the smallest (minimum) value that a function can take.

Since the advent of automated history matching, it has used many optimization methods in its framework. In the early years, gradient descent methods were popular (Slater and Durrer 1971, Gavalas, Shah and Seinfeld 1976). In these algorithms the next step is decided based on the value and direction of function gradient at the current step. These methods usually are too slow and often not able to converge to the global optimum when there are number of local minima in the solution space. In addition, sometimes calculating the gradient of objective function which is required in these methods is not easy task to perform (Anterion, Eymard and Karcher 1989). By starting the modern era in history matching at the beginning of 90's, global optimization methods, such as simulated annealing, came to help find the global optima in history matching (Ouenes, Meunier and Moegen 1992a, Ouenes, Fasanino and Lee 1993a, Ouenes, Brefort, et al. 1993b). Later evolutionary algorithms such as genetic algorithms and evolutionary strategies replaced simulated annealing due to their performance and the way they handle the high number of parameters (Sen, et al. 1995, Romero, Carter and Zimmerman, et al. 2000a, Romero, Carter and Gringarten, et al. 2000b, Schulze-Riegert, et al. 2001, Williams, et al. 2004, Al-Shamma and Teigland 2006). As the new century started many researchers tried to experiment with other types of optimizations methods in history matching. Almost all of these methods were already used in the other majors and passed their test. Particle Swarm Optimization (Eberhart and Yuhui Shi 2001, Mohamed, Christie and Demyanov, Comparison of Stochastic Sampling Algorithms for Uncertainty Quantification 2009, 2010, Rwechungura, Dadashpour and Kleppe 2011, Kathrada 2009), Ant Colony Optimization (Razavi and Jalai-Farahani 2008, Hajizadeh, Christie and Demyanov 2009, 2010), Neighborhood algorithm (Christie, MacBeth and Subbey 2002, Stephen, et al. 2006, Rotondi, et al. 2006, Subbey and Christie 2003), Tabu search (Yang, Ngheim and Card 2007), etc. were among those.

Recently, a new optimization method known as Differential Evolution (DE) which belongs to the class of evolutionary algorithms has been used in numerous case studies of history matching (Hajizadeh, Christie and Demyanov 2009, 2010, Wang and Gao 2010, Wang, Gao and Yang, et al. 2011, Mirzabozorg, et al. 2013, Okano 2013). This optimization method has been tried in many other problems outside of the oil industry and showed multiple strengths over the other global optimization methods. For instance, compared to other global 
optimization algorithms (such as genetic algorithm) the convergence rate is faster and needs fewer control parameters. It is easy to develop (program) and utilize DE. Like most of other global optimizers it can be fit in a parallel computing workflow. DE is an evolutionary algorithm and like similar approaches is inspired from the evolution mechanism. In continuation, we try to go through the details of evolutionary algorithm and then elaborate more on DE.

\subsubsection{Evolutionary Algorithm (EA)}

Evolutionary Algorithms are inspired by biological mechanisms of evolution related to theory of Charles Darwin (Wikipedia 2014). Interestingly the adaptation of Darwinian Theory in optimization problems is older than the advent of computational machines (Wikipedia 2014). The theory indicates that in a population of individuals those survive who can adapt themselves according to environmental conditions (survival of the fittest). For each environment obviously the fitness is different. Depending on the quality of each individual in population, the individual can stay in the population for the next generation or get eliminated.

Evolutionary Algorithms (as well as DE) are classified as stochastic population based systems (Price and Storn 1997). These optimization methods are called population based because they consist of multiple individuals, in which the interaction among them will lead to improvements in the quality of solutions.

Evolutionary algorithms differ from traditional techniques of optimization in the learning phase. While traditional methods do not have a learning step, evolutionary algorithms utilize the experiences of the unsatisfied and satisfied candidates in the previous generations. Therefore, evolutionary algorithms are considered as a subfield of artificial intelligence (Eiben and Smith 2003).

Different techniques of evolutionary algorithms have a general concept in common. There is always a population of individuals within an environment which compete for the limited resources. Based on the evolutionary mechanism the individuals will be passed to next generation which can adapt themselves according to environmental conditions. In this case, the candidate solutions in an optimization problem are the individuals and the fitness function is the environmental conditions (the higher the better). Survival of the fittest happens based on the fitness function and the stronger individuals are selected and will be passed to the new generation (selection). Therefore, the selection mechanism assures the increase in the quality. The process of creating new individuals for new generations makes sure to force the diversity 
to facilitate the novelty (through the mutation and recombination steps). Evolutionary algorithm is a stochastic optimization method because the variations happening during creation of new generation are randomly chosen. Furthermore, the selection process could be either deterministic or stochastic. For example, consider the chance for weak individuals (by random) to be passed to the new generation.

To summarize the process of EAs, let us assume we wish to find the optimum values of a given objective function. The first generation of individuals (initial set of solutions) is randomly selected. The objective functions are calculated for this set of individuals. The selection criteria are based on fitness function--the higher the better. Therefore, the fitness function allows us to select the candidates which are better than the parents for the new generation. The new individuals are created based on applying mutation and recombination rules on the parents. In this process recombination is an operator applied to two or more selected elements (old generation) and results in one or more new candidates (the children). Also mutation is applied to one candidate and results in one new candidate. This process will repeat until the candidates meet the termination (stoppage) criteria. The termination criteria could be after a number of generations or time or obtaining a value of objective function.

The following items are the general steps in an evolutionary algorithm (Eiben and Smith 2003):

1. Initial generation: Create the initial population of individuals randomly.

2. Fitness evaluation: Calculate the fitness value for each individual in the population.

3. Repeat the following tasks on the generation until the termination criteria are met:

a) Selection of parents: Choose the best-fit individuals for reproduction.

b) New generation: Breed new individuals through recombination (crossover) and mutation operations to give birth to offspring.

c) Function evaluation: Evaluate the individual fitness of new individuals.

d) Survival of the fittest: Replace least fit population with new individuals.

Figure 11 describes the process in a schematic way. 


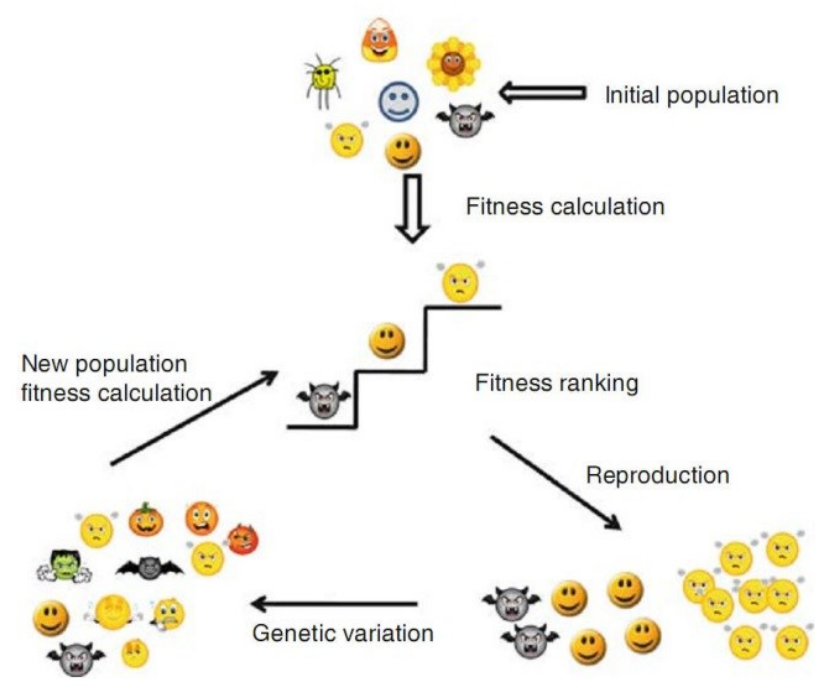

Figure 11: Schematic for evolutionary algorithms.

\subsubsection{Differential Evolution}

The Differential evolution (DE) algorithm was developed by Storn and Price (1995) as a stochastic population based algorithm for continuous and real-valued numerical optimization problems (Storn and Price 1997, Price and Storn 1997, Price, Storn and Lampinen 2005) . As it was mentioned earlier, DE belongs to the category of evolutionary algorithms and, like other EA methods (such as genetic algorithms), it has mutation, recombination, and selection steps. Because of simple mathematical structure, DE is considered as a very effective global optimization algorithm. Since the advent of DE, it has been applied to many engineering problems (Thomas and Vernon 1997, R. Storn 1996, R. Storn 1999, Liu and Lampinen 2002). Similar to a genetic algorithm DE is able to be used in a parallel processing framework. But unlike a genetic algorithm, DE is famous for fast convergence (Price, Storn and Lampinen 2005).

In the following, we go through the details of different steps in DE algorithm:

Let us assume we have a function with D real parameters, which we wish to find the optimum values. To start assume we select the size of population as $\mathrm{N}_{\mathrm{p}}$. Since some mutation and recombination rules require at least four individuals, $\mathrm{N}_{\mathrm{p}}$ must be at least four. Therefore, the parameter vectors are like:

Equation 2: Parameter vector in DE algorithm.

$$
x_{i, G}=\left[x_{1, i, G}, x_{2, i, G}, \ldots, x_{D, i, G}\right] .
$$

Which $\mathrm{i}=1,2, \ldots, \mathrm{N}_{\mathrm{p}}$. and $\mathrm{G}$ is the generation number. Also, again, $\mathrm{D}$ is the dimension of each vector (number of parameters). 


\section{Initialization and fitness evaluation}

For this step, the upper and lower bounds of each parameter should be defined. Then individuals in the initial generation are selected randomly within the intervals of $\left[\mathrm{x}_{\mathrm{j}}^{\mathrm{L}}, \mathrm{x}_{\mathrm{j}}^{\mathrm{U}}\right]$.

$$
\mathbf{x}_{\mathbf{j}}^{\mathbf{L}} \leq \mathbf{x}_{\mathbf{j}, \mathbf{i}, \mathbf{1}} \leq \mathbf{x}_{\mathbf{j}}^{\mathbf{U}}
$$

Equation 3: Randomly selection of initial generation.

$$
\mathbf{x}_{\mathbf{j}, \mathbf{i}, \mathbf{0}}=\mathbf{x}_{\mathbf{j}, \min }+\operatorname{rand}_{\mathbf{i}, \mathbf{j}}[\mathbf{0}, 1] .\left(\mathbf{x}_{\mathbf{j}, \max }-\mathbf{x}_{\mathbf{j}, \min }\right) .
$$

Here rand $[0,1]$ is a random real number between zero and one. Also $x_{j, m i n}$ and $x_{j, \max }$ are minimum and maximum of $\mathrm{j}^{\text {th }}$ parameter in vector $\mathrm{x}$. Figure 12 summarizes the initialization step in DE algorithm.

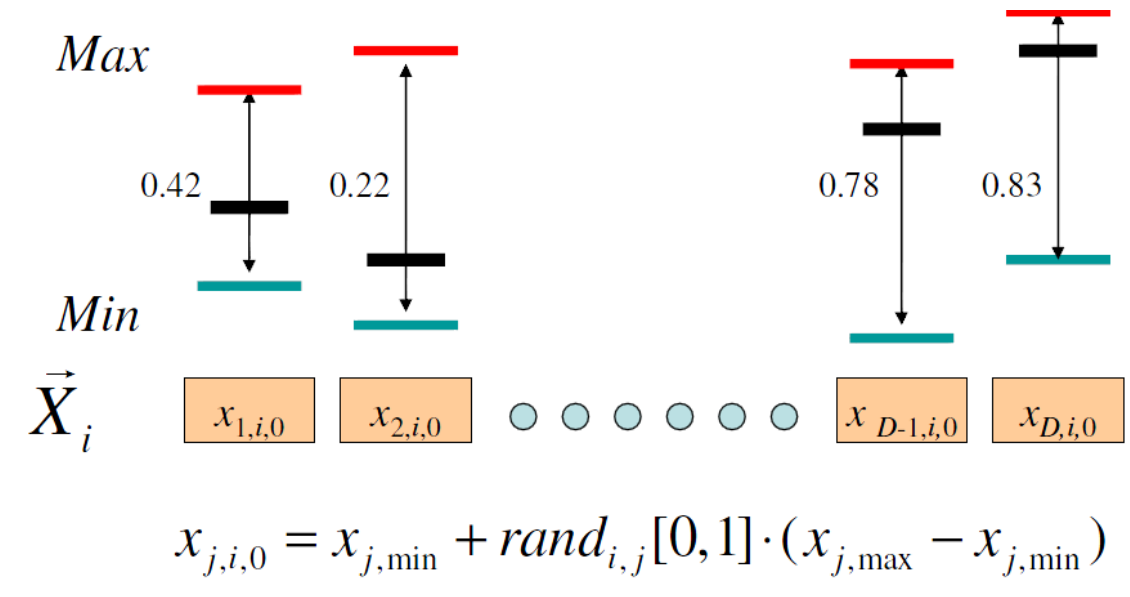

Figure 12: Initialization step in DE algorithm.

\section{Mutation}

So far we have the initial generation randomly chosen over the range of each parameter. Mutation and recombination rules are the attempt to create the options for new generation. Particularly, mutation tries to expand the search space. For a given parameter vector $\mathrm{x}_{\mathrm{i}, \mathrm{G}}$, we randomly select three other vectors (therefore there should be at least four vectors in the population). We assume these vectors are $\mathrm{x}_{\mathrm{r} 1, \mathrm{G}}, \mathrm{x}_{\mathrm{r} 2, \mathrm{G}}$ and $\mathrm{x}_{\mathrm{r} 3, \mathrm{G}}$. The indices $\mathrm{i}, \mathrm{r} 1, \mathrm{r} 2$ and $\mathrm{r} 3$ are different. The donor vector is defined based on the following equation:

Equation 4: Defining the donor vector.

$$
\mathbf{v}_{\mathbf{i}, \mathbf{G}+1}=\mathbf{x}_{\mathbf{r} 1, \mathrm{G}}+\mathbf{F} .\left(\mathbf{x}_{\mathbf{r} 2, \mathrm{G}}-\mathbf{x}_{\mathbf{r} 3, \mathbf{G}}\right)
$$

The F, known as mutation factor, is a constant from $[0,2]$. The donor vector is used in the recombination step.

\section{Recombination}

Recombination incorporates successful solutions from the previous generation. In this step a trial vector, $u_{i, G+1}$ is developed. The elements of the trial vector are from the components of 
the target (parent) vector, $\mathrm{x}_{\mathrm{i}, \mathrm{G}}$, and donor vector, $\mathrm{v}_{\mathrm{i}, \mathrm{G}+1}$. Elements of the donor vector enter the trial vector with probability of CR (crossover).

Therefore we have the trial vector as:

Equation 5: Forming the trial vector.

$$
\mathbf{u}_{\mathbf{j}, \mathbf{i}, \mathbf{G}+\mathbf{1}}= \begin{cases}\mathbf{v}_{\mathbf{j}, \mathbf{i}, \mathbf{G}+1} & \text { if } \operatorname{rand}_{\mathbf{j}, \mathbf{i}} \leq \operatorname{CR} \text { or } \mathbf{j}=\mathbf{I}_{\text {rand }} \\ \mathbf{X}_{\mathbf{j}, \mathbf{i}, \mathbf{G}} & \text { if } \operatorname{rand}_{\mathbf{j}, \mathbf{i}}>\operatorname{CR} \text { or } j \neq I_{\text {rand }}\end{cases}
$$

$I_{\text {rand }}$, rand $d_{j, i}$ and CR are the parameters which determine the source of parameter elements. There are two options for the source: the donor vector or the target (parent) vector.

The $\operatorname{rand}_{\mathrm{j}, \mathrm{i}}$ is selected from the standard normal distribution, $\mathrm{U}[0,1]$, and CR is compared to this value. If CR is greater than or equal to this random number, then the trial parameter is copied from the donor vector; otherwise the parameter is inherited from the parent vector.

The crossover constant, CR, is a probability constant defined by user from $[0,1]$. Basically this constant controls how much of the parameter are taken from the target (parent) vector or the donor vector. A higher CR increases the probability of selecting the element from the donor vector and a lower CR leans toward the element from the parent vector. For example $\mathrm{CR}=$ 1 leads to the selection of the element from the donor vector regardless of $\operatorname{rand}_{\mathrm{j}, \mathrm{i}}$ 's value and with $\mathrm{CR}=0$ it is guaranteed that the element comes from the parent vector. $\mathrm{I}_{\text {rand }}$ is a random integer from $[1,2, \ldots, \mathrm{D}] . \mathrm{I}_{\text {rand }}$ makes sure that the donor vector and the target are not identical. In addition, $\mathrm{I}_{\text {rand }}$ assures having at least one parameter to be taken from the donor vector, which guarantees the diversity in the population.

\section{Selection}

At this step there are two vectors, the target (parent) vector, $\mathrm{x}_{\mathrm{i}, \mathrm{G}}$, and the trial vector, $\mathrm{v}_{\mathrm{i}, \mathrm{G}+1}$. Here the fitness values for these vectors are evaluated and the one with lowest function value is admitted to the next generation.

Equation 6: Selection step of DE algorithm.

$$
\mathbf{x}_{i, G+1}=\left\{\begin{array}{lc}
\mathbf{u}_{i, G+1} & \text { if } f\left(u_{i, G+1}\right) \leq f\left(x_{i, G}\right) \\
x_{i, G} & \text { otherwise }
\end{array}\right.
$$

Mutation, recombination, and selection are repeated until it meets the stoppage criteria. The main steps of DE are described in Figure 13. Also Figure 14 illustrates the different steps of DE on a solution space. 


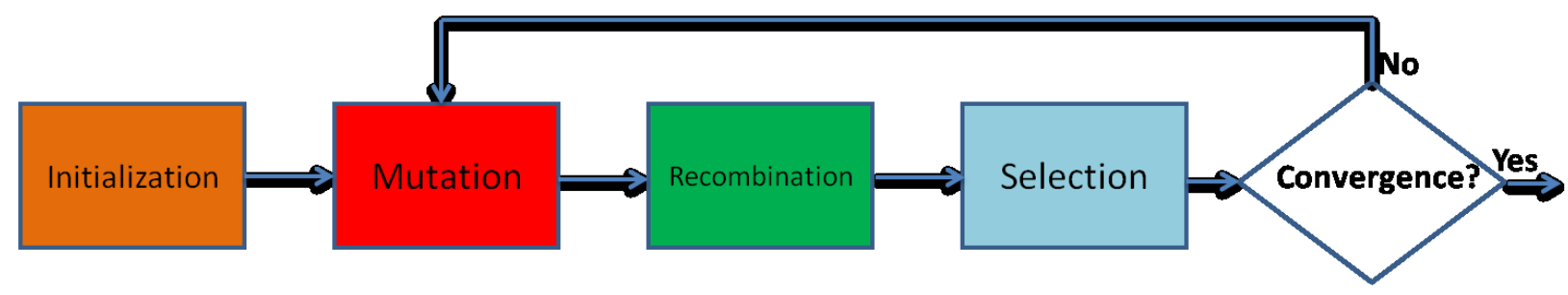

Figure 13: Flowchart of DE.

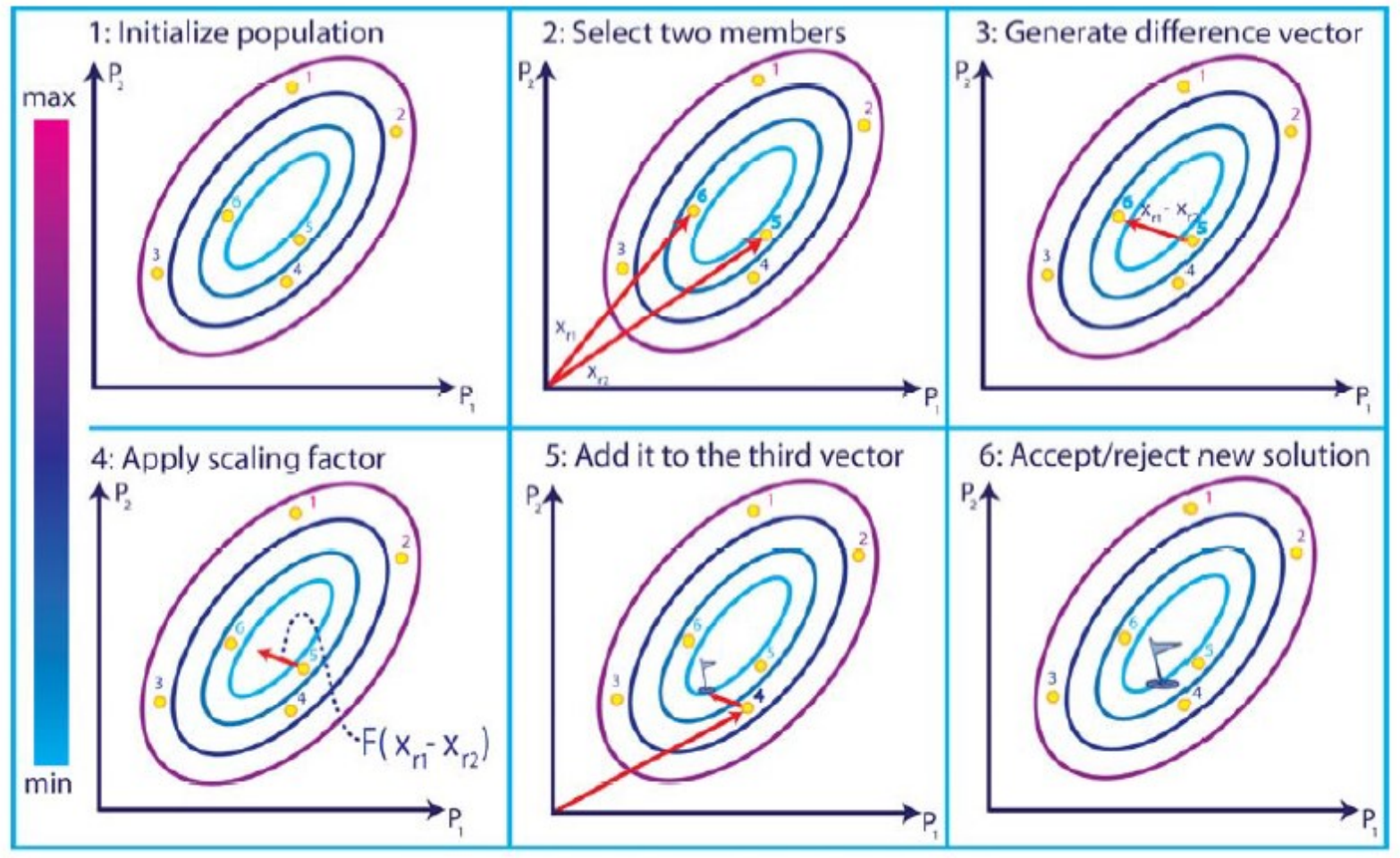

Figure 14: The steps of DE (Hajizadeh, Christie and Demyanov 2009).

One of the most important strengths of DE among other evolutionary strategies is its strong mutation strategies (Storn and Price 1997, Price and Storn, Differential evolution 1997). There are different types of $\mathrm{DE}$ that vary in the way that mutation and recombination rules are defined. The general naming rule used for the different mutation approaches is " $D E / x / y / z$ ", where $\mathrm{x}$ is the vector to mutate, $\mathrm{y}$ represents the number of difference vectors required in mutation equation, and $\mathrm{z}$ stands for the type of crossover used in the algorithm. There are two kinds of crossover schemes which can be used in the DE algorithm: exponential and binomial (Storn and Price 1997, Ferrante and Ville 2010, Price and Storn 1997, R. Storn 1999, Liu and Lampinen 2005, Shahryar, Hamid and Magdy 2008). There are five most commonly used strategies for the mutation step in the DE algorithm (Shahryar, Hamid and Magdy 2008, Das and Suganthan 2009, Kaelo and Ali 2006, Swagatam, et al. 2009, Janez, et al. 2007, Price, Storn and Lampinen 2005). In following we review these five strategies: 


\section{DE $/$ rand $/ 1$}

In this strategy, three randomly selected vectors are used. The difference of two vectors are multiplied by mutation factor, $\mathrm{F}$, and then added to the base vector.

Equation 7: First mutation strategy DE/rand/1.

$$
\mathbf{V}_{i}(t)=X_{r_{1}^{i}}(t)+F \cdot\left(X_{r_{2}^{i}}(t)-X_{r_{3}^{i}}(t)\right)
$$

\section{DE $/$ rand $/ 2$}

It is similar to the DE/rand/1, because the vectors are selected randomly; but there are two difference vectors and mutation factors in this strategy.

Equation 8: Second mutation strategy DE/rand/2.

$$
\mathbf{V}_{\mathrm{i}}(t)=\mathbf{X}_{\mathrm{r}_{1}^{\mathrm{i}}}(t)+\mathbf{F}_{1} \cdot\left(\mathbf{X}_{\mathrm{r}_{2}^{\mathrm{i}}}(t)-\mathbf{X}_{\mathrm{r}_{3}^{\mathrm{i}}}(t)\right)+\mathbf{F}_{2} \cdot\left(\mathbf{X}_{\mathrm{r}_{4}^{\mathrm{i}}}(\mathbf{t})-\mathbf{X}_{\mathrm{r}_{5}^{\mathrm{i}}}(\mathbf{t})\right)
$$

\section{DE/best/1}

Similar to the DE/rand/1, there is one difference vector. But here the algorithm chooses the best vector with lowest fitness function value as the base vector.

Equation 9: Third mutation strategy DE/best/1.

$$
\mathbf{V}_{\mathbf{i}}(\mathbf{t})=\mathbf{X}_{\mathbf{b e s t}}(\mathbf{t})+\mathbf{F} \cdot\left(\mathbf{X}_{\mathrm{r}_{1}^{\mathrm{i}}}(\mathbf{t})-\mathbf{X}_{\mathrm{r}_{2}^{\mathrm{i}}}(\mathbf{t})\right)
$$

\section{DE/best/2}

Like the DE/best/1, the base vector is the best vector with lowest fitness function value. But instead of one difference vector, two difference vectors are added to base vector.

Equation 10: Fourth mutation strategy DE/best/2.

$$
\mathbf{V}_{\mathbf{i}}(\mathbf{t})=\mathbf{X}_{\text {best }}(\mathbf{t})+\mathbf{F} \cdot\left(\mathbf{X}_{\mathrm{r}_{1}^{\mathrm{i}}}(\mathbf{t})-\mathbf{X}_{\mathrm{r}_{2}^{\mathrm{i}}}(\mathbf{t})\right)+\mathbf{F} \cdot\left(\mathbf{X}_{\mathrm{r}_{3}^{\mathrm{i}}}(\mathbf{t})-\mathbf{X}_{\mathrm{r}_{4}^{\mathrm{i}}}(\mathbf{t})\right)
$$

DE/target - to - best/1

In this strategy the base vector is the target (parent) vector and there are two difference vectors in the equation. The first difference vector is the difference between the target vector and the vector with best fitness function value. The second difference vector is the difference between two randomly selected vectors.

Equation 11: Fifth mutation strategy DE/target - to - best/1.

$$
\mathbf{V}_{\mathbf{i}}(\mathbf{t})=\mathbf{X}_{\mathbf{i}}(\mathbf{t})+\mathbf{F} \cdot\left(\mathbf{X}_{\text {best }}(\mathbf{t})-\mathbf{X}_{\mathbf{i}}(\mathbf{t})\right)+\mathbf{F} \cdot\left(\mathbf{X}_{\mathrm{r}_{1}^{\mathrm{i}}}(\mathbf{t})-\mathbf{X}_{\mathrm{r}_{2}^{\mathrm{i}}}(\mathbf{t})\right)
$$

\subsubsection{Performance of DE}

Although the efficiency of DE has been shown on a large range of classic optimization problems (Price, Storn and Lampinen 2005), there is no proof of convergence for DE. Storn and Price (1997) showed that the performance of DE is more effective than simulated annealing and genetic algorithms. In another study, Ali and Torn (2004) illustrated case 
studies that DE was more accurate and effective than controlled a random search and a modified version of genetic algorithm.

DE is famous for its simple structure. The three control parameters in DE are the size of population, $\mathrm{N}_{\mathrm{p}}$, the mutation factor, $\mathrm{F}$, and crossover, CR. The low number of control parameters makes DE simple, fast, and easy to apply. There are elaborate references on DE algorithm and parameters (Ferrante and Ville 2010, Kaelo and Ali 2006, Das and Suganthan 2009). DE has been effectively applied in different areas of engineering. Successful examples of DE application can be found in electric and electrical engineering (Qing 2009, Qing and Lee 2010), aerospace engineering (Madavan 2004), civil and urban engineering (Suribabu 2010), environmental engineering (Arunachalam 2008), material science and applied mechanics (Maciejewski, Myszka and Ziętek 2007), chemical engineering (Babu and Sastry 1999), and etcetera.

However the application of DE in petroleum engineering is relatively new. Decker and Mauldon (2006) coupled DE with two statistical methods to find the size and shape of fractures from trace data. Jahangiri (2007) utilized differential evolution to optimize smart well operations in order to maximize oil recovery. Wang et al. (2010) used DE for seismic waveform inversion and applied it to enhance the resolution of seismic data. Wang and his colleagues (2011) also used DE for waveform inversion of cross-well data. For the history matching purpose, Wang and Buckley (2006) deployed DE to find the relative permeabilities of oil and water to match the data from core flooding experiment.

Hajizadeh et al. (2010) used DE to achieve a multiple history matched model of a reservoir located in the Gulf of Mexico. Mirzabozorg et al. (2013) applied DE approach in history matching of a two dimensional SAGD case. They compared the performance of DE with Particle Swarm Optimization (PSO) method and showed that DE is faster and more accurate.

Okano (2013) used DE for the evaluation of $\mathrm{CO}_{2}$ injection test in a tight oil reservoir. The adjustable parameters were involved in the in-place volume and the connectivity between the wells. DE showed better performance in the required number of runs than PSO.

\subsection{Summary}

The topic of this chapter includes a new type of modeling which learns from the available examples and tries to find the hidden patterns and trends through these examples. These models gain their capabilities from artificial intelligence and data mining techniques. A famous example of these models is surrogate reservoir models. The characteristics associated with SRMs make them an attractive tool for reservoir modelers who struggle with the 
shortcomings of time-consuming and complicated reservoir simulation models. In this study, SRMs are built based on artificial neural networks. ANNs are non-linear data driven, fact and example based and most importantly a self-adaptive approach. These characteristics make them an ideal modeling tool for petroleum engineering problems.

SRMs are approximations of full field reservoir simulation models which are created just based on a few realizations of simulation models. Fast track modeling abilities of SRMs suits the necessity of having models with high resolution, accuracy, and pace in the reservoir management workflow. Since the advent of SRMs in 2006 many successful examples of their use have been published. These examples address different tasks of reservoir study such as uncertainty analysis and production optimization which require hundreds realizations of the reservoir model. Depending on the objective of study, SRMs can be developed to reproduce the outputs of simulation models at the well or grid level. For a history matching case, the outputs are at the well location and therefore the SRM is referred as well-based SRM. If the outputs are at grid level (such as pressure and saturation at grid block) they are known as gridbased SRM. Also depending on the objective of the study, the training realizations (required for SRM development) are varied in geological properties or operational conditions. For instance, a history matching study requires changing the geological characteristics and a production optimization analysis needs variation in operational conditions. An uncertainty assessment study can include both types of these properties.

In order to have a successful surrogate reservoir model, there are important points that should be considered. Preparation and assembling the realizations of reservoir simulation in a way that suits the features of AI\&DM techniques are really critical. The skill and knowledge of the user in reservoir engineering as well as the basics of AI\&DM techniques play an important role for this purpose. The details of different steps required for SRM development will be covered in the next chapter.

In order to have an automated history matching workflow, the reservoir model (it could be a simulation model or an approximation of it like SRMs) should be coupled with an optimization algorithm. Different methods of optimization have been utilized in history matching, as reviewed in the previous chapter. For this study, an evolutionary algorithm optimization method known as differential evolution (DE) was chosen. Recently DE has showed prosperous results for petroleum engineering applications. Because of its simple mathematical structure, DE is considered as a very effective global optimization algorithm. The low number of control parameters, makes DE simple, fast, and easy to apply. 


\section{Chapter 4: Surrogate Reservoir Model based history matching: A proof of concept investigation and a feasibility study}

\section{Overview}

In order to accomplish the objectives of this dissertation, a three step process were designed. At the beginning a proof of concept study was planned to show the potential of SRM to perform a successful history matching. Like many proof of concept studies, we start with a simple problem. Therefore an SRM was developed by considering only one uncertain property (permeability). However this property is heterogeneously distributed throughout the selected reservoir model. The next part of the project was a feasibility study. The objective of the second part is to demonstrate the robustness of SRM in handling higher degrees of uncertainty. Therefore, the number of uncertain properties increased (porosity, thickness, and permeability distributions at all grid blocks). SRM was trained, calibrated, and validated using a few number of simulation runs. The developed SRM was coupled with an optimization algorithm (DE). Finally the automated SRM-based history matching was performed by setting up the objective functions.

The third part of this project was to apply the lessons learned to a real-life case study. A reservoir model known as PUNQ-S3 model was selected. PUNQ-S3 model covers different aspects of a real-life case study. The model includes multiple layers, multi-phase fluids, faults, aquifer, and strikes of high porosity/permeability. The data for this model is available for the researchers who want to examine the novel methods in history matching and uncertainty assessment. Different realizations of this model were generated by altering porosity and permeability (vertical and horizontal) distributions. The SRM was developed (trained/calibrated/validated) and then coupled with DE in order to perform the automated SRM-based history matching. Figure 15 summarizes three steps designed in this study. 
Primarily this chapter reviews the general steps required for building an SRM. Afterward it discusses the first two steps planned for accomplishing the objectives of this dissertation (1Proof of concept 2- Feasibility study). We leave the third part of this research for the next chapter.

This chapter can be divided into three main sections. The first section reviews the general steps in the development of an SRM and also the application of SRM for history matching. In the second section the proof of concept study will be reviewed and some brief results will be shown. The third and last part of this chapter is a report on the feasibility study of SRM in accomplishment of a history matching project. Each one of these sections will have different sub-sections.

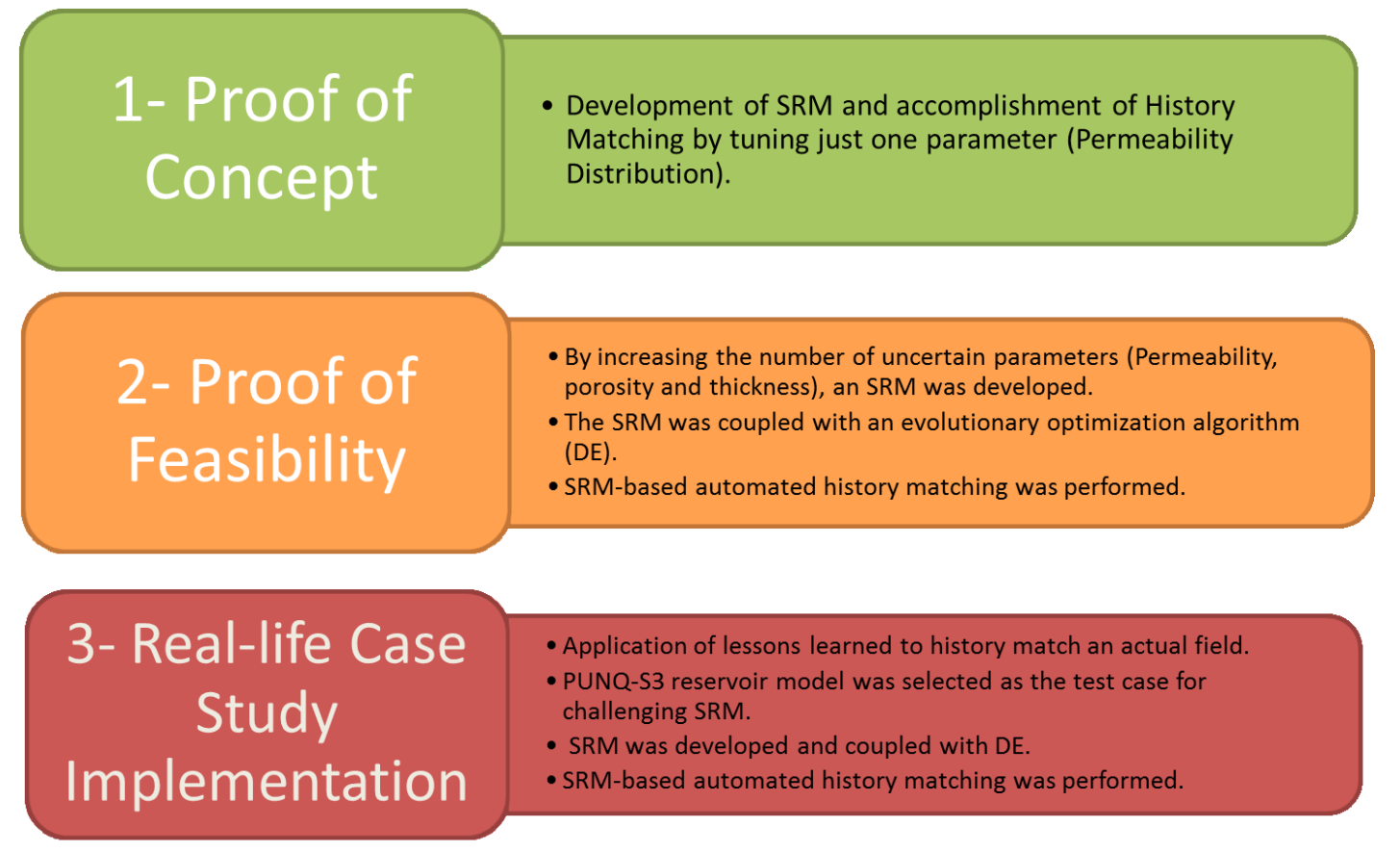

Figure 15: Three steps were defined to prove the concept of SRM, show the feasibility of the technology and apply the SRM to a real-life problem.

\subsection{General steps in development and application of SRM for history matching}

In the first part of this chapter, the general steps required for developing a surrogate reservoir model are explained. These steps have been performed for the three case studies in this project. There are three main steps in development and application of an SRM for history matching purposes. However each one of these steps includes sub-steps. These general steps are: 
- Model generation and spatio-temporal database preparation

- SRM development

- Using SRM to perform history matching

Figure 16 lists the general steps and sub-steps in developing an SRM to perform a history matching project. In continuation we explain each one of these steps.

\section{1- Model Generation \& Spatio-temporal Database Preparation}

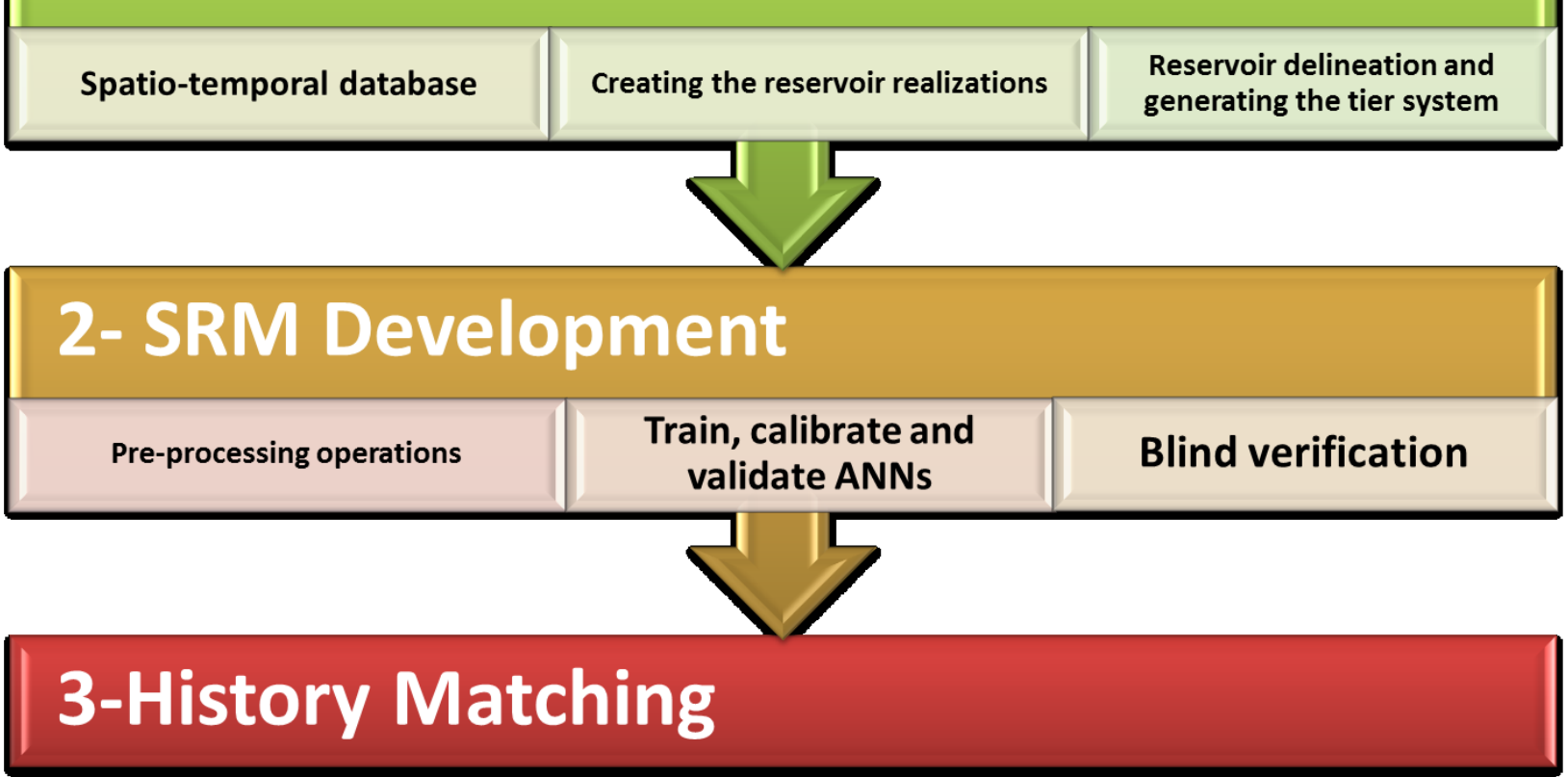

Figure 16: The steps in developing an SRM for history matching purpose.

\subsection{Model generation and spatio-temporal database preparation}

\subsubsection{Spatio-temporal database}

The artificial intelligence based reservoir models including SRMs are constructed based on a spatio-temporal database. Depending on the objective of study this database contains different types of data. The source of data is from different realizations of the reservoir simulation model. The data are extracted from these realizations to create the database. The main goal of this database is to teach the model the process of fluid flow phenomena in the reservoir. From one point of view the data in this database can be categorized as static and dynamic data. As was mentioned earlier, the static data refer to the properties which are constant overtime such as porosity, permeability, top depth and thickness. Also dynamic data are those ones which are 
not necessarily fixed overtime like operational constraints, the production at wells, pressure and phase saturations at the grid blocks, etcetera. Figure 17 is an example of different types of data in a spatio-temporal database.

As was discussed earlier, the SRMs can be categorized based on the outputs. Well-based SRMs are the ones developed to estimate the properties at well level. Similarly, the grid-based SRMs predict the properties at grid block level. The type of SRMs has a great influence on the way that the spatio-temporal database is arranged. For instance, if we have a well-based SRM (like this study) the information mainly concentrates on the well performance. Therefore, a row in this database represents a well at a time step and the columns are static and dynamic data (S. D. Mohaghegh, J. S. Liu, et al. 2012b). On the other hand, a grid-based SRM focuses on the grid level. The row mainly shows the properties at grid block and the output is a property at grid block (such as pressure or phase saturation) (Amini, Mohaghegh, et al. 2012, 2014, Shahkarami, et al. 2014).

We can conclude the spatio-temporal database is responsible to teach the SRM the behavior of reservoir. The quality of SRM performance mainly depends on how well this database has been prepared.

\begin{tabular}{|c|c|c|}
\hline \multirow{3}{*}{ 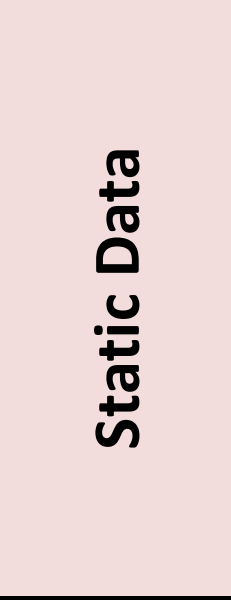 } & Identifier & $\begin{array}{c}\text { Index } \\
\text { Well Name } \\
\text { Run Number }\end{array}$ \\
\hline & Well Location & $\begin{array}{l}\mathrm{i} \\
\mathrm{j} \\
\mathrm{k} \\
\mathrm{X} \\
\mathrm{Y} \\
\mathrm{Z}\end{array}$ \\
\hline & $\begin{array}{l}\text { Static Reservoir Property } \\
\text { (at } 4 \text { tiers and offset wells) }\end{array}$ & $\begin{array}{c}\text { Top } \\
\text { Porosity } \\
\text { Permeability } \\
\text { Grid Thickness }\end{array}$ \\
\hline \multirow[b]{2}{*}{ Dynamic Data } & $\begin{array}{c}\text { Time } \\
\text { Onerational Constraint }\end{array}$ & Rottam-hole nrecsure \\
\hline & Production Rate & $\begin{array}{c}\text { Oil Rate at } \mathrm{t} \\
\text { Oil Rate at a time step behind }(\mathrm{t}-1)\end{array}$ \\
\hline
\end{tabular}

Figure 17: Different types of data in the spatio-temporal database. 


\subsubsection{Informative simulation runs representation of reservoir uncertainties}

The source of information in spatio-temporal database is the different realizations of the reservoir simulation model. Again, depending on the goal of study, the preparation of these realizations would be different. These realizations differ from each other in the variable uncertain properties. These uncertain properties are the variables which study would like to investigate their impacts on the output of reservoir model. For example in a history matching study which the adjustable parameters are porosity and permeability distributions, the realizations vary in distributions of these properties (S. D. Mohaghegh, J. S. Liu, et al. 2012b). Another example could be an uncertainty analysis of operational constraints. For this study we need realizations of reservoir model with different operational conditions (Amini, Mohaghegh, et al. 2012, 2014, Shahkarami, et al. 2014).

Although SRM does not need a high number of simulation runs, there are no rules to identify the exact number of realizations required to have a perfect SRM. Many criteria can increase or decrease the number of runs required for developing an SRM. The complexity of the problem, particularly the level of reservoir heterogeneity, is an important factor. Here the pattern recognition ability of SRM plays a vital role. For geo-statistical proxy models a homogenous reservoir is the ideal problem to solve because of the simplicity and linearity of the problem. Therefore, the number of runs to create these models decreases. But once the heterogeneity of reservoir increases the problem gets more complex and a high number of runs are required to capture the relationship among the parameters by the proxy model. However, this process is totally different in SRMs. Due to capabilities of AI\&DM techniques to understand the nonlinearity relations among the parameters, reservoir models such as SRMs do not require a high number of simulation runs to be developed. When it comes to a homogenous reservoir model (for example a model with constant value for permeability-no distribution) there is not much different information throughout the reservoir compared to a heterogeneous case. In other words, for a heterogeneous reservoir model, due to heterogeneity throughout the reservoir, each one of the grid blocks represents a different behavior of fluid flow. Therefore, although a heterogeneous reservoir is more complicated, it contains more information regarding the fluid flow behavior than a homogenous reservoir simulation model. Consequently a low number of simulation runs provides enough information for building an SRM. In other words heterogeneity is a friend of proxy models such as SRM. 


\subsubsection{Reservoir delineation and tier system}

After deciding about the number of informative realizations of reservoir, the static and dynamic data are extracted in order to build the representative spatio-temporal database. Presenting these data to the SRM is an important task to do. The huge amount of data creates a very high dimensional database. Data summarization is essential task in this part. One way of data summarization is to delineate the reservoir to different segments and make an average of data over the segments of reservoir. The delineation and segmentation of the reservoir is an important job in the process of developing an SRM. For a well-based SRM the delineation process is based on a famous theory known as Voronoi graph theory (Erwig 2000, Gomez, et al. 2009).The concept behind this delineation is simple. Let us assume having an ideal and homogenous reservoir, which also all the wells are producing under a same rate and at the same time. For this example, after a specific time the drainage area for each well is a polygon, where its boundaries have equal distance to the adjacent wells.

Figure 18 depicts a sample of reservoir delineation based on this theory. The drainage area assigned to each well is one Voronoi polygon. For each well in the spatio-temporal database the static data (reservoir characteristics) corresponding to its drainage area is considered.

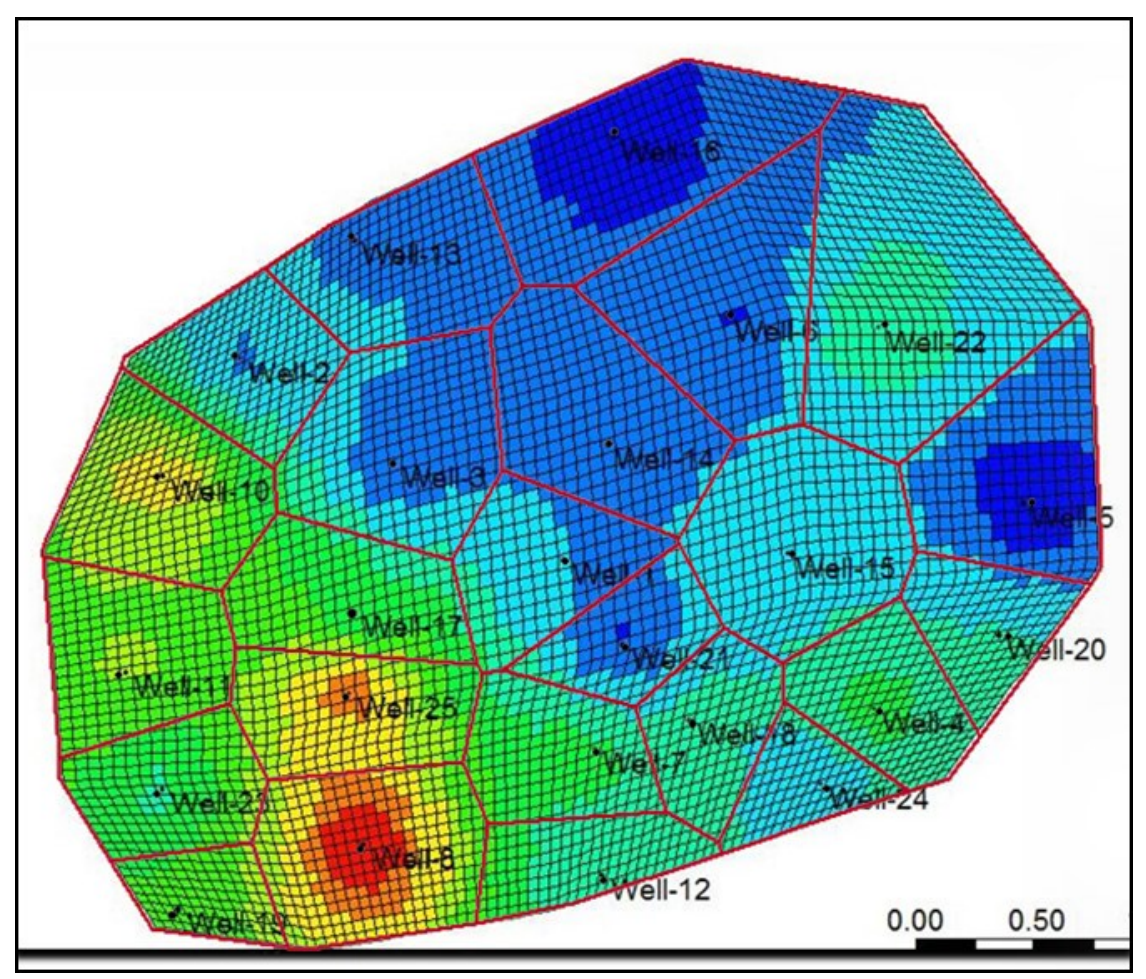

Figure 18: Reservoir delineation based on Voronoi graph theory. 
Considering a single drainage area, a reservoir segmentation (tier) system is generated. The main purpose of this system is to summarize the information of each grid block based on their impact on the well performance. For example, if we consider the Euclidian distance of grid blocks from the production well, obviously a closer grid block to the well has higher impacts on the well production. The criteria for creating the tiers are subjective and mainly based on the rule of thumb. After setting up the tier system, the average reservoir properties are calculated in each tier. Finally in the spatio-temporal database, the reservoir properties affecting the well performance (production/injection/well bottom-hole pressure) are represented by the average value for each tier. Figure 19 demonstrates a tier system used in the first part of this study (Proof of concept study). The first tier includes the well block and the second tier has eight grid blocks around the well block. The third tier consists of the next 16 grid blocks and last (fourth) tier in this system includes the rest of grid blocks in the drainage area.

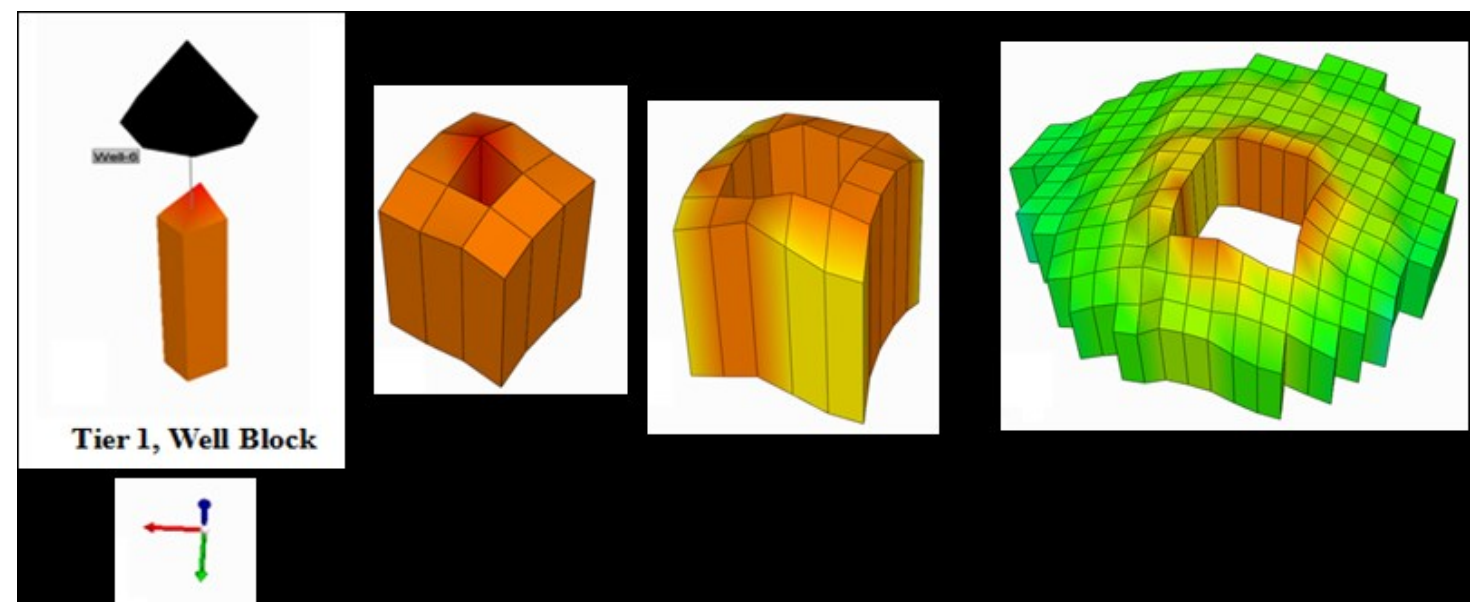

Figure 19: Dividing each Voronoi polygon (drainage area) to tiers. This tier system includes four tiers.

Sometimes in order to consider the effect of nearby wells on the performance of a specific well, the closest wells are assigned as the offset wells. Offset wells are the neighbor wells in which their performance have impacts on the behavior of the target well. The offset well effect is more highlighted in a mature (under production for a while) field when the wells have interference on each other's performance.

Generally, there are a few facts which affect the number of the offset wells in the database. The first one is the conductivity of the reservoir, which is a function of the reservoir characteristics, particularly permeability. It is much easier for the fluid to flow in a conduct reservoir. Therefore, the fluid sees the further distances of the reservoir faster. Therefore, the number of offset wells will be higher compared to a tight reservoir. The second fact is the 
background of the reservoir. In a brown (mature) field with long period of production, the reservoir has felt the further distance compared to a green field, which still is at the initial levels of production. Finally, the measureable impact (practical observation) of other wells performance should be considered. Based on the diffusivity equation, the effect of a change in a part of system can be observed simultaneously all over the system.

So far the realizations of the reservoir model are generated and the dynamic and static data are extracted. The reservoir is delineated using the Voronoi graph theory and based on the designed tier system the average values of static properties are calculated and assigned to the tiers. At this time, the spatio-temporal database is ready.

\subsection{SRM development}

An SRM is an ensemble of neuro-fuzzy systems that are trained, calibrated, and tested using the information provided in the spatio-temporal database. The neuro-fuzzy systems in SRM are used to identify the hidden patterns and trends in the database. This process happens mainly in two parts. The first part is a preprocessing step and the second one is during training and calibration of SRM. As was mentioned earlier, the main neuro-fuzzy systems used in this study are artificial neural networks. However, a fuzzy pattern recognition algorithm was used as a preprocessing tool.

\subsubsection{Preprocessing operations}

The spatio-temporal database usually contains a high number of parameters (static and dynamic). It is a fact that all the parameters in the database do not necessarily have an equal impact on the output of the SRM (Mohaghegh, Modavi, et al., Development of Surrogate Reservoir Model (SRM) for Fast Track Analysis of a Complex Reservoir 2009). Before training the SRM, preprocessing operations can be performed to identify the most important parameters in the database and decrease the dimensionality of the problem. The data preprocessing not only improves the efficiency of SRM (during training and implementation), but it also helps to have a better understanding of the problem behavior.

There are different types of preprocessing operations. These operations could be very simple such as computing differences between and taking ratios of inputs or scaling the data. On other hand, the operations could be very complicated; for example using fuzzy pattern recognition techniques to find the hidden patterns and trends among the data. Statistical based methods 
such as regression analysis are also common operations for proxy models which are based on statistical approaches.

The preprocessing step in this study is accomplished by using fuzzy pattern recognition techniques (S. D. Mohaghegh, Virtual intelligence applications in petroleum engineering: part 3 - fuzzy logic 2000, Intelligent Solutions, Inc. 2014). The technology is used to find the Key Performance Indicators (KPIs). The KPI analysis quantifies the influence of each parameter as well as the combinations of parameters on the target output. At the end of this process we have a ranking of the most influence parameters in our database. This ranking can serve as a useful guide to select the inputs of the SRM for training part.

Another important step before training the SRM is input data selection. Not all of the parameters extracted from the realizations could be helpful during the training process. Input data selection can be a demanding and complicated task. Surrogate reservoir models are based on data-driven modeling techniques and therefore they can be as good as the input data used to train them. Missing the important inputs in this step can have a significant effect on the SRM performance. As a result, developing an applicable surrogate reservoir model without a solid understanding of the problem domain could be a tough job to do. During the input selection, the petroleum engineering knowledge of the user plays an important role to in understanding the relations among the inputs. The SRM does not contribute to a particular form of analysis; it will attempt to utilize all of the input information available to model the fluid flow behavior. Therefore using fundamental data besides the technical data can improve the general performance of the SRM. This is an important point in building SRM which is ignored during

development of other types of proxy models. In other words, the SRM tries to mimic the exact behavior of the simulation model; therefore, the fundamental information, which may be fixed in different realizations (such as top depth), should be included in the database.

\subsubsection{Train, calibrate, and validate ANNs}

The training process of an SRM includes three different steps: training (learning), calibration, and validation procedures. Based on that, the spatio-temporal database is divided into three sets: the training or learning set, calibration set, and validation or verification set. The training set is part of the data shown to the ANNs during the training process. The ANNs are adapted to this set to match the provided outputs (reservoir simulation results). On the other hand, the calibration set is not used to adjust the outputs. This set is utilized to assure that any increase in accuracy over the training data set will lead to an increase in accuracy over a data set that 
has not been seen by ANNs. This set of data is helpful in determining when the training should be stopped. Finally, the verification set is a part of the database used to verify the predictability of the trained ANNs, and, subsequently, this data set is not used to train the ANNs. It is worth mentioning that the elapsed time to perform the training process (learning, calibration, and verification) is negligible when is compared to the reservoir simulation runtime. Another important point is that an SRM may be a collection of several ANNs that are trained, matched, and verified in order to generate different results (results can be at grid or well and then combined to generate the full field model outputs).

\subsubsection{Blind verification}

A further validation step is applied to test the robustness of the SRM. This step is referred to as "Blind Verification". It is called "blind" because it is a set of realization(s) that has not been used during the training process. These blind testing sets are complete realizations of the reservoir, while the verification set used in the training process is a randomly selected portion of the spatio-temporal database.

\subsection{History matching}

In this step the SRM is trained, calibrated, and validated. The further validation step of blind verification is performed and the performance of the SRM on a complete blind set is tested. Therefore, the SRM is ready to substitute the reservoir simulation model in the history matching process. In order to perform the history matching, the first order of business is to define the objective functions. The following equations are among the most common objective functions used for the history match process (CMG 2013).

This objective function calculates the relative difference between the SRM results and the measured production data. Equation 12 computes the relative differences at the well level. The subscripts $\mathrm{i}$ and $\mathrm{t}$ represent well and time respectively. $\mathrm{Nt}(\mathrm{i}, \mathrm{j})$ is the total number of measured data points for each well I and property $j . \mathrm{Y}_{\mathrm{i}, \mathrm{t}}^{\mathrm{S}}$ are the predicted production by SRM and $\mathrm{Y}_{\mathrm{i}, \mathrm{t}}^{\mathrm{m}}$ are the measured production data. $\Delta \mathrm{Y}_{\mathrm{i}}^{\mathrm{m}}$ is the scale calculated by subtracting the maximum and minimum of measured production data for well $\mathrm{i} . \mathrm{N}(\mathrm{i})$ is also the total number of properties required to be matched (for example oil production, gas production, water cut). Although for a real case, there is measurement error and it should be considered in the calculation, we assume there is not this kind of error in this study. In practice, it is common to consider that the quality and importance of measured data may be different for some specific properties and wells. Therefore some weighting factors $\left(\mathrm{tw}_{\mathrm{i}, \mathrm{j}}\right)$ are present in these equations. 
Equation 12: Individual well objective function.

$$
O F_{i}=\frac{1}{\sum_{j=1}^{N(i)} t w_{i, j}} \sum_{j=1}^{N(i)} \frac{\sqrt{\frac{\sum_{t=1}^{N t(i, j)}\left(Y_{i, j, t}^{s}-Y_{i, j, t}^{m}\right)^{2}}{N t(i, j)}}}{\Delta Y_{i, j}^{m}} .100 \% \cdot t_{i, j}
$$

It is also common to define a global objective function in order to have calculations in the field level. Equation 13 describes the global objective function using the well level objective function, which we defined in Equation 12. Here $\mathrm{OF}_{\text {global }}$ is the global objective function, $\mathrm{OF}_{\mathrm{i}}$ is the objective function for well $\mathrm{i}$, and $\mathrm{N}_{\mathrm{w}}$ is the total number of wells. $\mathrm{w}_{\mathrm{i}}$ is the defined weight for well i.

Equation 13: Global (Field) objective function.

$$
\mathbf{O F}_{\text {global }}=\frac{1}{\sum_{\mathrm{i}=1}^{N_{w}} \mathbf{w}_{\mathrm{i}}} \sum_{\mathrm{i}=1}^{\mathrm{N}_{\mathrm{w}}} \mathbf{w}_{\mathrm{i}} \mathbf{O} \mathrm{F}_{\mathrm{i}}
$$

Once the objective functions are set, the misfit between surrogate reservoir model outputs and measured data can be calculated. Thus, the misfit value is used in the history matching workflow. In this dissertation a manual history matching was used for the first part of the study. For the second and third parts of this research the surrogate reservoir model was coupled with the optimization method (DE).

The rest of this chapter will review the two main steps used to accomplish the objectives of this dissertation.

\subsection{Proof of concept study}

\subsubsection{Objective}

The objective of the first part of this study is to examine the utility of the surrogate reservoir model as an effective tool for history matching. In this step, an SRM was created for a synthetic but highly heterogeneous and complex oil field, with 24 production wells and 30 years of production data. The goal is to match the annual production data by tuning permeability distribution. The SRM was trained using ten heterogeneous realizations and then validated by a blind simulation run. Finally, the full field model was substituted by the trained SRM in the history match process. 
In order to develop the SRM, different steps mentioned in the previous section were followed. The reservoir was divided into different tiers and the spatio-temporal database was generated. It included static and dynamic data extracted from ten heterogeneous realizations of the reservoir. The uncertain property at this step of study was permeability distribution. In addition, one more realization was built to validate the trained SRM. KPI analysis was performed and the ANNs were trained, calibrated, and validated using the spatio-temporal database.

The developed SRM substituted the reservoir simulation model to tune permeability distribution at the created tiers in order to match the past performance of the reservoir. The SRM was used to calculate the oil production of 24 wells during 30 years (2000 to end of 2029). Consequently, the oil rate production of all 24 wells was history matched in a short period of time by modifying the permeability distribution throughout the reservoir. The final output of history matching is a permeability distribution that is compared to the original permeability distribution. The original (actual) permeability distribution was just used for comparison purposes after the completion of the history matching process.

\subsubsection{Model generation and spatio-temporal database preparation}

\subsubsection{Simulation model}

The reservoir model used in this study is a synthetic replica of a highly heterogeneous oil field, with 24 production wells and 30 years of production history. The base simulation model is a single porosity oil reservoir, which was constructed in $\mathrm{CMG}^{-B U I L D E R}{ }^{\mathrm{TM} 1}$ (CMG 2013). The reservoir has been divided into 4800 non-orthogonal grid blocks, 80 in $\mathrm{X}$ direction and 60 in $\mathrm{Y}$ direction. The reservoir has a single layer and thickness values are variable in different gird blocks. The field is producing oil at the initial pressure of 13,789.5 kilopascals (2000 psi) and bubble point pressure of 2,068.4 kilopascals (300 psi), therefore it is expected that the candidate reservoir will be producing oil for a long time in an under-saturated condition. The model is synthetic and does not represent a real field. Figure 20 shows three and two dimensional views of the reservoir structure. Figure 21 and Figure 22 illustrate three and two dimensional views of porosity and grid thickness distributions for the base reservoir model.

The given permeability range for the base model is from 10 to $75 \mathrm{md}$ (Figure 23). In addition, the geological information from the field identifies a high permeable zone. 24 production wells have been drilled in the field and they produce oil for 30 years. Minimum bottom-hole

\footnotetext{
${ }^{1}$ Computer Modeling Group
} 
pressure (BHP) is set as the production constraint which varies through time. The wells produce for 30 years, starting in 2000/01/01. The available historical data include oil rate production for all the wells.
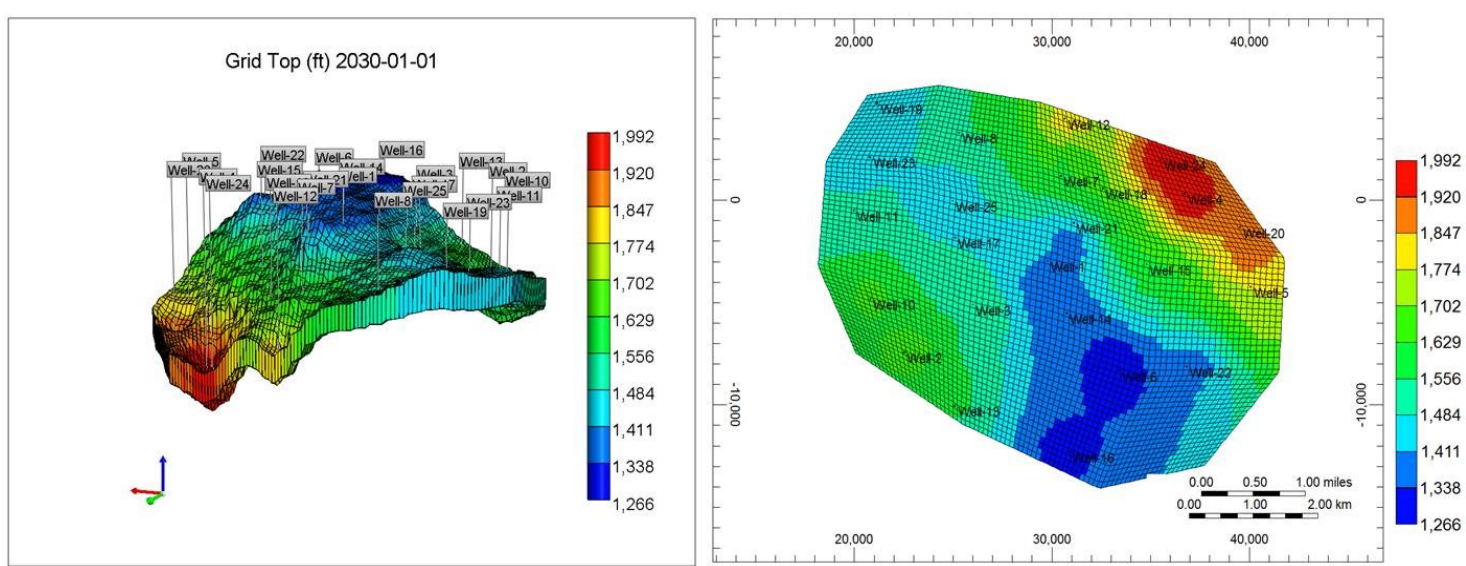

Figure 20: Three and two dimensional top views of simulation model.
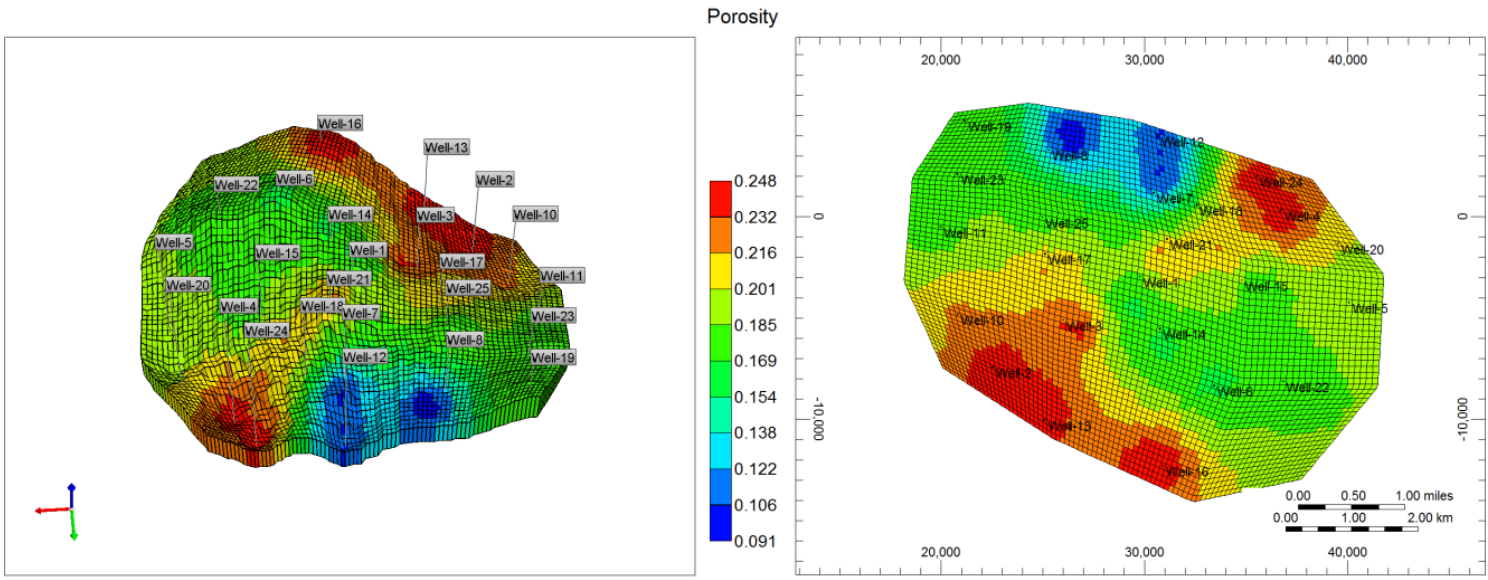

Figure 21: Three and two dimensional views of porosity distribution.
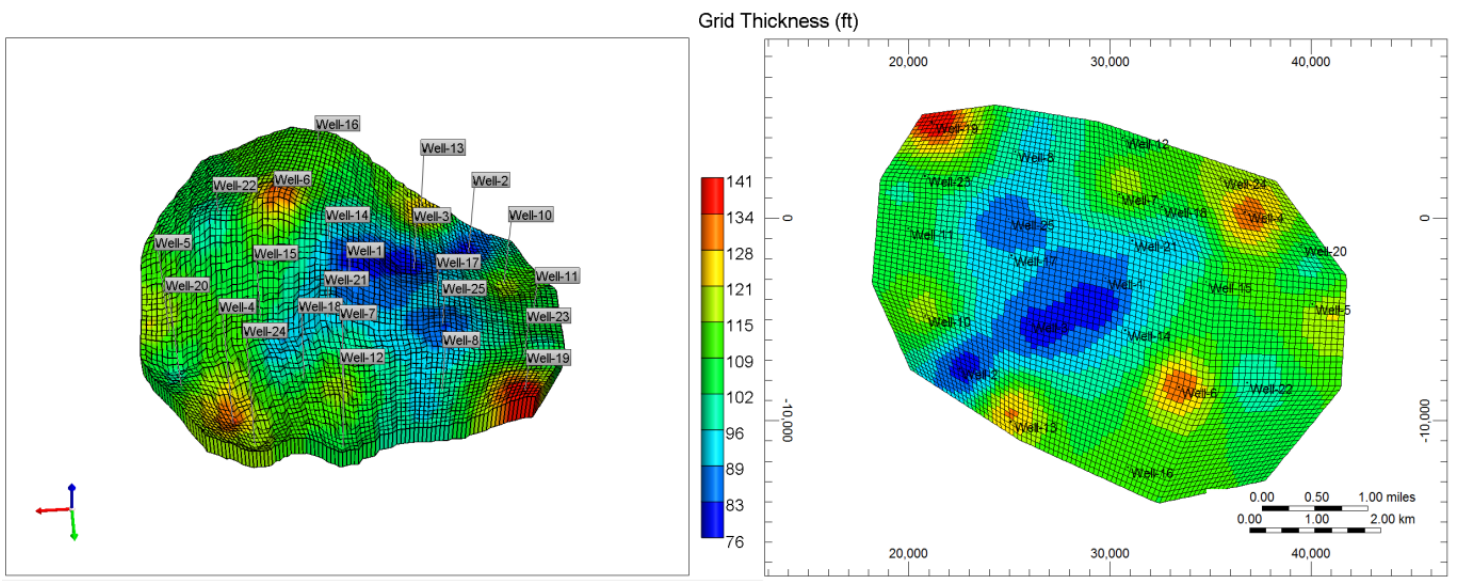

Figure 22: Three and two dimensional views of thickness map. 


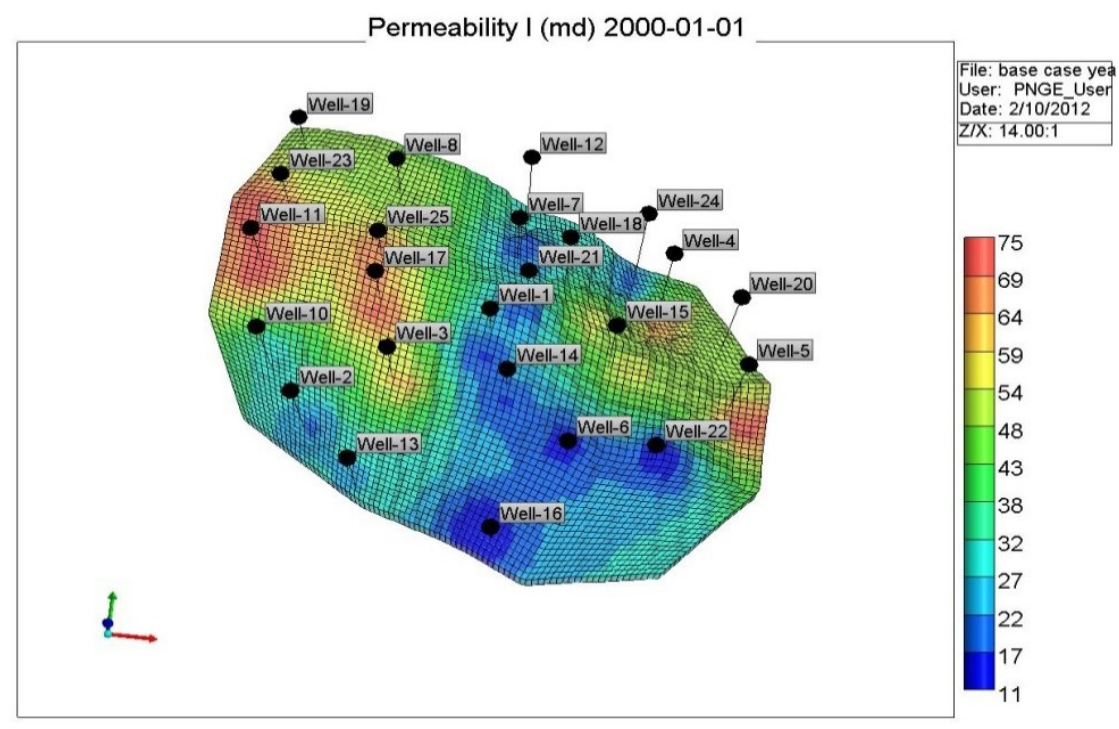

Figure 23: Given permeability map for the base case.

24 production wells have been drilled in the field and they produce oil for 30 years. Minimum bottom-hole pressure (BHP) is the production constraints. Figure 24 demonstrates the recorded monthly bottom-hole pressure for a couple of wells. Table 3 contains the values of porosity, thickness, and permeability in the base model at the well sites.

Well Constraints- Monthly BHP (psi)

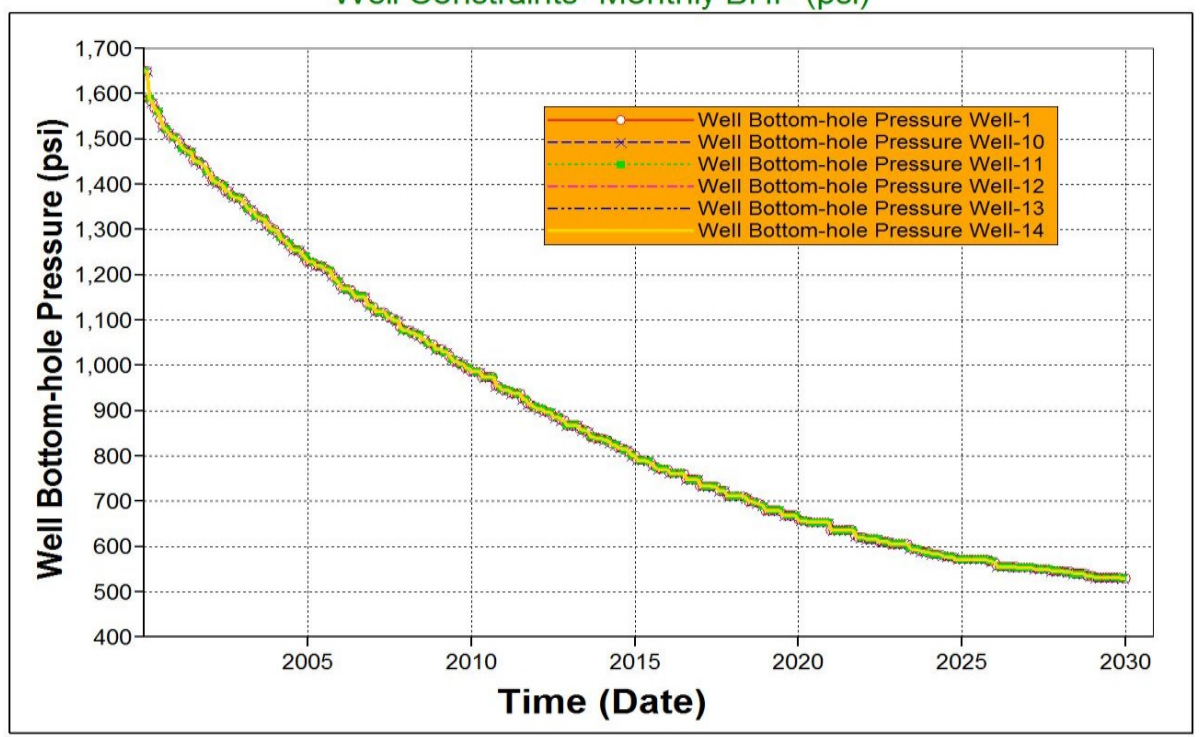

Figure 24: Bottom-hole pressure as constraints for the production wells. 
Table 3: Porosity, thickness and permeability values at the well sites.

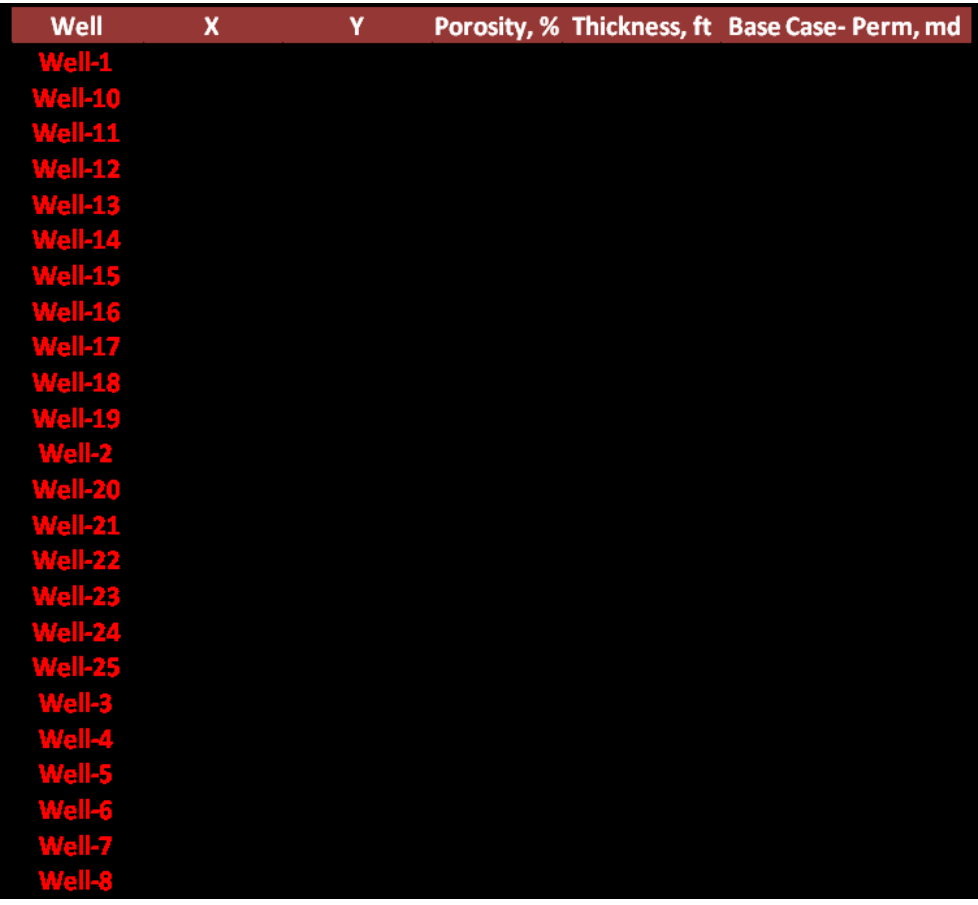

The wells produce oil for 30 years, starting in 2000/01/01. The available historical data include oil rate production and cumulative oil production for all the wells. Figure 25 shows the oil rate and cumulative oil for four wells. The complete historical data are available in the Appendix A (section a).

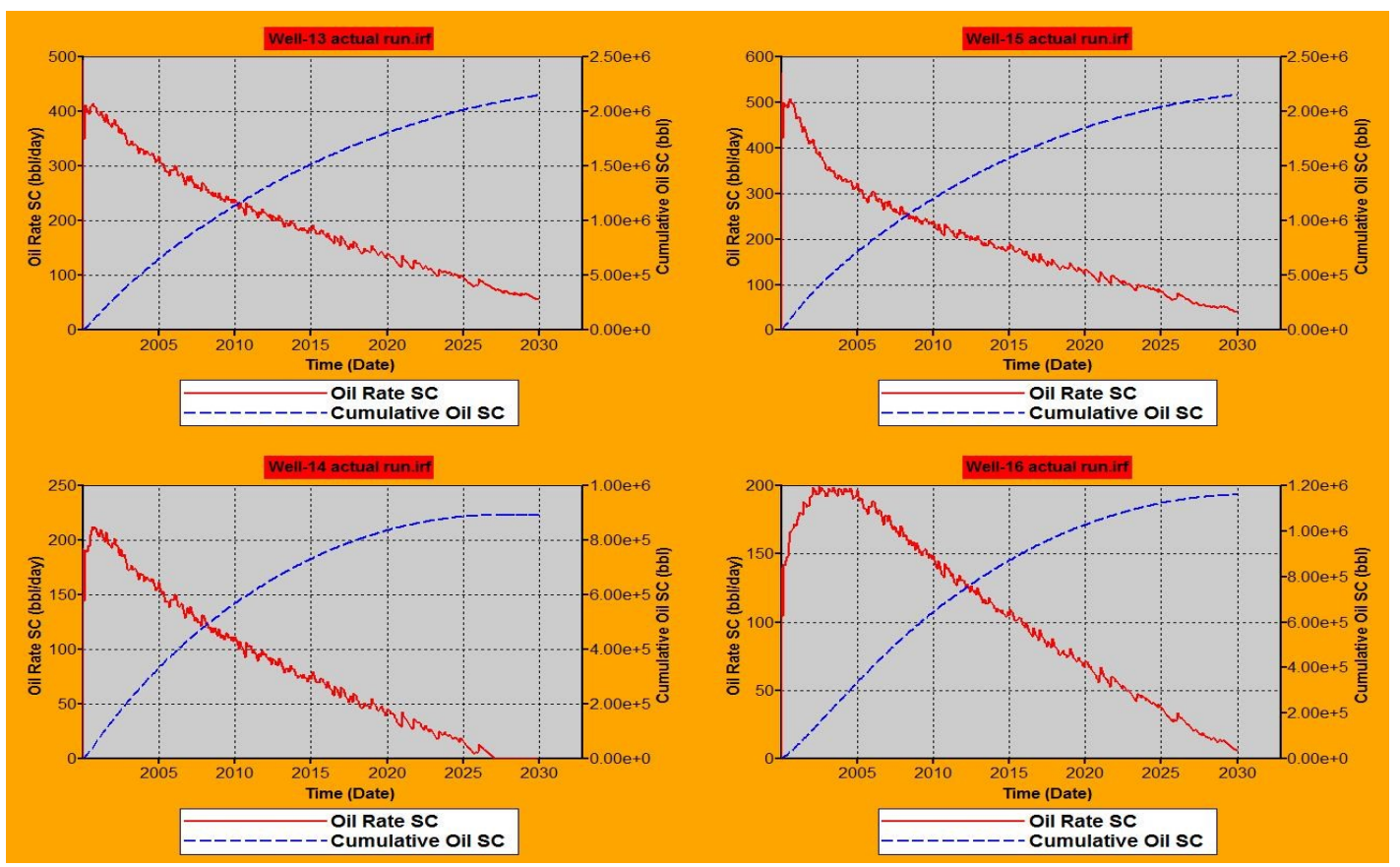

Figure 25: Production history data for some wells, the rest of wells are available in Appendix A (section a). 


\subsubsection{Informative simulation runs representation of reservoir uncertainties}

In order to introduce the uncertainties involved in the reservoir simulation model to SRM, a small number of simulation runs should be made. For the proof of concept part of the study ten different realizations of the base model were designed to develop the SRM. Using the permeability map from base reservoir model, ten different permeability maps were generated. The range of permeability for the base model is from 10 to $70 \mathrm{md}$. Due to the uncertainty involved in reservoir properties, a range from 10 to $200 \mathrm{md}$ was considered to create the permeability distributions. Afterward, to create ten different cases of permeability values at the well location, an experimental method (Latin Hypercube) was used. Table 4 summarizes generated permeability values at the well locations.

Table 4: Permeability (md) values designed at well locations for generating permeability maps. The permeability values for each well have been ranged by color, which red and blue represent minimum and maximum values, respectively.

\begin{tabular}{|c|c|c|c|c|c|c|c|c|c|c|}
\hline \multirow{2}{*}{ Well } & \multicolumn{9}{|c|}{ Permeabilty @ Well' Location for 10 Runs applied in Training part } \\
\cline { 2 - 12 } & Run 1 & Run 2 & Run 3 & Run 4 & Run 5 & Run 6 & Run 7 & Run 8 & Run 9 & Run 10 \\
\hline Well-1 & 44.6 & 21.6 & 21.0 & 38.7 & 60.5 & 67.1 & 31.3 & 66.4 & 44.2 & 89.7 \\
\hline Well-10 & 40.3 & 29.8 & 51.5 & 140.8 & 49.7 & 142.8 & 146.9 & 87.5 & 113.6 & 107.3 \\
\hline Well-11 & 41.7 & 85.8 & 32.6 & 83.8 & 138.6 & 39.0 & 122.6 & 117.7 & 97.1 & 33.0 \\
\hline Well-12 & 33.4 & 18.0 & 44.7 & 51.9 & 40.1 & 21.2 & 89.8 & 73.9 & 75.5 & 34.9 \\
\hline Well-13 & 64.3 & 41.3 & 64.3 & 61.0 & 74.9 & 69.2 & 54.7 & 60.9 & 118.4 & 104.9 \\
\hline Well-14 & 17.6 & 42.8 & 30.0 & 31.0 & 38.1 & 28.4 & 43.1 & 56.1 & 35.2 & 62.9 \\
\hline Well-15 & 18.9 & 44.9 & 37.5 & 47.4 & 55.8 & 32.7 & 92.0 & 93.1 & 91.2 & 59.6 \\
\hline Well-16 & 21.7 & 40.0 & 29.9 & 32.5 & 41.1 & 33.9 & 43.0 & 57.8 & 38.8 & 66.8 \\
\hline Well-17 & 78.1 & 45.0 & 27.7 & 44.6 & 84.1 & 102.4 & 93.8 & 143.9 & 158.9 & 152.5 \\
\hline Well-18 & 34.6 & 24.6 & 40.2 & 56.8 & 29.5 & 44.1 & 75.7 & 74.9 & 54.6 & 81.3 \\
\hline Well-19 & 76.8 & 60.3 & 95.6 & 38.9 & 54.0 & 81.7 & 107.5 & 172.6 & 49.3 & 61.4 \\
\hline Well-2 & 58.8 & 42.9 & 52.6 & 92.2 & 78.0 & 105.4 & 93.7 & 81.6 & 107.2 & 119.5 \\
\hline Well-20 & 41.2 & 27.3 & 14.4 & 53.9 & 70.7 & 27.4 & 18.9 & 37.4 & 88.0 & 21.9 \\
\hline Well-21 & 19.5 & 22.4 & 39.5 & 27.4 & 19.6 & 47.5 & 50.5 & 63.4 & 60.7 & 89.6 \\
\hline Well-22 & 30.0 & 36.0 & 31.9 & 42.4 & 46.1 & 43.3 & 63.6 & 61.3 & 70.1 & 57.4 \\
\hline Well-23 & 58.6 & 67.8 & 60.4 & 117.6 & 109.0 & 59.6 & 82.4 & 55.8 & 47.6 & 73.2 \\
\hline Well-24 & 29.2 & 31.6 & 21.5 & 44.0 & 28.2 & 57.5 & 17.5 & 77.4 & 69.4 & 27.8 \\
\hline Well-25 & 29.5 & 88.8 & 46.1 & 68.4 & 31.7 & 109.8 & 159.9 & 112.4 & 182.8 & 153.0 \\
\hline Well-3 & 67.0 & 38.5 & 76.5 & 55.4 & 72.5 & 53.2 & 36.8 & 43.1 & 123.5 & 94.0 \\
\hline Well-4 & 26.5 & 37.5 & 32.9 & 71.0 & 66.7 & 42.0 & 31.6 & 29.8 & 22.8 & 19.0 \\
\hline Well-5 & 43.2 & 30.2 & 31.7 & 15.9 & 26.4 & 66.3 & 60.4 & 86.2 & 83.2 & 72.5 \\
\hline Well-6 & 24.5 & 38.1 & 34.9 & 40.3 & 46.5 & 38.5 & 62.3 & 63.4 & 56.7 & 61.7 \\
\hline Well-7 & 41.6 & 15.9 & 30.7 & 65.1 & 39.2 & 20.6 & 44.5 & 49.6 & 50.4 & 57.0 \\
\hline Well-8 & 54.1 & 79.0 & 73.4 & 112.9 & 101.4 & 71.8 & 193.9 & 161.8 & 140.1 & 131.8 \\
\hline
\end{tabular}

Figure 26 shows three different permeability distributions, and the rest of the training realizations are shown in the Appendix A (section b). In these realizations, permeability distributions as well as the range of permeability values are different. 


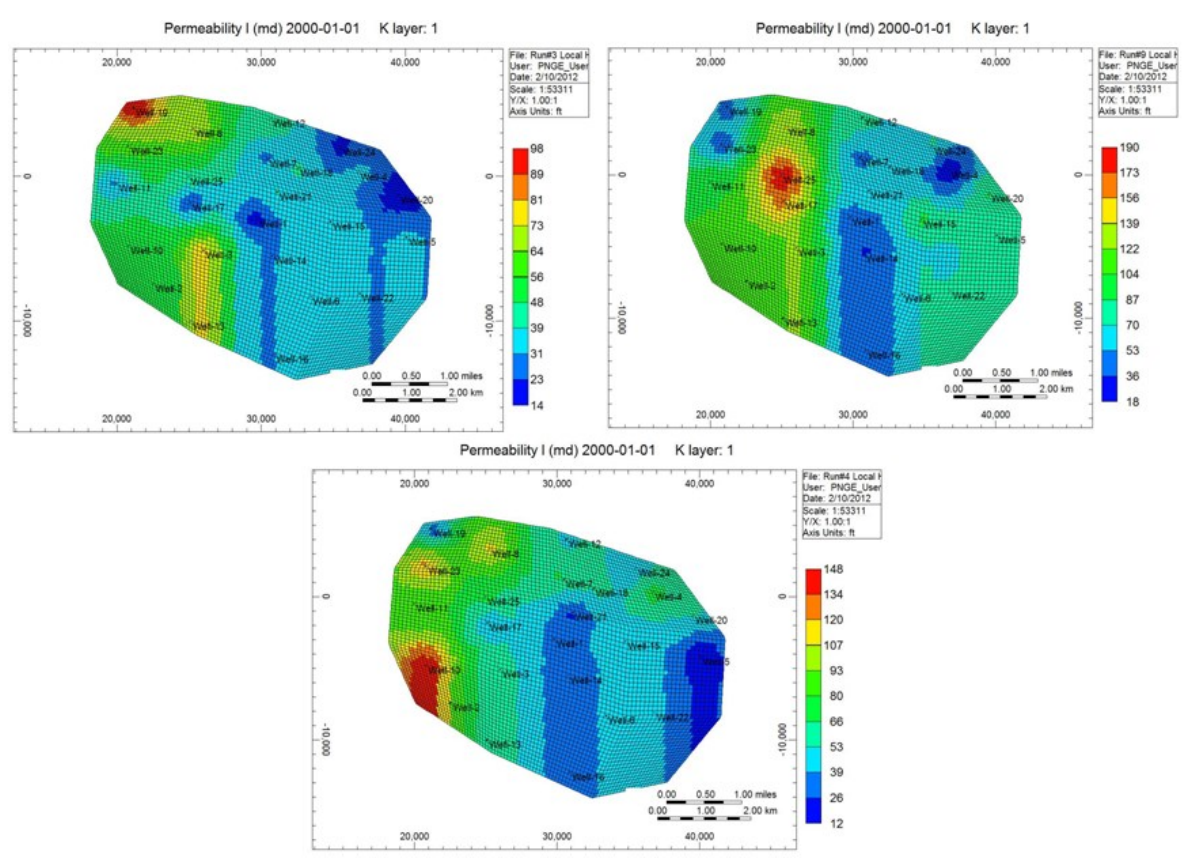

Figure 26: Different permeabilty distributions created to train the SRM (th rest of maps are available in the Appendix A-section b.)

\subsubsection{Reservoir delineation and tier system}

Using Voronoi graph theory, the drainage area for each well was designed. Figure 18 in the previous section is the designed Voronoi polygons for this reservoir model. In addition, in order to include the static data of adjacent grid blocks of a well into the spatio-temporal database, a tier system was generated. Figure 19 depicts the tier system used for this part of study. Finally to consider the impact of the adjacent wells on the production of target well, three offset wells were assigned to the target well. The selection criterion was based on the Euclidian distance. In the database the static and dynamic data of these offset wells were considered for the target well.

\subsubsection{SRM development}

\subsubsection{Preprocessing operations}

Up to this point, the required simulation runs have been designed and executed, the reservoir was divided into the desired segments and three offset wells were recognized for each well. In this step, the inputs to develop the spatio-temporal database are selected. As was mentioned, the objective of study is an important factor in this step (Amini, Mohaghegh, et al. 2012, Mohaghegh, Liu, et al., Application of Surrogate Reservoir Models (SRM) to an Onshore Green Field in Saudi Arabia; Case Study 2012a). Figure 17 lists different types of data in the database for this step of study. Basically the database is a table in which the rows are the wells 
at different time steps (here we have 24 wells and 30 time steps; therefore the database has $24 * 30=720$ rows). The columns of this database are the properties (static and dynamic) and also the identification parameters (such as well index, realization indices, etc.). Figure 27 demonstrate the general format of the spatio-temporal database.

Number of Columns = (Number of identifiers) + (number of static and dynamic properties)

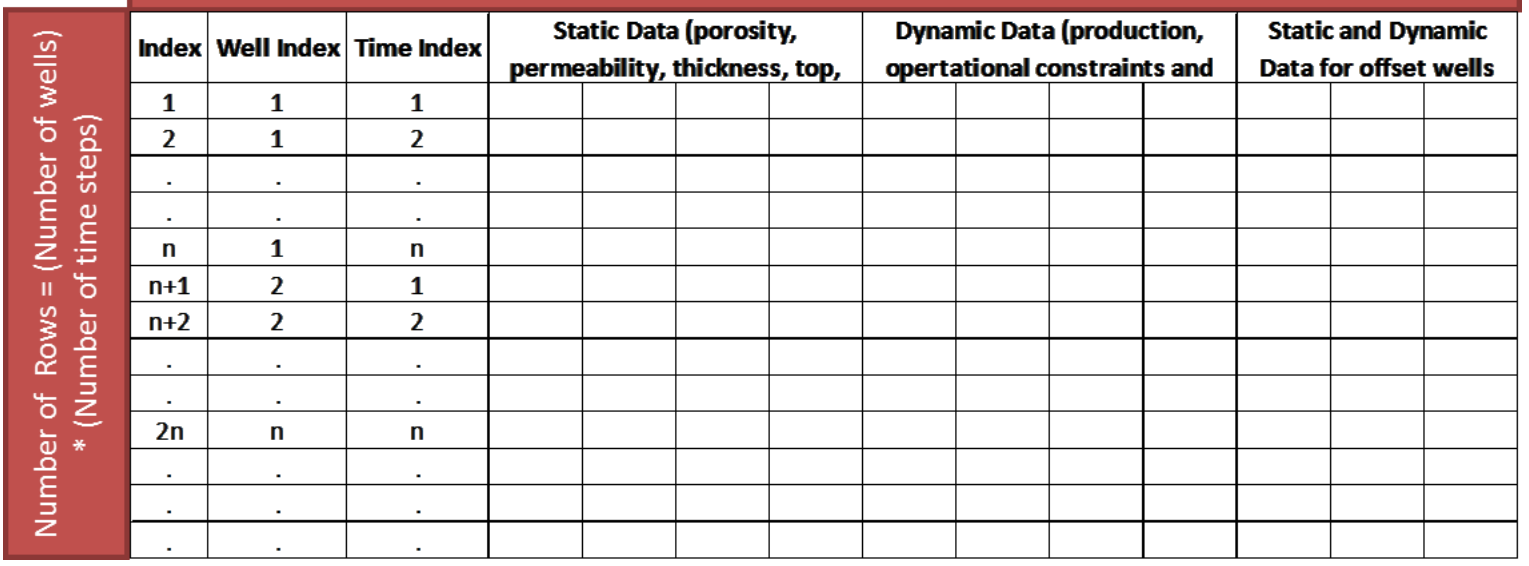

Figure 27: General format of a spatio-temporal database.

KPI analysis as a pre-modeling operation was performed to identify the most influential parameters in the database and provide a base for the input data selection before training the ANNs. KPI analysis and generating the neural networks in this study were performed by using a data-driven software known as IDEA ${ }^{\mathrm{TM}}$. This software is developed particularly for building artificial intelligence based models for petroleum engineering applications by Intelligent Solutions Inc. (ISI) (Intelligent Solutions, Inc. 2014).

In the proof of concept study, the SRM consists of two ANNs. These networks vary in selection of inputs and outputs. The output of first ANN, we call it "Initialization", is the annual oil production rate at the end of first year of production (2000/31/01). While the output of second network, named "Main", is the annual oil production rate for the other years of production. The inputs to train these ANNs are also different from each other.

Table 5 and Table 6 summarize the results of KPI analysis for this step of the study. Table 5 shows the results for the initialization network. As expected, the most high-ranking parameters for this model are permeability values for the target well and the offset wells. Table 6 shows the results of KPI analysis for the main ANN. For this network dynamic parameters such as bottom-hole pressure, time, and oil production rate are the most important properties. 
The KPI analyses can provide helpful feedback for selecting the final input parameters for the training part. However, the selection of inputs is not just limited to KPI analysis. The reservoir engineering knowledge could provide good judgment for adding some inputs. Table 7 and Table 8 list the final inputs selected for initialization and main networks respectively.

Table 5: The results of KPI analysis for the Initialization ANN.

\begin{tabular}{|c|c|c|}
\hline Rank & Feature & \% Degree of Influence \\
\hline 1 & Horizontal Permeability, Tier \# 1 & 100 \\
\hline 2 & Horizontal Permeability, Tier \# 2 & 98 \\
\hline 3 & Horizontal Permeability, Tier \# 3 & 82 \\
\hline 4 & Horizontal Permeability, Tier \# 4 & 57 \\
\hline 5 & Horizontal Permeability, Offset well \# 1, Tier \# 4 & 37 \\
\hline 6 & Horizontal Permeability, Offset well \# 1, Tier \# 3 & 33 \\
\hline 7 & Latitude (X) & 31 \\
\hline 8 & Latitude (X) Offset well \# 1 & 31 \\
\hline 9 & Horizontal Permeability, Offset well \# 3, Tier \# 4 & 28 \\
\hline 10 & Horizontal Permeability, Offset well \# 1, Tier \# 2 & 25 \\
\hline 11 & Horizontal Permeability, Offset well \# 3, Tier \# 3 & 25 \\
\hline 12 & Top, Tier \# 1 & 24 \\
\hline 13 & Top, Tier \# 2 & 23 \\
\hline 14 & Horizontal Permeability, Offset well \# 2, Tier \# 3 & 21 \\
\hline 15 & Horizontal Permeability, Offset well \# 1, Tier \# 1 & 21 \\
\hline 16 & Top, Tier \# 3 & 21 \\
\hline 17 & Top Offset well \# 1 Tier \#1 & 21 \\
\hline 18 & Top Offset well \# 1 Tier \#2 & 20 \\
\hline 19 & Horizontal Permeability, Offset well \# 2, Tier \# 4 & 20 \\
\hline 20 & Other parameters & Less than 20\% \\
\hline
\end{tabular}

Table 6: The results of KPI analysis for the Main ANN.

\begin{tabular}{|c|c|c|}
\hline Rank & Feature & \% Degree of Influence \\
\hline 1 & Oil production rate @ a year before & 100 \\
\hline 2 & Bottom-hole Pressure (psi) & 54 \\
\hline 3 & time & 52 \\
\hline 4 & Oil production rate @ (t-1) Offset well \# 3 & 42 \\
\hline 5 & Oil production rate @ (t-1) Offset well \# 1 & 41 \\
\hline 6 & Oil production rate @ (t-1) Offset well \# 2 & 35 \\
\hline 7 & Top, Tier \# 1 & 13 \\
\hline 8 & Top, Tier \# 2 & 12 \\
\hline 9 & Horizontal Permeability, Tier \# 1 & 12 \\
\hline 10 & Top, Tier \# 3 & 11 \\
\hline 11 & Horizontal Permeability, Tier \# 1 & 11 \\
\hline 12 & Latitude (X) Offset well \# 1 & 11 \\
\hline 13 & Thickness, Tier \# 2 & 11 \\
\hline 14 & Thickness, Tier \# 1 & 10 \\
\hline 15 & Top Offset well \# 1 Tier \# 4 & 10 \\
\hline 16 & Top Offset well \# 1 Tier \# 1 & 10 \\
\hline 17 & Porosity, Tier \#4 & 10 \\
\hline 18 & Top Offset well \# 1 Tier \#2 & 9 \\
\hline 19 & Thickness, Tier \#4 & 9 \\
\hline 20 & Other parameters & Less than 9\% \\
\hline
\end{tabular}


Table 7: Selected inputs for the Initialization ANN.

Table 8: Selected inputs for the Main ANN.

\begin{tabular}{|c|c|}
\hline & Static Inputs \\
\hline \multirow{18}{*}{ 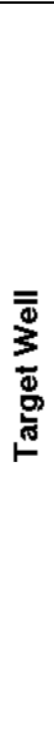 } & Well Latitude (X) \\
\hline & Well Langitude $(\mathrm{Y})$ \\
\hline & Porosity, Tier \# 1 \\
\hline & Horizontal Permeability, Tier \# 1 \\
\hline & Thickness, Tier \# 1 \\
\hline & Top, Tier \# 1 \\
\hline & Porosity, Tier \# 2 \\
\hline & Horizontal Permeability, Tier \# 2 \\
\hline & Thickness, Tier \# 2 \\
\hline & Top, Tier \# 2 \\
\hline & Porosity, Tier \# 3 \\
\hline & Horizontal Permeability, Tier \# 3 \\
\hline & Thickness, Tier \# 3 \\
\hline & Top, Tier \# 3 \\
\hline & Porosity, Tier \# 4 \\
\hline & Horizontal Permeability, Tier \# 4 \\
\hline & Thickness, Tier \# 4 \\
\hline & Top, Tier \# 4 \\
\hline \multirow{3}{*}{ 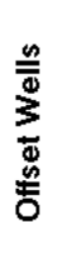 } & $\begin{array}{c}\text { Horizontal Permeability, Tier \# 4, } \\
\text { Offset Well \# } 1\end{array}$ \\
\hline & $\begin{array}{c}\text { Horizontal Permeability, Tier \# 4, } \\
\text { Offset Well \# } 2\end{array}$ \\
\hline & $\begin{array}{c}\text { Horizontal Permeability, Tier \# 4, } \\
\text { Offset Well \# } 3\end{array}$ \\
\hline
\end{tabular}

\begin{tabular}{|c|c|c|}
\hline & Static Inputs & Dynamic Inputs \\
\hline \multirow{18}{*}{$\begin{array}{l}\overline{\bar{\Phi}} \\
3 \\
\bar{\Phi} \\
\bar{\sigma} \\
\stackrel{5}{-}\end{array}$} & Well Latitude (X) & \multirow{5}{*}{ Bottom-hole Pressure } \\
\hline & Well Langitude $(\mathrm{Y})$ & \\
\hline & Horizontal Permeability, Tier \# 1 & \\
\hline & Thickness, Tier \# 1 & \\
\hline & Top, Tier \# 1 & \\
\hline & Porosity, Tier \# 2 & \multirow{6}{*}{ Time } \\
\hline & Horizontal Permeability, Tier \# 2 & \\
\hline & Thickness, Tier \# 2 & \\
\hline & Top, Tier \# 2 & \\
\hline & Porosity, Tier \# 3 & \\
\hline & Horizontal Permeability, Tier \# 3 & \\
\hline & Thickness, Tier \# 3 & \multirow{7}{*}{$\begin{array}{c}\text { Oil production rate @ } \\
\text { a year before }\end{array}$} \\
\hline & Top, Tier \# 3 & \\
\hline & Porosity, Tier \# 4 & \\
\hline & Horizontal Permeability, Tier \#4 & \\
\hline & Thickness, Tier \# 4 & \\
\hline & Top, Tier \# 4 & \\
\hline & Top,T4 & \\
\hline \multirow{4}{*}{ 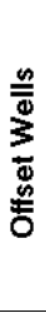 } & Well Latitude $(X)$ & $\begin{array}{c}\text { Oil production rate @ (t-1) } \\
\text { Offset well \# } 1\end{array}$ \\
\hline & Well Langitude $(\mathrm{Y})$ & $\begin{array}{c}\text { Oil production rate @ (t-1) } \\
\text { Offset well \# } 2 \\
\end{array}$ \\
\hline & Horizontal Permeability, Tier \# 4 & \multirow{2}{*}{$\begin{array}{c}\text { Oil production rate @ (t-1) } \\
\text { Offset well \# } 3\end{array}$} \\
\hline & Thickness, Tier \#4 & \\
\hline
\end{tabular}

\subsubsection{Train, calibrate, and validate the ANNS}

$\mathrm{The}_{\text {IDEA }}^{\mathrm{TM}}$ provides a data partitioning algorithm which selects training, calibration, and verification sets randomly. As mentioned, the spatio-temporal database was built based on the information from ten simulation runs. The training, calibration, and verification set include $80 \%, 10 \%$, and $10 \%$ of the data in the database respectively. It should be noted that the size of each partition, like many other things in the development of SRMs, is a function of the complexity of the problem at hand. There is no "one size fit all" approach, but there are general rules that can be followed.

Up to this point two different ANNs have been initiated. The inputs are selected and the database is divided into training, calibration, and verification sets. At this step, the ANNs are ready to get into the training procedure. The training algorithm used in this study is a back propagation method (Fausett 1993, Rojas and Feldman. 1996). Back propagation networks always consist of at least one hidden layer. Krose and Smagt (1996) have stated that only one hidden layer is enough to approximate any function with finitely many discontinuities to arbitrary precision. Therefore, one hidden layer is selected in this study. 
The number of neurons in the hidden layer is completely our choice and there is no rule to determine the best possible number. If the number is too large, it will encourage the network to memorize the input patterns instead of learning the prototype of reservoir simulation. On the other hand, a hidden layer with too small a number of neurons will drastically increase the number of iterations essential to train the network. The software has a suggestion for the number of neurons, which is a function of the inputs number. In this study, the recommended numbers of IDEA $^{\mathrm{TM}}$ have been used which are 20 and 50 for the initialization and main networks respectively.

Figure 28 shows the ANN structure for the initialization and main networks. These graphs portray three different layers of ANNs: input, hidden, and output layers. Input layer includes the parameters chosen in the input selection part. The hidden neurons were suggested by the software and the output of models is annual oil production rate. Different layers are connected by the weight vectors; the weights are tuned in an iteration process during the training step to match the known outputs (reservoir simulation results).
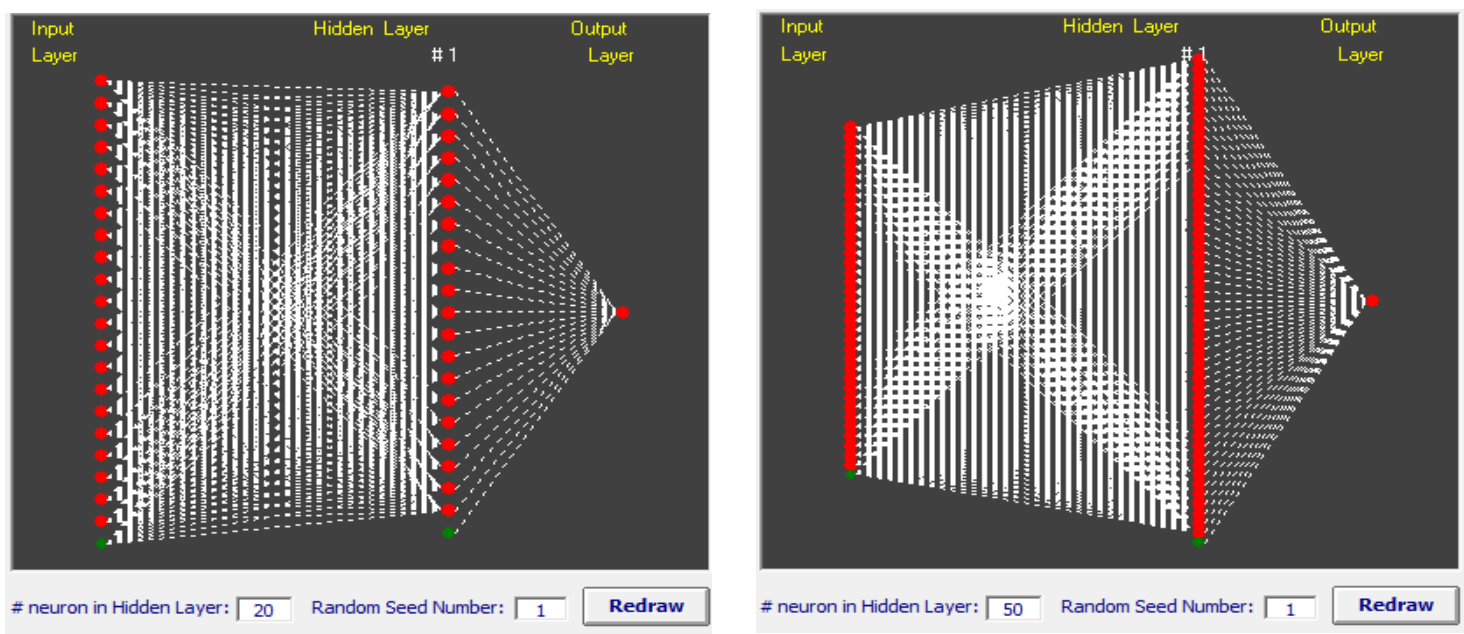

Figure 28: ANN structure for the Initialization (left) and Main (right) networks.

Figure 29 is a snapshot of a dynamic training procedure. The graph shows the error profile for training (right) and calibration (left) sets. The best results are saved based on the best values achieved for the calibration set. Figure 30 and Figure 31 demonstrates the scatter plots for training, calibration, and verification sets after training process for initialization and main networks respectively. 


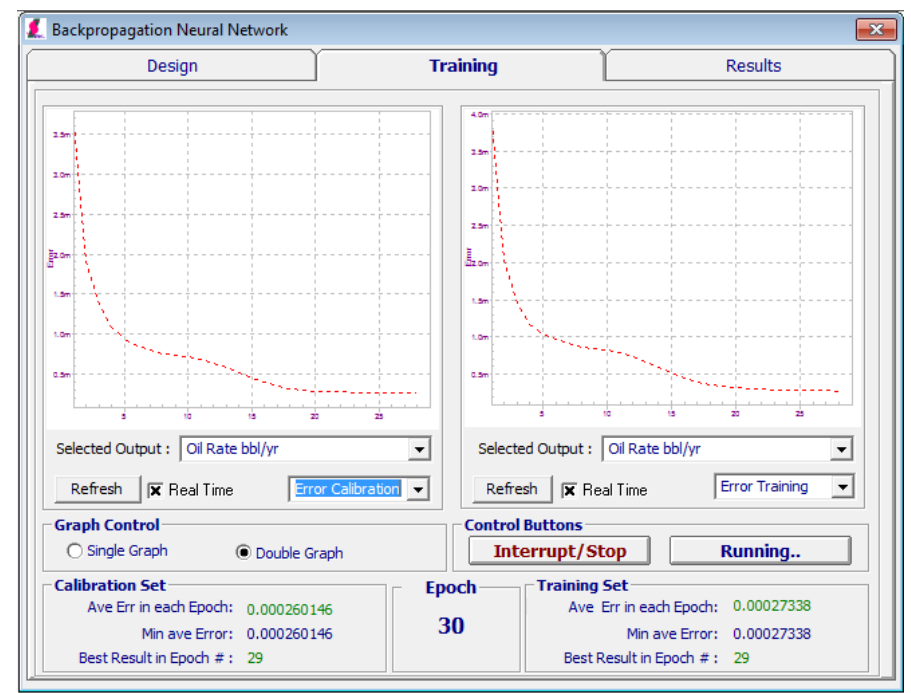

Figure 29: A snapshot of the training procedure.
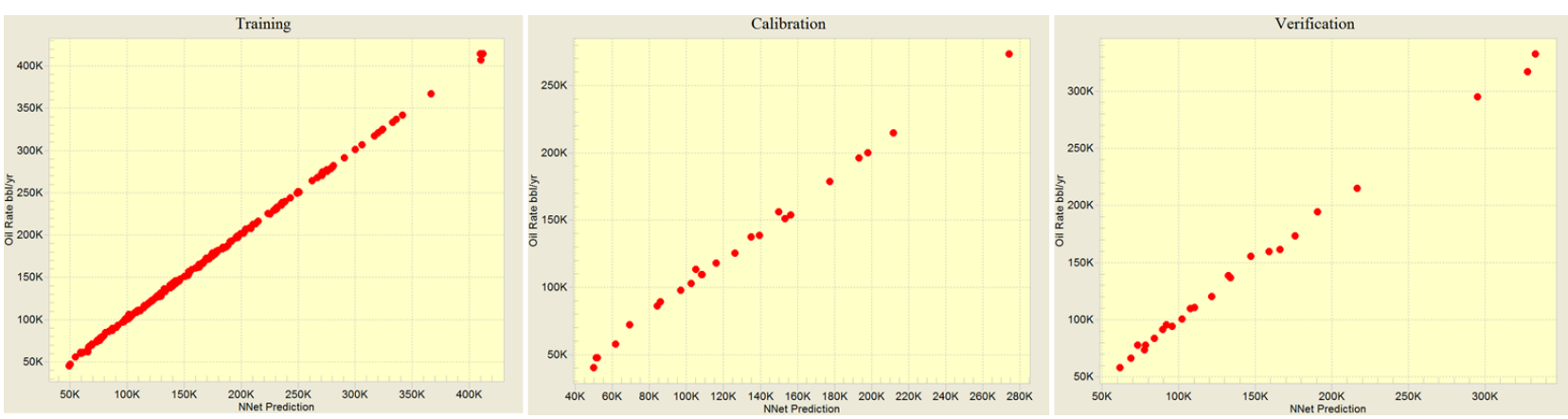

Figure 30: Scatter plots for the training, calibration and verification sets during the training process of the initialization ANN.
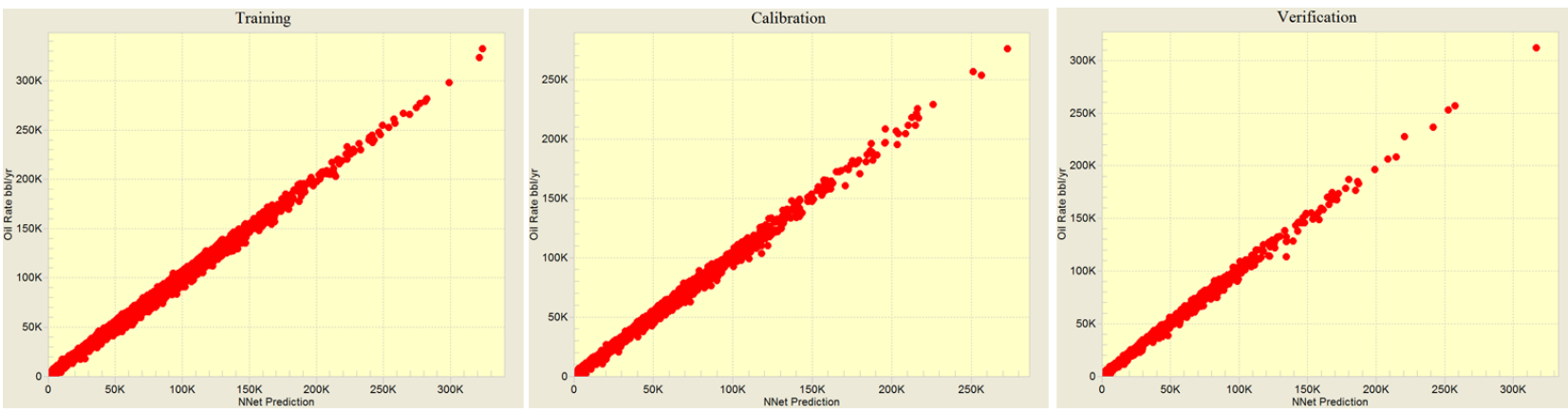

Figure 31: Scatter plots for the training, calibration, and verification sets during the training process of the main ANN.

\subsubsection{Blind verification}

Although there is a verification procedure during the training process, the ANNs are going through an extra verification step. The trained ANNs are validated against a complete blind realization of the reservoir. Therefore the $11^{\text {th }}$ simulation run was made. The permeability 
distribution for this realization is completely different from the ten runs used for SRM training. This blind testing set is a complete realization of the reservoir, while the verification set used in the training process was a randomly selected portion $(10 \%)$ of ten realizations.

It should be noted that this blind realization should honor the range of permeability, which was used to train the ANNs. Finally, the trained ANNs were applied to predict the oil rate from the blind realization inputs. Figure 32 depicts the permeability distribution for the blind realization. The results of the blind verification run compared to the reservoir simulator values will be presented in the results section.
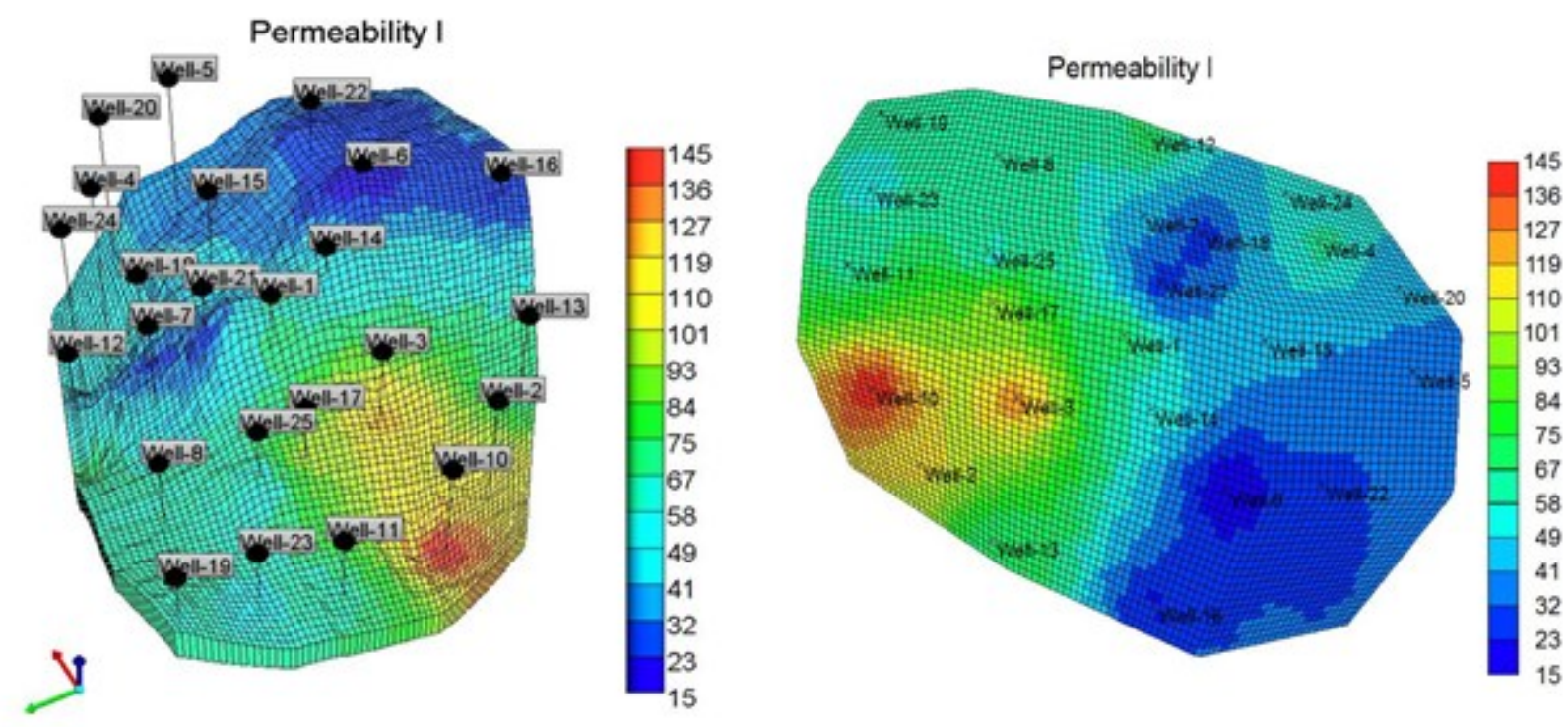

Figure 32: Three (left) and two (right) dimensional views of permeability distribution for the blind verification realization.

\subsubsection{History matching}

After the blind verification step, the surrogate reservoir model is ready to be used in the history matching process. In the first step of this project we started with just one uncertain property, permeability. In order to accomplish the history matching, permeability values at each defined tier was adjusted. Then the defined objective functions were used to compare the oil rates predicted by the SRM against the real production rates. This procedure was repeated until an acceptable match in each well was obtained.

\subsubsection{Results}

In this section we present the results of one well (well \#20) for different steps of construction and application of SRM in the proof of concept section. The results for rest of the wells are available in Appendix A (Appendix A: section c shows the results of run \#1, section d presents 
the results of blind verification run, and part e shows the results of history matching). Figure 33 shows the results after the training process; the chart portrays the oil rate's profile for 30 years of production, comparing SRM results with the simulator outputs. In this picture, the blue squares represent the SRM and the red line with stars shows the simulator results. It can be seen that SRM can reproduce the simulator results with high accuracy.

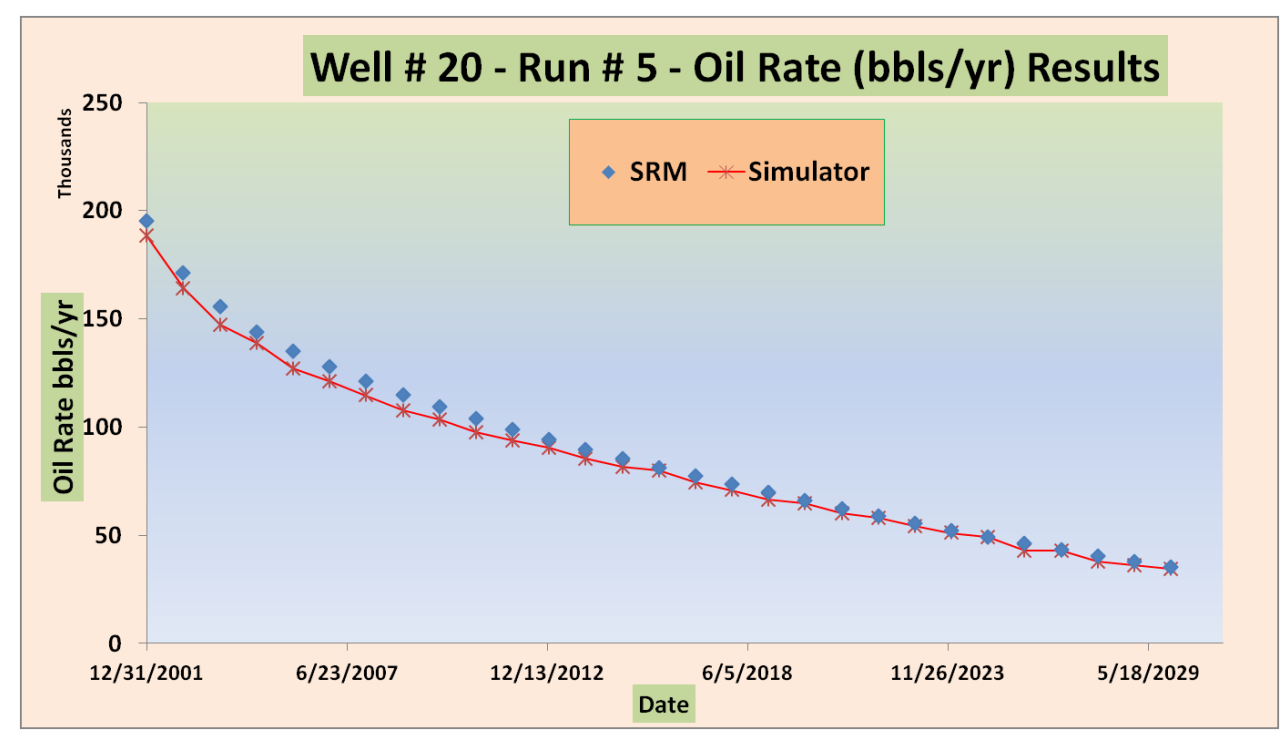

Figure 33: Comparison between the results of SRM and simulator for well \#20 in a training realization.

Figure 34 shows the results of blind verification realization. As was mentioned before, a blind realization was used for testing the SRM with a realization set which has not seen by SRM. Therefore, this graph shows the potential of SRM to predict a realization performance out of the training dataset.

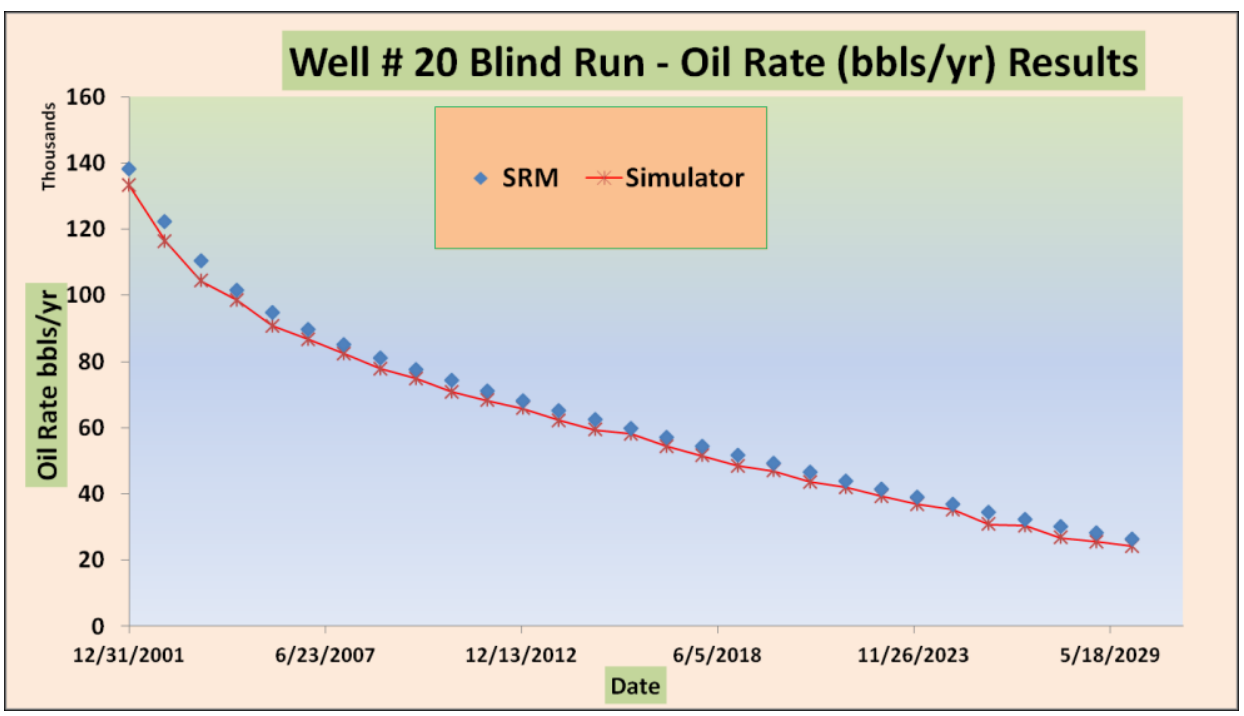

Figure 34: Comparison between the results of SRM and simulator for well \#20 in a blind verification run. 
Finally Figure 35 is a snapshot of the history match results for this well. This graph is the comparison of the SRM with the measured production data.

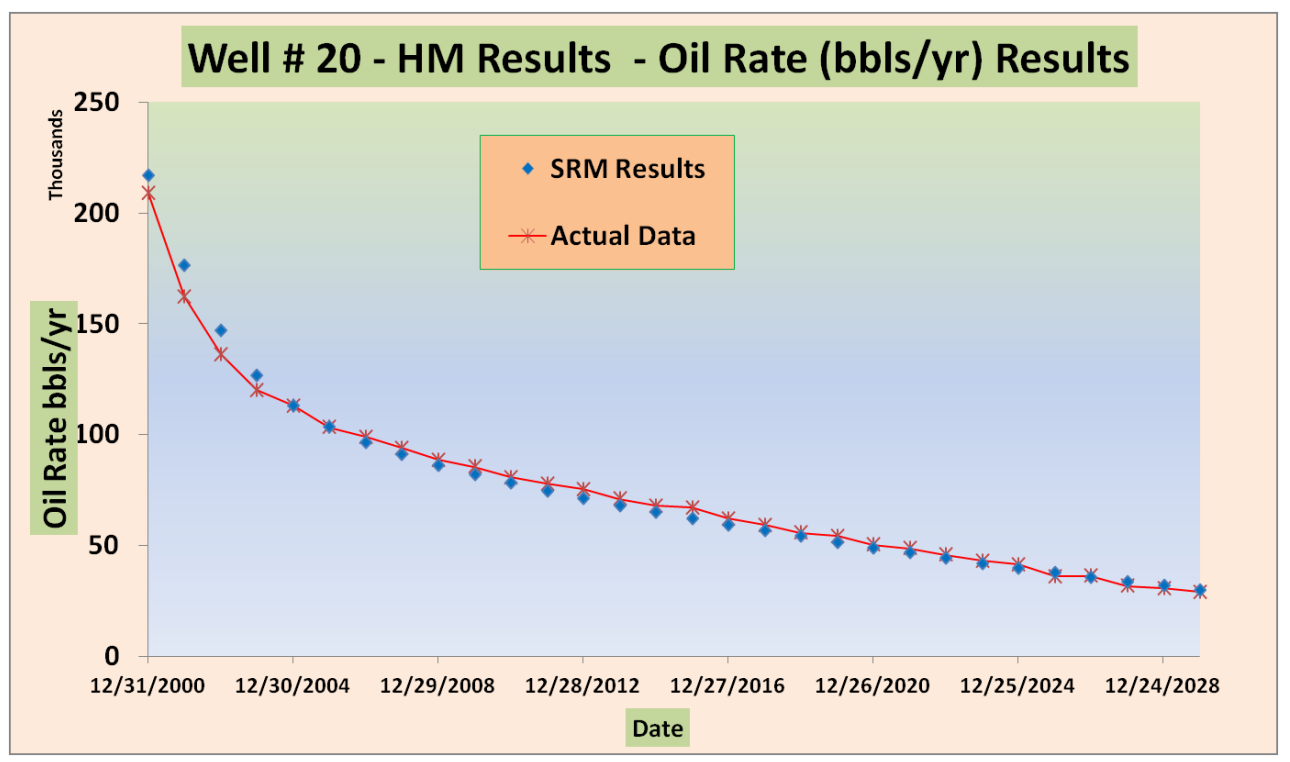

Figure 35: The outcome of history matching for well \#20 comparing the best match (SRM results- blue squares) and actual data (red line).

The matched permeability values are shown as a permeability distribution map in Figure 36. The right side of this figure pictures some shots of the matched permeability map, while the left side shots are the actual permeability distribution. In addition, Figure 37 shows the error between the actual permeability and the matched one.

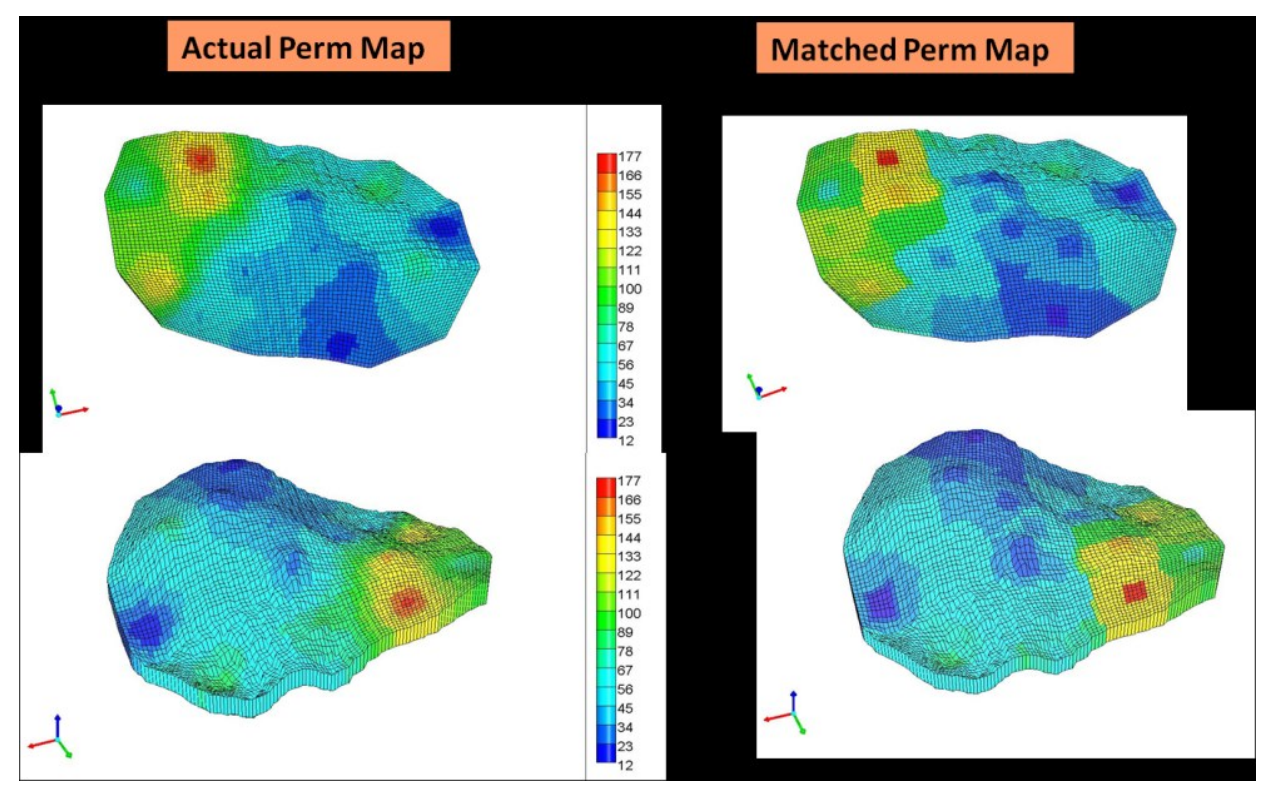

Figure 36: Comparison of matched and actual Permeability distributions. 


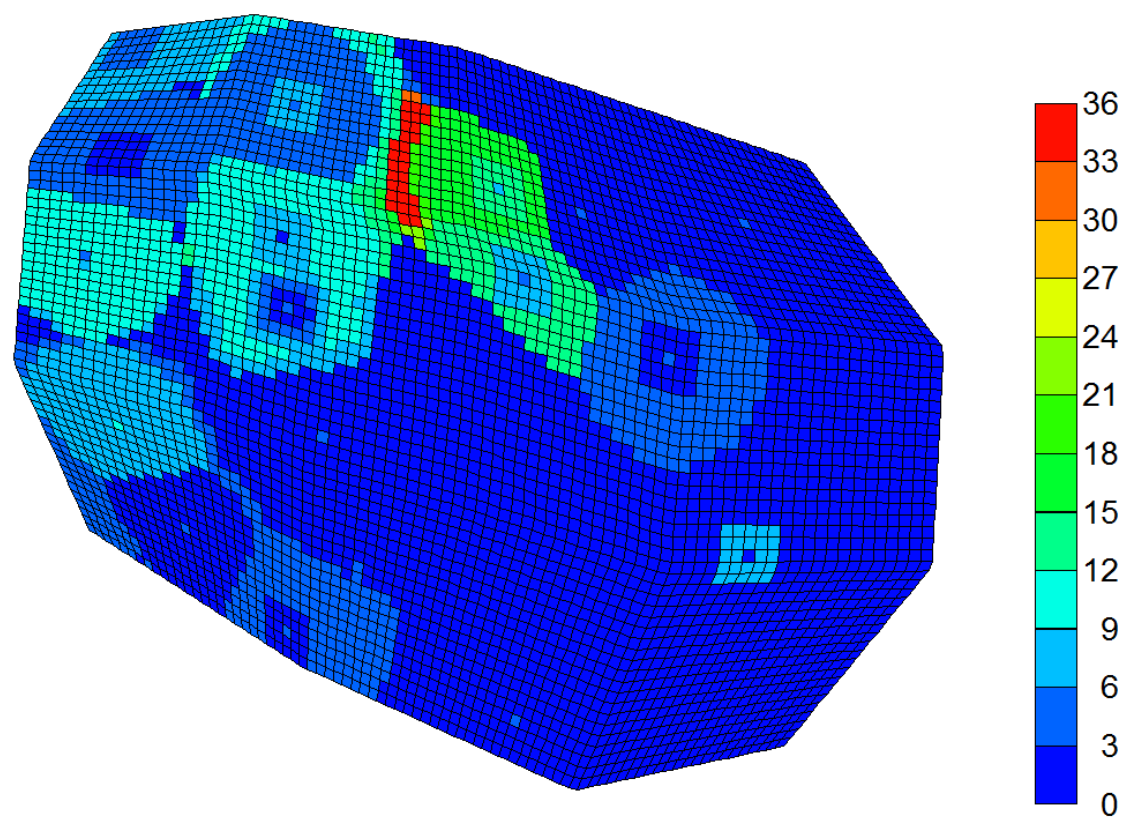

Figure 37: A snapshot of error distribution of permeability.

\subsection{Feasibility study}

\subsubsection{Objective}

The first part of this study was to show that the SRM is able to be an efficient substitute of reservoir simulation model in the history matching workflow. The results of the first part were a proof of SRM applicability to perform history matching. Like many other proof of concept studies, we started with a simple and controlled problem. The study was accomplished by adjusting one uncertain property (permeability). In the second part of study, we decided to step forward and study the performance of the SRM when the problem gets more complicated. Therefore, the number of uncertain properties increased to three properties (porosity, permeability, and thickness). The SRM was developed by having 20 different realizations of the reservoir. The distributions of porosity, permeability, and thickness are different in these realizations. The dynamic and static data were then extracted from these simulation runs in order to construct the spatio-temporal database. Once the SRM is trained, calibrated, and validated, two extra simulations runs (runs $21^{\text {st }}$ and $22^{\text {nd }}$ ) were used as the blind verification realizations.

Another important improvement in this step of study was to couple the SRM with a global optimization algorithm (DE). Nowadays, although manual history matching still exists in the industry, the trend is to develop automated systems which are able to repeatedly adjust the uncertain parameters, run the model, achieve the results, and report the best matches. These 
goals are obtained in this step of study by having an optimization algorithm which selects the parameters within the given ranges, calls the developed SRM, runs it, calculates the misfit through the defined objective functions, and finally reports the best matches between the SRM and actual case.

In this section of chapter four we go through the steps required to develop the SRM and then use it in the history matching process. Some preliminary results also will be reviewed. The complete results are provided in Appendix B.

\subsubsection{Model generation and spatio-temporal database preparation}

\subsubsection{Simulation model}

The reservoir model used in this part is the same as the reservoir model explained in the proof of concept study section. Therefore, we skip the simulation model section here and the readers can refer to the section 4.5.2.1.

\subsubsection{Informative simulation runs representation of reservoir uncertainties}

20 realizations of the reservoir simulation model were generated to train the SRM. These realizations are different in porosity, permeability, and thickness distributions. An experimental design method was utilized over the properties range to construct combinations of the input parameter values such that the maximum information can be obtained from the minimum number of simulation runs. Latin hypercube sampling (LHS) is the experimental design method in this study. Latin hypercube sampling has enjoyed popularity as a widely used sampling technique for the propagation of uncertainty in analyses of complex systems (J.C. Helton, and F.J. Davis 2002).

Using the experimental design method, the range of properties is constrained to the base model (with some expansion in range for adding some uncertainties, for example the range of permeability in the base model is 10-70 md, therefore we assumed a range of 5-200 md for the training realizations). The distribution of properties changes for different realizations. It is assumed the property values at the well locations are available (in reality they are coming from the core data or log information); therefore, using an interpolation method (Inverse Distance Estimation provided in CMG-Builder ${ }^{\mathrm{TM}}$ (CMG 2013)), a distribution of property can be generated. Figure 38 explains the process of generating new realizations (altering property values at well locations). Figure 39 to Figure 41 compare the permeability, porosity, and thickness distributions of the first and second realizations. The property distributions for the rest of the realizations are provided in Appendix B (section a). 


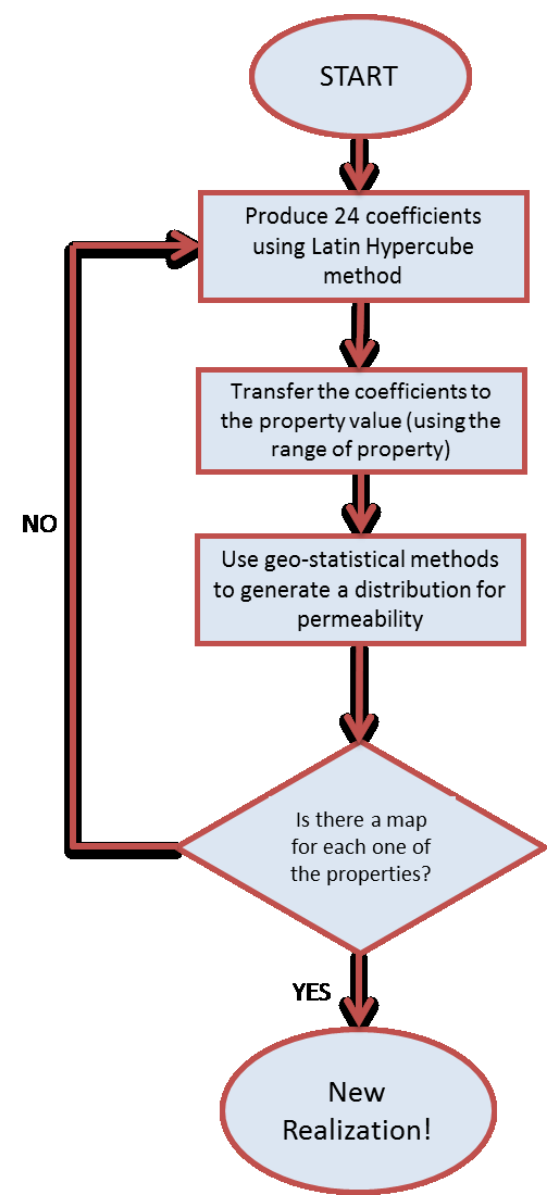

Figure 38: Flow chart to generate different realizations by altering property values at well locations.

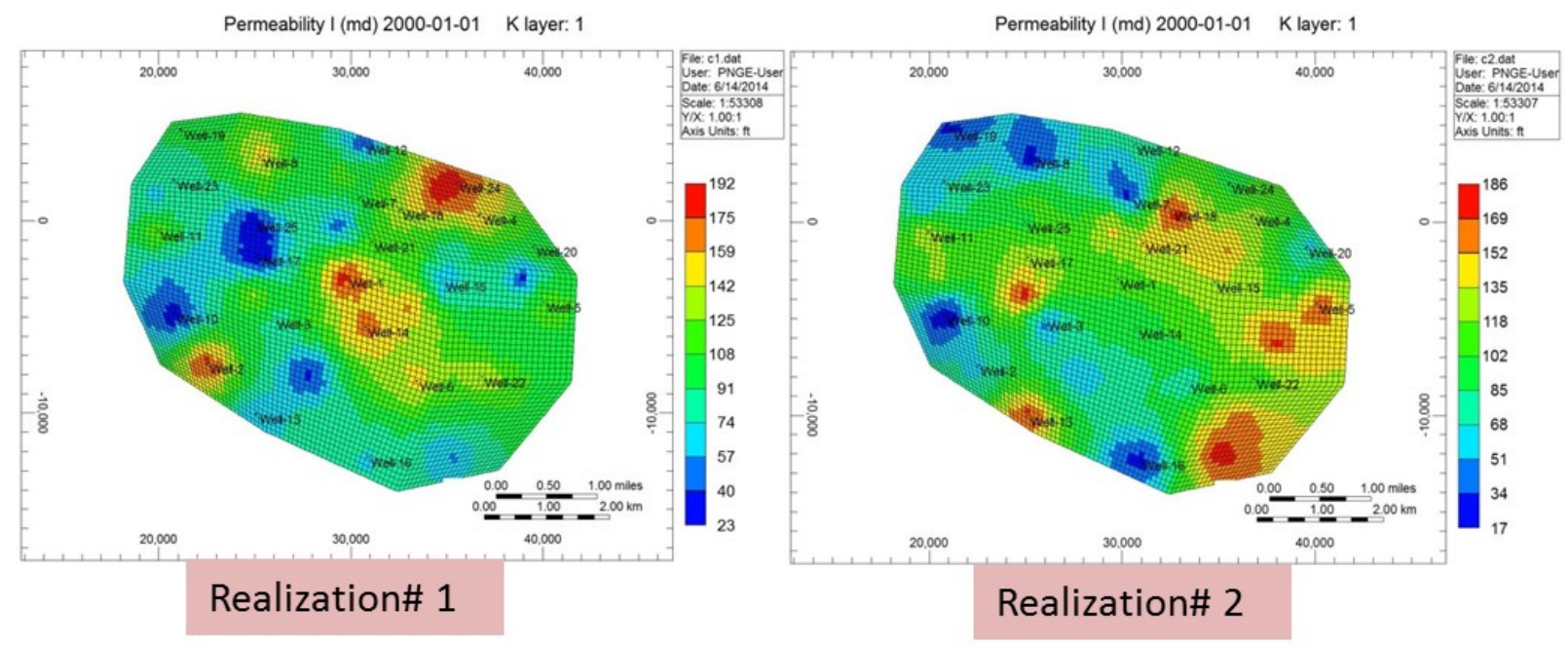

Figure 39: Comparison of permeability distributions between realizations \#1 and \#2. The property distributions for the rest of realizations are provided in Appendix B (section a). 


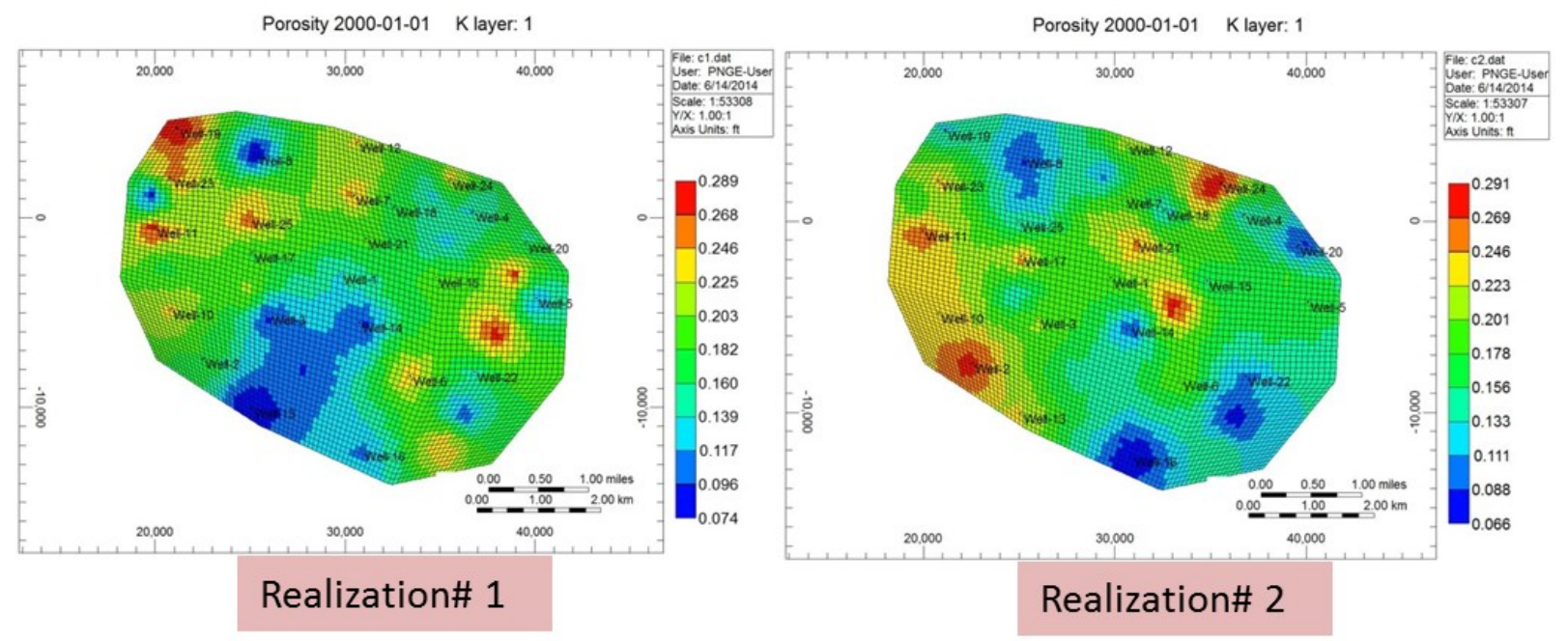

Figure 40: Comparison of porosity distributions between realizations \#1 and \#2.

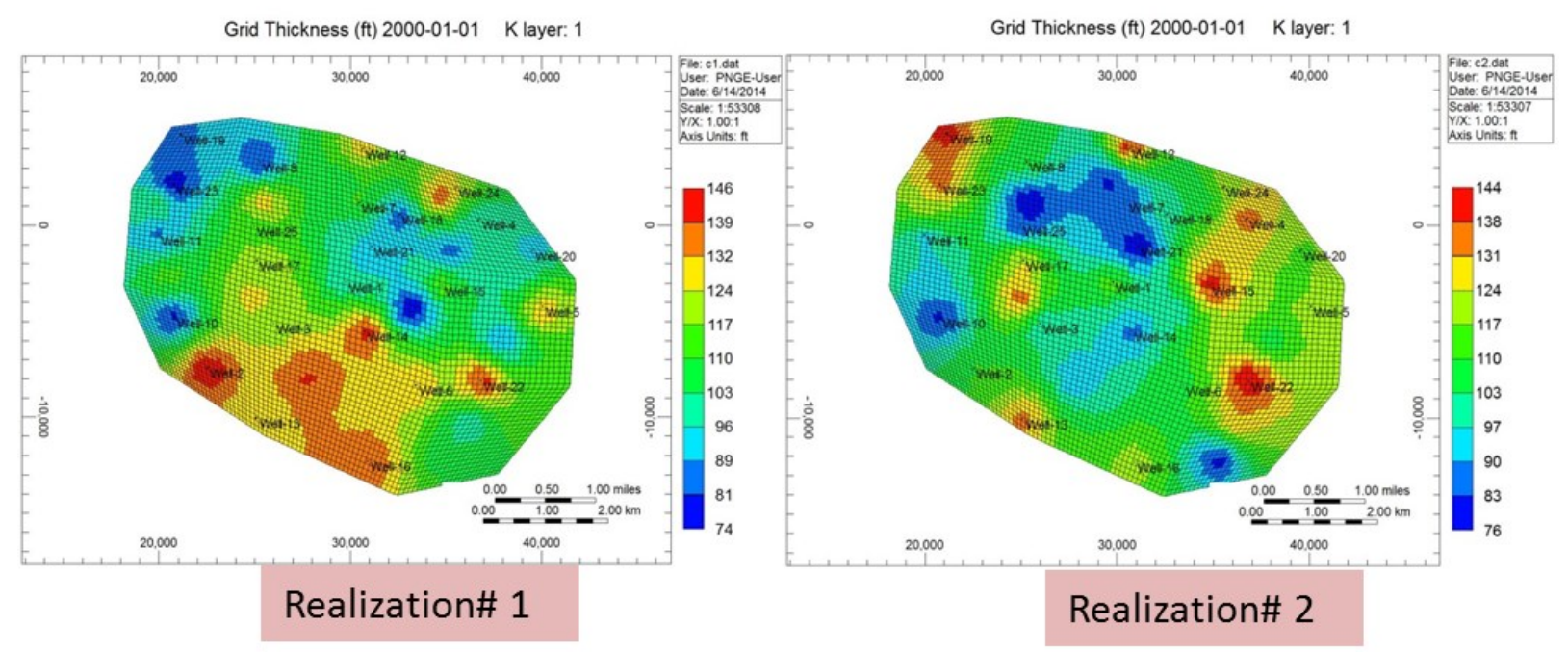

Figure 41: Comparison of thickness distributions between realizations \#1 and \#2.

In addition to the 20 training realizations, two extra realizations were created to be used as the blind verification runs. Figure 42 displays permeability, porosity, and thickness distributions for blind verification runs.

The $23^{\text {rd }}$ realization was created and assumed as the true case in which its output (oil production) is considered as the actual field data. Figure 43 depicts permeability, porosity, and thickness distributions for the actual case. 


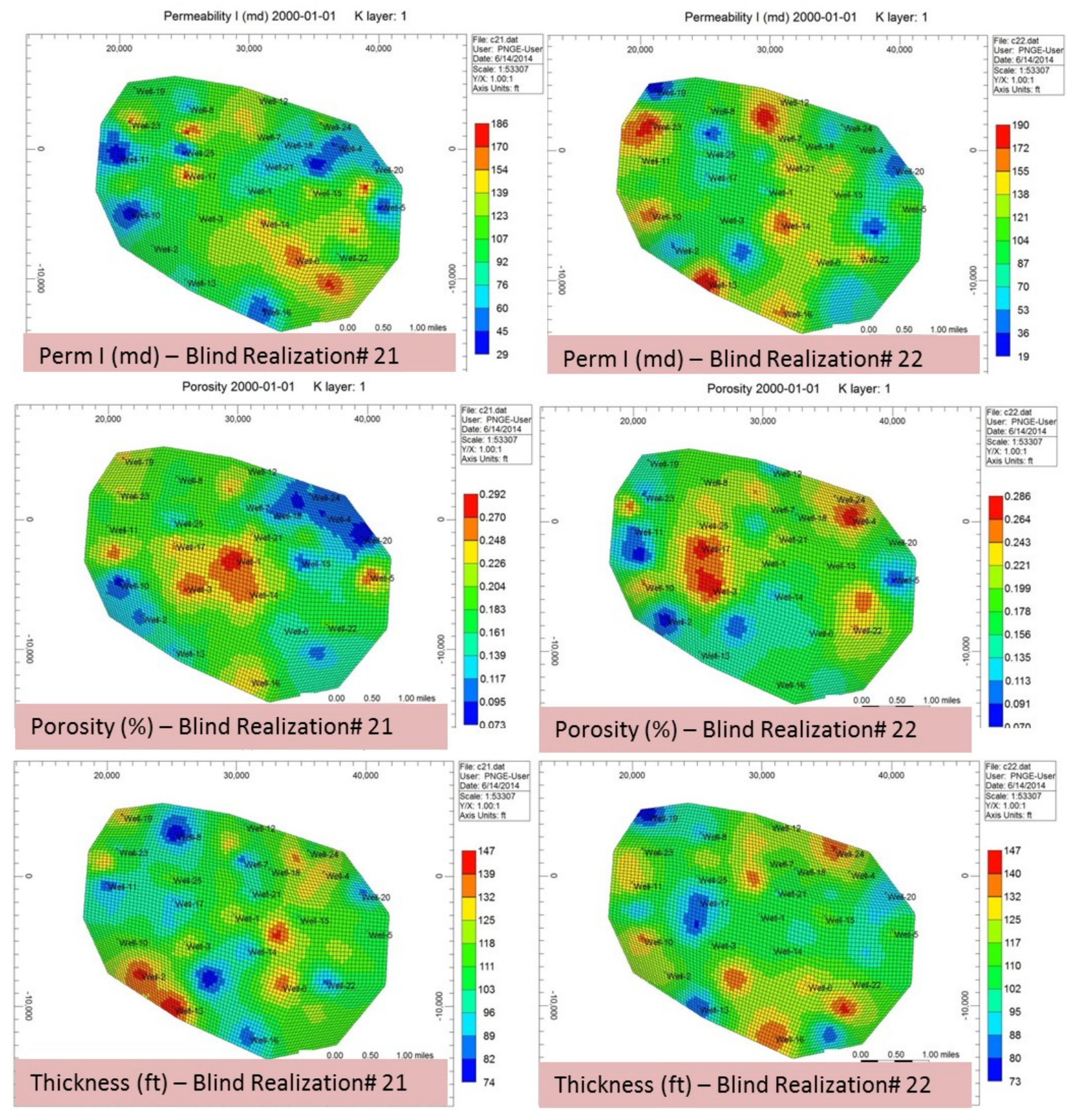

Figure 42: Permeability, porosity, and thickness distributions for two blind verification realizations. 


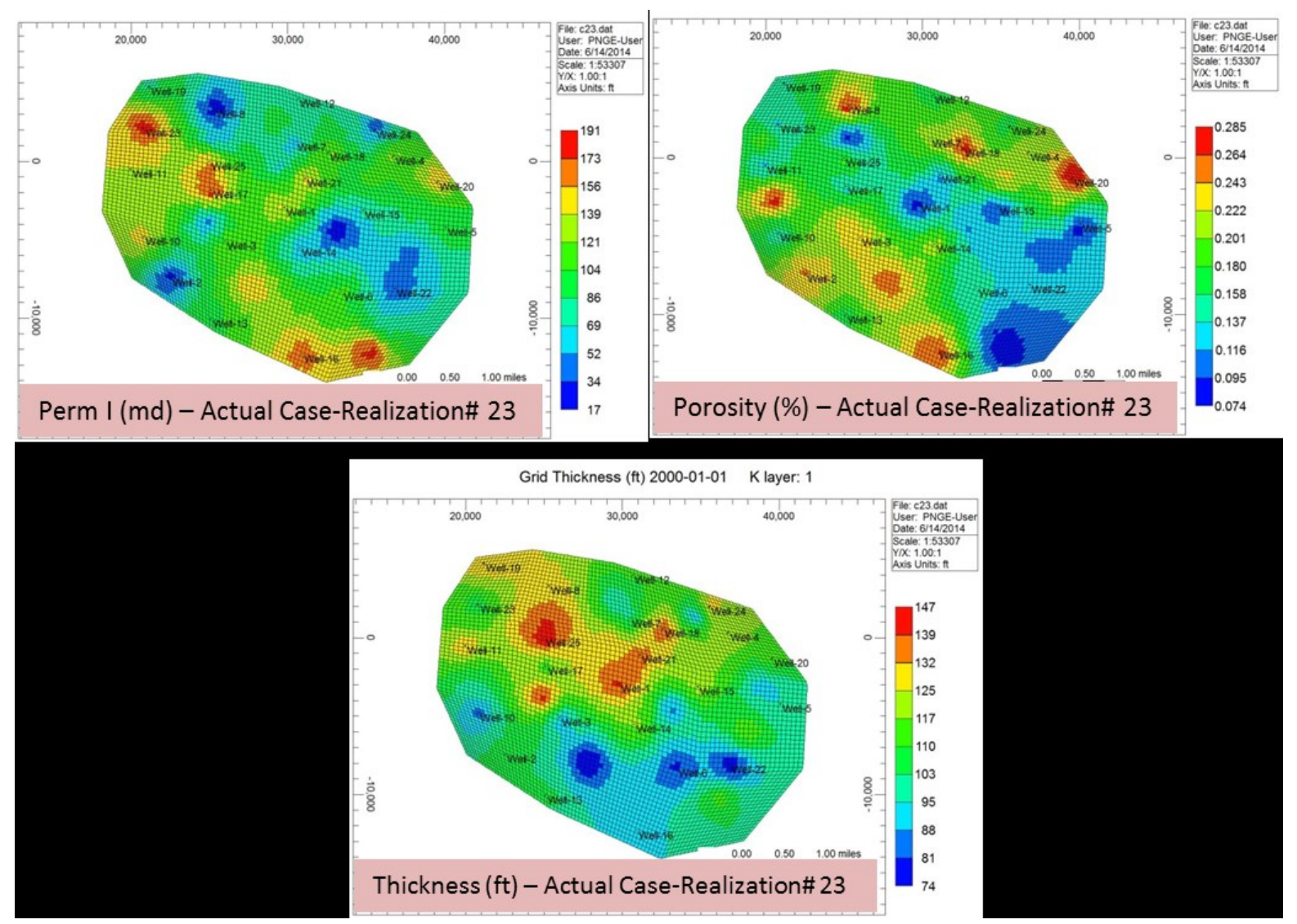

Figure 43: Permeability, porosity, and thickness distribution for actual simulation model, $23^{\text {rd }}$ realization.

\subsubsection{Reservoir delineation and tier system}

The reservoir was delineated to Voronoi polygons and then each polygon was divided into four tiers. The tier system used in this section is same as the one shown in Figure 19. After extracting the reservoir characteristics, the average value of properties in each tier was calculated. Also the dynamic data was extracted and imported into the database. At this stage the spatio-temporal database is ready to enter the training process.

\subsubsection{SRM development}

\subsubsection{Preprocessing operations}

As a pre-modeling process, KPI analysis was performed to find the most important parameters in the database. Table 9 is the result of KPI analysis for this part of study. The high ranking parameters in this list are the dynamic properties, and then the next are static parameters such as top and permeability. In this study one neural network was trained to estimate the annual oil rate production. Table 10 shows the selected inputs for the training part of SRM. The latitude (X) and longitude (Y) of the wells, the variable parameters (porosity, permeability, and thickness), and the top values at each tier are the static parameters. The dynamic parameters 
include well bottom-hole pressure (target well and offset wells), time, and oil production rate during the year before.

Table 9: The results of KPI analysis for the SRM in the "Feasibility Study".

\begin{tabular}{|c|c|c|}
\hline Rank & Feature & \% Degree of Influence \\
\hline 1 & Well Bottom-hole Pressure & 100 \\
\hline 2 & Well Bottom-hole Pressure, Offset well \# 2 & 100 \\
\hline 3 & Well Bottom-hole Pressure, Offset well \# 1 & 99 \\
\hline 4 & Well Bottom-hole Pressure, Offset well \# 3 & 98 \\
\hline 5 & Time, (day) & 92 \\
\hline 6 & Annual Production (t-1), bbl/yr & 91 \\
\hline 7 & Oil Rate bbl/day (t-1) Offset well \# 3 & 29 \\
\hline 8 & Oil Rate bbl/day (t-1) Offset Well \# 2 & 28 \\
\hline 9 & Top, Tier \#3 & 25 \\
\hline 10 & Top, Tier \#1 & 25 \\
\hline 11 & Top, Tier \#2 & 25 \\
\hline 12 & Horizontal Permeability, Tier \#2 & 23 \\
\hline 13 & Oil Rate bbl/day (t-1), Offset well \# 1 & 23 \\
\hline 14 & Horizontal Permeability, Tier \#1 & 21 \\
\hline 15 & Top, Tier \#4 & 21 \\
\hline 16 & Horizontal Permeability, Tier \#3 & 19 \\
\hline 17 & Top Offset well \#2 Tier \#4 & 15 \\
\hline 18 & Top Offset well \#2 Tier \#1 & 14 \\
\hline 19 & Top Offset well \#2 Tier \#3 & 14 \\
\hline 20 & Other parameters & Less than 14 \% \\
\hline
\end{tabular}

Table 10: Selected inputs for training the SRM in the "Feasibility Study".

\begin{tabular}{|c|c|}
\hline Static Inputs & Dynamic Inputs \\
\hline Well Latitude $(\mathrm{X})$ & \multirow{3}{*}{ Bottom-hole Pressure } \\
\hline Well Langitude $(\mathrm{Y})$ & \\
\hline Horizontal Permeability, Tier \# 1 & \\
\hline Thickness, Tier \# 1 & \multirow{3}{*}{ Time } \\
\hline Top, Tier \# 1 & \\
\hline Porosity, Tier \# 2 & \\
\hline Horizontal Permeability, Tier \# 2 & \multirow{3}{*}{ Oil production rate @ a year before } \\
\hline Thickness, Tier \# 2 & \\
\hline Top, Tier \# 2 & \\
\hline Porosity, Tier \# 3 & \multirow{3}{*}{ Well Bottom-hole Pressure, Offset well \# 1} \\
\hline Horizontal Permeability, Tier \# 3 & \\
\hline Thickness, Tier \# 3 & \\
\hline Top, Tier \# 3 & \multirow{3}{*}{ Well Bottom-hole Pressure, Offset well \# 2} \\
\hline Porosity, Tier \# 4 & \\
\hline Horizontal Permeability, Tier \# 4 & \\
\hline Thickness, Tier \# 4 & \multirow{3}{*}{ Well Bottom-hole Pressure, Offset well \# 3} \\
\hline Top, Tier \# 4 & \\
\hline Top,T4 & \\
\hline
\end{tabular}

\subsubsection{Train, calibrate, and validate the ANNS}

After the inputs selection is made, using IDEA $^{\mathrm{TM}}$ the database is divided into training, calibration, and verification sets (with $80 \%, 10 \%$, and $10 \%$ shares of database respectively). 
A back propagation training algorithm was used to train the data. Figure 44 displays the structure of the ANN used in this section. There is one hidden layer in this structure which contains 29 neurons. The hidden layer is connected to the inputs and output layers. The inputs are based on the selected parameters in the previous section and the output of network is annual oil production rate.

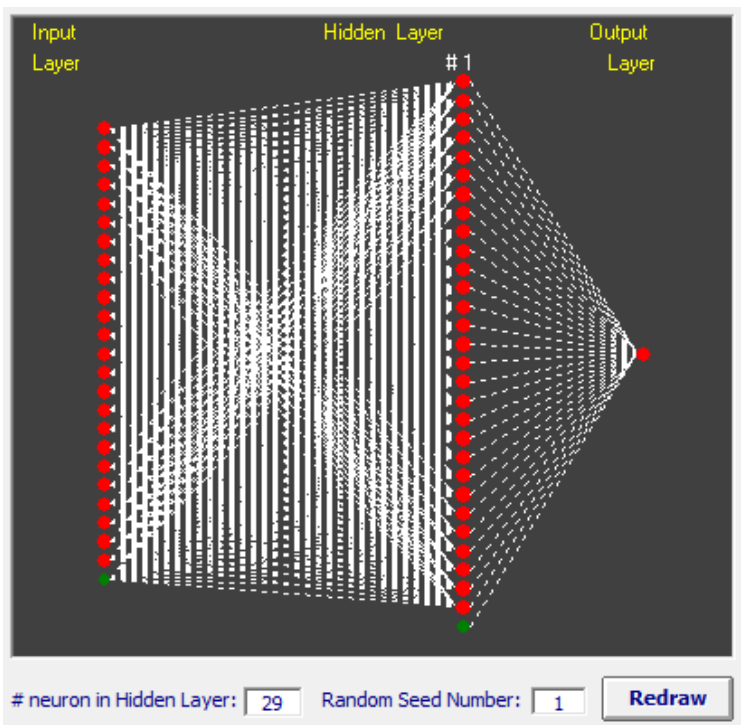

Figure 44: ANN structure.

Figure 45 shows the results in scatter plots for training, calibration, and verification sets during the training process.
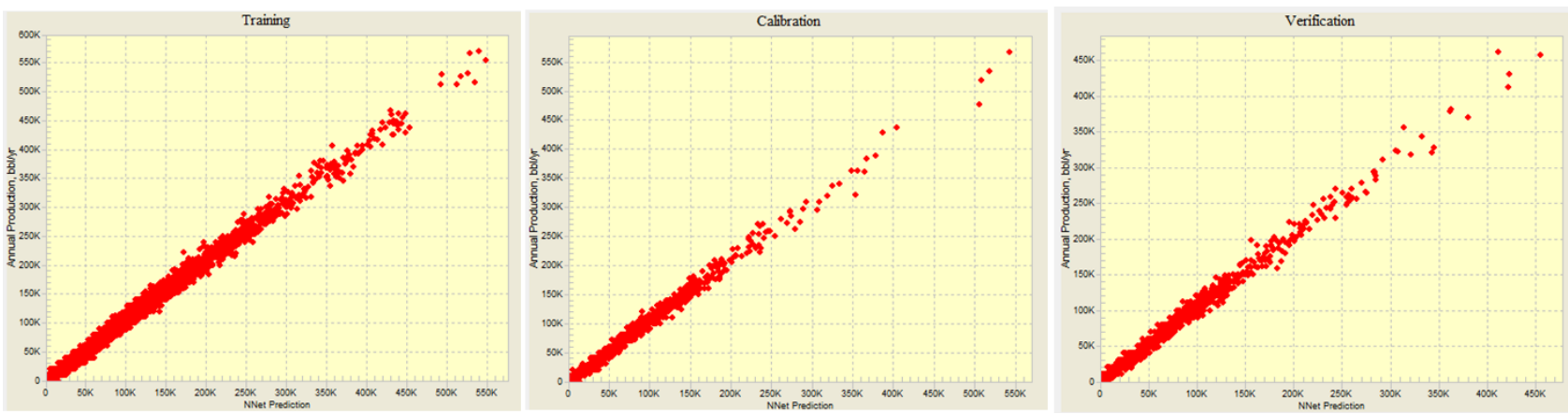

Figure 45: Scatter plots for the training, calibration and verification sets during the training process.

\subsubsection{Blind verification}

When the SRM is trained, its robustness is tested using the blind verification runs. As it was mentioned earlier, two extra runs were generated as the blind verification realizations. These realizations have completely different distributions of permeability, porosity, and thickness (Figure 42). 


\subsubsection{Automated SRM-based history matching}

The developed surrogate reservoir model was further validated using the blind verification runs. After passing the testing step, the SRM is ready to get into the history matching process. For this purpose the SRM was coupled with DE optimization algorithm to perform the history matching. Coupling SRM with DE provides an automated workflow. The range of properties and the objective functions are the only items provided by the user in this workflow. Then DE selects the first set of parameters and calls the developed SRM. The SRM calculates the outputs (oil production) and the misfit value are measured using the objective functions. The misfit values and selected parameters are saved in the memory of computer. This process is carried out automatically and repeated until the stoppage criteria are met. The stoppage criteria here are a constant value for misfit or after calling SRM for 3000 times.

\subsubsection{Results}

Similar to the other section, we do not show the results for all the wells here. A single well (well \#2) was selected in this section to present the results at the different steps of development and application of SRM. The results of the other wells are available in Appendix $\mathrm{B}$ (sections $\mathrm{b}$ and $\mathrm{c}$ ). Figure 46 shows a comparison of the results between the performance of SRM and simulator (CMG) for well \#2 in a training realization. In addition, Figure 47 shows the same comparison for a blind verification run. The SRM shows a good performance over the training and blind realizations.

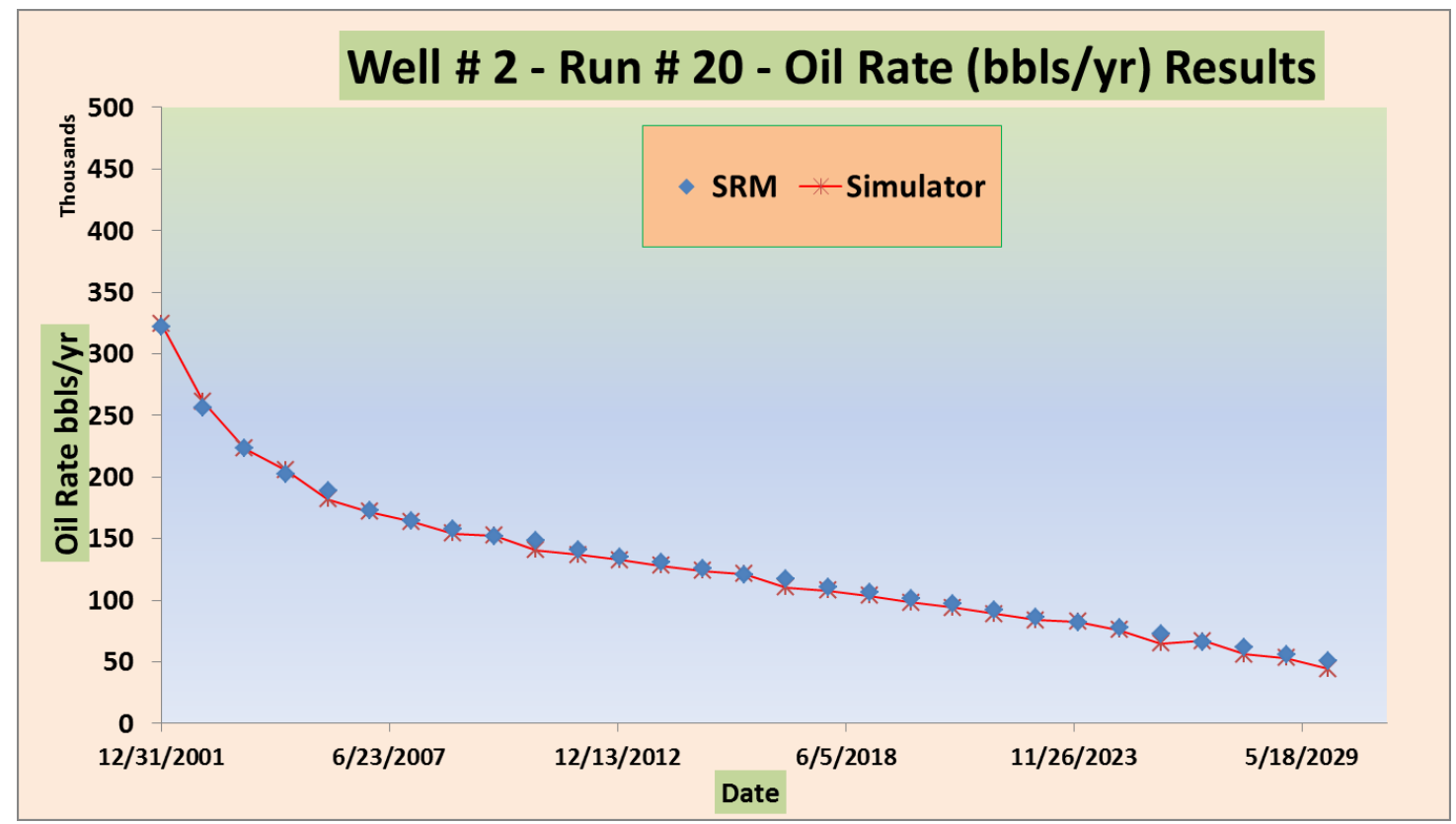

Figure 46: Comparison between the results of SRM and simulator for well \#2 in a training realization. 


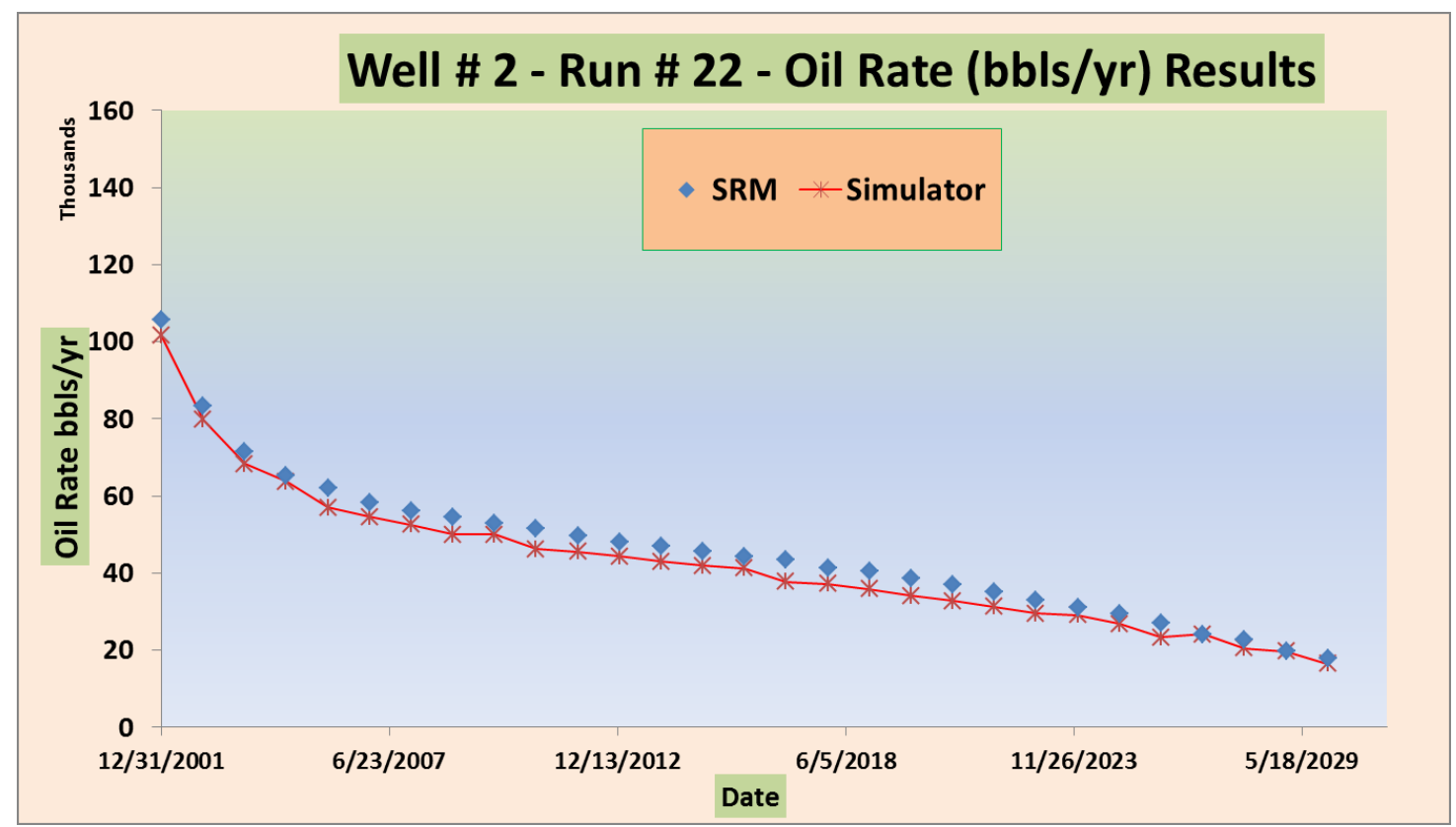

Figure 47: Comparison between the results of SRM and simulator for well \#2 in a blind verification run.

Figure 48 is the results of history matching for well \#2 comparing the ten best achieved matches with the actual data. The blue lines in this figure represent the ten best matches and the red stars are the actual data. Offering multiple matched realizations is an advantage provided in this automated history matching workflow.

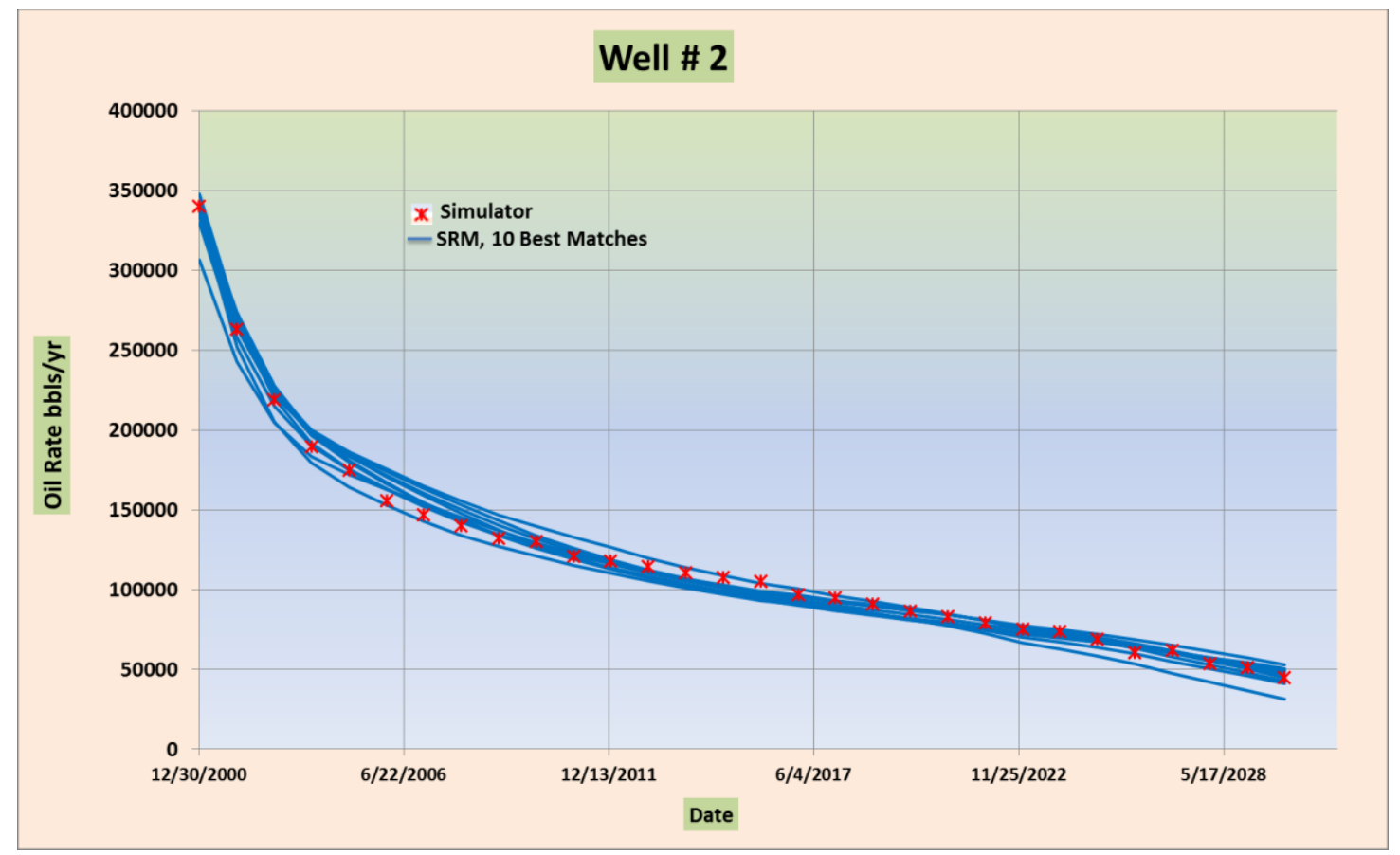

Figure 48: The outcome of history matching for well \#2 comparison between ten best matches (blue lines) and actual data (red stars). 


\subsection{Discussion and concluding remarks}

In this chapter we reviewed the general steps required to develop a surrogate reservoir model (SRM) in order to utilize it in a history matching process. Based on the explained instructions, two examples of SRM were constructed. The first example was an attempt to show the potential of SRM to replace the reservoir simulation model in history matching workflow. The second case tried to increase the complexity of problem to challenge the SRM performance. Furthermore, the SRM was connected to an optimization algorithm to set up an automated history matching package. In both cases, the surrogate reservoir models were built using a few realizations of the base reservoir model. The number of simulation runs to build the SRMs is far less than the number of runs required for history matching. The SRMs were able to accurately match the behavior of the reservoir models through the provided spatio-temporal databases. Moreover, the robustness of the SRMs was further verified by applying them to extra realizations of the reservoir models. These realizations, called blind verification runs, were not seen by SRMs during the training steps.

Once the SRMs were ready, they substituted the reservoir simulation models. In the first example the goal was to achieve a match of production history by tuning permeability distribution. Using the SRM in this example accomplished a satisfying match.

In the next step, the number of uncertain properties increased. The SRM was created by altering the distributions of porosity, permeability, and thickness. These properties were the uncertain reservoir characteristics to match the field production. The developed SRM showed a significant performance in an automated history matching workflow. Based on this approach the SRM was connected to an evolutionary algorithm optimization method. This workflow is able to produce multiple realizations which match the past performance of reservoir.

The applications of SRM to reproduce the results of reservoir simulation model in manual and automated history matching workflows were tested. The results of history matching demonstrate the efficiency of SRM to be used in the history matching process. Although the running time for the case studies of reservoir model used in this study is not the concern, the number of simulation runs to attain a desired match is time and power consuming. In a numerical reservoir simulator, by increasing the size and complexity of the components, the run-time can increase in orders of magnitude. Nevertheless, due to pattern recognition capability of SRM, it will not be an issue using this technology. 
The results of two case studies in this chapter showed the potential of this novel technology (SRM) to assist the history matching process. Implementing the technology on a more sophisticated (and real-life) case study is the topic of the next chapter of this dissertation. 


\section{Chapter 5: A real-life case study, implementation of SRM on PUNQ-S3 problem}

\section{Overview}

In the previous chapter we developed two surrogate reservoir models in order to replace the reservoir simulation model in history matching case studies. We started with simple assumptions. Initially an SRM was made by tuning just one property, and then the number of uncertain properties increased. In the first case, history matching was completed in a manual workflow. But in the second case we had an automated workflow benefiting from an optimization algorithm. In both cases the objective was to match the oil production history. However, in reality we deal with more complicated cases. There are usually various types of field data to match (for example matching oil, gas, and water productions or well bottom-hole pressure).

The ideas behind the SRM as well as some details on how to develop SRM were covered in chapters 3 and 4 . This chapter intends to demonstrate the development and application of an SRM for a more realistic case. The chapter starts with introducing the reservoir model. Then the steps to develop the surrogate reservoir model are presented. The automated history matching workflow for this case is reviewed and finally representative results are shown.

\subsection{Real-life case study - PUNQ Model}

In the literature we can find various approaches to perform history matching. All these methods are usually associated with synthetic or real case examples trying to demonstrate their capabilities. However, most of time, it is tough to compare these methods with each other because they are very subjective to the case that they have been tested on. One way suggested to surpass this problem is to test different methods on a similar and unique example. This is a common practice in different areas of applied science. In the area of reservoir management there are a couple famous standard case studies which have been utilized to test and compare the results of different workflows (Floris, et al. 2001, Chen and Oliver 2010). PUNQ reservoir 
model, designed for PUNQ project, is one of these examples. In continuation, we will talk about this project and characteristics of the reservoir model used in this study.

The PUNQ project, (PUNQ stands for Production forecasting with Uncertainty Quantification), is a mutual study supported by European Union and conducted by 10 European companies, universities, and research centers (Figure 49) (Floris, et al. 2001). The main objective of the project was to compare the methods for quantifying uncertainty assessment in history matching. In order to achieve the objective of the project a simple reservoir model (PUNQ-S) was constructed. PUNQ-S was originally based on a real field operated by Elf Exploration and Production (Floris, et al. 2001, Barker, Cuypers and Holden 2001). Three different versions were adopted from PUNQ-S model known as: PUNQ-S1, S2, and S3. These models vary in the way the distributions of porosity and permeability were created. Since the creation of PUNQ models, many researchers have studied different methods of history matching and uncertainty assessment using these models (mainly working on PUNQ-S3 model) (Floris, et al. 2001, Barker, Cuypers and Holden 2001, Gu and Oliver 2005, Gao, Zafari and Reynolds 2005, Abdollahzadeh, et al. 2011). Figure 50 summarizes the search result of "PUNQ Model" in the database belonging to the Society of Petroleum Engineers (SPE) (OnePetro 2014).

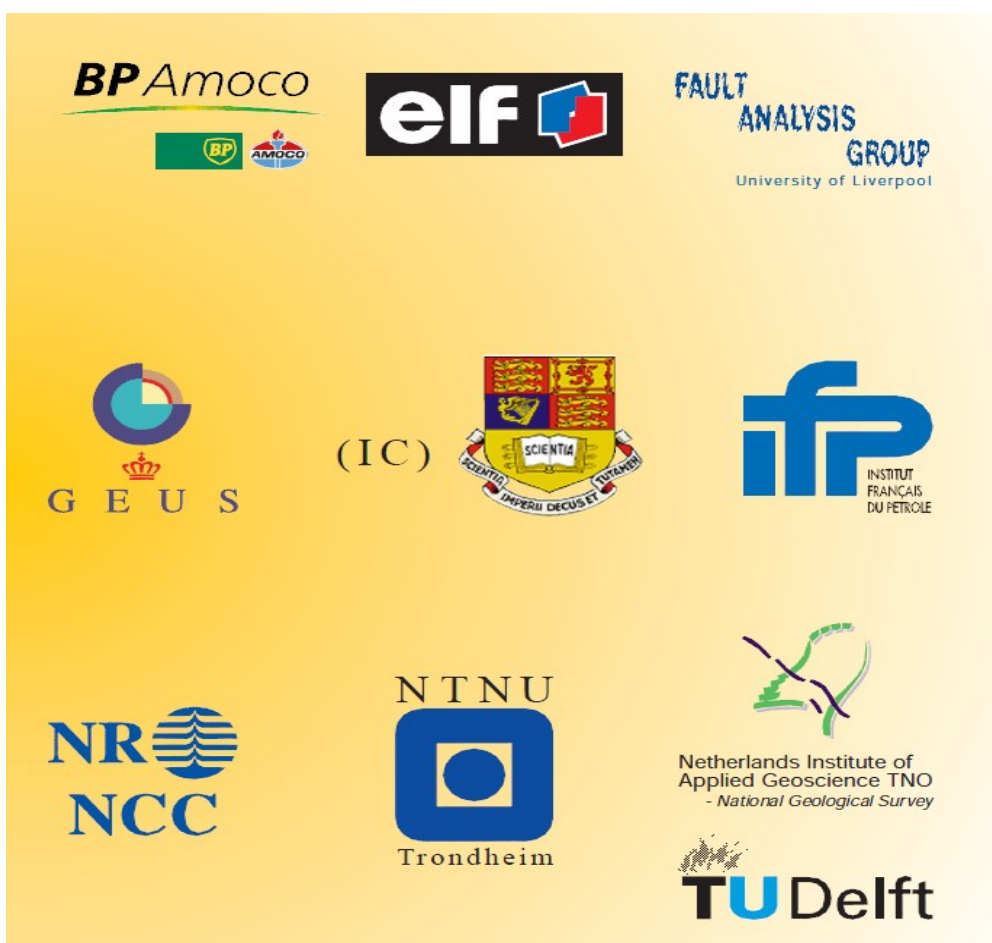

Figure 49: European companies, universities, and research centers involved in the PUNQ project (Floris, et al. 2001). 

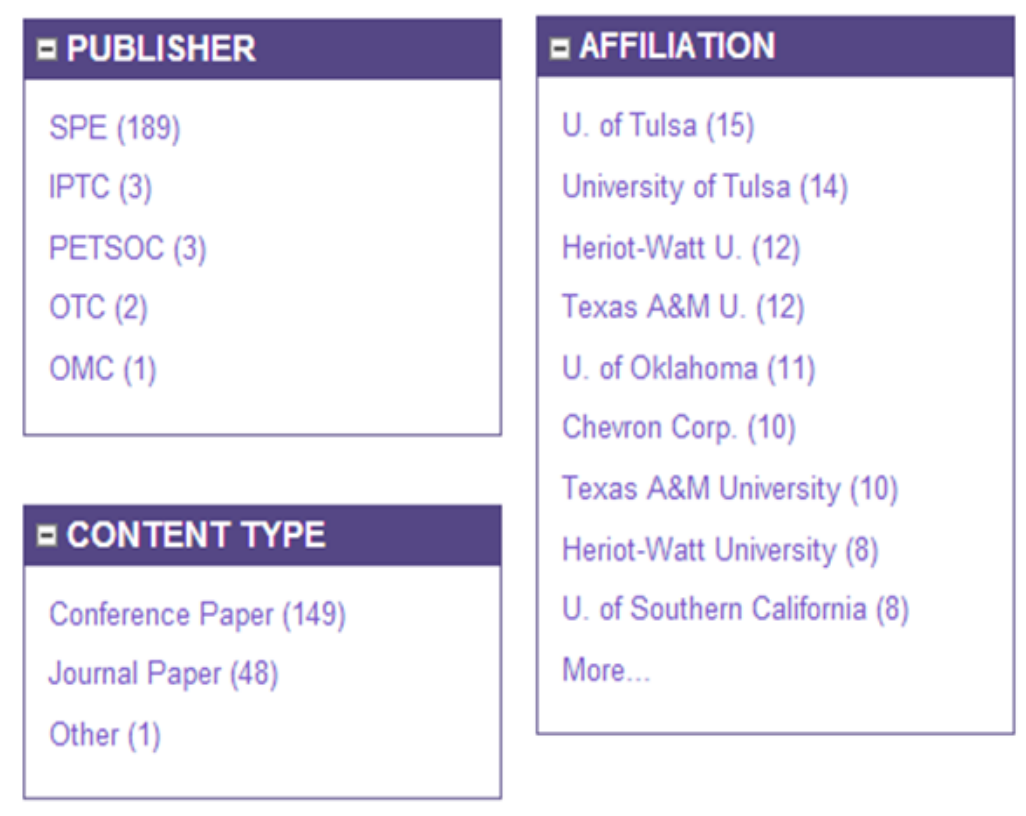

\section{E AUTHOR}

Dongxiao Zhang (13)

Gaoming Li (12)

Albert C. Reynolds (10)

Albert Coburn Reynolds (9)

Geir Naevdal (8)

Michael A. Christie (8)

Akhil Datta-Gupta (7)

Dean S. Oliver (7)

Geir Evensen (7)

More.

Figure 50: Search results for "PUNQ model" key word in the SPE database (access date: March 2014). The figure summarizes the number of publications by different publishers, the type of publications, the affiliation of authors, and the authors involved in highest number of publications (OnePetro 2014).

In this study we use the third version of PUNQ-S model, known as PUNQ-S3 reservoir simulation model. PUNQ-S3 reservoir simulation model is different from PUNQ-S1 and S2 because of the stochastic correlation between porosity and permeability and also the added random noise to the static and dynamic well data (Boss 1999). This simulation model is considered a small-size industrial reservoir engineering model and it is widely accepted as a standard synthetic test case to investigate the ability of different methods of history matching and uncertainty quantification. The model consists of $19 \times 28 \times 5$ grid blocks (180 $\mathrm{m}$ by $180 \mathrm{~m}$ ), of which a total of 1,761 grid blocks are active. The geometry of the field has been modeled using corner-point geometry. The field is bounded to the east and south by a fault. There is also a fairly strong aquifer located in the north and west of reservoir. Due to presence of aquifer and providing the pressure support, no injection plan was designed. In addition there is a small gas cap in the first layer and in the center of the dome shaped structure. In order to avoid the gas production from the gas cap no well has been perforated in the first layer.

Figure 51 demonstrates the top structure of the PUNQ-S3 reservoir model. As it is shown in this figure, there are six production wells drilled in the reservoir. Layers one and two are left without perforation. The other layers are completed for different wells: wells PRO-1, PRO-4 and PRO-12 are perforated in layers 4 and 5. The wells PRO-5 and PRO-11 have been completed in layers 3 and 4 and well PRO-15 has been perforated only in layer 4 . 


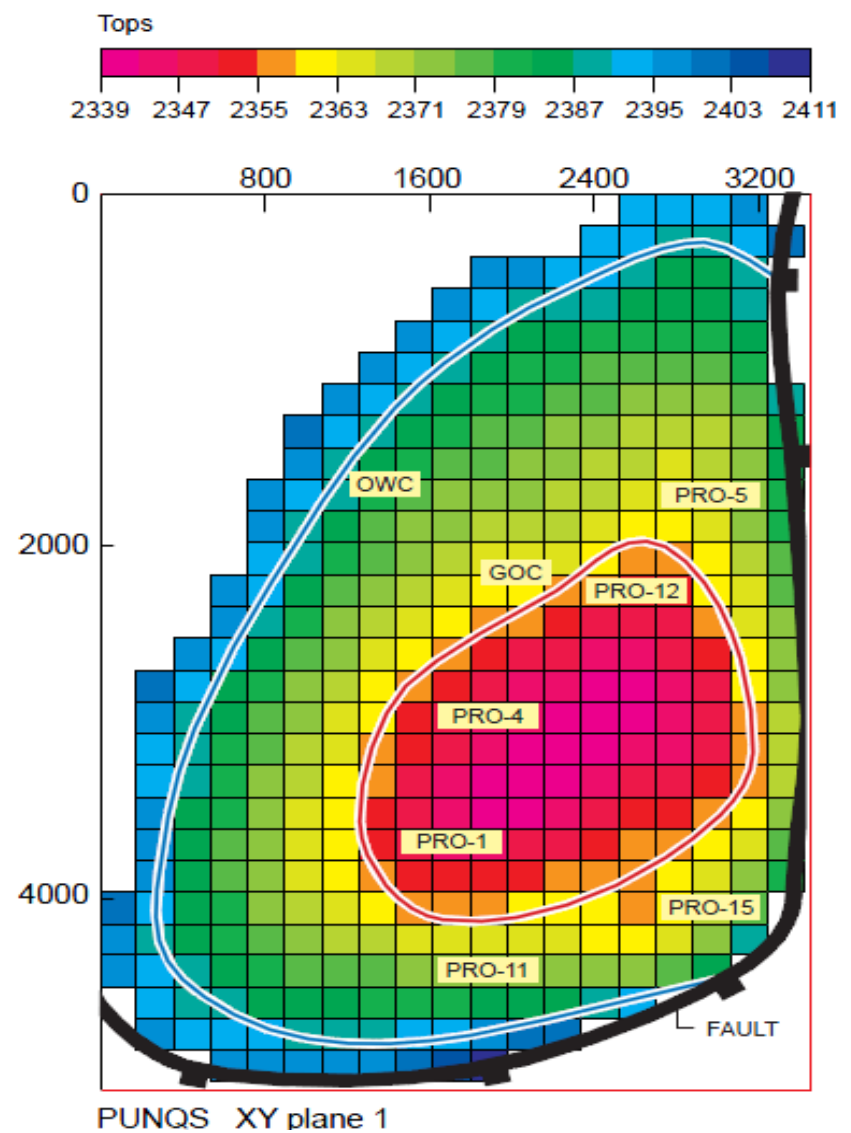

Figure 51: The top structure of PUNQ-S3 model. The field is bounded to the east and south by a fault, and links to the north and west to a fairly strong aquifer. In addition there is a small gas cap in the center of the dome shaped structure (Floris, et al. 2001).

\subsubsection{True case}

In order to provide similar and identical data for everybody, a "true" case has been designed. The synthetic actual data is provided by executing the true model. Then these actual data are used to compare the results of different methods of history matching and uncertainty assessment. The main characteristics to generate the true case are porosity and permeability (horizontal and vertical) distributions. The values of these properties at well sites are taken from the original model. The comprehensive procedure of creating the porosity and permeability distributions for true case can be found somewhere else (Barker, Cuypers and Holden 2001, Petroleum Engineering \& Rock Mechanics Group (PERM) 2014). Figure 52 and Figure 53 show the horizontal and vertical permeability distributions for the true case. Figure 54 also depicts the porosity distributions. Then the outputs of the true case are considered as the actual historical data. Eight years of production and 16.5 years cumulative production are available. Eight years of production will be used to match the model and 16.5 years of cumulative production will be utilized to perform the future forecast and uncertainty quantification. 

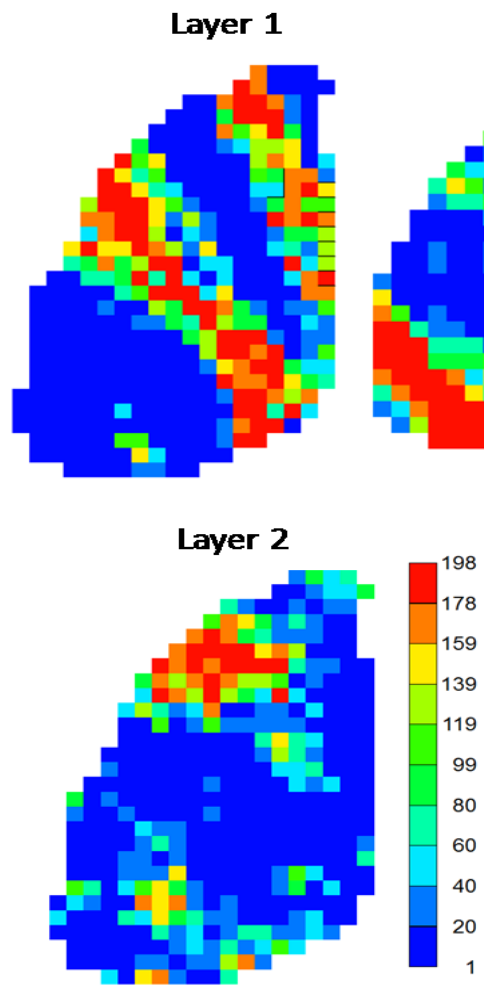

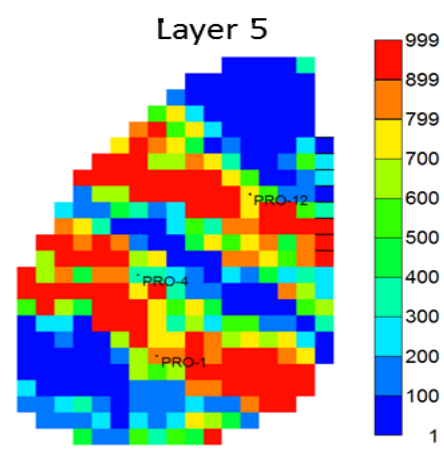

Layer 4

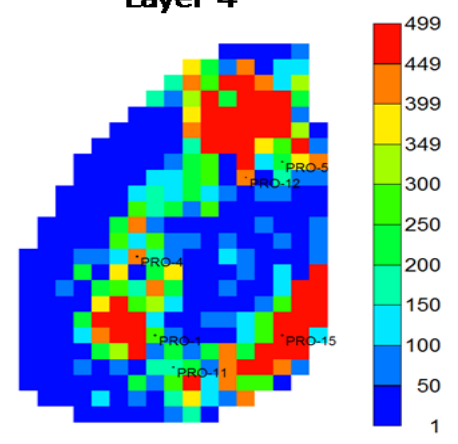

Figure 52: Horizontal permeability distributions for PUNQ-S3 true case.

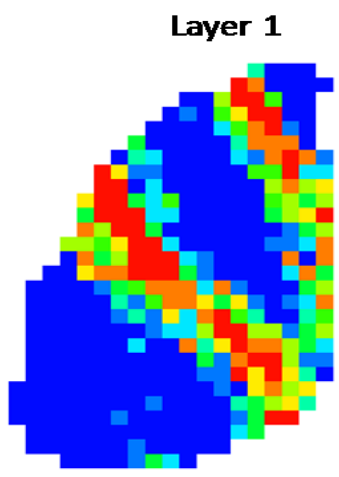

Layer 2

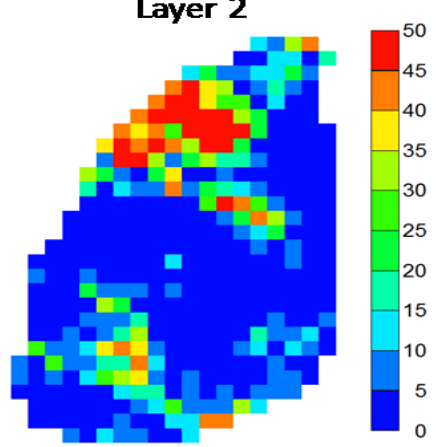

Layer 3
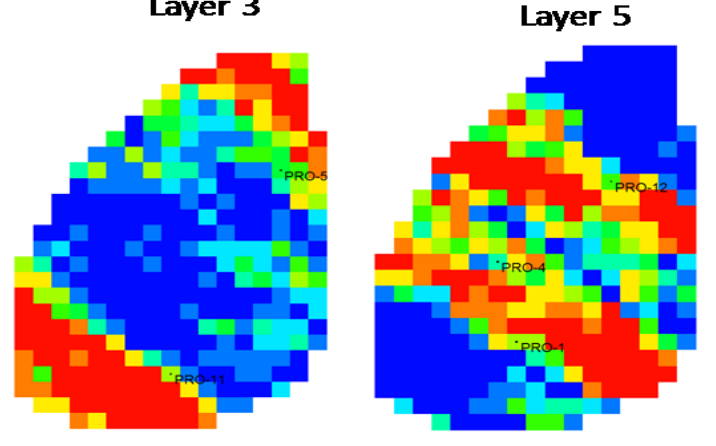

Layer 4

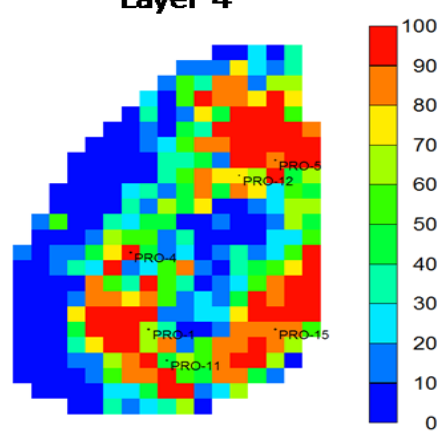

Figure 53: Vertical permeability distributions for PUNQ-S3 true case. 


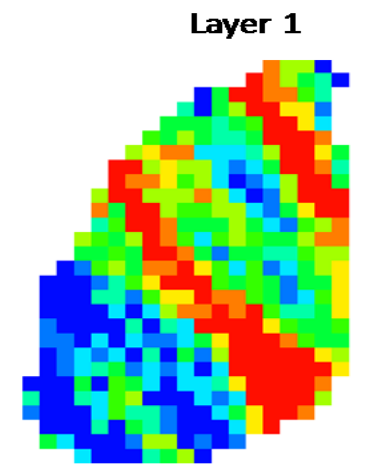

Layer $\mathbf{2}$

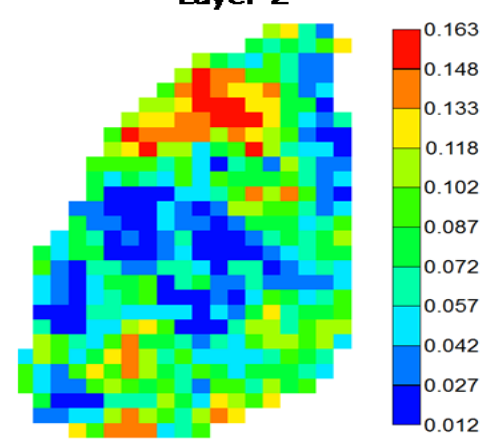

Layer 3
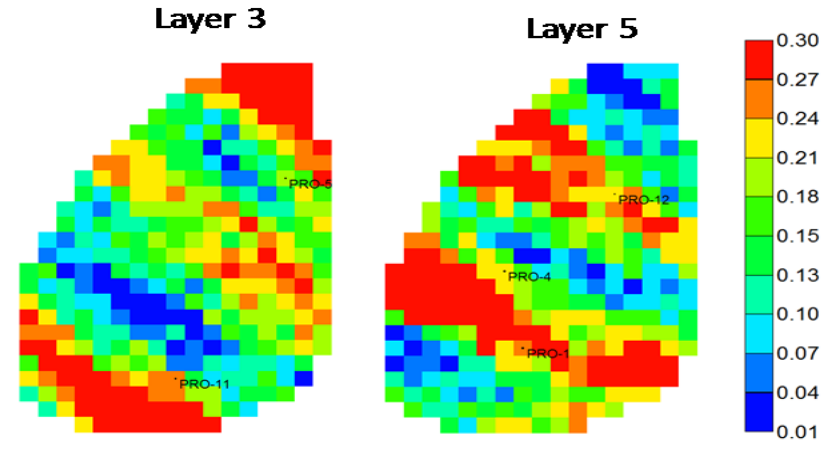

Layer 4

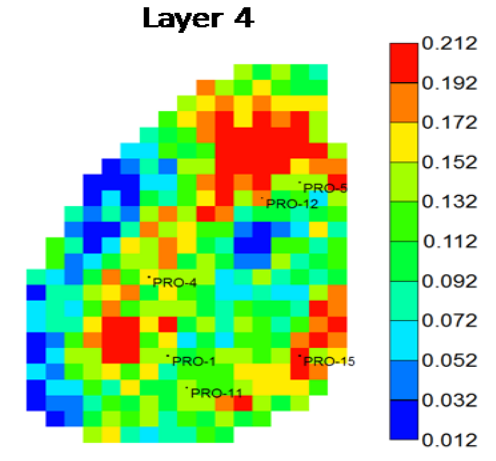

Figure 54: Porosity distributions for PUNQ-S3 true case.

\subsubsection{Geological description}

Along with presenting the simulation model, there are some geological descriptions available for each layer of PUNQ-S3 reservoir model. Layers 1, 3, and 5 have linear streaks (channels) of highly porous sandstone. The second layer is mainly low porous, shaly sediment, with some irregular patches of somewhat higher porosity. Layer 4 includes an intermediate porosity region with an approximate lobate shape embedded in a low-porosity matrix. Complete detailed geological description is presented in the work by Floris et al. (2001). The PVT and aquifer data for the true model are also available. The relative permeability values are based on the power law relative permeability functions and it is assumed there is no capillary pressure (Floris, et al. 2001, Barker, Cuypers and Holden 2001). The gas oil contact (GOC = $2355.0 \mathrm{~m})$ and water oil contact $(\mathrm{WOC}=2397.4)$ values are also given.

\subsubsection{Field production}

The production schedule is similar to what happened in the original model and is based on the following schedule (Floris, et al. 2001):

- First year extended well testing divided into four three-monthly production periods.

- Three years of shut-in.

- Twelve and half years of field production. 
- Every year of production includes two weeks shut-in test in order to gather the shut-in pressure data.

As it was mentioned previously due to the strong aquifer there is no injection plan. Well PRO4 is perforated close to the aquifer; therefore, we see water breakthrough in the seventh year. The production constraint is a constant oil rate $\left(150 \frac{\mathrm{m}^{3}}{d a y}\right)$ until the bottom-hole pressure reaches below a fixed value $(12000 \mathrm{kPa})$, which they start producing with a constant bottomhole pressure $(12000 \mathrm{kPa})$. The true model was completed in a commercial simulator (ECLIPSE $^{\mathrm{TM} 1}$ (Schlumberger 2014)). After running the model, the first eight years of production and pressure data was considered as the "actual" data for history matching purpose. In addition cumulative production (total oil recovery) was provided after 16.5 years for future forecast comparison and uncertainty assessments study. The synthetic actual data include oil, gas, and water production as well as bottom-hole pressure for each well. Figure 55 demonstrates a sample of production and pressure curves. The complete data set designed for ECLIPSE $^{\mathrm{TM}}$ is available for the public here (Petroleum Engineering \& Rock Mechanics Group (PERM) 2014). Using this simulator, the cumulative production after 16.5 years was reported $3.87 \times 10^{6} \mathrm{Sm}^{3}$ (Floris, et al. 2001, Barker, Cuypers and Holden 2001). However the

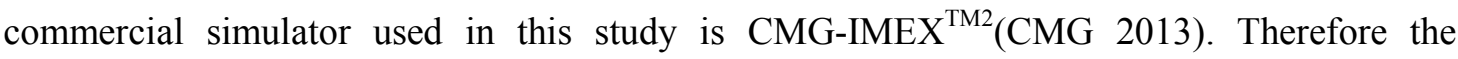
cumulative production using this simulator is $3.92 \times 10^{6} \mathrm{Sm}^{3}$, which there is a relative difference of $1.29 \%$ compared to what ECLIPSE $^{\mathrm{TM}}$ produced. In order to mimic the real-life measurement errors, some noise was added to porosity/permeability values as well as the synthetic field data. The details of adding noise to the data can be found here (Floris, et al. 2001, Barker, Cuypers and Holden 2001).

To summarize what we have covered so far, the available data for PUNQ-S3 reservoir model could be listed as the following:

- Porosity and permeability values at well locations.

- Geological descriptions for each layer.

- Production history for the first 8 years (for history matching study).

- Cumulative production (total oil recovery) after 16.5 years (for uncertainty quantification and production forecast study).

\footnotetext{
${ }^{1}$ Schlumberger

${ }^{2}$ Computer Modeling Group
} 
- $\quad$ PVT, relative permeability and Carter-Tracy aquifer dataset all taken from original field data.

- There is no capillary pressure function.

- Gas oil contact (GOC) and water oil contact (WOC).

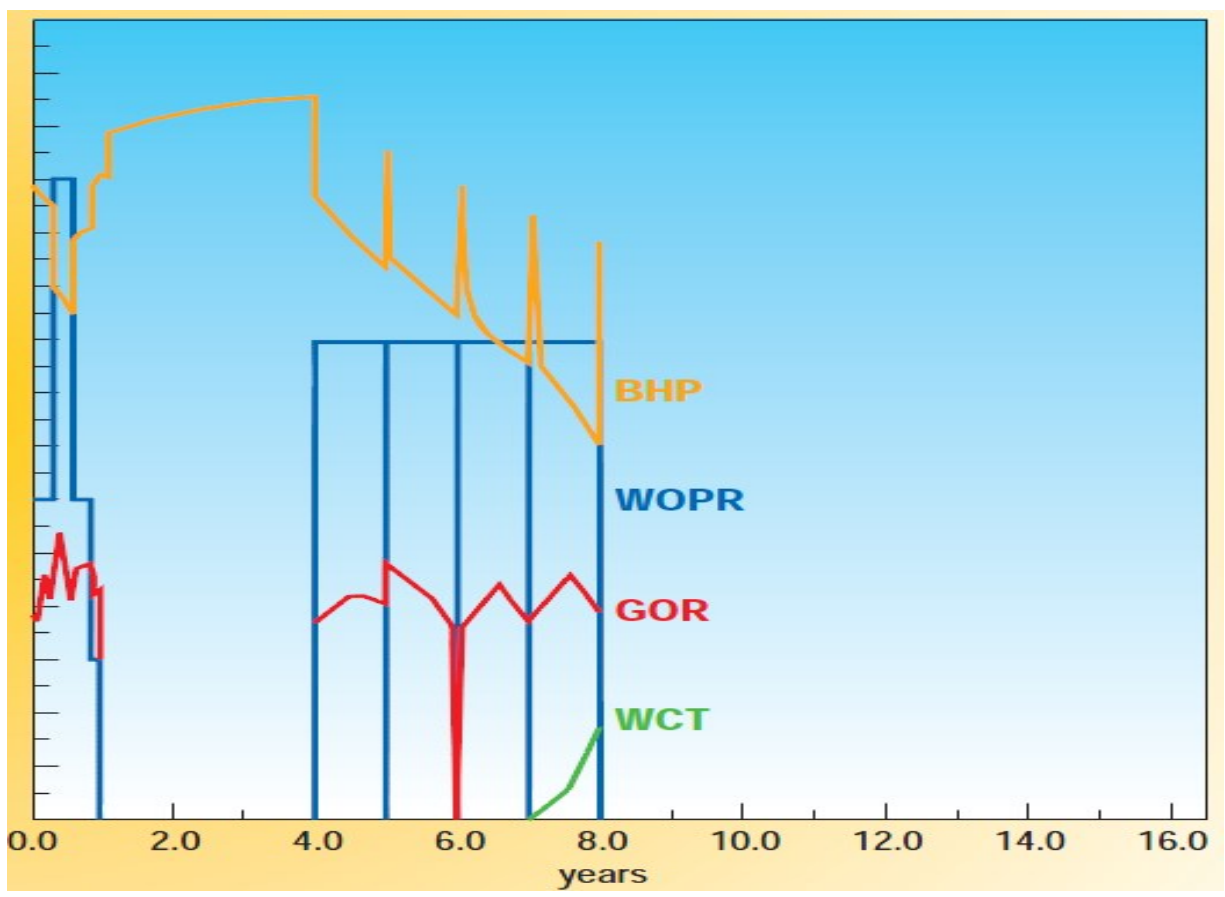

Figure 55: Provided field performance for PUNQ-S3 reservoir model. Bottom-hole pressure (BHP), well oil production (WOPR), gas oil ratio (GOR), and water cut (WCT) for each well and field are available for eight years (Floris, et al. 2001).

\subsubsection{Literature review on PUNQ-S3 reservoir model}

In the literature of petroleum engineering, PUNQ-S3 reservoir model is considered as a widely favored standard model to challenge the new methods of history matching and uncertainty assessment. This model is appropriate as a scale to compare the results of new approaches with the previous methods. Many studies have been conducted using PUNQ-S3 reservoir model. Floris et al. (2001) summarized the results of eleven different approaches performed by the research groups involved in the PUNQ project. These eleven approaches were distinguished by the following features:

- Reservoir parameterization: For instance using homogenous layers, homogeneous drainage area regions, pilot points selected, etc.

- Number and type of adjustable parameters to match the field data: For example different approaches vary in selecting porosity and permeability (horizontal and vertical) as the uncertain properties. 
- Spatial technique for generating the distributions of porosity/permeability such as Kriging, Gaussian Random Fields (GRF), etc.

- The optimization algorithms used for history matching such as genetic algorithm, Gauss-Newton, etc.

- Three different kinds of reservoir simulator.

Each one of these approaches and their results has been discussed in the article published by Floris et al. (2001). Figure 56 displays low, median, and high ranges of total oil recovery after 16.5 years for all eleven approaches described by Floris et al. (2001).

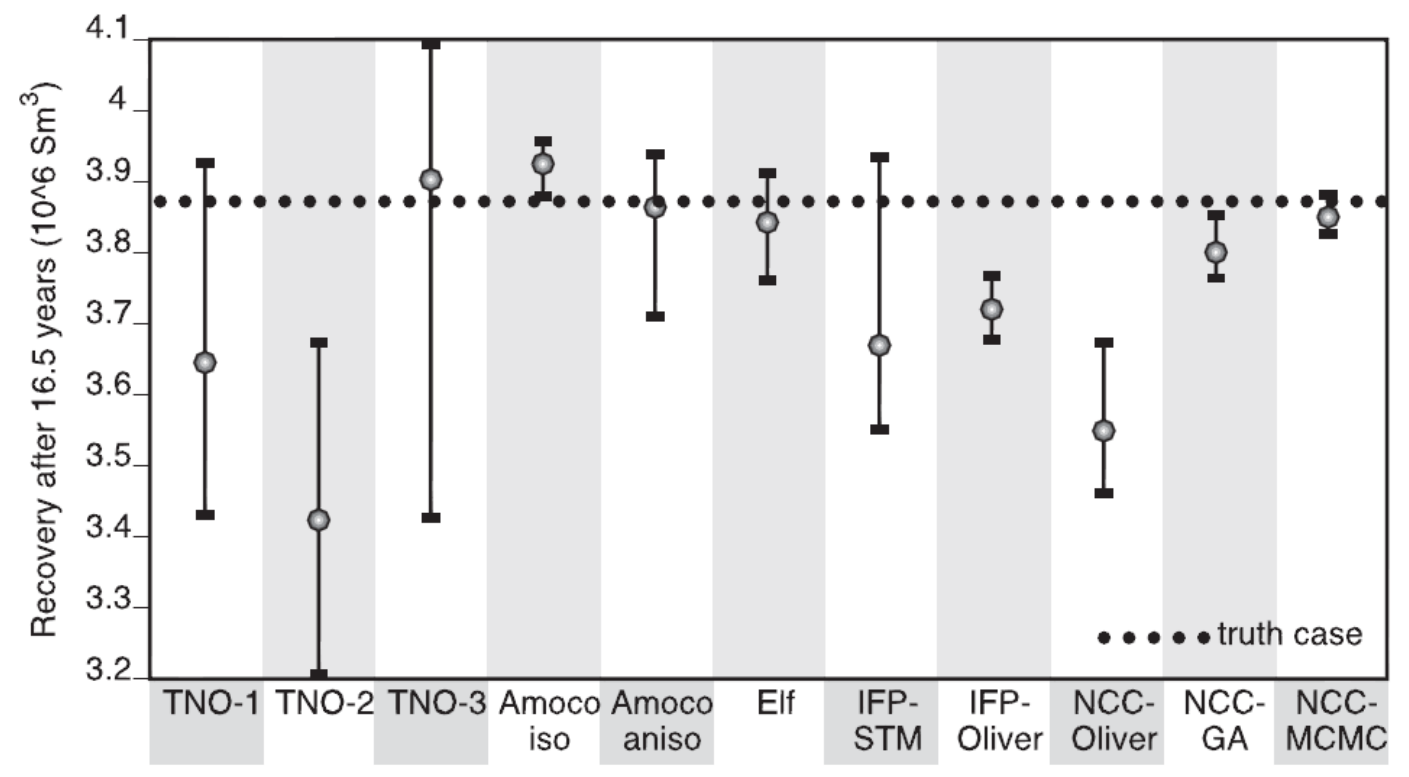

Figure 56: Comparing the total oil recovery of the true model with low-median-high ranges of cumulative production after 16.5 years for all the approaches in Floris et al. article. Floris et al. (2001) published the description and results of eleven approaches using PUNQ-S3 as the test model.

PUNQ-S3 model was used by Soleng (1999) as the test model to investigate a genetic algorithm approach for history matching. Manceau et al. (2001) applied a combination of experimental design and joint modeling methods to this model. Later Barker et al. (2001), who were a partner group in the PUNQ project, published some additional results for the problem of PUNQ-S3 model and compared them with the ones reported by Floris and coworkers.

Gu and Oliver (2005) applied ensemble Kalman filter (EnKF) to PUNQ-S3 reservoir model. Using this model to test the different versions of EnKF method is popular. Gao et al. (2005) used a modified EnKF method and randomized maximum likelihood (RML) to history match 
the PUNQ-S3 model. They concluded that EnKF and RML methods give relatively good results for this problem.

Hajizadeh et al. (2010, 2009) particularly used PUNQ-S3 model to study different stochastic global optimization methods such as ant colony optimization, differential evolution, and neighborhood algorithm. Li and Yang (2011) applied an ensemble-based history matching technique (EnKF) to PUNQ-S3 reservoir model. They defined four different scenarios mainly differ in adjustable properties (porosity, permeability, and relative permeability), tuning parameter number and characteristics of the ensemble. Then they compared the results of these scenarios with those ones described by Floris et al. They reported overall progress using their method. Abdollahzadeh et al. (2011) applied different population-based optimization algorithms originated in the Evolutionary Computation field to solve the history matching and uncertainty quantification problem of PUNQ-S3.

From all these studies, it can be concluded that the main characteristics to tune in order to match the field performance include: porosity and permeability (horizontal and vertical). However, in some works they tried to play with the coefficients of relative permeability functions (Li and Yang 2011).

\subsection{Model generation and spatio-temporal database preparation}

In this section we go through the preparation steps of the SRM for PUNQ-S3 reservoir model. The uncertain properties for developing the SRM and matching the field performance include: porosity, vertical, and horizontal permeabilities. These properties are the most common uncertain reservoir characteristics used in the literature to match history data of this model. Similar to what happens in reality, it is assumed that these properties are measured at the well locations (well logging and core data samples). Also the geological descriptions of this model indicate the streaks of high porosity/permeability profiles in the reservoir (Floris, et al. 2001, Barker, Cuypers and Holden 2001). These types of information were used to generate training realizations.

\subsubsection{Informative simulation runs representation of reservoir uncertainties}

Based on the property values provided at well sites and also the geological descriptions, ten different realizations of the reservoir were created. In order to create these realization a sampling method (Latin Hypercube) was utilized. The process of creating a new realization of 
reservoir was the same as the way we explained in the previous chapter (section 4.6.2.2). It is assumed we have the values of reservoir characteristics (porosity and permeability) at the well spots. Then these values are fed to an interpolation method provided by CMG-Builder ${ }^{\mathrm{TM}}$ to create the property distributions. Different realizations differ in the property values at well block. In order to assure capturing the maximum information form ten realizations, the Latin Hypercube was used. Figure 57 to Figure 59 are the designed property distributions for the first realization. The created maps for the rest of realizations are available in Appendix C (sections a and b). Figure 60 and Figure 61 show the results of well bottom-hole pressure and gas rate production for the well PRO-1 in the training and blind realizations. They are also compared with the observed data. The comparison for the rest of the wells are available in Appendix C (section ??). For the eight years of history data, water breakthrough happens for just one well (PRO-11). Figure 62 demonstrates the results of water rate production for this well for training realizations, blind case, and observed data.

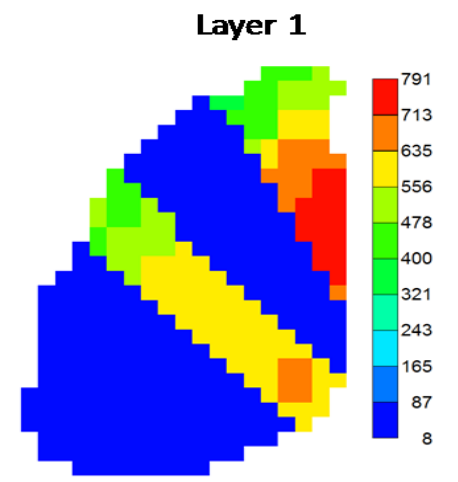

Layer 2

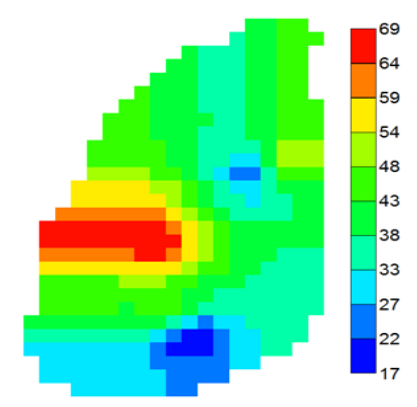

Layer 3

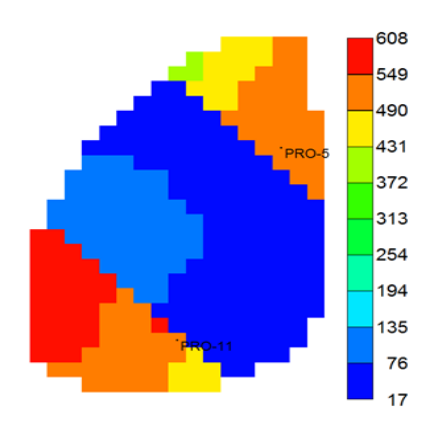

Layer 4
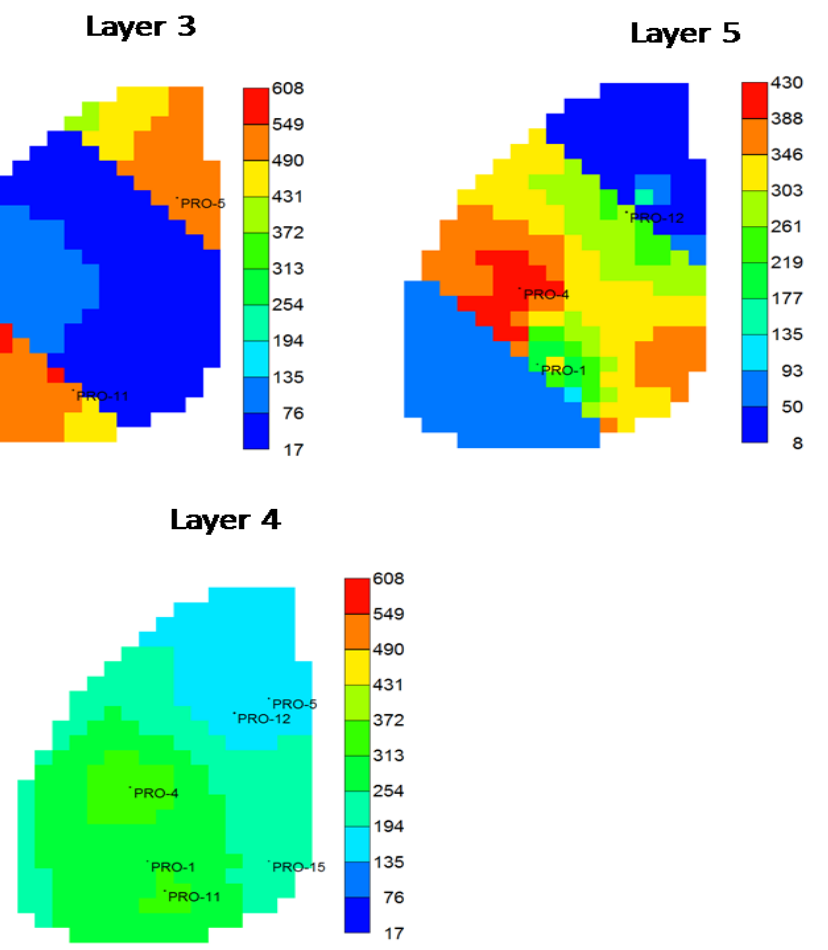

\section{Horizontal Permeability (md) - Realization \# 1}

Figure 57: Horizontal permeability distributions for the realization \#1. 


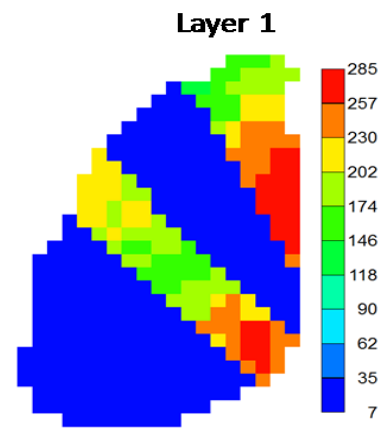

Layer 2

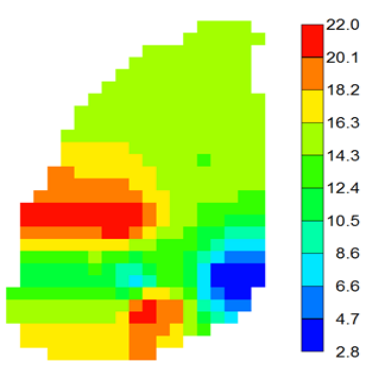

Layer 3

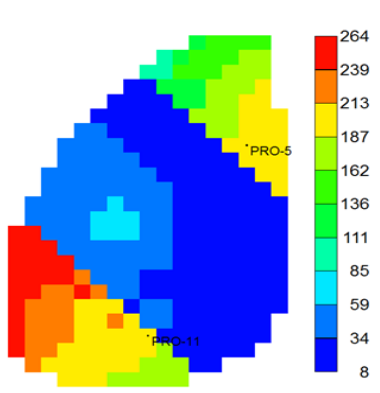

Layer 4

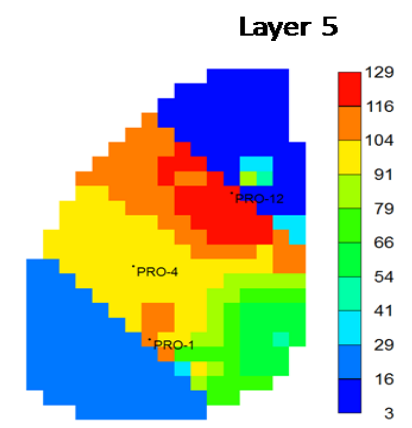

\section{Vertical Permeability (md) - Realization \# 1}

Figure 58: Vertical permeability distributions for the realization \#1.
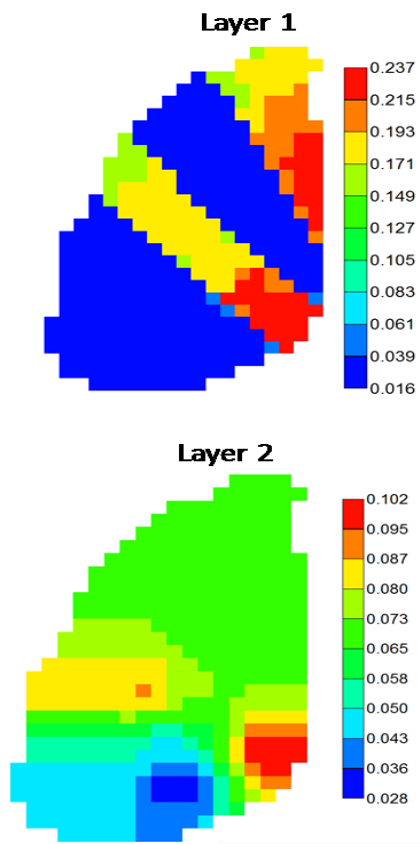

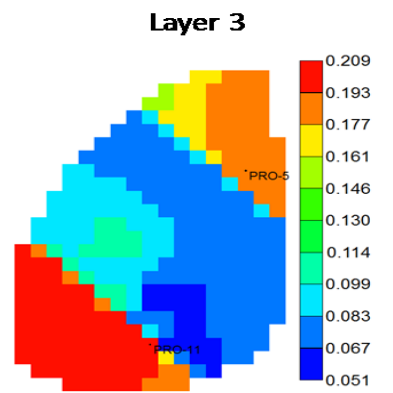

Layer 4

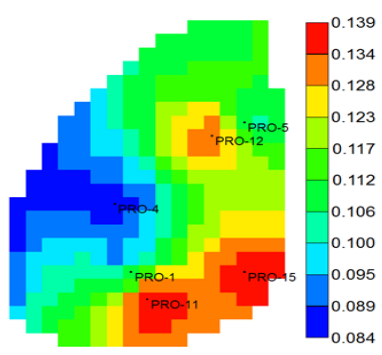

Layer 5

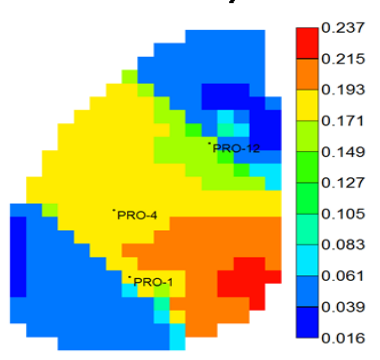

$$
\text { Porosity (\%) - Realization \# } 1
$$

Figure 59: Porosity distributions for the realization \#1. 
Well PRO-1

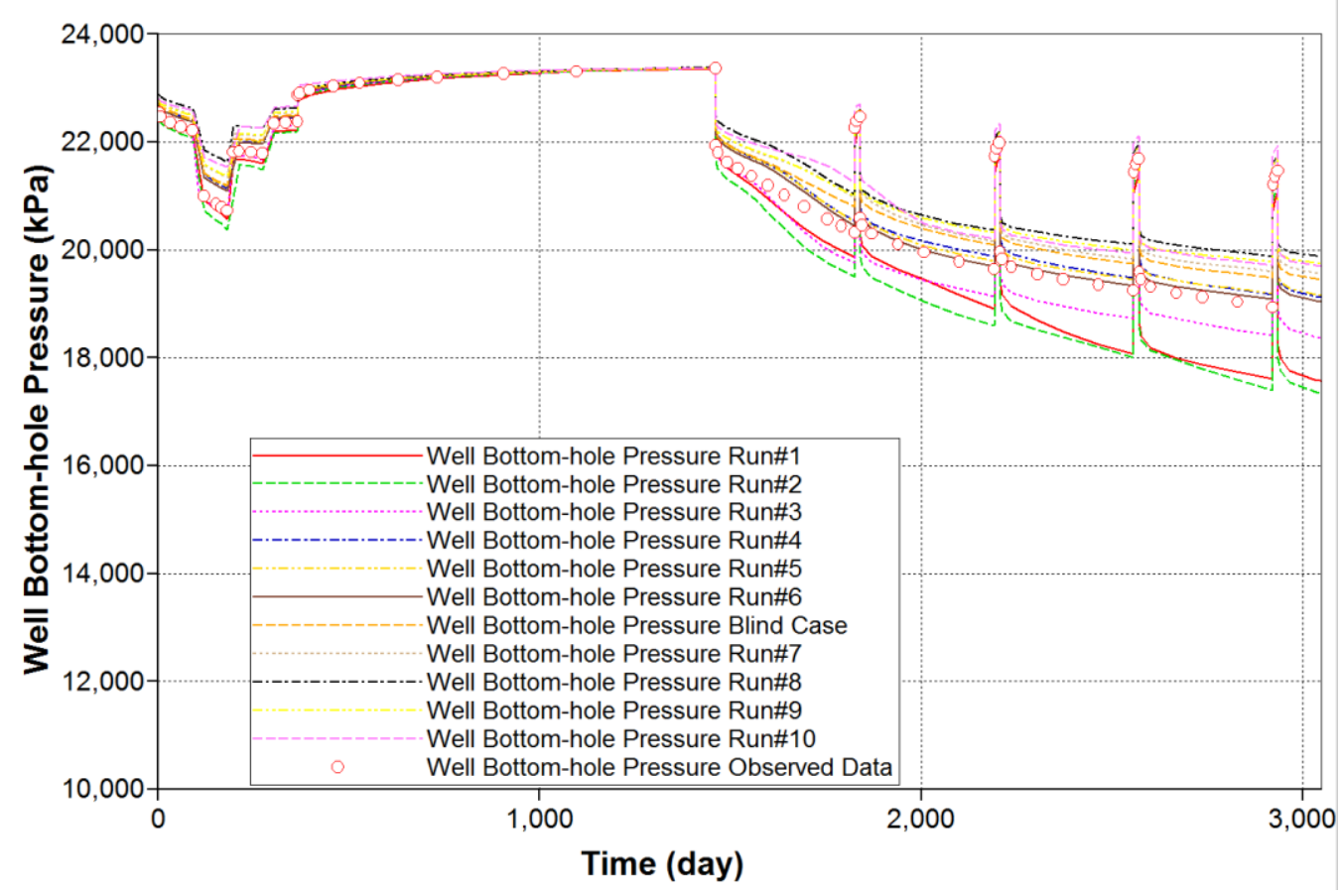

Figure 60: Comparison of well bottom-hole pressure for the ten training realizations and blind case with observed data- well PRO-1.

\section{Well PRO-1}

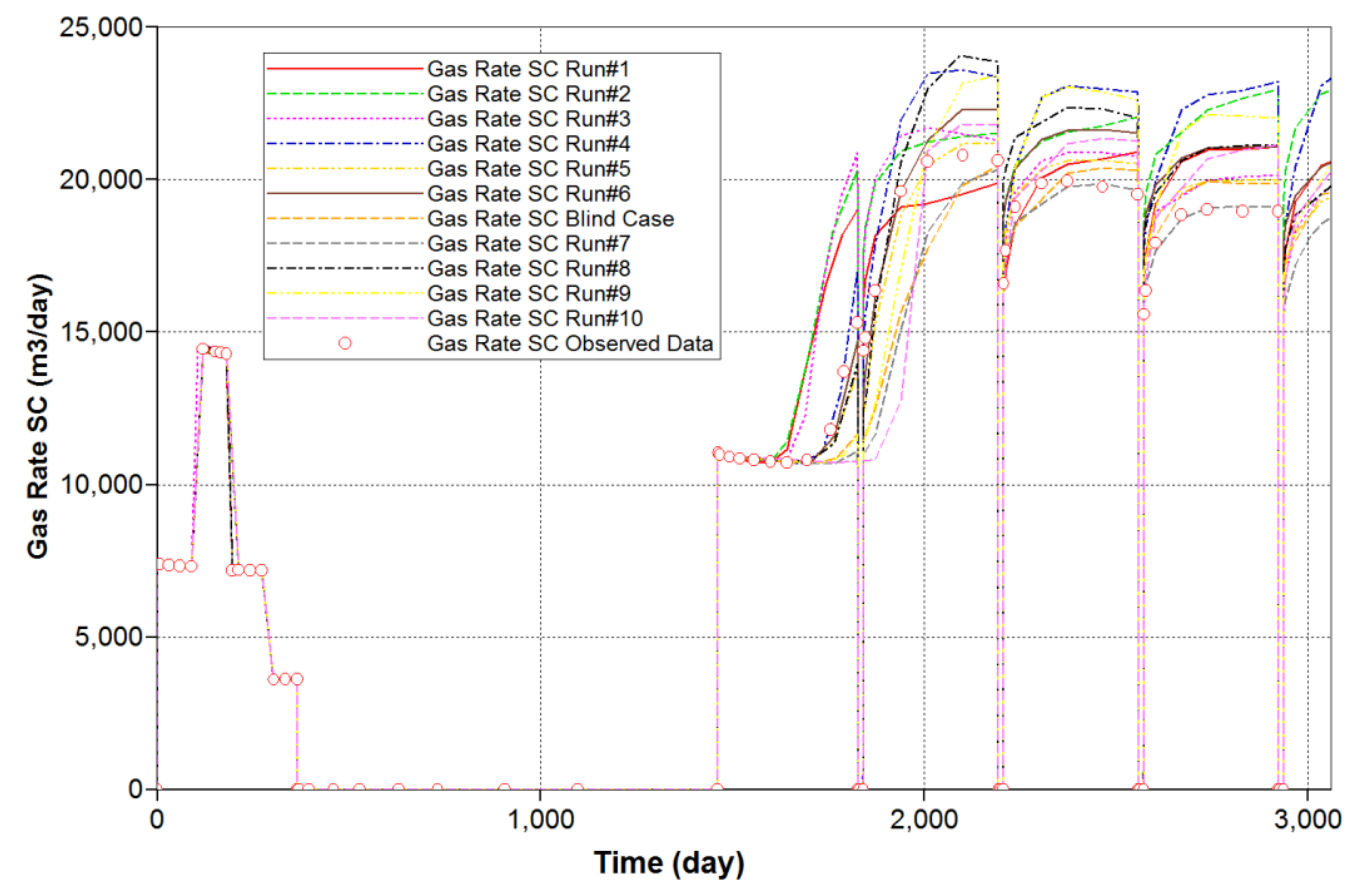

Figure 61: Comparison of gas rate production for the ten training realizations and blind case with the observed data- well PRO-1. 
Well PRO-11

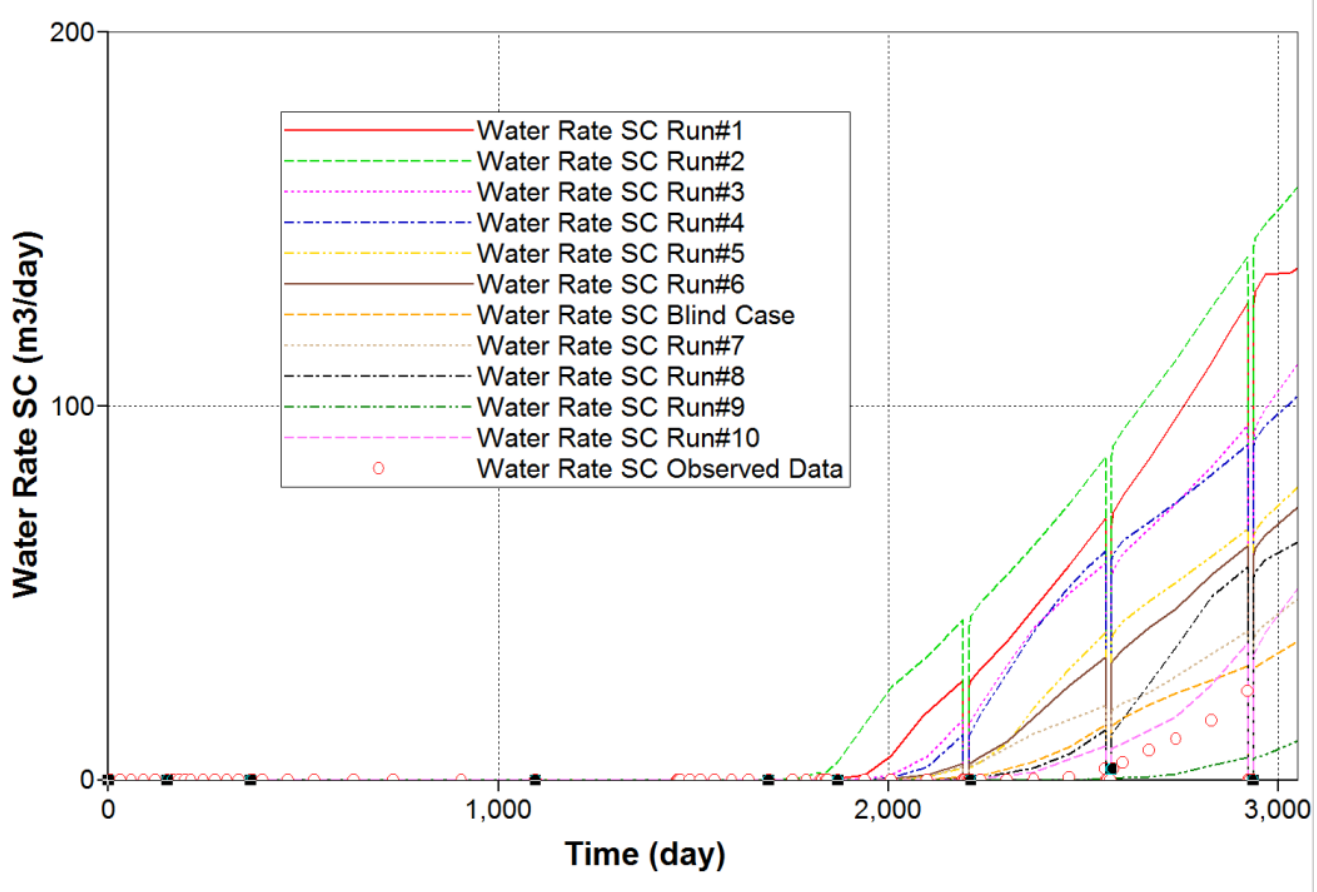

Figure 62: Comparison of water rate production for the ten training realizations and blind case with the observed data- well PRO-11.

\subsubsection{Reservoir delineation and tier system}

After designing and running ten realizations, the static and dynamic data are extracted to generate the spatio-temporal database. In order to summarize and fit the static data into the database, the reservoir was delineated into drainage areas. Recalling from the previous chapter, Voronoi graph theory was used to perform this task. In the previous case studies we had just one layer and no channels of high porosity/permeability, so simply a Voronoi polygon was considered as the drainage area for a well. PUNQ-S3 reservoir model consists of five layers. The given geological description indicates high porosity/permeability channels located in the layers one, three, and five. Therefore, for these layers we consider a modified version of Voronoi polygons. Also based on the given geological description, layers two and four have no channels. Therefore we consider a regular Voronoi diagram for these layers like what we had for the previous two reservoir models. Figure 63 depicts the designed Voronoi diagrams for the second and fourth layers. 

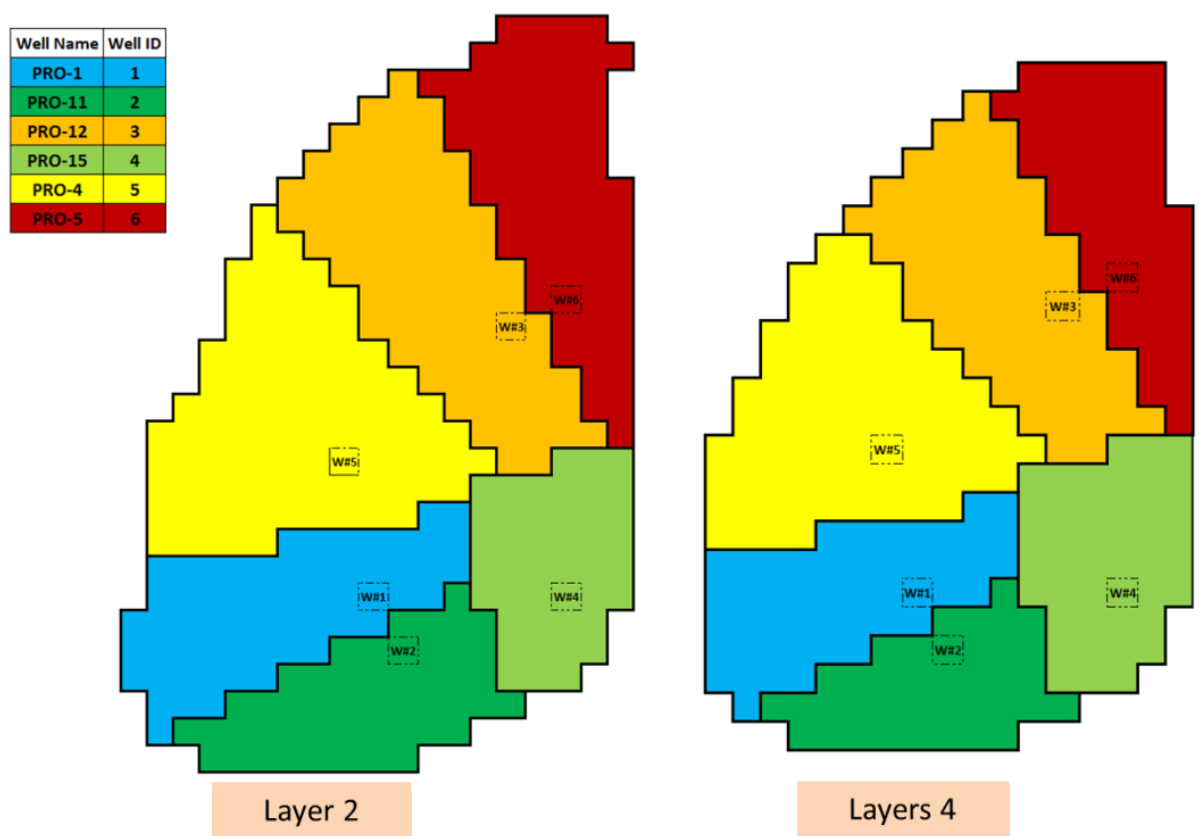

Figure 63: The drainage areas assigned to the wells in layers two and four based on Voronoi diagram.

Considering the information provided about the channels and also looking at the values of porosity and permeability at well blocks, we decided a modified version of Voronoi polygons as well drainage areas for layers one, three, and five. Figure 64 demonstrates the drainage areas designed for these layers.
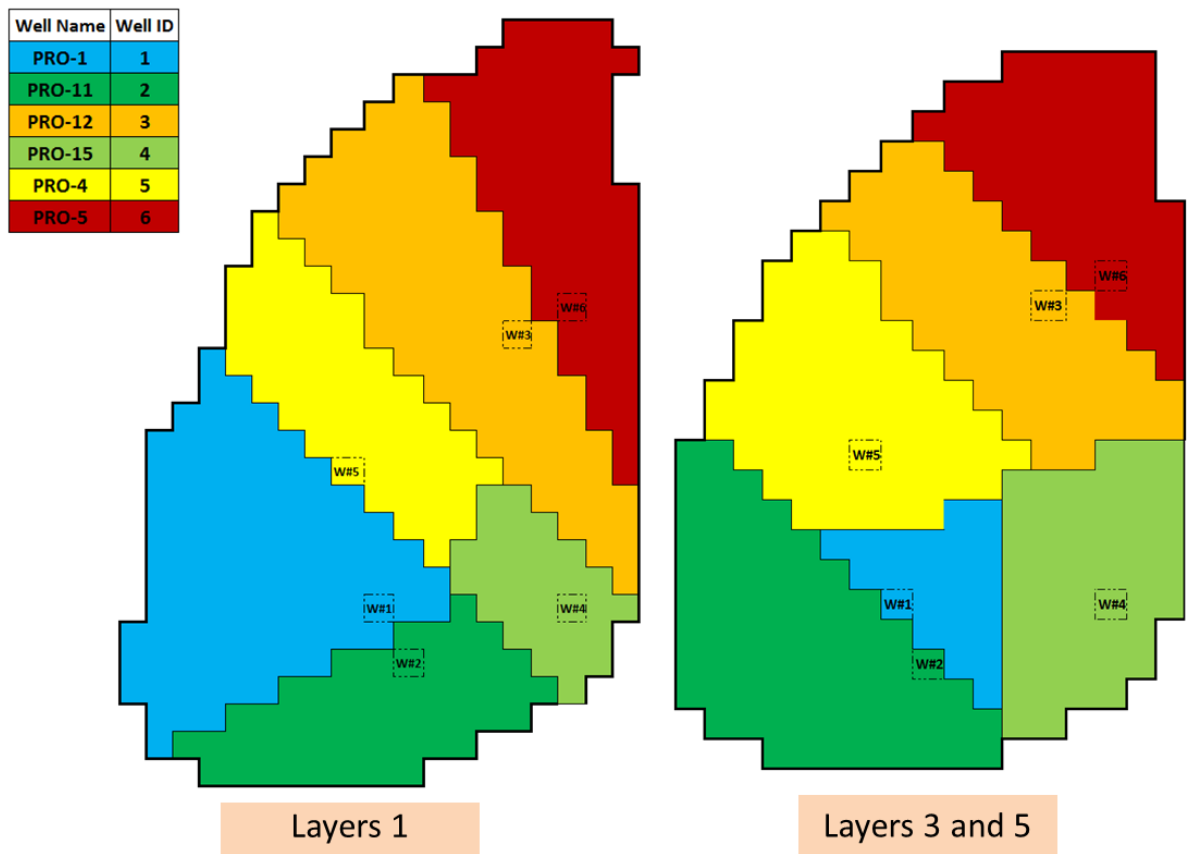

Figure 64: A modified version of Voronoi diagram used to define the drainage areas for the wells in layers one, three and five. 
Each drainage area then is divided into four tiers. The first tier is the well block, which has a significant impact on the well behavior. The second tier includes the first row of grid blocks around the well block. The third tier is the next row of grid blocks around the second tier. Finally the rest of grid blocks in the drainage area are summed up in the fourth tier. The average value of reservoir characteristics was calculated at each tier and assigned to the corresponding well and tiers in the database. Figure 65 is a scheme of the designed tier system in this study.

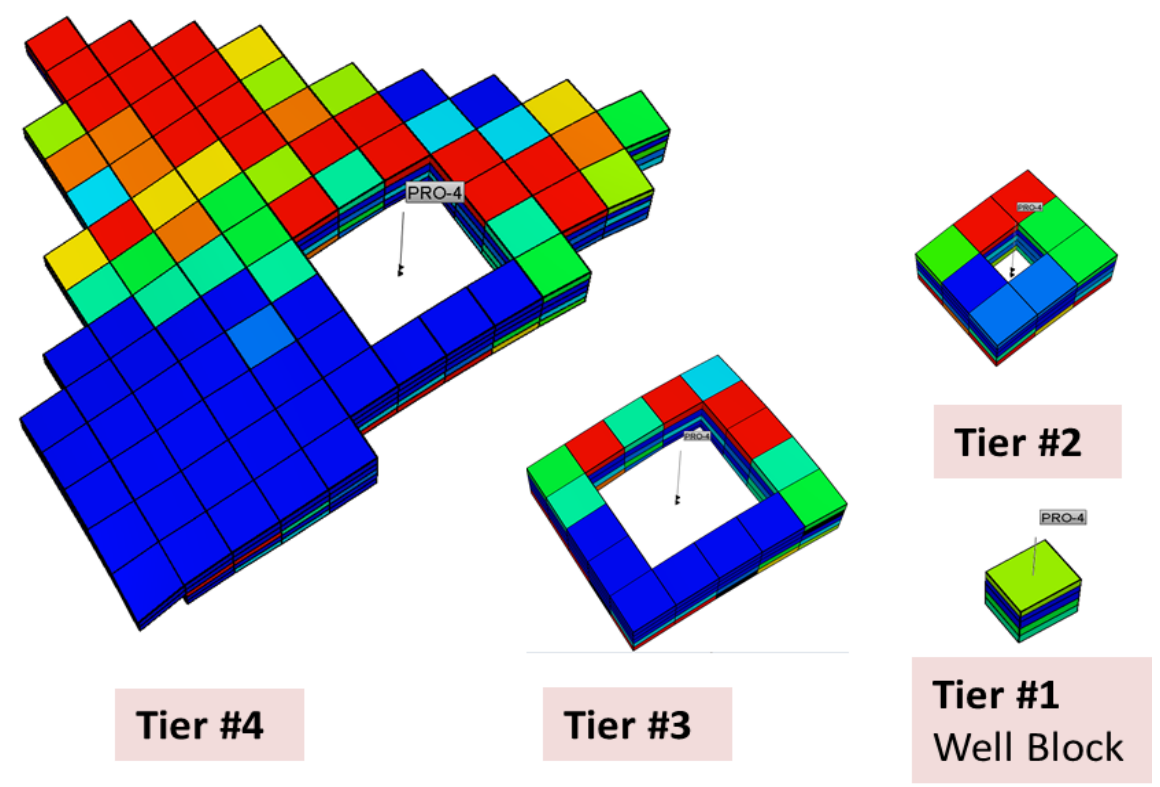

Figure 65: The designed tier system for the PUNQ-S3 reservoir model.

\subsection{SRM development}

Due to complexity of PUNQ-S3 reservoir model we face a much higher number of input parameters in this case study compared to the previous examples. The oil production rate is the main constraint during the eight years of production for this reservoir. Thus the outputs of this model, which need to be matched, are well bottom-hole pressure, gas production rate, and water production rate. For each one these outputs one ANN was created(totally three ANNs).

PUNQ-S3 model has five layers and a total of six wells. Each drainage area was divided into four tiers and also we have three uncertain parameters (porosity, vertical, and horizontal permeability). Consequently there are 360 uncertain (adjustable) parameters (5 layers $\times$ 4 tiers $\times 6$ wells $\times 3$ properties) which can be tuned in order to match the history data. In order to build the SRM, just considering a single well we need to at least include 60 parameters for each well. These do not contain the other types of data such as thickness and top for each tier. Generally we have a database with more than 120 inputs. Figure 66 
summarizes the types of input and output in the spatio-temporal database for PUNQ-S3 reservoir model. Making sure to select the right inputs is not an easy task to do. Many of artificial based models fail in this step (Zubarev 2009, Mohaghegh, Liu, et al., Application of Surrogate Reservoir Models (SRM) to an Onshore Green Field in Saudi Arabia; Case Study 2012a).

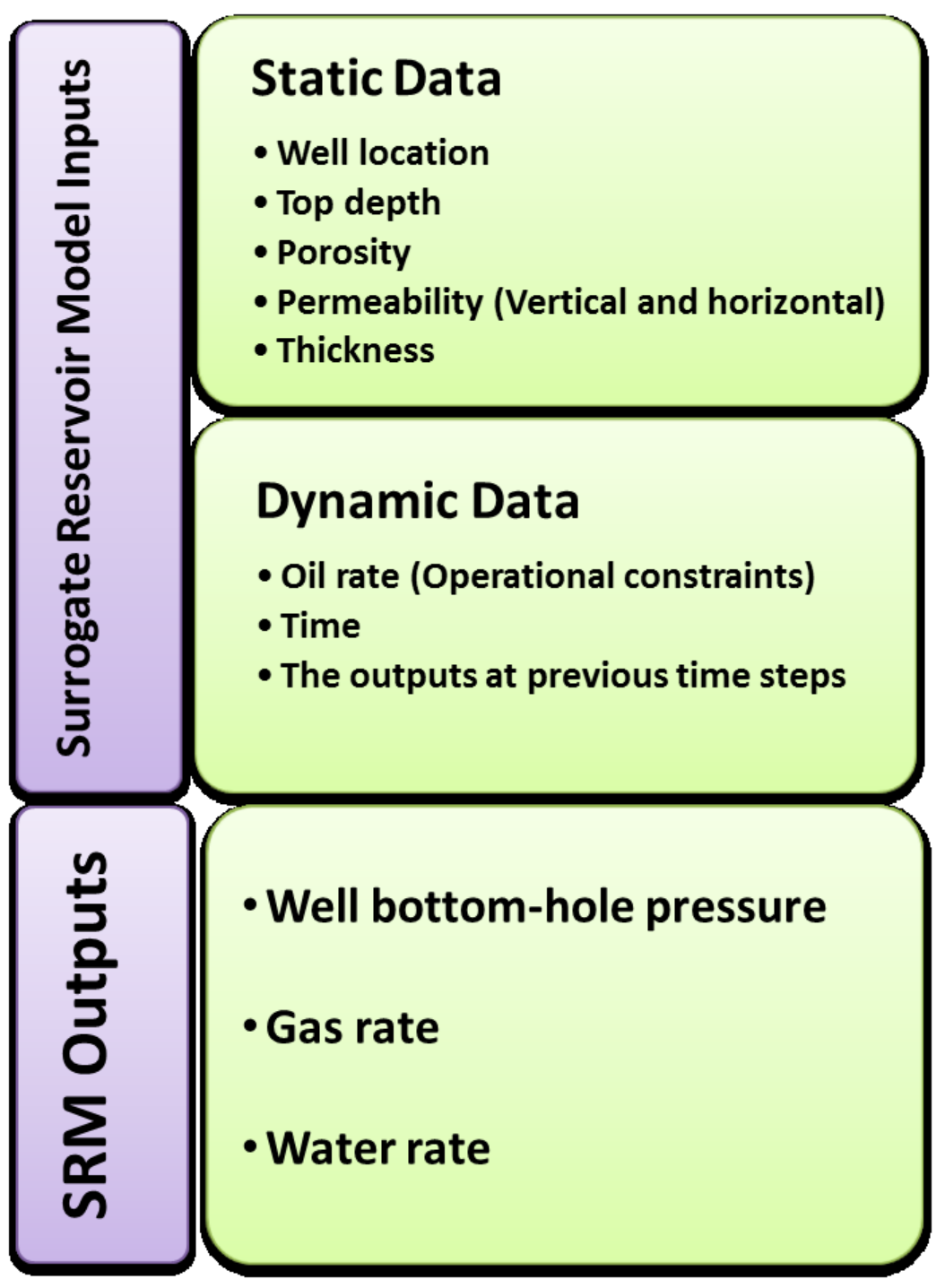

Figure 66: Inputs and outputs available in the spatio-temporal database for PUNQ-S3 SRM.

\subsubsection{Preprocessing operations}

In order to select the best parameters for the training step KPI analysis was performed. The results can be used as a guide for input selection; however, choosing inputs are not limited just to this analysis. Table 11 lists the most important parameters identified by KPI analysis tool box in IDEA $^{\mathrm{TM}}$. Table 11 shows the KPI analysis results for the network which estimates well 
bottom-hole pressure. There was around 120 parameters which were not all listed in this table. Though obviously the most important parameters, which decide the fluid flow to the wellbore, are the dynamic inputs such as oil rate (which is set as the constraint), well bottom-hole pressure at the time steps behind and time. The important static data include the horizontal permeability and porosity. These results were expected since dynamic data impose the production in the field. The porosity represents the storage capacity and permeability shows the conductivity of the reservoir.

Table 11: The results of KPI analysis for the SRM in the PUNQ-S3 problem.

\begin{tabular}{|c|c|c|}
\hline Rank & Feature & $\%$ Degree of Influence \\
\hline 1 & Oil Rate (Production Constraint) & 100 \\
\hline 2 & Well Bottom-hole Pressure @ (t-1) & 60 \\
\hline 3 & Time & 56 \\
\hline 4 & Well Bottom-hole Pressure @ (t-2) & 40 \\
\hline 5 & Hirozontal Permeability-Tier \#3- Layer \#4 & 33 \\
\hline 6 & Porosity -Tier \#4- Layer \#4 & 31 \\
\hline 7 & Hirozontal Permeability-Tier \#2- Layer \#4 & 30 \\
\hline 8 & Hirozontal Permeability-Tier \#4- Layer \#4 & 28 \\
\hline 9 & Hirozontal Permeability-Tier \#1- Layer \#4 & 27 \\
\hline 10 & Porosity -Tier \#2- Layer \#3 & 27 \\
\hline 11 & Top-Tier \#4- Layer \#3 & 27 \\
\hline 12 & Top-Tier \#2- Layer \#2 & 25 \\
\hline 13 & Porosity - Tier \#4- Layer \#3 & 24 \\
\hline 14 & Top-Tier \#1- Layer \#5 & 24 \\
\hline 15 & Vertical Permeability-Tier \#4- Layer \#4 & 23 \\
\hline 16 & Porosity -Tier \#1- Layer \#3 & 23 \\
\hline 17 & Top-Tier \#2- Layer \#5 & 23 \\
\hline 18 & Top-Tier \#4- Layer \#1 & 22 \\
\hline 19 & Top-Tier \#4- Layer \#2 & 22 \\
\hline \multirow[t]{2}{*}{20} & Hirozontal Permeability-Tier \#4- Layer \#3 & 22 \\
\hline & Other Parameters & Less than $22 \%$ \\
\hline
\end{tabular}

In addition to the KPI results, the reservoir engineering judgment and experience were used to choose the inputs. Table 12 to Table 14 are the selected inputs for three networks in this study. The inputs generally include the static and dynamic data. The static data contain the uncertain properties which are tuned to match the history data, thickness, and top depth. Time and oil production rate are common inputs for different networks. However, depending on the output, we use the value of the output for time steps behind. For example, if we would like to predict well bottom-hole pressure at time $t$, the value of well bottom-hole pressure at one and two time steps behind are used as input of network. 
Table 12: Selected inputs for the well bottom-hole pressure network.

\begin{tabular}{|c|c|c|c|}
\hline \multicolumn{2}{|c|}{ Static Inputs } & \multirow{3}{*}{$\begin{array}{c}\text { Dynamic Inputs } \\
\text { Well bottom-hole } \\
\text { Pressure @ (t-1) and (t-2) }\end{array}$} & Output \\
\hline Latitude $(\mathrm{X})$ & ( ) Well Block & & \multirow{6}{*}{$\begin{array}{c}\text { Well bottom-hole } \\
\text { Pressure }\end{array}$} \\
\hline Langitude $(\mathrm{Y})$ & @ Well Block & & \\
\hline Horizontal Permeability & @ 5 Layers and 4 Tiers & \multirow{2}{*}{ Time } & \\
\hline Vertical Permeability & @ 5 Layers and 4 Tiers & & \\
\hline Thickness & @ 5 Layers and 4 Tiers & \multirow{2}{*}{$\begin{array}{c}\text { Oil production rate } \\
\text { (operational constraints) }\end{array}$} & \\
\hline Top & @ 5 Layers and 4 Tiers & & \\
\hline
\end{tabular}

Table 13: Selected inputs for the gas production rate network.

\begin{tabular}{|c|c|c|c|}
\hline \multicolumn{2}{|c|}{ Static Inputs } & \multirow{3}{*}{$\begin{array}{c}\text { Dynamic Inputs } \\
\text { Gas production rate } \\
@(t-1) \text { and }(t-2)\end{array}$} & Output \\
\hline Latitude $(\mathrm{X})$ & @ Well Block & & \multirow{6}{*}{$\begin{array}{c}\text { Gas production } \\
\text { rate }\end{array}$} \\
\hline Langitude $(\mathrm{Y})$ & @ vvell ВIоск & & \\
\hline Horizontal Permeability & @ 5 Layers and 4 Tiers & \multirow{2}{*}{ Time } & \\
\hline Vertical Permeability & @ 5 Layers and 4 Tiers & & \\
\hline Thickness & @ 5 Layers and 4 Tiers & \multirow{2}{*}{$\begin{array}{c}\text { Oil production rate } \\
\text { (operational constraints) }\end{array}$} & \\
\hline Top & @ 5 Layers and 4 Tiers & & \\
\hline
\end{tabular}

Table 14: Selected inputs for the water production rate network.

\begin{tabular}{|c|c|c|c|}
\hline \multicolumn{2}{|c|}{ Static Inputs } & \multirow{3}{*}{$\begin{array}{c}\text { Dynamic Inputs } \\
\text { Water production rate } \\
@(\mathrm{t}-1) \text { and }(\mathrm{t}-2)\end{array}$} & Output \\
\hline Latitude $(\mathrm{X})$ & ค MLل Plot & & \multirow{6}{*}{$\begin{array}{l}\text { Water } \\
\text { oduction rate }\end{array}$} \\
\hline Langitude $(\mathrm{Y})$ & @ vvell BIOCr & & \\
\hline Horizontal Permeability & @ 5 Layers and 4 Tiers & \multirow{2}{*}{ Time } & \\
\hline Vertical Permeability & @ 5 Layers and 4 Tiers & & \\
\hline Thickness & @ 5 Layers and 4 Tiers & \multirow{2}{*}{$\begin{array}{c}\text { Oil production rate } \\
\text { (operational constraints) }\end{array}$} & \\
\hline Top & @ 5 Layers and 4 Tiers & & \\
\hline
\end{tabular}

\subsubsection{Train, calibrate, and validate the ANNs}

Once the inputs were selected, $80 \%$ of the database was used as training set. The share of calibration and validation sets is also $10 \%$ for each one. For each output we have one network; therefore, a total three networks are set. All three networks contain one hidden layer (Figure 67). Consequently the SRM is created by integrating three neural networks after the training process is completed. 


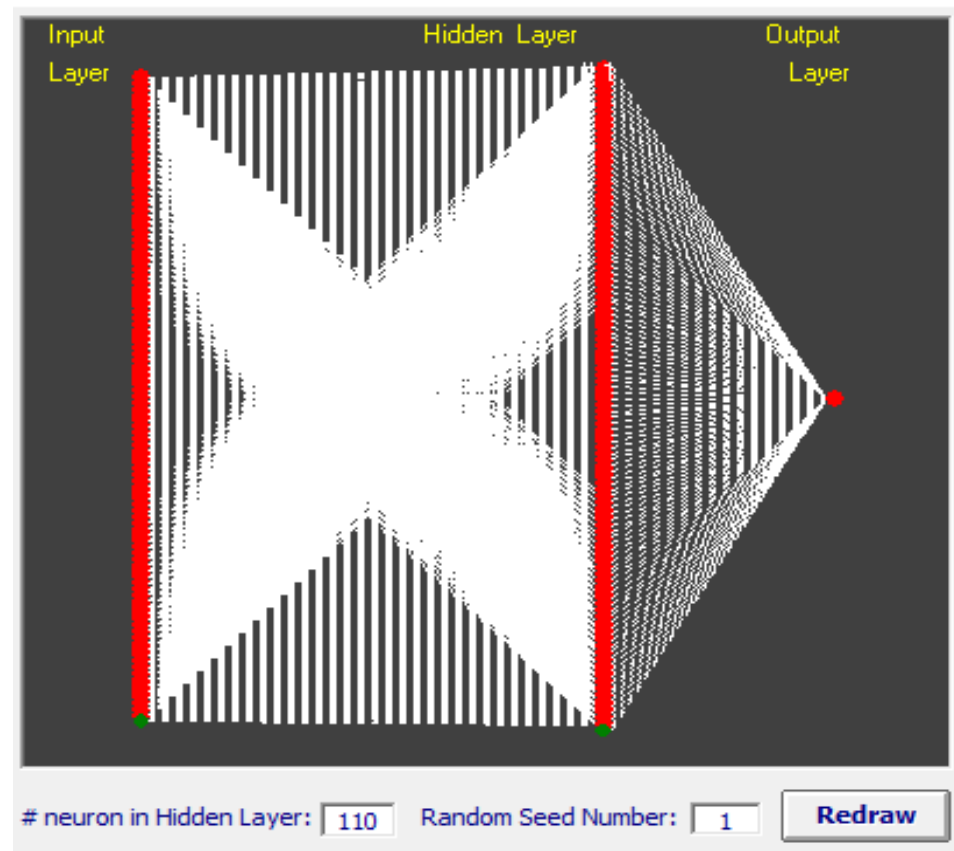

Figure 67: ANN structure used for training the SRM.

\subsubsection{Blind verification}

An extra simulation run (eleventh realization) was created in order to validate the developed surrogate reservoir model.

\subsection{Automated SRM-based history matching}

The surrogate reservoir model then combined with differential evolution optimization method to build up the automated workflow for history matching. The objective functions defined in the previous chapter were used to estimate the misfits between SRM estimation and actual data. In order to start the automated workflow, the ranges of properties are given to the optimization algorithm. The ranges are taken from the geological description provided for PUNQ-S3 model. The automated workflow was set to be stopped automatically after 3000 runs of SRM (stoppage condition). However, the process can be stopped at any time or reaching a specific value of misfit.

\subsection{Results}

In this section we present some results for development and application of SRM for PUNQ-S3 problem. The first set of results belongs to a training realization used to train the SRM. Figure 68 is the comparison of well bottom-hole pressure results generated by SRM and similar results from simulator. The figure portrays six profiles corresponding to six wells. Blue markers are SRM results over eight years (almost 3000 days) of reservoir life compared to simulator (CMG) outputs (red line). As it was mentioned earlier, the oil rate is the main 
constraint during this period. Therefore, we have well bottom-hole pressure, gas rate, and water rate date to match.
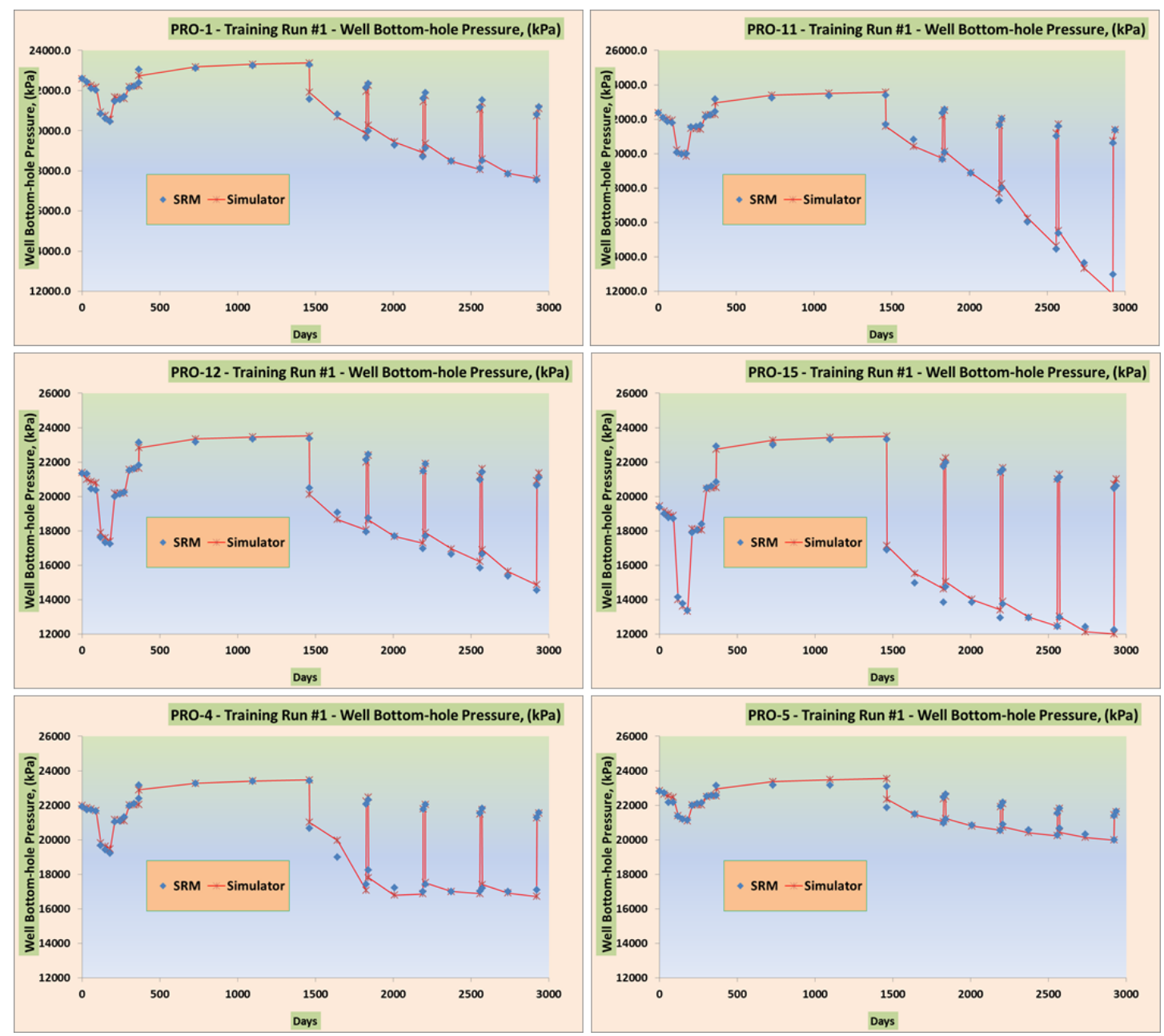

Figure 68: Comparison of well bottom-hole pressure profile generated by the SRM (blue markers) with the similar results from a numerical simulator (CMG) for a training realization of PUNQ-S3 reservoir model.

Figure 69 depicts the results of gas rate production for a training realization. It is a comparison between the results of SRM and simulator for six wells in the PUNQ-S3 reservoir model. For eight years of history data, we have just one well (well PRO-11) having water breakthrough. Figure 70 compares the results of water rate production generated by SRM and same results from simulator. For the first five years there is no water production, and then slowly we observe some water production. However, SRM is able to properly capture the zero water production. 


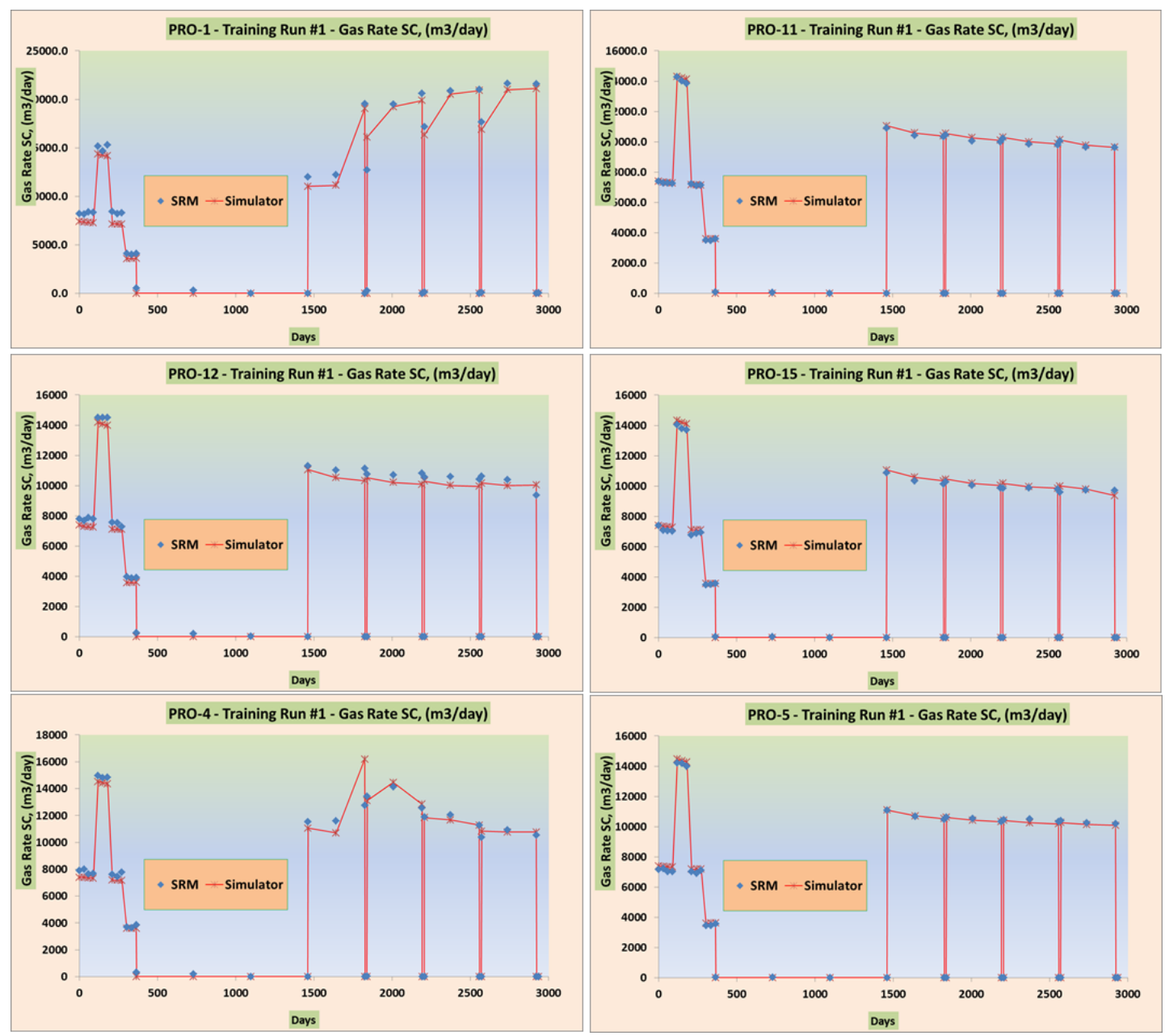

Figure 69: Comparison of gas production rates generated by the SRM (blue markers) with the similar results from a numerical simulator (CMG) for a training realization of PUNQ-S3 reservoir model.

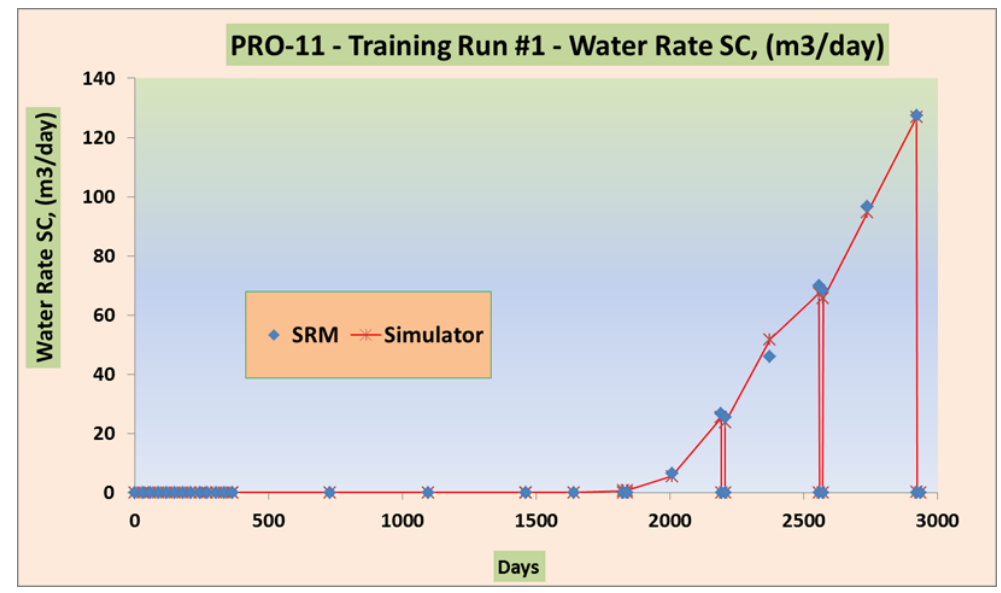

Figure 70: Comparison of water production rate generated by the SRM (blue markers) with the similar results from a numerical simulator (CMG) for a training realization of PUNQ-S3 reservoir model.

The training results show the good performance of SRM during the training process. It seems like the SRM has learnt the behavior of wells through the database. However, it should be 
made sure that the SRM has same quality over the realizations which have not been used during the training process. For this purpose the SRM was applied on a completely blind realization. Figure 71 is the validation results for well bottom-hole pressure. Similar to the training results, the SRM is represented by blue markers compared with simulator outcome (red line).
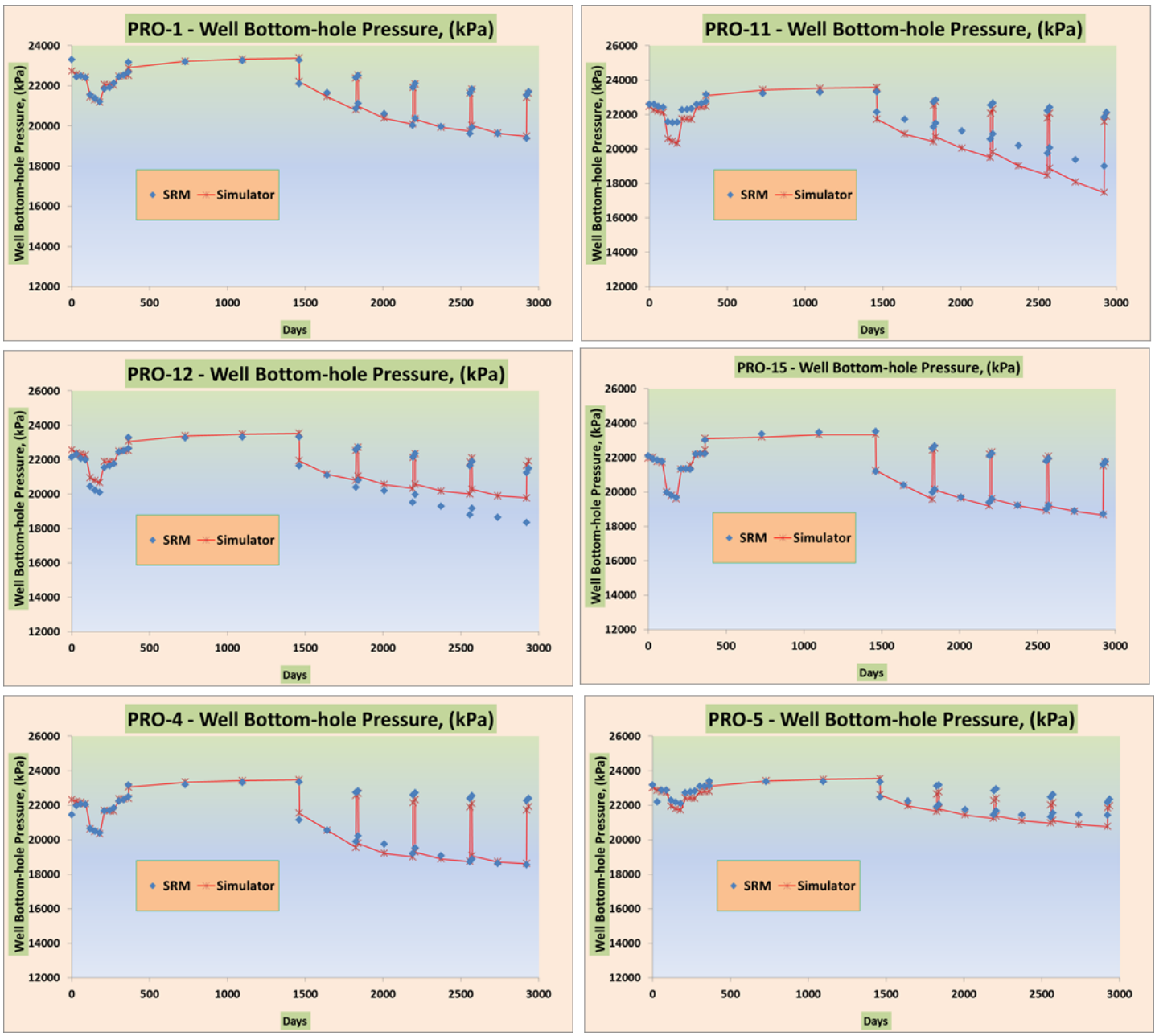

Figure 71: Validating the SRM using a blind run. The comparison of well bottom-hole pressure from the SRM with the results of numerical simulation model for PUNQ-S3 problem.

Figure 72 and Figure 73 are the validation results for gas production and water production rates respectively. 

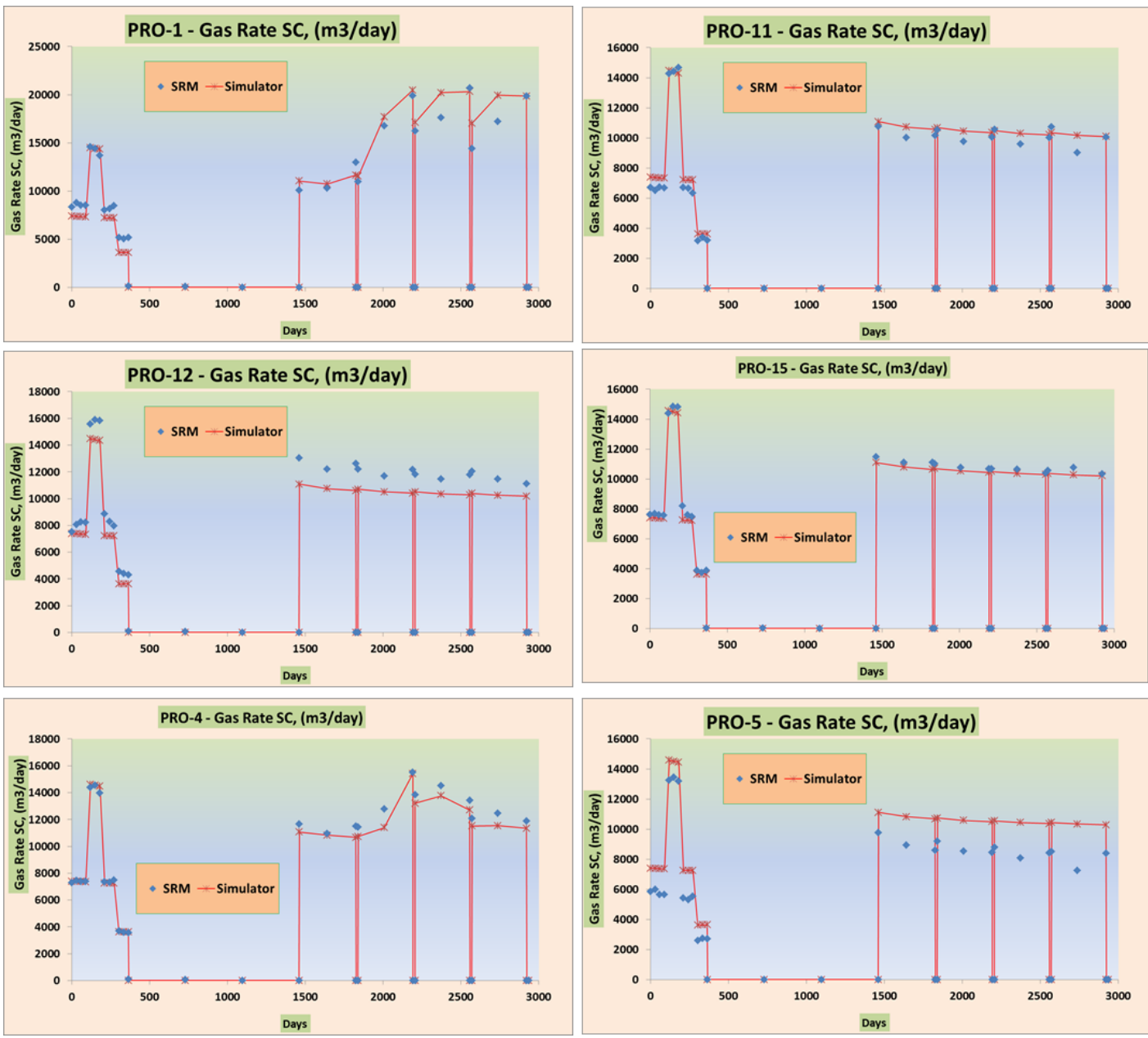

Figure 72: Validating the SRM using a blind run. The comparison of gas production rate profile from the SRM with the results of numerical simulation model for PUNQ-S3 problem.

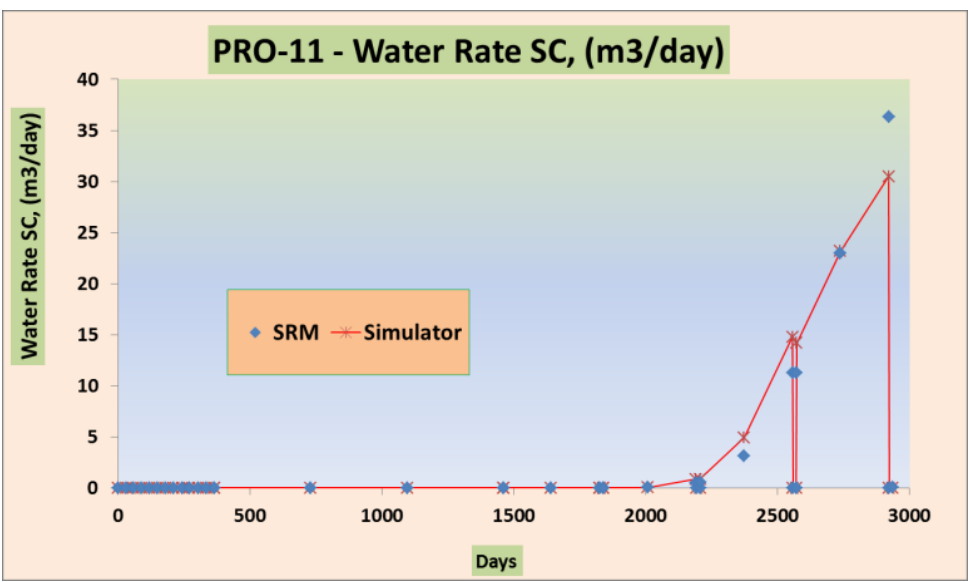

Figure 73: Validating the SRM using a blind run. The comparison of water production rate profile (Well PRO-11) from the SRM with the results of numerical simulation model for PUNQ-S3 problem.

Once the robustness of SRM was assured, it is ready to be used in history matching process. One advantage of the automated history matching workflow is to offer multiple realizations 
which match the field data. In this study we selected top ten best matches. Figure 74 displays the results of history matching for well bottom-hole pressure. There are six profiles of bottomhole pressure for six wells present in PUNQ-S3. Each diagram compares the results of top ten matches (blue lines) with the actual data (red circles).
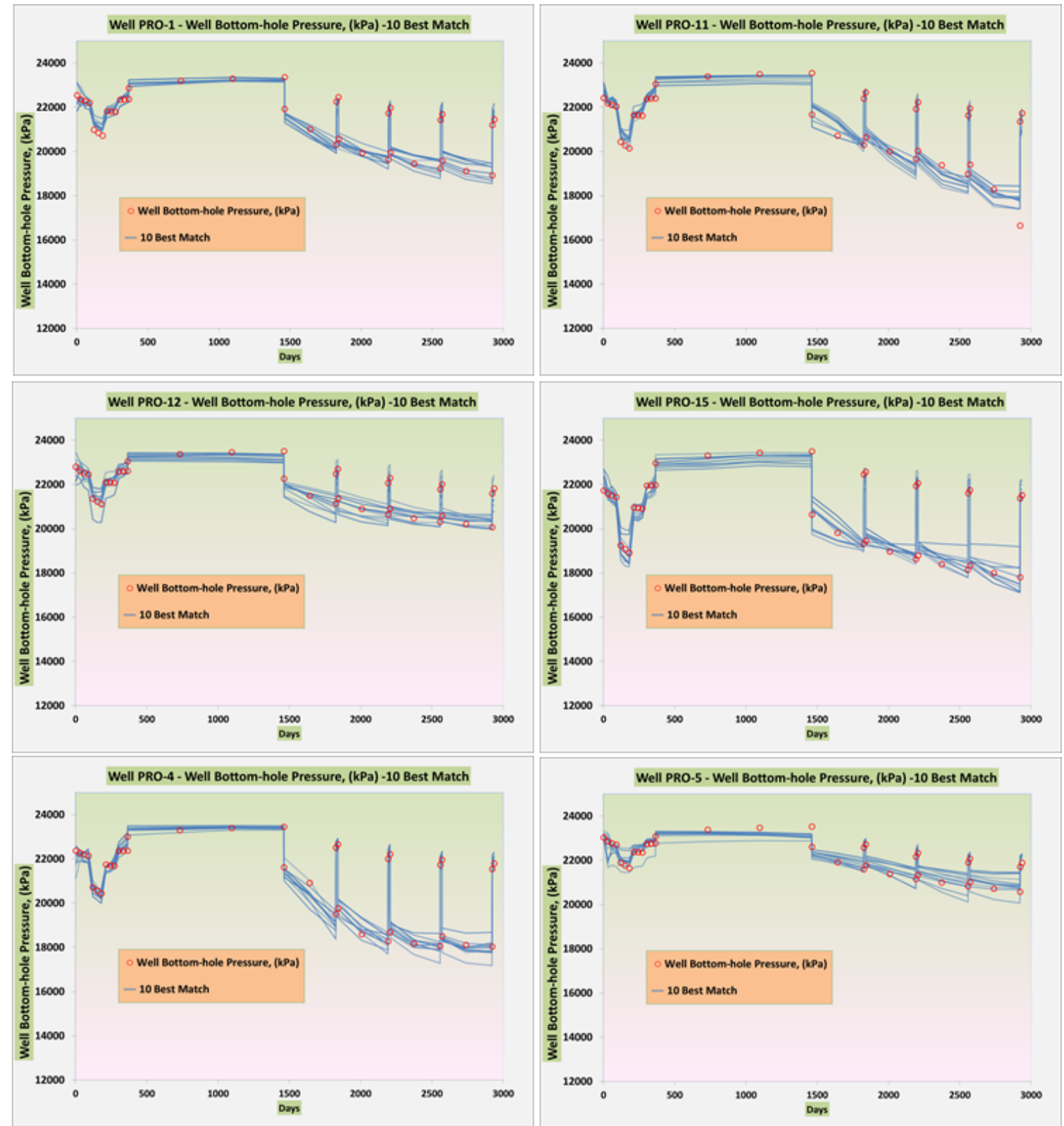

Figure 74: History matching results of well bottom-hole pressure. The comparision of 10 best matches (blue lines) coming from SRM with the actual data (red circles).

Figure 75 and Figure 76 demonstrate the comparison between the ten best matches (blue lines) and the actual data (red circles) for gas production. On the left side we have the gas production rate and the right side diagrams show the cumulative gas production. 

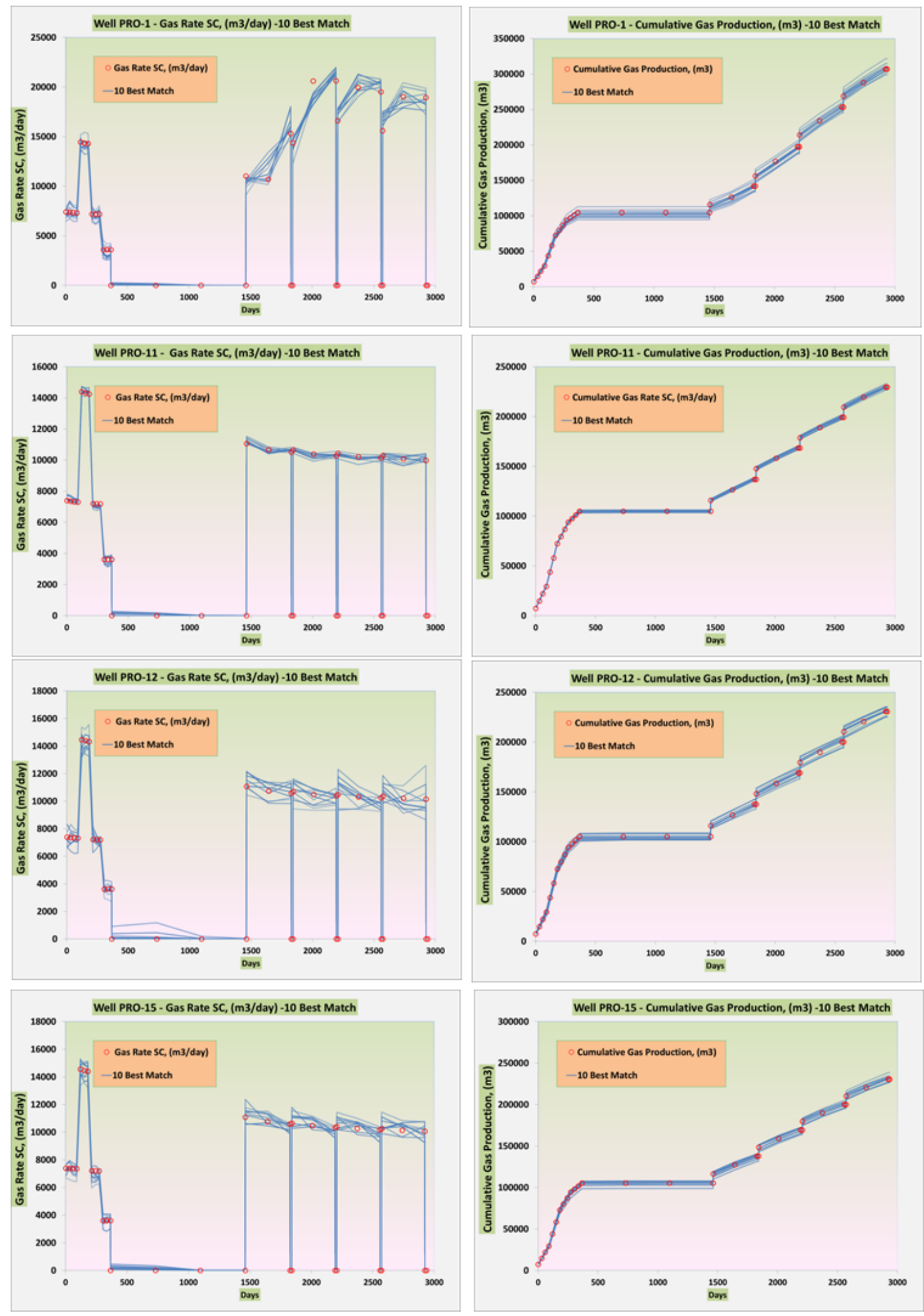

Figure 75: History matching results of gas production (rates are on the left side and cumulative production are on the right side of the figure). Comparision of 10 best matches (blue lines) coming from the SRM with the actual data (red circles). The data belong to the wells PRO-1, PRO-11, PRO-12 and PRO-15. 

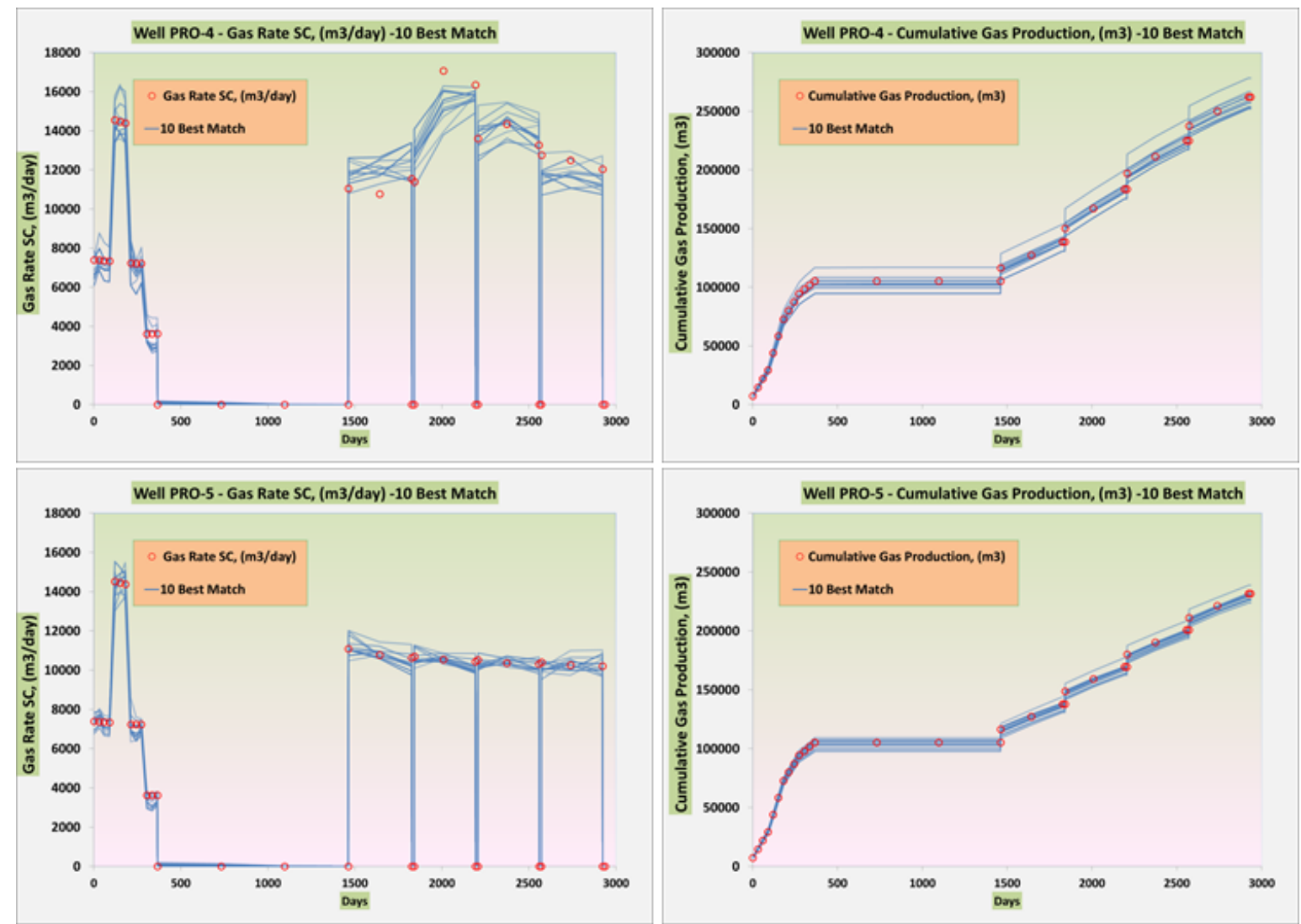

Figure 76: History matching results for gas production (rates are on the left side and cumulative production are on the right side of the figure). Comparision of 10 best matches (blue lines) coming from the SRM with the actual data (red circles). The data belong to wells PRO-4 and PRO-5.

Finally, Figure 77 compares the results of the top ten matches (blue lines) with the actual data (red circles) for water production. In this figure, the diagram on the left is water rate production and the right side depicts the cumulative gas production both for well PRO-11.
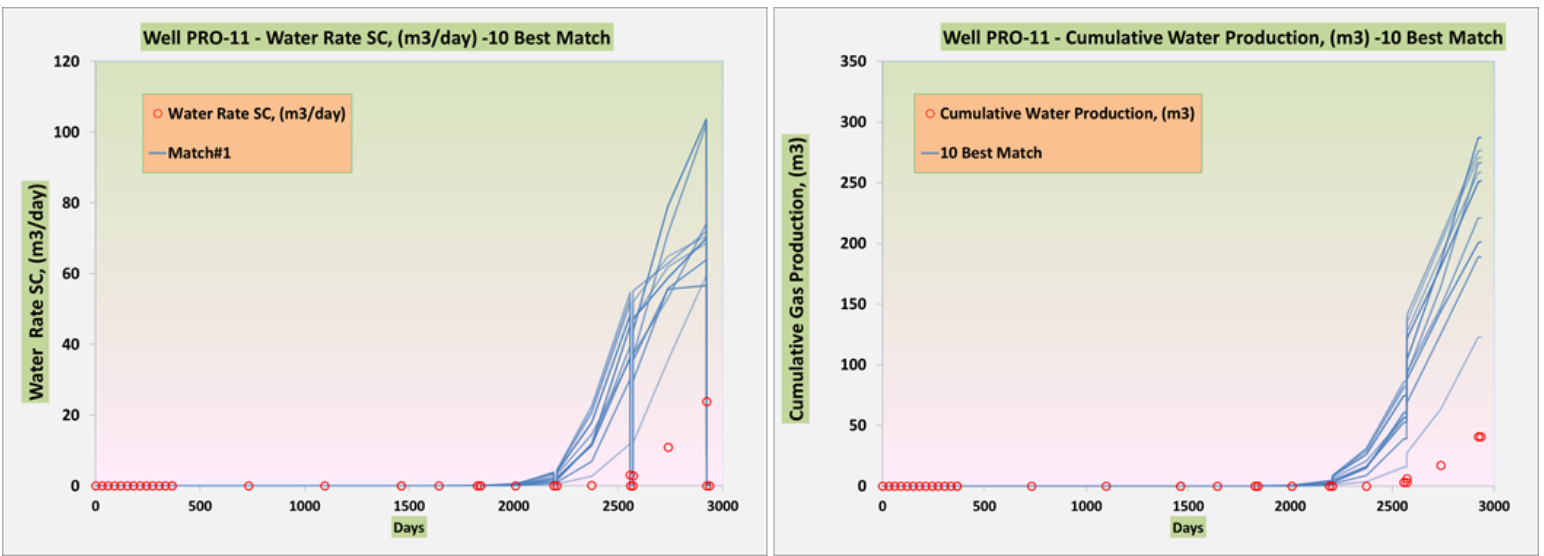

Figure 77: History matching results of water production (rates are on the left side and cumulative production are on the right side of the figure). Comparision of 10 best matches (blue lines) coming from the SRM with the actual data (red circles). In this study we have just one well (PRO-11) with water breakthrough during eight years of production history. 


\subsubsection{Importing the matched characteristics into the simulator}

In this dissertation, SRMs were made to substitute an industrial reservoir simulator (CMG$\mathrm{IMEX}^{\mathrm{TM}}$ ) in the history matching process. An automated SRM-based history matching workflow was designed. This workflow is able to provide multiple realizations of reservoir which match the actual data. In this study we chose to have ten best matches. These ten realizations were imported into the simulator in order to observe the performance of simulator with the inputs coming from the SRM. Figure 78 demonstrates the field cumulative oil production results of the simulator after importing the matched properties (match \# 1) from the SRM into the simulator. This graph compares the simulator results (red line) with the actual field cumulative oil production (blue circles). As it was mentioned earlier there are eight years of field data available for the history matching purposes. In addition to eight years of history data, the field cumulative oil production after 16.5 years has been reported. This value can be used for future forecast comparison. Keep in mind this information was not used in the history matching process. In the Figure 78 the green dot represents this value.

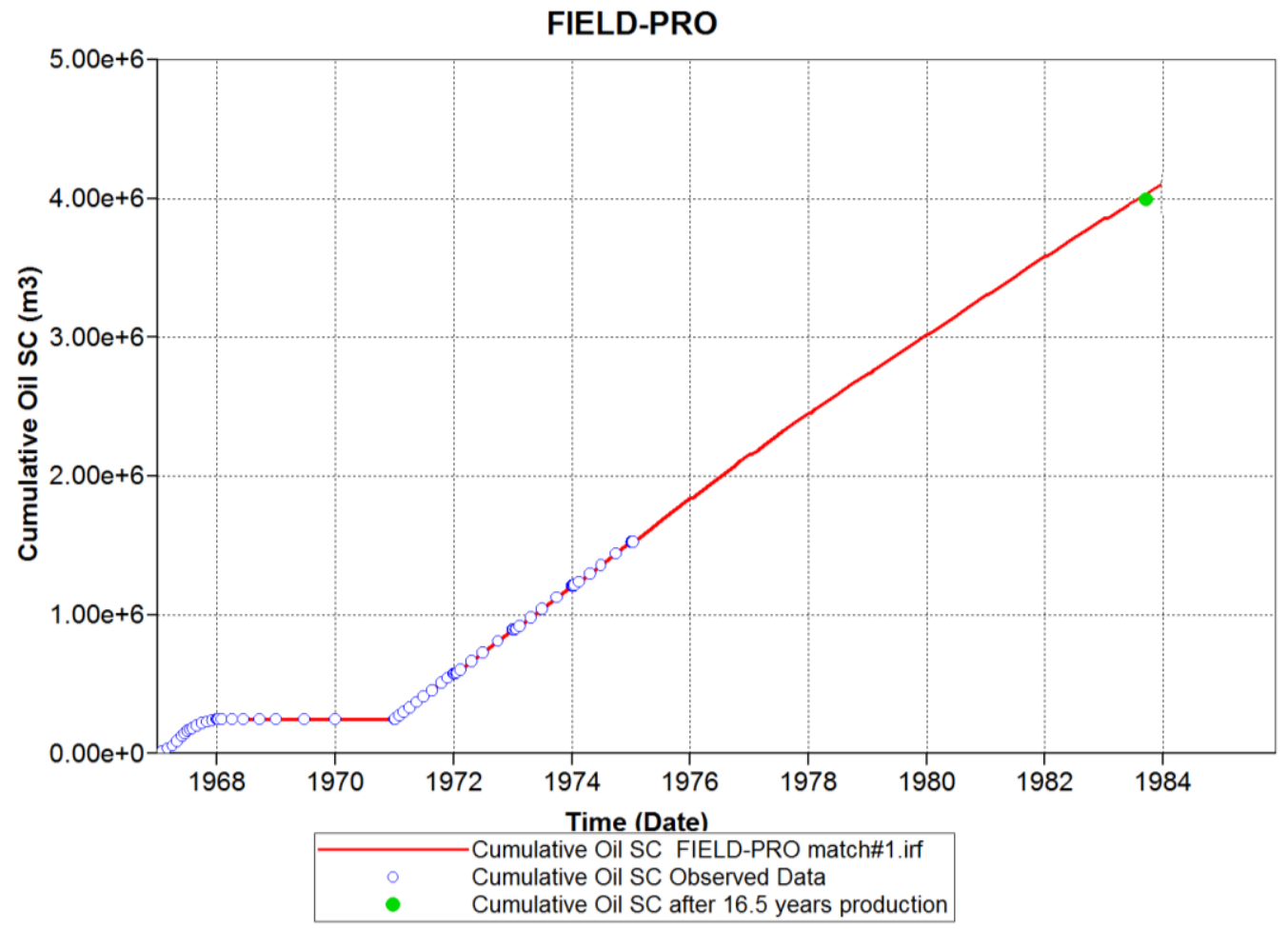

Figure 78: Comparison between the SRM based history matching results (match \#1) and actual data for cumulative oil production. The red line represents the matched realization and the blue circles are the actual field data (eight years of production history). The green dot also displays the cumulative production for the true case after 16.5 years. 
Similar to the oil production, Figure 79 shows the comparison between the simulator results and actual field data for the cumulative gas production.

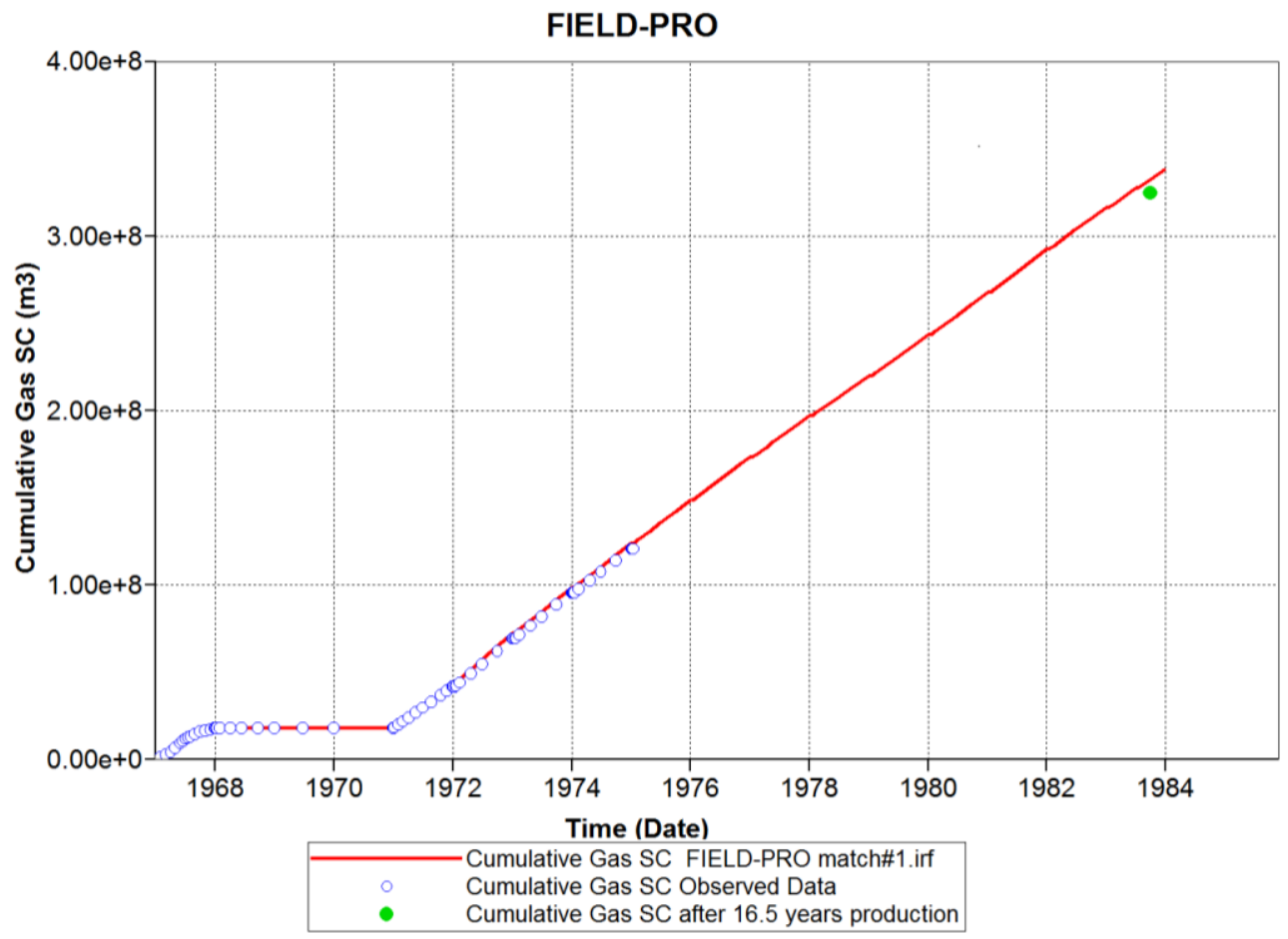

Figure 79: Comparison between the SRM based history matching results (match \#1) and actual data for cumulative gas production. The red line represents the matched realization and the blue circles are the actual field data (eight years of production history). The green dot also displays the cumulative production for the true case after 16.5 years.

As it was mentioned the water breakthrough happens at the seventh year for just one well (PRO-11). Therefore, except for the well PRO-11 which produces water, the other wells have a very little water production. Figure 80 compares the cumulative water production between the simulator and actual data. 
FIELD-PRO

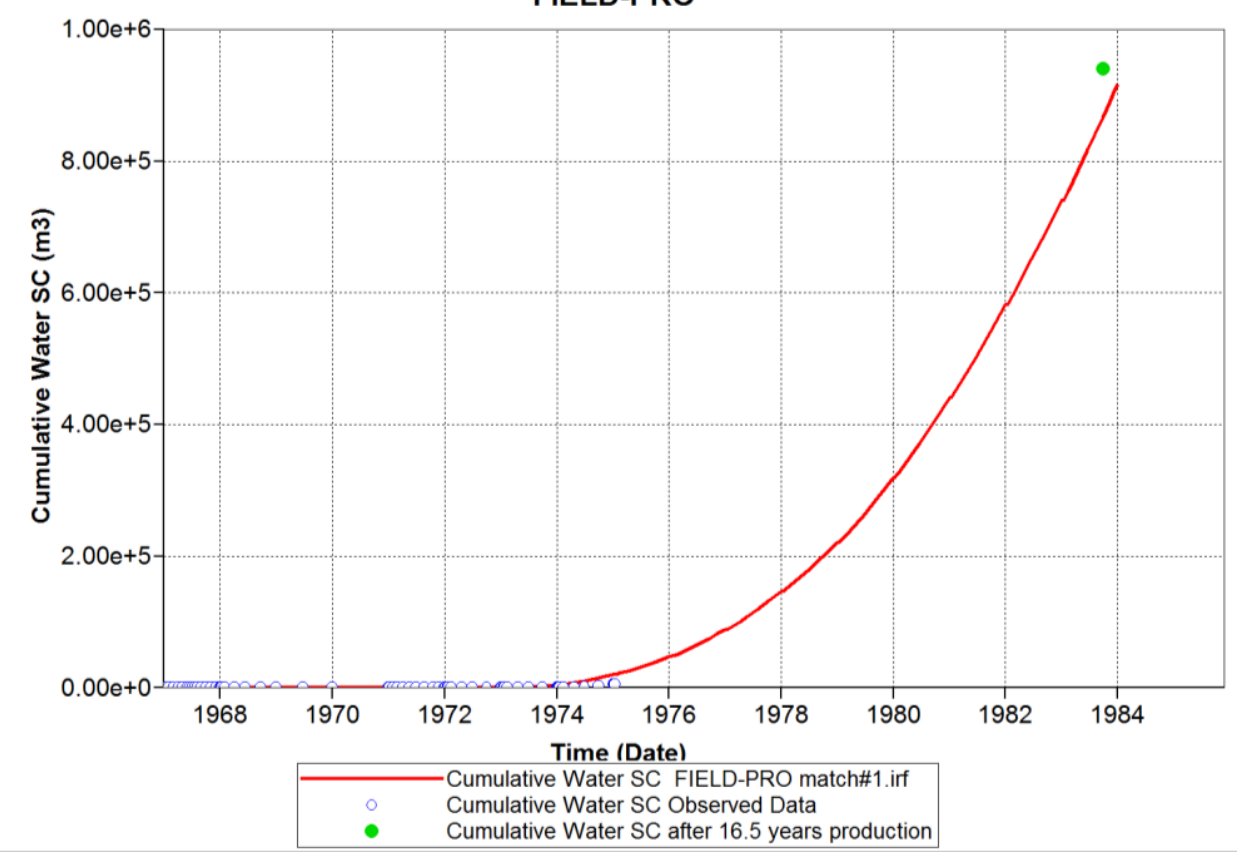

Figure 80: Comparison between the SRM based history matching results (match \#1) and actual data for cumulative water production. The red line represents the matched realization and the blue circles are the actual field data (eight years of production history). The green dot also displays the cumulative production for the true case after 16.5 years.

Figure 81 shows the gas rate and cumulative gas production for the wells in PUNQ-S3 reservoir. These results belong to the reservoir model which was built using the matched properties of the best match obtained by the SRM. This graph compares the results of simulator and available actual data. Figure 82 is the same comparison for the well bottom-hole pressure. Finally Figure 83 compares the water rate and cumulative water production. 


\section{Gas Rate (m3/day) and Cumulative Gas (m3)- Match \#1}
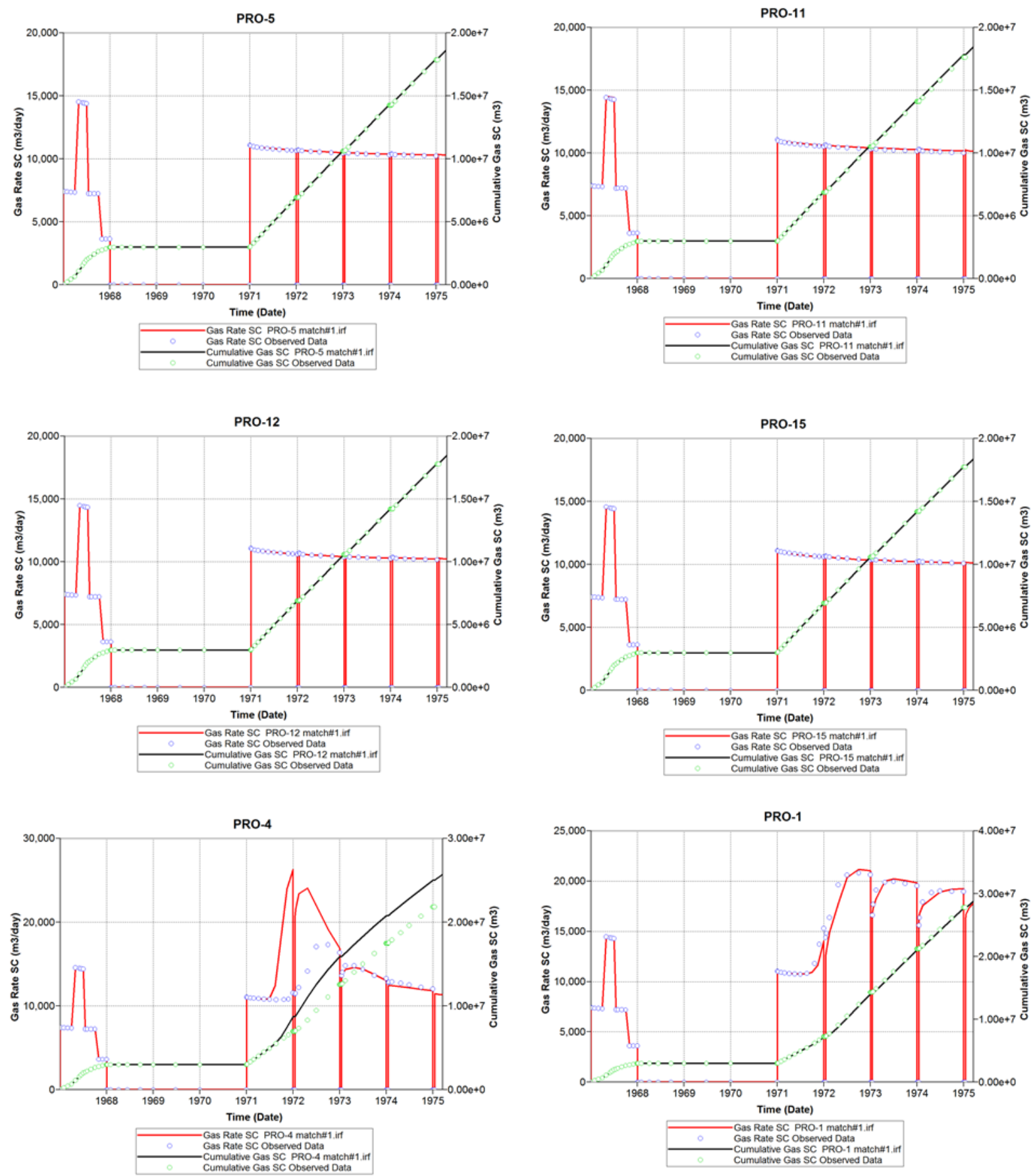

Figure 81: Comparison between the SRM history matching results and actual data. The graph compares the gas rate and cumulative gas production for eight years of available field data for different wells in the PUNQ-S3 reservoir model. 


\section{Bottom-hole Pressure (kPa) - Match \#1}
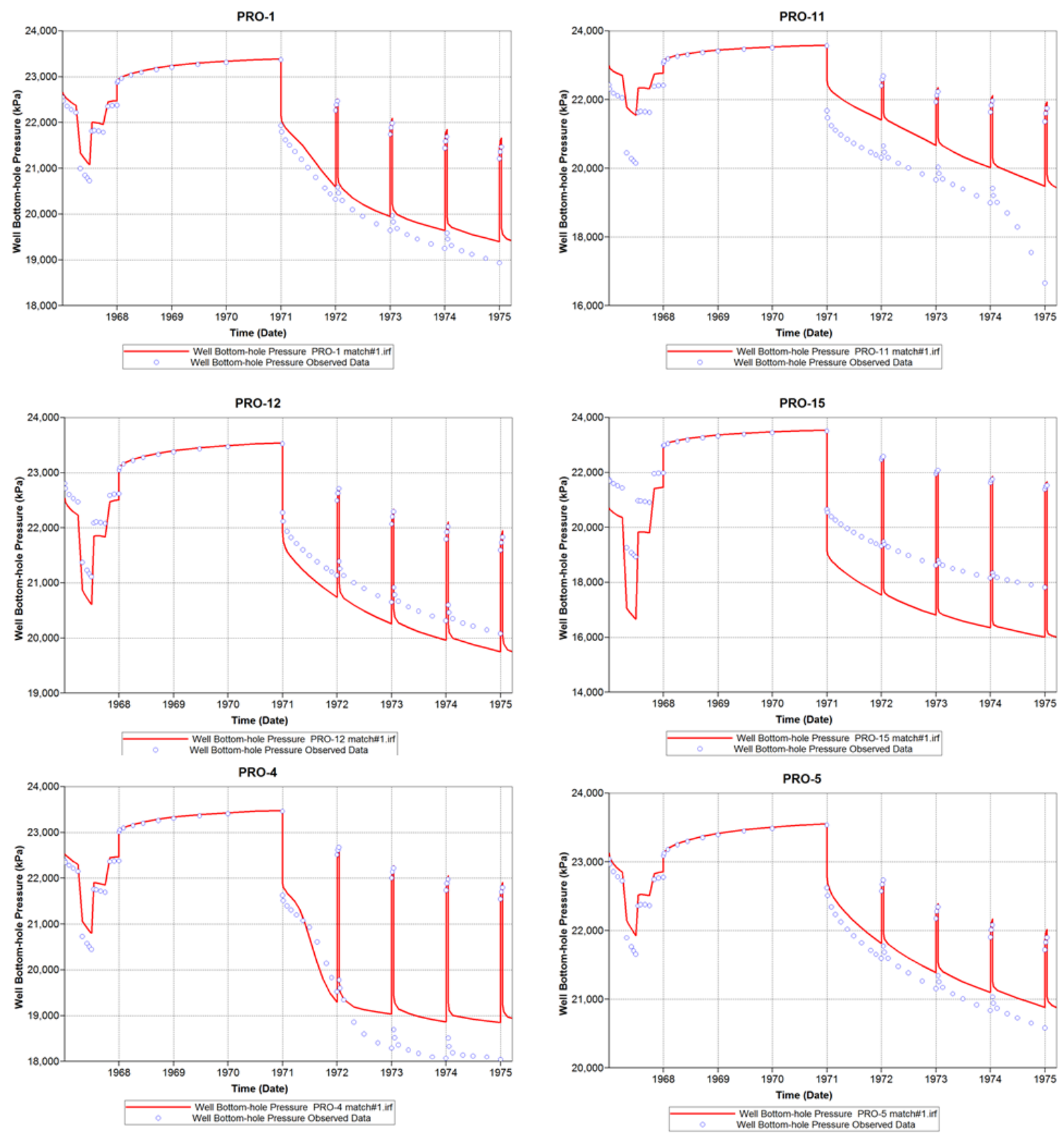

Figure 82: Comparison between the SRM history matching results and actual data. The graph compares the well bottom-hole pressure for eight years of available field data for different wells in the PUNQ-S3 reservoir model. 


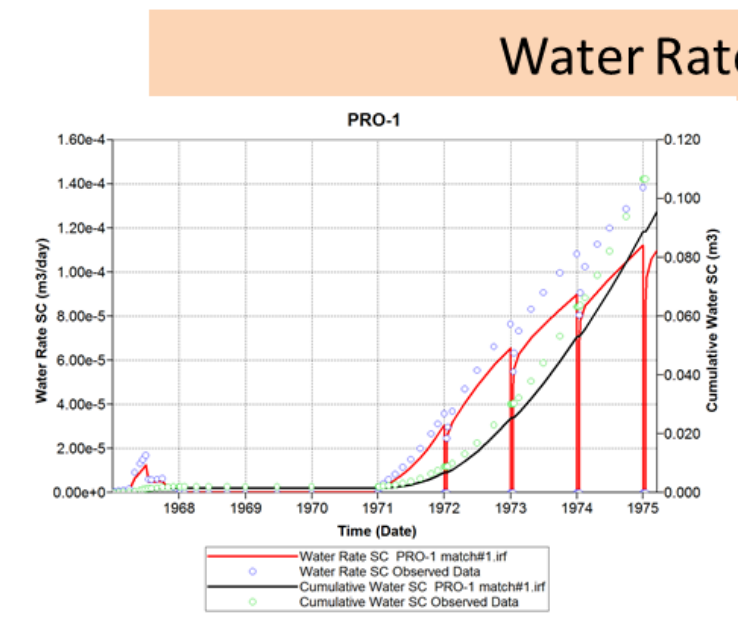

(m3)- Match \#1
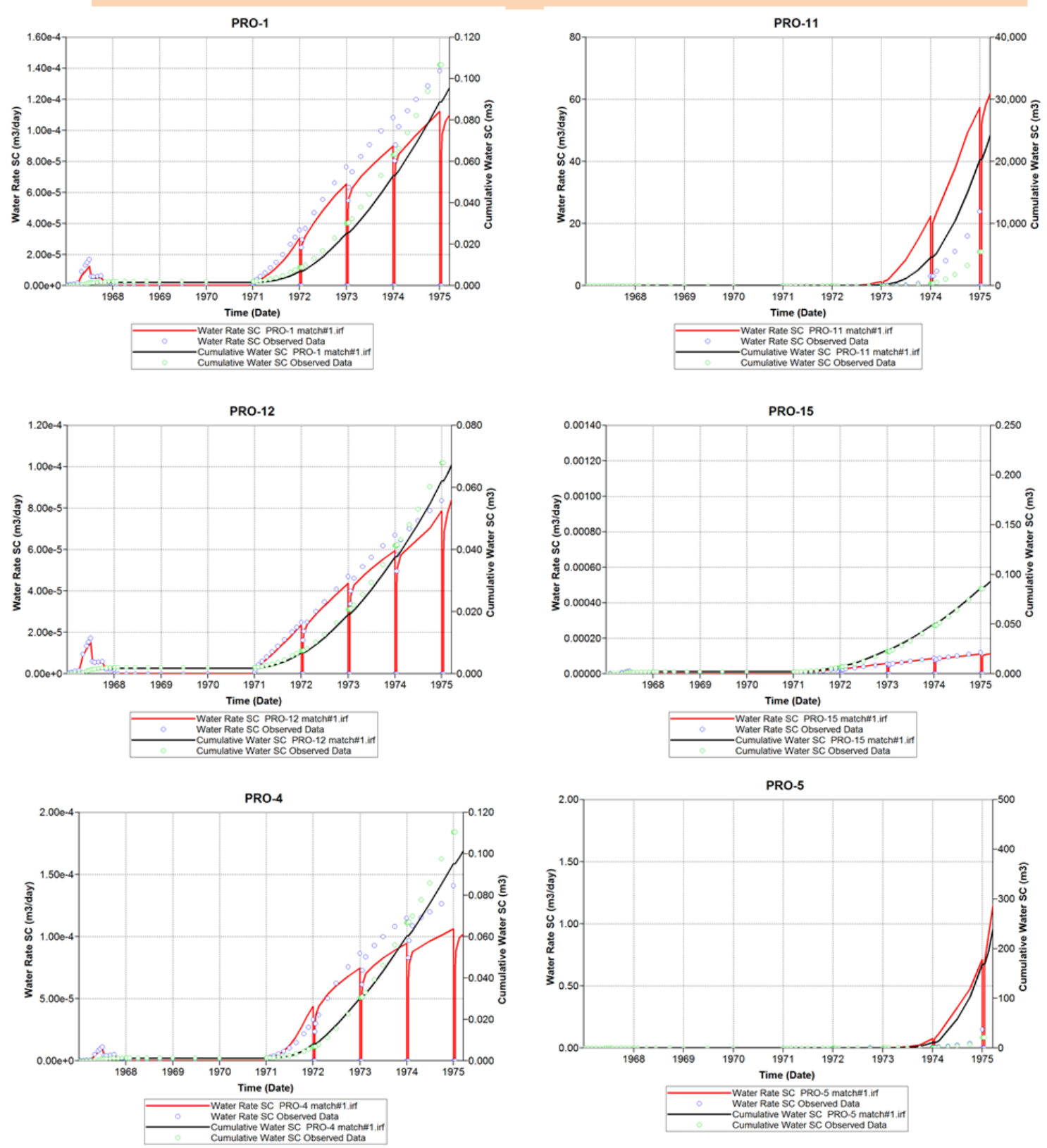

Figure 83: Comparison between the SRM history matching results and actual data. The graph compares the water rate and cumulative water production for eight years of available field data for different wells in the PUNQ-S3 reservoir model.

The ten best matched obtained from the history matching were used to generate another realization of the PUNQ-S3 reservoir model. The characteristics of this realization are the average values of ten best matches. Figure 84 presents the field cumulative oil production and compares the results of simulator (red line) with the actual data (blue circles). Also it shows the simulator forecast compared to the actual cumulative oil production. Figure 85 is the same 
results for the field cumulative gas production. Furthermore, Figure 86 is the results for the field cumulative water production.

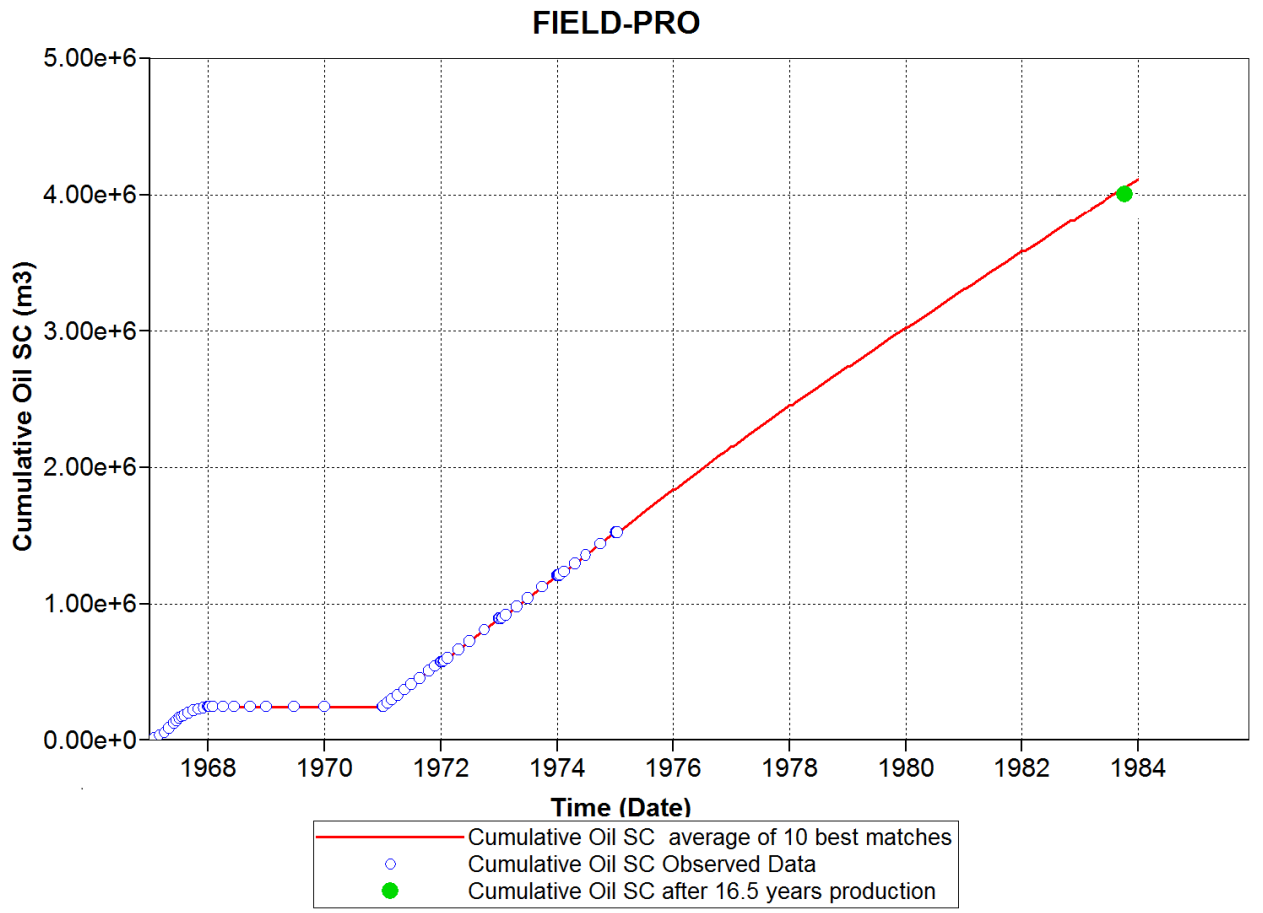

Figure 84: Comparison of actual data and the result of a realization of PUNQ-S3 reservoir model. This realization was made using the average properties values of ten best matches. This graph represents the field cumulative oil production, comparing the actual data (blue circles) with the simulator results (red line). Also the green dot is the actual cumulative oil production after 16.5 years, provided in PUNQ project for the forecast comparison. 


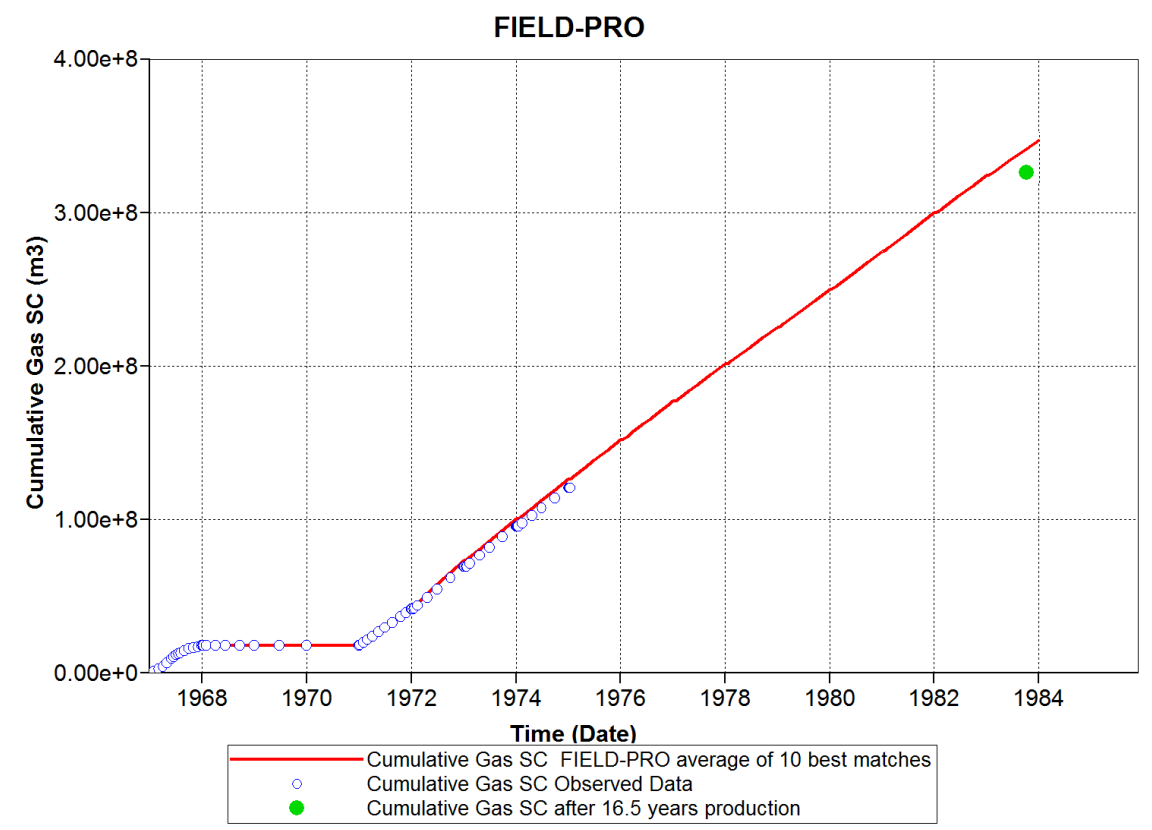

Figure 85: Comparison of actual data and the result of a realization of PUNQ-S3 reservoir model. This realization was made using the average properties values of ten best matches. This graph represents the field cumulative gas production, comparing the actual data (blue circles) with the simulator results (red line). Also the green dot is the actual cumulative gas production after 16.5 years, provided in PUNQ project for the forecast comparison.

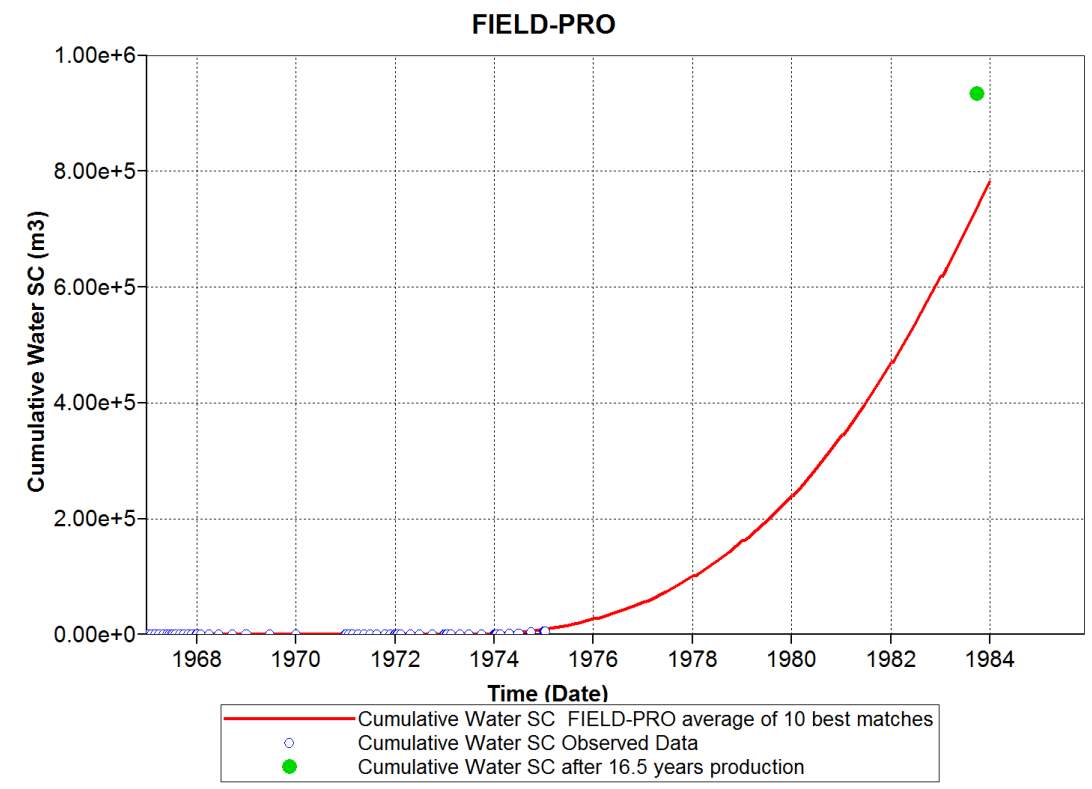

Figure 86: Comparison of actual data and the result of a realization of PUNQ-S3 reservoir model. This realization was made using the average properties values of ten best matches. This graph represents the field cumulative water production, comparing the actual data (blue circles) with the simulator results (red line). Also the green dot is the actual cumulative water production after 16.5 years, provided in PUNQ project for the forecast comparison. 


\section{Gas Rate (m3/day) and Cumulative Gas (m3)- Average of 10 Best Matches}
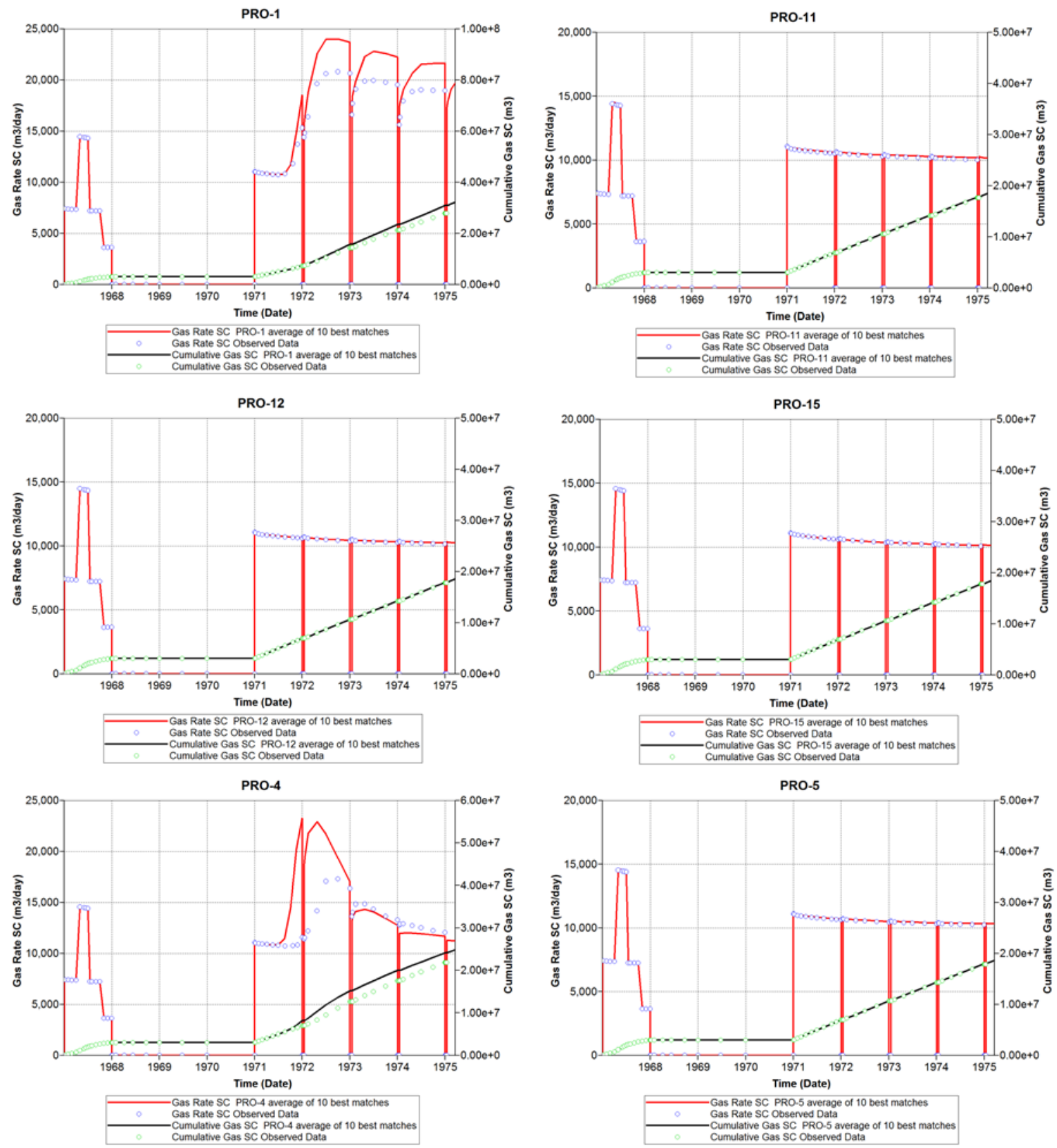

Figure 87: Comparison between the SRM history matching results and actual data. The graph compares the gas rate and cumulative gas production for eight years of available field data for different wells in the PUNQ-S3 reservoir model. The results belong to a realization of PUNQ-S3 reservoir which its characteristics were the average of properties of ten best matches. 


\section{Bottom-hole Pressure ( $\mathrm{kPa})$ - Average of 10 Best Matches}
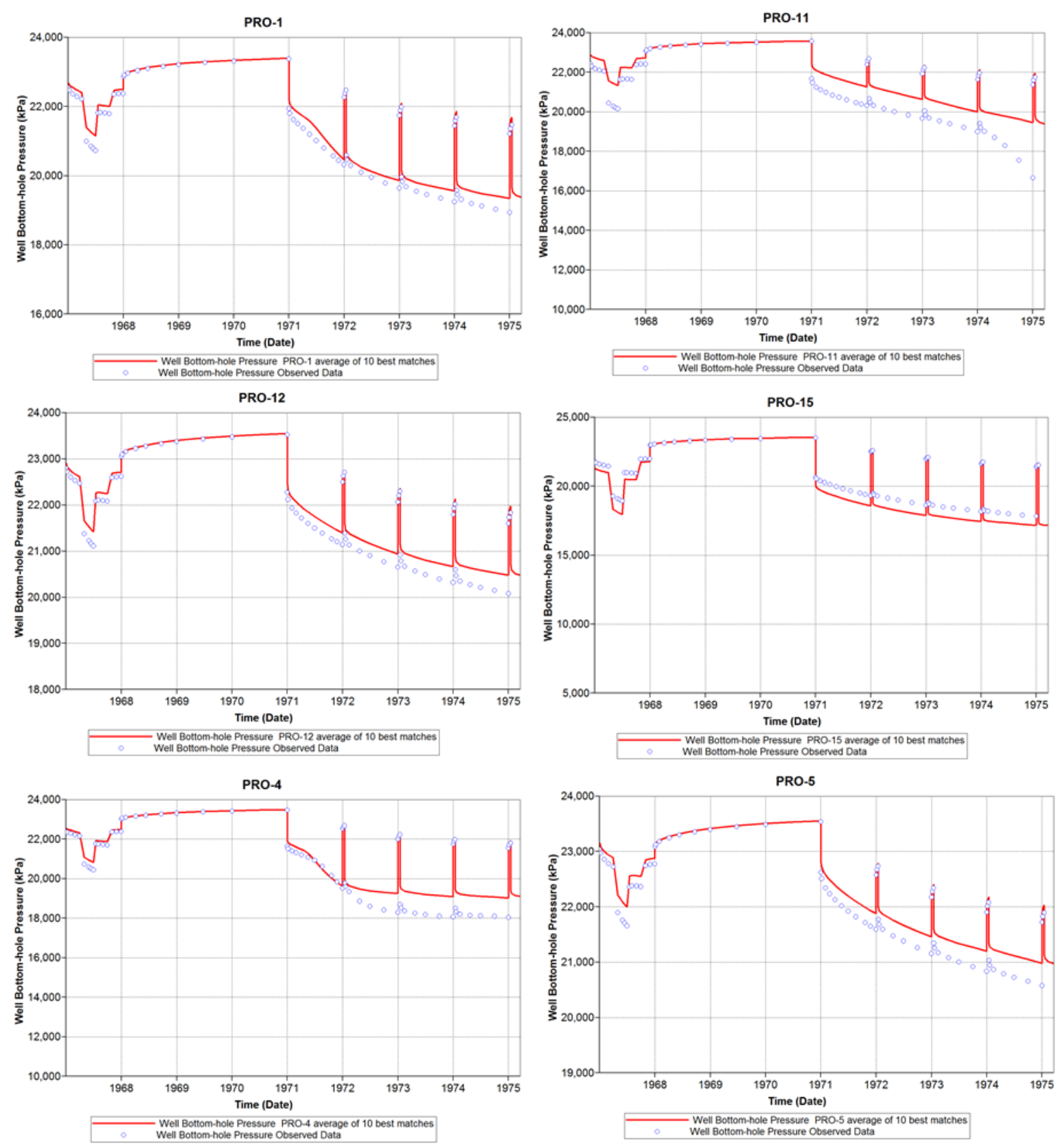

Figure 88: Comparison between the SRM history matching results and actual data. The graph compares the well bottom-hole pressure for eight years of available field data for different wells in the PUNQ-S3 reservoir model. The results belong to a realization of PUNQ-S3 reservoir which its characteristics were the average of properties of ten best matches. 


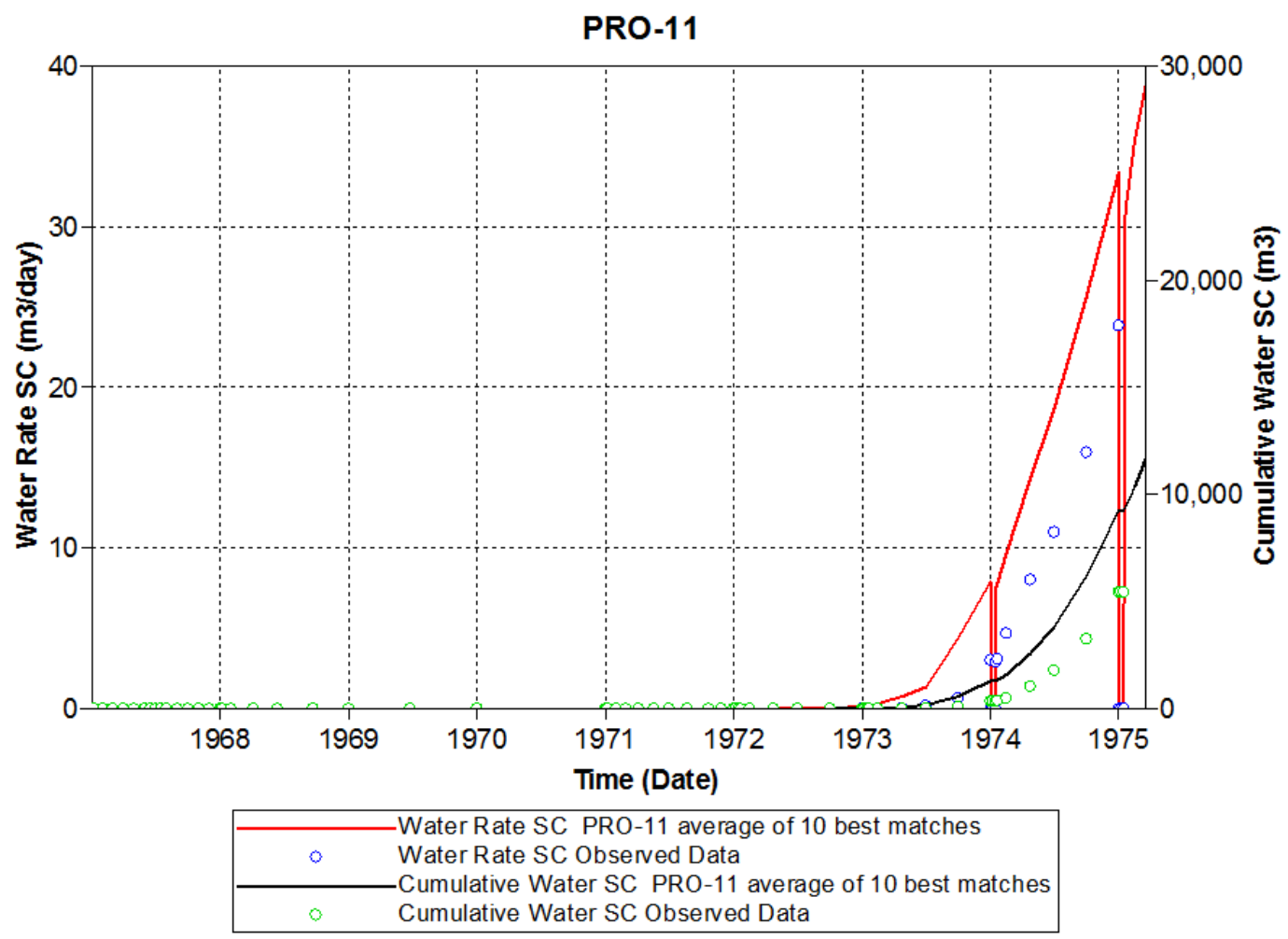

Figure 89: Comparison between the SRM history matching results and actual data. The graph compares the water rate and cumulative water production for eight years of available field data for the well PRO-11 in PUNQ-S3 reservoir model. The results belong to a realization of PUNQ-S3 reservoir which its characteristics were the average of properties of ten best matches.

For the future prediction, the ten best matches and the average of these matches (total 11 matches) were executed to capture the field cumulative oil production after 16.5 years. Figure 90 describes the outputs of these realizations and compares them to the value reported as the true case result. The red line is the oil recovery for the true case, the blue markers are the ten best matches, and finally the green marker shows the value of oil recovery for the realization which is the average of ten best matches.

Figure 91 is the box plot of oil recovery for the 11 selected matches. The upper level of the box in this graph shows P90, the middle of box is P50 (median) and the bottom level of the box describes $\mathrm{P} 10$ of the results. 


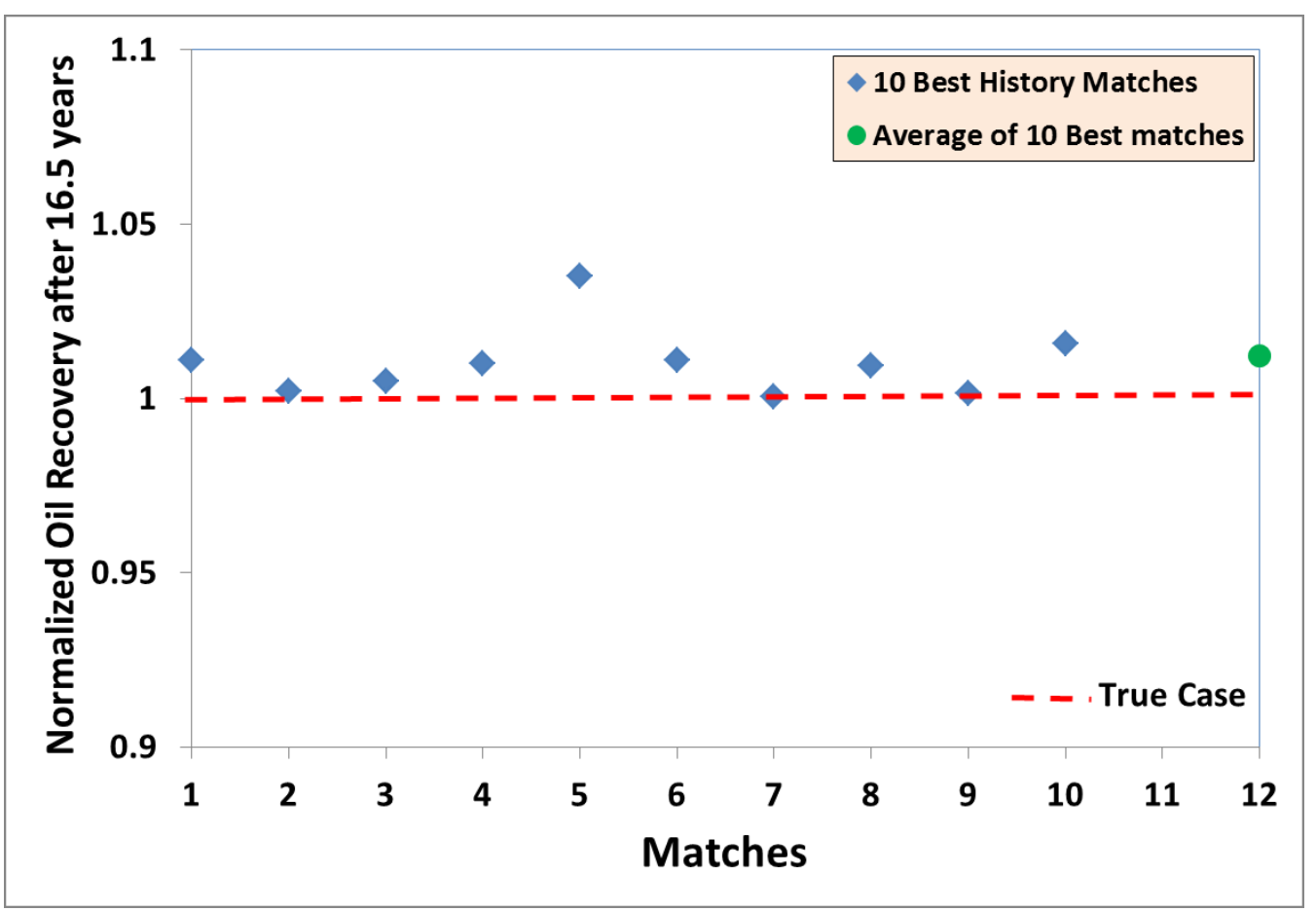

Figure 90: Comparison of future production forecast of the best matched models with the true case.

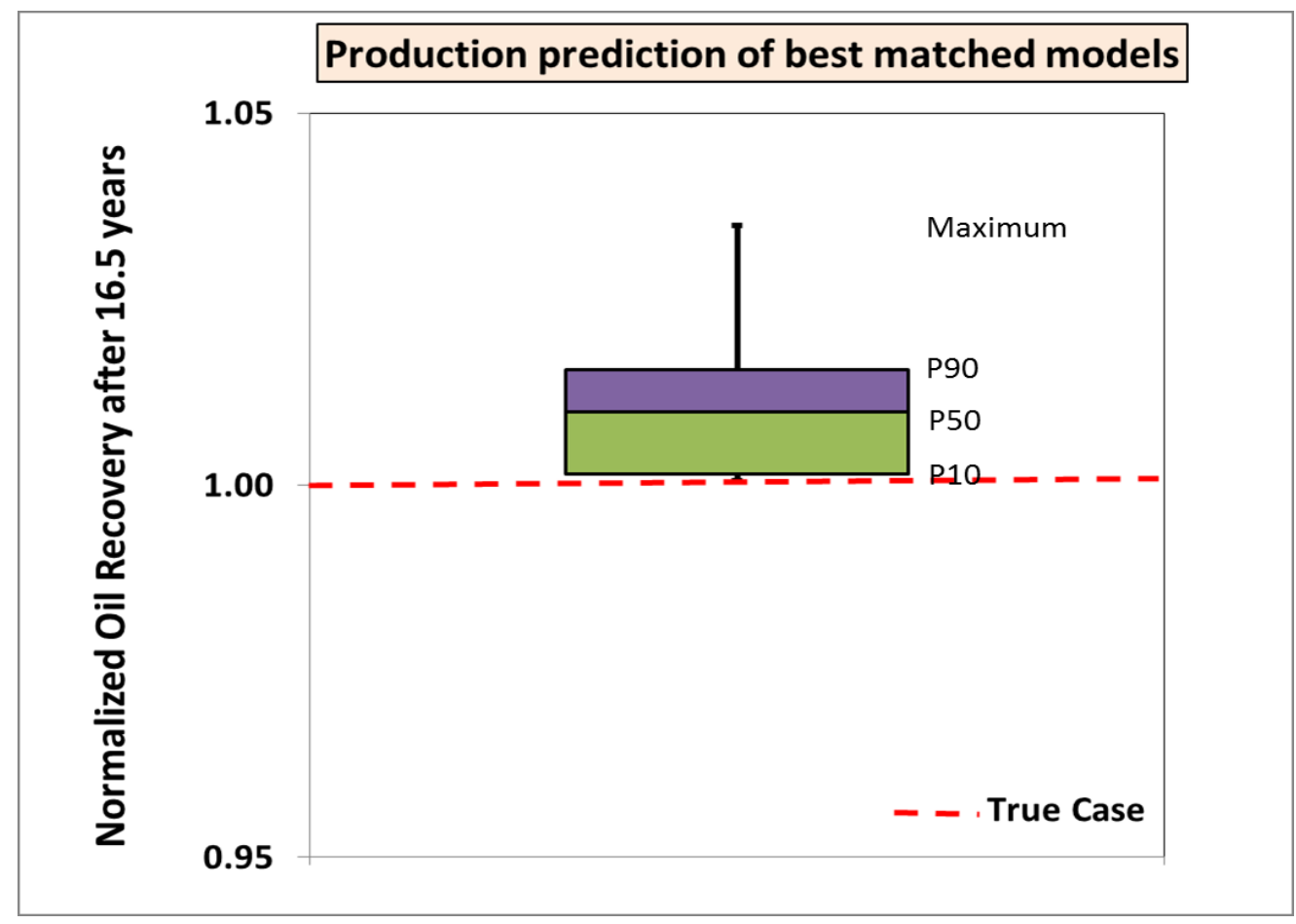

Figure 91: Box plot of future production forecast for 11 selected matches (ten best matches and the average of these matches). The upper level of box represents P90, the middle is P50 and the bottom of the box is P10. 


\subsection{Discussion and concluding remarks}

In this chapter a surrogate reservoir model was developed for the PUNQ-S3 reservoir simulation model. The PUNQ-S3 model is widely accepted as a standard reservoir simulation model to test the ability of different methods on history matching and uncertainty quantification. The characteristics of this model make PUNQ-S3 model a unique case to study the potential of SRM for history matching. The variable properties to create the SRM are porosity and permeability (horizontal and vertical) distributions. In order to train the SRM, ten realizations of PUNQ-S3 simulation model were generated. An extra realization $\left(11^{\text {th }}\right.$ case) was used to verify the trained SRM.

One important feature of an effective history matching workflow is the automation ability. Therefore, the developed SRM was coupled with the DE optimization algorithm. The objective functions were created to calculate the misfit values between the actual data and measured results (SRM). The goal of history matching was to match eight years of history data available for three different properties. These properties include the well bottom-hole pressure, gas production rate, and water production rate. The stoppage condition for the automated history matching workflow was 3,000 times calling the SRM. Also this workflow was able to report multiple realizations of the reservoir which match the actual data. Thus, ten best matches were selected to be used for the future forecast. The future forecast consists of predicting the field cumulative oil production after 16.5 years production. Beside the eight years of history data, the PUNQ project provides the field cumulative oil production after 16.5 years for the purpose of future production comparison.

Figure 68, Figure 69, and Figure 70 show the results of SRM during the training process for the well bottom-hole pressure, gas production rate, and water production rate, respectively. These graphs portray the comparison between the SRM results with the outputs of simulator. The significant match between the results of SRM and simulator proves that SRM has been well-trained. The ability of SRM to capture the zero values of gas and water production rates is clear in the Figure 69 and Figure 70.

Figure 71, Figure 72, and Figure 73 depict the performance of SRM on a completely unseen realization of reservoir during the training process. This step, referred to as blind realization, shows the robustness of the SRM. The quality of the match for the blind case, as it is seen in these graphs, is not as good as the training realizations (Figure 68, Figure 69, and Figure 70). This is a normal and expected reaction of the SRM to the set of data which are not used in the SRM training. 
The trained and verified SRM was used to perform the history matching. As it was discussed earlier, the history matching was accomplished in an automated workflow. Furthermore, this workflow produced eleven realizations of the reservoir which able to match the history of the field. Figure 74, Figure 75, Figure 76, and Figure 77 are the results of ten best history matched realizations. The results are the outputs of SRM. These graphs are the comparison between the ten best matches with the actual data for the well bottom-hole pressure, gas production rate, cumulative gas production, water production rate, and cumulative water production. The quality of matches for the well bottom-hole pressure and gas production rate are superb. For the water production rate (Figure 77), the matches are acceptable. The SRM matched the zero values of water production very well; however for the non-zero values, the quality of matches decrease (although they are still acceptable).

The average values of characteristics of ten best matches were used to construct another realization. Therefore, total 11 realizations of PUNQ-S3 are the outputs of this part of study. The characteristics of these realizations were imported into the simulator. The goal was to observe the performance of simulator and compare it with the actual data. Particularly these matches were used to predict the field cumulative production after 16.5 years. The PUNQ project has provided the value of field cumulative oil production after 16.5 years.

Figure 78 displays the results of the best achieved match imported into the simulator. This figure compares the results of this realization with the actual data for the field cumulative oil production. The results show a good match for the eight years of available history. Also, this graph predicts the field cumulative oil production for the next 8.5 years. At the end of this time period, the prediction performance has been compared with the reported value. Although the match shows an excellent quality, the prediction is slightly overestimating the future production. Figure 79 and Figure 80 are the same comparison for the field cumulative gas and water production. For the gas production, we see same overestimating behavior; however water production has been slightly underestimated.

Figure 90 and Figure 91 summarize the production prediction of the selected eleven matches for the oil recovery. These figures quantify the uncertainty involved in the production prediction. The prediction of field cumulative oil production is very close to what has been reported as the true case (red line in these figures).

The general results in this chapter demonstrate the robustness of SRM in history matching and future prediction for the PUNQ-S3 problem. Numerous studies have used the PUNQ-S3 
reservoir model to test the methods on history matching. Many of these studies are the investigation of different optimization methods for automated history matching. Generally these optimization algorithms have been coupled with a commercial simulator. The reported numbers of simulation runs for history matching the PUNQ-S3 reservoir model are in the order of thousands realizations (Hajizadeh, Christie and Demyanov 2010, 2009, Abdollahzadeh, et al. 2011). In this study, the required simulation runs to create and validate the SRM (eleven runs) are extremely less. Although, the run-time is not an issue for the PUNQ-S3 reservoir simulation model; in reality, a typical reservoir simulation model is more time-consuming to run and requires higher computational cost. In such a case using a numerical reservoir simulator for history matching would be a huge computational issue. In conclusion, the application of SRM for history matching purposes would be a great asset in the reservoir management workflow. 


\section{Chapter 6: Summary, conclusions and recommendations}

\subsection{Summary}

Reservoir simulation and modeling is utilized throughout the field development in different capacities. Sensitivity analysis, history matching, operations optimization, and uncertainty assessment are the conventional analyses in full field model studies. Realistic modeling of the complexities of a reservoir requires a large number of grid blocks. As the complexity of a reservoir increases and consequently the number of grid blocks, so does the time required to accomplish the abovementioned tasks. A relatively new technology known as Surrogate Reservoir Model (SRM) is introduced as a tool for addressing many time-consuming operations performed with the reservoir simulation models. SRMs are the replicas of full field models that run in matters of seconds.

History matching techniques are used in reservoir modeling to fine-tune the reservoir properties such as porosity and permeability by matching the measured production data (pressure and production profiles at each well). During the last two decades there have been many attempts to improve history matching approaches in a way that could be applicable and practical in the real world. Despite all the efforts, due to the increasing rate of complexity and resulotion in the reservoir models, there is still hesitation about the practicality and potential of these methods to handle highly complicated real reservoir models.

In this study, efforts were made in order to show the proofs that SRM is an efficient tool to replicate the reservoir simulator performance in the history matching process. This replica results in a faster performance and with a reasonable accuracy in order to address the practical issues of reservoir simulation models in the history matching workflow.

Accomplishments of the objectives of this study included a three step process:

1. Part one, a proof of concept study: The goal of first step was to prove that SRM is able to substitute the reservoir simulation model in a history matching project. In this project, a SRM was created for a synthetic but heterogeneous and under-saturated oil field, with 24 production wells and 30 years of production history.

The history match was accomplished by tuning only one property (permeability) throughout the reservoir. As a result, an SRM was trained, calibrated, and validated 
using ten geological realizations of the reservoir simulation model. Then, the SRM was further validated using a complete blind (eleventh) realization of the reservoir. Finally the trained and validated SRM substituted the reservoir simulation model in the history matching process. The oil production rate of all the 24 wells was history matched in a short period of time by modifying the permeability distribution throughout the reservoir.

2. Part two, a feasibility study: The objective of the second part was to demonstrate the robustness of SRM in handling higher degrees of uncertainty. Therefore, the number of uncertain reservoir properties increased to three properties (permeability, porosity, and thickness). The SRM was trained, calibrated, and validated using a few geological realizations of the base reservoir model.

An additional feature in this part of the study was coupling the SRM with an optimization algorithm (DE). DE optimization method is considered as a novel and robust optimization algorithm from the class of evolutionary algorithm methods. Coupling the SRM with DE created an automated history matching workflow. This workflow is able to select the parameters within the given ranges, call the developed SRM, run it, calculate the misfit through the defined objective functions, and finally report multiple realizations of the reservoir which match the history data.

3. Part three, a real-life implementation: The third part of this research was to apply the lessons learned to a real-life case study. For this purpose, a reservoir model, known as PUNQ-S3 model, was selected. The PUNQ-S3 model covers different aspects of a real-life case study and has been used by numerous researchers to test the different methods of history matching and uncertainty quantification. The model includes multiple layers, multi-phase fluids, faults, aquifer, and strikes of high porosity/permeability. Eight years of history data are available for the well bottomhole pressure, gas production rate, and water production rate. These data are used for history matching. In addition, the field cumulative oil production after 16.5 years is given to compare the future prediction.

Ten realizations of this model were generated by altering porosity and permeability (vertical and horizontal) distributions. A spatio-temporal database was constructed using the data extracted from the ten realizations of PUNQ-S3 reservoir simulation model. The SRM was developed (trained/calibrated/validated) based on the spatiotemporal database. A further validation step was accomplished by utilizing an extra realization of PUNQ-S3 reservoir model (blind verification). Finally, the SRM was 
coupled with DE in order to perform the automated SRM-based history matching. Ten best matches were selected as the output of the history matching process. The average values of these ten best matches formed another realization. Consequently, the characteristics these eleven matches were imported into the reservoir simulator to predict the future performance.

\subsection{Conclusions}

The major conclusions that can be drawn from this dissertation are summarized as the following:

1. The surrogate reservoir models were proved to be an effective tool in accomplishment of history matching projects. The unique features of AI\&DM techniques, utilized to develop the SRMs, give exceptional characteristics to the SRMs. These techniques are able to learn the specific behaviors of a system through a few examples. Particularly, in this study, SRMs were made using a very few realizations of the original system (reservoir simulation models). The results of SRMs in different steps of development and application proved and demonstrated the potential of SRMs in history matching process. The following items are concluded in order to accomplish a successful SRM:

a. SRMs are built based on the provided examples of the reservoir simulation model. These examples are the main source of information for a spatiotemporal database which is used to train the SRMs. In order to develop a successful SRM, meticulous efforts should be dedicated to the preparation of this database. The quality of the SRM mainly depends on the quality of the provided database. The objective of study is the main item in shaping the spatio-temporal database. For instance, depending on the goal of project, the outputs and inputs of SRMs might vary. For a history matching project, like this study, we are seeking to estimate the performance of the reservoir simulation model at the well. This performance could be a production profile or the well bottom-hole pressure at the well site. The SRM created for this type of study is known as well-based SRM.

b. The purpose of having different realizations of a reservoir simulation model is to introduce the uncertainties involved in the model to the SRM. Although the SRM does not need a high number of the simulation runs, selecting a right number of runs is delicate. If the number of simulation runs is too small, then the SRM may not be able to capture the uncertainty and the variation in the 
parameters. Therefore, the SRM does not show a good performance on the data not used in the training examples. This justifies the use of blind verification step in the SRM development. In the other words, the validation examples will expose the lack of required information in the training samples. On the other hand, if the number of simulation runs is too large, then there is no reason to develop an SRM since the solution is close to the original problem, which is a high number of simulation runs.

c. Data summarization techniques are very important to decrease the high dimension of the spatio-temporal database. One way of data summarization is to delineate the reservoir into the different segments (tiers) and make an average of the reservoir property values over the grid blocks in the designed segments. The Voronoi graph theory (Erwig 2000, Gomez, et al. 2009) was proved to be useful in delineating the reservoir to the drainage areas. Each one of these drainage areas were divided into multiple tiers based on the impacts of different segments of the reservoir on the performance of wells.

d. Input data selection is another important step for having an efficient SRM. The preprocessing operations could be a great help for selecting the right inputs to train the SRMs. These operations could vary from very simple statistical procedures to complicated data mining techniques. Furthermore, the reservoir engineering knowledge of the user plays an important role in selecting the right inputs. This is a point which is usually neglected in developing the AI-based reservoir models.

2. In this dissertation, the SRMs were developed for different reservoir models. Initially, an SRM was created for a reservoir model by tuning just one property. In the next step, the number of uncertain properties increased. In both cases, the SRMs showed a great performance in the history matching processes.

3. Then, the SRM was applied to a standard test case, the PUNQ-S3 reservoir model. The SRM showed significant results in history matching different properties of this reservoir model. The results achieved by SRM were imported into the reservoir simulator to predict the future performance of the reservoir. The future forecast showed a great match with the reported value.

4. The SRM was able to easily couple with an optimization algorithm. The most important feature added by the optimization algorithm (DE), was automating the history matching workflow. A very important objective of having optimization 
methods in history matching approaches is to decrease the number of simulation runs in order to achieve the desired matches. However, in a SRM-based history matching workflow this is not the issue.

5. The results of this dissertation prove the ability of SRMs in history matching process; nevertheless, these achievements benefit the other time-consuming operations in the reservoir management workflow such as sensitivity analysis, production optimization, and uncertainty assessment.

\subsection{Recommendations for future studies}

The AI\&DM techniques have showed their practical capabilities in many aspects of different areas of science and engineering (some examples are shown in Table 2). These techniques are proved approaches in those areas and have been utilized frequently. On the other hand, it is difficult to make a similar conclusion for the oil and gas industry. AI\&DM based reservoir models such as SRMs are relatively new applications in the petroleum engineering and this area of engineering is considered as completely a new territory for the AI\&DM techniques.

An important characteristic of petroleum engineering problems is that these types of problems are very subjective to their own properties. In other words, each problem in petroleum engineering is associated with very particular specifications. These specifications make each one of these problems a unique case. For this reason it is not possible to create a general simulation model suitable for different reservoirs. This is a characteristic which perfectly suits the features of AI\&DM techniques.

In this dissertation the development and applications of SRMs for three case studies of history matching were discussed. However, there is a long way ahead for the petroleum industry to accept these types of technologies as a new tool of modeling. In order to increase the rate of acceptance the applications of these methods should be further analyzed on different types of problems. Therefore, it is highly recommended trying to repeat the accomplishments of this study on the new and more complicated history matching problems. The run-time was not the issue for the reservoir simulation models in this study; however, in realty, real history matching problems include reservoir simulation models which are time-consuming and with high computational cost. The continuation for this study could be implementing the developed technology on a real-life size reservoir simulation model.

The PUNQ-S3 reservoir simulation model included many aspects of complexities involved in a reservoir simulation model. These complexities were such as multi layers, multi-phase 
fluids, faults, aquifer, and channels of high porosity/permeability. However, increasing the complexity involved in the reservoir simulation models could be another step for the future studies. These complexities could be increasing the number of wells, adding injection scenarios, working with different types of operational constraints, having horizontal wells in the systems, and etc.

An aspect of modern era of history matching is the utilization of optimization algorithms. In this study DE was the chosen optimization method. The DE has a simple structure and that makes it easy to develop and apply in a history matching workflow. In addition to DE, a long list of optimizations methods has been proposed for the history matching purposes. An additional study regarding the future works could be application and comparison of different methods of optimizations in couple with the SRM. 


\section{References}

A. A., Adebiyi, Ayo C. K., Adebiyi M. O., and Otokiti and O. S. "Stock Price Prediction using Neural Network with Hybridized Market Indicators." Journal of Emerging Trends in Computing and Information Sciences, 2012.

Aanonsen, Sigurd I., Geir Nævdal, Dean S. Oliver, Albert C. Reynolds, and Brice Vallès. "The Ensemble Kalman Filter in Reservoir Engineering--a Review." SPE Journal (Society of Petroleum Engineers) 14, no. 03 (2009): 393 - 412.

Abdollahzadeh, Asaad, Alan Reynolds, Michael A. Christie, David Corne, Brian James Davies, and Glyn Williams. "Bayesian Optimization Algorithm Applied to Uncertainty Quantification." SPE EUROPEC/EAGE Annual Conference and Exhibition. Vienna, Austria: Society of Petroleum Engineers, 2011.

Agarwal, Bijan, and Martin Blunt. "A Streamline-Based Method for Assisted History Matching Applied to an Arabian Gulf Field." SPE Annual Technical Conference and Exhibition. Denver, Colorado: Society of Petroleum Engineers, 2003.

Agarwal, Ram G., David C. Gardner, Stanley W. Kleinsteiber, and Del D. Fussell. "Analyzing Well Production Data Using Combined-Type-Curve and Decline-Curve Analysis Concepts." SPE Reservoir Evaluation \& Engineering (Society of Petroleum Engineers) 2, no. 02 (1999): 478 - 486.

AGH University of Science and Technology webpage. 2014. http://home.agh.edu.pl/ vlsi/AI/intro/ (accessed 5 01, 2014).

Ahmed, T., C.A. Link, K.W. Porter, C.J. Wideman, P. Himmer, and J. Braun. "Application of Neural Network Parameter Prediction in Reservoir Characterization and Simulation A Case History: The Rabbit Hills Field." Latin American and Caribbean Petroleum Engineering Conference. Rio de Janeiro, Brazil, 1997.

Alessio, Laurent Didier, Laurent Marc Bourdon, and Spencer Coca. "Experimental Design as a Framework for Multiple Realisation History Matching: F6 Further Development Studies." SPE Asia Pacific Oil and Gas Conference and Exhibition. Jakarta, Indonesia: Society of Petroleum Engineers, 2005.

Ali, J K. "Neural Networks: A New Tool for the Petroleum Industry?" European Petroleum Computer Conference. Aberdeen, United Kingdom: Society of Petroleum Engineers, 1994.

Ali, MM, and A Torn. "Population set-based global optimization algorithms: some modifications and numerical studies." Comput Oper Res 31, no. 10 (2004): 17031725.

Al-Kaabi, A U, and W J Lee. "An Artificial Neural Network Approach To Identify the Well Test Interpretation Model: Applications." SPE Annual Technical Conference and Exhibition. New Orleans, Louisiana: Society of Petroleum Engineers, 1990. 
Al-Shamma, B, and R Teigland. "History Matching of the Valhall Field Using a Global Optimization Method and Uncertainty Assessment." SPE Annual Technical Conference and Exhibition. San Antonio, Texas: Society of Petroleum Engineers, 2006.

Amini, Shohreh, Shahab D. Mohaghegh, Razi Gaskari, and Grant Bromhal. "Pattern Recognition and Data-Driven Analytics for Fast and Accurate Replication of Complex Numerical Reservoir Models at the Grid Block Level." SPE Intelligent Energy Conference \& Exhibition. Utrecht, The Netherlands: Society of Petroleum Engineers, 2014.

—. "Uncertainty Analysis of a CO2 Sequestration Project Using Surrogate Reservoir Modeling Technique." SPE Western Regional Meeting,. Bakersfield, California, USA: Society of Petroleum Engineers, 2012.

—. "Uncertainty Analysis of a CO2 Sequestration Project Using Surrogate Reservoir Modeling Technique." SPE Western Regional Meeting,. Bakersfield, California: Society of Petroleum Engineers, 2012.

Aminzadeh, Fred, and Paul deGroot. "A Neural Networks Based Seismic Object Detection Technique." SEG Annual Meeting. Houston, Texas, 2005.

Amsallem, David, and Charbel Farhat. "Interpolation method for adapting reduced-order models and application to aeroelasticity." AIAA Journal, 2008: 1803-1813.

Anterion, F., R. Eymard, and B Karcher. "Use of Parameter Gradients for Reservoir History Matching." Symposium on Reservoir Simulation. Houston,Texas, USA: Society of Petroleum Engineers, 1989.

Arps, J.J. "Analysis of Decline Curves." Transactions of the AIME (Society of Petroleum Engineers) 160, no. 01 (1945): 228 - 247.

Arunachalam, Vasan. "Water Resources Research Report - Optimization Using Differential Evolution." The University of Western Ontario Department of Civil and Environmental Engineering, 2008.

Arwini, Saleh, and Karl D. Stephen. "Combining Experimental Design with Proxy Derived Sensitivities to Improve Convergence Rates in Seismic History Matching." SPE EUROPEC/EAGE Annual Conference and Exhibition. Vienna, Austria: Society of Petroleum Engineers, 2011.

Athichanagorn, S, and R N Horne. "Automatic Parameter Estimation From Well Test Data Using Artificial Neural Network." SPE Annual Technical Conference and Exhibition. Dallas, Texas: Society of Petroleum Engineers, 1995.

B. Baesens, R. Setiono, C. Mues, and J Vanthienen. "Using Neural Network Rule Extraction and Decision Tables for Credit-Risk Evaluation." Management Science, 2003: 312329. 
Babu, B.V., and K.K.N. Sastry. "Estimation of heat transfer parameters in a trickle-bed reactor using differential evolution and orthogonal collocation." Computers and Chemical Engineering, 1999: 327-339.

Baker, Richard. "Streamline Technology: Reservoir History Matching and Forecasting = Its Success, Limitations, and Future." Journal of Canadian Petroleum Technology 40, no. 4 (2001): 23-27.

Baldwin, J L, D N Otte, and C L Whealtley. "Computer Emulation of Human Mental Processes: Application of Neural Network Simulators to Problems in Well Log Interpretation." SPE Annual Technical Conference and Exhibition. San Antonio, Texas, 1989.

Ballester, P., and J Carter. "A Parallel Real-Coded Genetic Algorithm for History Matching and Its Application to a Real Petroleum Reservoir." Journal of Petroleum Science \& Engineering 59 (2007): 157-168.

Barker, J.W., M. Cuypers, and L Holden. "Quantifying Uncertainty in Production Forecasts: Another Look at the PUNQ-S3 Problem." SPE Journal 6, no. 4 (2001): 433-441.

Batycky, Roderick Panko, Andrew C. Seto, and Darryl Hyde Fenwick. "Assisted History Matching of a 1.4-Million-Cell Simulation Model for Judy Creek--A Pool Waterflood/HCMF Using a Streamline-Based Workflow." SPE Annual Technical Conference and Exhibition. Anaheim, California: Society of Petroleum Engineers, 2007.

Bazargan, Hamid, and Mike Christie. "Efficient Polynomial Chaos Proxy-based History Matching and Uncertainty Quantification for Complex Geological Structures." SPE Kuwait International Petroleum Conference and Exhibition. Kuwait City, Kuwait: Society of Petroleum Engineers, 2012.

Bazargan, Hamid, Mike Christie, and Hamdi Tchelepi. "Efficient Markov Chain Monte Carlo Sampling Using Polynomial Chaos Expansion." SPE Reservoir Simulation Symposium. The Woodlands, Texas: Society of Petroleum Engineers, 2013.

Boss, C. Production forecasting with UNcertainty Quantification. Netherlands Institute of Applied Geoscience TNO, 1999.

Box, G. E. P., and K.B. Wilson. "On the Experimental Attainment of Optimum Conditions (with discussion)." Journal of the Royal Statistical Society Series B 13, no. 1 (1951): $1-45$.

Briones, M F, G.A. Rojas, J.A. Moreno, and E R Martinez. "Application of Neural Networks in the Prediction of Reservoir Hydrocarbon Mixture Composition From Production Data." SPE Annual Technical Conference and Exhibition. New Orleans, Louisiana: Society of Petroleum Engineers, 1994. 
Bush, M.D., and J.N. Carter. "Application of a Modified Genetic Algorithm to Parameter Estimation in Petroleum Industry." Intelligent Engineering Systems through Artificial Neural Networks, 1996: volume 6, P 397.

Cardoso, Marco A. "Reduced-Order Models for Reservoir Simulation." SPE Annual Technical Conference and Exhibition. New Orleans, Louisiana: Society of Petroleum Engineers, 2009.

Cardoso, Marco A., and Louis J. Durlofsky. "Use of Reduced-Order Modeling Procedures for Production Optimization." SPE Journal, 2010: 426 - 435.

Carreras, P.E.,Turner, S.E. and Wilkinson, G.T. "Tahiti: Development Strategy Assessment Using Design of Experiments and Response Surface Methods." SPE Western Regional/AAPG Pacific Section/GSA Cordilleran Section Joint Meeting. Alaska, 2006.

Castellini, A. "Quantifying Uncertainty in Production Forecast for Fields with Significant History: A West African Case Study." International Petroleum Technology Conference. Doha, Qatar, 2005.

Castellini, A., B. Yeten, U. Singh, A. Vahedi, and R Sawiris. "History Matching and Uncertainty Quantification Assisted by Global Optimization Techniques." 10th European Conference on the Mathematics of Oil Recovery. Amsterdam, The Netherlands, 2006.

Černý, V. "Thermodynamical approach to the traveling salesman problem: An efficient simulation algorithm." Journal of Optimization Theory and Applications 45 (1985): $41-51$.

Chen, W.H., G.R. Gavalas, J. H. Seinfeld, and M. L. Wasserman. "A New Algorithm for Automatic History Matching." Society of Petroleum Engineers Journal (Society of Petroleum Engineers) 14, no. 06 (1974): 593 - 608.

Chen, Yan, and Dean S. Oliver. "Ensemble-Based Closed-Loop Optimization Applied to Brugge Field." SPE Reservoir Evaluation \& Engineering (Society of Petroleum Engineers) 13, no. 01 (2010): 56 - 71.

Cheng, Hao, Kaveh Dehghani, and Travis C. Billiter. "A Structured Approach for Probabilistic-Assisted History Matching Using Evolutionary Algorithms: Tengiz Field Applications." SPE Annual Technical Conference and Exhibition. Denver, Colorado: Society of Petroleum Engineers, 2008.

Cheng, Yueming, W. John Lee, and Duane A. McVay. "Improving Reserves Estimates From Decline-Curve Analysis of Tight and Multilayer Gas Wells." Society of Petroleum Engineers 11, no. 05 (2008): 912 - 920.

Cheng-Dang, Zhou, Shi-Ling Wu, Zhang Chao Mo, De Hai Zhu, and Zhang Van Xu. "Direct Identification of Hydrocarbon From Well Logs: A Neural Network Interpretation 
Approach." Annual Technical Meeting. Calgary, Alberta, Canada: Petroleum Society of Canada, 1994.

Cheong, Yaw Peng, and Ritu Gupta. "Experimental Design and Analysis Methods for Assessing Volumetric Uncertainties." SPE Journal (Society of Petroleum Engineers) 10, no. 03 (2005): 324 - 335.

Christie, M., C. MacBeth, and S. Subbey. "Multiple History-Matched Models for Teal South." The Leading Edge 21, no. 3 (2002): 286-289.

CMG, Computer Modelling Group. "Computer Modelling Group Manual." 2013.

Coats, K., J. Dempsey, and J. Henderson. "A New Technique for Determining Reservoir Description from Field Performance Data." Society of Petroleum Engineers Journal, 1970: 66 - 74 .

Cosentino, Luca. Integrated Reservoir Studies. Paris: Institut Français du Pétrole Publications, 2001.

D.H.F. Yip, E.L. Hines, and W.W.H. Yu. "Application of artificial neural networks in sales forecasting." International Conference on Neural Networks. Houston, TX, 1997. 2121 $-2124$.

Damsleth, E., A. Hage, and R Volden. "Maximum Information at Minimum Cost: Development Study With an Experimental Design." Journal of Petroleum Technology, 1992: 1350-1356.

Das, S, and P Suganthan. "Differential evolution: a survey of the state-of-the-art." IEEE Trans Evol Comput 15, no. 1 (2009): 4-31.

David, H. J. "Seismic Attribute Calibration Using Neural Networks." SEG Annual Meeting. Washington, DC: Society of Exploration Geophysicists, 1993.

Decker, J.B., and M. Mauldon. "Determining Size and Shape of Fractures from Trace Data using a Differential Evolution Algorithm." The 41st U.S. Symposium on Rock Mechanics (USRMS): "50 Years of Rock Mechanics - Landmarks and Future Challenges.". Golden, Colorado: American Rock Mechanics Association, 2006.

Dougherty, E.L., and D. Khairkhah. "History Matching of Gas Simulation Models Using Optimal Control Theory." SPE California Regional Meeting. Ventura, California: Society of Petroleum Engineers, 1975.

Eberhart, R.C., and Yuhui Shi. "Particle swarm optimization: developments, applications and resources." Proceedings of the 2001 Congress on on Evolutionary Computation. Seoul, South Korea, 2001. 81-86.

Eiben, A.E., and J.E. Smith. Introduction to Evolutionary Computing. Springer, Natural Computing Series, 2003. 
Eide, A., L. Holden, E. Reiso, and S Aanonsen. "Automatic History Matching by Use of Response Surfaces and Experimental Design." 4th European Conference on the Mathematics of Oil Recovery. Roros, Norway, 1994.

Emanuel, Alan S., and William J. Milliken. "History Matching Finite Difference Models With 3D Streamlines." SPE Annual Technical Conference and Exhibition. New Orleans, Louisiana: Society of Petroleum Engineers, 1998.

Erbas, D., and M. Christie. "Effect of Sampling Strategies on Prediction Uncertainty Estimation." SPE Reservoir Simulation Symposium. Houston, Texas, 2007.

Ershaghi, Iaj, Xuehai Li, Mahnaz Hassibi, and Yusuf Shikari. "A Robust Neural Network Model for Pattern Recognition of Pressure Transient Test Data." SPE Annual Technical Conference and Exhibition. Houston, Texas: Society of Petroleum Engineers, 1993.

Ertekin, T, J. H. Abou-Kassem, and G. R. King. "Basic Applied Reservoir Simulation." In Basic Applied Reservoir Simulation, by Turgay Ertekin, Jamal H. Abou-Kassem and Gregory R. King. Richardson, Texas: Society of Petroleum Engineers, 2001.

Erwig, M. "The graph Voronoi diagram with applications." s.l. : John Wiley \& Soncs, Inc., 2000.

Evensen, G. "The ensemble Kalman filter: Theoretical formulation and practical implementation." Ocean Dynamics, 2003.

F. Amato, A. López, E. María Peña-Méndez, P. Vaňhara, and A. Hampl. "Artificial neural networks in medical diagnosis." Journal of Applied Biomedicine, 2013: 47-58.

Fanchi, John R. Principles of Applied Reservoir Simulation. Publisher: Elsevier Science and Technology Books, Inc, 2006.

Fasanino, G., E. Molinard, G. Marsily, and V Pelce. "Inverse Modeling in Gas Reservoirs." SPE Annual Technical Conference and Exhibition. Society of Petroleum Engineers, 1986.

Fausett, L. V. Fundamentals of Neural Networks: Architectures, Algorithms And Applications. Prentice Hall, 1993.

Fenwick, Darryl Hyde, Marco Roberto Thiele, Mohammed Alawi Agil, Ahmed Hussain, Fahad A. Al-Humam, and Jef Karel Caers. "Reconciling Prior Geologic Information With Production Data Using Streamlines: Application to a Giant Middle-Eastern Oil Field." SPE Annual Technical Conference and Exhibition. Dallas, Texas: Society of Petroleum Engineers, 2005.

Ferrante, N, and T Ville. "Recent advances in differential evolution: a survey and experimental analysis." Artif Intell Rev 33, no. 1-2 (2010): 61-106. 
Fetkovich, M.J. "Decline Curve Analysis Using Type Curves." Journal of Petroleum Technology (Society of Petroleum Engineers) 32, no. 06 (1980): 1,065 - 1,077.

Fetkovich, M.J., M.E. Vienot, M.D. Bradley, and U.G. Kiesow. "Decline Curve Analysis Using Type Curves: Case Histories." (Society of Petroleum Engineers) 2, no. 04 (1987): 637 - 656.

Floris, F.J.T., M.D. Bush, M. Cuypers, F. Roggero, and A Syversveen. "Methods for Quantifying the Uncertainty of Production Forecasts." Petroleum Geoscience 7, no. 10 (2001): 87-96.

Forrester, Alexander I.J., and Andy J. Keane. "Recent advances in surrogate-based optimization." Progress in Aerospace Sciences 45, no. 1-3 (2009): 50-79.

Friedmann, F., A. Chawathe, and D.K. Larue. "Assessing Uncertainty in Channelized Reservoirs Using Experimental Designs." SPE Reservoir Evaluation \& Engineering (Society of Petroleum Engineers) 6, no. 04 (2003): 264 - 274.

G.Glan Devadhas, S. Pushpakumar, and D.M.Mary Synthia Regis Prabha. "ANN Based MARC Controller Design for an Industrial Chemical Process." International Conference on Computing, Electronics and Electrical Technologies. 2012.

Gao, G., M. Zafari, and C.A Reynolds. "Quantifying Uncertainty for the PUNQ-S3 Problem in a Bayesian Setting with RML and EnKF." Reservoir Simulation Symposium. Houston, Texas: Society of Petroleum Engineers, 2005.

Gavalas, G.R., P.C. Shah, and J.H. Seinfeld. "Reservoir History Matching by Bayesian Estimation." Society of Petroleum Engineers Journal 16, no. 06 (1976): 337 - 350.

Gharbi, R.B., and A.M., Elsharkawy. "Neural Network Model for Estimating The PVT Properties of Middle East Crude Oils." Middle East Oil Show and Conference. Bahrain, 1997.

Gildin, Eduardo, Ashraf Ibrahim, and Mohammadreza Ghasemi. "Reduced Order Modeling in Reservoir Simulation Using the Bilinear Approximation Techniques." SPE Latin America and Caribbean Petroleum Engineering Conference. Maracaibo, Venezuela: Society of Petroleum Engineers, 2014.

Gomez, Yorgi, Yasaman Khazaeni, Shahab D. Mohaghegh, and Razi Gaskari. "Top Down Intelligent Reservoir Modeling." SPE Annual Technical Conference and Exhibition. New Orleans, Louisiana: Society of Petroleum Engineers, 2009.

Gómez-Hernánez, J.J., A Sahuquillo, and J Capilla. "Stochastic simulation of transmissivity fields conditional to both transmissivity and piezometric data-I. Theory." Journal of Hydrology 203 (1997): 162-174.

Goodwin, Nigel, and Mark Powell. "Simulation and Uncertainty: Lessons From Other Industries." The Way Ahead, 2012: 20-21. 
Gu, Y., and D. Oliver. "History Matching of the PUNQ-S3 Reservoir Model Using the Ensemble Kalman Filter." SPE Journal 10, no. 2 (2005): 217-224.

Gupta, Ritu, Roger Collinson, Gregory Charles Smith, Scott Andrew Ryan, and Jai Pragash Louis. "History Matching Of Field Production Using Design Of Experiments." SPE Asia Pacific Oil and Gas Conference and Exhibition. Perth, Australia: Society of Petroleum Engineers, 2008.

Hagan, M.T., H.B. Demuth, and M.H. Beale. Neural Network Design first edition. Boston, MA, USA.: PWS Publishing Co, 1996.

Hajizadeh, Y, M Christie, and V Demyanov. "Application of Differential Evolution as a New Method for Automatic History Matching." 2009 Kuwait International Petroleum Conference and Exhibition. Kuwait City, Kuwait: Society of Petroleum Engineers, 2009.

Hajizadeh, Y. "Ants Can Do History Matching." SPE Annual Technical Conference and Exhibition. Florence, Italy: Society of Petroleum Engineers, 2010.

Hajizadeh, Y., M. Christie, and V. Demyanov. "Ant Colony Optimization for History Matching." SPE EUROPEC/EAGE Annual Conference and Exhibition. Amsterdam, The Netherlands: Society of Petroleum Engineers, 2009.

—. "History Matching with Differential Evolution Approach; a Look at New Search Strategies." SPE EUROPEC/EAGE Annual Conference and Exhibition. Barcelona, Spain: Society of Petroleum Engineers, 2010.

Hanea, Remus Gabriel, Justyna Katarzyna Przybysz-Jarnut, Mariya Krymskaya, Arnold Heemink, and Feb Dirk Jansen. "The Choice Of The "Best" Assisted Hisotry Matching Algorithm For Subsurface Characterization In Reservoir Engineering." SPE EUROPEC/EAGE Annual Conference and Exhibition. Barcelona, Spain: Society of Petroleum Engineers, 2010.

Haugen, Vibeke Eilen Jensen, Lars-Jorgen Natvik, Geir Evensen, Aina Margrethe Berg, Kristin Margrethe Flornes, and Geir Naevdal. "History Matching Using the Ensemble Kalman Filter on a North Sea Field Case." SPE Annual Technical Conference and Exhibition. San Antonio, Texas, USA: Society of Petroleum Engineers, 2006.

Haukas, Jarle, Ivar Aavatsmark, and Magne Espedal. "A Black-Oil and Compositional Impsat Simulator with Improved Compositional Convergence." 9th European Conference on the Mathematics of Oil Recovery. Cannes, France, 2004.

Haykin, Simon. Neural Networks and Learning Machines. Prentice Hall, 2008.

He, Jincong, Pallav Sarma, and Louis J. Durlofsky. "Use of Reduced-order Models for Improved Data Assimilation within an EnKF Context." SPE Reservoir Simulation Symposium. The Woodlands, Texas, USA: Society of Petroleum Engineers, 2011.

Hurst, William. "The Material Balance Equation." Society of Petroleum Engineers, 1974. 
Ilk, Dilhan, David M. Anderson, Garth W.J. Stotts, Louis Mattar, and Thomas Blasingame. "Production Data Analysis--Challenges, Pitfalls, Diagnostics." (Society of Petroleum Engineers) 13, no. 03 (2010): 538 - 552.

"Intelligent Solutions, Inc." Intelligent Solutions, Inc. 2014.

http://www.intelligentsolutionsinc.com/products.htm (accessed 03 01, 2014).

J.C. Helton, and F.J. Davis. Latin Hypercube Sampling and the Propagation of Uncertainty in Analyses of Complex Systems. Sandia report. New Mexico: Sandia National Laboratories, 2002.

Jacquard, P. "Theory of the interpretation of pressure measurements." Revue IFP 19, no. 3 (March 1964): 297-338.

Jacquard, P., and C. Jain. "Permeability Distribution From Field Pressure Data." SPE, 1965: 281-294.

Jahangiri, H. R. "Production Optimization using Smart Well Technology with Differential Evolution Algorithm." Graduate Student Symposium, University of Southern California. 2007.

Jahns, H.O. "A Rapid Method for Obtaining a Two-Dimensional Reservoir Description From Well Pressure Response Data." SPE, 1966: 315-327.

Jain, Anil K. "Data Clustering: 50 Years Beyond K-Means." Pattern Recognition Letters, 2009.

Jalali, Jalal, and Shahab D. Mohaghegh. "Reservoir Simulation and Uncertainty Analysis of Enhanced CBM Production Using Artificial Neural Networks." SPE Eastern Regional Meeting. Charleston, West Virginia: Society of Petroleum Engineers, 2009.

Janez, B, B Borko, G Saso, and et al. "erformance comparison of self-adaptive and adaptive differential evolution algorithms." Soft Comput 11, no. 7 (2007): 617-629.

Jansen, Jan-Dirk, Roald Brouwer, and Sippe G. Douma. "Closed Loop Reservoir Management." SPE Reservoir Simulation Symposium. Woodlands, Texas: Society of Petroleum Engineers, 2009.

Jong-Se, L, and K. Jungwhan. "Reservoir Porosity and Permeability Estimation from Well Logs using Fuzzy Logic and Neural Networks." SPE Asia Pacific Oil and Gas Conference and Exhibition. Perth, Australia, 2004.

Kaelo, P, and MM Ali. "A numerical study of some modified differential evolution algorithms." Eur J Oper 169, no. 3 (2006): 108-132.

Kalam, M.Z., S.M. Al-Alawi, and M. Al-Mukheini. "Assessment of Formation Damage Using Artificial Neural Networks." SPE Formation Damage Control Symposium. Lafayette, Louisiana, 1996. 
Kathrada, M. Uncertainty Evaluation of Reservoir Simulation Models using Particle Swarms and Hierarchical Clustering. Edinburgh: PhD thesis, Heriot Watt University, 2009.

Key, S.C., H.H. Nielsen, Claude Signer, Lars Sønneland, Ketil Waagbø, and Helene H. Veire. "Fault And Fracture Classification Using Artifical Neural Networks - Case Study From the Ekofisk Field." SEG Annual Meeting. Dallas, Texas, 1997.

Kirkpatrick, S., C. D. Gelatt Jr, and M. P. Vecchi. "Optimization by Simulated Annealing." Science 220, no. 4598 (1983): 671-680.

Kriesel, David. A Brief Introduction to Neural Networks. dkriesel.com, 2011.

Krose, Ben, and Patrick van der Smagt. An introduction to neural networks. Amsterdam, The netherlands: The University of Amsterdam, 1996.

Kruger, W. D. "Determining Areal Permeability Distribution by Calculations." J. Pet. Tech. $691,1961$.

Kruger, W.D. "Determining Areal Permeability Distribution by Calculations." 35th Annual Fall Meeting. Denver: SPE, 1960.

Kumar, Koushal, and Gour Sundar Mitra Thakur. "Advanced Applications of Neural Networks and Artificial Intelligence: A Review." International Journal of Information Technology and Computer Science(IJITCS), 2012: 57-68.

L.Thomas, Andrew, and Paul R. La Pointe. "Conductive fracture identification using neural networks." The 35th U.S. Symposium on Rock Mechanics (USRMS). Reno, NV, 1995.

Landa, J. L., and B. Güyagüler. "A Methodology for History Matching and the Assessment of Uncertainties Associated with Flow Prediction." SPE Annual Technical Conference and Exhibition. Denver, Colorado: Society of Petroleum Engineers, 2003.

Li, B.,Friedmann,F. "A Novel Response Surface Methodology Based on "Amplitude Factor" Analysis for Modeling Nonlinear Responses Caused by Both Reservoir and Controllable Factors." SPE Annual Technical Conference and Exhibition. Dallas, Texas, 2005.

Li, Eldon Y. "Artificial neural networks and their business applications." Information \& Management, 1994: 303-313.

Li, Heng, and Daoyong Tony Yang. "Estimation of Multiple Petrophysical Parameters for the PUNQ-S3 Model Using Ensemble-Based History Matching." SPE EUROPEC/EAGE Annual Conference and Exhibition. Vienna, Austria: Society of Petroleum Engineers, 2011.

Li, Kewen, and Roland N. Horne. "A Decline Curve Analysis Model Based on Fluid Flow Mechanisms." SPE Western Regional/AAPG Pacific Section Joint Meeting. Long Beach, California: Society of Petroleum Engineers, 2003. 
Liu, J, and J Lampinen. "A fuzzy adaptive differential evolution algorithm." 2002 IEEE Region 10 Conference on Computers, Communications, Control and Power. 2002. 606-611.

Liu, J, and J Lampinen. "A fuzzy adaptive differential evolution algorithm." Soft Comput 9, no. 6 (2005): $448-462$.

M. Chattopadhyay, P. K. Dan, S. Majumdar, and P. S.Chakraborty. "Application of artificial neural network in market segmentation: A review on recent trends." Management Science Letters , 2012: 425-438.

M. Yalcintas, S. Akkurt. "Artificial neural networks applications in building energy predictions and a case study for tropical climates." INTERNATIONAL JOURNAL OF ENERGY RESEARCH, 2005: 891-901.

Maciejewski, L, W Myszka, and G Ziętek. "Application of differential evolution algorithm for identification of experimental data." The Archive of Mechanical Engineering, 2007: 327-337.

Madavan, N.K. "On Improving Efficiency of Differential Evolution for Aerodynamic Shape Optimization Applications." 10th AIAA/ISSMO Multidisciplinary Analysis and Optimization Conference. Albany, NY, 2004.

Manceau, E., M. Mezghani, I. Zabala, and F. Roggero. "Combination of Experimental Design and Joint Modeling Methods for Quantifying the Risk Associated with Deterministic and Stochastic Uncertainties - An Integrated Test Study." Annual Technical Conference and Exhibition. New Orleans, Louisiana: Society of Petroleum Engineers, 2001.

Manceau, E., M. Mezghani, I. Zabalza-Mezghani, and F. Roggero. "Combination of Experimental Design and Joint Modeling Methods for Quantifying the Risk Associated With Deterministic and Stochastic Uncertainties - An Integrated Test Study." SPE Annual Technical Conference and Exhibition. New Orleans, Louisiana: Society of Petroleum Engineers, 2001.

Mantica, S. Cominelli, A. Mantica, G. "Combining Global and Local Optimization Techniques for Automatic History Matching Production and Seismic Data." SPE Journal, 2002: 123-130.

Marsily, G., G. Lavedan, M. Boucher, and G Fasanino. "Interpretation of Inference Tests in a Well Field Using Geostatistical Techniques to Fit the Permeability Distribution in a Reservoir Model." Geostatistics for Natural Resources Characterization, Part 2 (Reidel Publishing Company), 1987: 831-849.

Masoud, N. "Neural Network Knowledge-Based Modeling of Rock Properties Based on Well Log Databases." SPE Western Regional Meeting. Bakersfield, California, 1998. 
Mattax, Calvin C, and Robert L. Dalton. Reservoir Simulation. Richardson, TX: Society of Petroleum Engineer, 1990.

Maucec, M. Dourma, S. Hohl, D, and Leguijt, J. "Streamline-based history matching and uncertainty, Markov-chain Monte Carlo study of an offshore oil field." SPE Annual Technical Conference and Exhibition. Anaheim, California, 2007.

Merriam Webster Dictionery Online. 2014. http://www.merriam-webster.com/ (accessed 05 01, 2014).

Mirzabozorg, A., L. Nghiem, Z. Chen, and C. Yang. "Differential Evolution for Assisted History Matching Process: SAGD Case Study." SPE Heavy Oil Conference Canada. Calgary, Alberta, Canada: Society of Petroleum Engineers, 2013.

Mohaghegh, Shahab D. "Artificial Intelligence and Data Mining: Enabling Technology for Smart Fields." SPE's The Way Ahead Journal, 2009: 14-19.

Mohaghegh, Shahab D. "Converting detail reservoir simulation models intoeffective reservoir management tools using SRMs;case study - three green fields in Saudi Arabia." Int. J. Oil, Gas and Coal Technology 7, no. 2 (2014): 115 - 131.

Mohaghegh, Shahab D. "Neural Network: What It Can Do for Petroleum Engineers." Journal of Petroleum Technology, 1995.

—. "Reservoir Simulation and Modeling Based on Pattern Recognition." SPE Digital Energy Conference and Exhibition. Woodlands, Texas, 2011.

Mohaghegh, Shahab D. "Virtual intelligence applications in petroleum engineering: part 3 fuzzy logic." Journal of Petroleum Technology, 2000: 82-87.

Mohaghegh, Shahab D., Cyrus Abdallah Modavi, Hafez H. Hafez, Masoud Haajizadeh, Maher Mahmoud Kenawy, and Srikanth Guruswamy. "Development of Surrogate Reservoir Model (SRM) for Fast Track Analysis of a Complex Reservoir." International Journal of Oil, Gas and Coal Technology, 2009: 2-23.

—. "Development of Surrogate Reservoir Models (SRM) For Fast Track Analysis of Complex Reservoirs." Intelligent Energy Conference and Exhibition. Amsterdam, The Netherlands: Society of Petroleum Engineers, 2006.

Mohaghegh, Shahab D., Jim S. Liu, Razi Gaskari, Mohammad Maysami, and Olugbenga A. Olukoko. "Application of Surrogate Reservoir Models (SRM) to an Onshore Green Field in Saudi Arabia; Case Study." North Africa Technical Conference and Exhibition. Cairo, Egypt: Society of Petroleum Engineers, 2012a.

—. "Application of Well-Base Surrogate Reservoir Models (SRMs) to Two Offshore Fields in Saudi Arabia, Case Study." SPE Western Regional Meeting. Bakersfield, California: Society of Petroleum Engineers, $2012 \mathrm{~b}$. 
Mohaghegh, Shahab, Reza Arefi, Samuel Ameri, and D. Rose. "Design and Development of An Artificial Neural Network for Estimation of Formation Permeability." SPE Computer Applications 7, no. 06 (1995): 151 - 154.

Mohamed, Lina, Michael A. Christie, and Vasily Demyanov. "Comparison of Stochastic Sampling Algorithms for Uncertainty Quantification." Reservoir Simulation Symposium. Woodlands, Texas: Society of Petroleum Engineers, 2009.

—. "Reservoir Model History Matching with Particle Swarms: Variants Study." SPE Oil and Gas India Conference and Exhibition. Mumbai, India: Society of Petroleum Engineers, 2010.

Nikravesh, M., A.R. Kovscek, R.M. Johnston, and T.W. Patzek. "Prediction of Formation Damage During Fluid Injection into Fractured, Low Permeability Reservoirs via Neural Networks." SPE Formation Damage Control Symposium. Lafayette, Louisiana: Society of Petroleum Engineers, 1996.

Ojo, K.P., and S.O. Osisanya. "Material Balance Revisited." Nigeria Annual International Conference and Exhibition. Abuja, Nigeria: Society of Petroleum Engineers, 2006.

Okano, H. "Reservoir Model History-Matching and Uncertainty Quantification in Reservoir Performance Forecast Using Bayesian Framework." SPE Reservoir Characterisation and Simulation Conference and Exhibition. Abu Dhabi, UAE: Society of Petroleum Engineers, 2013.

Oloso, Munirudeen A., Amar Khoukhi, Abdulazeez Abdulraheem, and Moustafa Elshafei. "Prediction of Crude Oil Viscosity and Gas/Oil Ratio Curves Using Recent Advances to Neural Networks." SPE/EAGE Reservoir Characterization and Simulation Conference. Abu Dhabi, UAE, 2009.

OnePetro. 2014. https://www.onepetro.org/ (accessed March 2014).

Osman, E A, O A Abdel-Wahhab, and M A Al-Marhoun. "Prediction of Oil PVT Properties Using Neural Networks." SPE Middle East Oil Show. Bahrain, 2001.

Ouenes, A., B. Brefort, G. Meunier, and S Dupere. "A New Algorithm for Automatic History Matching: Application of Simulated Annealing Method (SAM) to Reservoir Inverse Modeling." Society of Petroleum Engineers, 1993b.

Ouenes, A., G. Fasanino, and R Lee. "Simulated Annealing for Interpreting Gas/Water Laboratory." 67th Annual Technical Conference and Exhibition. Washington D.C.: Society of Petroleum Engineers, 1993a.

Ouenes, A., G. Meunier, and H Moegen. "Application of Simulated Annealing Method (SAM) to Gas Storage Reservoir Characterization." 96th Annual AIChE National Spring Meeting. New Orleans, Louisiana, 1992a. 
—. "Application of Simulated Annealing Method (SAM) to Gas Storage Reservoir Characterization." 96th Annual AIChE National Spring Meeting. New Orleans, Louisiana, 1992b.

Palatnic, B., L. Zakirov, S. Haugen, and J. van Roosmalen. "New Approaches to Multiple History Matching." Seventh European Symposium on Improved Oil Recovery. Moscow, Russia, 1993.

Petroleum Engineering \& Rock Mechanics Group (PERM). 2014. http://www3.imperial.ac.uk/earthscienceandengineering/research/perm/punqs3model/truthcase/procedure (accessed March 2014).

Price, K, and S Storn. "Differential evolution." Dr Dobbs J 22, no. 4 (1997): 18-24.

Price, K, R Storn, and J Lampinen. "Differential evolution: a practical approach to global optimization." Springer, 2005.

Pruess, K., O. Weres, R. Schroeder, R. Marconcini, and G Neri. "History Match Simulation of Serrazzano Geothermal Reservoir." 55th Annual Fall Technical Conference and Exhibition. Dallas, Texas: Society of Petroleum Engineers, 1980.

Qing, A. Differential Evolution: Fundamentals and Applications in Electrical Engineering. Wiley-IEEE Press, 2009.

Qing, A, and C. K. Lee. Differential Evolution in Electromagnetics (Google eBook). Springer, 2010.

Queipo, Nestor V., Raphael T. Haftka, Wei Shyy, Tushar Goel, Rajkumar Vaidyanathan, and P. Kevin Tucker. "Surrogate-based analysis and optimization." Progress in Aerospace Sciences 41, no. 1 (2005): 1-28.

R. O. Duda, P. E. Hart, and D. G. Stork. Pattern Classification, 2nd Editixon. Wiley, John \& Sons, Incorporated, 2001.

RamaRao, B.S., A.M. LaVenue, G.D. Marsily, and M.G. Marietta. "Pilot Point Methodology for Automated Calibration of an Ensemble of conditionally Simulated Transmissivity Fields: 1. Theory and Computational Experiments." Water Resources Research ( American Geophysical Union) 31, no. 3 (March 1995): 475-493.

Razavi, F., and F Jalai-Farahani. "Ant Colony Optimization: A Leading Algorithm in Future Optimization of Petroleum Engineering Processes." 9th International Conference on Artificial Intelligence and Soft Computing. Zakopane, Poland, 2008.

Roberts, Eric. "Image form this webpage Neural Networks." 2000. http://cs.stanford.edu/people/eroberts/courses/soco/projects/neuralnetworks/index.html (accessed 05 01, 2014).

Rojas, Raul, and J. Feldman. Neural Networks: A Systematic Introduction. Springer, 1996. 
Romero, C., J. Carter, A. Gringarten, and R Zimmerman. "A Modified Genetic Algorithm for Reservoir Characterization." International Oil and Gas Conference and Exhibition. Beijing, China: Society of Petroleum Engineers, $2000 \mathrm{~b}$.

Romero, C., J. Carter, R. Zimmerman, and A Gringarten. "Improved Reservoir Characterization Through Evolutionary Computation." Annual Technical Conference and Exhibition. Dallas, Texas: Society of Petroleum Engineers, 2000a.

Romeu, Régis Kruel. "Advanced Reservoir-Management Workflow EnKF-Based AssistedHistory-Matching Method." JPT, 2010: 81-88.

Roth, Gunter, and Albert Tarantoia. "Inversion of Seismic Waveforms Using Neural Networks." SEG Annual Meeting. New Orleans, Louisiana: Society of Exploration Geophysicists, 1992.

Rotondi, Marco, Giovanna Nicotra, Antonella Godi, F. Michela Contento, Martin Julian Blunt, and Mike Christie. "Hydrocarbon Production Forecast and Uncertainty Quantification: A Field Application." SPE Annual Technical Conference and Exhibition. San Antonio, Texas, USA: Society of Petroleum Engineers, 2006.

Rwechungura, Richard Wilfred, Mohsen Dadashpour, and Jon Kleppe. "Advanced History Matching Techniques Reviewed." SPE Middle East Oil and Gas Show and Conference. Manama, Bahrain: Society of Petroleum Engineers, 2011.

—. "Application of Particle Swarm Optimization for Parameter Estimation Integrating Production and Time Lapse Seismic Data." Offshore Europe. Aberdeen, UK: Society of Petroleum Engineers, 2011.

S. Aless, R. Sarcià , G. Cantone, and V. R. Basili. "A STATISTICAL NEURAL NETWORK FRAMEWORK FOR RISK MANAGEMENT PROCESS From the Proposal to its Preliminary Validation for Efficiency." ISOFT, 2007: 168-177.

S. Lei and W. Xing-cheng. "Artificial Neural Networks:Current Applications in Modern Medicine." International Conference on Computer and Communication Technologies in Agriculture Engineering. 2010.

Sadiq, T., and I.S. Nashawi. "Using Neural Networks for Prediction of Formation Fracture Gradie." SPE/CIM International Conference on Horizontal Well Technology. Calgary, Alberta, Canada, 2000.

Saleri, Nansen G. "Reservoir Performance Forecasting: Acceleration by Parallel Planning." Journal of Petroleum Technology 45, no. 07 (1993): 652 - 657.

Salhi, M.A., Van rijen, M. "Structured Uncertainty Assessment for Fahud Field through the Application of Experimental Design and Response Surface Methods." SPE Middle East Oil and Gas Show and Conference. Kingdom of Bahrain, 2005.

Schlumberger. "ECLIPSE Reservoir Simulator." 2014. 
Schulze-Riegert, R., J. Axmann, O. Haase, D. Rian, and Y You. "Optimization Methods for History Matching of Complex Reservoirs." Reservoir Simulation Symposium. Houston, Texas, USA: Society of Petroleum Engineers, 2001.

Sen, M., A. Datta-Gupta, P. Stoffa, L. Lake, and G Pope. "Stochastic Reservoir Modeling Using Simulated Annealing and Genetic Algorithms." SPE Formation Evaluation 10, no. 1 (1995): 49-55.

Shahkarami, Alireza, Shahab D. Mohaghegh, Vida Gholami, Alireza Haghighat, and Daniel Moreno. "Modeling pressure and saturation distribution in a $\mathrm{CO} 2$ storage project using a Surrogate Reservoir Model (SRM)." Greenhouse Gas Sci Technol (Society of Chemical Industry and John Wiley \& Sons, Ltd) 04 (2014): 289-315.

Shahryar, R, RT Hamid, and MAS Magdy. "Opposition-based differential evolution." IEEE Trans Evol Comput 12, no. 1 (2008): 64-79.

Singh, Virendra, Pramod Kumar Painuly, Anil Kumar Srivastava, Devendra Nath Tiwary, and Mahesh Chandra. "Neural Networks And Their Applications In Lithostratigraphic Interpretation of Seismic Data For Reservoir Characterization." World Petroleum Congress. Madrid, Spain, 2008.

Slater, G., and E. Durrer. "Adjustment of Reservoir Simulation Models to Match Field Performance." Society of Petroleum Engineers Journal (Society of Petroleum Engineers), 1971: 295 - 305.

Soleng, H. "Oil Reservoir Production Forecasting with Uncertainty Estimation Using Genetic Algorithms." IEEE Evolutionary Computation Conference (CEC). Washington D.C., 1999.

Solorzano, L. N., S. E. Arredondo, and E. Sergio. "Method for Automatic History Matching of Reservoir Simulation Models." Fall Meeting of the Society of Petroleum Engineers of AIME. Las Vegas, Nevada: Society of Petroleum Engineers, 1973.

Sousa, S. "Scatter search metaheuristic applied to the history matching problem." SPE Annual Technical Conference and Exhibition. Anaheim, California, U.S.A., 2007.

Stephen, K.D., and S. Arwini. "Improving Stochastic Inversion Methods in History Matching Using Proxy Models." 12th European Conference on the Mathematics of Oil Recovery . Oxford, United Kingdom, 2010.

Stephen, Karl D., Juan Soldo, Colin Macbeth, and Mike A. Christie. "Multiple Model Seismic and Production History Matching: A Case Study." SPE Journal (Society of Petroleum Engineers) 11, no. 04 (2006): 418 - 430.

Storn, R, and K Price. "Differential evolution - a simple and efficient heuristic for global optimization over." J Glob Optim 11, no. 4 (1997): 341-359.

Storn, R. "Differential evolution design of an IIR-filter with requirements of magnitude and group delay." IEEE Conference on Evolutionary Computation. 1996. 268-273. 
Storn, R. "System Design by Constraint Adaptation and Differential Evolution." IEEE Trans. on Evolutionary Computation 3, no. 1 (1999): 22-34.

Storn, R., and K Price. "Differential Evolution - A Simple and Efficient Adaptive Scheme for Global Optimization over Continuous Spaces." Technical Report for International Computer Science Institute, Berkeley, 1995.

Subbey, S., and M. Christie. "A Strategy for Rapid Quantification of Uncertainty in Reservoir Performance Prediction." SPE Reservoir Simulation Symposium. Houston, Texas, 2003.

Sultanp, Mir Asif, and Abdulaziz U. Al-Kaabi. "Application of Neural Network to the Determination of Well-Test Interpretation Model for Horizontal Wells." SPE Asia Pacific Oil and Gas Conference and Exhibition. Melbourne, Australia, 2002.

Suribabu, C.R. "Differential Evolution Algorithm for Optimal Design of Water Distribution Networks." Journal of Hydroinformatics 12, no. 1 (2010): 66-82.

Swagatam, D, A Ajith, KC Uday, and et al. "Differential evolution using a neighborhoodbased mutation operator." IEEE Trans Evol Comput 13, no. 3 (2009): 526-553.

Szklarz, Slawomir, Remus Gabriel Hanea, and Elisabeth Peters. "A Case Study of the History Matching Of A Sector Of The Norne Field Using the Ensemble Kalman Filter." SPE EUROPEC/EAGE Annual Conference and Exhibition. Vienna, Austria: Society of Petroleum Engineers, 2011.

Tan, C. N. W. An Artificial Neural Networks Primer with Financial Applications Examples in Financial Distress Predictions and Foreign Exchange Hybrid Trading System. Published in internet (http://www.smartquant.com/references/NeuralNetworks/neural28.pdf), 2004.

Thiele, M.R., R.P. Batycky, and D.H. Fenwick. "Streamline Simulation for Modern ReservoirEngineering Workflows." Journal of Petroleum Technology 62, no. 01 (2010): 64-70.

Thomas, P, and D. Vernon. "Image registration by differential evolution." Irish Machine Vision and Image Processing Conference. 1997. 221-225.

Tokuda, N, S Takahashi, M Watanabe, and T Kurose. "Application of Genetic Algorithm to history matching for core flooding." SPE Asia Pacific Oil and Gas Conference and Exhibition. Perth, Australia: Society of Petroleum Engineers, 2004.

Toronyi, RM, and NG Saleri. "Engineering control on reservoir engineering, Part 2." Society of Petroleum Engineers, 1988.

Tyler, K.J., Tarald Svanes, and Sturle Omdal. "Faster History Matching and Uncertainty in Predicted Production Profiles With Stochastic Modeling." 68th Annual Technical Conference and Exhibition of the Society of Petroleum Engineers. Houston: Society of Petroleum Engineers, 1993. 
Vakili-Ghahani, S. Ali, and Jan-Dirk Jansen. "Control-Relevant Upscaling." SPE Journal (Society of Petroleum Engineers) 15, no. 02 (2010): 471 - 479.

Valladao, Davi M., Ruben R. Torrado, Bruno Flach, and Sonia Embid. "On The Stochastic Response Surface Methodology For The Determination Of The Development Plan Of An Oil \& Gas Field." SPE Middle East Intelligent Energy Conference and Exhibition. Manama, Bahrain: Society of Petroleum Engineers, 2013.

Van Leeuwen, P. J. "Comment on "Data assimilation using an ensemble Kalman filter technique." Monthly Weather Review 127, no. 6 (1999): 1374-1377.

Vasco, D.W., S. Yoon, and A. Datta-Gupta. "Integrating Dynamic Data Into High-Resolution Reservoir Models Using Streamline-Based Analytic Sensitivity Coefficients." SPE Annual Technical Conference and Exhibition. New Orleans, Louisiana: Society of Petroleum Engineers, 1998.

W.S. McCulloch and W.H. Pitts. "A logical calculus of the ideas immanent in nervous activity ." Bulletin of Mathematical Biophysics, 1943: Vol. 5, 115-133.

Wahl, W.L., L.D. Mullins, R.H. Barham, and W.R. Bartlett. "Matching the Performance of Saudi Arabian Oil Fields With an Electrical Model." Journal of Petroleum Technology (Society of Petroleum Engineers) 14, no. 11 (1962): 1275 - 1282.

Wang, C., and J. Gao. "Enhancing resolution of seismic data based on waveform inversion with cooperative coevolutionary differential evolution algorithm." SEG Denver 2010 Annual Meeting. Denver, CO, 2010.

Wang, C., J. Gao, H. Yang, W. Zhao, and Z. Zhu. "Waveform inversion of cross-well data with cooperative coevolutionary differential evolution algorithm." SEG San Antonio 2011 Annual Meeting. San Antonio, Texas, 2011.

Wang, J., and J. S. Buckley. "Automatic History Matching using Differential Evolution Algorithm." International Symposium of the Society of Core Analysts. Trondheim, Norway, 2006.

Wang, Yuandong, and Anthony R. Kovscek. "A Streamline Approach for History-Matching Production Data." SPE/DOE Improved Oil Recovery Symposium. Tulsa, Oklahoma: Society of Petroleum Engineers, 2000.

Wasserman, M.L., A.S. Emanuel, and J.H. Seinfeld. "Practical Applications of OptimalControl Theory to History-Matching Multiphase Simulator Models." Society of Petroleum Engineers Journal 15, no. 04 (1975): 347 - 355.

Watkins, A., R . Parish, and A Modine. "A Stochastic Role for Reservoir Engineering Input to Reservoir History Matching." Second Latin American Petroleum Engineering Conference. Caracas, Venezuela: Society of Petroleum Engineers, 1992. 
Watson, A., and W Lee. "A New Algorithm for Automatic History Matching Production Data." Unconventional Gas Technology Symposium. louisville, Kentucky: Society of Petroleum Engineers, 1986.

Watson, A.T., J.H. Seinfeld, G.R. Gavalas, and P.T. Woo. "History Matching in Two-Phase Petroleum Reservoirs." Society of Petroleum Engineers Journal (Society of Petroleum Engineers) 20, no. 06 (1980): 521 - 532.

Watts, J.W. "Reservoir Simulation: Past, Present, and Future." SPE Computer Applications, 1997: 171-176.

White, Christopher D., and Steve A. Royer. "Experimental Design as a Framework for Reservoir Studies." SPE Reservoir Simulation Symposium. Houston, Texas: Society of Petroleum Engineers, 2003.

Wikipedia. 2014. http://en.wikipedia.org/wiki/Mathematical_optimization (accessed 05 01, 2014).

Wikipedia. 2014. http://en.wikipedia.org/wiki/Evolutionary_algorithms (accessed 05 02, 2014).

Wikipedia. 2014.http://en.wikipedia.org/wiki/Evolutionary_computation (accessed 0501 , 2014).

Williams, G., M. Mansfield, D. MacDonald, and M Bush. "Top-down Reservoir Modeling." Annual Technical Conference and Exhibition. Houston, Texas: Society of Petroleum Engineers, 2004.

Wu, Xiao-hui, Linfeng Bi, Yahan Yang, and Xiaochen Wang. "Multiphase Upscaling Using Approximation Techniques." SPE Reservoir Simulation Symposium. The Woodlands, Texas, USA: Society of Petroleum Engineers, 2013.

Yang, C., L Ngheim, and C. Card. "Reservoir Model Uncertainty Quantification through Computer-Assisted History Matching." SPE Annual Technical Conference and Exhibition. Anaheim, California, U.S.A., 2007.

Yang, H.Z., and Kou-Yuan Huang. "Hybrid Neural Network For Seismic Pattern Recognition." SEG Annual Meeting. Houston, Texas: Society of Exploration Geophysicists, 1991.

Yang, P., and A Watson. "Automatic History Matching with Variable-Metric Methods." SPE Reservoir Engineering (Society of Petroleum Engineers) 3, no. 3 (1988): 995-1001.

Yang, Yahan, Jeffrey Edward Davidson, David James Fenter, Ozgur Ozen, and Barbara A. Boyett. "Reservoir Development Modeling Using Full Physics And Proxy Simulations." International Petroleum Technology Conference. Doha, Qatar: International Petroleum Technology Conference, 2009. 
Zabalza, I., G. Blanc, D. Collmobier, and M. Mezghani. "Use of Experimental Design in Resolving Inverse Problems: Application to History Matching." ECMOR, European Conference on the Mathematics of Oil Recovery. Baveno, Italy, 2000.

Zhangxin, Chen, Huan Guanren, and Li Baoyan. "An Improved IMPES Method for TwoPhase Flow in Porous Media." Transport in Porous Media 54 (2004): 361-376.

Zubarev, D.I. "Pros and Cons of Applying Proxy-models as a Substitute for Full Reservoir Simulations." New Orleans, Louisiana: Society of Petroleum Engineers, 2009.

Zuber, M., W. Sawyer, R. Schraufnagel, and V Kuuskaa. "The Use of Simulation and History Matching Determine Critical Coalbed Methane Reservoir Properties." Low Permeability Reservoirs Symposium. Denver, Colorado: Society of Petroleum Engineers, 1987. 


\section{Appendix A: Proof of concept study}

\section{a. Production history performance for the reservoir model in proof of concept and feasibility investigation study}
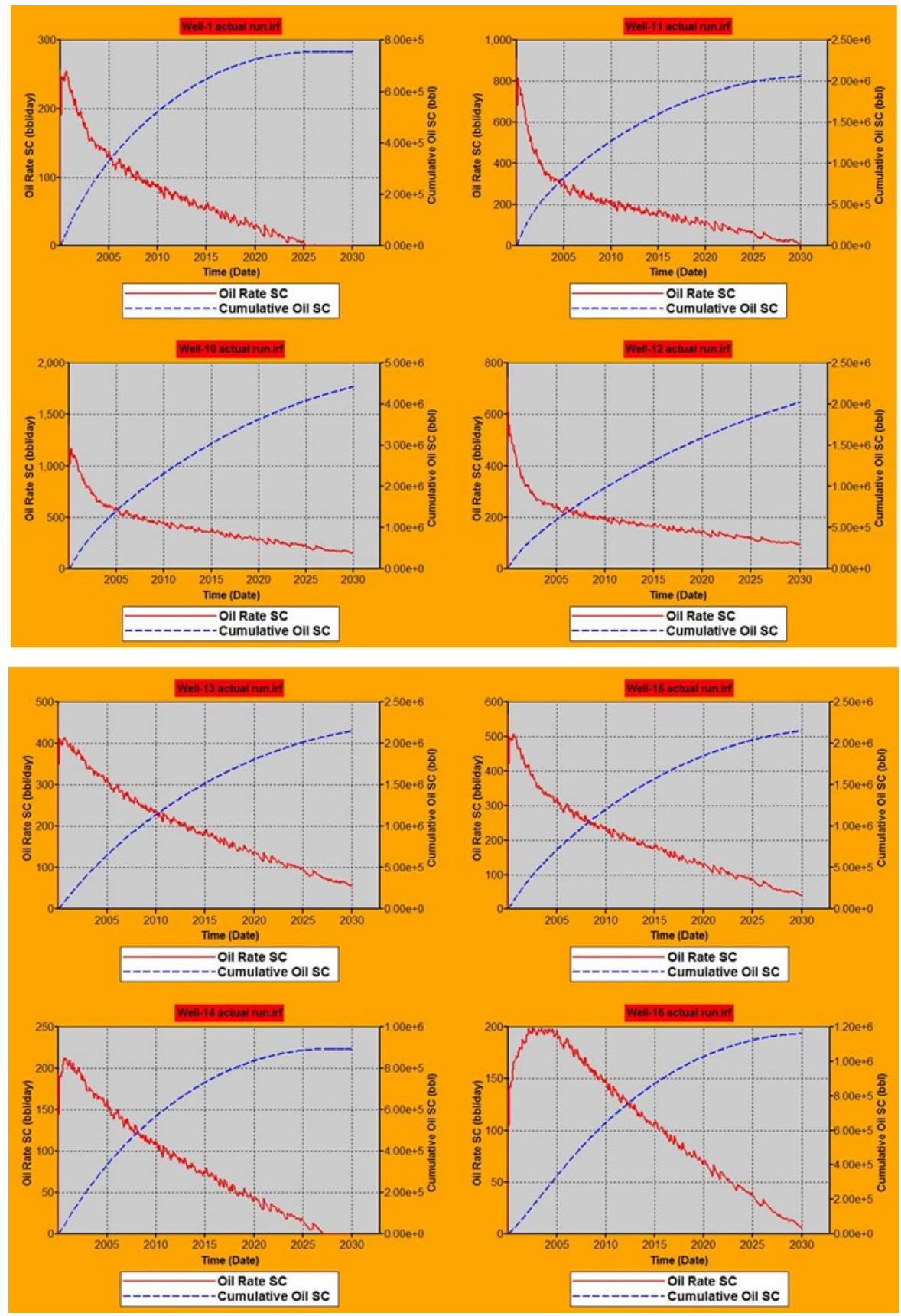

Figure 92: Actual oil rate and cumulative production for different wells. 

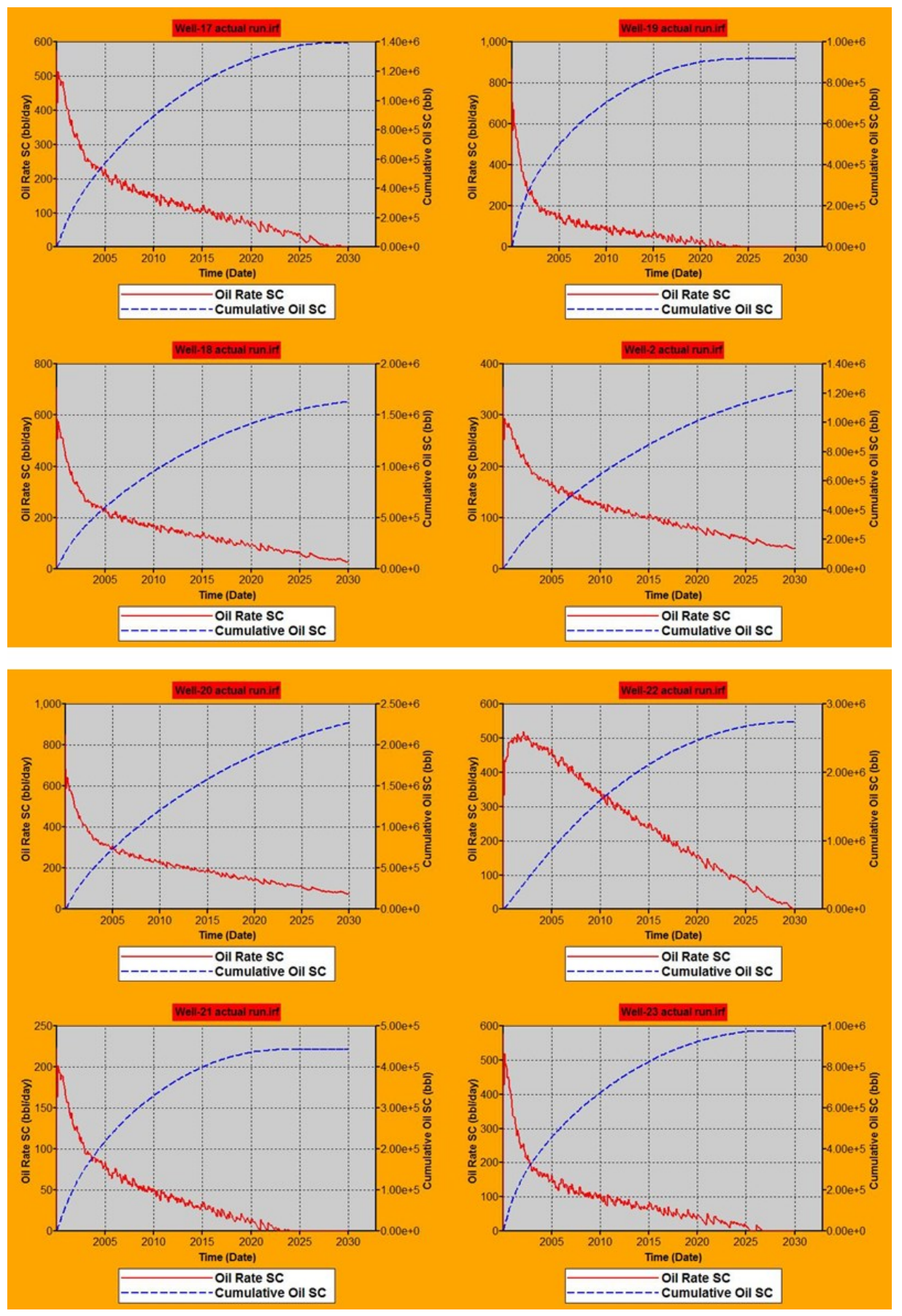

Figure 93: Actual oil rate and cumulative production for different wells. 

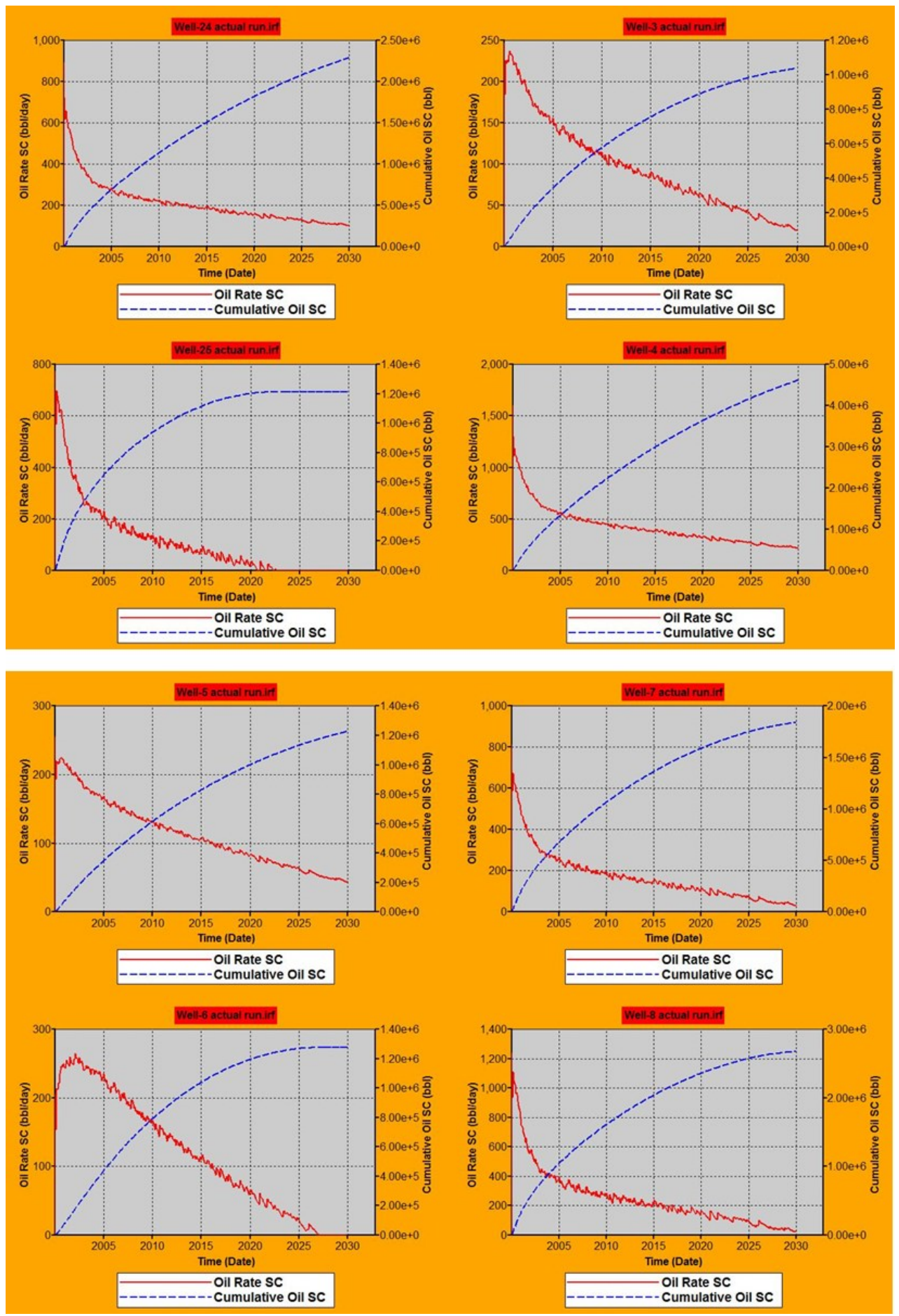

Figure 94: Actual oil rate and cumulative production for different wells. 


\section{b. Ten created permeability distributions for training the SRM}
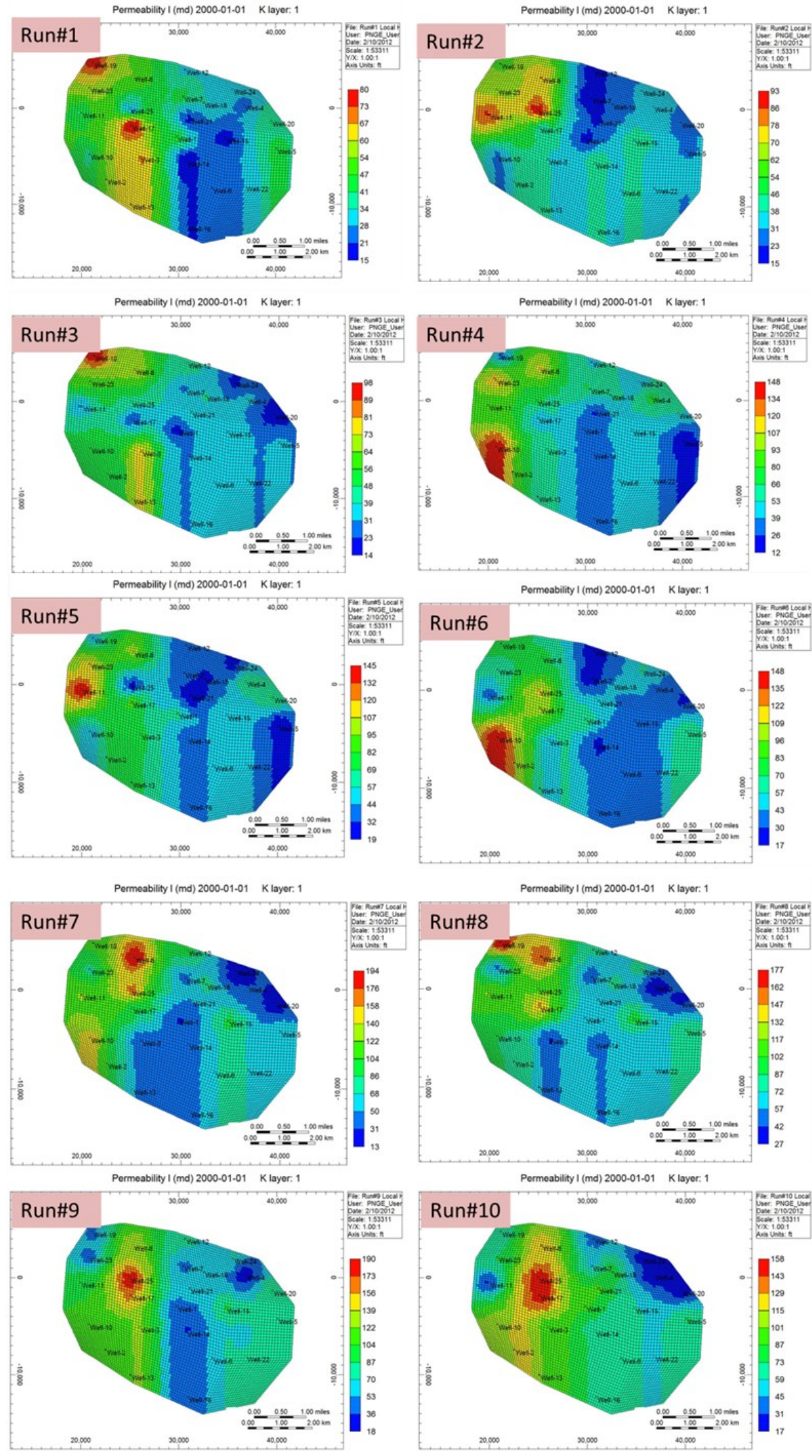

Figure 95: Permeability Map for different Training Realizations. 


\section{c. The results of SRM for a training realizations- Run \# 1}
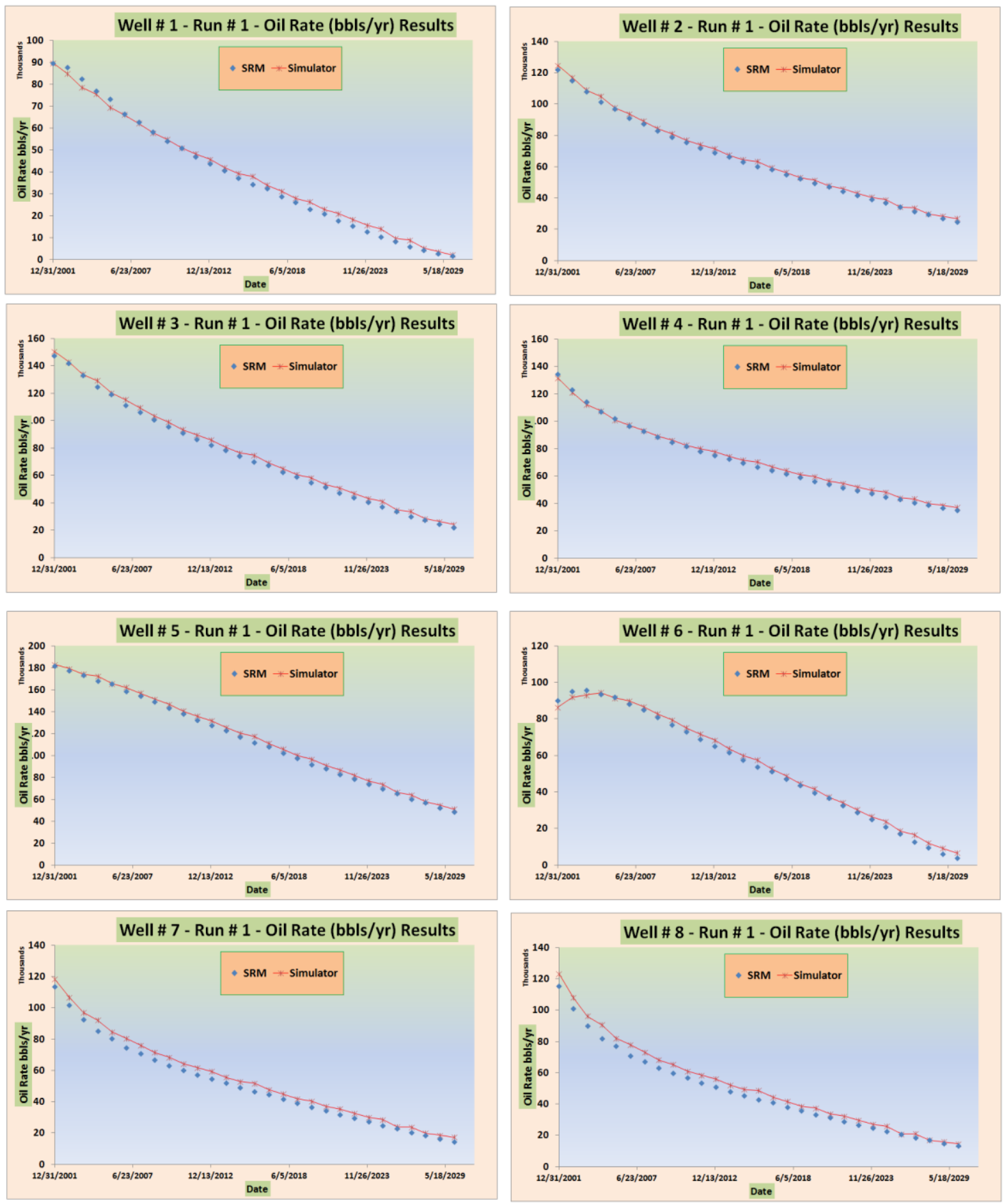

Figure 96: Comparison of the SRM results with simulator outputs after the training process- Run \# 1. 

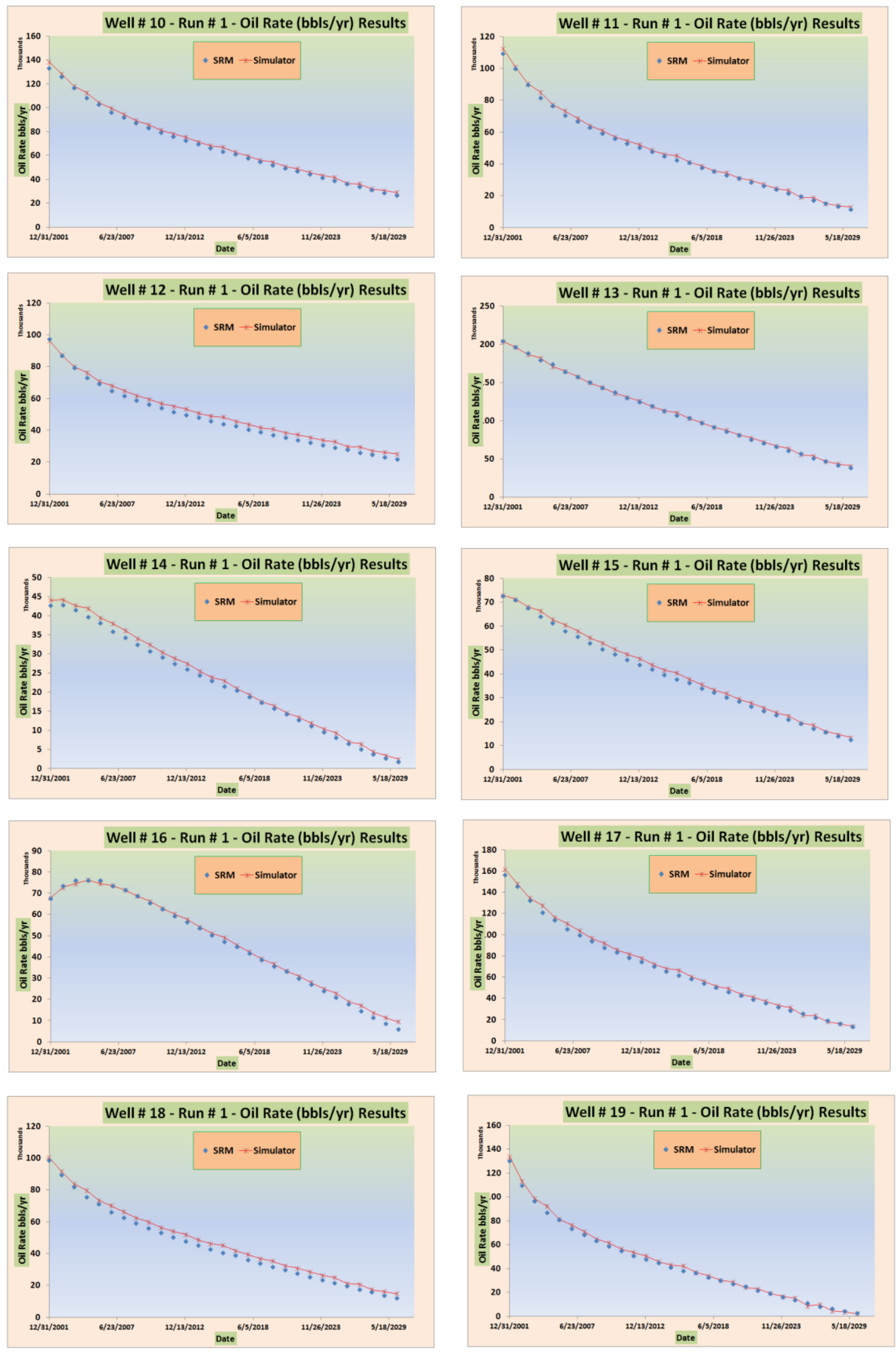

Figure 97: Comparison of the SRM results with simulator outputs after the training process- Run \# 1. 

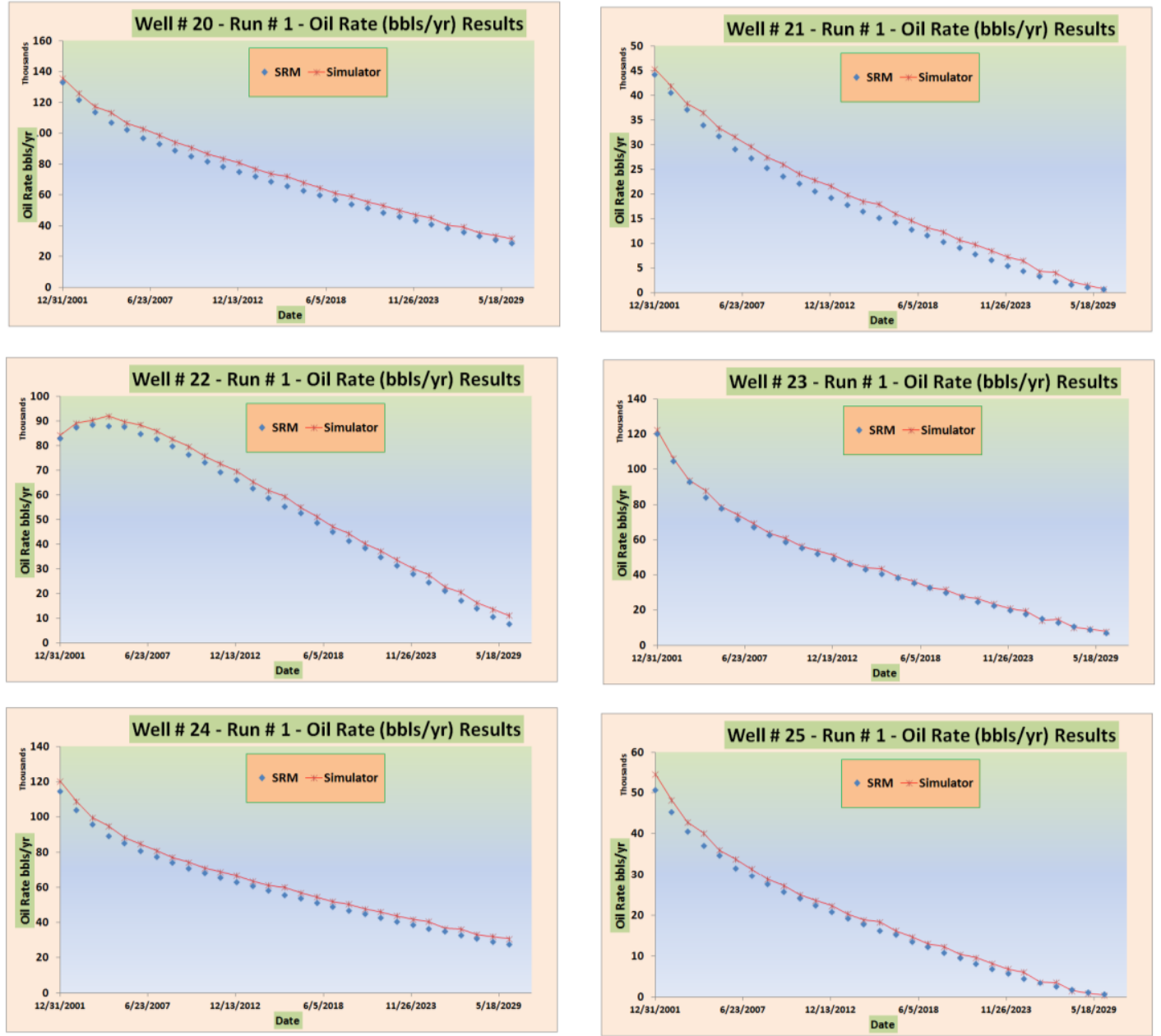

Figure 98: Comparison of the SRM results with simulator outputs after the training process- Run \# 1. 


\section{d. The results of SRM - blind verification realization}
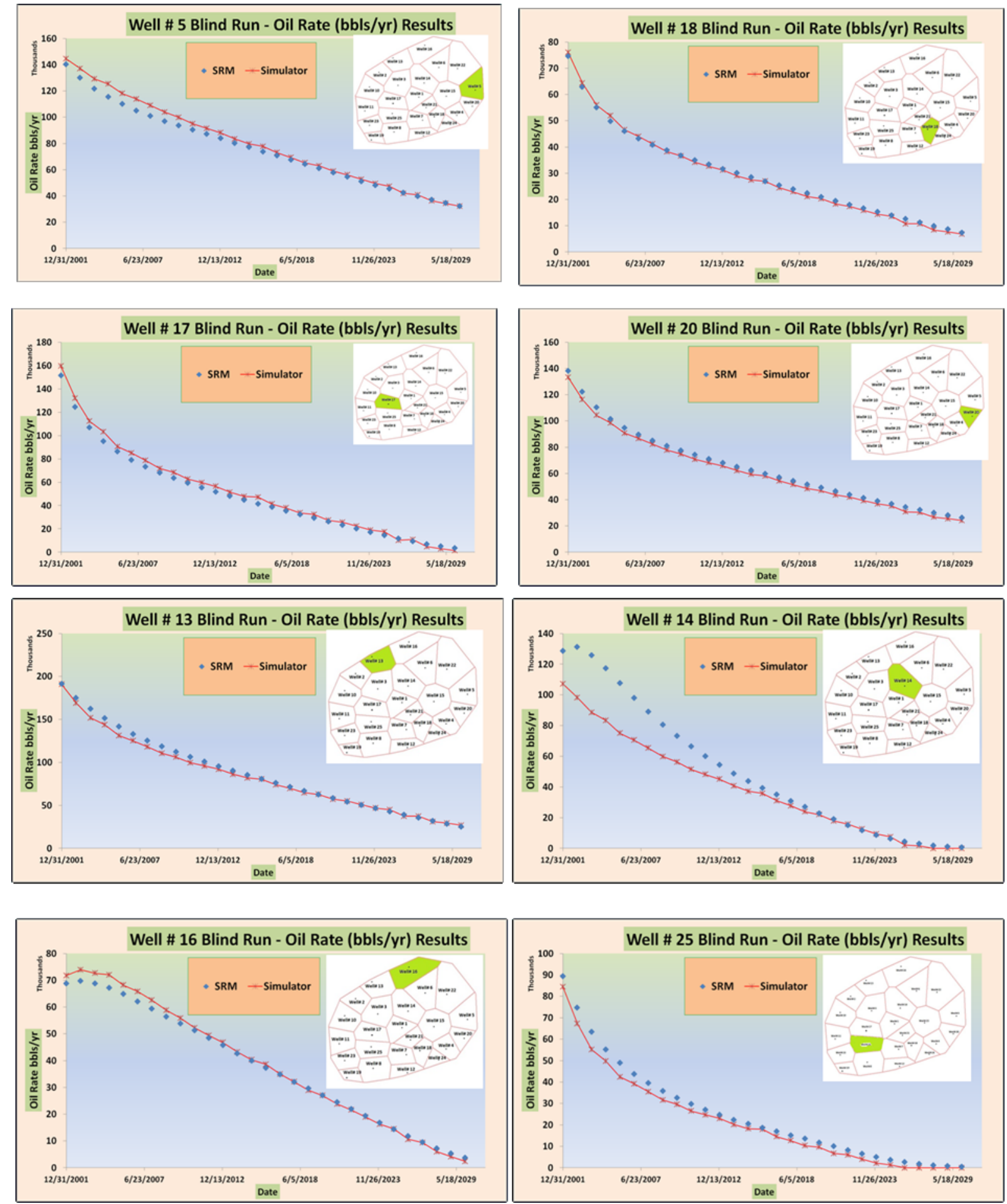

Figure 99: Comparison of the SRM results with simulator outputs, the blind verification run. 

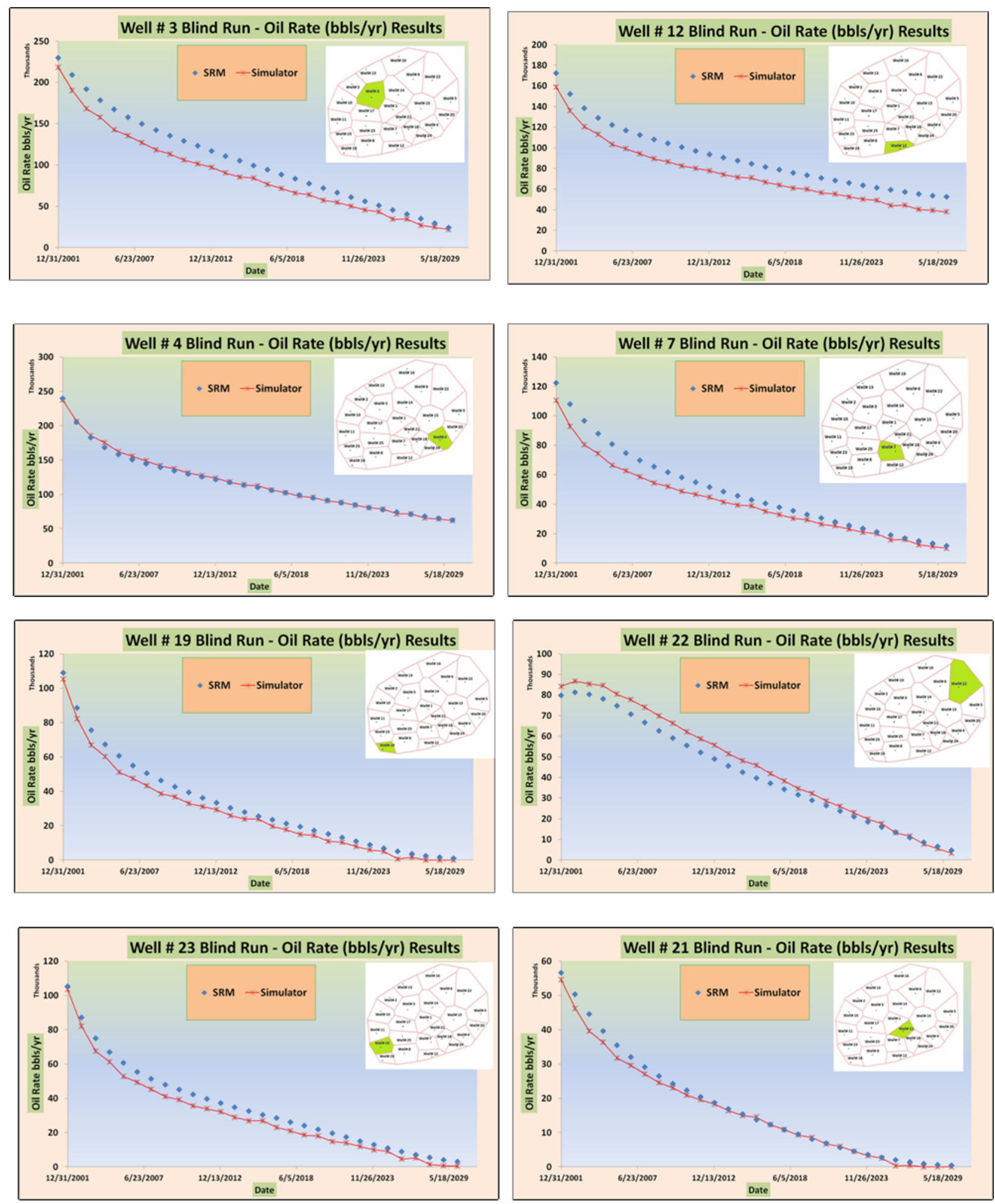

Figure 100: Comparison of the SRM results with simulator outputs, the blind verification run. 

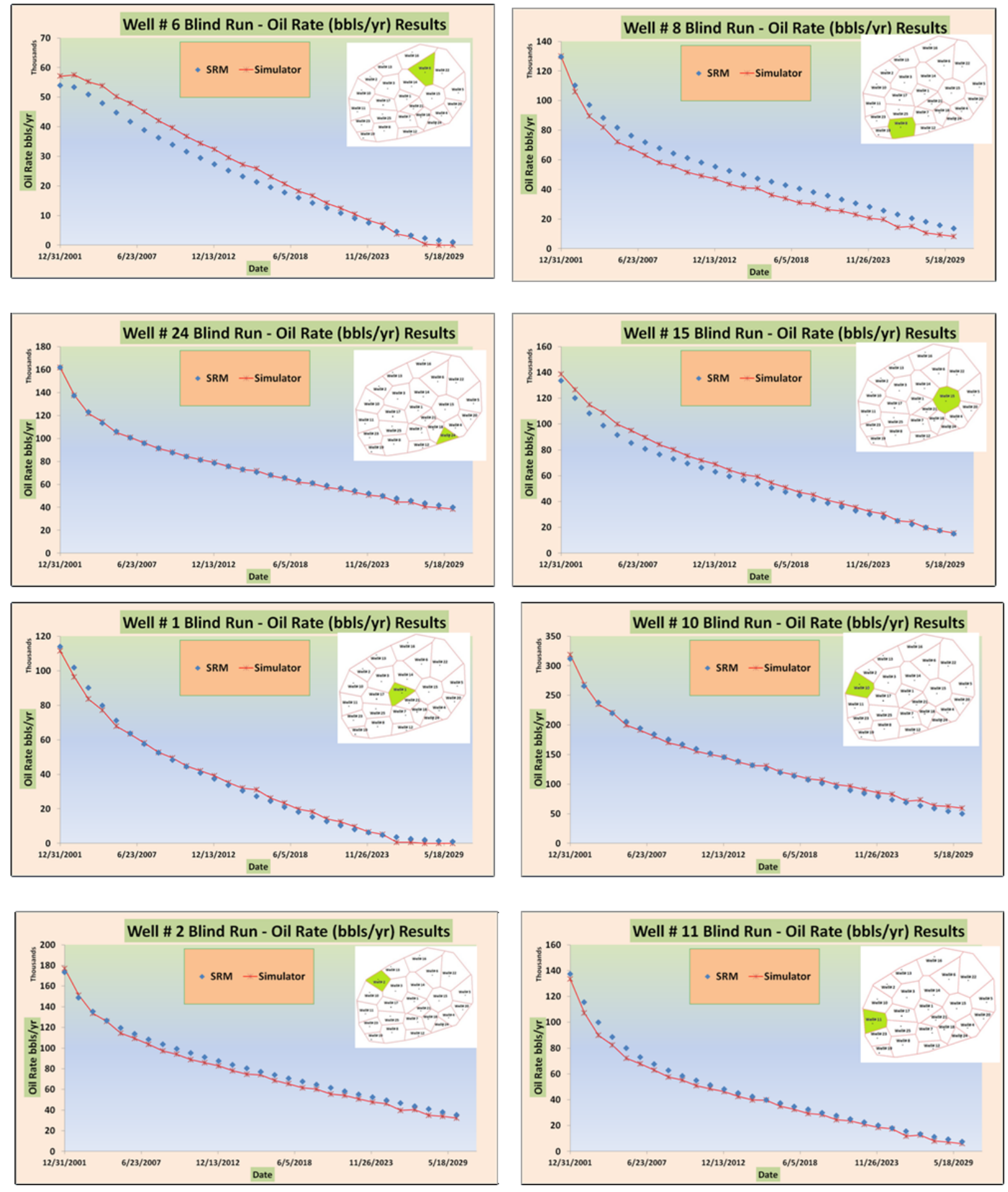

Figure 101: Comparison of the SRM results with simulator outputs, the blind verification run. 


\section{e. The results of SRM- history match results}
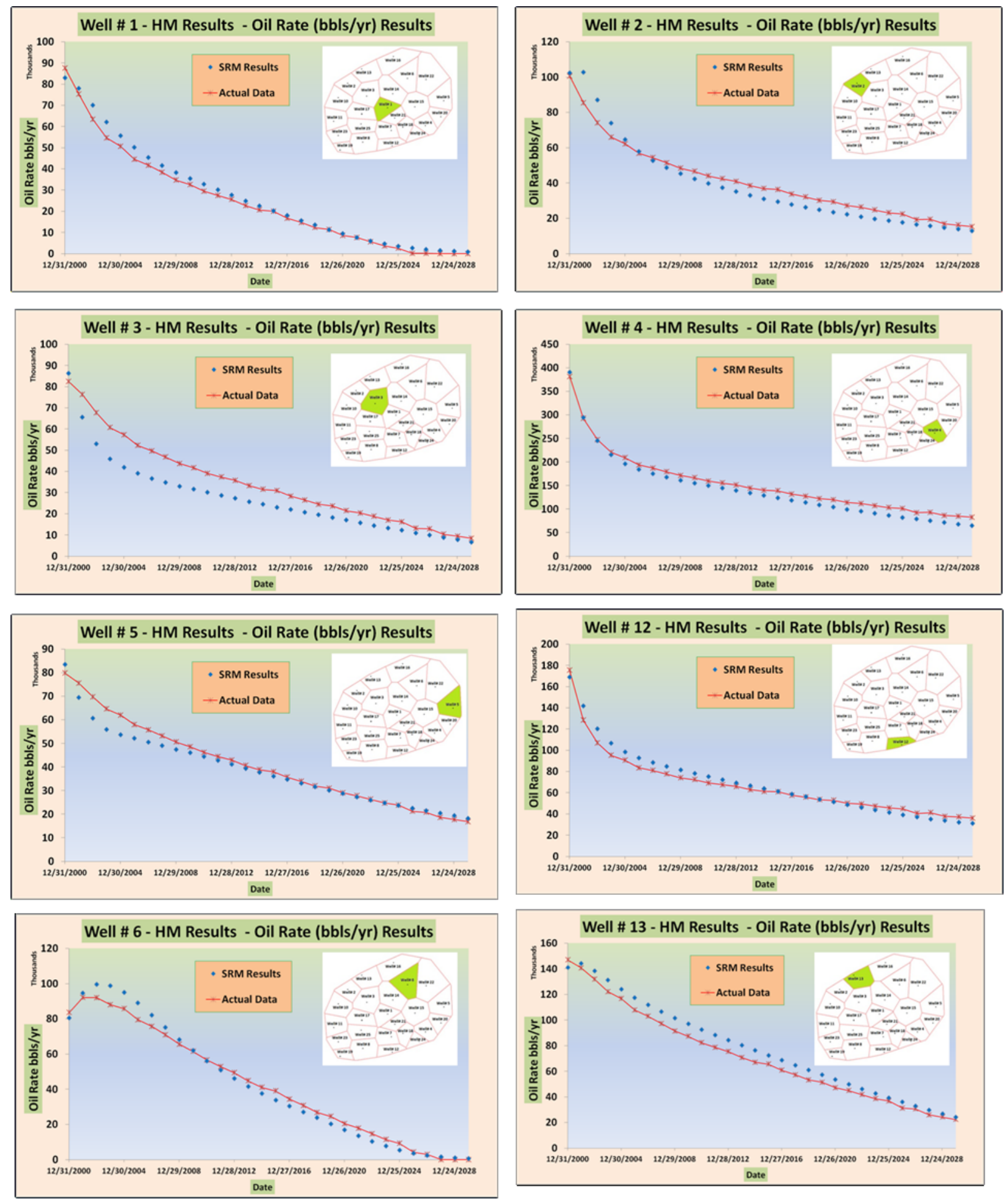

Figure 102: Comparison of the SRM results with field data, history match results. 

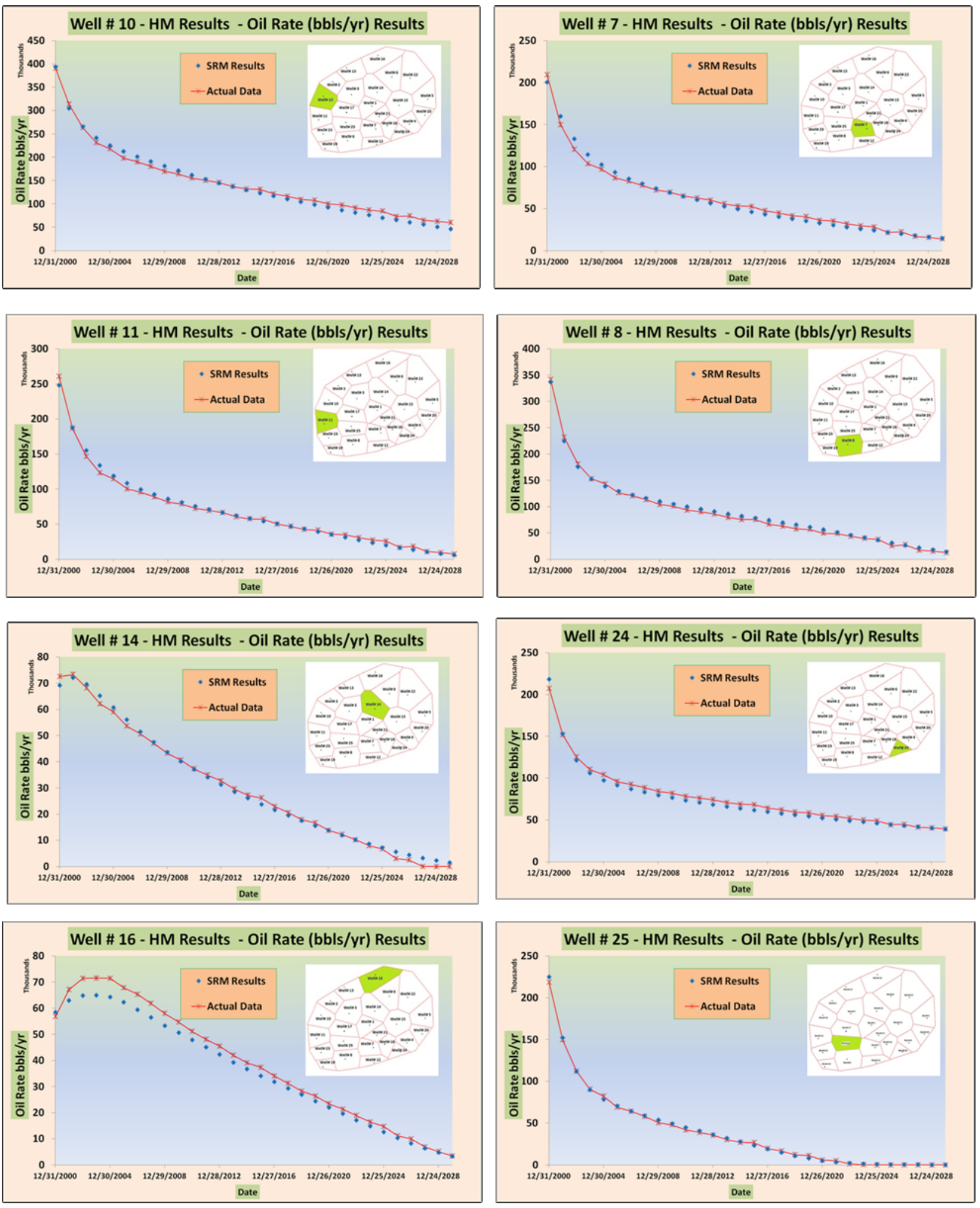

Figure 103: Comparison of the SRM results with field data, history match results. 

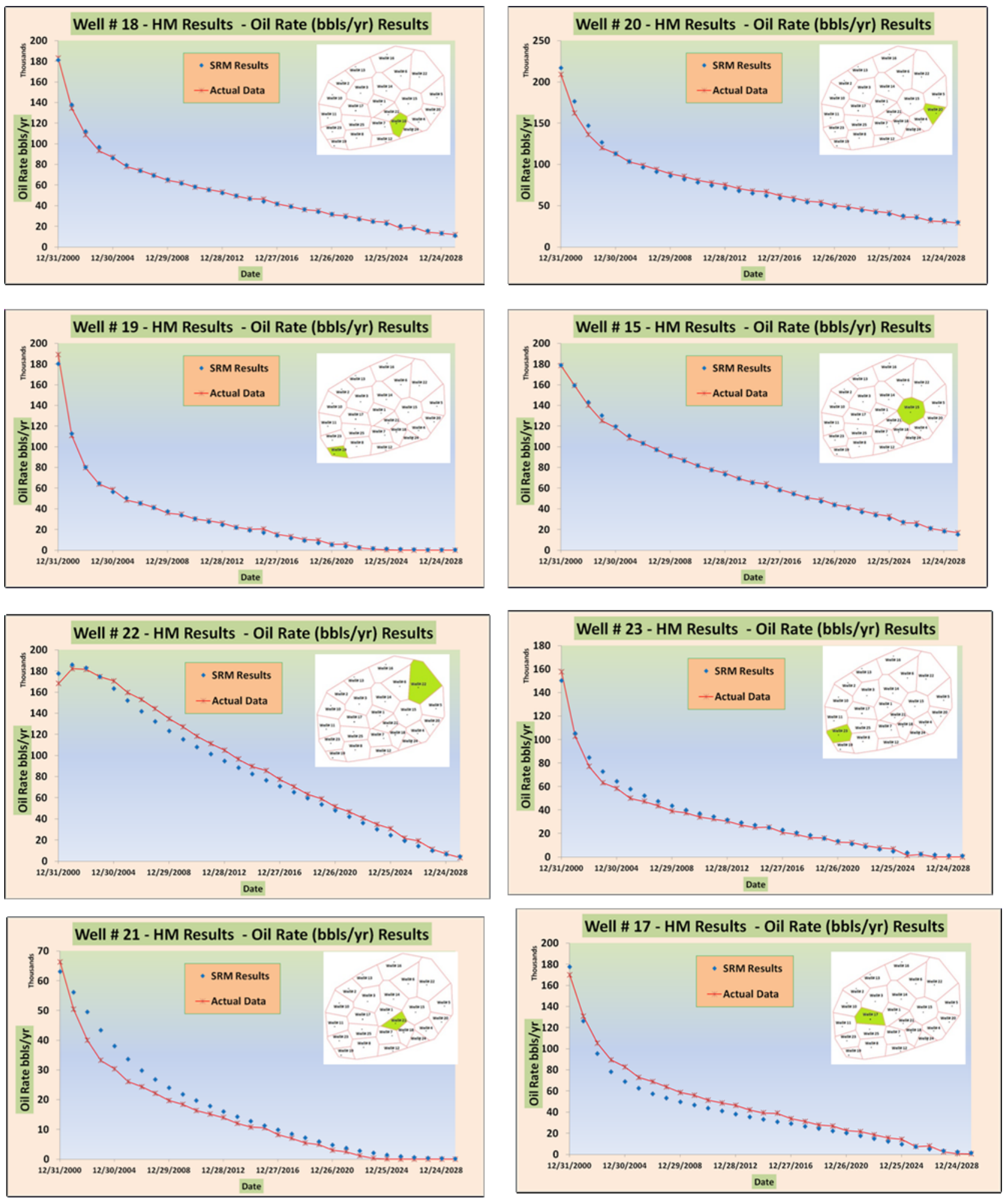

Figure 104: Comparison of the SRM results with field data, history match results. 


\section{Appendix B: Feasibility investigation study}

\section{a. Variable reservoir characteristics for training realizations}

\section{a.1 Permeability distributions}

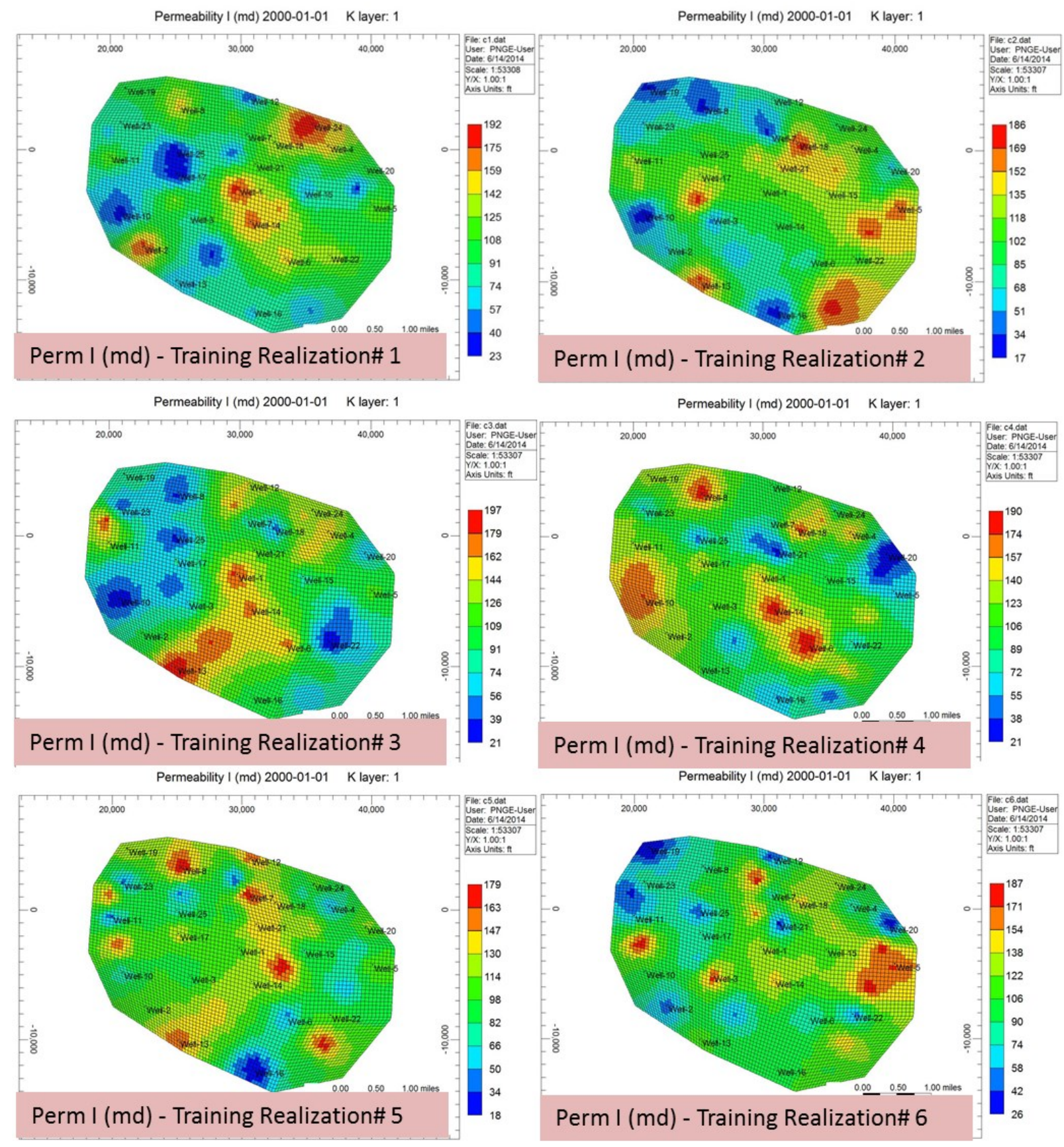

Figure 105: Permeability distributions for training realizations. 


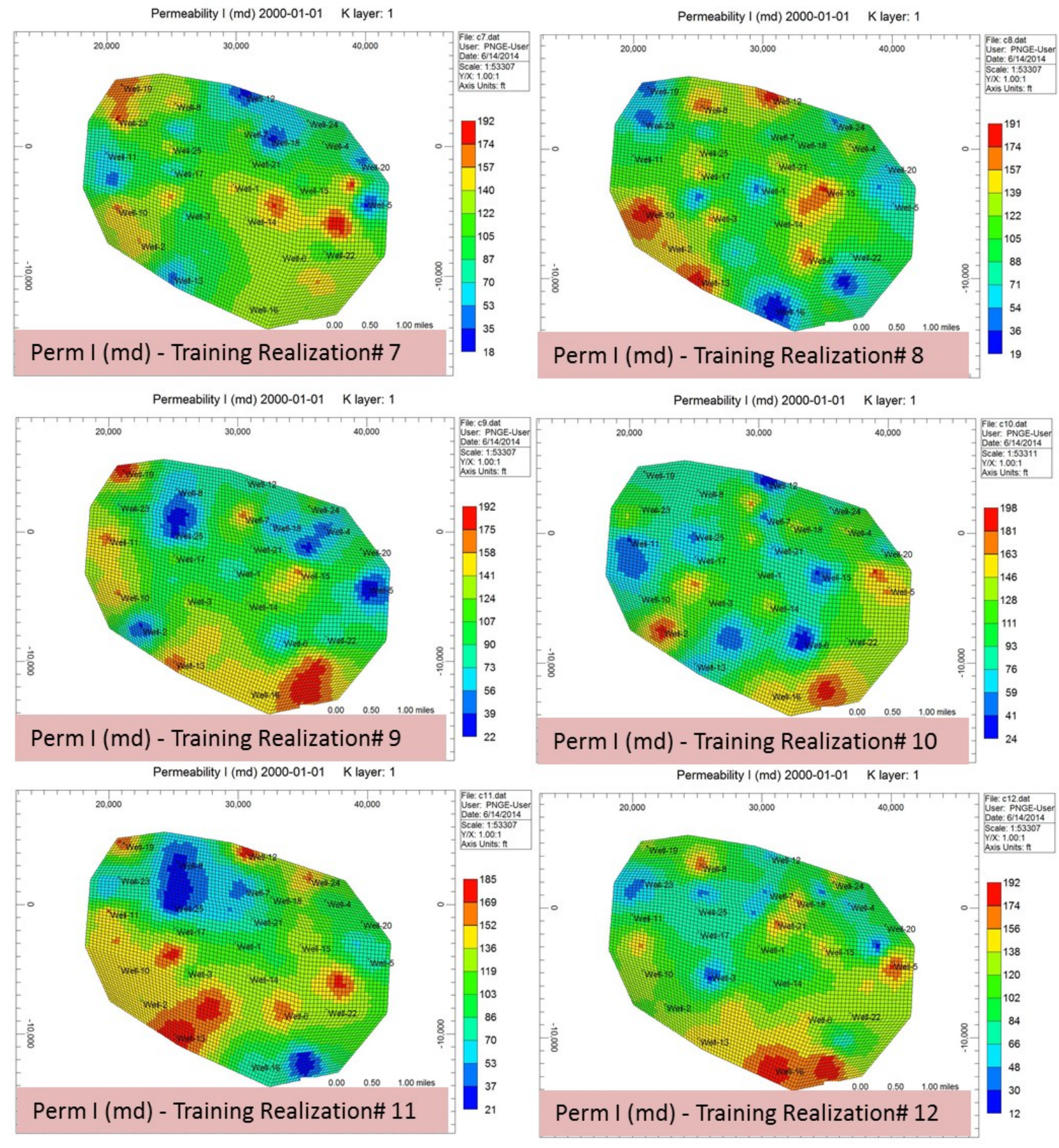

Figure 106: Permeability distributions for training realizations. 


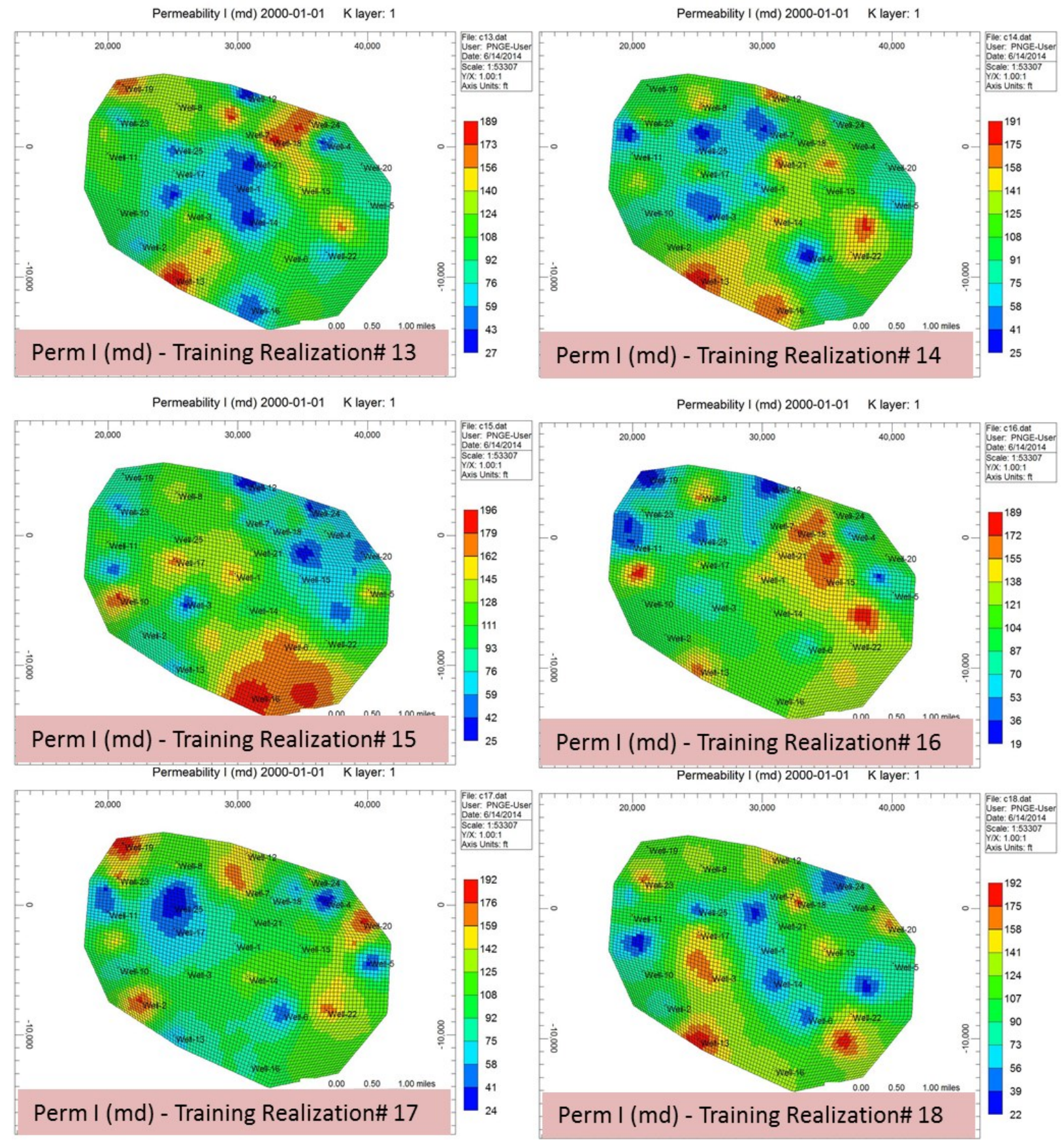

Figure 107: Permeability distributions for training realizations. 


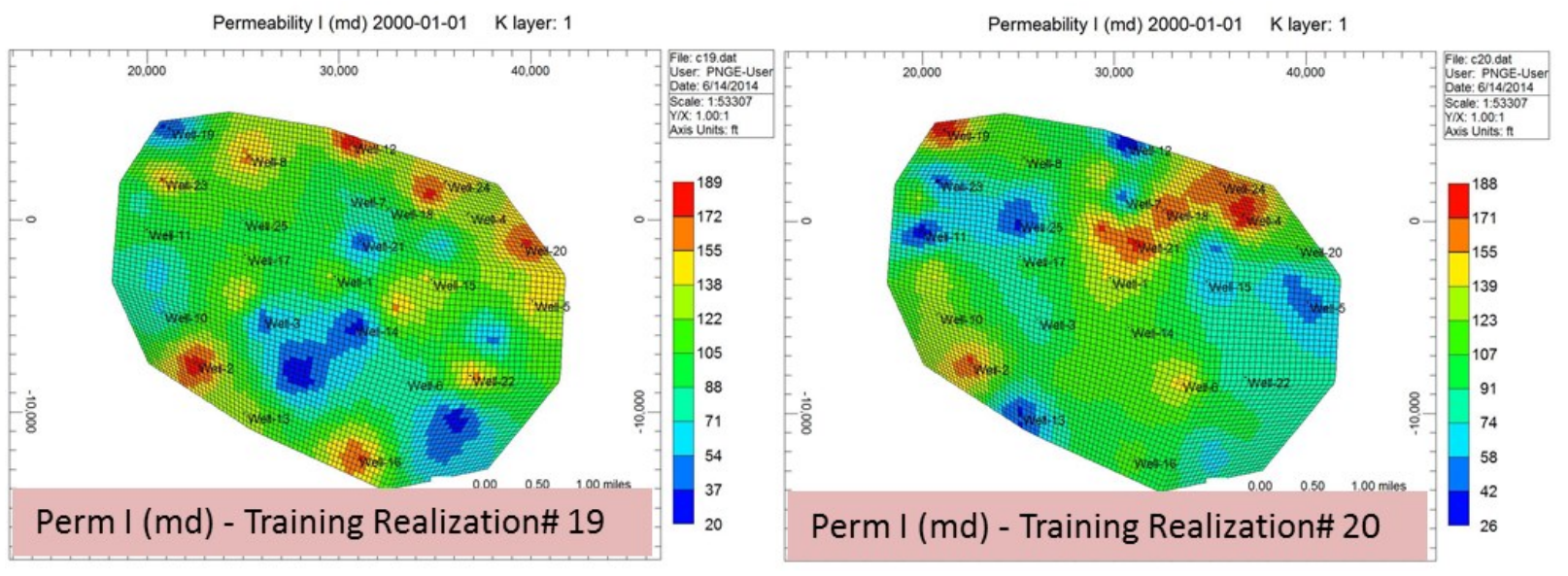

Figure 108: Permeability distributions for training realizations.

\section{a.2 Porosity distributions}
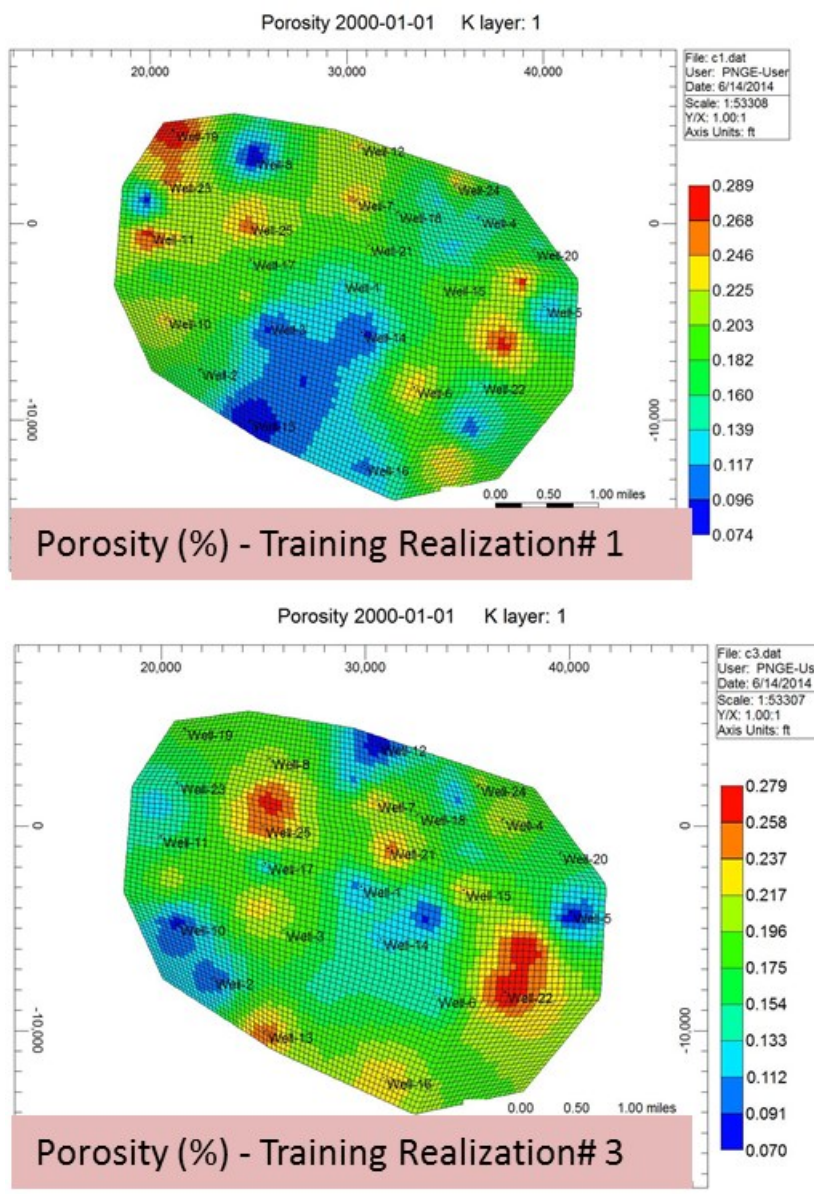
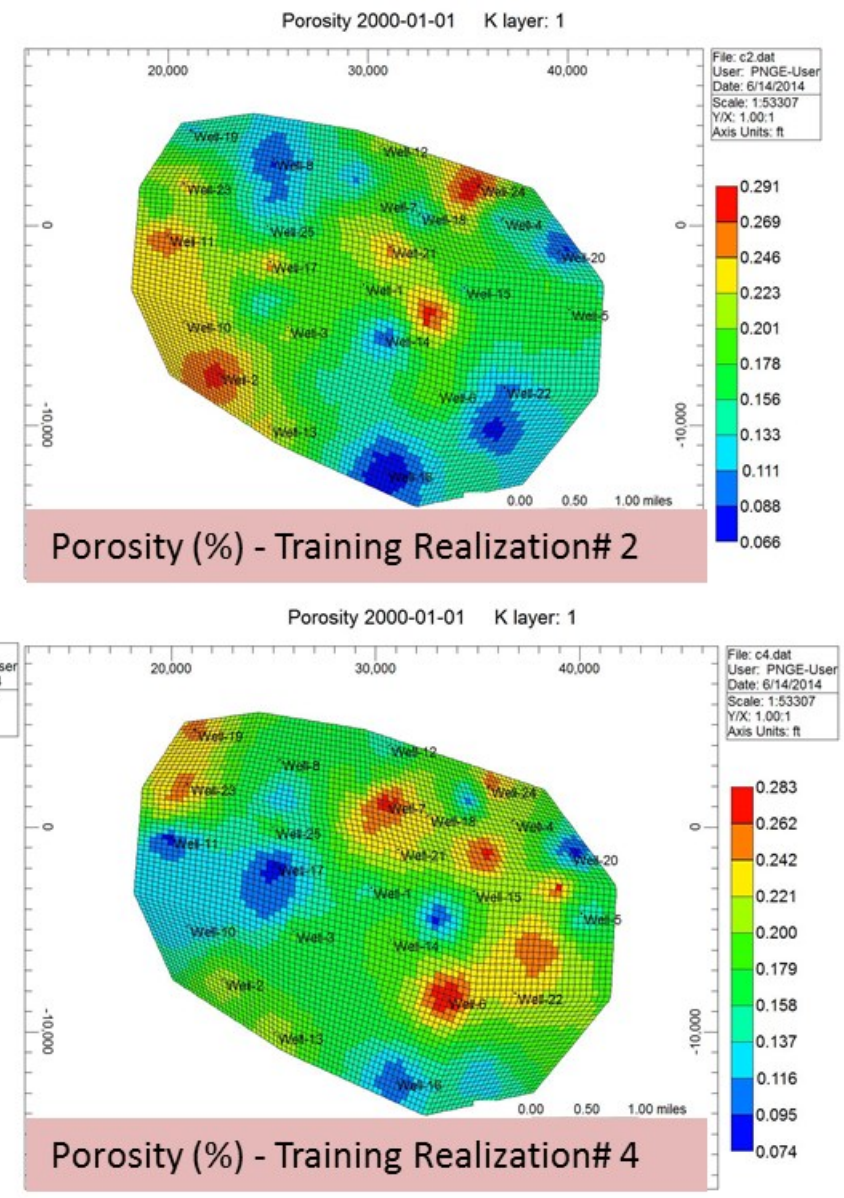

Figure 109: Porosity distributions for training realizations. 


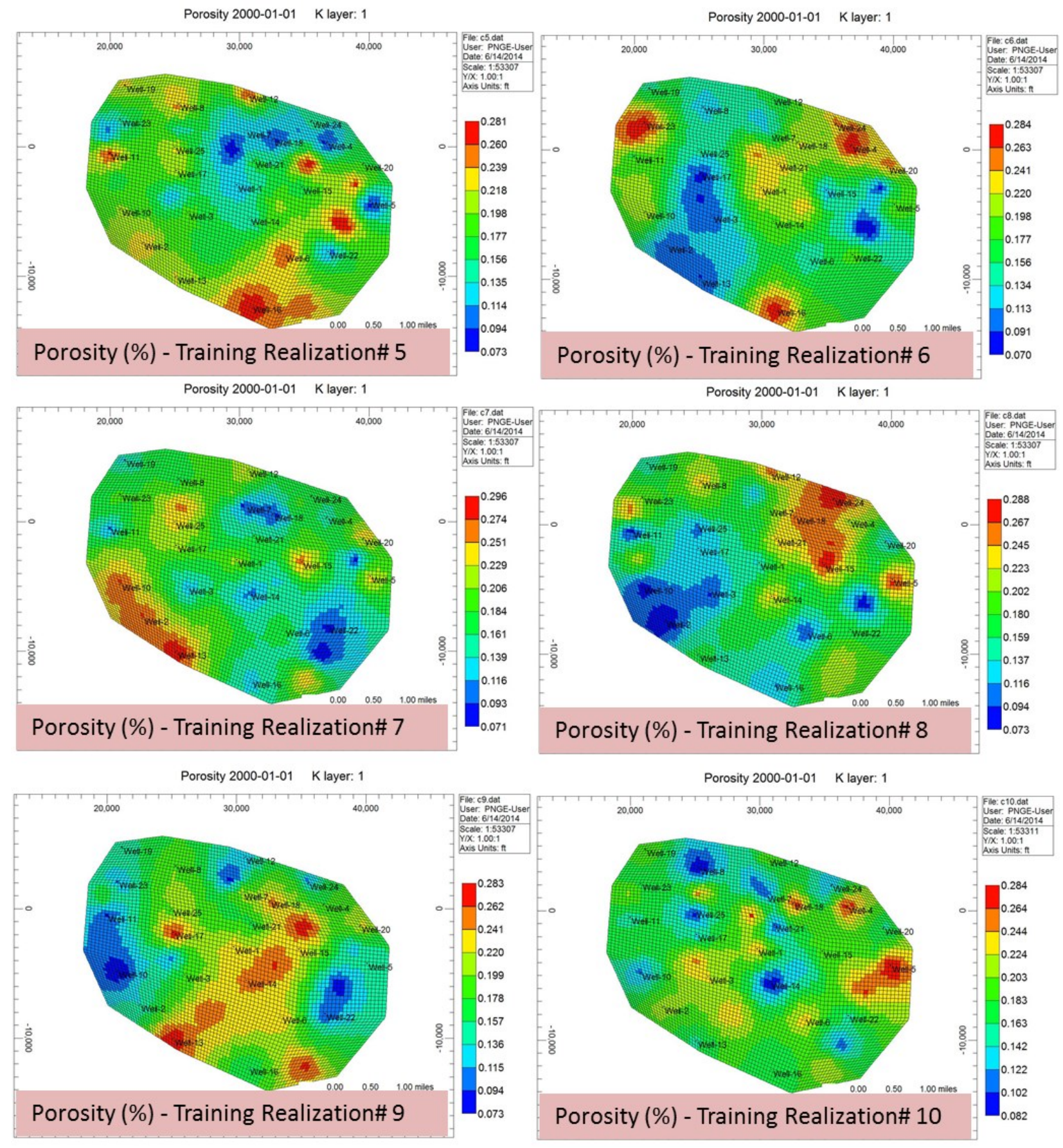

Figure 110: Porosity distributions for training realizations. 


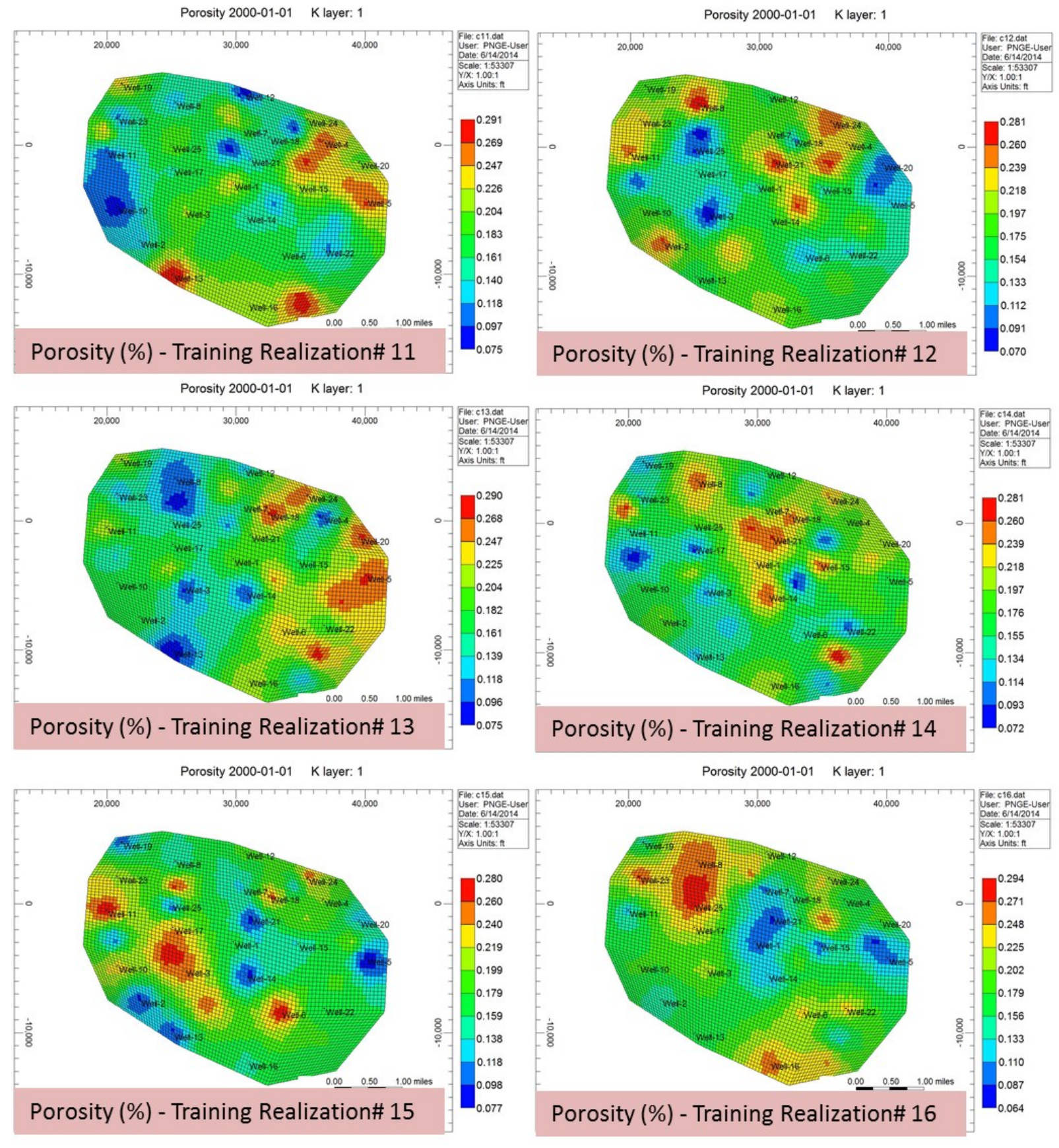

Figure 111: Porosity distributions for training realizations. 


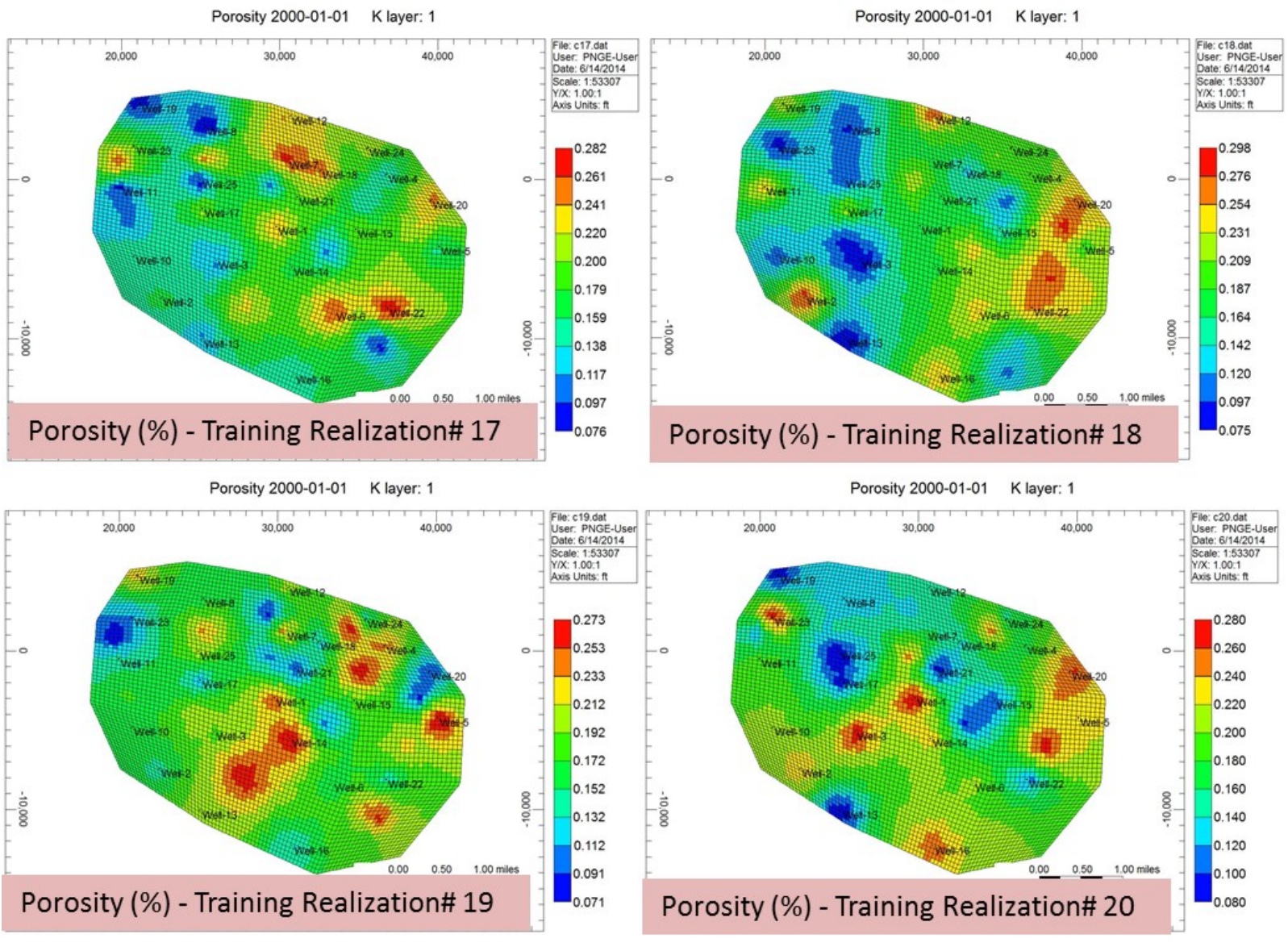

Figure 112: Porosity distributions for training realizations.

\section{a.3 Thickness distributions}

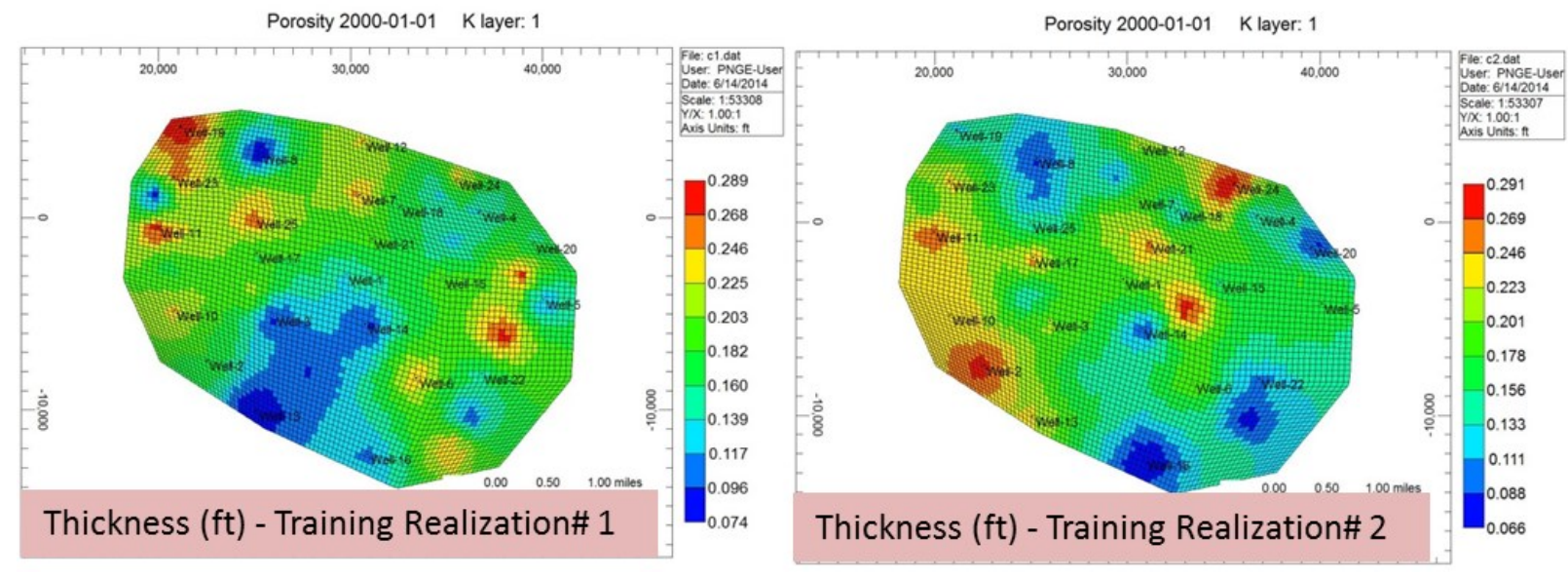

Figure 113: Thickness distributions for training realizations. 


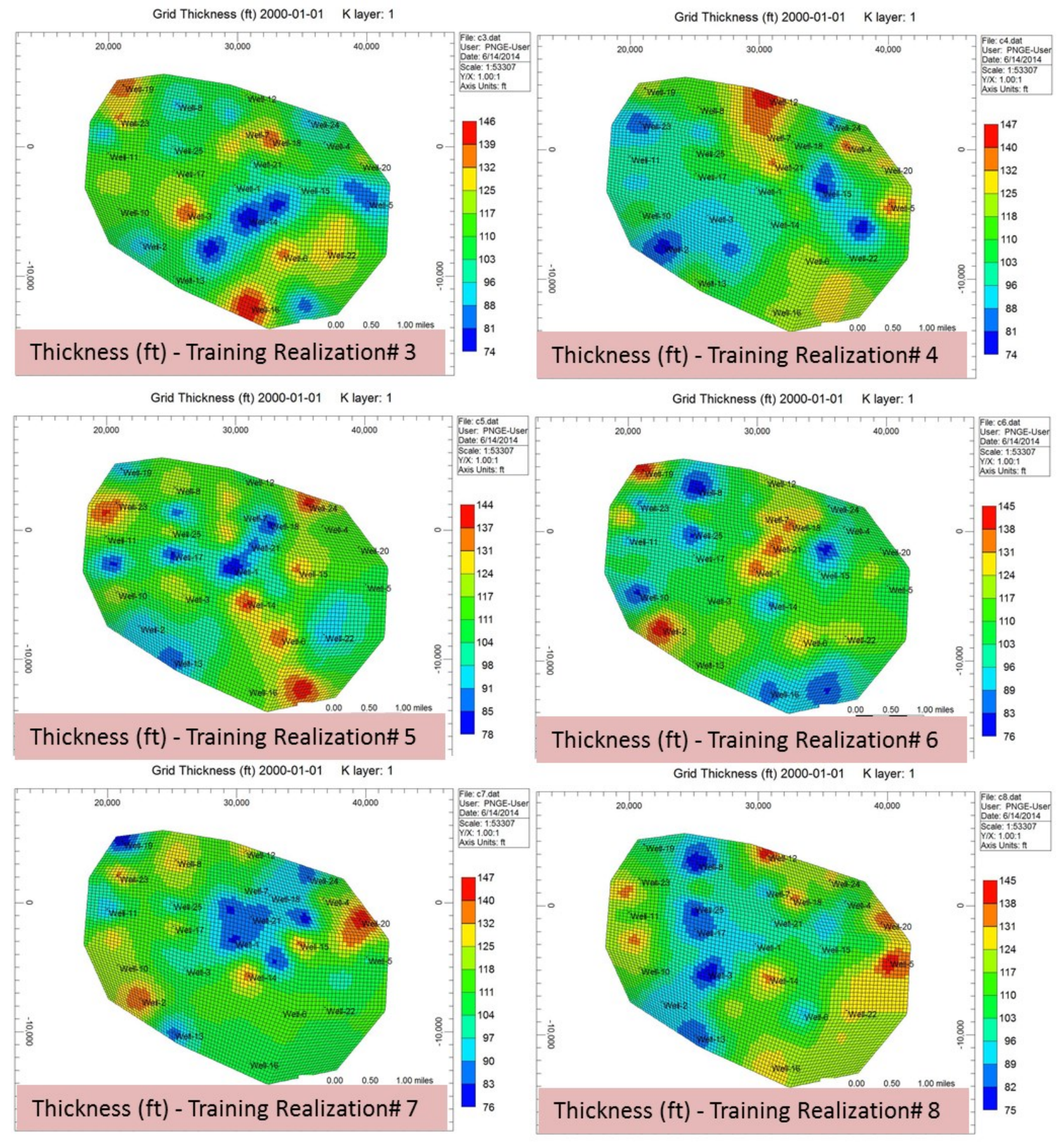

Figure 114: Thickness distributions for training realizations. 


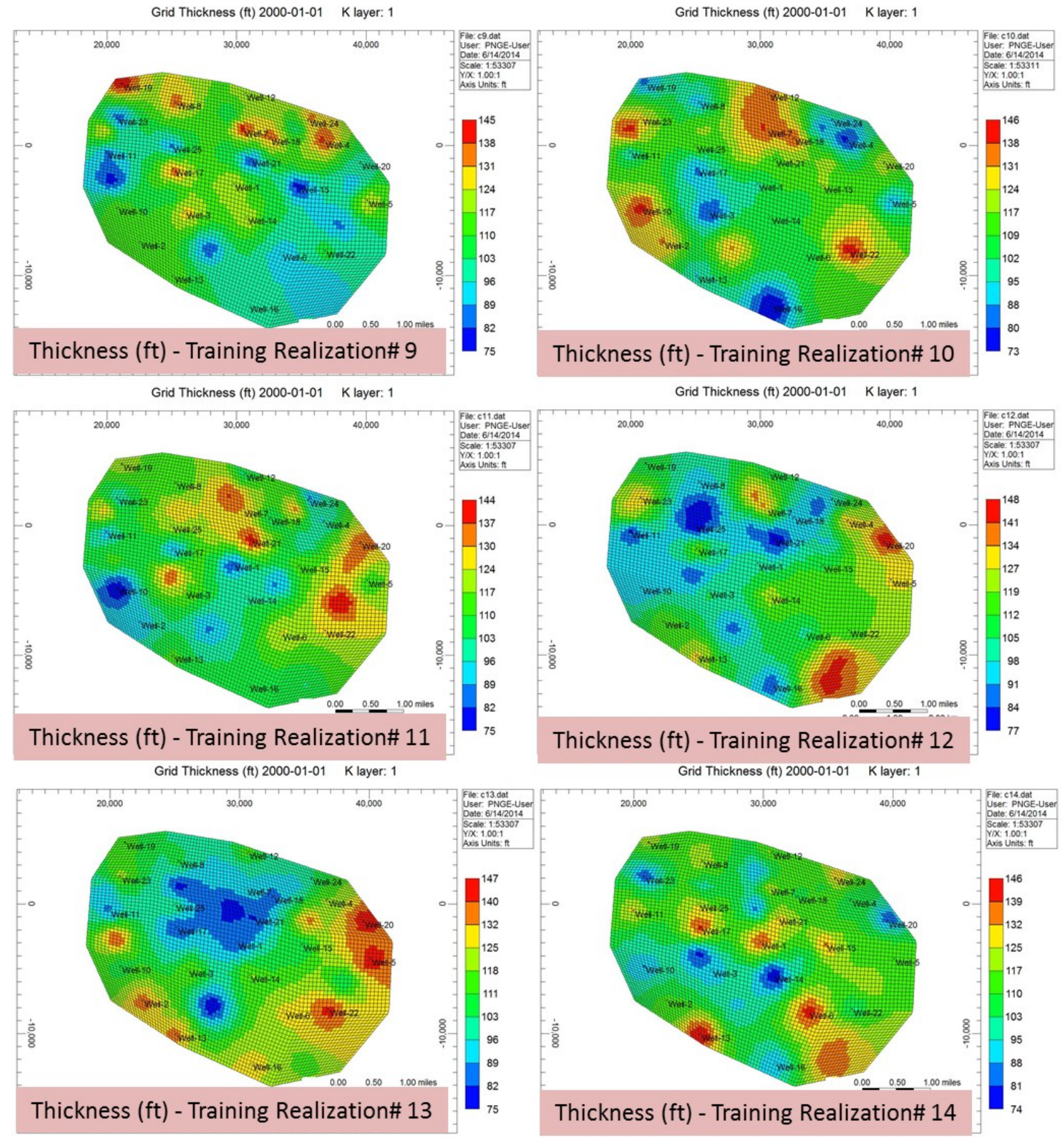

Figure 115: Thickness distributions for training realizations. 


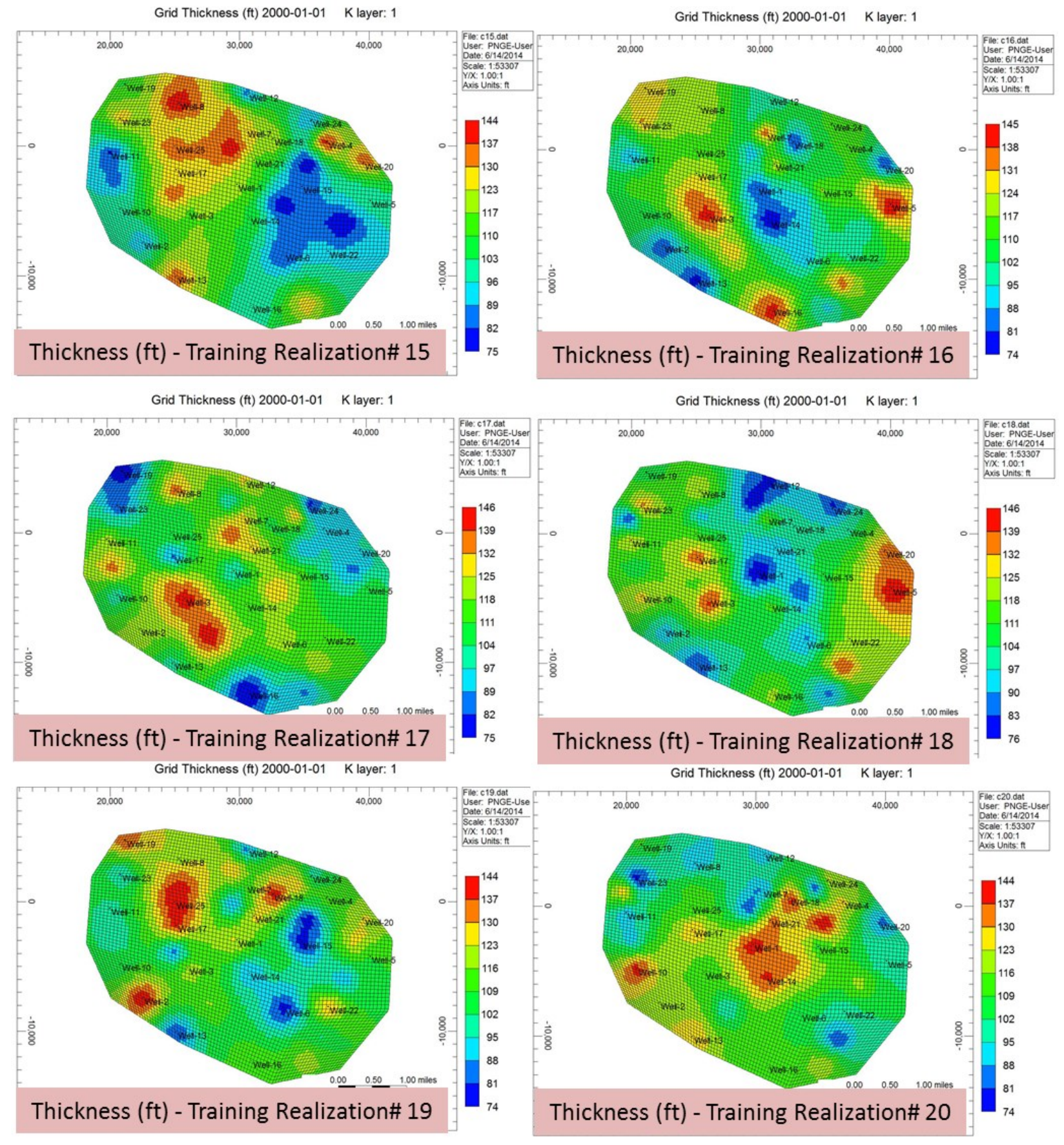

Figure 116: Thickness distributions for training realizations. 


\section{b. The results of SRM for a training realization- Run \#1}
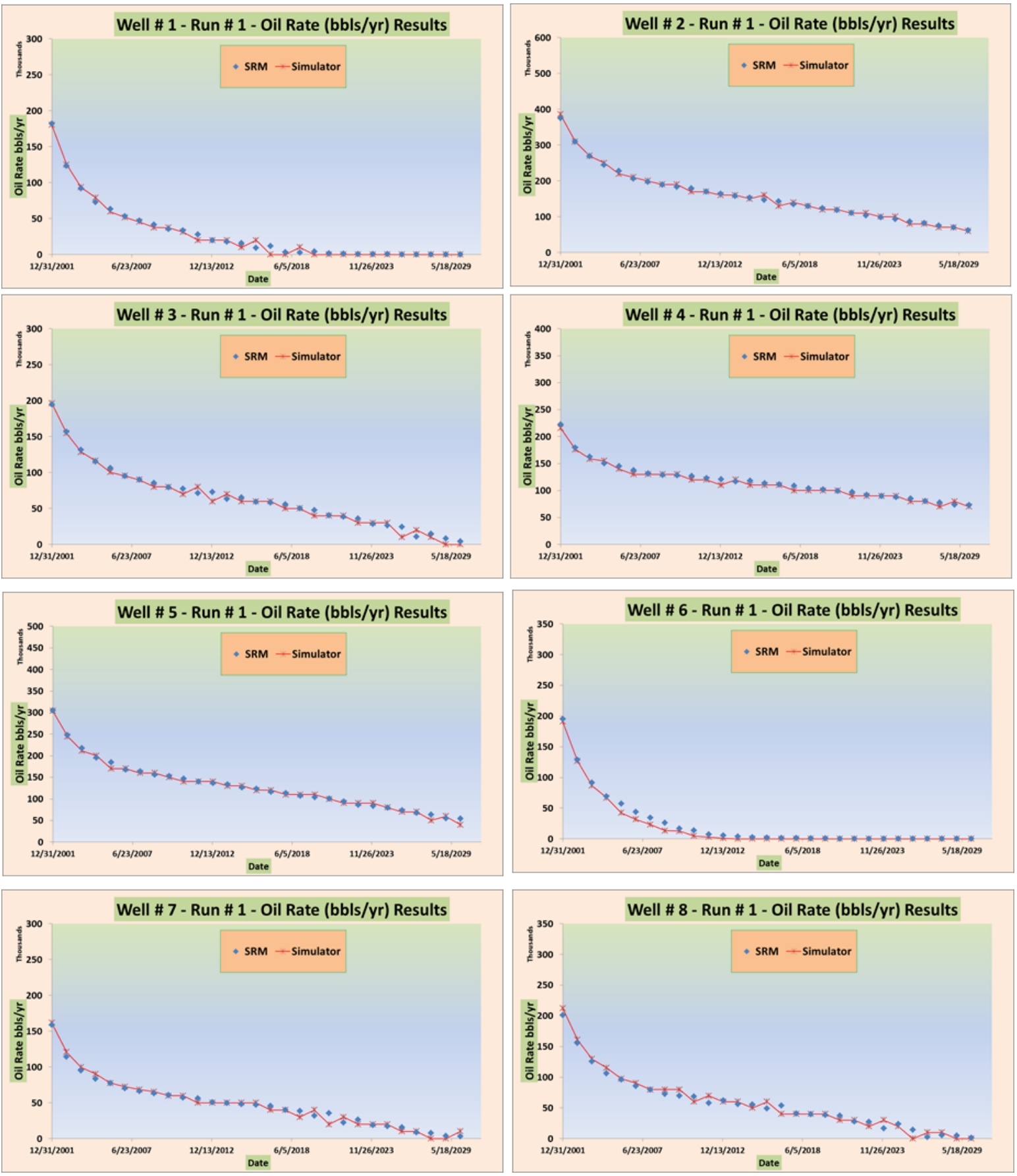

Figure 117: Comparison between the results of SRM and simulator for wells in a training realization. 

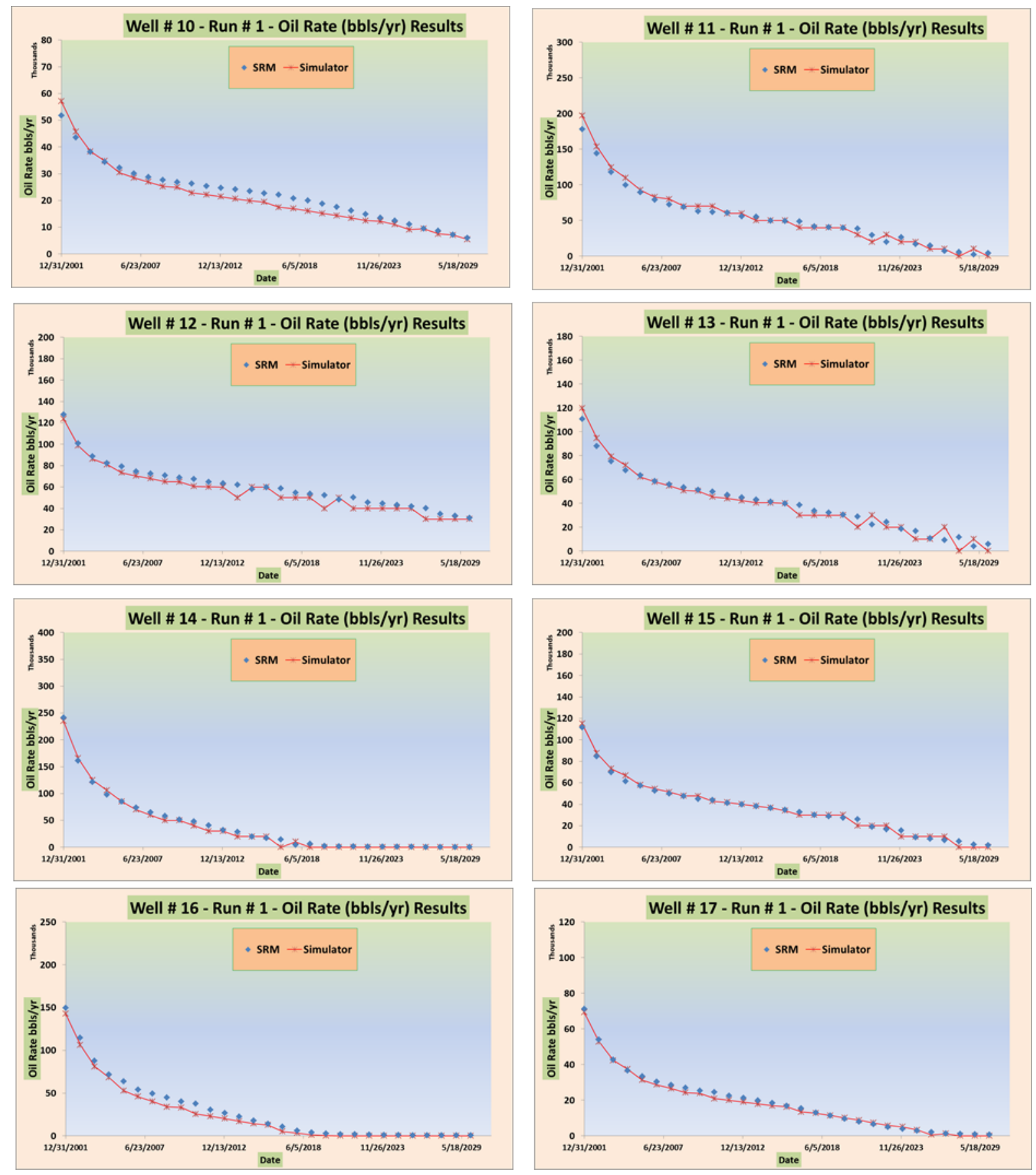

Figure 118: Comparison between the results of SRM and simulator for wells in a training realization. 

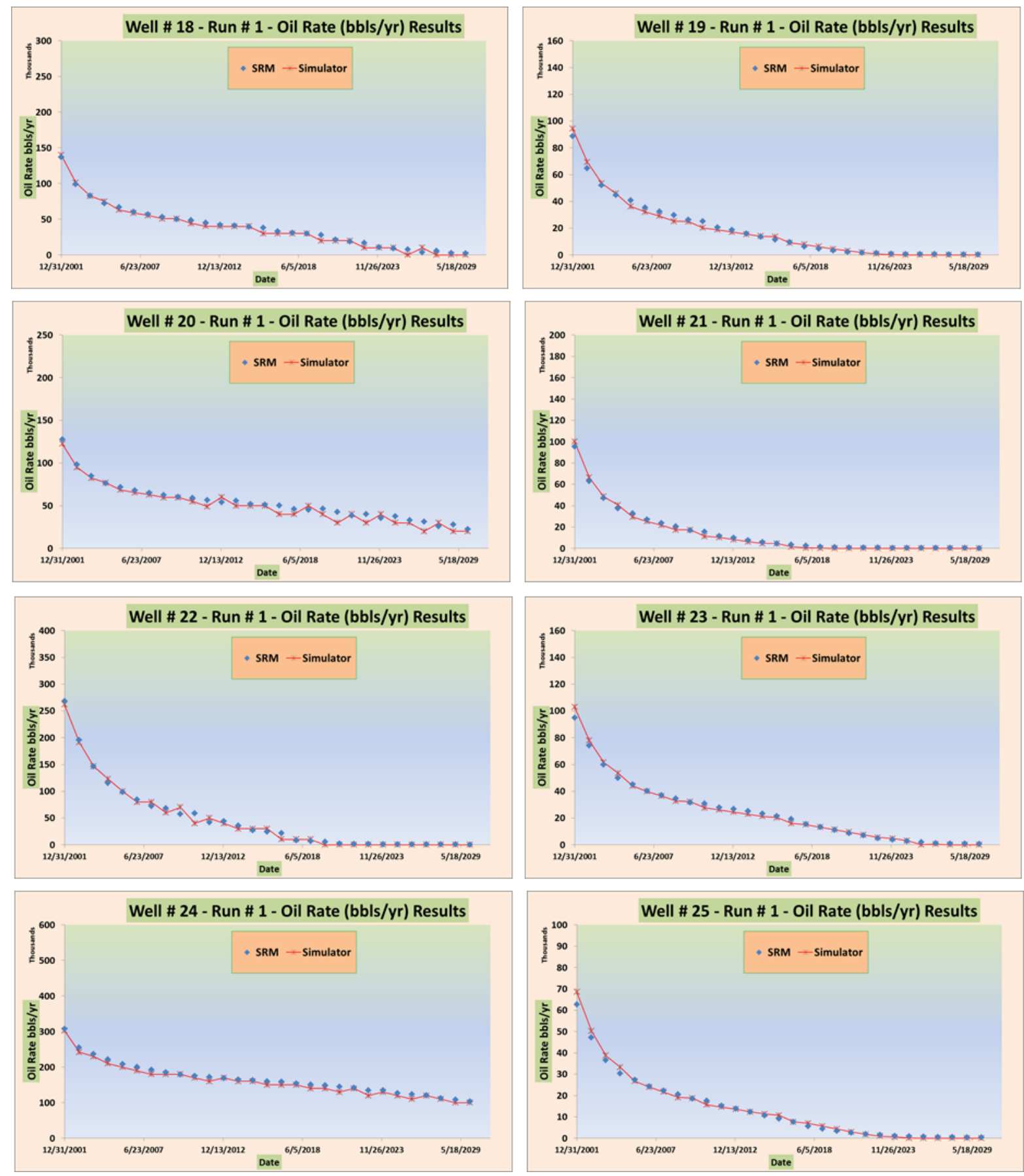

Figure 119: Comparison between the results of SRM and simulator for wells in a training realization. 


\section{c. The results of SRM - blind verification realization}
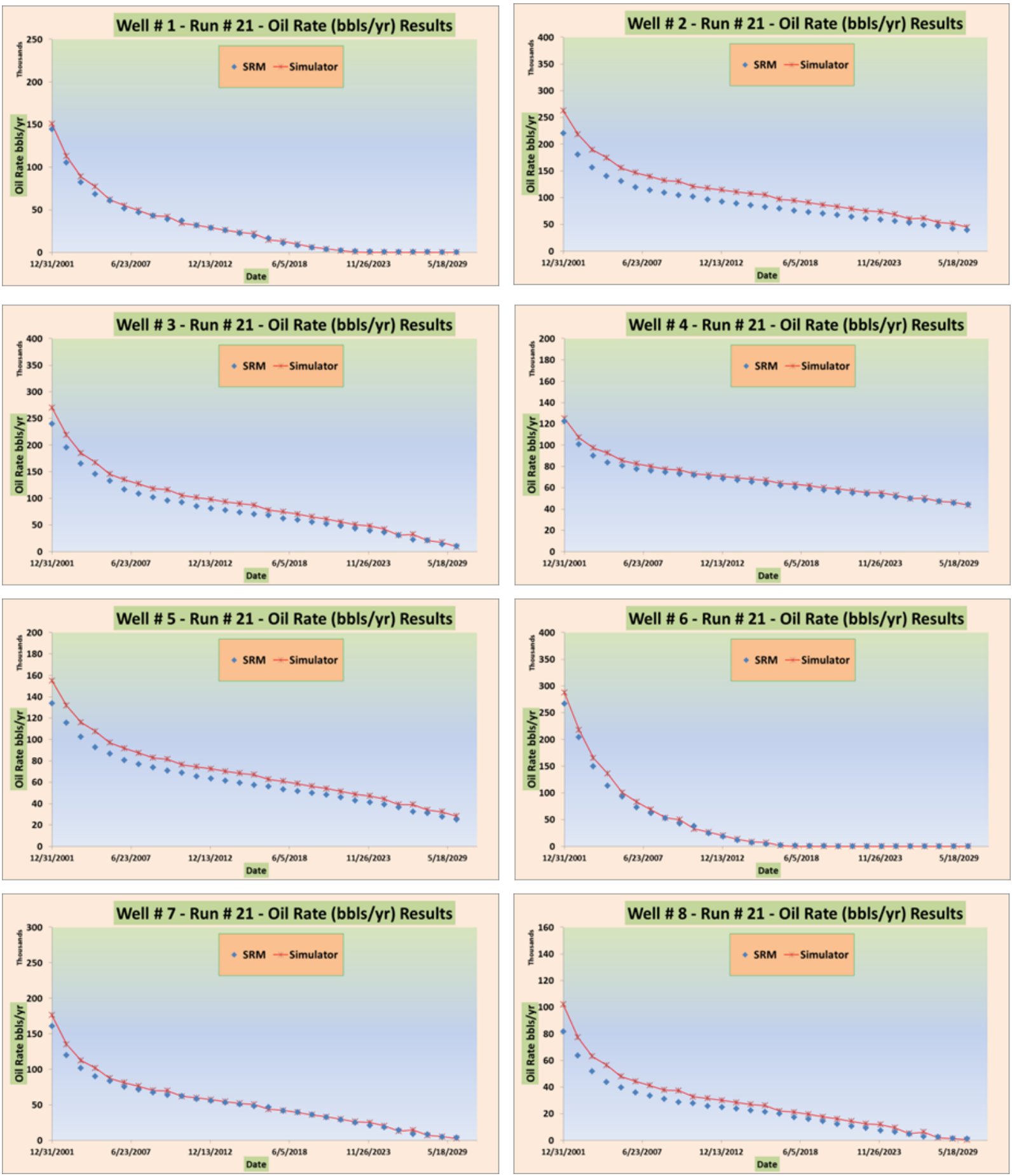

Figure 120: Comparison between the results of SRM and simulator for wells in a blind realization. 

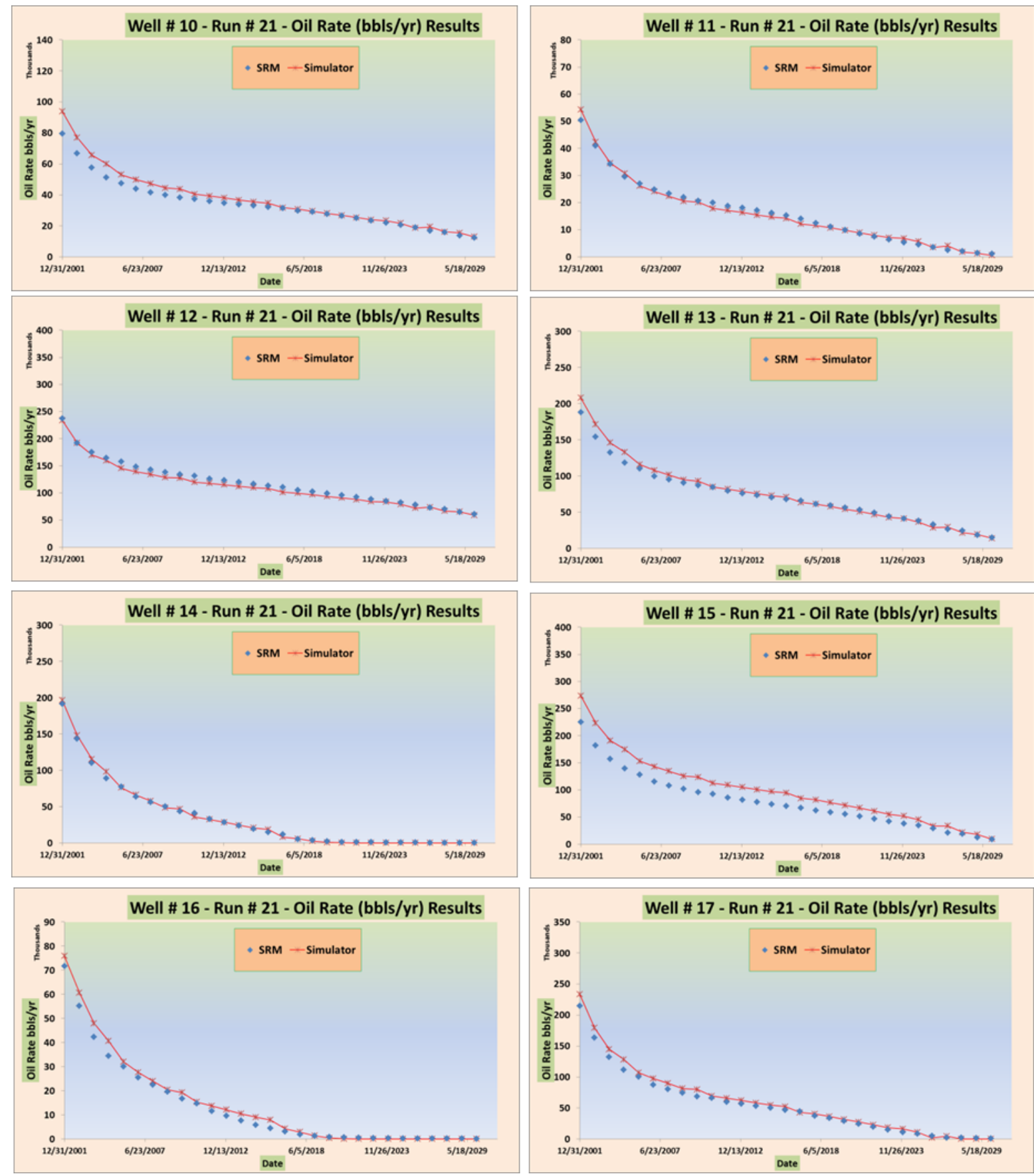

Figure 121: Comparison between the results of SRM and simulator for wells in a blind realization. 

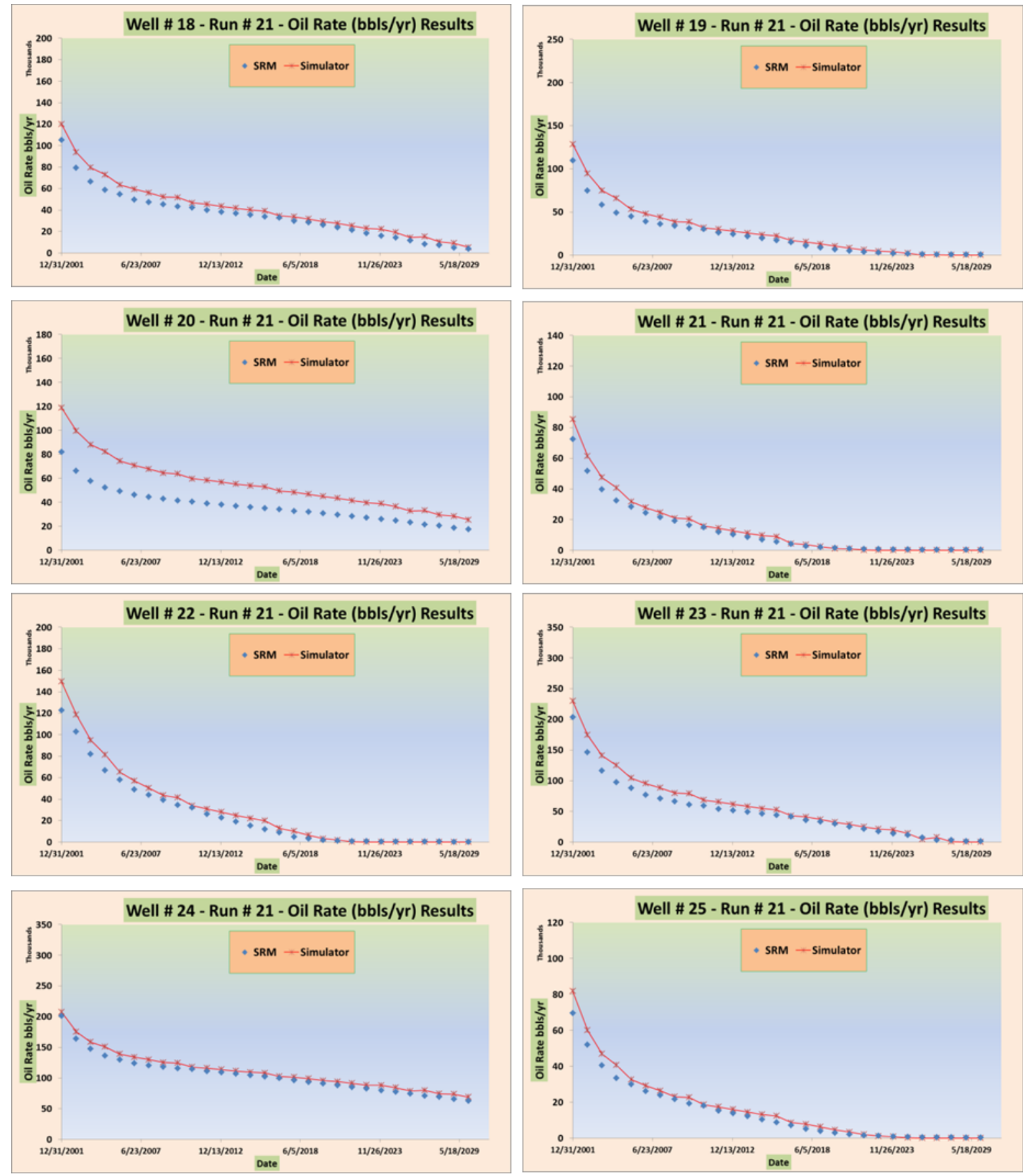

Figure 122: Comparison between the results of SRM and simulator for wells in a blind realization. 

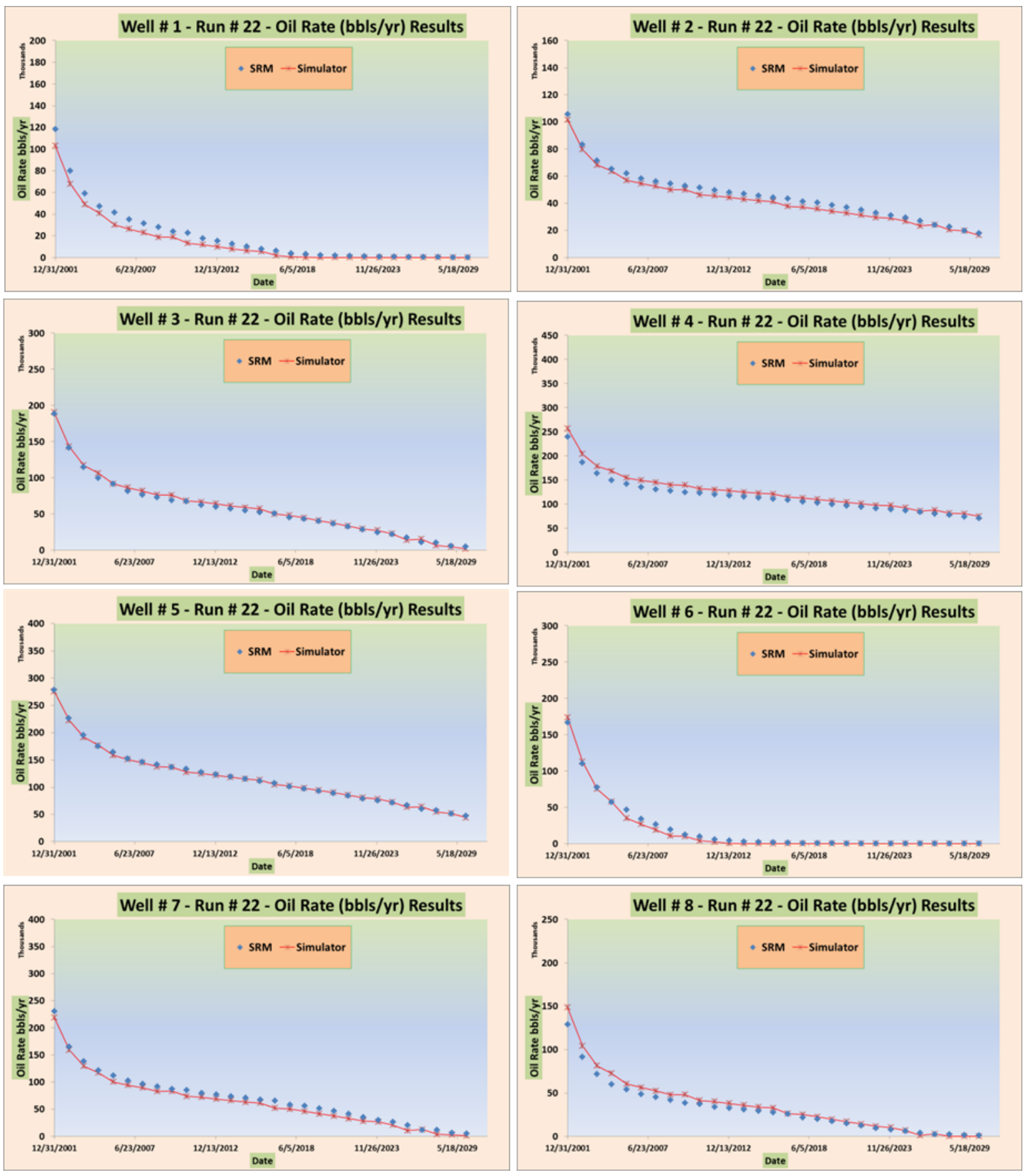

Figure 123: Comparison between the results of SRM and simulator for wells in a blind realization. 

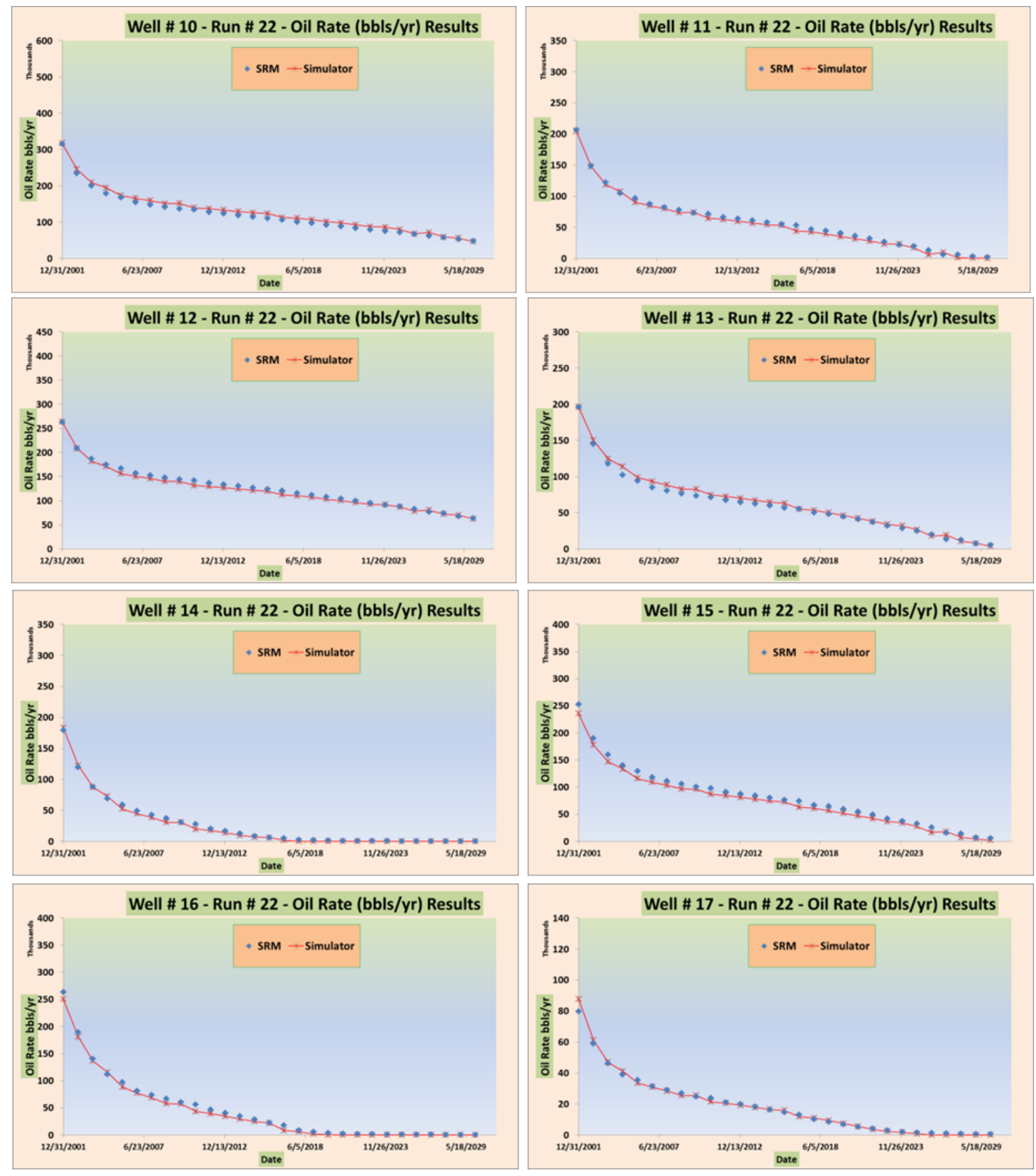

Figure 124: Comparison between the results of SRM and simulator for wells in a blind realization. 

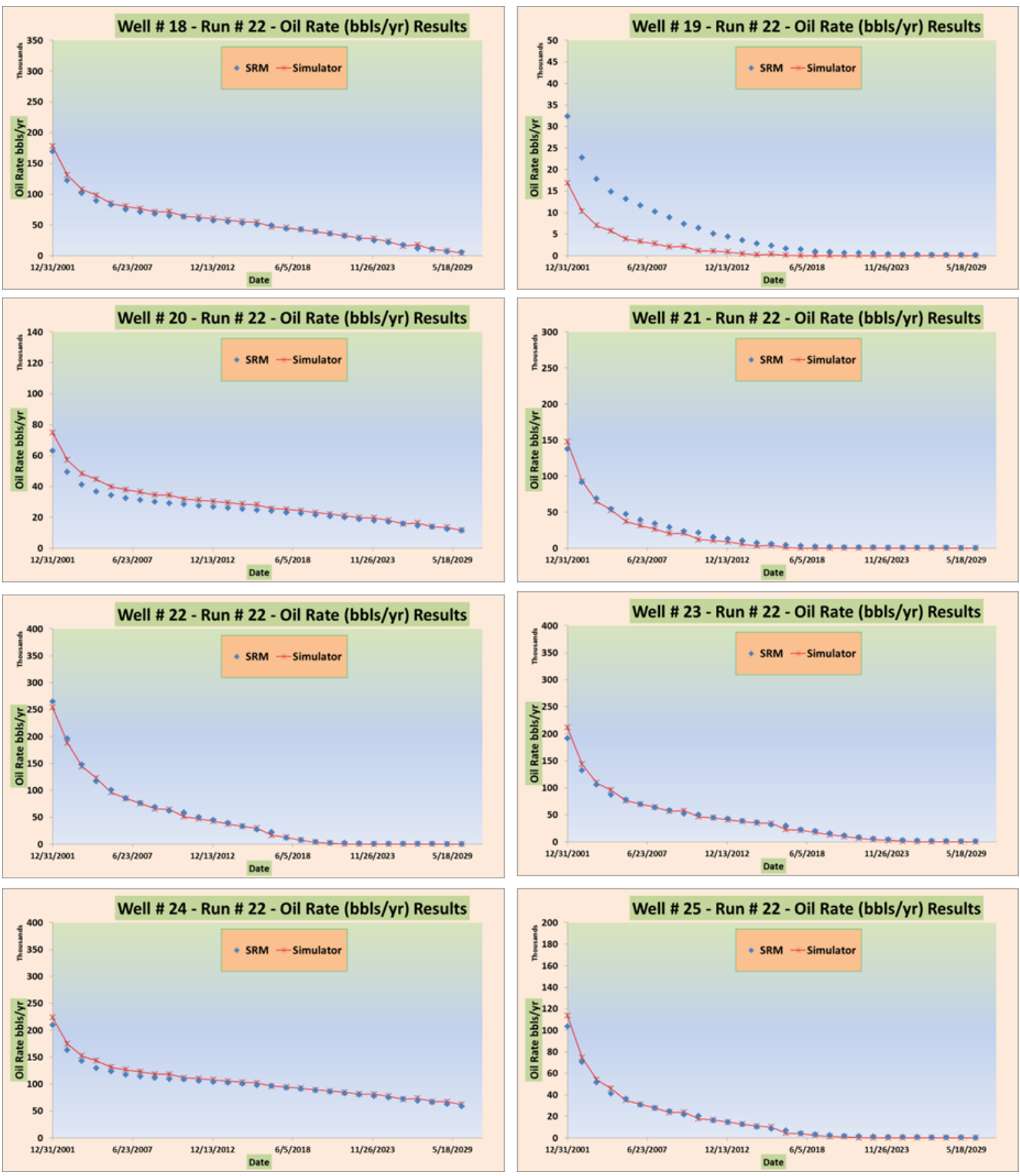

Figure 125: Comparison between the results of SRM and simulator for wells in a blind realization. 


\section{d. The results of SRM - automated history matching results}
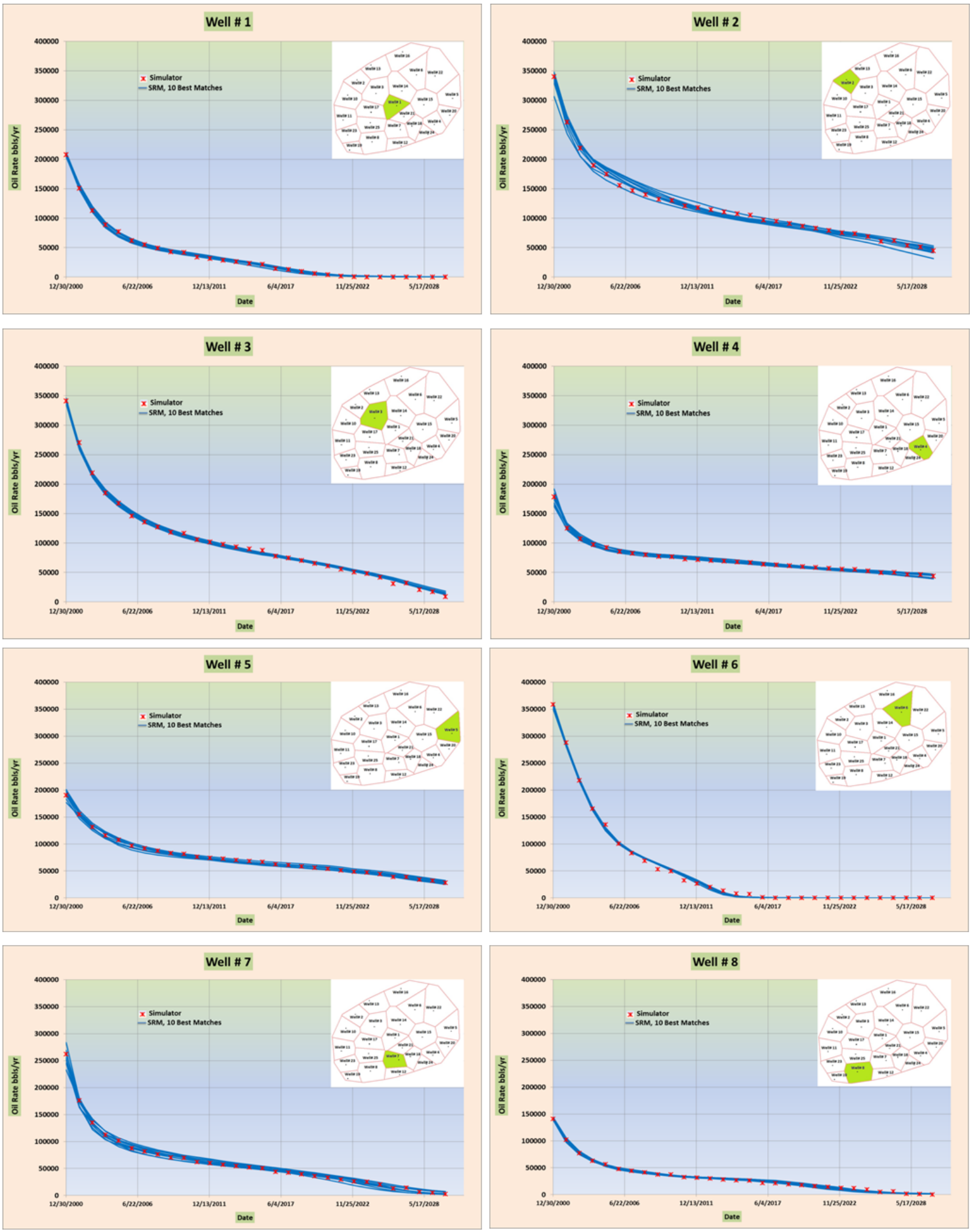

Figure 126: Comparison between ten best history matching cases (blue lines) and actual data (red stars) for different wells. 

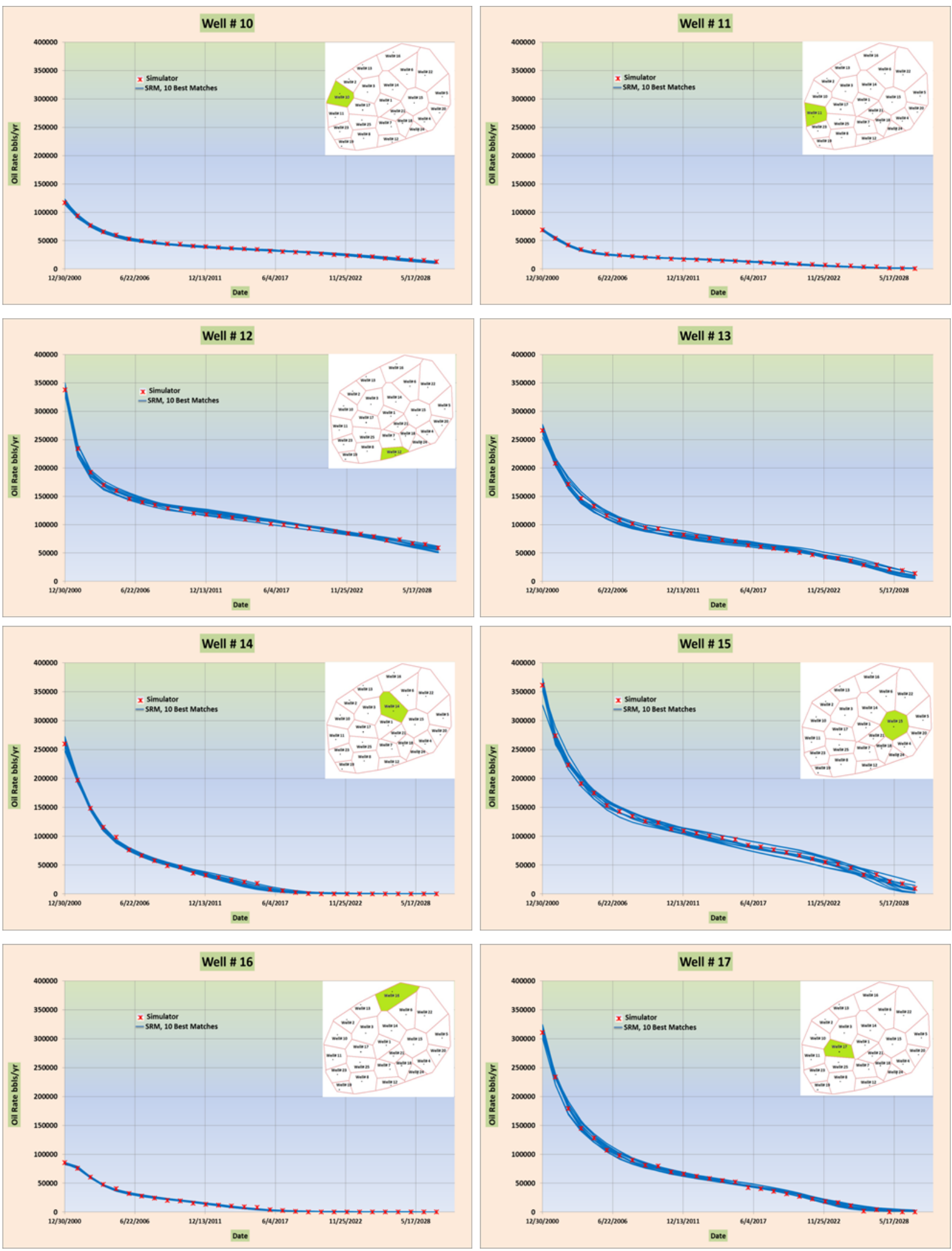

Figure 127: Comparison between ten best history matching cases (blue lines) and actual data (red stars) for different wells. 

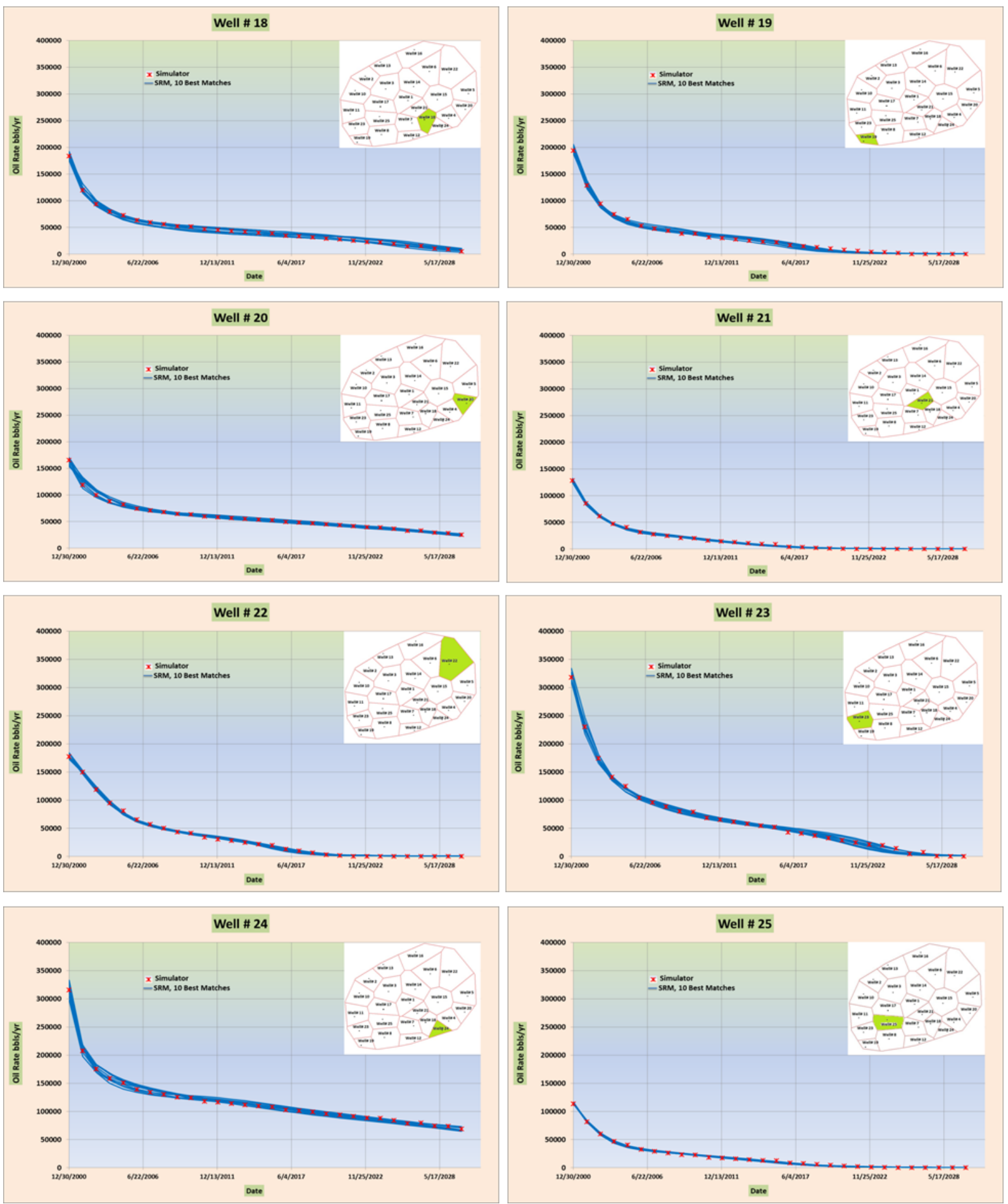

Figure 128: Comparison between ten best history matching cases (blue lines) and actual data (red stars) for different wells. 


\section{Appendix C: A real-life case study, implementation of SRM on PUNQ-S3 problem}

a. Variable reservoir characteristics for training realizations

a.1 Horizontal permeability distributions

Layer 2
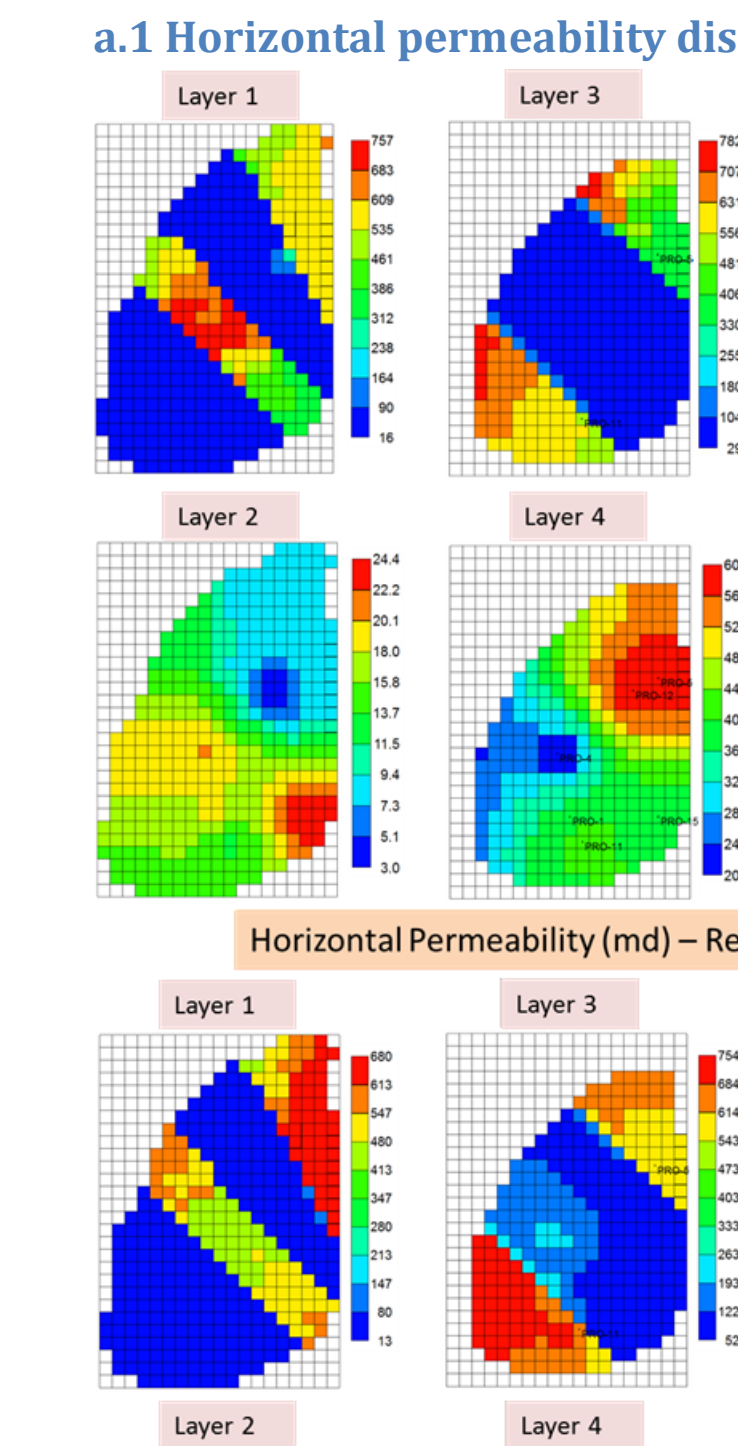

Layer 3

Layer 4
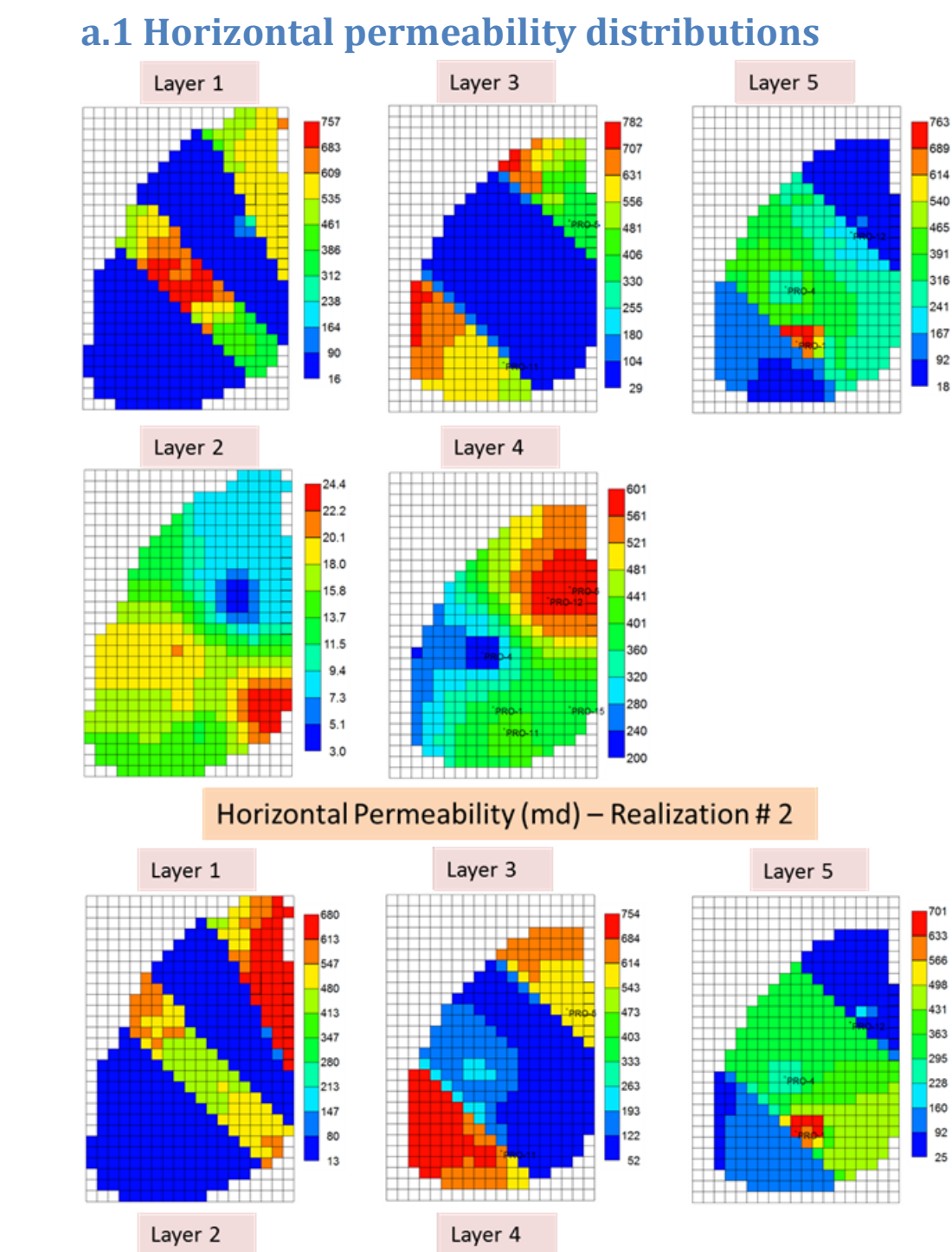

Horizontal Permeability (md) - Realization \# 2

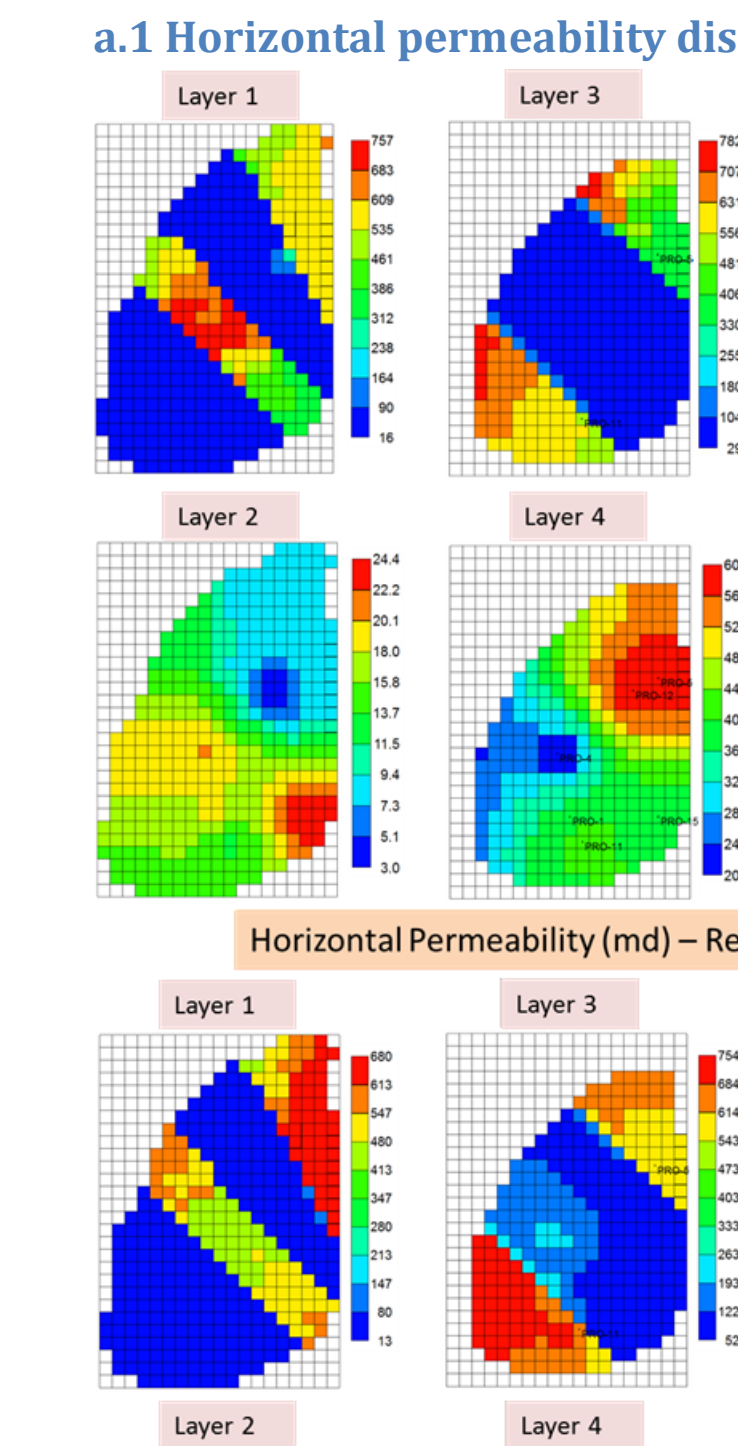

Layer 2

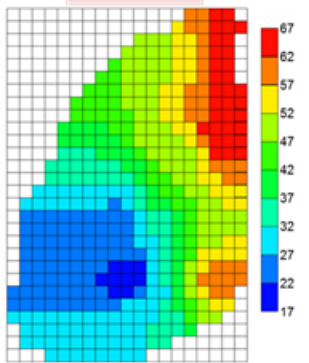

Layer 4

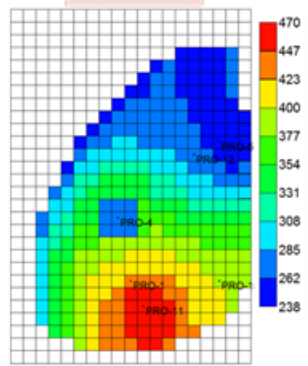

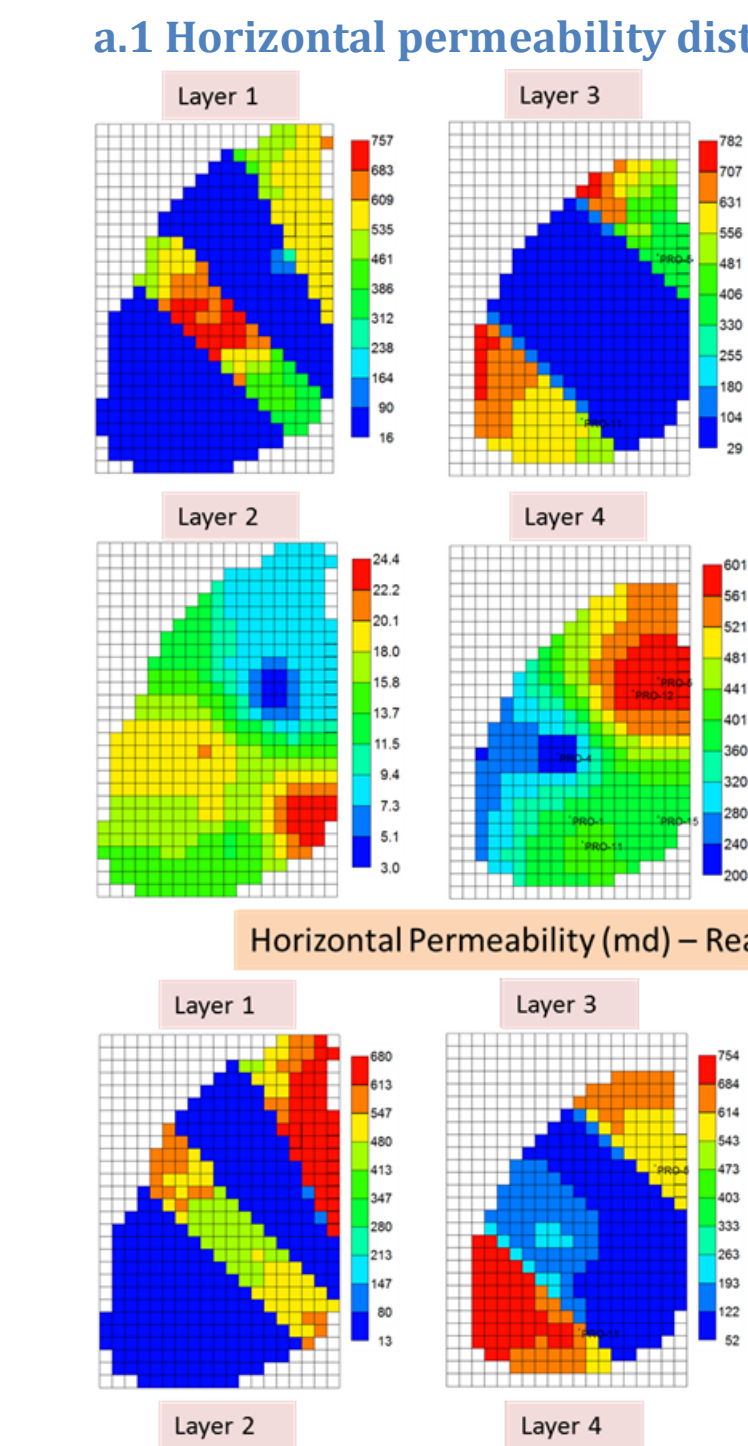

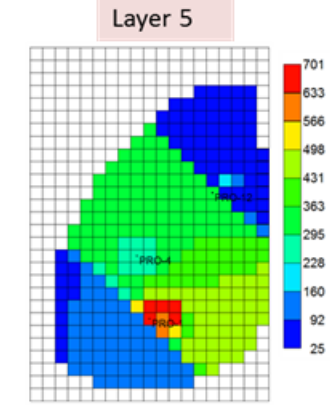

\section{tril}



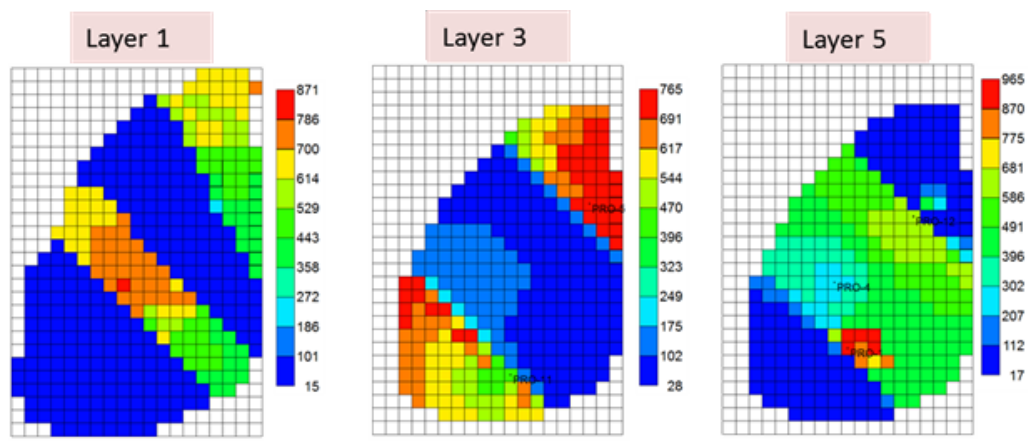

Layer 2

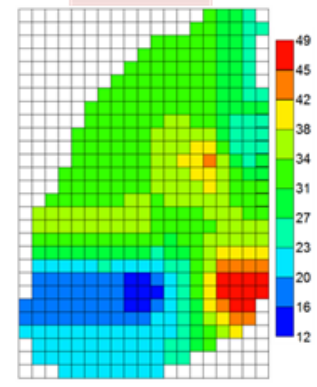

Layer 4

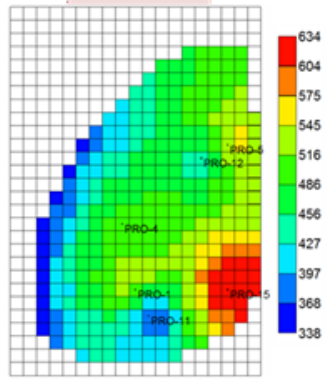

Horizontal Permeability (md) - Realization \# 4

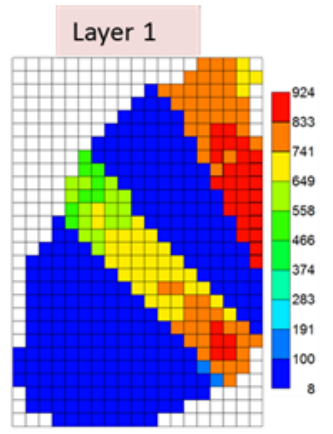

Layer 2

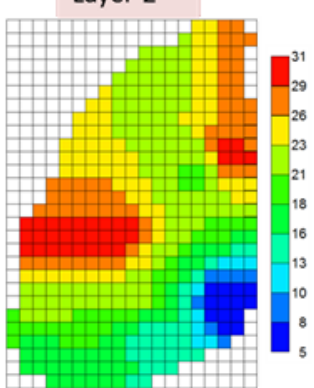

Horizontal Permeability (md) - Realization \# 5
Layer 3

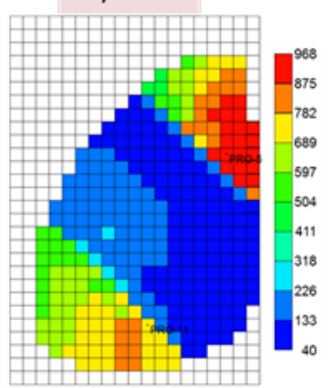

Layer 4

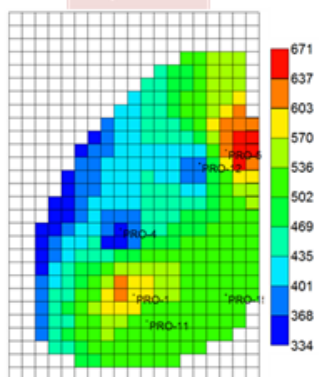

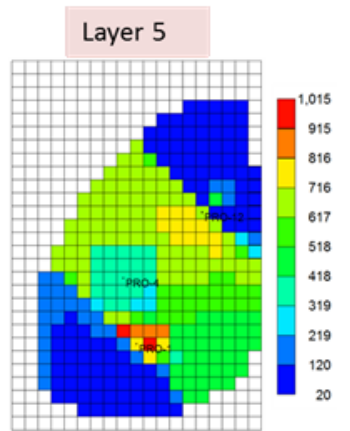

15
915
816
716
517
518
418
319
219
120
20

Figure 130: Horizontal permeability distributions for the training realizations. 

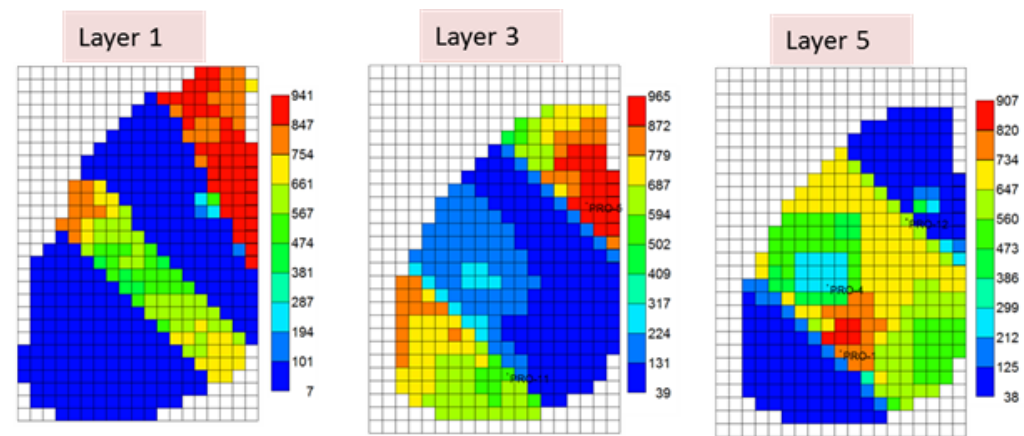

Layer 2

Layer 4
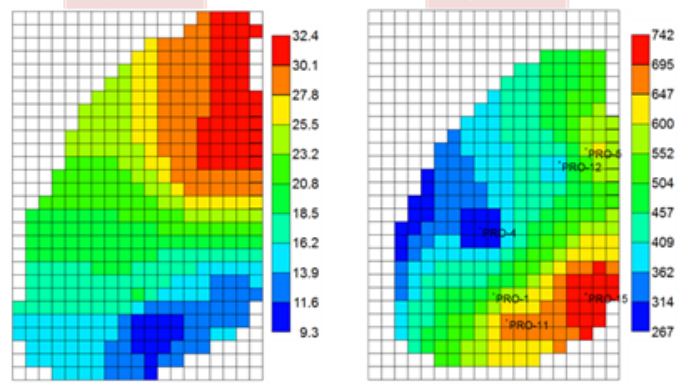

Horizontal Permeability (md) - Realization \# 6

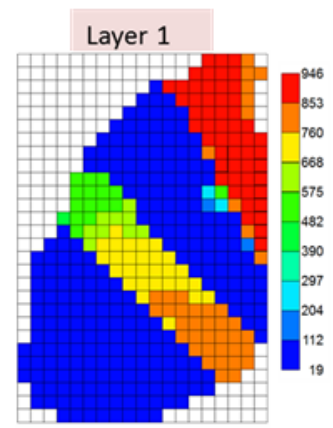

Layer 3

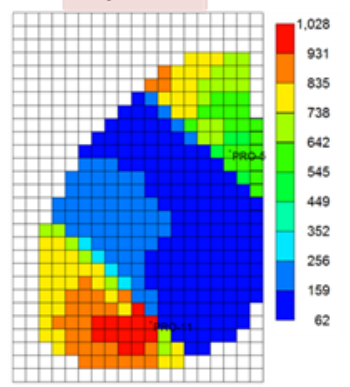

Layer 4

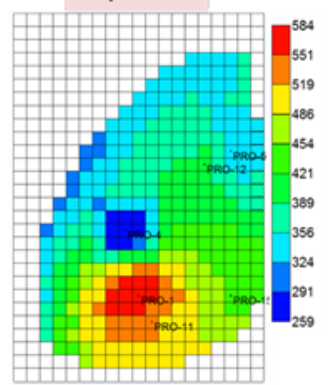

Layer 2

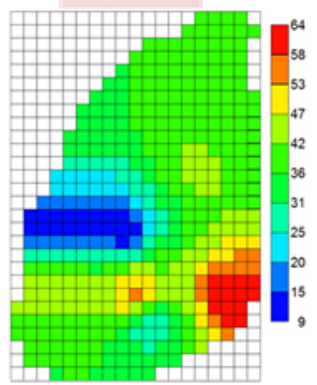

Layer 5

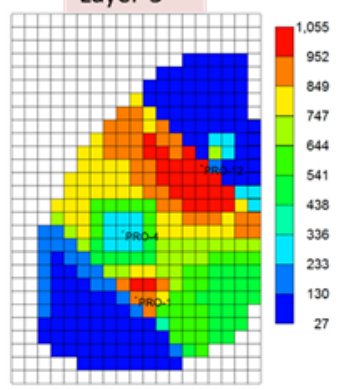

Horizontal Permeability (md) - Realization \# 7

Figure 131: Horizontal permeability distributions for the training realizations. 

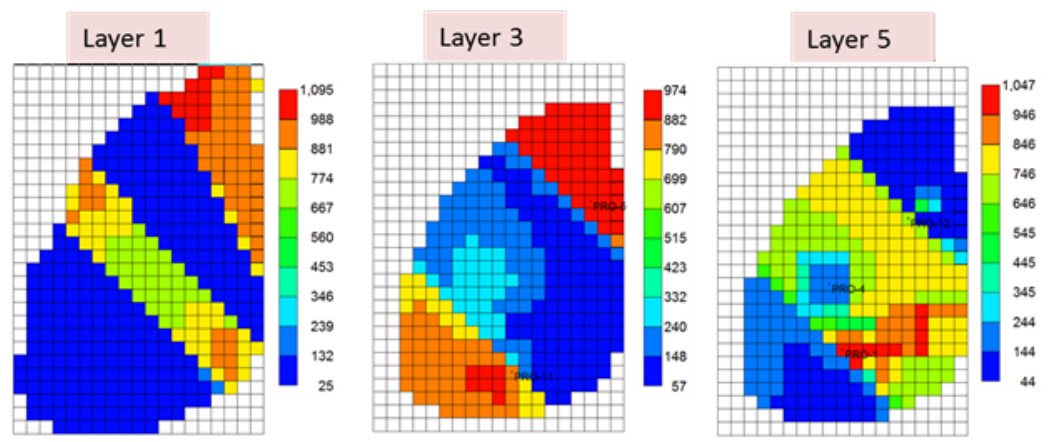

Layer 2

Layer 4
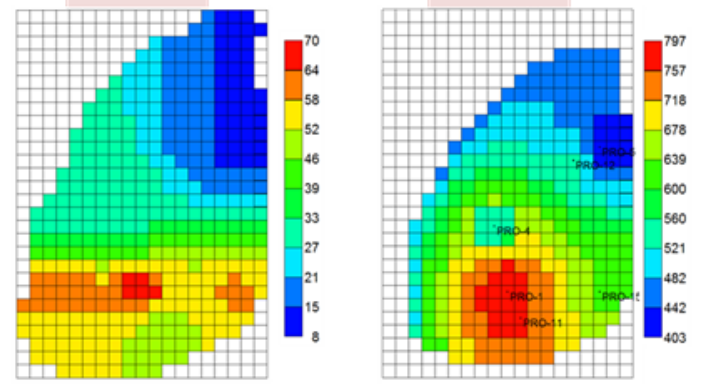

Horizontal Permeability (md) - Realization \# 8

Layer 1

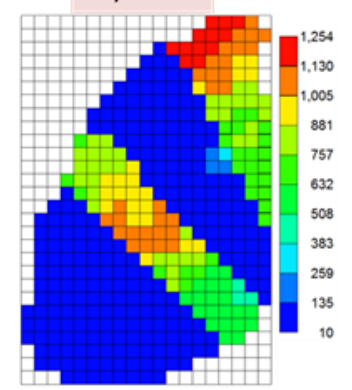

Layer 2

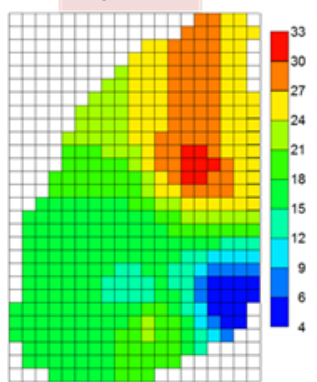

Layer 3

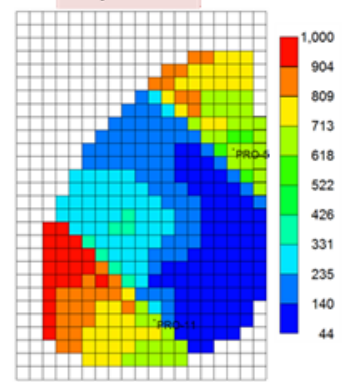

Layer 4

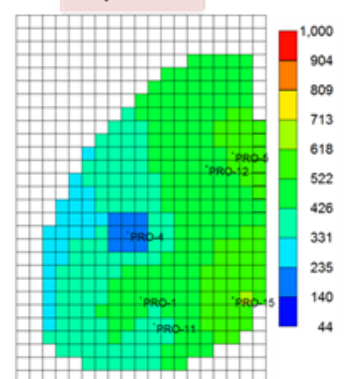

Horizontal Permeability (md) - Realization \# 9

Figure 132: Horizontal permeability distributions for the training realizations. 

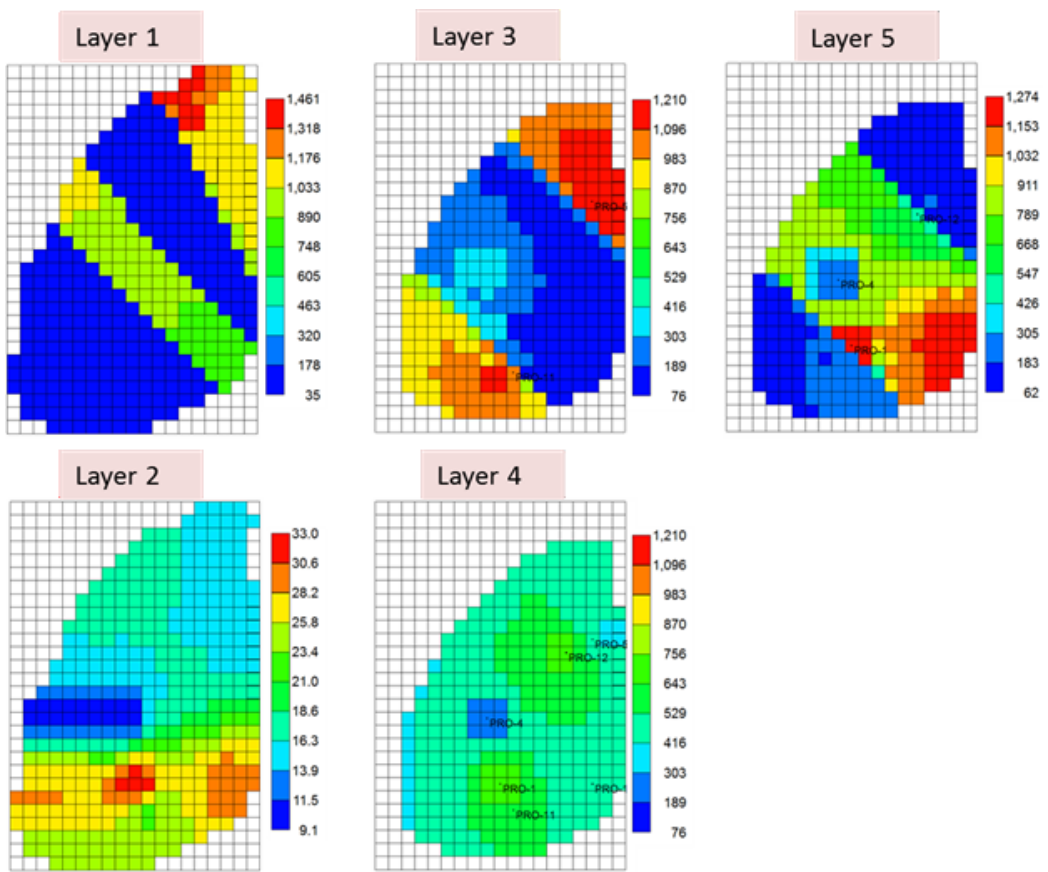

Horizontal Permeability (md) - Realization \# 10

Figure 133: Horizontal permeability distributions for the training realizations. 
a.2 Vertical permeability distributions
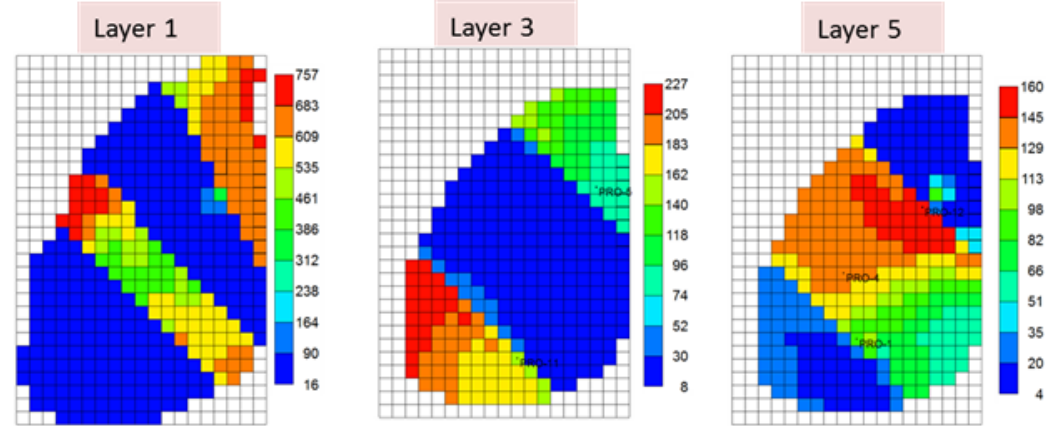

Layer 2
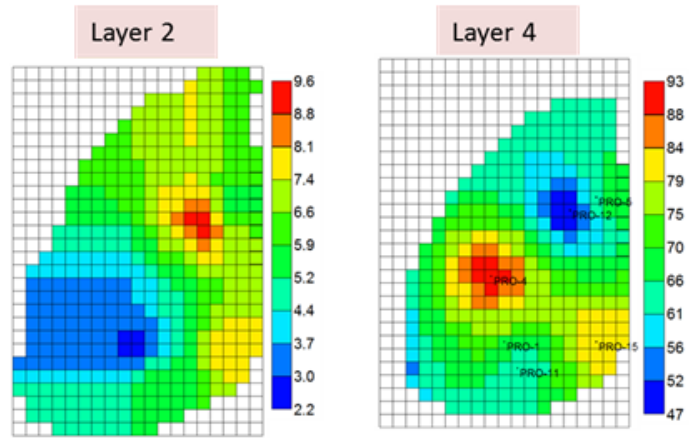

\section{Vertical Permeability (md) - Realization \# 2}

Layer 1

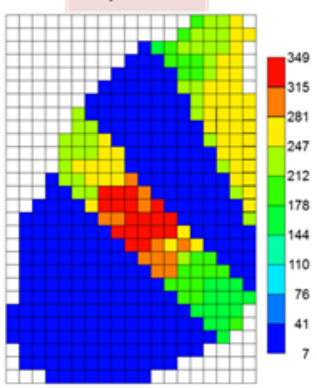

Layer 2

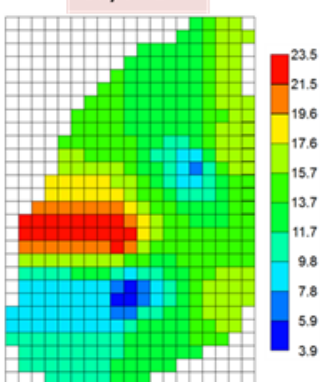

Layer 3

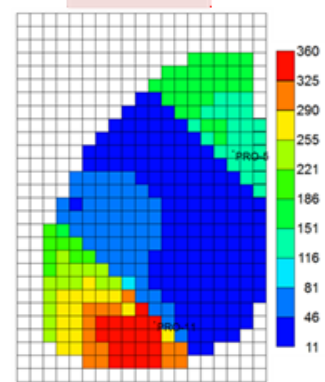

Layer 4

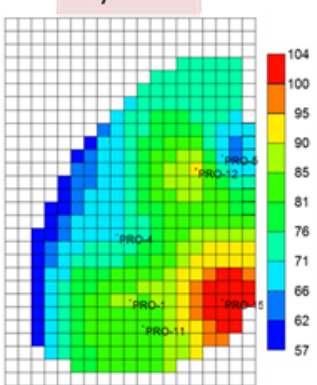

Vertical Permeability (md) - Realization \# 3

Figure 134: Vertical permeability distributions for the training realizations. 

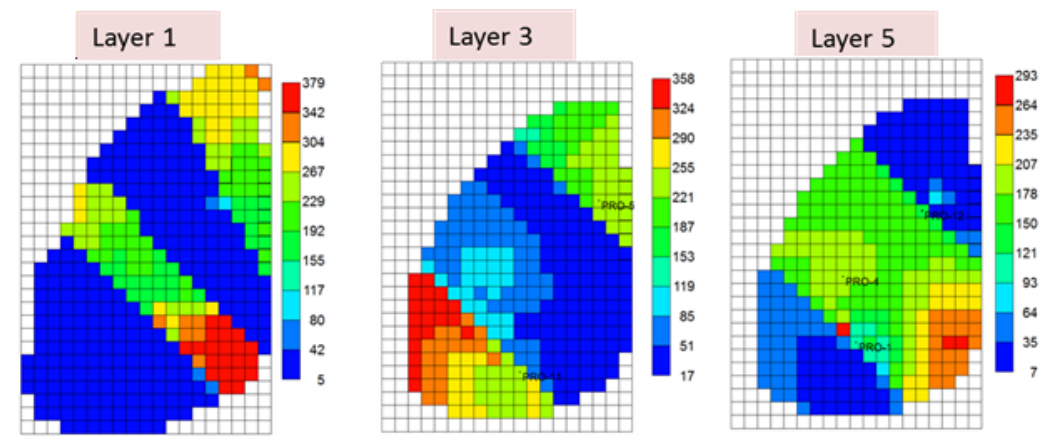

Layer 2
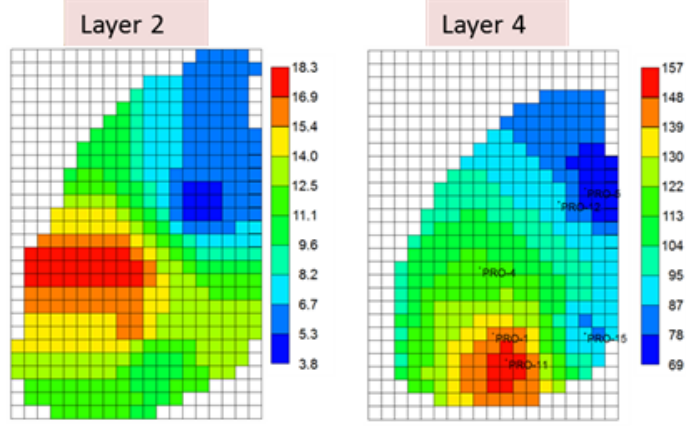

Vertical Permeability (md) - Realization \# 4

Layer 1

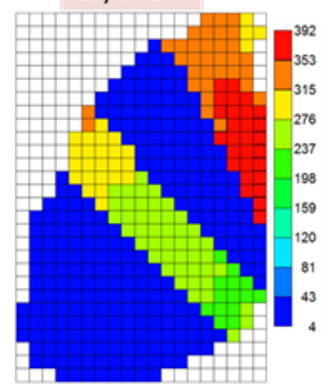

Layer 2

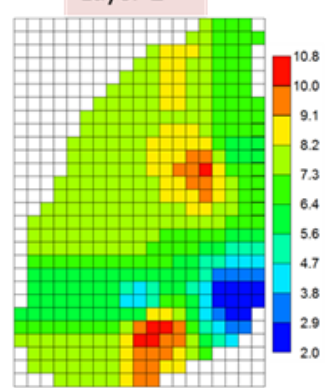

Layer 3

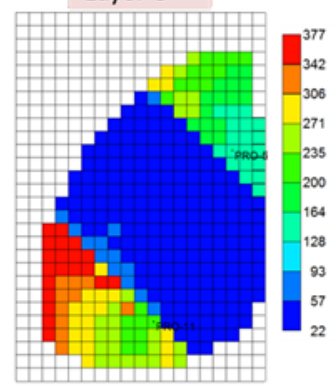

Layer 4

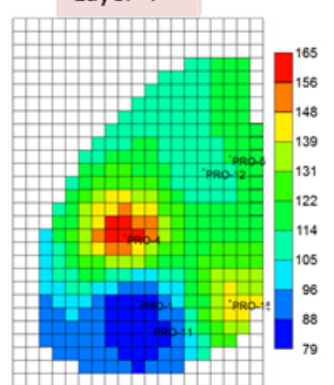

Layer 5

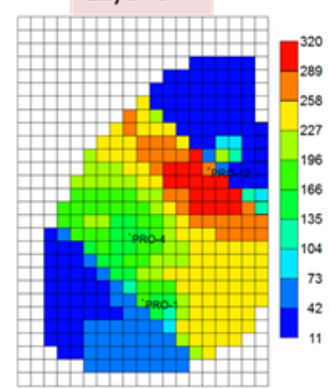

Vertical Permeability (md) - Realization \# 5

Figure 135: Vertical permeability distributions for the training realizations. 

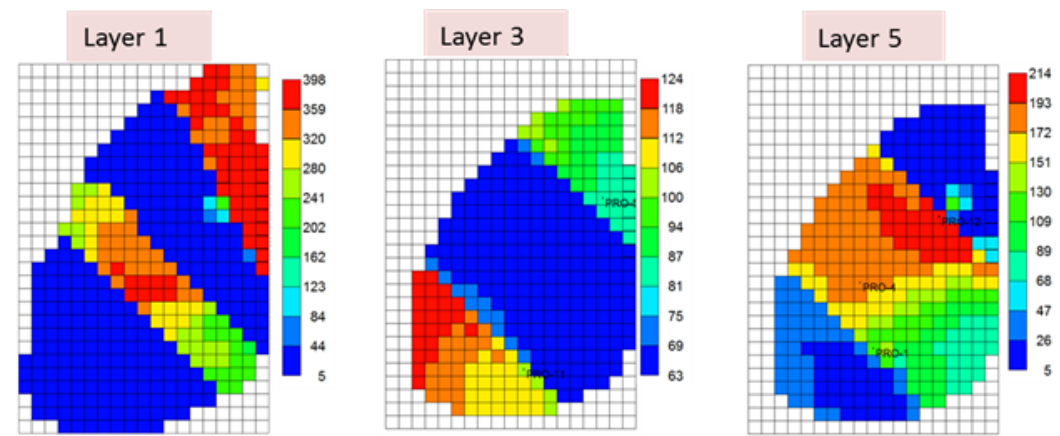

Layer 2
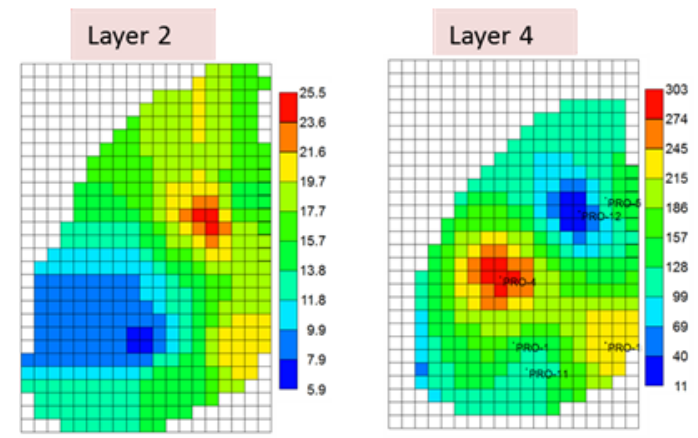

Vertical Permeability (md) - Realization \# 6
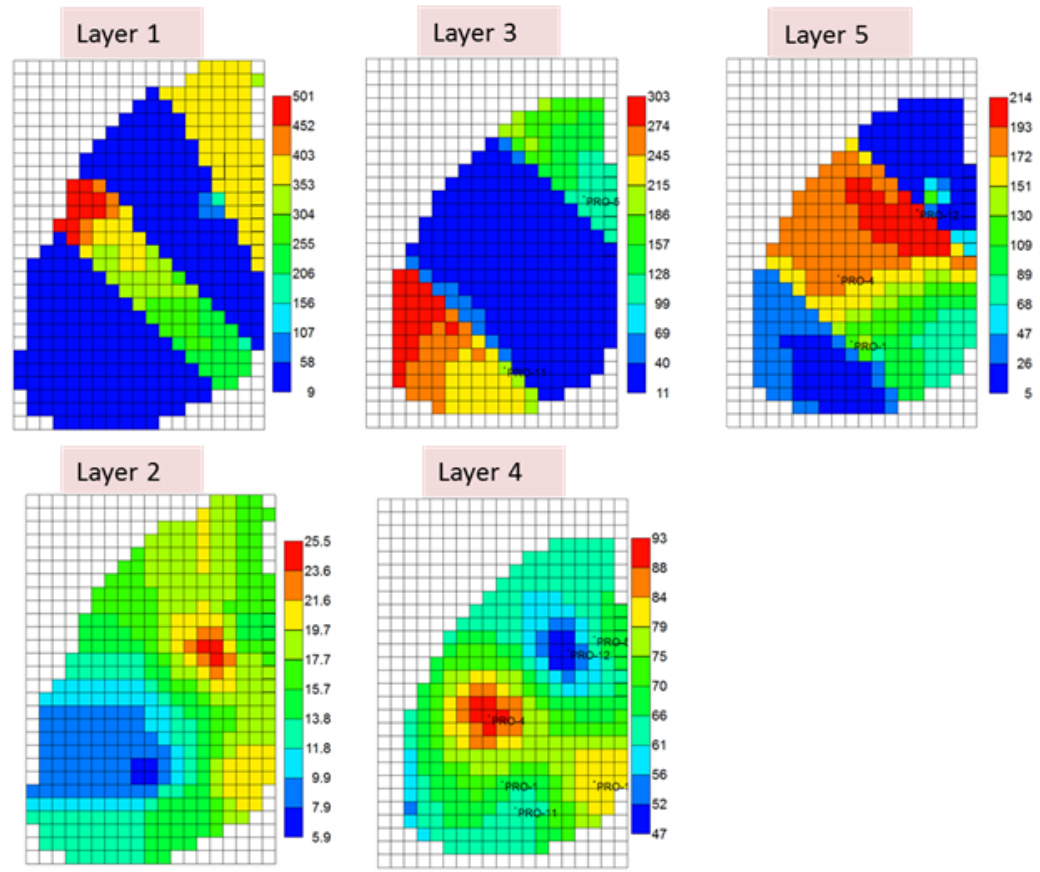

Vertical Permeability (md) - Realization \# 7

Figure 136: Vertical permeability distributions for the training realizations. 


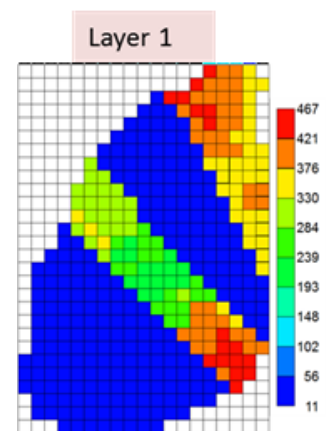

Layer 2

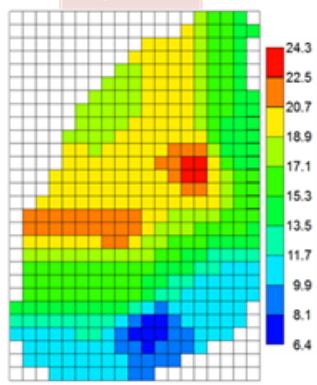

Vertical Permeability (md) - Realization \# 8

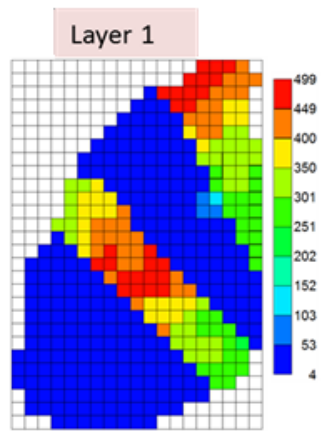

Layer 2

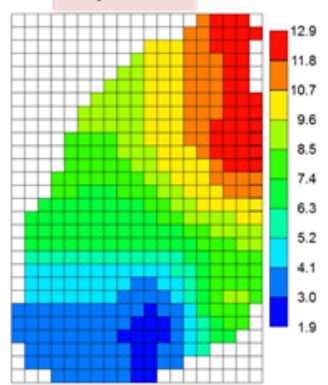

Vertical Permeability (md) - Realization \# 9

Layer 3

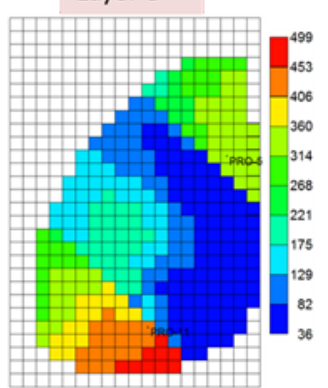

Layer 4

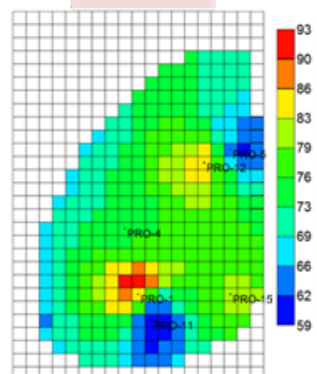$$
\text { (n) }
$$$$
\text { . }
$$

Layer 3

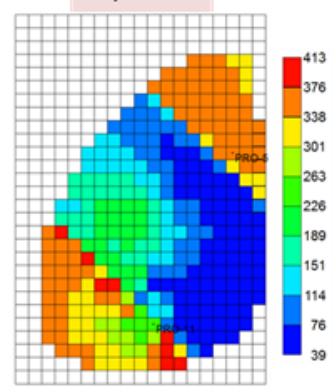

Layer 4

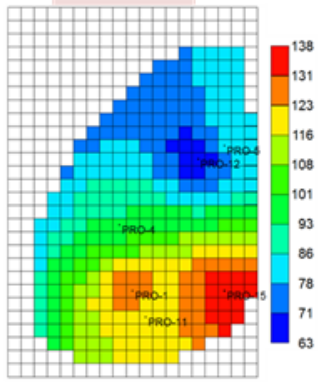

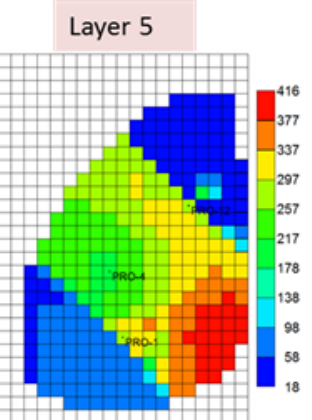

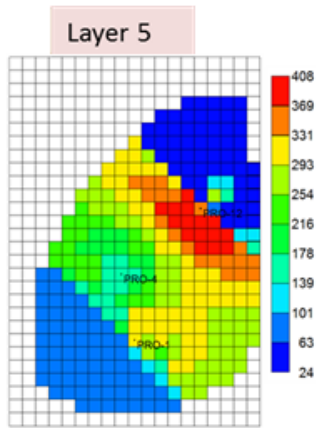



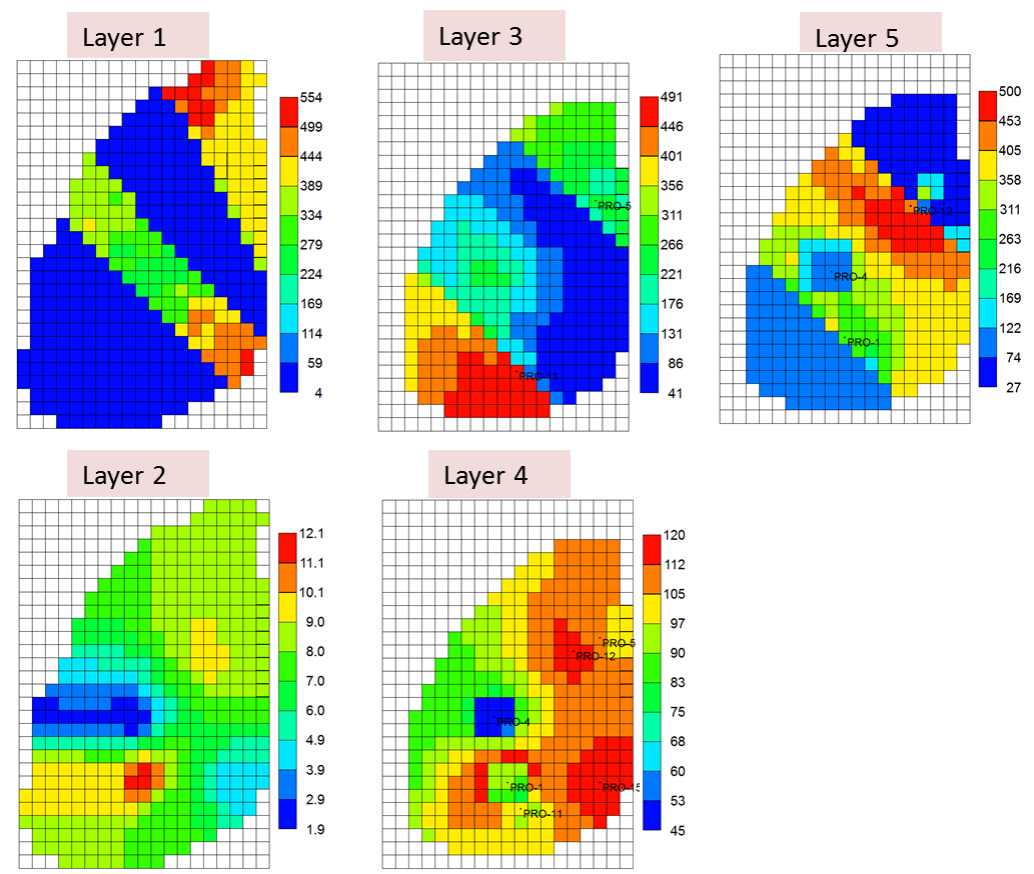

Vertical Permeability (md) - Realization \# 10

Figure 138: Vertical permeability distributions for the training realizations. 
a.3 Porosity distributions

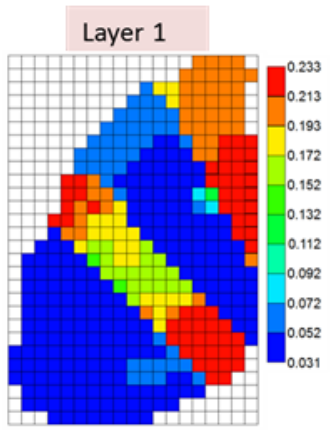

Layer 2

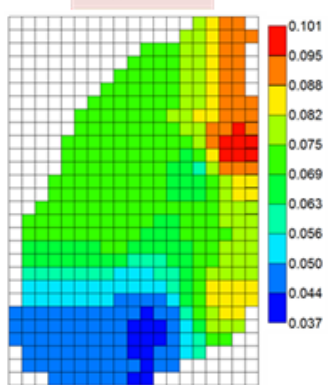

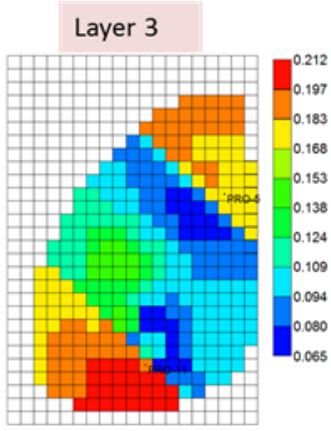

Layer 4

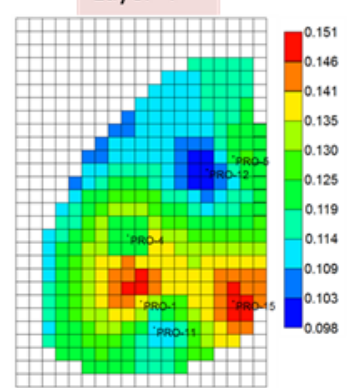

$\begin{array}{r}151 \\ 146 \\ 141 \\ 135 \\ 130 \\ .25 \\ .19 \\ 1.14 \\ 109 \\ 103 \\ \hline 98 \\ \hline\end{array}$

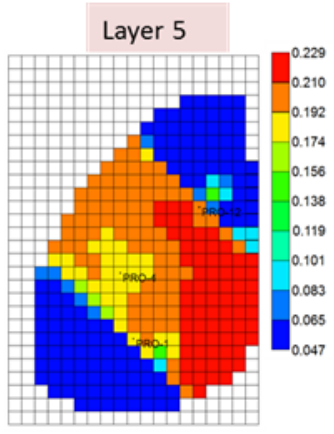

Porosity (\%) - Realization \# 2
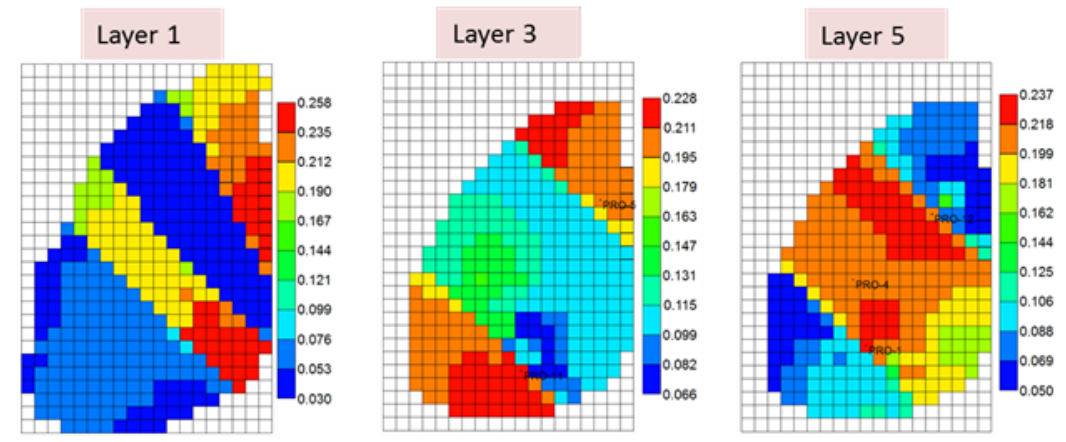

Layer 2

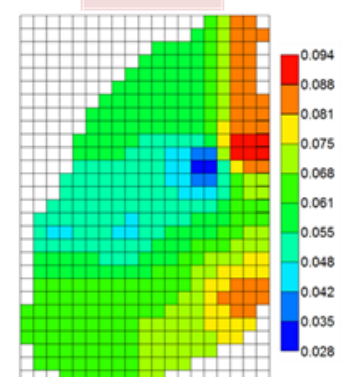

Layer 4

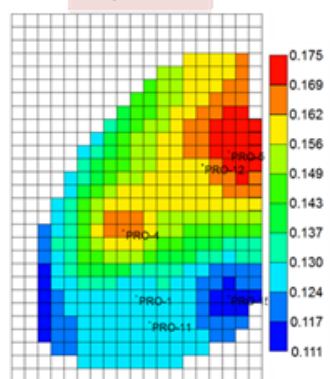

Porosity (\%) - Realization \# 3

Figure 139: Porosity distributions for the training realizations. 

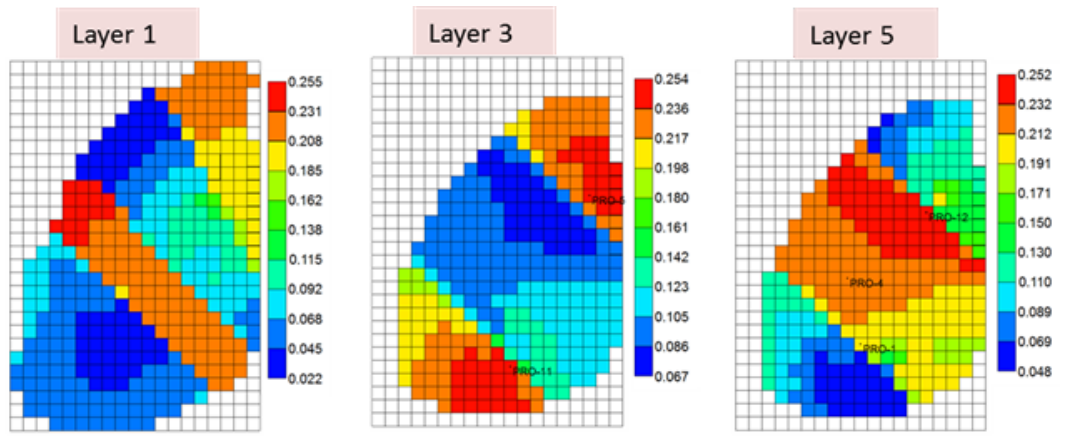

Layer 2

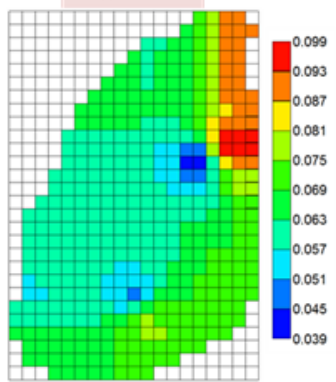

Layer 4

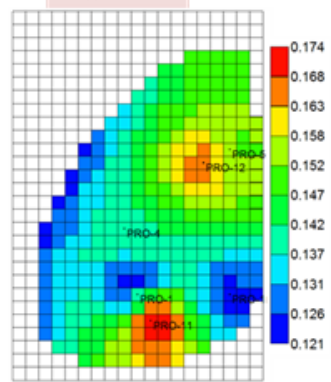

Porosity (\%) - Realization \# 4
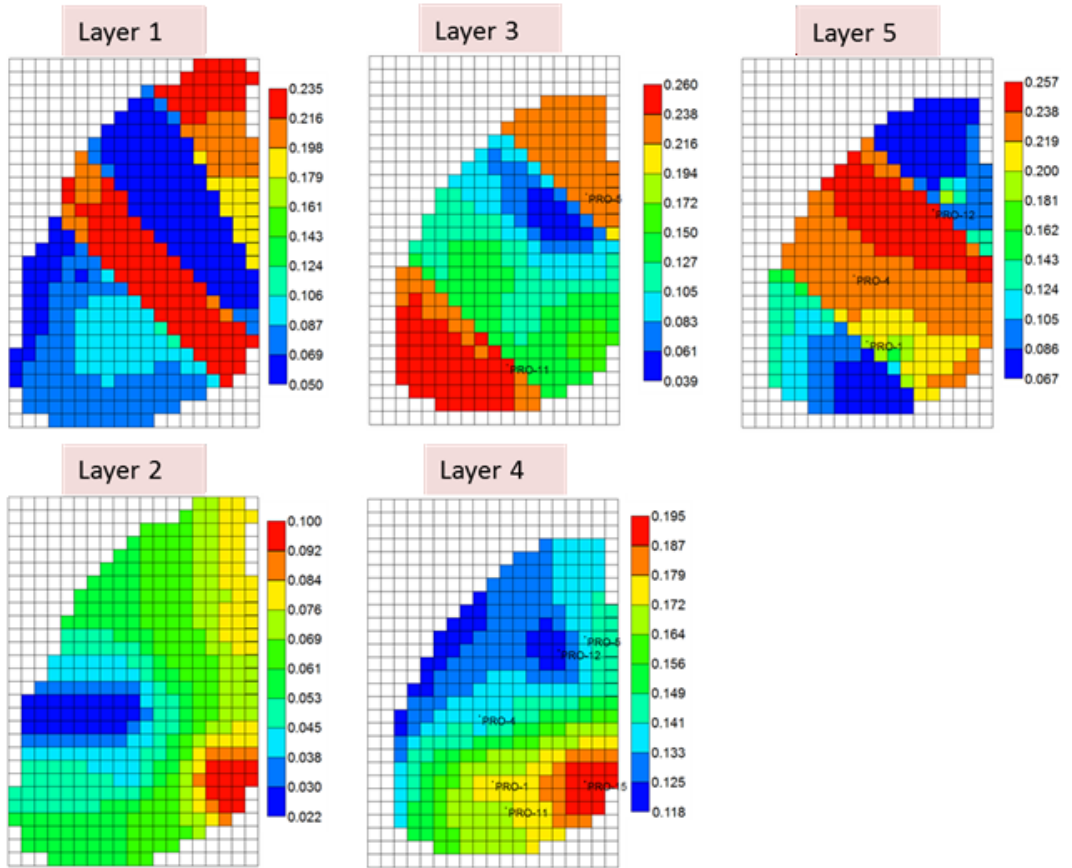

\section{Porosity (\%) - Realization \# 5}

Figure 140: Porosity distributions for the training realizations. 

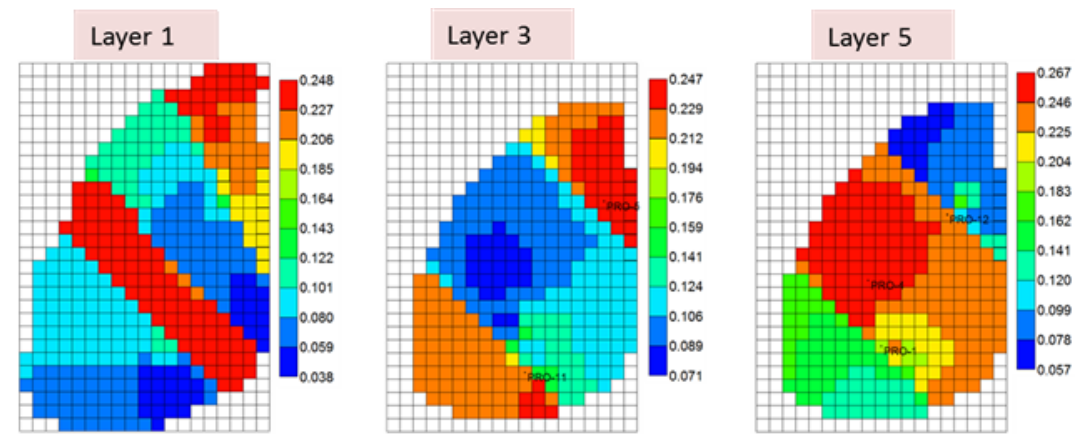

Layer 2

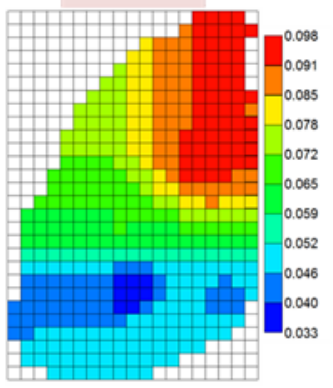

Layer 4

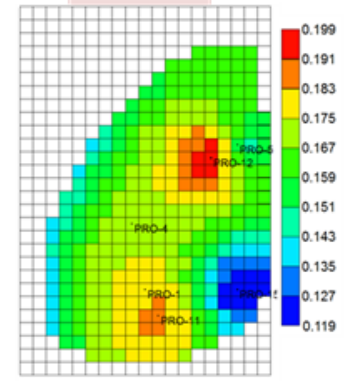

Porosity (\%) - Realization \# 6
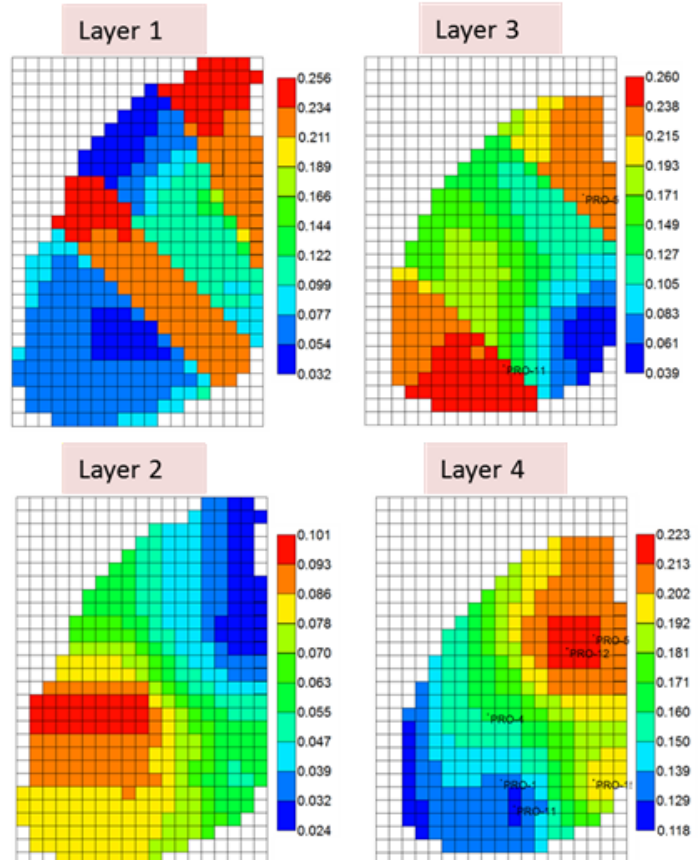

Layer 3

Layer 4

Layer 5
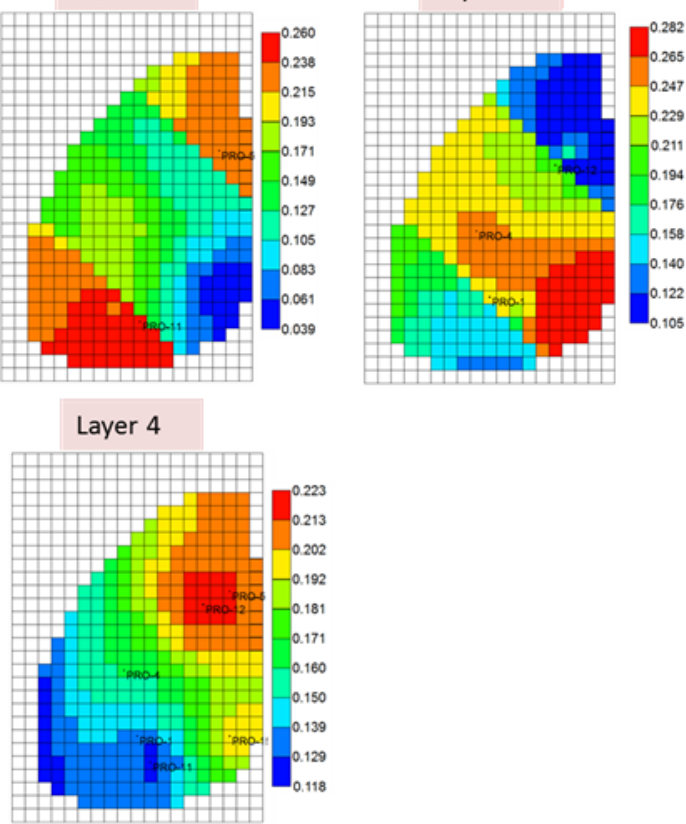

Porosity (\%) - Realization \# 7

Figure 141: Porosity distributions for the training realizations. 

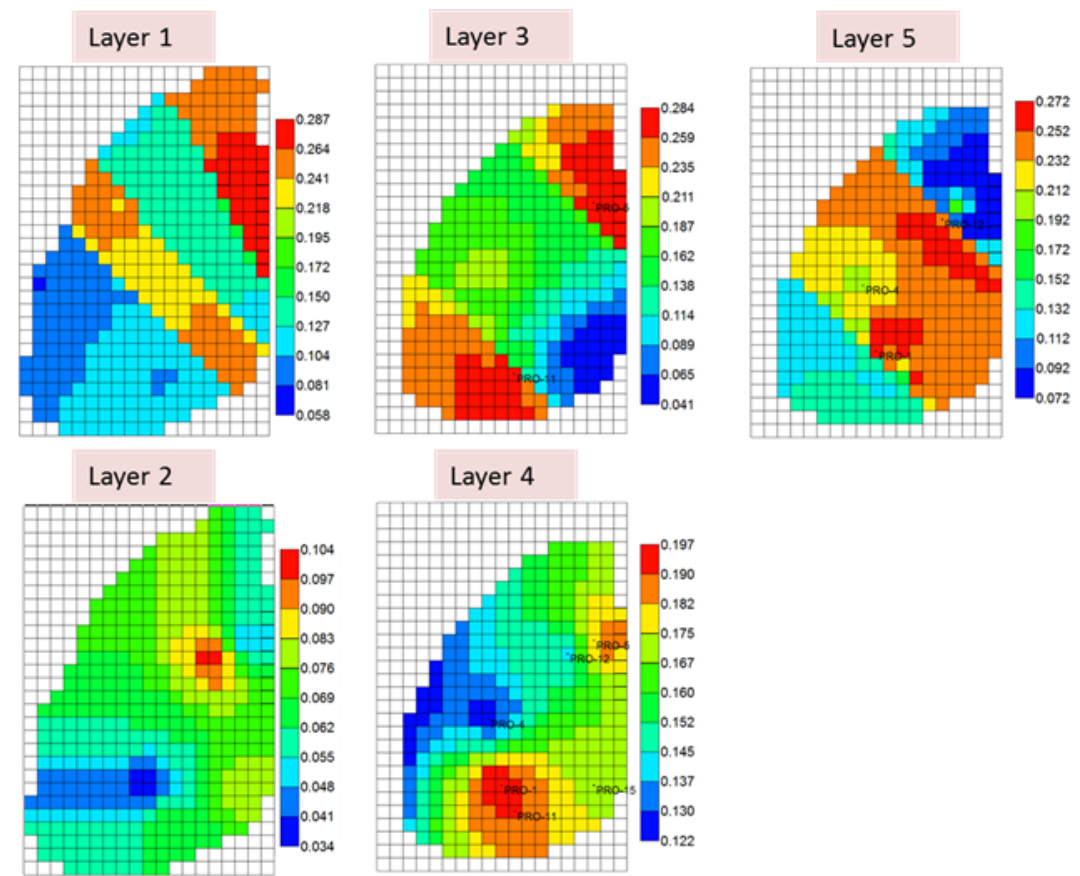

\section{Porosity (\%) - Realization \# 8}

Layer 1

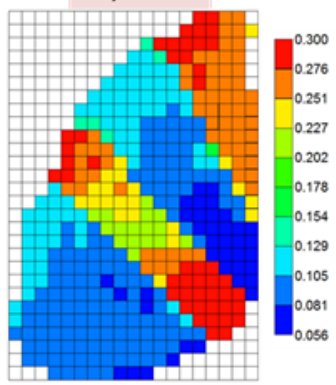

Layer 2

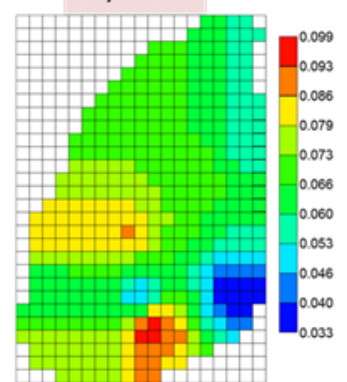

Layer 3

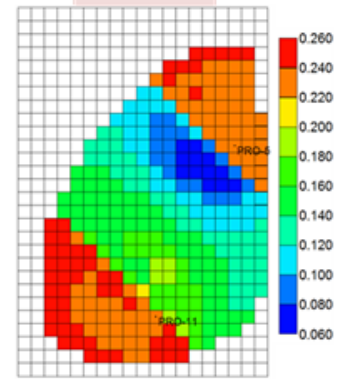

Layer 4

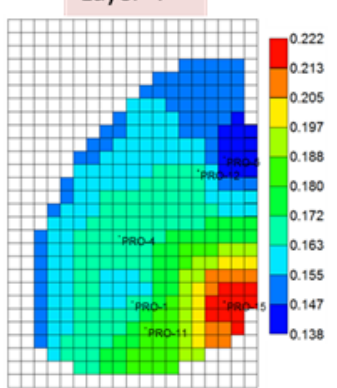

Layer 5

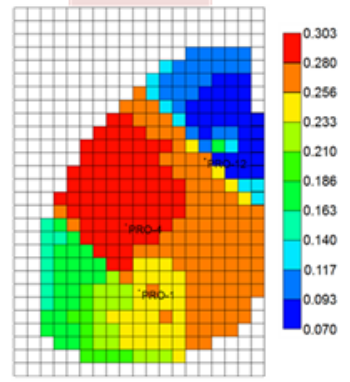

\section{Porosity (\%) - Realization \# 9}

Figure 142: Porosity distributions for the training realizations. 

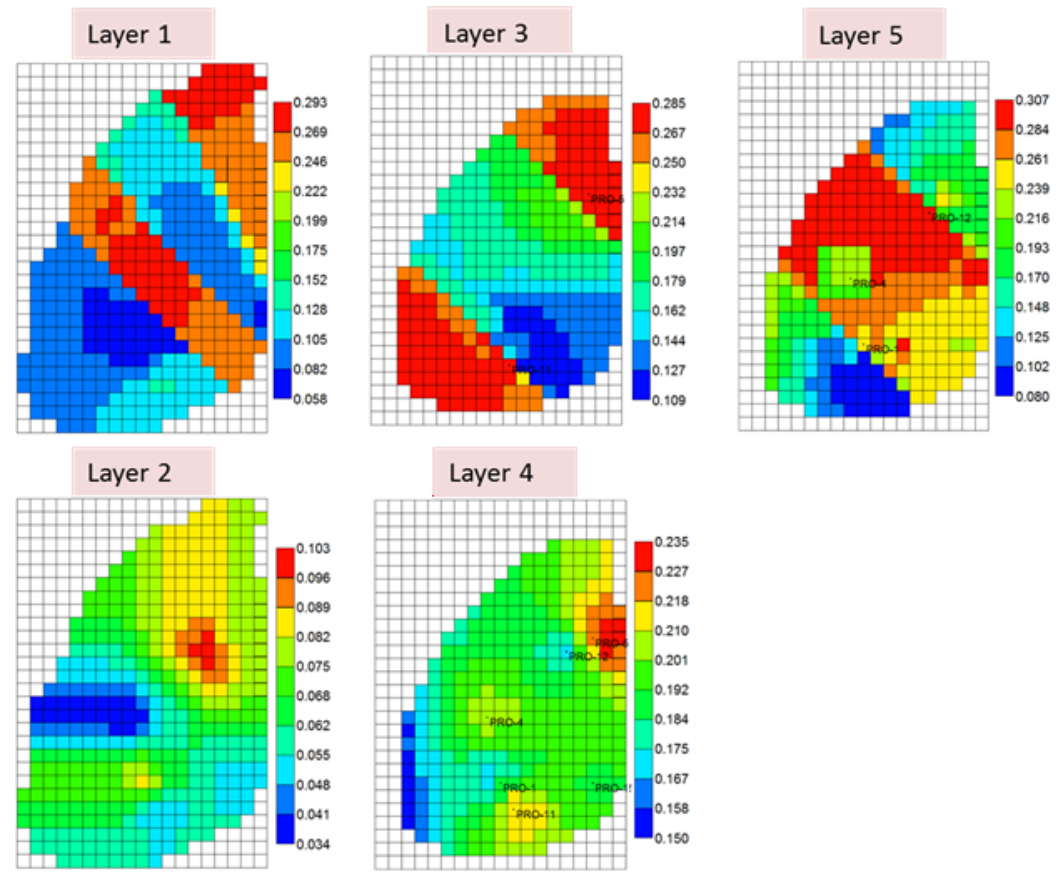

Porosity (\%) - Realization \# 10

Figure 143: Porosity distributions for the training realizations. 
b. Variable reservoir characteristics for the blind realization

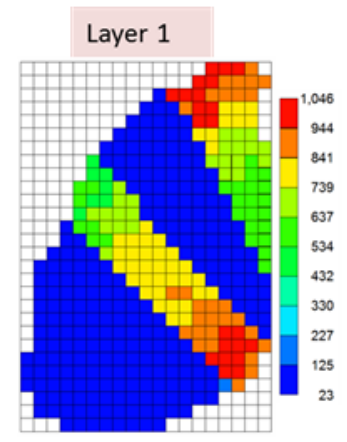

Layer 2

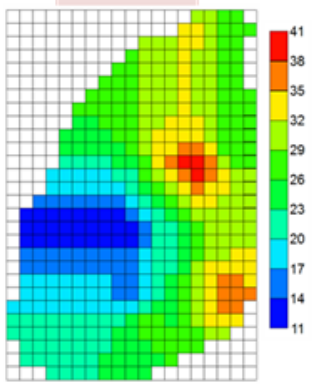

Layer 3

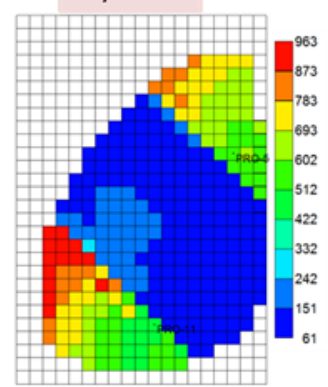

Layer 4

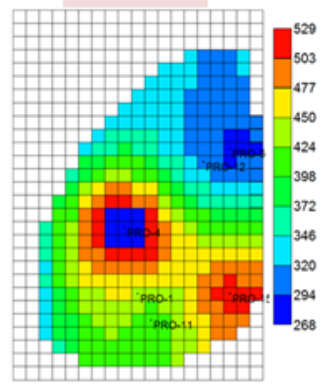

Horizontal Permeability (md) - Blind Realization

Figure 144: Horizontal permeability distributions for the blind realization.

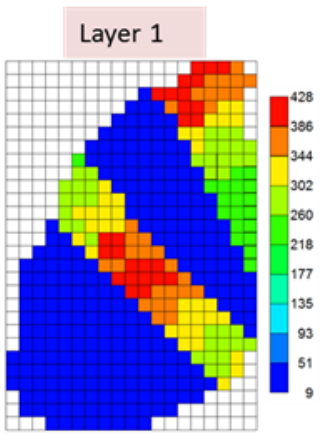

Layer 2

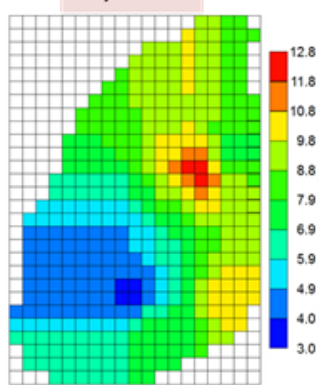

Layer 3

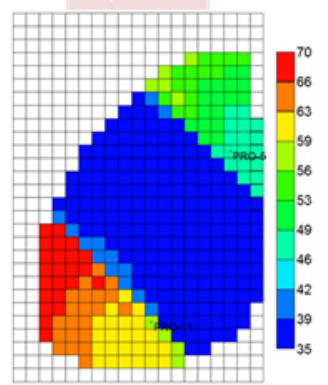

Layer 4

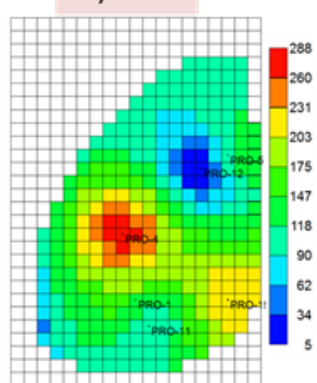

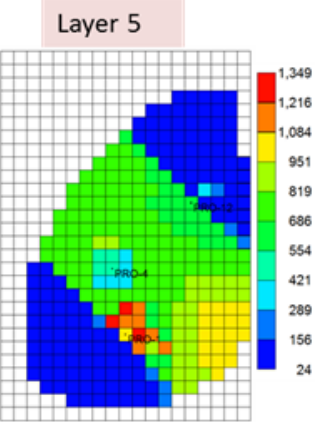



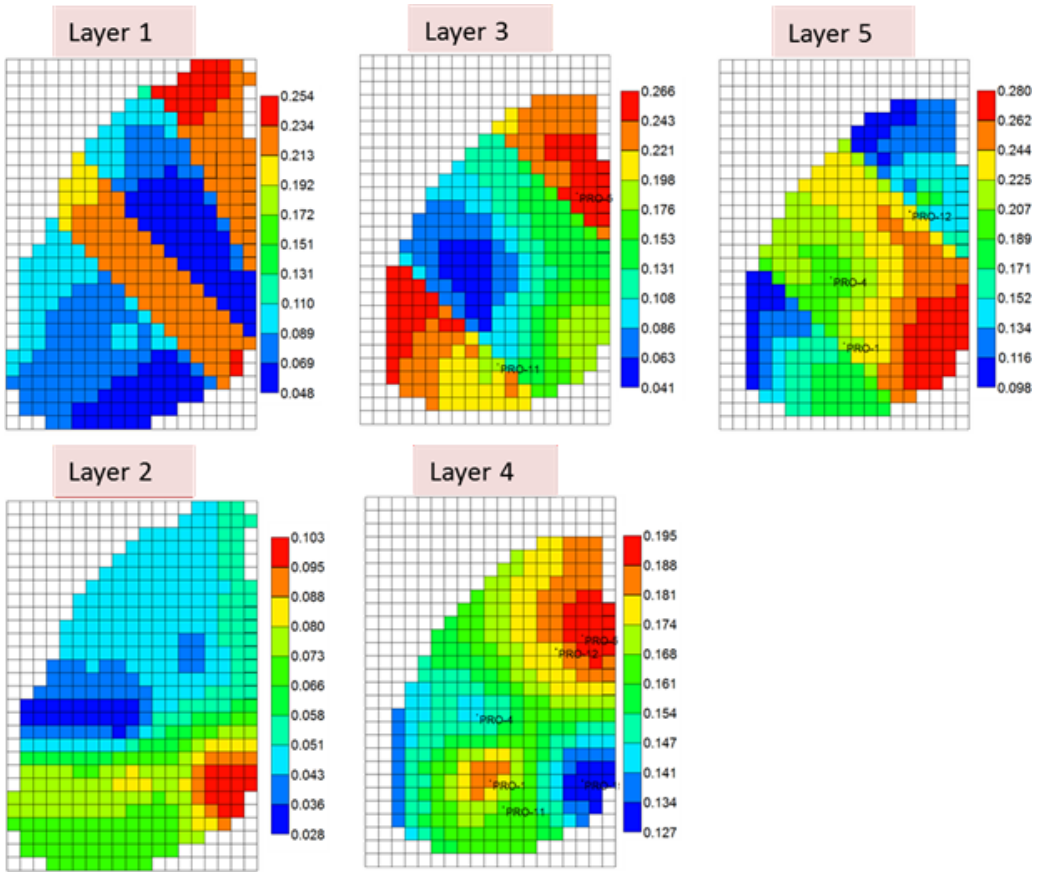

Porosity (\%) - Blind Realization

Figure 146: Porosity distributions for the blind realization. 


\section{c. The results of training and blind realizations compared with the observed data}

\section{c.1 Well bottom-hole pressure}

\section{Well PRO-11}

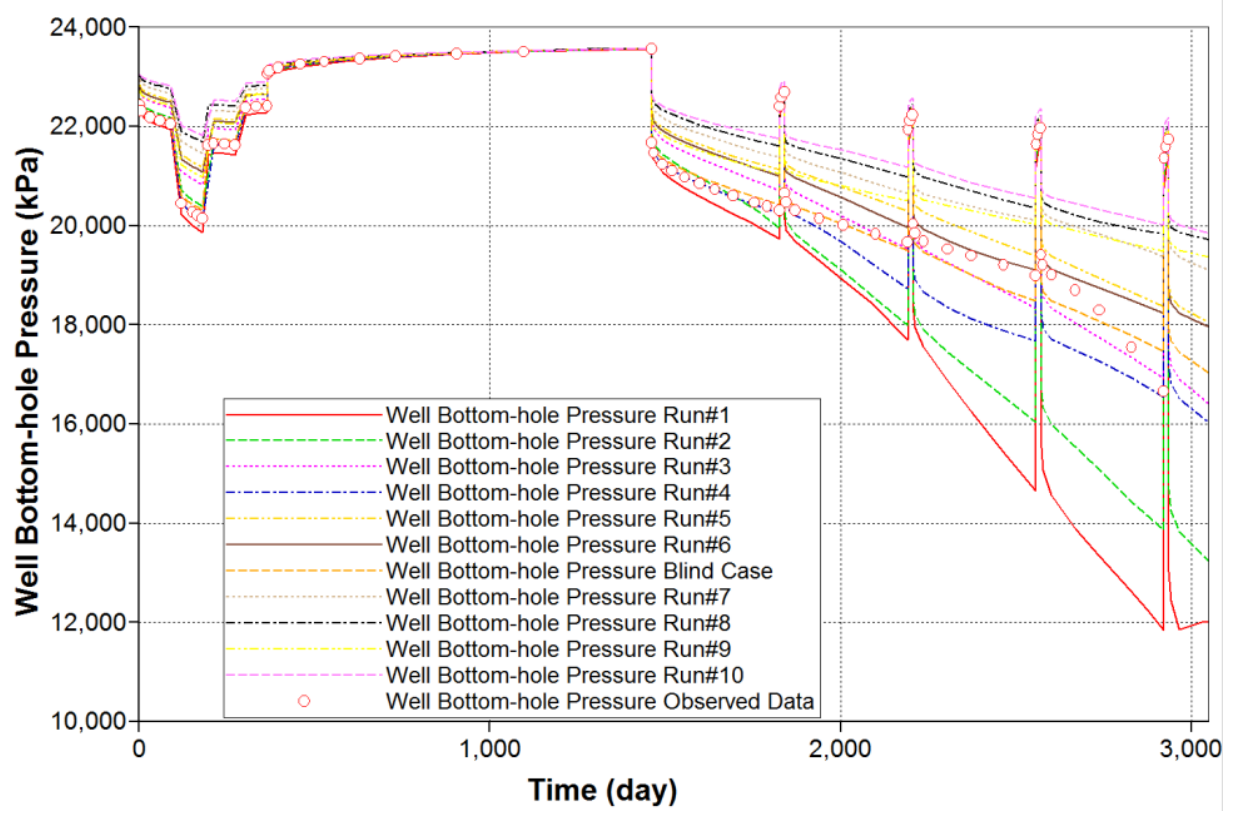

Figure 147: Comparison of well bottom-hole pressure for the ten training realizations and blind case with observed data- well PRO-11.

Well PRO-12

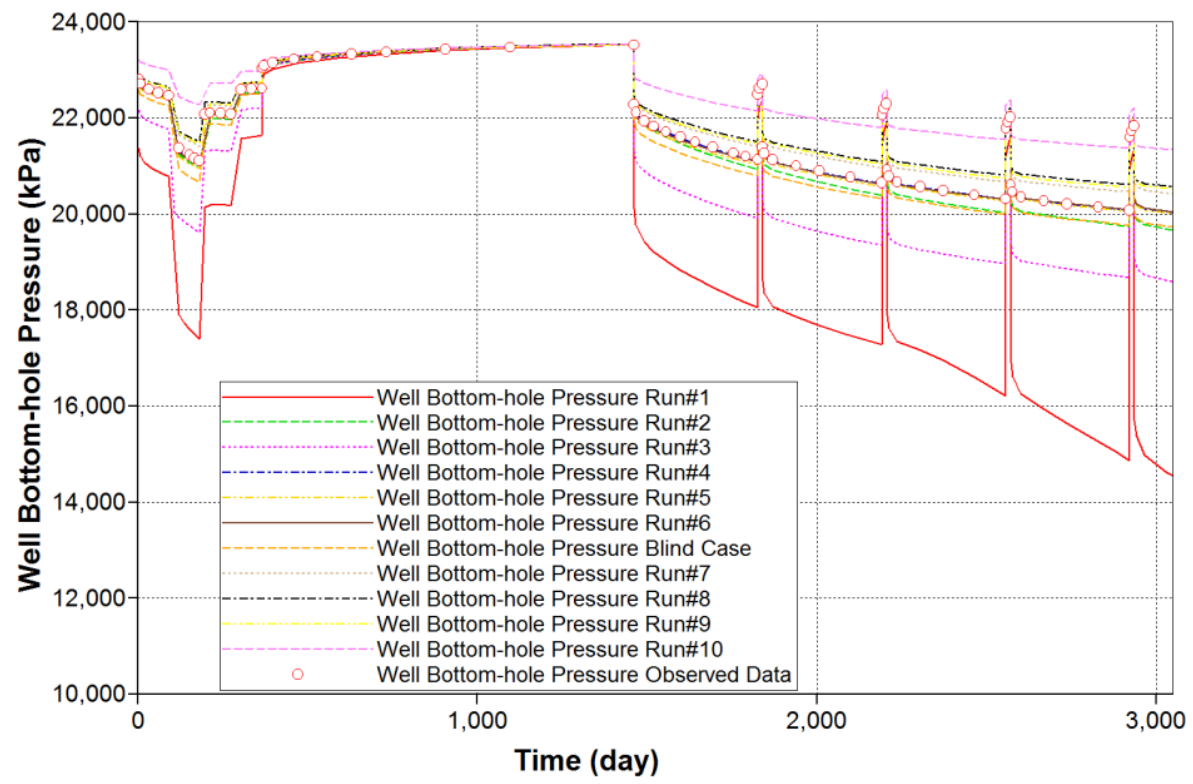

Figure 148: Comparison of well bottom-hole pressure for the ten training realizations and blind case with observed data- well PRO-12. 
Well PRO-15

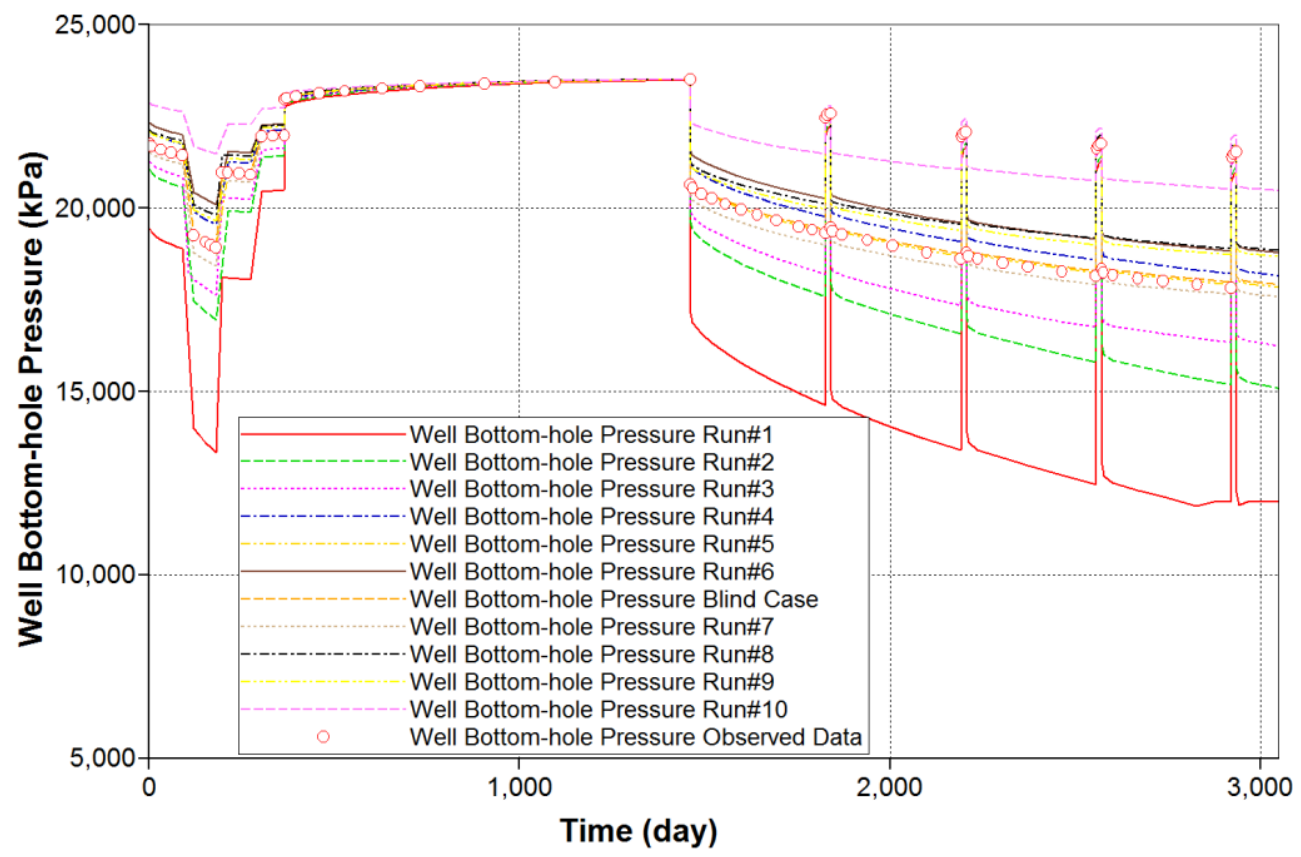

Figure 149: Comparison of well bottom-hole pressure for the ten training realizations and blind case with observed data- well PRO-15.

Well PRO-4

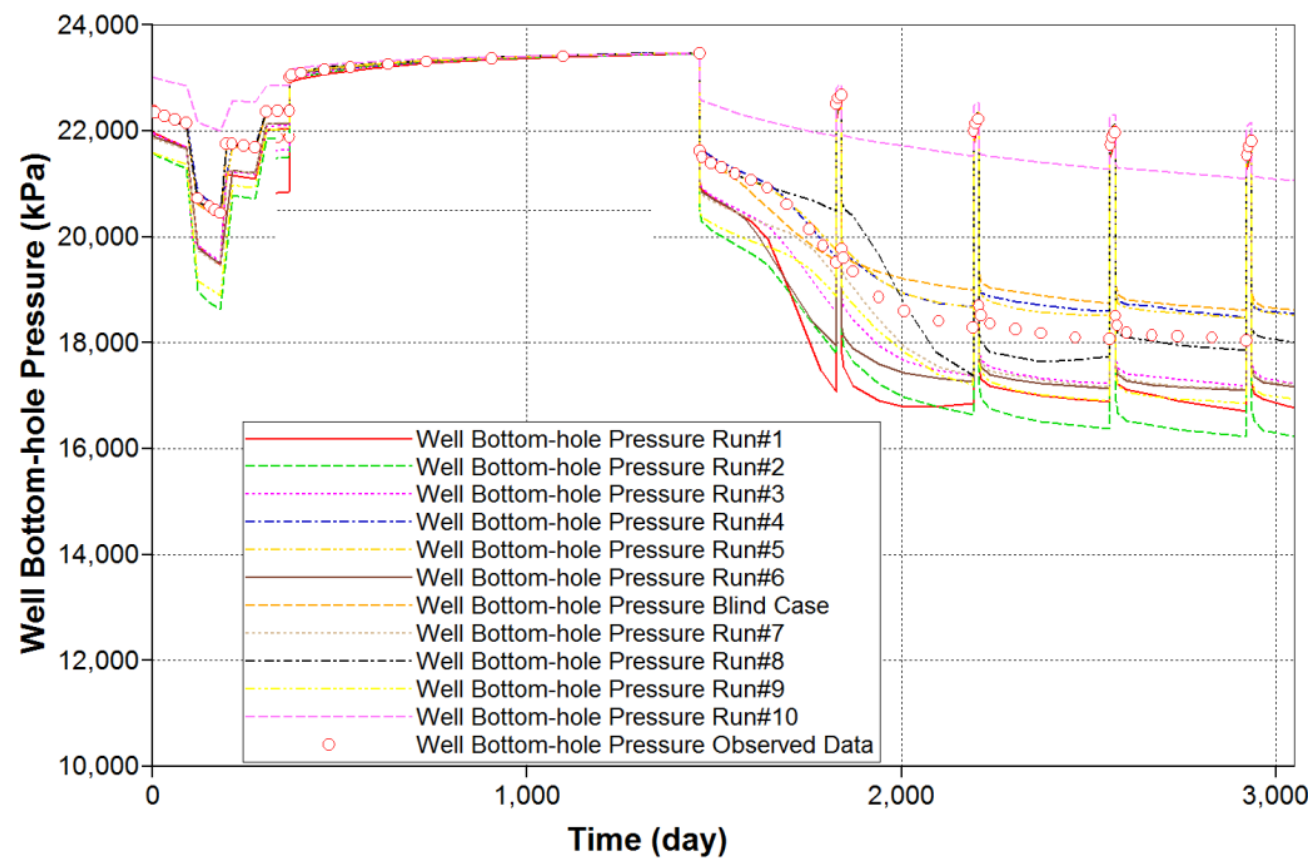

Figure 150: Comparison of well bottom-hole pressure for the ten training realizations and blind case with observed data- well PRO-4. 


\section{Well PRO-5}

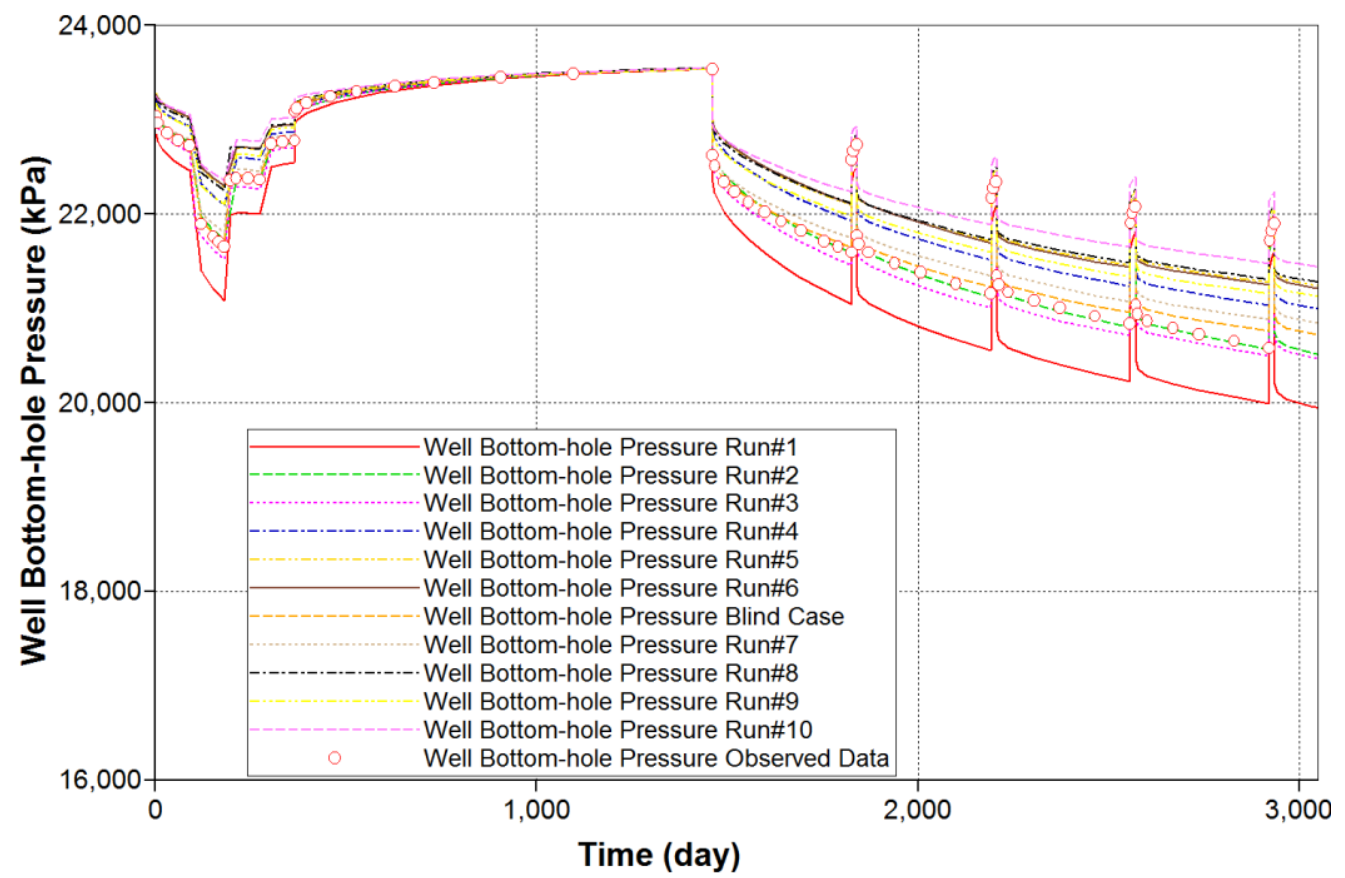

Figure 151: Comparison of well bottom-hole pressure for the ten training realizations and blind case with observed data- well PRO-5. 


\section{c.2 Gas production rate}

\section{Well PRO-11}

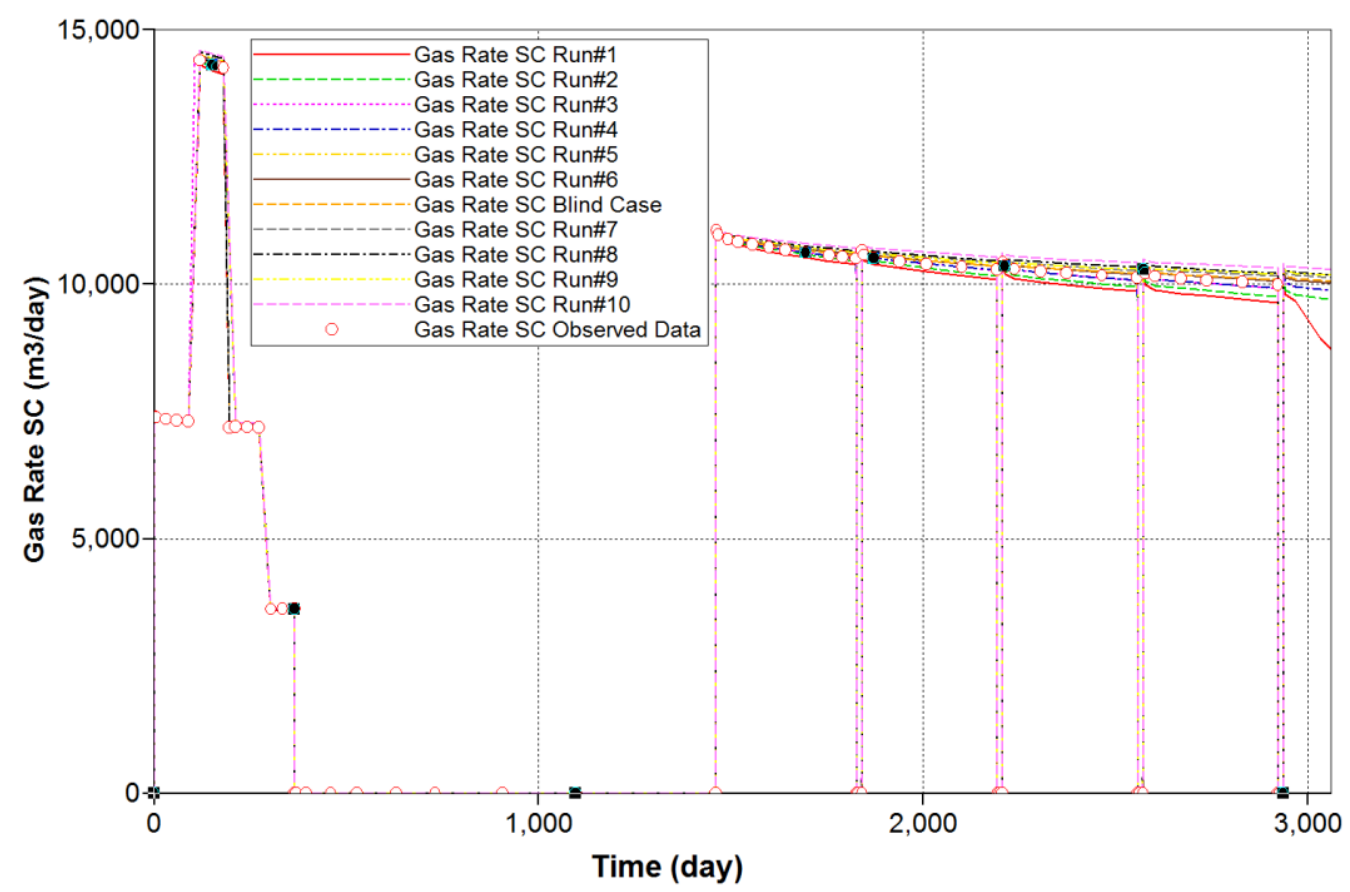

Figure 152: Comparison of gas rate production for the ten training realizations and blind case with the observed data- well PRO-11.

Well PRO-12

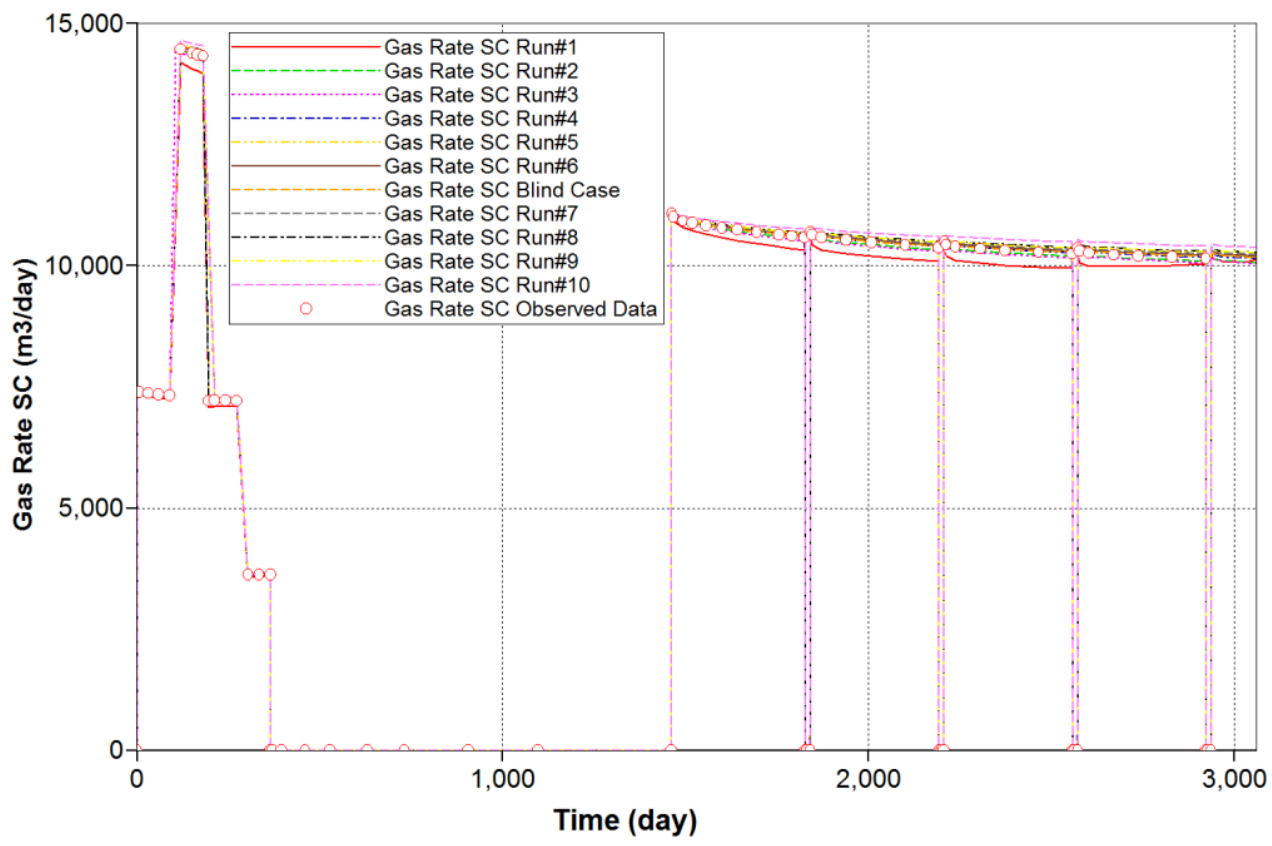

Figure 153: Comparison of gas rate production for the ten training realizations and blind case with the observed data- well PRO-12. 


\section{Well PRO-15}

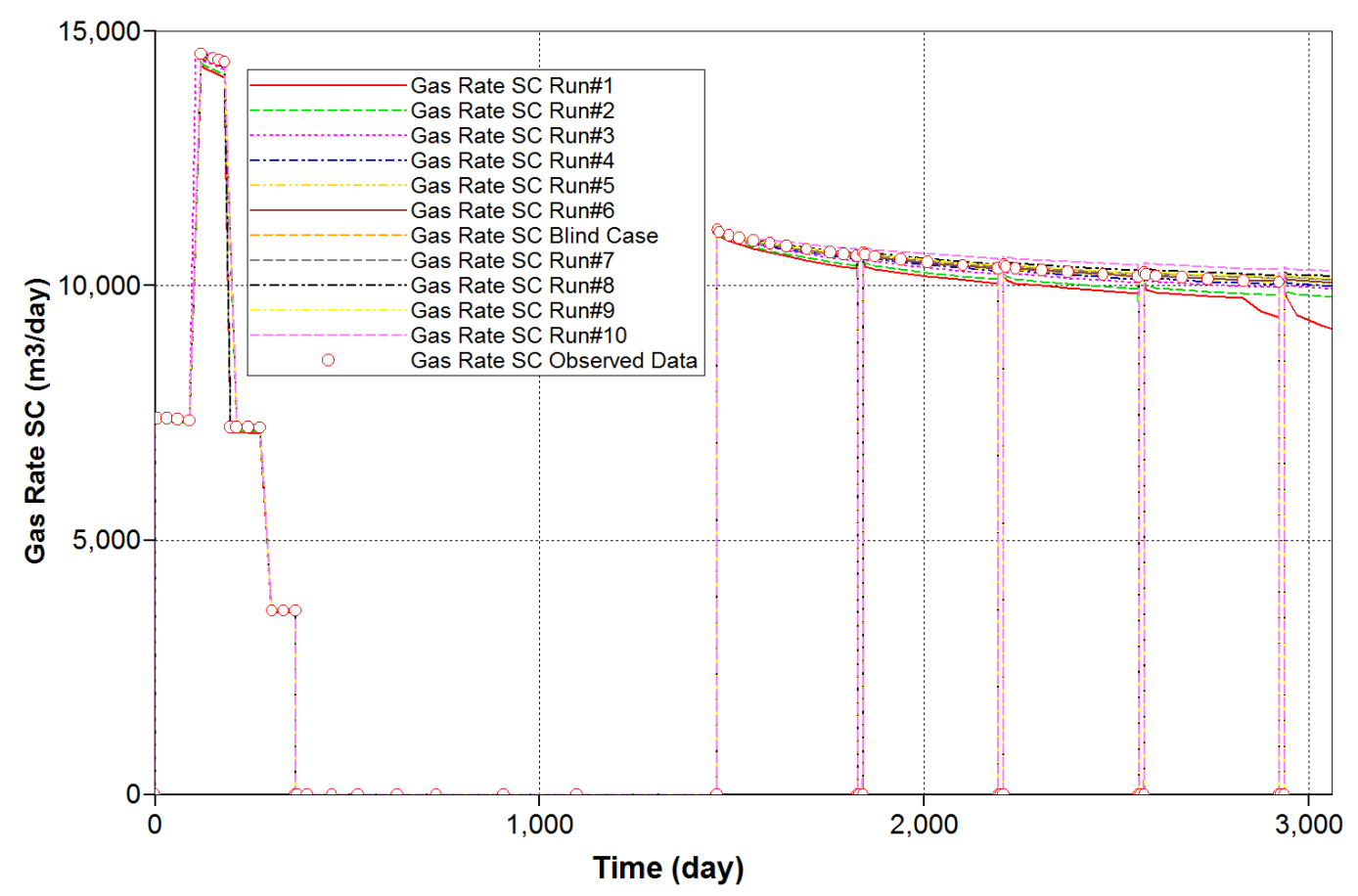

Figure 154: Comparison of gas rate production for the ten training realizations and blind case with the observed data- well PRO-15.

\section{Well PRO-4}

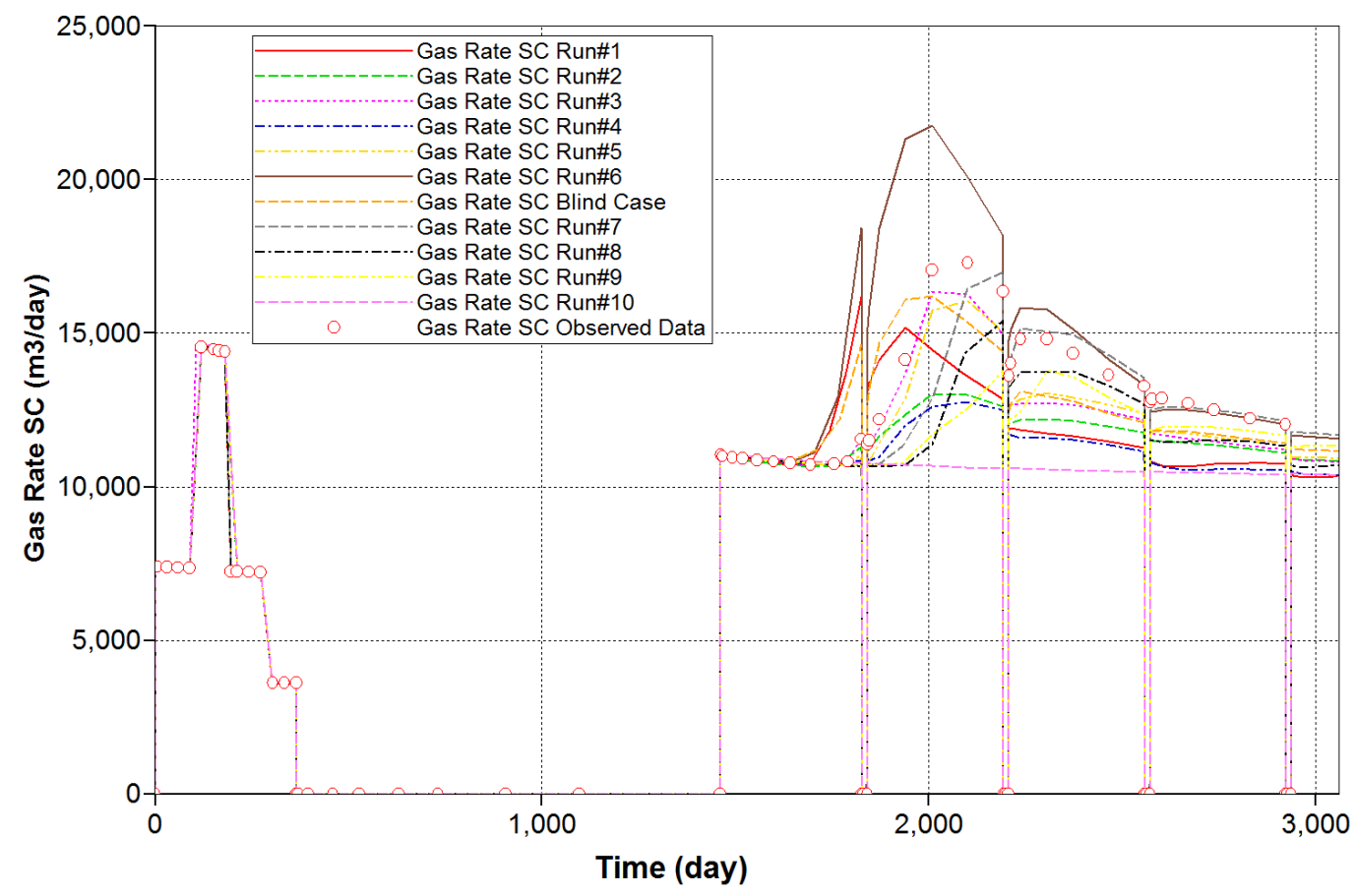

Figure 155: Comparison of gas rate production for the ten training realizations and blind case with the observed data- well PRO-4. 


\section{Well PRO-5}

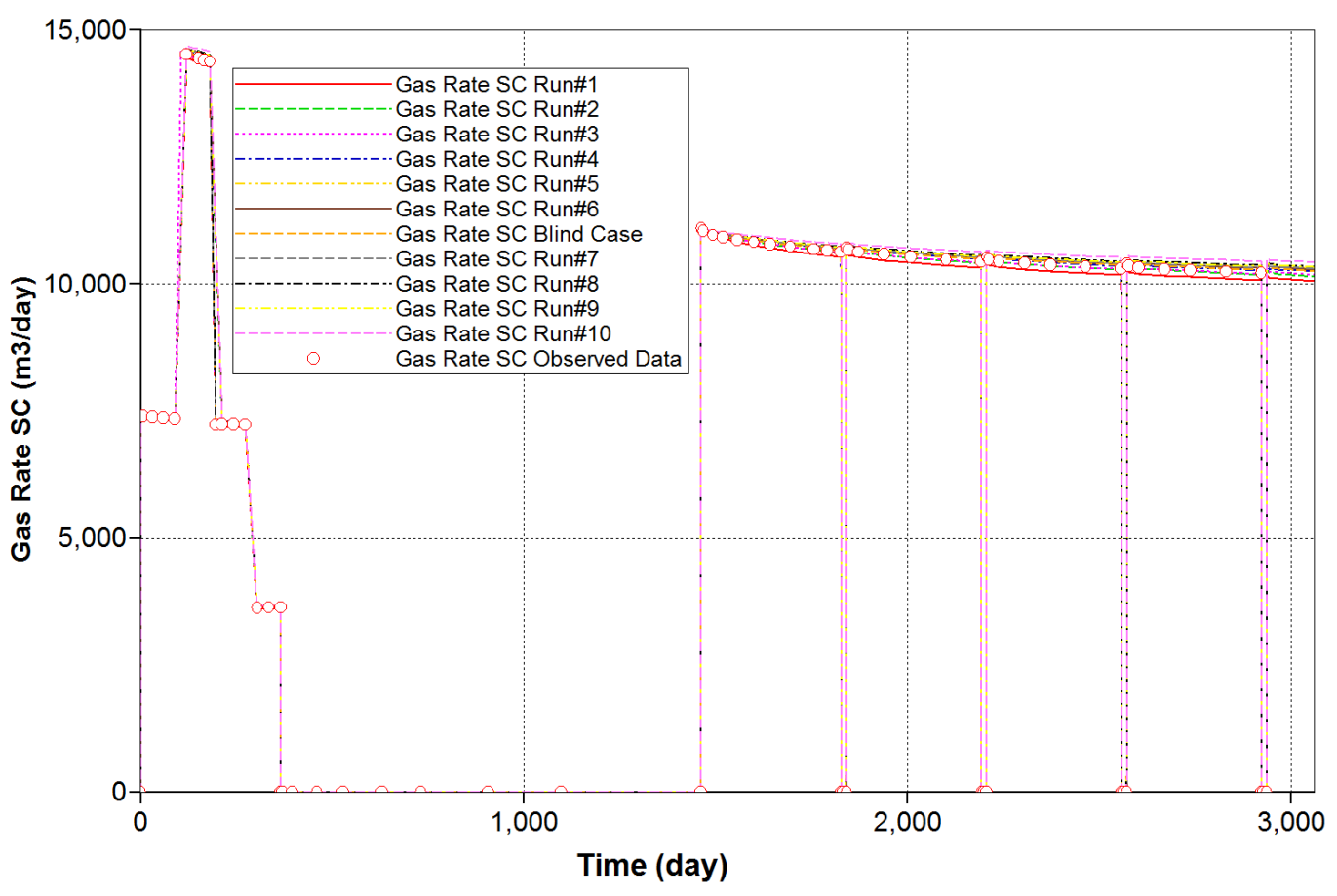

Figure 156: Comparison of gas rate production for the ten training realizations and blind case with the observed data- well PRO-5. 


\section{d. The results of SRM for the training realizations}

\section{d.1 Well bottom-hole pressure}

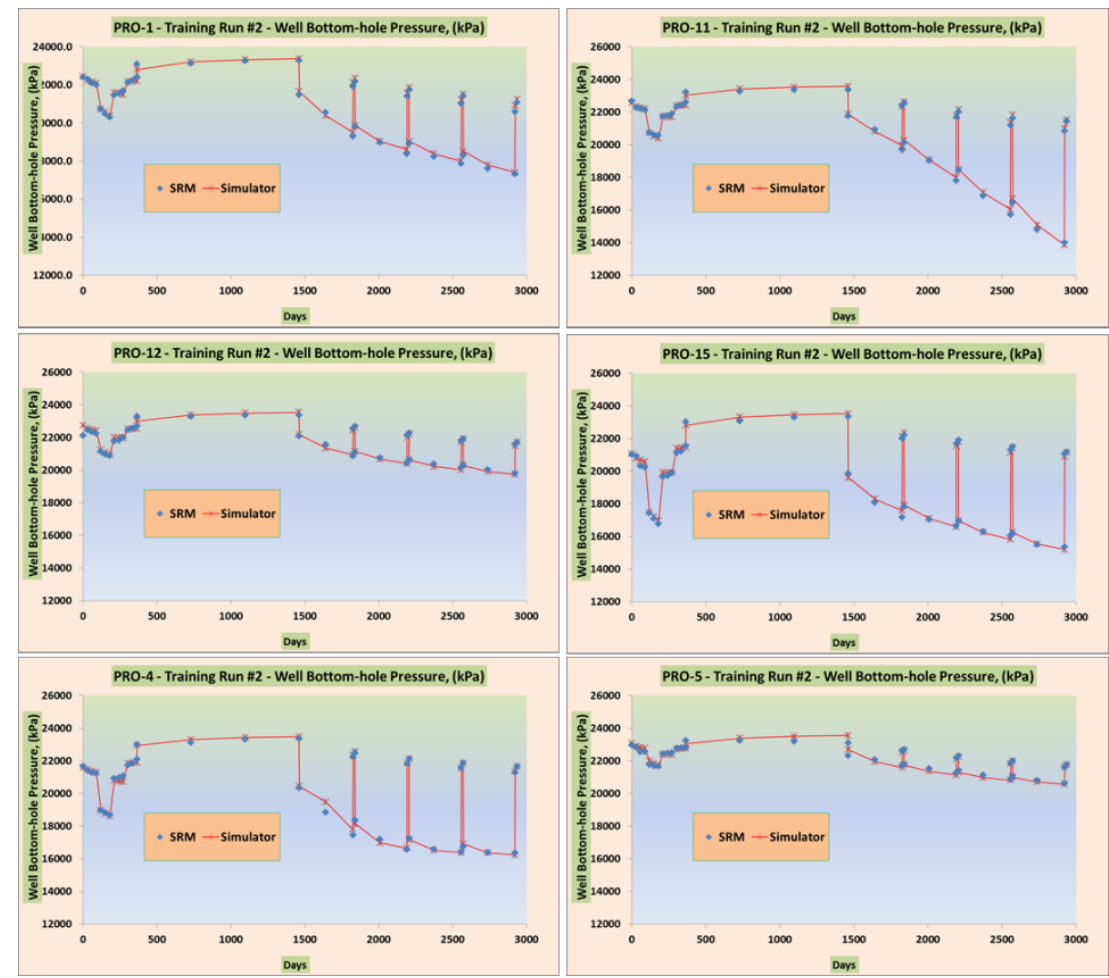

Figure 157: Comparison of the well bottom-hole pressure results for SRM and simulator for training run \#2. 

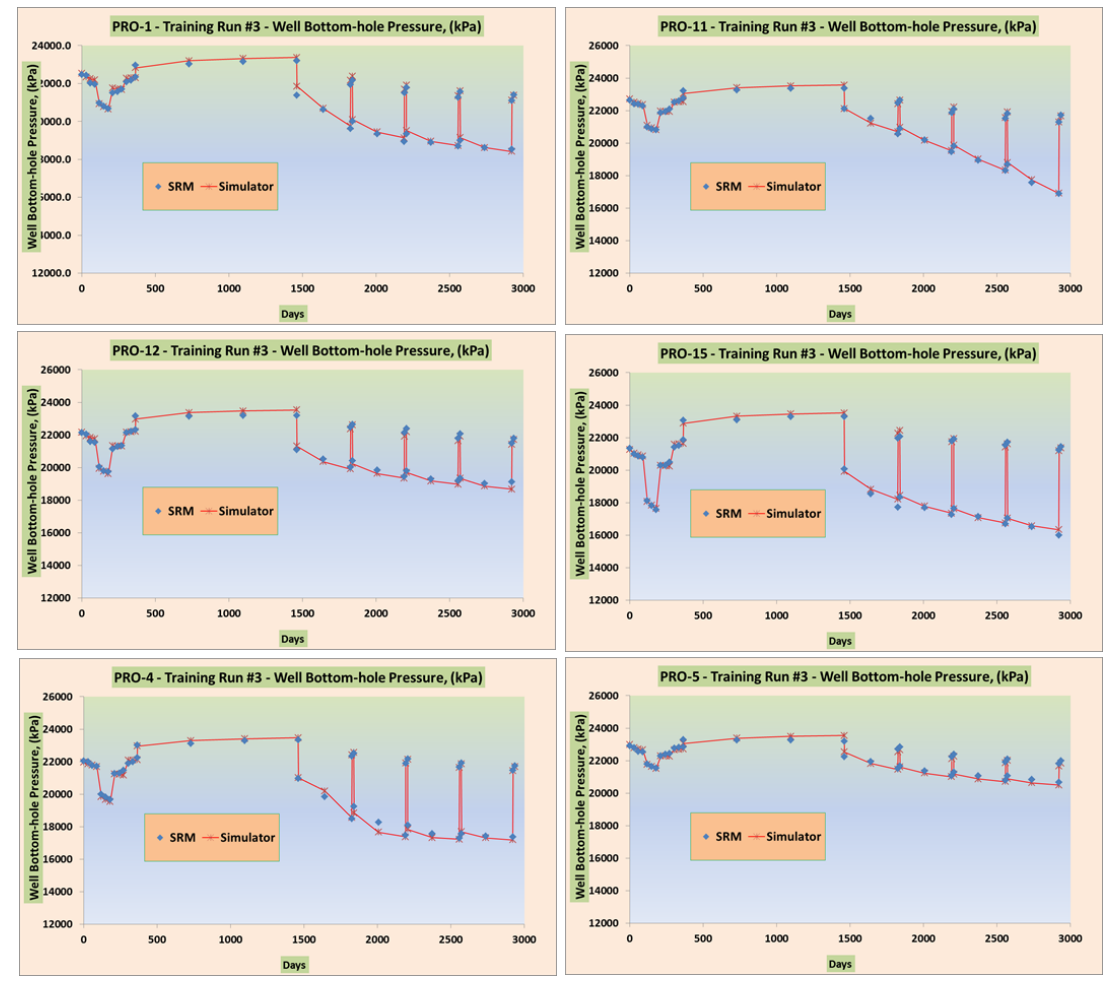

Figure 158: Comparison of the well bottom-hole pressure results for SRM and simulator for training run \#3.
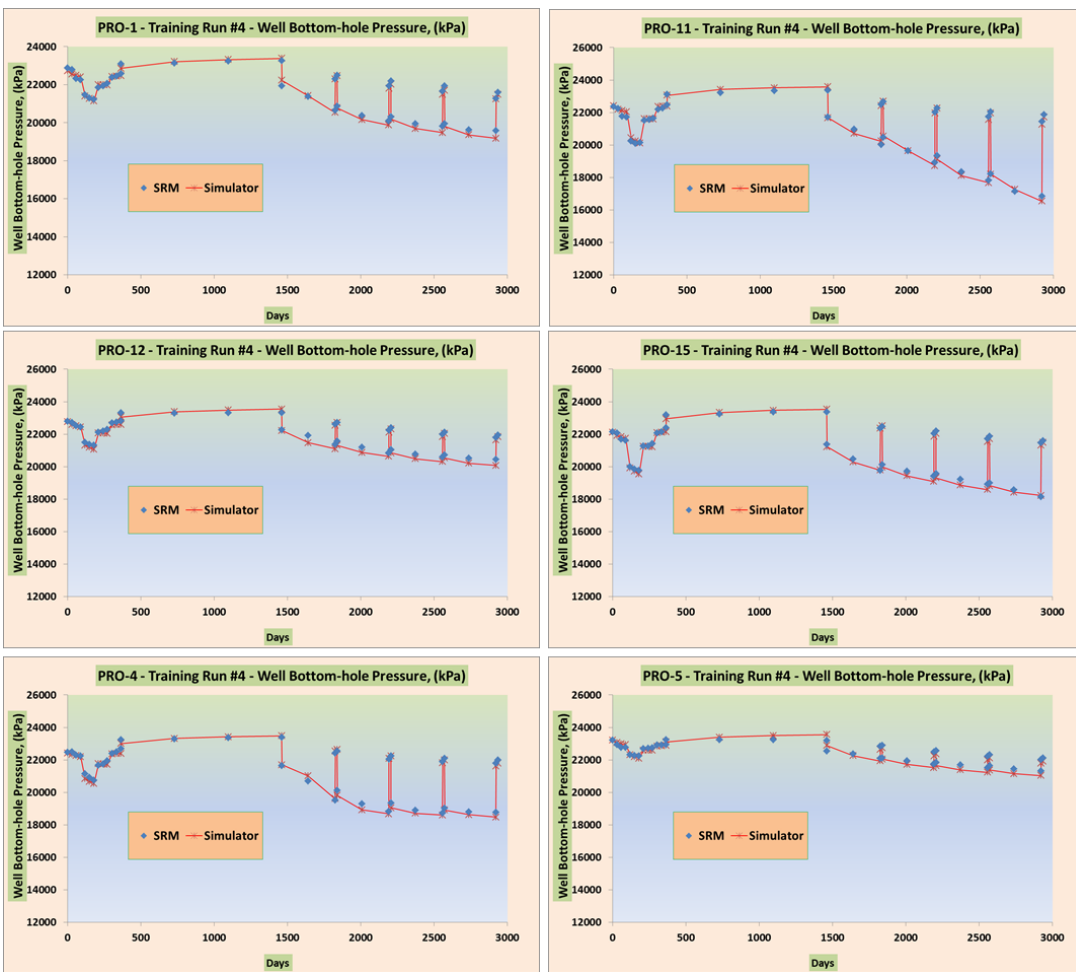

Figure 159: Comparison of the well bottom-hole pressure results for SRM and simulator for training run \#4. 

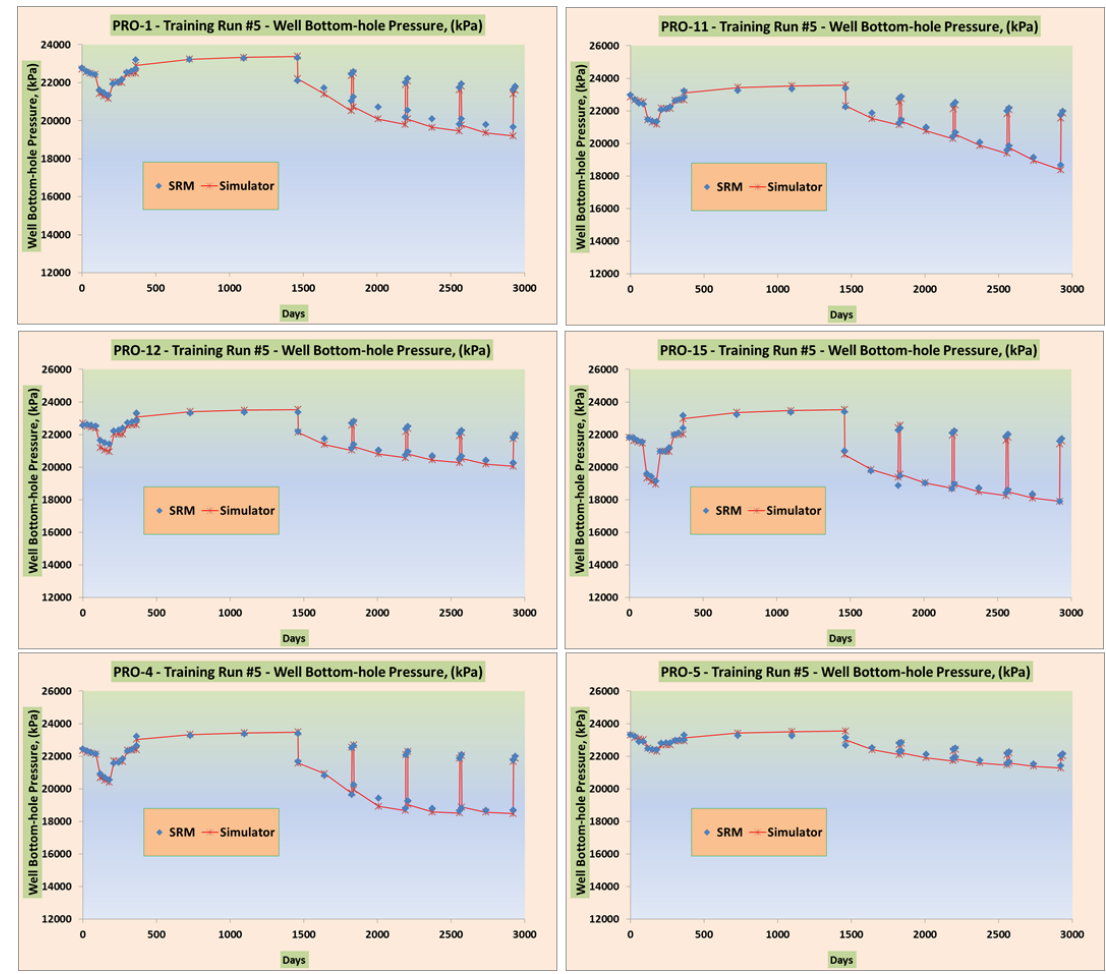

Figure 160: Comparison of the well bottom-hole pressure results for SRM and simulator for training run \#5. 

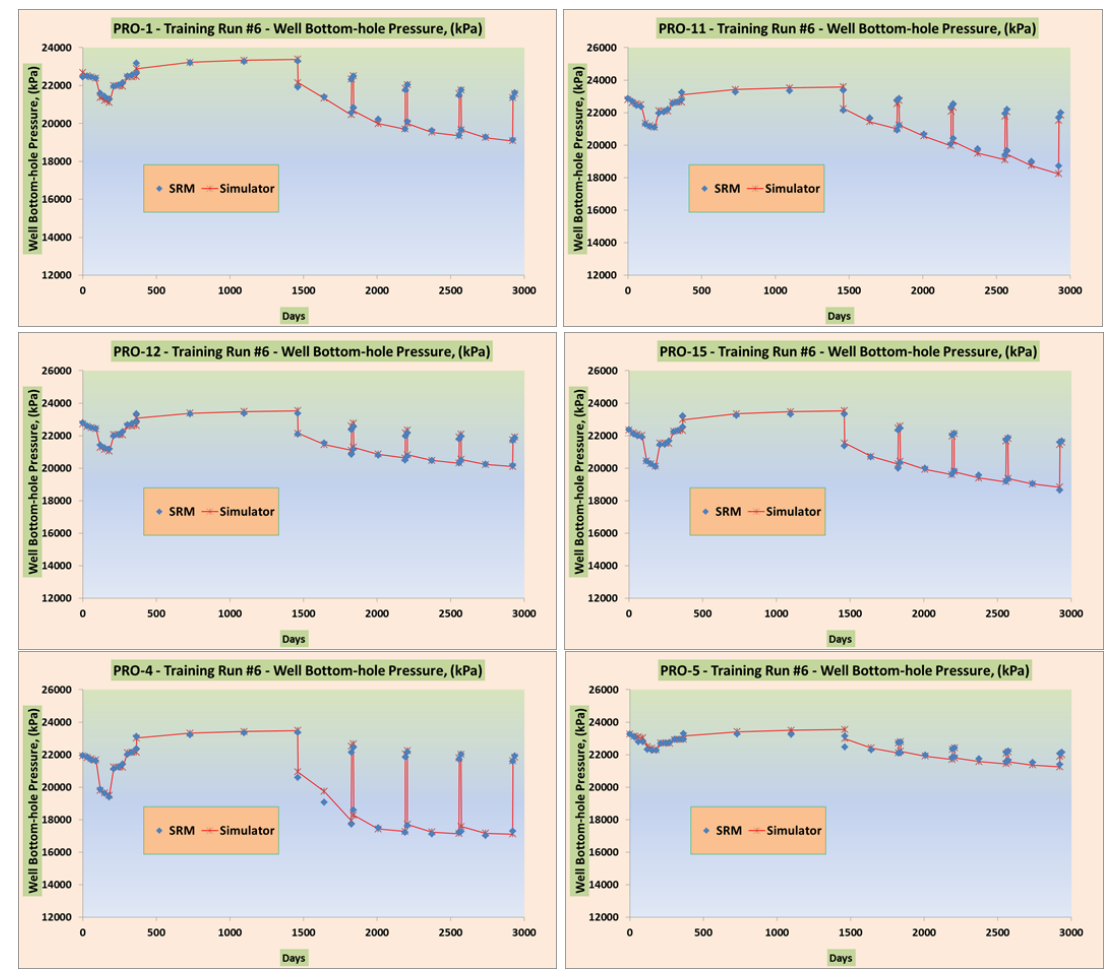

Figure 161: Comparison of the well bottom-hole pressure results for SRM and simulator for training run \#6.
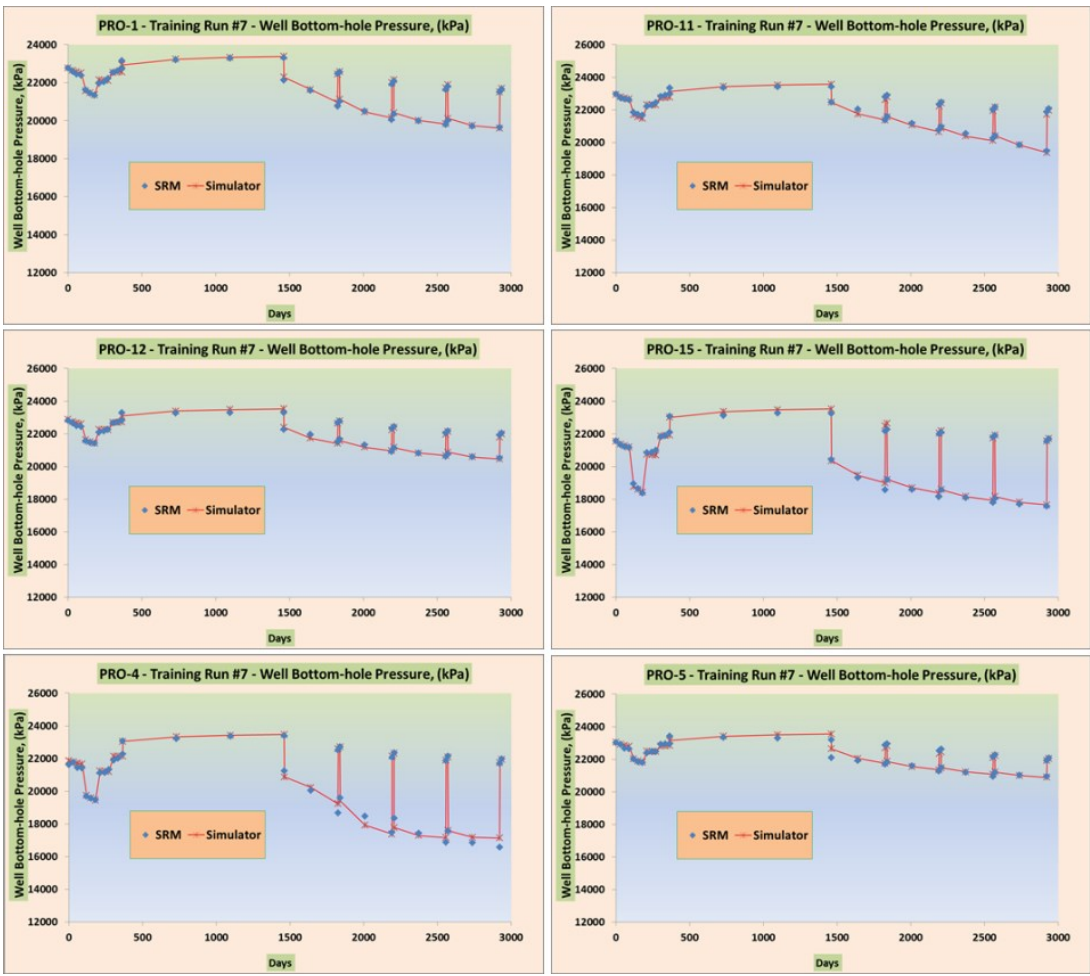

Figure 162: Comparison of the well bottom-hole pressure results for SRM and simulator for training run \#7. 

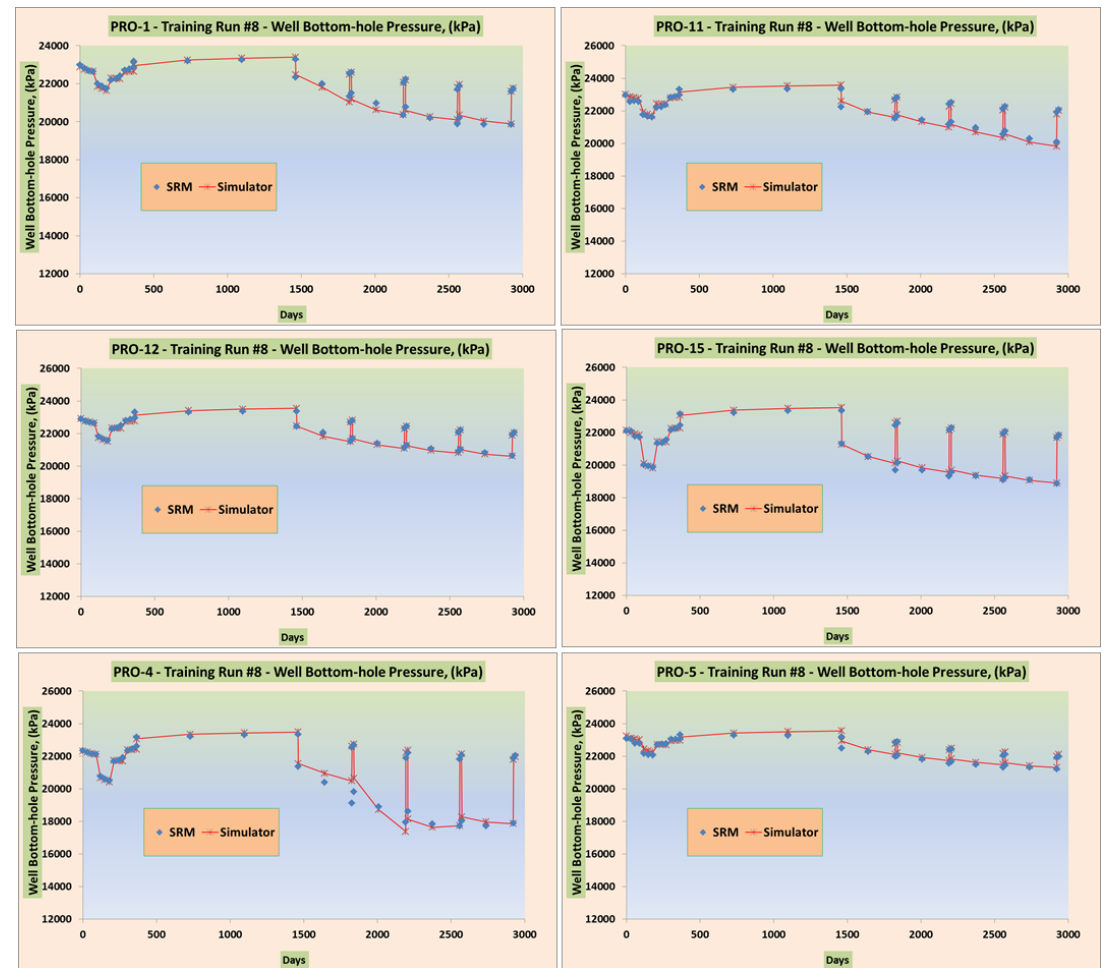

Figure 163: Comparison of the well bottom-hole pressure results for SRM and simulator for training run \#8.
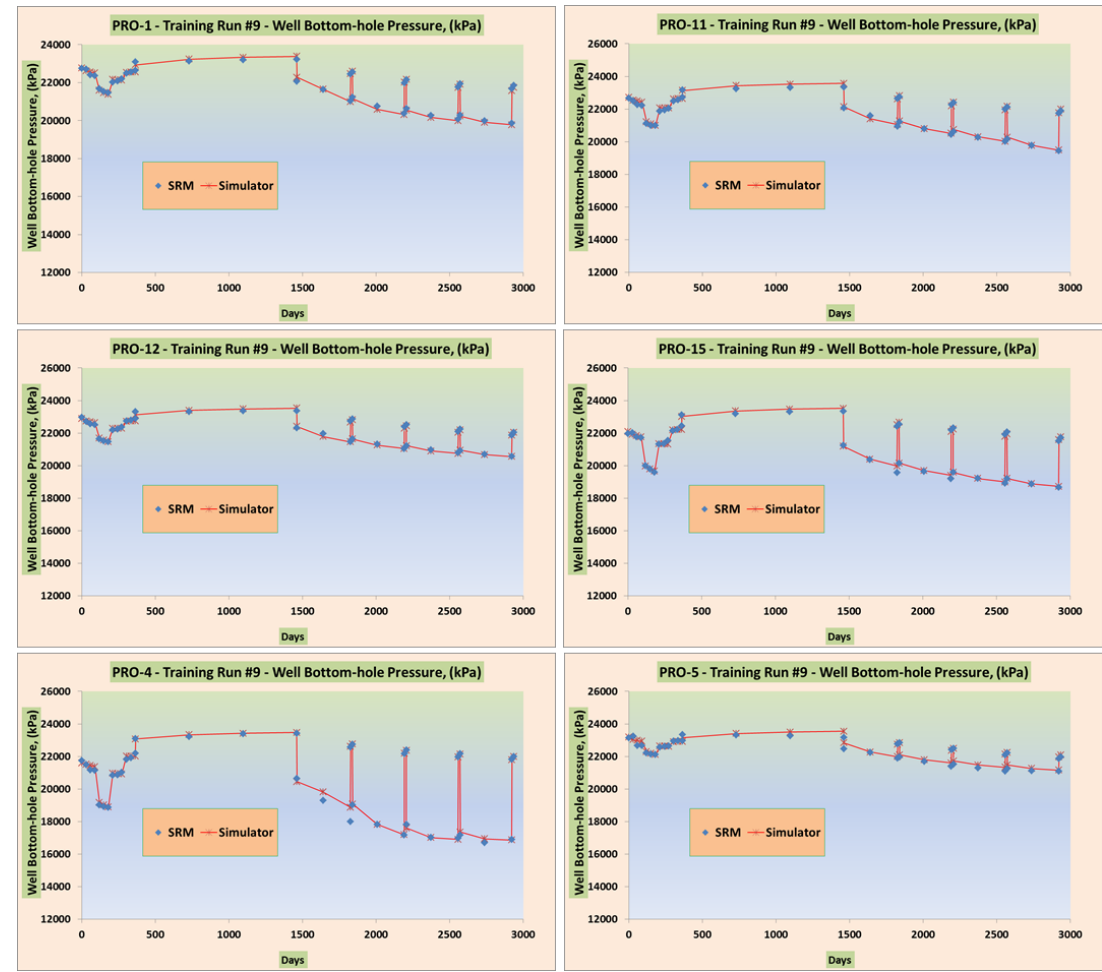

Figure 164: Comparison of the well bottom-hole pressure results for SRM and simulator for training run \#9. 

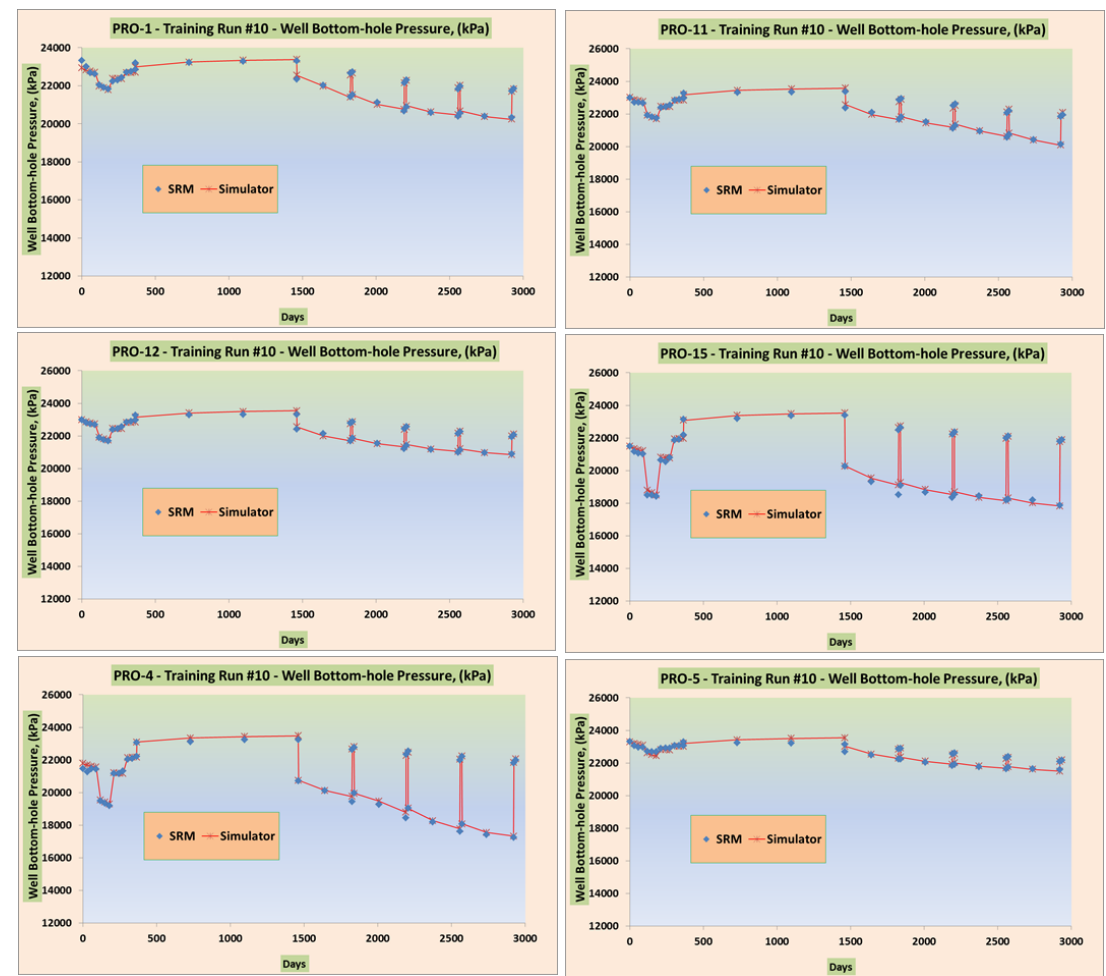

Figure 165: Comparison of the well bottom-hole pressure results for SRM and simulator for training run \#10. 


\section{d.2 Gas production rate}

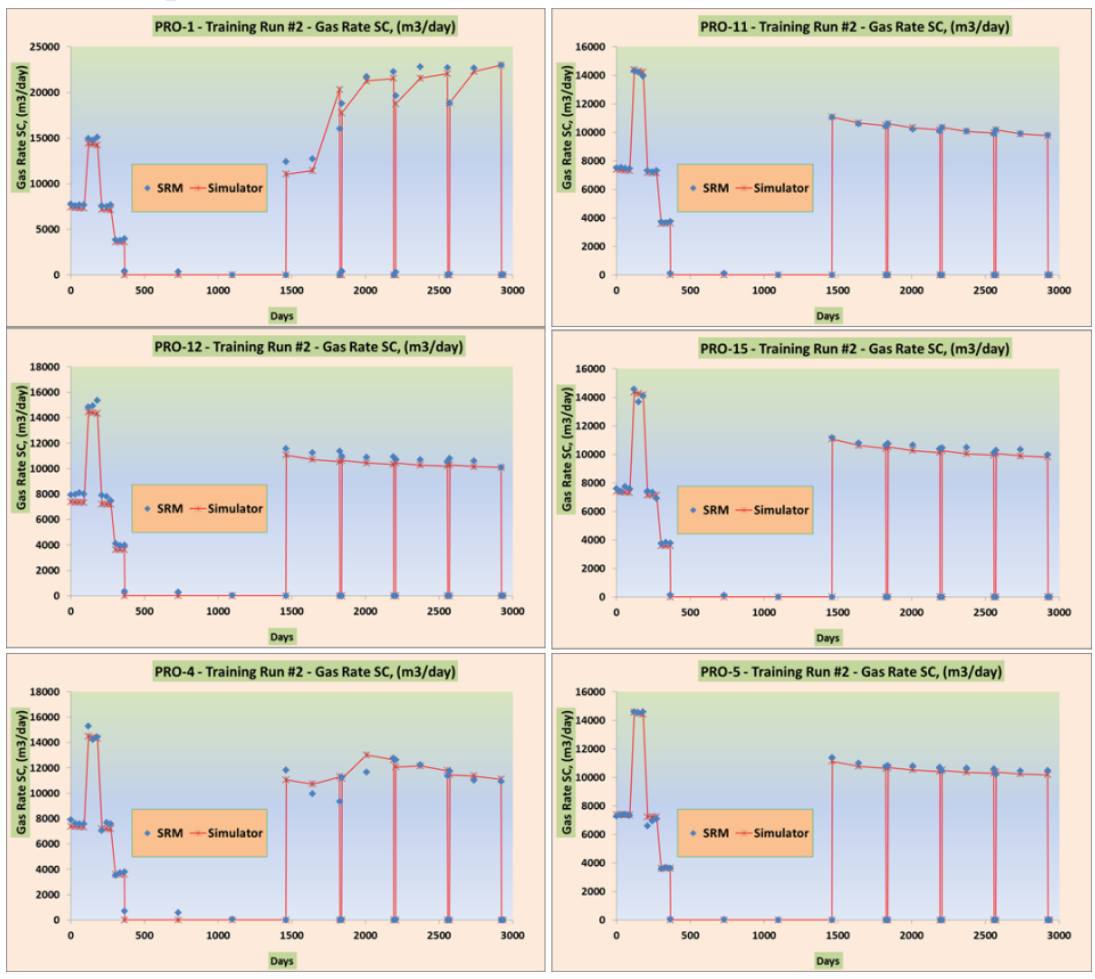

Figure 166: Comparison of the gas production rate results for SRM and simulator for training run \#2.
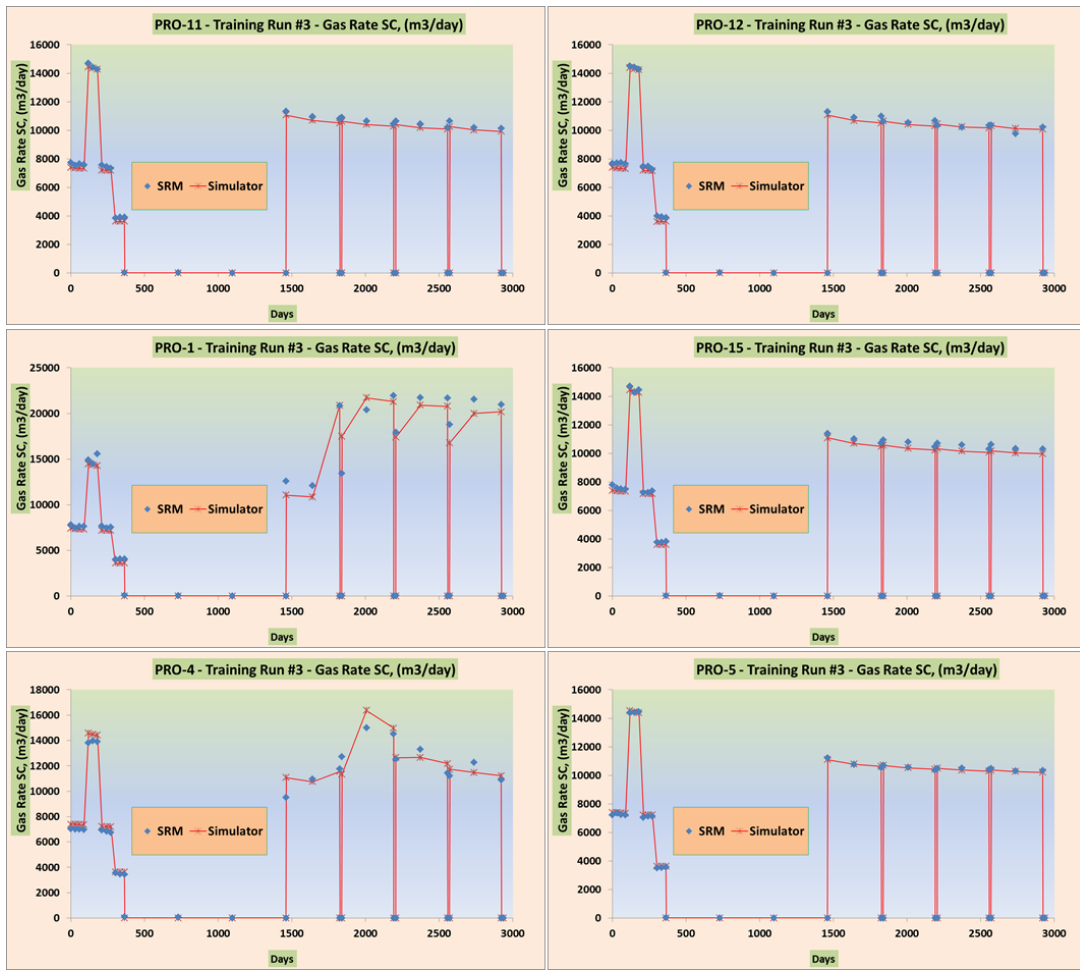

Figure 167: Comparison of the gas production rate results for SRM and simulator for training run \#3. 

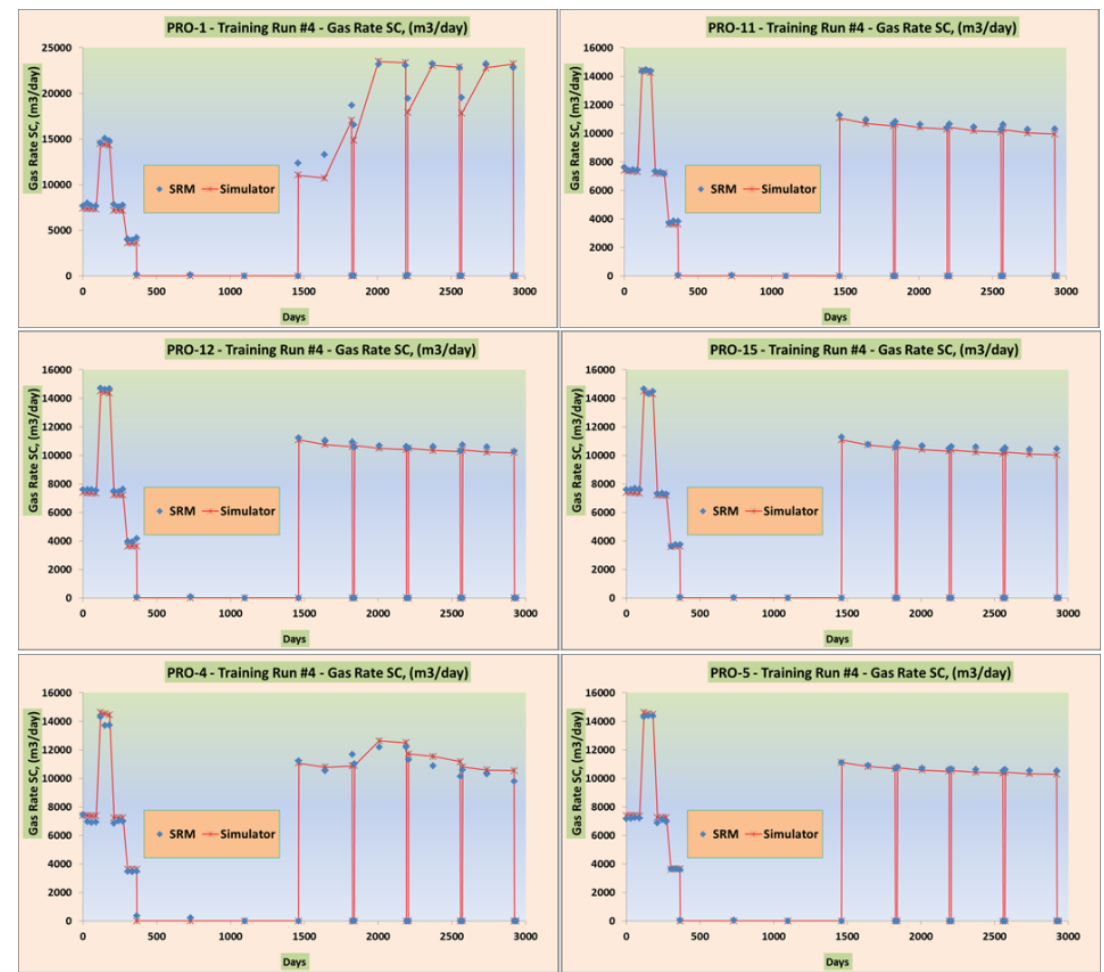

Figure 168: Comparison of the gas production rate results for SRM and simulator for training run \#4.
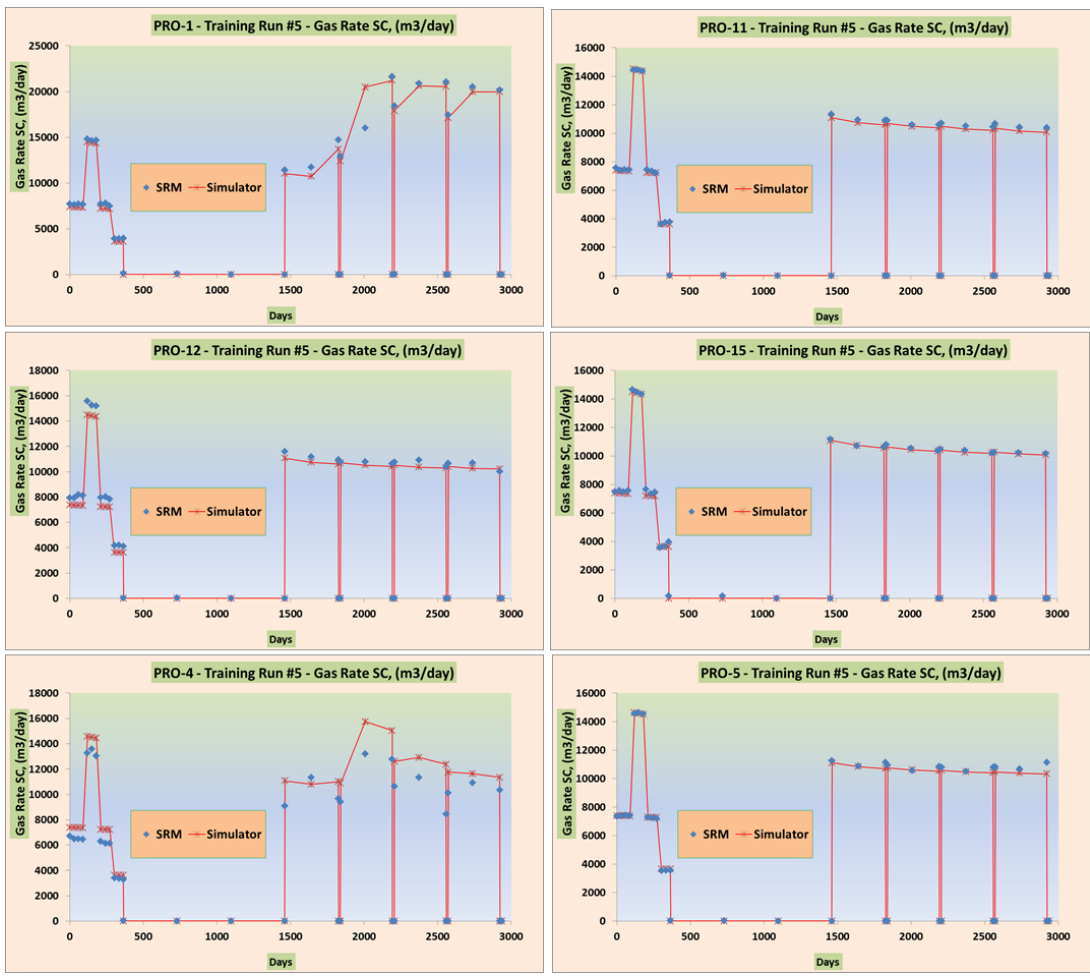

Figure 169: Comparison of the gas production rate results for SRM and simulator for training run $\# 5$. 

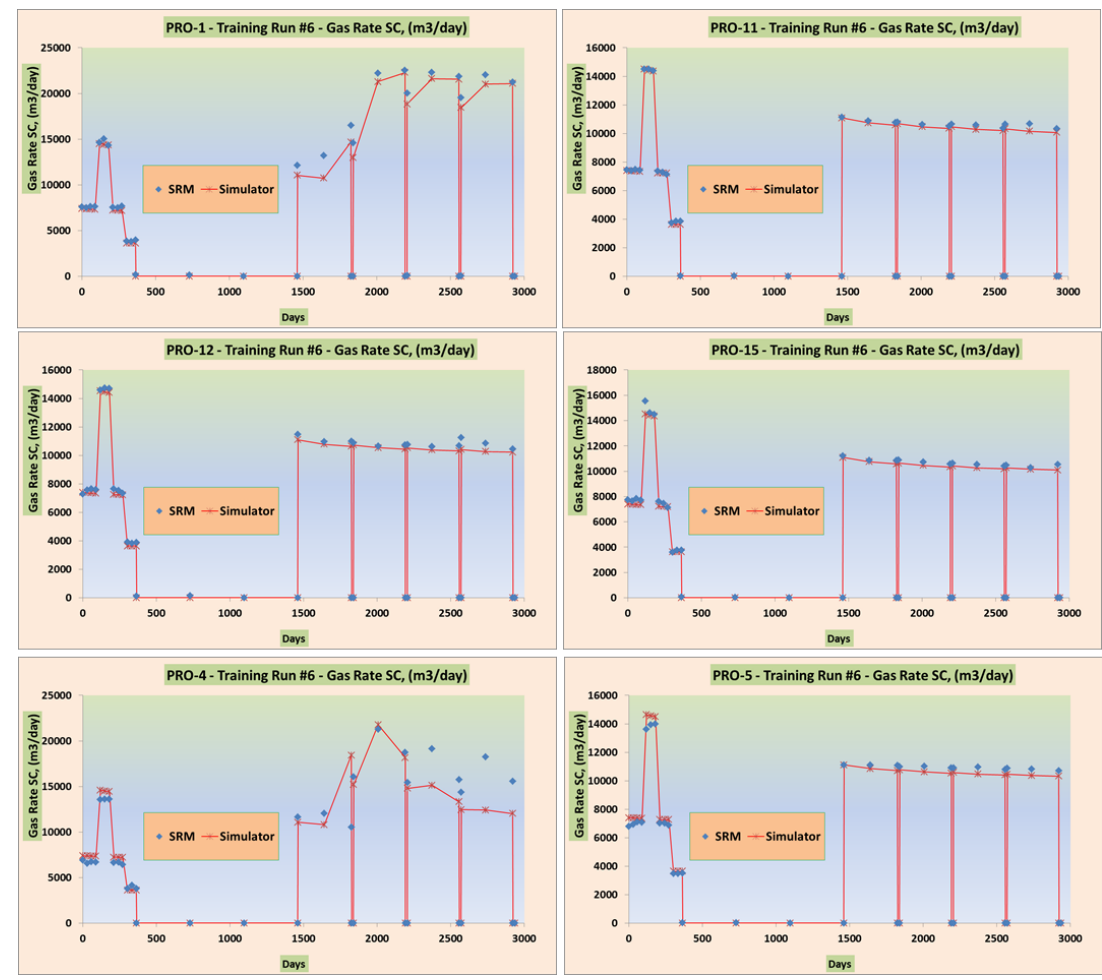

Figure 170: Comparison of the gas production rate results for SRM and simulator for training run \#6.
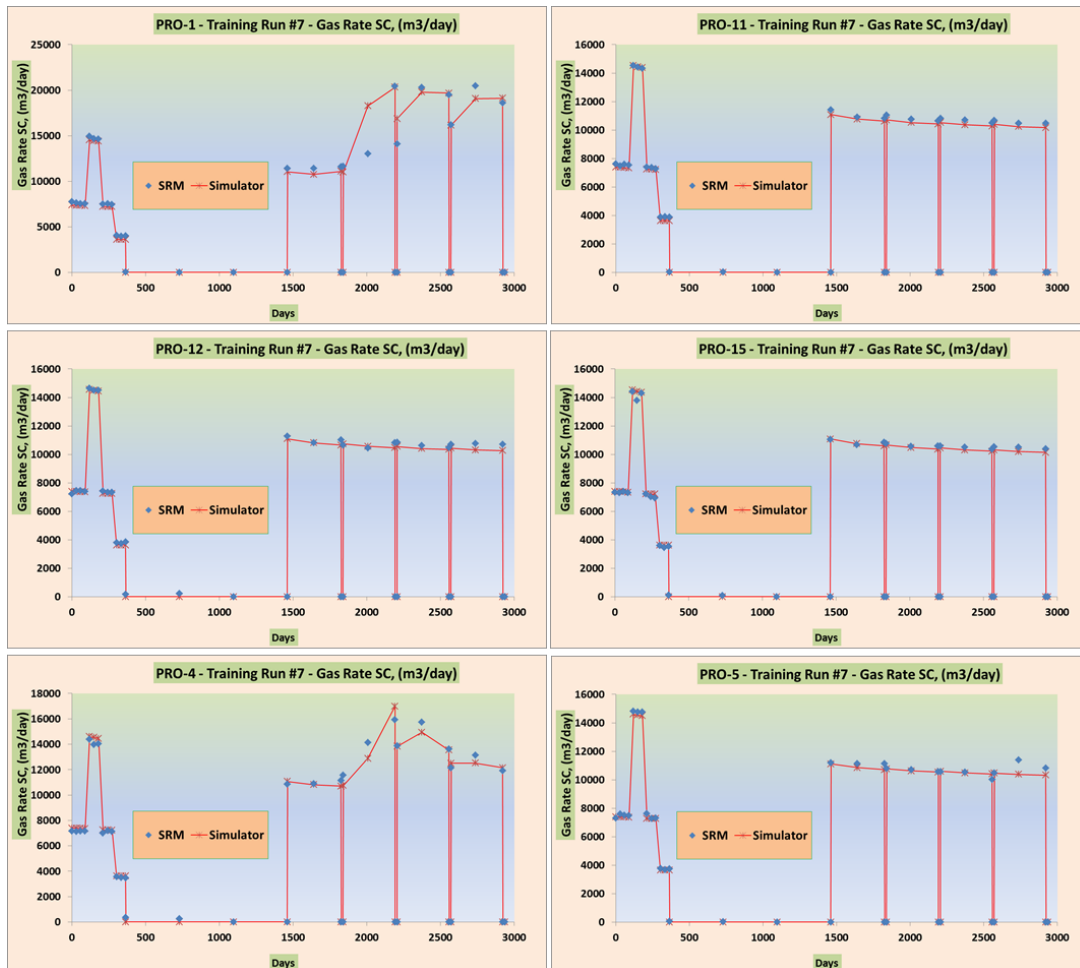

Figure 171: Comparison of the gas production rate results for SRM and simulator for training run \#7. 

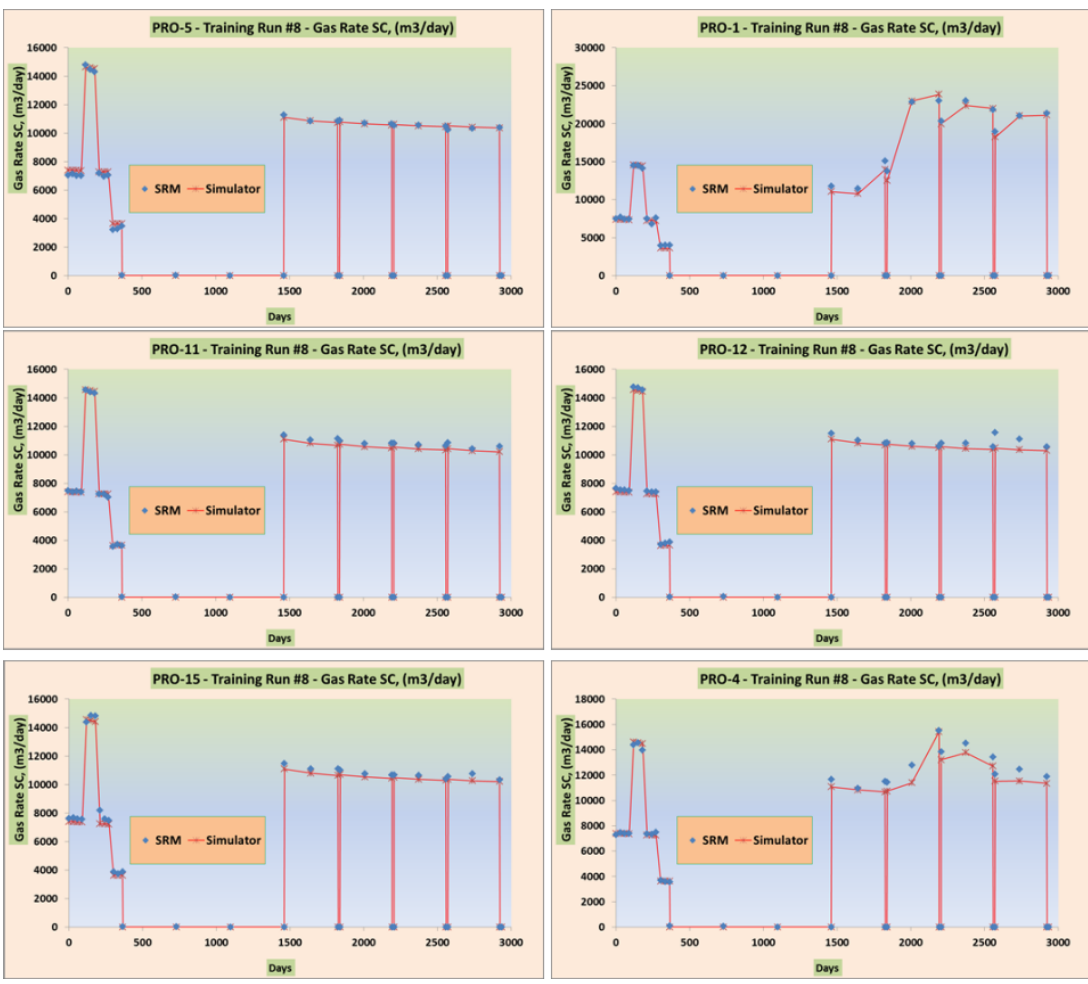

Figure 172: Comparison of the gas production rate results for SRM and simulator for training run \#8.
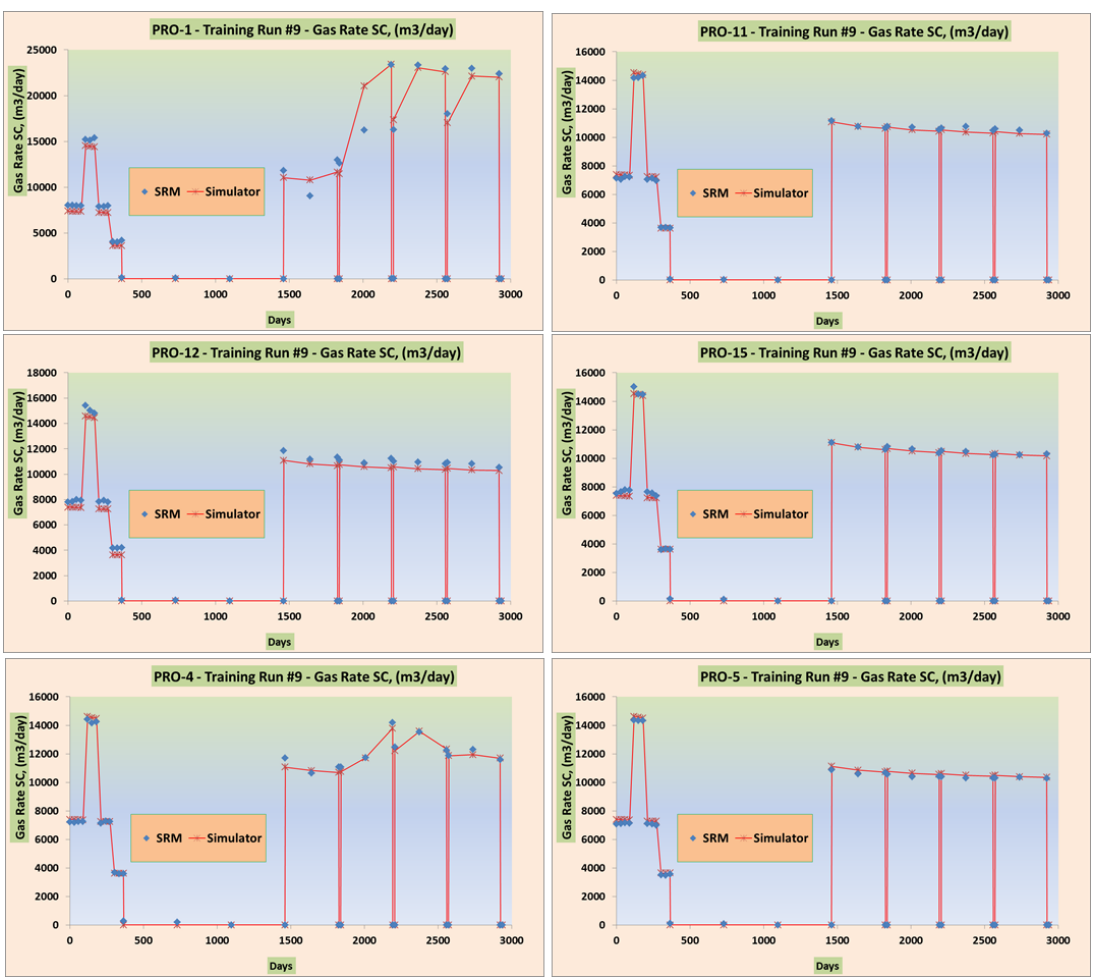

Figure 173: Comparison of the gas production rate results for SRM and simulator for training run \#9. 

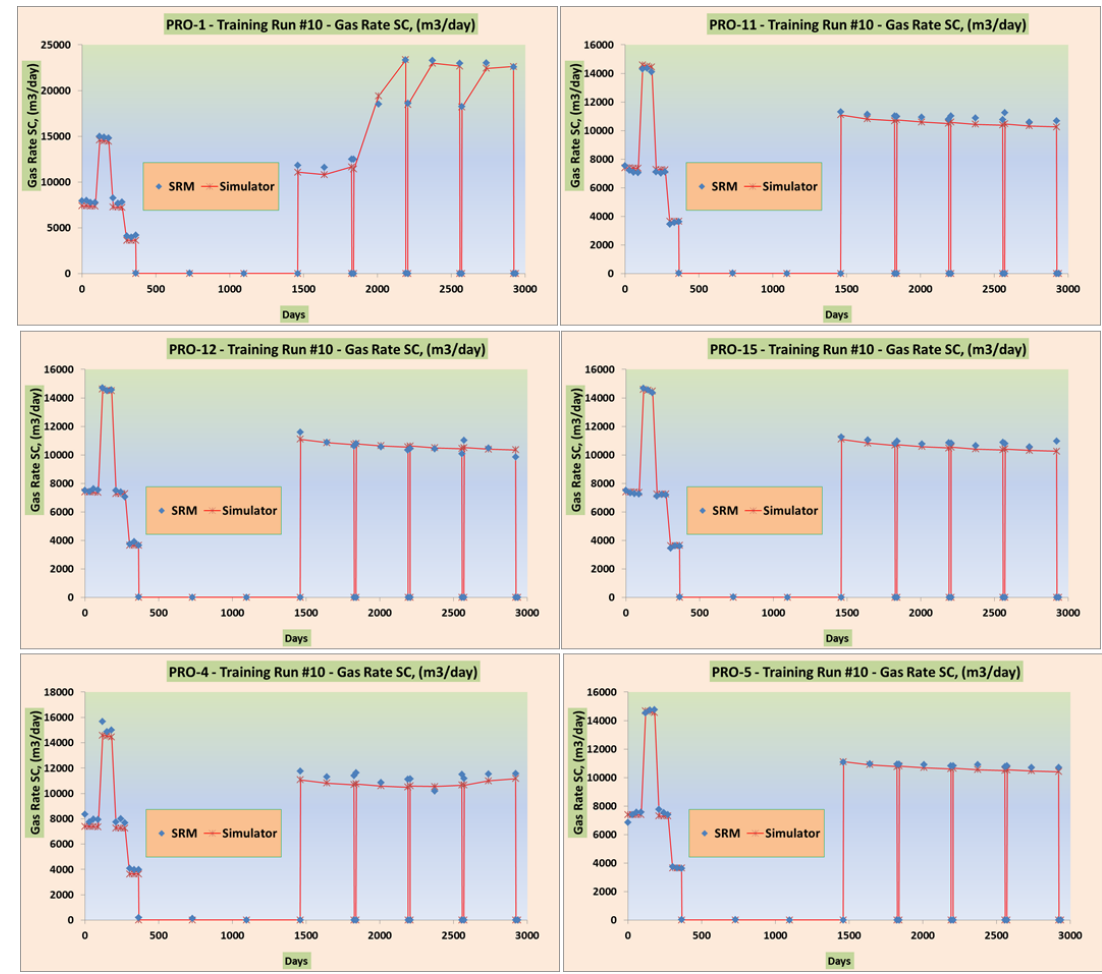

Figure 174: Comparison of the gas production rate results for SRM and simulator for training run \#10. 


\section{d.3 Water production rate}
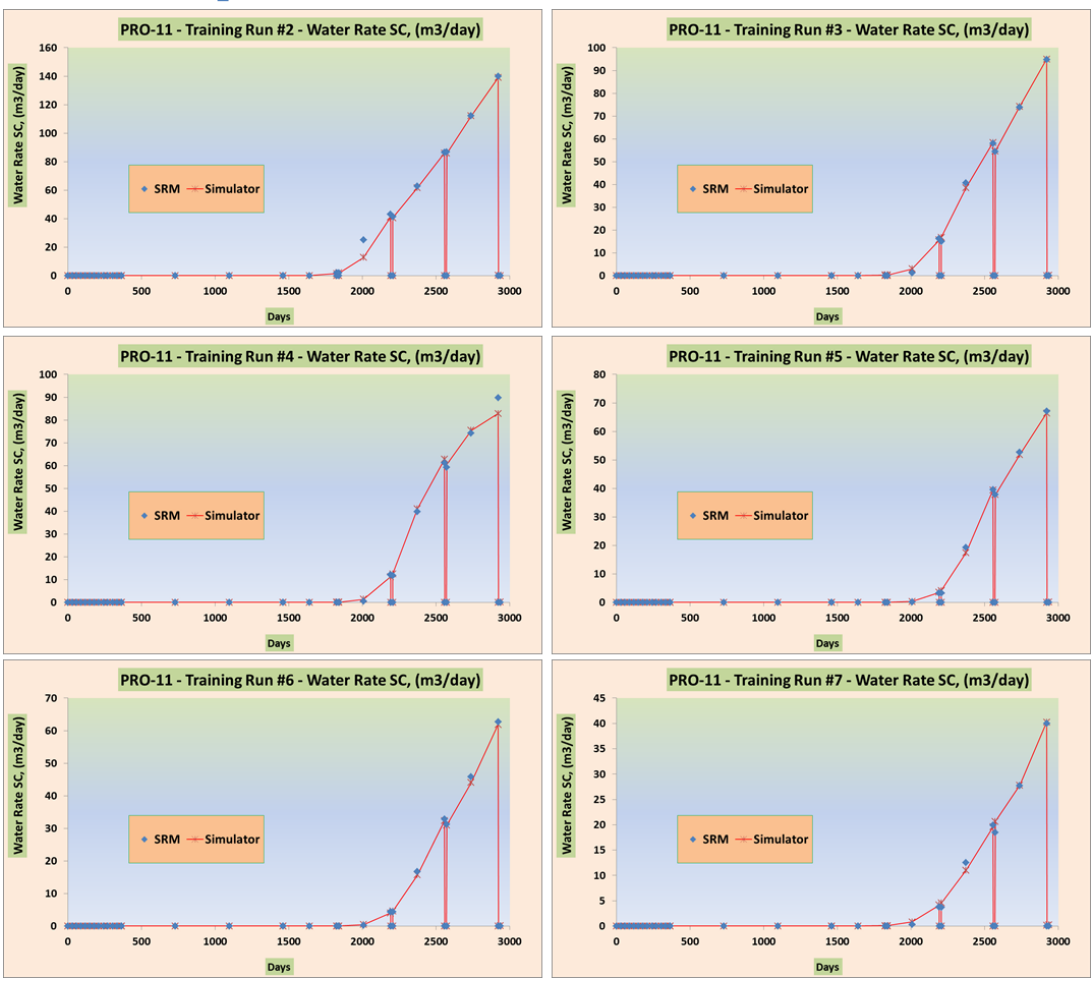

Figure 175: Comparison of the water production rate results for SRM and simulator for training runs \#2 to \#7.
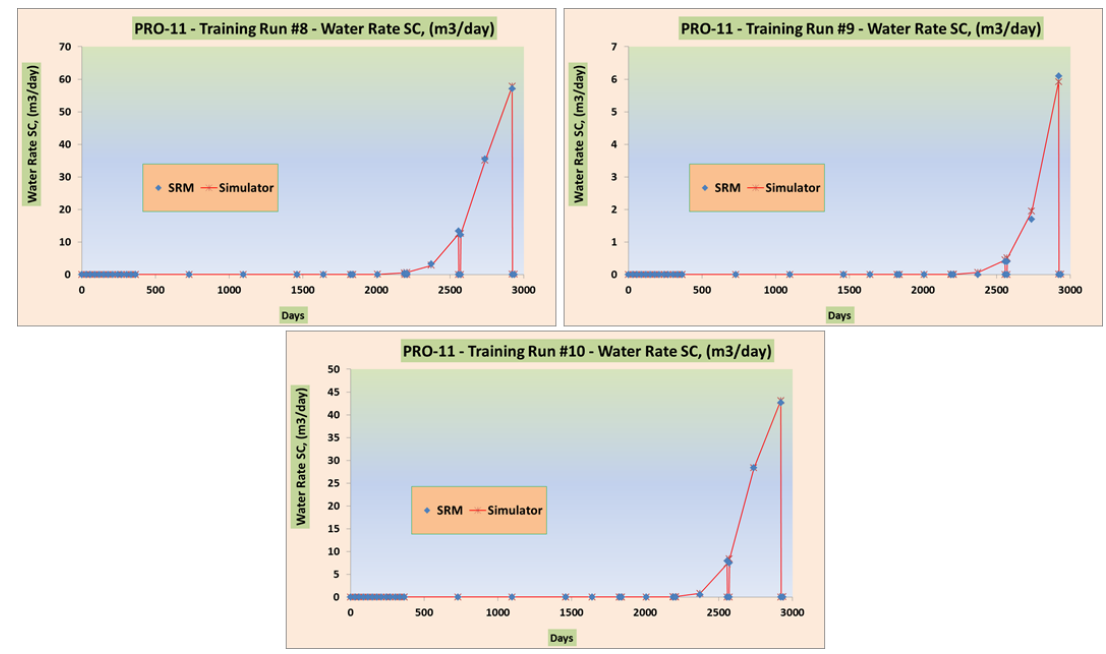

Figure 176: Comparison of the water production rate results for SRM and simulator for training runs \#8 to \#10. 